\title{
BRAIN STIMULATION AND BEHAVIORAL CHANGE
}

EDITED BY: Claudio Lucchiari, Nicholas J. Kelley, Maria E. Vanutelli and Roberta Ferrucci

PUBLISHED IN: Frontiers in Behavioral Neuroscience

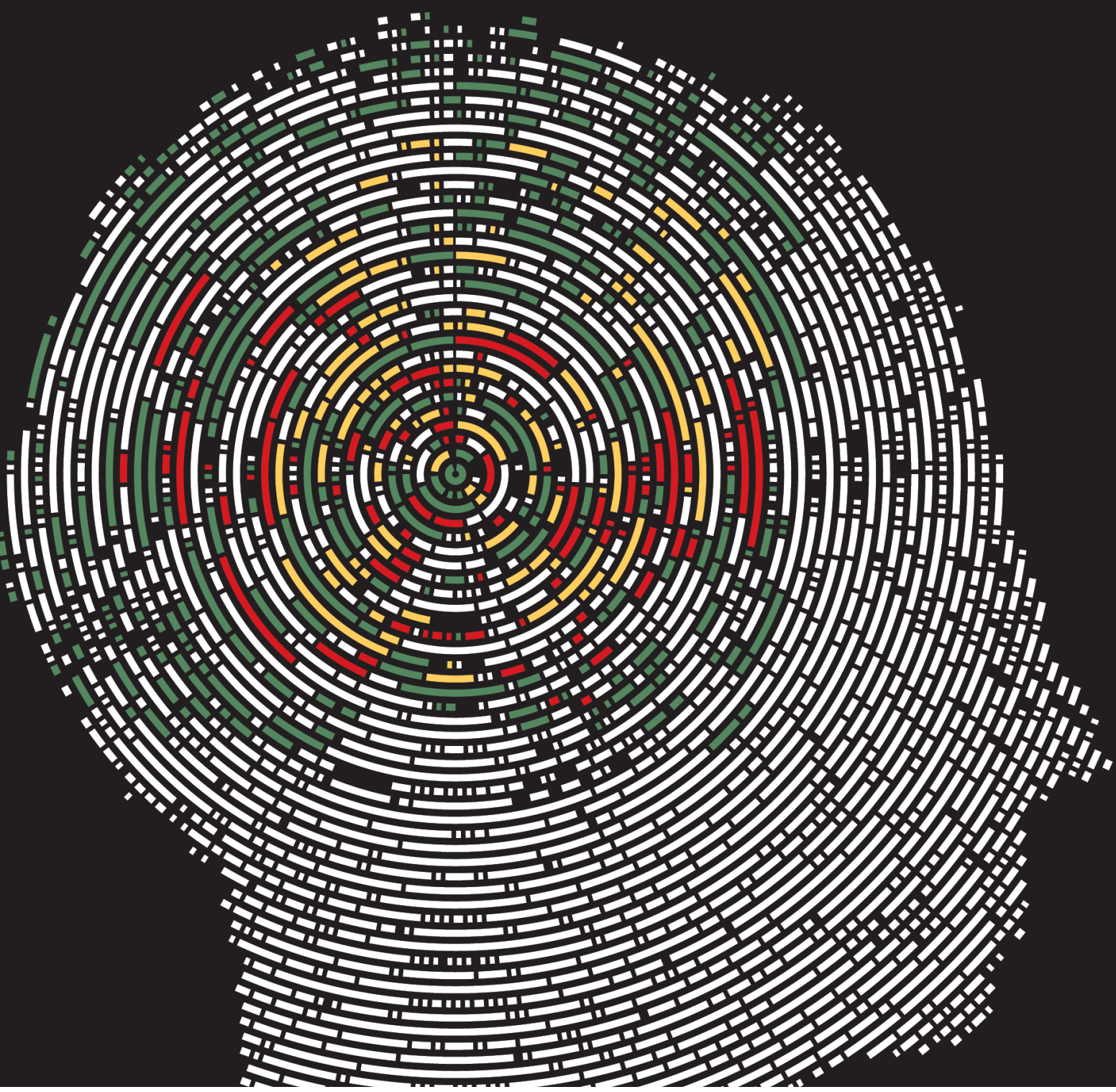




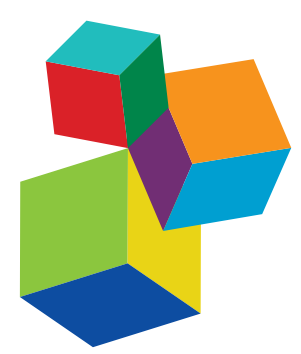

Frontiers Copyright Statement

C Copyright 2007-2019 Frontiers Media SA. All rights reserved.

All content included on this site, such as text, graphics, logos, button

icons, images, video/audio clips, downloads, data compilations and

software, is the property of or is

licensed to Frontiers Media SA

("Frontiers") or its licensees and/or subcontractors. The copyright in the text of individual articles is the property of their respective authors, subject to a license granted to Frontiers.

The compilation of articles constituting

this e-book, wherever published,

as well as the compilation of all other

content on this site, is the exclusive property of Frontiers. For the

conditions for downloading and

copying of e-books from Frontiers

website, please see the Terms for

Website Use. If purchasing Frontiers

e-books from other websites

or sources, the conditions of the website concerned apply.

Images and graphics not forming part

of user-contributed materials may not be downloaded or copied without permission

Individual articles may be downloaded and reproduced in accordance

with the principles of the CC-BY

licence subject to any copyright or other notices. They may not be re-sold as an e-book.

As author or other contributor you grant a CC-BY licence to others to reproduce your articles, including any

graphics and third-party materials supplied by you, in accordance with the Conditions for Website Use and subject to any copyright notices which you include in connection with your articles and materials.

All copyright, and all rights therein, are protected by national and international copyright laws.

The above represents a summary only.

For the full conditions see the Conditions for Authors and the Conditions for Website Use. ISSN 1664-8714 ISBN 978-2-88945-838-7 DOI 10.3389/978-2-88945-838-7

\section{About Frontiers}

Frontiers is more than just an open-access publisher of scholarly articles: it is a pioneering approach to the world of academia, radically improving the way scholarly research is managed. The grand vision of Frontiers is a world where all people have an equal opportunity to seek, share and generate knowledge. Frontiers provides immediate and permanent online open access to all its publications, but this alone is not enough to realize our grand goals.

\section{Frontiers Journal Series}

The Frontiers Journal Series is a multi-tier and interdisciplinary set of open-access, online journals, promising a paradigm shift from the current review, selection and dissemination processes in academic publishing. All Frontiers journals are driven by researchers for researchers; therefore, they constitute a service to the scholarly community. At the same time, the Frontiers Journal Series operates on a revolutionary invention, the tiered publishing system, initially addressing specific communities of scholars, and gradually climbing up to broader public understanding, thus serving the interests of the lay society, too.

\section{Dedication to Quality}

Each Frontiers article is a landmark of the highest quality, thanks to genuinely collaborative interactions between authors and review editors, who include some of the world's best academicians. Research must be certified by peers before entering a stream of knowledge that may eventually reach the public - and shape society; therefore, Frontiers only applies the most rigorous and unbiased reviews.

Frontiers revolutionizes research publishing by freely delivering the most outstanding research, evaluated with no bias from both the academic and social point of view. By applying the most advanced information technologies, Frontiers is catapulting scholarly publishing into a new generation.

\section{What are Frontiers Research Topics?}

Frontiers Research Topics are very popular trademarks of the Frontiers Journals Series: they are collections of at least ten articles, all centered on a particular subject. With their unique mix of varied contributions from Original Research to Review Articles, Frontiers Research Topics unify the most influential researchers, the latest key findings and historical advances in a hot research area! Find out more on how to host your own Frontiers Research Topic or contribute to one as an author by contacting the Frontiers Editorial Office: researchtopics@frontiersin.org 


\section{BRAIN STIMULATION AND BEHAVIORAL CHANGE}

Topic Editors:

Claudio Lucchiari, Università degli Studi di Milano, Italy Nicholas J. Kelley, Northwestern University, United States

Maria E. Vanutelli, Università degli Studi di Milano, Italy

Roberta Ferrucci, IRCCS Ca 'Granda Foundation Maggiore Policlinico Hospital (IRCCS), Italy

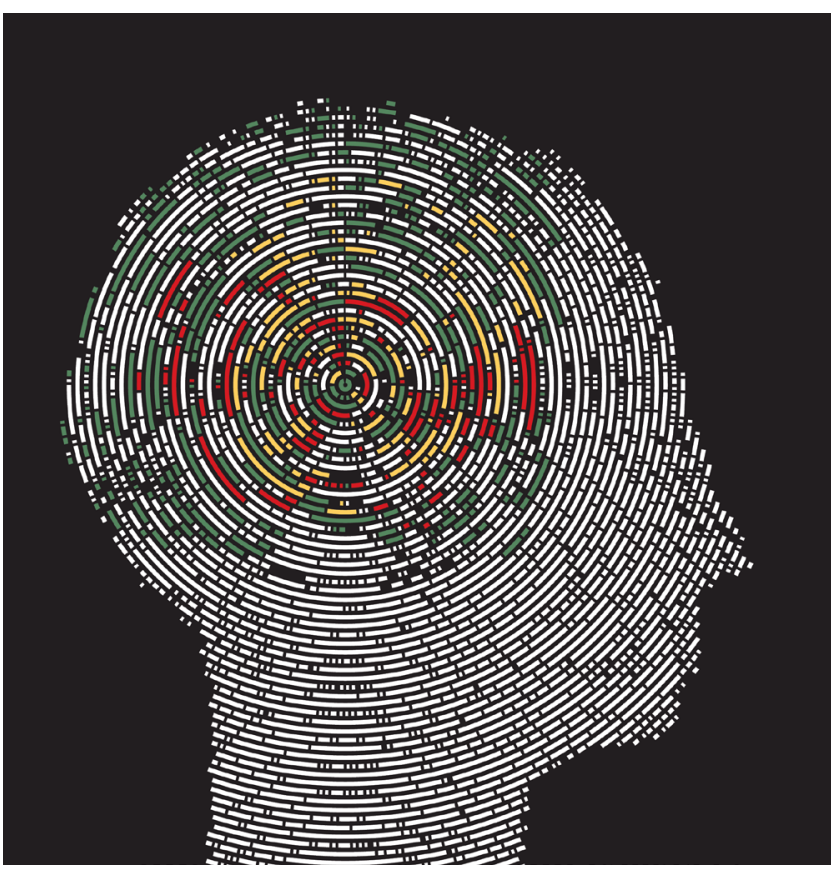

Image: igor kisselev/Shutterstock.com

The eBook entitled "Brain Stimulation and Behavioral Change" is a collection of articles about the use of transcranial electric stimulation (tES) to change behaviors and face pathological conditions, to enhance cognition and to explore cerebral functions using safe and non-invasive brain modulation techniques. The tESs include transcranial direct current stimulation (tDCS), transcranial alternate current stimulation (tACS), and, due to the way it induces changes in the brain, Transcranial Magnetic Stimulation (TMS). The use of tES has recently exploded. Certainly, one reason for this explosion of research is that it is a cheap way to change behavior. However, on the other hand, we still know very little about the neural mechanisms that underlie tES. The present eBook includes both original studies and reviews. It covers a wide scope of arguments, including studies aimed at testing the potential ability of tES in mitigating physical and psychiatric symptoms, and to support 
neurological rehabilitation, enhancing reading abilities, motor abilities, and creativity. Furthermore, some contributions about the role of tES in discovering and mapping the neuro-functional correlates of higher cognitive functions are also included. The common background of the contributions included in the eBook lies in the idea that we need sound scientific evidence about how to move these techniques from labs to real-life contexts. Addressing these issues and understanding the real potentialities of tES in clinical and non-clinical applications require a significant cross-fertilization between disciplines. We hope this eBook will be able to boost the discussion on this vital topic.

Citation: Lucchiari, C., Kelley, N. J., Vanutelli, M. E., Ferrucci, R., eds. (2019). Brain Stimulation and Behavioral Change. Lausanne: Frontiers Media.

doi: 10.3389/978-2-88945-838-7 


\section{Table of Contents}

\section{INTRODUCTION}

06 Editorial: Brain Stimulation and Behavioral Change

Claudio Lucchiari, Nicholas J. Kelley, Maria Elide Vanutelli and Roberta Ferrucci

\section{BRAIN STIMULATION TO IMPROVE PATHOLOGICAL CONDITIONS}

09 At-Home Transcranial Direct Current Stimulation (tDCS) With Telehealth Support for Symptom Control in Chronically-Ill Patients With Multiple Symptoms

Alexa Riggs, Vaishali Patel, Bhaskar Paneri, Russell K. Portenoy, Marom Bikson and Helena Knotkova

\subsection{NEUROLOGICAL APPLICATIONS}

19 Behavioral and Neurophysiological Effects of Transcranial Direct Current Stimulation (tDCS) in Fronto-Temporal Dementia

Roberta Ferrucci, Simona Mrakic-Sposta, Simona Gardini, Fabiana Ruggiero, Maurizio Vergari, Francesca Mameli, Andrea Arighi, Marco Spallazzi, Federica Barocco, Giovanni Michelini, Anna Margherita Pietroboni, Laura Ghezzi, Giorgio Giulio Fumagalli, Giordano D'Urso, Paolo Caffarra, Elio Scarpini, Alberto Priori and Sara Marceglia

30 Unilateral Application of Cathodal tDCS Reduces Transcallosal Inhibition and Improves Visual Acuity in Amblyopic Patients

Tommaso Bocci, Francesco Nasini, Matteo Caleo, Laura Restani, Davide Barloscio, Gianluca Ardolino, Alberto Priori, Lamberto Maffei, Marco Nardi and Ferdinando Sartucci

39 Effects of Multi-Session Repetitive Transcranial Magnetic Stimulation on Motor Control and Spontaneous Brain Activity in Multiple System Atrophy: A Pilot Study

Zhu Liu, Huizi Ma, Victoria Poole, Xuemei Wang, Zhan Wang, Yaqin Yang, Lanxi Meng, Brad Manor, Junhong Zhou and Tao Feng

46 Cathodal tDCS Over Motor Cortex Does not Improve Tourette Syndrome: Lessons Learned From a Case Series

Nora Behler, Bianka Leitner, Eva Mezger, Elif Weidinger, Richard Musil, Bernhard Blum, Beatrice Kirsch, Linda Wulf, Lisa Löhrs, Christine Winter, Frank Padberg and Ulrich Palm

\subsection{PSYCHIATRIC CONDITIONS}

53 Transcranial Direct Current Stimulation, Symptomatology, and Cognition in Psychosis: A Qualitative Review

Tina Gupta, Nicholas J. Kelley, Andrea Pelletier-Baldelli and Vijay A. Mittal

63 New Treatment Perspectives in Adolescents With Anorexia Nervosa: The Efficacy of Non-invasive Brain-Directed Treatment

Floriana Costanzo, Deny Menghini, Antonella Maritato, Maria C. Castiglioni, Alberta Mereu, Cristiana Varuzza, Valeria Zanna and Stefano Vicari 
76 Augmentation of Fear Extinction by Transcranial Direct Current Stimulation (tDCS)

Natalie Dittert, Sandrina Hüttner, Thomas Polak and Martin J. Herrmann

\section{BRAIN STIMULATION AND MOTOR SKILLS}

92 Brain Oscillatory and Hemodynamic Activity in a Bimanual Coordination Task Following Transcranial Alternating Current Stimulation (tACS): $A$ Combined EEG-fNIRS Study

Alisa Berger, Nils H. Pixa, Fabian Steinberg and Michael Doppelmayr

109 Effects of tDCS on Bimanual Motor Skills: A Brief Review

Nils H. Pixa and Bettina Pollok

\section{BRAIN STIMULATION TO ENHANCE COGNITION}

118 The Effects of Transcranial Direct Current Stimulation (tDCS) on Psychomotor and Visual Perception Functions Related to Driving Skills

Alexander Brunnauer, Felix M. Segmiller, Sabine Löschner, Valérie Grun, Frank Padberg and Ulrich Palm

124 tDCS Modulatory Effect on Reading Processes: A Review of Studies on Typical Readers and Individuals With Dyslexia

Alice Cancer and Alessandro Antonietti

136 Effect of Transcranial Direct Current Stimulation of the Medial Prefrontal Cortex on the Gratitude of Individuals With Heterogeneous Ability in an Experimental Labor Market

Pengcheng Wang, Guangrong Wang, Xiaofei Niu, Huiliang Shang and Jianbiao Li

145 Promoting Creativity Through Transcranial Direct Current Stimulation (tDCS). A Critical Review

Claudio Lucchiari, Paola Maria Sala and Maria Elide Vanutelli

\section{BRAIN STIMULATION AS A TECHNIQUE TO STUDY HIGHER} COGNITIVE FUNCTIONS

156 The Agent Brain: A Review of Non-invasive Brain Stimulation Studies on Sensing Agency

Davide Crivelli and Michela Balconi

162 Stimulating Self-Regulation: A Review of Non-invasive Brain Stimulation Studies of Goal-Directed Behavior

Nicholas J. Kelley, Alessia Gallucci, Paolo Riva, Leonor Josefina Romero Lauro and Brandon J. Schmeichel 


\title{
Editorial: Brain Stimulation and Behavioral Change
}

\author{
Claudio Lucchiari ${ }^{*}$, Nicholas J. Kelley ${ }^{2}$, Maria Elide Vanutelli ${ }^{1}$ and Roberta Ferrucci ${ }^{3,4}$ \\ ${ }^{1}$ Department of Philosophy, Università degli Studi di Milano, Milan, Italy, ${ }^{2}$ Department of Psychology, Northwestern University, \\ Evanston, IL, United States, ${ }^{3}$ Fondazione Ca' Granda, IRCCS Ospedale Maggiore Policlinico, Milan, Italy, "A Aldo Ravelli" \\ Research Center for Neurotechnology and Experimental Brain Therapeutics, University of Milan Medical School, Milan, Italy
}

Keywords: brain stimulation, tDCS, TMS, behavioral change, neuro-cognitive correlates

\section{Editorial on the Research Topic}

\section{Brain Stimulation and Behavioral Change}

The use of brain stimulation techniques has recently exploded. Certainly, one reason for this explosion of research is that it is a cheap way to change behavior. However, on the other hand we still know very little about the underlying neural mechanisms. In the research topic "Brain stimulation and behavioral change" we highlight both empirical research and theoretical reviews demonstrating the ability of non-invasive brain stimulation techniques to affect behavior change in a variety of domains including: psychiatric symptoms (e.g., anorexia, psychosis), neurological rehabilitation, motor control, visual perception, self-regulation, and social cognition. This research topic highlights the unique opportunity brain stimulation provides for understanding neuro-functional brain networks, testing theoretical constructs, assessing the presence of cognitive deficits or potentials, mitigating symptoms of disease, and promoting health.

Along this path, heterogeneous by its nature but also rich in suggestions and unexpected connections, Ferrucci et al. described a transcranial direct current stimulation (tDCS) study on patients with fronto-temporal dementia. The authors, by using a stimulation protocol of 5 consecutive days, found an improvement in the visuo-attentive abilities of the patients following an anodal stimulation applied over the fronto-temporal cortex after 1 month from the end of the treatment, as well as a short-term improvement of some neuropsychiatric symptoms. Although the study needs further developments on larger samples, it is worth noting the correlation found between the decrease of the frontal slow waves and the cognitive improvement of the patients. This finding confirms that transcranial stimulation can target not only a specific symptom but also neuro-functional correlations, thus allowing a highly focused evidence-based treatment with a limited impact on the patient's quality of life.

Similarly, the study performed by Bocci et al. in patients with amblyopia (strabismus) shows how the cathodal application of tDCS on the primary visual cortex contralateral to the defective eye reduces transcallosal inhibition as measured through visual evoked potentials (VEPs) and compared to that of healthy subjects, thus allowing an increase in visual acuity. In this case, the study not only allows us to hypothesize future treatments targeted at adult patients, but it also permits to support the hypothesis of the role of the interhemispheric balance in the physiopathology of amblyopia.

In the same direction goes the review proposed by Gupta et al. which is aimed at summarizing the literature about the potential of transcranial stimulation to improve the symptoms and quality of life of psychotic patients. Neuropsychiatric contexts are increasingly benefiting from transcranial stimulation applications in support to the limited effects of pharmacotherapy and its side effects. The review shows how tDCS can be used safely and profitably both to mitigate positive psychiatric symptoms (hallucinations and delusions) and to enhance cognitive functioning. 
The experiment conducted by the group of Costanzo et al. also shows how tDCS can be a useful ally of the psychiatrist. In particular, the study shows the positive effect of a tDCS protocol in patients with anorexia nervosa (AN). The protocol, in fact, has proven to be superior to standard treatments in promoting food intake and stabilizing the weight of adolescents with AN. This finding is probably associated with the ability of tDCS to directly stimulate some nervous networks involved in the regulation of feeding and in reward and gratification which are usually difficult targets to reach with a standard intervention.

Still considering psychiatric applications, Dittert et al. showed how it is possible to apply tDCS to the ventromedial prefrontal cortex (vmPFC) to accelerate the extinction of conditioned learning. In this way, the authors investigated the use of a tDCS protocol with a large sample of healthy subjects in a controlled laboratory setting. However, the study also presents some critical aspects, since it was expected to increase the response to the stimulus but also to increase the reaction to the unconditioned one. This implies the necessity to better understand the mechanisms underlying vmPFC stimulation in conditioned learning before it can be applied in clinical settings.

The study by Behler et al. also shows critical aspects of tDCS application. In fact, the authors present a series of clinical cases with little significant effects in improving the symptoms associated with Tourette's syndrome.

In contrast, the protocol presented by Liu et al. based on transcranial magnetic stimulation (rTMS) of the cerebellum and the primary motor cortex (M1) proved to improve the motor symptoms of patients with Multiple System Atrophy. In particular, the improvement seems to be related to a normalization of the resting-state dynamics in the motor circuit (cerebellum-M1) targeted by rTMS, as demonstrated by fMRI. In this case, therefore, the intervention protocol has a very precise neuro-functional target that allows establishing a priori some experimentally verifiable hypotheses, both at a behavioral and neurophysiological level.

At this regard, it is particularly interesting the study proposed by Berger et al. that investigated the role of transcranial alternating current stimulation (tACS) on the oscillatory activity of subjects engaged in a bimanual coordination task by combining EEG and fNIRS. In this case, the authors demonstrated a specific neurophysiological effect (detectable by both EEG and fNIRS) of the tACS applied over the motor circuits involved in the chosen task within the parietal areas. Moreover, the choice of the experimental task proves to be increasingly relevant in evaluating the effect of specific transcranial stimulation protocols both in experimental and in applicative settings.

The review by Pixa and Pollok goes precisely in this direction. In fact, it describes the role of tDCS in enhancing motor learning, with reference to bimanual coordination tasks. The authors, in fact, report a generally positive impact of tDCS in promoting bimanual skills both in healthy subjects and patients with neurological disorders, showing the importance of using tDCS protocols where the stimulation is consistent with the motor tasks required.

However, transcranial stimulation, and particularly tDCS, can also be used at home to mitigate symptoms and improve the quality of life of patients with various diseases. In this way, remote treatment protocols can be hypothesized through the integration of stimulation procedures and e-health protocols, thus increasing patient compliance and reducing the costs of health institutions. In this sense, the study reported by Riggs et al. demonstrates the feasibility of remote-controlled tDCS protocols, as well as good patient compliance. This kind of applications will probably find wide diffusion in the near future.

Remaining in the field of non-traditional clinical applications of transcranial stimulation, Cancer and Antonietti showed how it is possible to design and implement tDCS protocols to improve some typical defects of learning disorders, such as reading speed. Stimulating the circuits involved in this process (left temporo-parietal cortex, right cerebellum, and left frontal cortices) improves the reading process (even if in a diversified way) both in adults and adolescents with dyslexia, but not in typical readers.

The study by Brunnauer et al. also aims to evaluate tDCS effects in non-traditional settings, such as the improvement of cognitive skills underlying driving skills. However, the study shows a poor impact of the left dorsolateral prefrontal cortex (DLPFC) stimulation on these skills.

The study by Wang et al. is similar to the previous one in terms of setting, but it is aimed at studying the neurophysiological mechanisms underlying gratitude. The authors used a tDCS protocol stimulating the $\mathrm{mPFC}$ during an economic game set in a working context. In the beginning, subjects were tested for their socio-cognitive skills. Then, they were required to pretend to be employees facing different choices. The cathodal stimulation over the mPFC proved to be capable of decreasing the effort of showing gratitude in employees with poor skills, while the anodal stimulation of the same area increased the effort in high-functioning employees. A further demonstration of how transcranial stimulation has differential effects according to the experimental setting but also according to the individual features.

Moreover, three reviews showed that transcranial stimulation can be used to study psychological constructs such as agency, goal-oriented behavior, and creativity. Specifically, Crivelli and Balconi have collected and discussed the literature related to the agent brain studied through transcranial stimulation, showing how this technique can be particularly useful in supporting neuroimaging data, as well as providing new lines of research. Also, the review by Kelley et al. discussed the findings obtained by stimulating the prefrontal cortices that may promote positive self-regulation. The principle is to alter the balance in the activity between the prefrontal cortex and the subcortical regions involved in emotion and reward processing.

Finally, the review by Lucchiari et al. summarized the existing literature on the promotion of creativity through tDCS. The critical discussion led to an explanatory model that correlates the stimulation of certain brain areas, such as the left lower frontal gyrus, and the balancing between the frontal cognitive control system and the default mode network. This balance can be considered the basis of the relationship between divergent and convergent thinking which transcranial stimulation can modify directly, thus promoting evident short-term effects.

In summary, the papers within this Research Topic suggest that the brain stimulation techniques play an important role in 
neuroscience, both as tools to improve our knowledge about the human mind, and to develop protocols to change dysfunctional behaviors, mitigate symptoms and improve cognitive and behavioral perfomance. However, results from original studies as well as from review articles highlight the importance of using specific and testable theoretical models about the neural circuits to be stimulated in order to improve the probability of success and prevent potential side effects before considering real-world applications.

\section{AUTHOR CONTRIBUTIONS}

All authors listed have made a substantial, direct and intellectual contribution to the work, and approved it for publication.

\section{ACKNOWLEDGMENTS}

To the Frontiers team and to all the reviewers who substantially contributed with their competence to the accomplishment of this Research Topic.

Conflict of Interest Statement: The authors declare that the research was conducted in the absence of any commercial or financial relationships that could be construed as a potential conflict of interest.

Copyright (c) 2019 Lucchiari, Kelley, Vanutelli and Ferrucci. This is an open-access article distributed under the terms of the Creative Commons Attribution License (CC $B Y)$. The use, distribution or reproduction in other forums is permitted, provided the original author(s) and the copyright owner(s) are credited and that the original publication in this journal is cited, in accordance with accepted academic practice. No use, distribution or reproduction is permitted which does not comply with these terms. 


\section{OPEN ACCESS}

Edited by:

Claudio Lucchiari,

Università degli Studi di Milano, Italy

Reviewed by: Martin J. Herrmann, Julius-Maximilians-Universität

Würzburg, Germany

Gábor Csifcsák,

University of Tromsø - The Arctic

University of Norway, Norway

${ }^{*}$ Correspondence:

Helena Knotkova

HKnotkov@mjhs.org

Received: 31 January 2018 Accepted: 23 April 2018 Published: 22 May 2018

Citation:

Riggs A, Patel V, Paneri B, Portenoy RK, Bikson $M$ and Knotkova H (2018) At-Home Transcranial Direct Current Stimulation (tDCS) With Telehealth Support for Symptom Control in Chronically-II

Patients With Multiple Symptoms.

Front. Behav. Neurosci. 12:93. doi: 10.3389/fnbeh.2018.00093

\section{At-Home Transcranial Direct Current Stimulation (tDCS) With Telehealth Support for Symptom Control in Chronically-III Patients With Multiple Symptoms}

\author{
Alexa Riggs ${ }^{1}$, Vaishali Patel ${ }^{1}$, Bhaskar Paneri ${ }^{2}$, Russell K. Portenoy ${ }^{1,3,4,5}$, Marom Bikson ${ }^{2}$ \\ and Helena Knotkova ${ }^{1,4 *}$
}

'MJHS Institute for Innovation in Palliative Care, New York, NY, United States, ${ }^{2}$ Department of Biomedical Engineering, Grove School of Engineering, The City College of New York, New York, NY, United States, ${ }^{3}$ MJHS Hospice and Palliative Care, New York, NY, United States, ${ }^{4}$ Department of Family and Social Medicine, Albert Einstein College of Medicine, New York, NY, United States, ${ }^{5}$ The Saul R. Korey Department of Neurology, Albert Einstein College of Medicine, New York, NY, United States

Transcranial direct current stimulation (tDCS) delivered in multiple sessions can reduce symptom burden, but access of chronically ill patients to tDCS studies is constrained by the burden of office-based tDCS administration. Expanded access to this therapy can be accomplished through the development of interventions that allow at-home tDCS applications.

Objective: We describe the development and initial feasibility assessment of a novel intervention for the chronically ill that combines at-home tDCS with telehealth support.

Methods: In the developmental phase, the tDCS procedure was adjusted for easy application by patients or their informal caregivers at home, and a tDCS protocol with specific elements for enhanced safety and remote adherence monitoring was created. Lay language instructional materials were written and revised based on expert feedback. The materials were loaded onto a tablet allowing for secure video-conferencing. The telehealth tablet was paired with an at-home tDCS device that allowed for remote dose control via electronic codes dispensed to patients prior to each session. tDCS was delivered in two phases: once daily on 10 consecutive days, followed by an as needed regimen for 20 days. Initial feasibility of this tDCS-telehealth system was evaluated in four patients with advanced chronic illness and multiple symptoms. Change in symptom burden and patient satisfaction were assessed with the Condensed Memorial Symptom Assessment Scale (CMSAS) and a tDCS user survey.

Results: The telehealth-tDCS protocol includes one home visit and has seven patienttailored elements and six elements enhancing safety monitoring. Replicable electrode placement at home without 10-20 EEG measurement is achieved via a headband that holds electrodes in a pre-determined position. There were no difficulties with patients' training, protocol adherence, or tolerability. A total of 60 tDCS sessions were applied. No session required discontinuation, and there were no adverse events. Data collection was 
feasible and there were no missing data. Satisfaction with the tDCS-telehealth procedure was high and the patients were comfortable using the system.

Conclusion: At-home tDCS with telehealth support appears to be a feasible approach for the management of symptom burden in patients with chronic illness. Further studies to evaluate and optimize the protocol effectiveness for symptom-control outcomes are warranted.

Keywords: non-invasive brain stimulation, transcranial direct current stimulation, patient-tailored protocol, chronic illness, symptom management, home settings, at-home tDCS

\section{INTRODUCTION}

In the United States, at least 175 million people are chronically ill (Centers for Medicare and Medicaid Services, 2012; Ortman et al., 2014). Most are elderly and receive care in the ambulatory setting or at home (Hasselman and Center for Health Care Strategies, 2013). Effective symptom management is essential but is often challenged by the occurrence of multiple symptoms and the need for polypharmacy, which may produce adverse effects in the medically fragile. For many patients, the need for frequent visits to a physician's office presents additional challenges. Innovative strategies for symptom management that could be provided at home and reduce the reliance on drug therapy would represent a significant advance in addressing the burden of illness among seriously ill patients.

Transcranial direct current stimulation (tDCS) is a noninvasive and non-pharmacological intervention that may address multiple symptoms of chronic illness (Nitsche and Paulus, 2000; Fregni et al., 2006a,b; Rigonatti et al., 2008; Valle et al., 2009; Loo et al., 2012; Knotkova et al., 2014a,b). tDCS is delivered via a battery-powered device with two or more electrodes that transfer electrical current of low intensity (usually 1-2 mA) to the surface of the head. The primary mechanism of tDCS is a subthreshold modulation of neuronal resting membrane potential (Nitsche and Paulus, 2000). If delivered for several minutes, tDCS can induce neuroplasticity of glutamatergic synapses, resulting in enduring alterations of neural excitability (Nitsche et al., 2003; Antal et al., 2010; Monte-Silva et al., 2013). Recent evidence suggests that tDCS interacts with various neurotransmitters in the brain, such as dopamine, acetylcholine, serotonin and gamma-aminobutyric acid (GABA; Nitsche et al., 2004a,b,c, 2006, 2009; Polania et al., 2011; Stefani et al., 2012; Stagg et al., 2013). The neurophysiologic effects of tDCS can be detected in cortical and subcortical areas distant from the site of stimulation, and recent findings suggest that tDCS can upregulate and downregulate functional connectivity within complex brain networks, such as those that are important for cognitive, motor, and pain processing or mood modulation (Stagg et al., 2013). The scientific rationale for tDCS studies in symptom management builds on findings from neurophysiological and neuroimaging studies indicating that the development and maintenance of symptoms frequently occurring in various chronic illnesses are associated with changes in cerebral networks and altered functional connectivity (e.g., Apkarian et al., 2013; Hemington et al., 2016). Earlier studies suggest that reversal of these potentially maladaptive changes in brain function is possible and can be associated with an improvement of symptoms (Maihöfner et al., 2003, 2004; Napadow et al., 2012; and others). Neuromodulation techniques, such as tDCS, can induce enduring alterations of neural activity and connectivity, and can be used to attempt reversal the maladaptive neuroplastic changes occurring in chronic illness (Lefaucheur, 2016; Stock et al., 2016; Woods et al., 2016).

Numerous randomized controlled trials suggest that tDCS can relieve symptoms common in chronic illness, such as pain, fatigue, sleep difficulties, or mood disturbance (Fregni et al., 2006a,b; Roizenblatt et al., 2007; Boggio et al., 2008; Valle et al., 2009; Loo et al., 2012; Brunoni et al., 2014; Ferrucci et al., 2014; Saiote et al., 2014; Fagerlund et al., 2015; and others). Although the extant evidence would support a trial of tDCS in chronically ill patients with multiple poorly controlled symptoms, the access to tDCS for those patients has been constrained. There are many potential reasons for this observation, among which may be the burden associated with the conventional tDCS approach, which requires consecutive daily visits to an office for 1 or more weeks. The development of a home-based tDCS intervention suitable for the chronically ill with complex symptoms would have the potential to expand access to this promising nonpharmacological method.

We have developed a novel intervention that combines athome tDCS and telehealth support; we have adapted the tDCS procedure and technology for easy home use by the chronically ill, and developed instructional materials, a comprehensive training plan, and a patient-tailored tDCS application protocol that allows for dose-control, and enhanced adherence and safety monitoring in remote. Here we describe the development of this intervention and feasibility testing in four patients with advanced chronic illness and multiple unrelieved symptoms.

\section{MATERIALS AND METHODS}

\section{Development}

The guiding principles for the development of the intervention were low burden for the patient, strict dose control, and enhanced monitoring for adherence and safety. To identify and adjust potentially difficult steps in the tDCS application for patients and their informal caregivers, analysis of the tDCS procedure was performed. Possible solutions were presented to a group of hospice and palliative care professionals in expert-feedback sessions that included in total six experts $(1 \mathrm{M}, 5 \mathrm{~F})$ whose experience in hospice and palliative care averaged 24 years and whose current positions were either managerial or clinical. After 
each expert provided consent, he or she was interviewed and asked to provide specific feedback on suitability of the adjusted tDCS procedure for patients and their informal caregivers and on versions of lay-language training materials - an instructional video and a step-by-step brochure.

The device for home-based tDCS was the "1X1 tDCS miniCT” device, model 1601 (Soterix Medical Inc., NY, United States), with two $5 \mathrm{~cm} \times 5 \mathrm{~cm}$ saline-soaked sponge electrodes. The device included a safeguard for dose control, consisting of electronic unlocking codes, that were provided to the patient or caregiver prior to each $\mathrm{tDCS}$ treatment. The device also generated an electronic post-treatment record that informed the tDCS technician about completion, interruption, or restart of the tDCS session.

The telehealth device paired with the tDCS unit was a Samsung Galaxy touchscreen tablet $6.93^{\prime \prime} \times 9.57^{\prime \prime}$. The tablet contained educational and instructional materials for the patient and informal caregiver and allowed secure videoconferencing with the patient for remote assistance and adherence monitoring by the tDCS technician.

\section{Pilot to Assess Feasibility}

To perform an initial evaluation of the feasibility of the at-home tDCS-telehealth intervention, four patients were recruited following referrals from MJHS Hospice and Palliative Care personnel. Patients were required to be at least 18 years of age, English-speaking, able to provide informed consent, have at least one chronic illness, and to report moderate to severe distress from one or more of the following symptoms: pain, fatigue, difficulties concentrating, worrying, feeling sad, or feeling nervous, as selfrated by the patient on the Condensed Memorial Symptom Assessment Scale (CMSAS; below).

Patients were excluded if they had metal implants in the head or in the neck; cancer affecting the head; history of seizures or seizure disorder; unstable acute medical condition; compromised integrity or sensitivity of the skin at or near locations where electrodes would be placed; or if they used another neurostimulation device.

Patients had an option to include their informal caregiver to assist with the tDCS procedures. To co-participate, the caregivers were required to be at least 18 years of age, able to follow instructions in English, and to provide informed consent.

The at-home tDCS was applied in accordance with a patient-tailored protocol (described in detail in section Results) in two phases: 10 daily tDCS sessions on 10 consecutive days were followed by as needed applications over 20 days, using either the "DLPFC" montage or "M1-SO" electrode montage. The montage selection was congruently informed by findings from previous studies and patient's report of most distressing symptom(s) at screening. Patients were considered for the study participation if they reported moderate to severe distress from one or more of the following symptoms: worrying, feeling sad, feeling nervous, difficulties concentrating, fatigue, or pain. Based on findings from previous studies, each of these symptoms can be addressed using the "DLPFC" montage with the anode on the left hemisphere over the area corresponding with the F3 point of the international 10-20 EEG system and the cathode over F4
(Valle et al., 2009; Brunoni et al., 2013; Saiote et al., 2014; Sandrini et al., 2014; Glaser et al., 2016; Manenti et al., 2017; and others). However, specifically for pain, the more common tDCS montage is the "M1-SO" montage, with the anode over the area of the motor cortex (C3 or C4 of the 10-20 EEG system) contralateral to pain-affected side of the body in unilateral pain, or over $\mathrm{C} 3$ in bilateral pain, with the cathode over the supraorbital region on the hemisphere contralateral to the anode placement (Fregni et al., 2006a,b; Valle et al., 2009; Mori et al., 2010; Kim et al., 2013; and others). In our study, the "M1-SO" montage was used if pain was reported as dominating most distressing symptom.

\section{Data Collection}

Patients' sociodemographic characteristics - age, race, marital status, living status (lives alone, does not live alone), education (high school $\sim 12$ years of education; some college $\sim 13-15$ years; undergraduate $\sim 16$ years; graduate school $\sim 18+$ years of education), were recorded. Performance status was assessed with the Karnofsky Performance Status scale (KPS) between 0 and 100 , with anchor points 0 (death) and 100 (no complaints/no evidence of disease) (Schag et al., 1984). Symptom distress was assessed with the CMSAS, a validated 14-item questionnaire used to evaluate the prevalence and distress associated with 11 physical and 3 psychological symptoms (Chang et al., 2004). Prevalence was indicated on a single item, which was checked if the symptom was experienced during the indicated timeframe. Distress due to each of the physical symptoms was measured on a Likerttype scale, which asked "How much did the symptom bother or distress you in the past 7 days?" and was graded using a 5-point scale: 0 (no distress at all), 1 (a little bit), 2 (somewhat), 3 (quite a bit), and 4 (very much). Distress due to each of the psychological symptoms was measured on a 4-point scale that asked "How frequently did the following symptom occur?" and was graded 1 (rarely), 2 (occasionally), 3 (frequently), 4 (almost constantly). The scores for the Global Distress and its two subscales Physical Symptom Distress and Psychological Symptom Distress were calculated as the sum of the scores reported on corresponding groups of physical and psychological symptoms. The assessment was carried out at the baseline, at the end of the first ten sessions, and again at the end of the twenty-day optional phase involving up to ten additional applications. Patient satisfaction with the tDCS system was determined from an 8-item user survey administered after the last tDCS application.

The study was carried out in accordance with the recommendations of the Handbook for Good Clinical Research Practice, WHO, 2002. The procedures were approved by the New England Independent Review Board (NEIRB), and all participants provided written informed consent in accordance with the Declaration of Helsinki.

\section{RESULTS}

\section{Development of the Intervention Adjustment of the tDCS Application Process}

A step-by-step analysis of tDCS application process informed by expert feedback identified major challenges associated with 
TABLE 1 | Analysis of the tDCS application process, with focus on potentially difficult steps in tDCS application for seriously ill patients and their informal caregivers; and possible solutions.

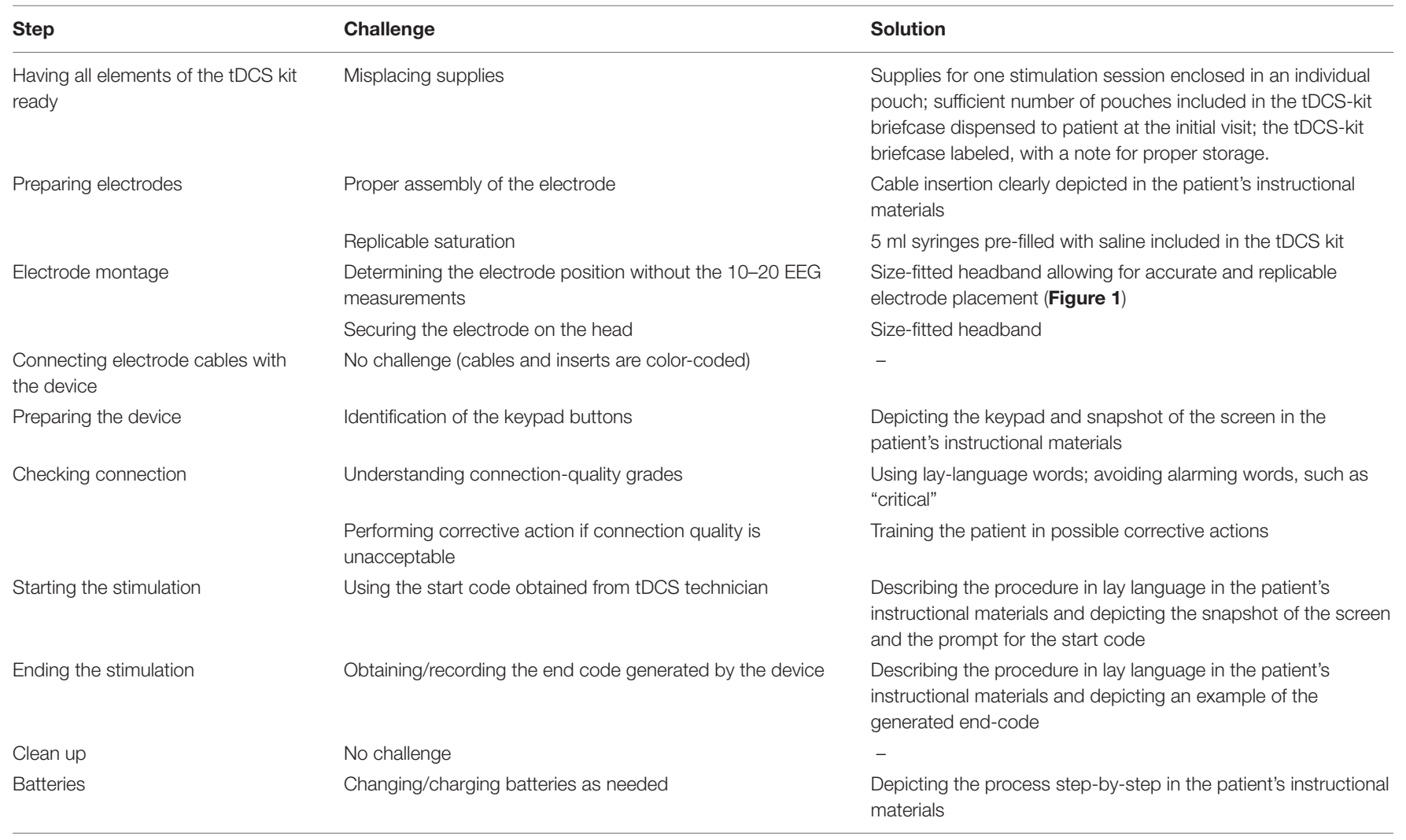

preparation of the electrodes and with the electrode montage (Table 1).

To assure replicable saturation of the electrode sponges, saline was pre-filed into $5 \mathrm{ml}$ syringes that were included in the tDCS kit dispensed to the patients. To achieve electrode montage without the 10-20 EEG measurements, electrodes were placed in a headband that held the electrodes in the desired position for the stimulation over the area of the primary motor cortex ("M1-SO" montage, Figure 1; Knotkova et al., 2017), or over the dorsolateral prefrontal cortex ("DLPFC"; Seibt et al., 2015), based on reported symptoms. The headband had a navigational mark that served as reference point for proper positioning and was size-fitted to the patient at the time of equipment dispense.

\section{Patient-Tailored Protocol}

The protocol included one home visit for consenting, eligibility screening, initial tDCS tolerability test, and in-person initiation of tDCS training. The training process (Figure 2) continued using instructional materials preloaded on the telehealth tablet (Figure 3) with remote assistance via videoconference as needed. The training was concluded with the tDCS Competency test. tDCS was applied in two phases; first, patients are encouraged to apply 10 sessions, one 20 -minute stimulation per day on 10 consecutive days. This build-up phase was followed by a fully optional 20-day period, during which the patient could apply tDCS as needed for up to ten additional sessions delivered once or twice per day. The number of applications in the "as-needed" regimen was guided by the patient judgment and the frequency was determined by the patient without input from the study personnel.

The protocol had seven specific elements that allowed for tailoring $(\mathrm{T})$ to the needs and preferences of the patient (Figure 4, T1-T7), and six elements that supported monitoring for safety and compliance with the protocol (C; Figure 4, C1-C6).

The elements for tailoring treatment included: (1) optional inclusion of assisting informal caregiver; (2) broad inclusion criteria that allow targeting of one or more of multiple symptoms; (3) tailored stimulation intensity (default of $1.5 \mathrm{~mA}$, but able to be decreased to $1 \mathrm{~mA}$ for patients who perceive $1.5 \mathrm{~mA}$ as unpleasant); (4) tailored tDCS training involving either the patient or a co-participating informal caregiver, or both, and mediated through instructional video, videoconference with tDCS technician and/or a step-by-step brochure; (5) versatility of remote assistance via a videoconference or by phone, with frequency and mode of assistance determined by the patient's needs; (6) a recommended regimen during the first block of stimulation (tDCS applications on consecutive days strongly encouraged but not mandated); and (7) optional applications in the second phase, potentially allowing twice daily stimulation.

The elements that supported safety and monitoring for the adherence with the protocol (Figure 4, C1-C6) included the following: (1) exclusion criteria that precluded tDCS initiation in patients with metal implants in the head/face, history of seizures or brain tumor, compromised skin integrity at the 

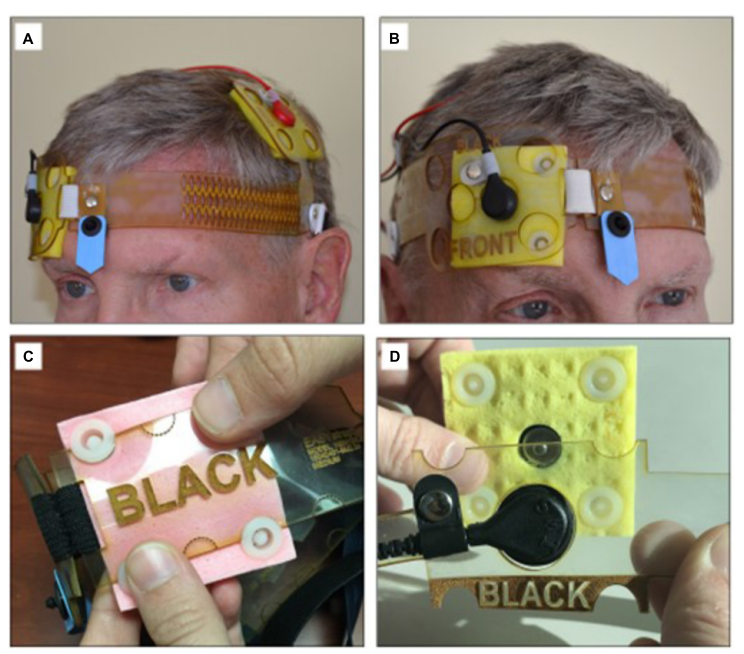

FIGURE 1 | tDCS headgear for the electrode M1-SO montage with the anode over the area of the motor cortex and the cathode over the contralateral supraorbital region. The headgear $(\mathbf{A}, \mathbf{B})$ does not require neuronavigational measurements. The center point (blue arrow) supports accurate self-placement by user. The headgear accommodates either conventional saline-soaked electrodes (C) or snap-on pre-moisturized ones (D). Panel AB is courtesy of HK. The person shown has given permission to publish this picture.

electrode-placement area, or an inability to provide consent or follow instructions; (2) initial tDCS tolerability test, which excluded participants who could not tolerate $1 \mathrm{~mA}$ of stimulation; (3) use of a competency test at the conclusion of training to assure preparedness of the tDCS operator (patient or assisting informal caregiver), and exclusion of patient if competency in the application process could not be demonstrated within 1 week after initiation of training; (4) monitoring for compliance with good practices in tDCS (including remote quality checks to evaluate if procedures for the electrode preparation and tDCS delivery were done properly and in accordance with training materials); (5) comprehensive safety monitoring that ensured that safety checks prior to and during stimulation were completed (e.g., intact skin in the electrode area and absence of adverse effects), and that stimulation be stopped if perceived as painful; and (6) electronic dose control: the device was electronically unlocked for each session at a pre-set dose and provided an end-of-session code indicating whether stimulation was completed, delivered without or with interruptions, or was aborted.

\section{Pilot to Assess Feasibility}

Four patients were recruited for the initial feasibility assessment. The patients' characteristics are summarized in Table 2.

Patient 1 was a 52-year-old Hispanic male, single, but not living alone, and not employed. He had advanced congenital myasthenia gravis associated with quadriplegia and chronic ventilator support. The patient experienced severe distress from chronic pain of the neck, lower back, and right lower limb for longer than 1 year. Other reported symptoms

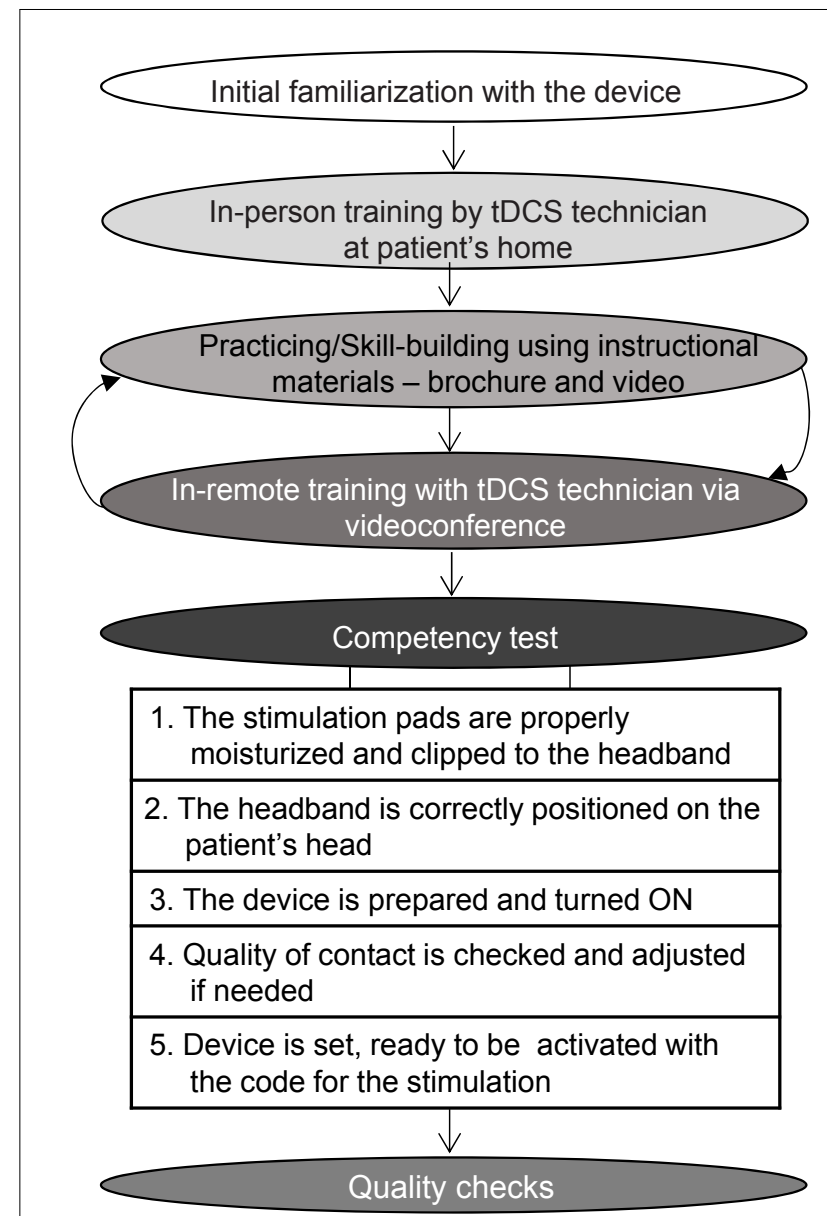

FIGURE 2 | A step-by-step training plan to build patients' and caregivers' competency for tDCS applications.

included fatigue, insomnia, difficulty concentrating, nervousness, worrying, feeling sad, lack of appetite, dry mouth, weight loss, and constipation. Symptoms were poorly controlled despite treatment with fentanyl and gabapentin. The patient was severely disabled, with the KPS score of 30 .

Patient 2 was a 44-year-old Hispanic female, single, but not living alone, and not employed. She had a longstanding indolent spinal tumor associated with back pain and depressed mood (worrying, feeling sad), and reported severe distress from each of these symptoms for longer than one year. Other reported symptoms included lack of energy, lack of appetite, dry mouth, feeling drowsy, constipation, nausea, difficulties sleeping, and difficulties concentrating. Symptom-directed medications included baclofen, hydromorphone, oxycodone-acetaminophen, escitalopram, docusate, diphenhydramine, and pantoprazole sodium. The patient had the KPS score of 80 , able to carry on normal activity with effort.

Patient 3 was a 58-year-old white female, married, and not employed. She had post-traumatic plexopathy and chronic pain in the left lower limb that caused moderate symptom distress for more than 1 year. Other reported symptoms were difficulty 


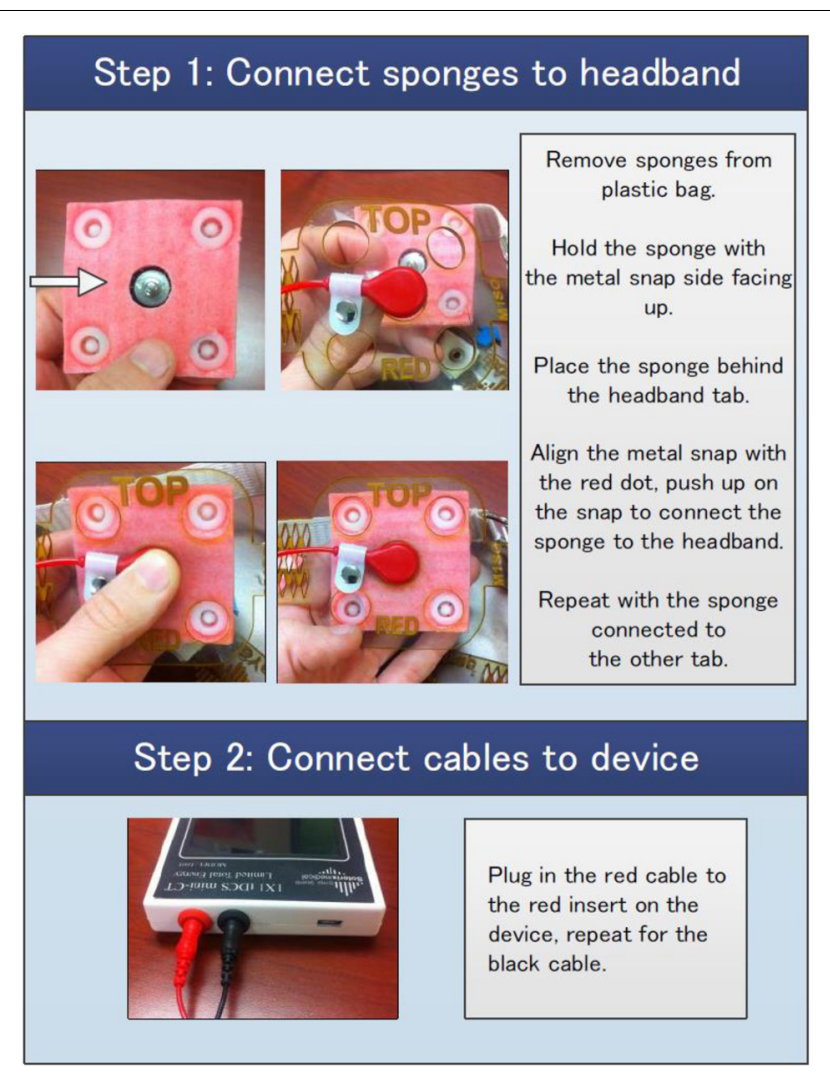

FIGURE 3 | A snapshot of the tDCS instructional brochure for patients.

concentrating and feeling nervous. Pain was poorly controlled despite hydromorphone. Her KPS was 90, able to carry on normal activity.

Patient 4 was a 63-year-old Hispanic male, married, and not employed. The patient had a history of stroke and associated left leg and arm paralysis and chronic pain. The pain was adequately controlled with gabapentin, but the patient experienced burden due to psychological symptoms - worrying, feeling sad, and feeling nervous for longer than three months. His KPS was 70, able to care for himself, but not able to carry on normal activity or do active work.

Patients \#1 and \#4 opted for an inclusion of informal caregiver to assist with $\mathrm{tDCS}$ administration.

At the initial visit at the patient's home, patients and coparticipating informal caregivers provided informed consent and were screened. Initial familiarization with the device proceeded without difficulties and patients received size-fitted headbands for electrode placement for the following montages: the "M1SO" with anode on the left (Patient \#1) or right (Patient \#3); and the "DLPFC" montage (Patients \#2 and 4). All patients underwent the initial acceptability test and found the intensity of stimulation at $1.5 \mathrm{~mA}$ acceptable. Training proceeded without difficulty and all designated tDCS operators (Patients \#2 and 3 themselves and assisting caregivers for Patients \#1 and \#4), passed the competency test and were allowed to proceed with tDCS applications.

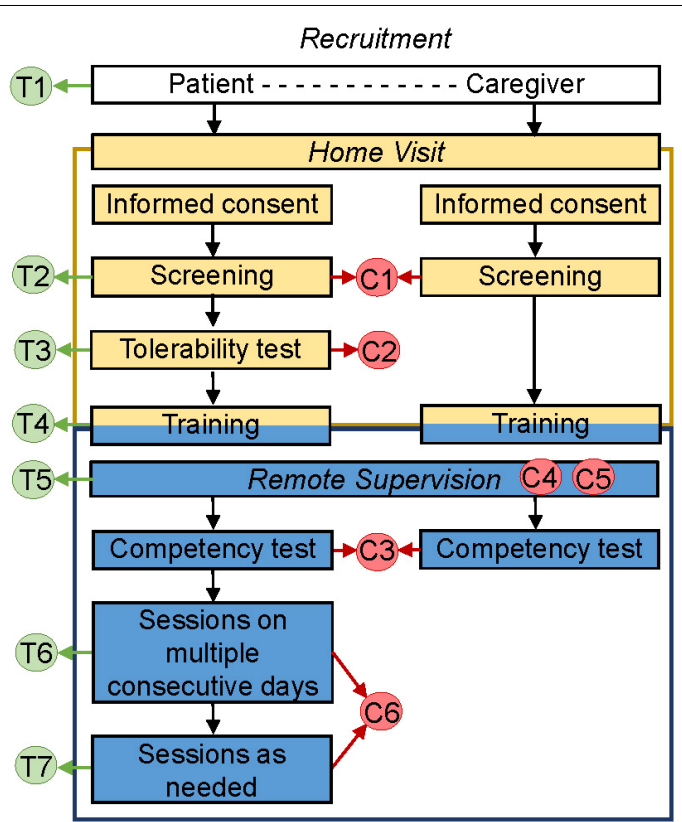

FIGURE 4 | Protocol for tDCS application by chronically ill patients at home. The protocol includes 1 at-home visit and has specific elements that allow for tailoring to the needs and preferences of the patient (T1-T7), as well as elements that support safety and adherence monitoring (C1-C6).

TABLE 2 | Patients' profiling characteristics.

\section{Profiling}

characteristics

\begin{tabular}{lllll}
\cline { 2 - 4 } & $\mathbf{1}$ & $\mathbf{2}$ & $\mathbf{3}$ & $\mathbf{4}$ \\
\hline Age & 52 & 44 & 58 & 63 \\
Race/ethnicity & Hispanic & Hispanic & White & Hispanic \\
Sex & Male & Female & Female & Male \\
Marital status & Single & Single & Married & Married \\
Living status & Doesn't live & Doesn't live & Doesn't live & Doesn't live \\
& alone & alone & alone & alone \\
Employment status & Not & Not & Not & Not \\
& employed & employed & employed & employed \\
Education in years & $13-15$ & $13-15$ & $18+$ & 12 \\
Karnofsky & 30 & 80 & 90 & 70
\end{tabular}

performance status

In the first tDCS phase, all patients received 10 applications on 10 consecutive days. In the second "as needed" phase, Patients $\# 1$ and 3 did not opt for additional applications during the subsequent period and Patients \#2 and 4 received another 10 sessions, no more than one session per day. No adverse events were reported.

Outcome data collection using the CMSAS was carried out without problems. There were no missing data and the results illustrate feasibility of tracking symptom scores. At the baseline, Patients \#1 and 2 had high Global Distress Score of 32.4 and 39.2, respectively; the score substantially declined to 9.6 and 14.0 , respectively after 10 consecutive tDCS applications, and 


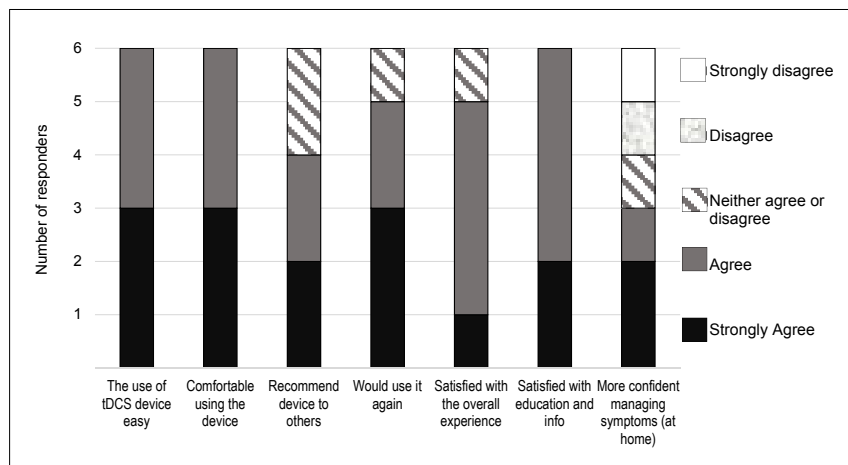

FIGURE 5 | Results of the tDCS satisfaction survey by the four patients and two co-participating caregivers.

remained low, 4.0 and 11.0, respectively, at the end of the as needed phase in which Patient \# 1 did not opt for any tDCS session and Patient \#2 received 10 additional applications. Patients \#3 and 4 had the baseline Global Distress Score low, 8.6 and 8.0, respectively, and no substantial change was noted in the course of tDCS treatment; the score was 7.6 and 8.6, respectively, after 10 tDCS applications, and 11.6 and 10.0, respectively, after the optional phase in which Patient \#3 has not received any tDCS application and Patient \#4 opted for 10 sessions. These results do not reflect efficacy given the small sample, lack of a comparator, and varied number of tDCS applications; they do, however, demonstrate the ability of symptom measurement to capture different response profiles during home-based tDCS trials.

On the satisfaction survey (Figure 5), all four patients and the two co-participating caregivers indicated "agreed/strongly agreed" in response to statements "I find the use of tDCS device easy," "I was comfortable using the tDCS device," and "I was satisfied with the education and information I received before using the device." Five users agreed/strongly agreed with statements "If I were offered this device and equipment in the future I would use it again," and "I was satisfied with the overall experience of using the tDCS device," four users would recommend the device to others, and three users agreed/strongly agreed with statement "With the help of the tDCS device I am more confident managing the symptoms at home."

\section{DISCUSSION}

We have developed a novel at-home tDCS intervention with telehealth support and have demonstrated initial feasibility in four patients with complex symptom control problems associated with chronic illness. There were no difficulties with the initial study visit at the patient's home, training or patients' acceptance of the procedure. The adherence to the protocol was excellent; no session required discontinuation, all patients received 10 tDCS sessions as recommended in the initial phase and proceeded to the as-needed phase. Data collection was without problems and there were no missing data. Although a decrease of posttDCS CMSAS scores was noted in two patients, no conclusions should be drawn from these findings as there was no control comparison, the number of subjects was low, and the study was not designed to evaluate efficacy of the procedure. Regardless, the patients' and caregivers' satisfaction with the procedure was high; they were satisfied with the training and were comfortable using the device, indicating very good acceptability of the intervention. Overall, our findings suggest that with proper training and enhanced remote monitoring, tDCS application in home-bound patients with multiple chronic symptoms is possible.

The approach allows for tailoring the procedures to the needs and preferences of the patient, as well as includes elements for enhanced safety and adherence monitoring, low burden, and ease of use. The instructional materials, training procedures, and quality check enable patients and their informal caregivers to develop skills necessary for tDCS application in accordance with good practices. The frequent contact with a tDCS technician via video-conference and telephone enables remote assistance as needed and enhances safety and adherence monitoring. Safety and adherence with the protocol are further supported by technical features of the equipment, which includes remote dose control, and simple easy-to-use headgear allows for reliable electrode montage without performing the 10-20 EEG measurements.

Importantly, this novel intervention comports with guidelines for tDCS use in clinical patient-populations in home settings (Charvet et al., 2015). The recommendations include: (1) training of staff in tDCS treatment and supervision; (2) assessment of the user's capability to participate in tDCS remotely; (3) training procedures and materials including assessments of the user and/or caregiver; (4) simple and fail-safe electrode preparation techniques and tDCS headgear; (5) strict dose control for each session; (6) ongoing monitoring of compliance/adherence to the protocol; (7) monitoring for treatment-emergent adverse effects; and (8) guidelines for discontinuation of a session and/or study participation (Charvet et al., 2015).

This novel non-pharmacological adjuvant tDCS-telehealth intervention is well positioned to address the needs of seriously ill patients in home settings and promote the national goal of more effective community-based care. It can also support the efforts to reduce the need for drug therapy, particularly in chronically ill older patients. Recent surveys indicate that almost one-third of Medicare beneficiaries take $\geq 5$ prescribed medications, and the polypharmacy is associated with higher risks for the patients when possible adverse effects of drug therapy may compound the risks associated with disease-related organ dysfunction (Centers for Medicare and Medicaid Services, 2012; Hasselman and Center for Health Care Strategies, 2013; Ortman et al., 2014). Although existing evidence pertaining to the tDCS potential to reduce medication intake stems from studies in postoperative settings (Borckardt et al., 2011, 2013; Glaser et al., 2016), this tDCS-telehealth intervention can facilitate future explorations of this potential for the chronically ill in home settings.

Each component of this novel intervention, tDCS and the telehealth support, has potential to substantially facilitate symptom control. Randomized controlled studies (Boggio et al., 2008; Hagenacker et al., 2014; Fagerlund et al., 2015; Lefaucheur, 2016; and others) have shown that tDCS delivered in 
several sessions can reduce symptoms common in chronic illness, such as pain, fatigue, sleeping difficulties, cognitive difficulties, worrying, or depression, and can result in decreased demand for symptom-directed medications (Borckardt et al., 2011, 2013; Glaser et al., 2016), while telehealth support may help the chronically ill get engaged in symptom monitoring, facilitate access to information about symptom management, or facilitate contact with the support team (Kearney et al., 2009; Kroenke et al., 2010; Head et al., 2011; Johnston et al., 2012; Ruland et al., 2013; and others). In the past decade, various telephone-based or computer-based systems have been tested to enhance reporting and monitoring symptoms in patients with serious illnesses, such as cancer, in both urban and rural areas, and preliminary results suggest high acceptance of this approach by patients and families (Davis et al., 2007; Weaver et al., 2007; Grubaugh et al., 2008; Kroenke et al., 2010; Head et al., 2011). Although it is impossible to circumvent the physical and psychological impact of chronic illness on patients and their families, telehealth technology provides innovative remote ways toward improving patient education, symptom assessment, communication and outreach by the support team.

Further, the protocol for application of this at-home tDCS-telehealth intervention can serve as a template or a generic protocol for future tDCS studies or tDCS clinical applications in the chronically ill. Various sets of outcome assessment tools can be easily incorporated in the protocol and the number of tDCS sessions in the two phases of tDCS applications can be adjusted as well. The tDCS-telehealth intervention offers many opportunities for further modification of the technology and the protocol to the needs of various patient populations.

Overall, we aimed for the development and initial feasibility testing of the novel tDCS-telehealth intervention for

\section{REFERENCES}

Antal, A., Paulus, W., and Nitsche, M. A. (2010). "Principle and mechanisms of transcranial direct current stimulation (tDCS)," in Pain Brain Stimulation in the Treatment of Pain, eds H. Knotkova, R. Cruciani, and J. Merrick (New York, NY: Nova), 129-142.

Apkarian, A. V., Baliki, M. N., and Farmer, M. A. (2013). Predicting transition to chronic pain. Curr. Opin. Neurol. 26, 360-367. doi: 10.1097/WCO. 0b013e32836336ad

Boggio, P. S., Rigonatti, S. P., Ribeiro, R. B., Myczkowski, M. L., Nitsche, M. A., Pascual-Leone, A., et al. (2008). A randomized, double-blind clinical trial on the efficacy of cortical direct current stimulation for the treatment of major depression. Int. J. Neuropsychopharmacol. 11, 249-254. doi: 10.1017/ S1461145707007833

Borckardt, J. J., Reeves, S. T., Robinson, S. M., May, J. T., Epperson, T. I., Gunselman, R. J., et al. (2013). Transcranial direct current stimulation (tDCS) reduces postsurgical opioid consumption in total knee arthroplasty (TKA). Clin. J. Pain 29, 925-928. doi: 10.1097/AJP.0b013e31827e32be

Borckardt, J. J., Romagnuolo, J., Reeves, S. T., Madan, A., Frohman, H., Beam, W., et al. (2011). Feasibility, safety, and effectiveness of transcranial direct current stimulation for decreasing post-ERCP pain: a randomized, sham-controlled, pilot study. Gastrointest. Endosc. 73, 1158-1164. doi: 10.1016/j.gie.2011.01.050

Brunoni, A. R., Boggio, P. S., De Raedt, R., Benseñor, I. M., Lotufo, P. A., Namur, V., et al. (2014). Cognitive control therapy and transcranial direct current stimulation for depression: a randomized, double-blinded, controlled trial. J. Affect. Disord. 162, 43-49. doi: 10.1016/j.jad.2014.03.026 home-bound chronically ill patients with multiple unrelieved symptoms. The pilot study was not intended to investigate the clinical efficacy of the intervention and no conclusions about the efficacy should be drawn from the reported results. Further, due to a low sample size, the findings on feasibility of the procedure in the home-bound chronically ill are preliminary, and larger studies are needed to further evaluate the intervention and to determine/optimize the protocol efficacy and effectiveness for symptom control outcomes.

\section{AUTHOR CONTRIBUTIONS}

AR contributed to the data collection, data evaluation, design of graphic materials, and the manuscript writing, with important intellectual input from the rest of the team. VP contributed to the development of instructional materials. BP contributed to the technical solutions. RP contributed to the development of design of the protocol and the manuscript writing, with important intellectual input from the rest of the team. MB contributed to the technical solutions and manuscript writing, with important intellectual input from the rest of the team. HK lead the design and development of the overall project, and contributed to the data evaluation and the manuscript writing, with important intellectual input from the rest of the team.

\section{ACKNOWLEDGMENTS}

The authors would like to thank Mr. Joshua Harounian for assistance during the expert feedback meetings and Ms. Shelita Clark for assistance with preparation of the manuscript.

Brunoni, A. R., Valiengo, L., Baccaro, A., Zanão, T. A., de Oliveira, J. F., Goulart, A., et al. (2013). The sertraline vs. electrical current therapy for treating depression clinical study: results from a factorial, randomized, controlled trial. JAMA Psychiatry 70, 383-391. doi: 10.1001/2013.jamapsychiatry.32

Centers for Medicare and Medicaid Services (2012). Chronic Conditions Among Medicare Beneficiaries. Baltimore, MD: Centers for Medicare and Medicaid Services, 1-30.

Chang, V. T., Hwang, S. S., Kasimis, B., and Thaler, H. T. (2004). Shorter symptom assessment instruments: the condensed memorial symptom assessment scale (CMSAS). Cancer Invest. 22, 526-536. doi: 10.1081/CNV-200026487

Charvet, L. E., Kasschau, M., Datta, A., Knotkova, H., Stevens, M. C., Alonzo, A., et al. (2015). Remotely-supervised transcranial direct current stimulation (tDCS) for clinical trials: guidelines for technology and protocols. Front. Syst. Neurosci. 17:26. doi: 10.3389/fnsys.2015.00026

Davis, K., Yount, S., Del Ciello, K., Whalen, M., Khan, S., Bass, M., et al. (2007). An innovative symptom monitoring tool for people with advanced lung cancer: a pilot demonstration. J. Support. Oncol. 5, 381-387.

Fagerlund, A. J., Hansen, O. A., and Aslaksen, P. M. (2015). Transcranial direct current stimulation as a treatment for patients with fibromyalgia: a randomized controlled trial. Pain 156, 62-71. doi: 10.1016/j.pain.00000000000 00006

Ferrucci, R., Vergari, M., Cogiamanian, F., Bocci, T., Ciocca, M., Tomasini, E., et al. (2014). Transcranial direct current stimulation (tDCS) for fatigue in multiple sclerosis. NeuroRehabilitation 34, 121-127. doi: 10.3233/NRE-131019

Fregni, F., Boggio, P. S., Lima, M. C., Ferreira, M. J., Wagner, T., Rigonatti, S. P., et al. (2006a). A sham-controlled, phase II trial of transcranial direct current 
stimulation for the treatment of central pain in traumatic spinal cord injury. Pain 122, 197-209.

Fregni, F., Gimenes, R., Valle, A. C., Ferreira, M. J., Rocha, R. R., Natalle, L., et al. (2006b). A randomized, sham-controlled, proof of principle study of transcranial direct current stimulation for the treatment of pain in fibromyalgia. Arthritis Rheum. 54, 3988-3998.

Glaser, J., Reeves, S. T., Stoll, W. D., Epperson, T. I., Hilbert, M., Madan, A., et al. (2016). Motor/Prefrontal transcranial direct current stimulation (tDCS) following lumbar surgery reduces postoperative analgesia use. Spine 41, 835-839. doi: 10.1097/BRS.0000000000001525

Grubaugh, A. L., Cain, G. D., Elhai, J. D., Patrick, S. L., and Frueh, B. C. (2008). Attitudes toward medical and mental health care delivered via telehealth applications among rural and urban primary care patients. J. Nerv. Ment. Dis. 196, 166-170. doi: 10.1097/NMD.0b013e318162aa2d

Hagenacker, T., Bude, V., Naegel, S., Holle, D., Katsarava, Z., Diener, H. C., et al. (2014). Patient-conducted anodal transcranial direct current stimulation of the motor cortex alleviates pain in trigeminal neuralgia. J. Headache Pain 15:78. doi: 10.1186/1129-2377-15-78

Hasselman, D., and Center for Health Care Strategies (2013). Super-Utilizer Summit: Common Themes from Innovation Complex Care Management Programs. 1-32. Available at: https://www.chcs.org/resource/super-utilizersummit-common-themes-from-innovative-complex-care-managementprograms/

Head, B. A., Keeney, C., Studts, J. L., Khayat, M., Bumpous, J., and Pfeifer, M. (2011). Feasibility and acceptance of a telehealth intervention to promote symptom management during treatment for head and neck cancer. J. Support. Oncol. 9, 1-11. doi: 10.1016/j.suponc.2010.12.006

Hemington, K. S., Wu, Q., Kucyi, A., Inman, R. D., and Davis, K. D. (2016). Abnormal cross-network functional connectivity in chronic pain and its association with clinical symptoms. Brain Struct. Funct. 221, 4203-4219. doi: 10.1007/s00429-015-1161-1

Johnston, B., Kidd, L., Wengstrom, Y., and Kearney, N. (2012). An evaluation of the use of telehealth within palliative care settings across Scotland. Palliat. Med. 26, 152-161. doi: 10.1177/0269216311398698

Kearney, N., McCann, L., Norrie, J., Taylor, L., Gray, P., McGee-Lennon, M., et al. (2009). Evaluation of a mobile phone-based, advanced symptom management system (ASyMS) in the management of chemotherapy-related toxicity. Support. Care Cancer 17, 437-444. doi: 10.1007/s00520-008-0515-0

Kim, Y. J., Ku, J., Kim, H. J., Im, D. J., Lee, H. S., Han, K. A., et al. (2013). Randomized, sham controlled trial of transcranial direct current stimulation for painful diabetic polyneuropathy. Ann. Rehabil. Med. 37, 766-776. doi: 10.5535/arm.2013.37.6.766

Knotkova, H., Leuschner, Z., Soto, E., Davoudzadeh, E., Greenberg, A., and Cruciani, R. (2014a). Evaluating outcomes of transcranial direct current stimulation (tDCS) in patients with chronic neuropathic pain. J. Pain 15:S69. doi: 10.1016/j.jpain.2014.01.284

Knotkova, H., Malamud, S. C., and Cruciani, R. A. (2014b). Transcranial direct current stimulation (TDCS) improved cognitive outcomes in a cancer survivor with chemotherapy-induced cognitive difficulties. Brain Stimul. 7, 767-768. doi: $10.1016 /$ j.brs.2014.05.007

Knotkova, H., Riggs, A., Patel, V., Truong, D., Arce, D., Bernstein, H., et al. (2017). A novel approach to determining M1 tDCS montage without neuronavigational measurements, suitable for patients in home settings. Brain Stimul. 10, 78-80. doi: 10.1016/j.brs.2017.04.117

Kroenke, K., Theobald, D., Wu, J., Norton, K., Morrison, G., Carpenter, J., et al. (2010). Effect of telecare management on pain and depression in patients with cancer: a randomized trial. JAMA 304, 163-171. doi: 10.1001/jama.2010.944

Lefaucheur, J. P. (2016). A comprehensive database of published tDCS clinical trials (2005-2016). Neurophysiol. Clin. 46, 319-398. doi: 10.1016/j.neucli.2016.10.002

Loo, C. K., Alonzo, A., Martin, D., Mitchell, P. B., Galvez, V., and Sachdev, P. (2012). Transcranial direct current stimulation for depression: 3-week, randomized, sham-controlled trial. Br. J. Psychiatry 200, 52-59. doi: 10.1192/ bjp.bp.111.097634

Maihöfner, C., Handwerker, H. O., Neundorfer, B., and Birklein, F. (2003). Patterns of cortical reorganization in complex regional pain syndrome. Neurology 61, 1715-1717. doi: 10.1212/01.WNL.0000098939.02752.8E

Maihöfner, C., Handwerker, H. O., Neundorfer, B., and Birklein, F. (2004). Cortical reorganization during recovery from complex regional pain syndrome. Neurology 63, 693-701. doi: 10.1212/01.WNL.0000134661.46 658.B0

Manenti, R., Sandrini, M., Gobbi, E., Cobelli, C., Brambilla, M., Binetti, G., et al. (2017). Strengthening of existing episodic memories through noninvasive stimulation of prefrontal cortex in older adults with subjective memory complaints. Front. Aging Neurosci. 5:401. doi: 10.3389/fnagi.2017.00401

Monte-Silva, K., Kuo, M. F., Hessenthaler, S., Fresnoza, S., Liebetanz, D., Paulus, W., et al. (2013). Induction of late LTP-like plasticity in the human motor cortex by repeated non-invasive brain stimulation. Brain Stimul. 6, 424-432. doi: 10.1016/j.brs.2012.04.011

Mori, F., Codecà, C., Kusayanagi, H., Monteleone, F., Buttari, F., Fiore, S., et al. (2010). Effects of anodal transcranial direct current stimulation on chronic neuropathic pain in patients with multiple sclerosis. J. Pain 11, 436-442. doi: 10.1016/j.jpain.2009.08.011

Napadow, V., Maeda, Y., Audette, J., and Kettner, N. (2012). "Chapter IX Neuroplasticity in carpal tunnel syndrome," in Neural Plasticity in Chronic Pain, eds H. Knotkova, R. Cruciani, and J. Merrick (New York, NY: Nova Science Publishers Inc.), 153-184.

Nitsche, M. A., Grundey, J., Liebetanz, D., Lang, N., Tergau, F., and Paulus, W. (2004a). Catecholaminergic consolidation of motor cortical neuroplasticity in humans. Cereb. Cortex 14, 1240-1245.

Nitsche, M. A., Jaussi, W., Liebetanz, D., Lang, N., Tergau, F., and Paulus, W. (2004b). Consolidation of human motor cortical neuroplasticity by D-cycloserine. Neuropsychopharmacology 29, 1573-1578.

Nitsche, M. A., Kuo, M. F., Karrasch, R., Wächter, B., Liebetanz, D., and Paulus, W. (2009). Serotonin affects transcranial direct current-induced neuroplasticity in humans. Biol. Psychiatry 66, 503-508. doi: 10.1016/j.biopsych.2009.03.022

Nitsche, M. A., Lampe, C., Antal, A., Liebetanz, D., Lang, N., Tergau, F., et al. (2006). Dopaminergic modulation of long-lasting direct current-induced cortical excitability changes in the human motor cortex. Eur. J. Neurosci. 23, 1651-1657. doi: 10.1111/j.1460-9568.2006.04676.x

Nitsche, M. A., Liebetanz, D., Antal, A., Lang, N., Tergau, F., and Paulus, W. (2003). Modulation of cortical excitability by weak direct current stimulationtechnical, safety and functional aspects. Suppl. Clin. Neurophysiol. 56, 255-276. doi: 10.1016/S1567-424X(09)70230-2

Nitsche, M. A., Liebetanz, D., Schlitterlau, A., Henschke, U., Fricke, K., Frommann, K., et al. (2004c). GABAergic modulation of DC stimulationinduced motor cortex excitability shifts in humans. Eur. J. Neurosci. 19, $2720-2726$.

Nitsche, M. A., and Paulus, W. (2000). Excitability changes induced in the human motor cortex by weak transcranial direct current stimulation. J. Physiol. 527, 633-639. doi: 10.1111/j.1469-7793.2000.t01-1-00633.x

Ortman, J. M., Velkoff, V. A., and Hogan, H. (2014). An Aging Nation: The Older Population in the United States, Current Population Reports. Available at: https: //www.census.gov/prod/2014pubs/p25-1140

Polania, R., Paulus, W., and Nitsche, M. A. (2011). Modulating cortico-striatal and thalamo-cortical functional connectivity with transcranial direct current stimulation. Hum. Brain Mapp. 33, 2499-2508. doi: 10.1002/hbm.21380

Rigonatti, S. P., Boggio, P. S., Myczkowski, M. L., Otta, E., Fiquer, J. T., Ribeiro, R. B., et al. (2008). Transcranial direct stimulation and fluoxetine for the treatment of depression. Eur. Psychiatry 23, 74-76. doi: 10.1016/j.eurpsy.2007. 09.006

Roizenblatt, S., Fregni, F., Gimenez, R., Wetzel, T., Rigonatti, S. P., Tufik, S., et al. (2007). Site-specific effects of transcranial direct current stimulation on sleep and pain in fibromyalgia: a randomized, sham-controlled study. Pain Pract. 7, 297-306. doi: 10.1111/j.1533-2500.2007.00152.x

Ruland, C. M., Maffei, R. M., Børøsund, E., Krahn, A., Andersen, T., and Grimsbø, G. H. (2013). Evaluation of different features of an eHealth application for personalized illness management support: cancer patients' use and appraisal of usefulness. Int. J. Med. Inform. 82, 593-603. doi: 10.1016/j.ijmedinf.2013.02.007

Saiote, C., Goldschmidt, T., Timäus, C., Steenwijk, M. D., Opitz, A., Antal, A., et al. (2014). Impact of transcranial direct current stimulation on fatigue in multiple sclerosis. Restor. Neurol. Neurosci. 32, 423-436. doi: 10.3233/RNN130372

Sandrini, M., Brambilla, M., Manenti, R., Rosini, S., Cohen, L. G., and Cotelli, M. (2014). Noninvasive stimulation of prefrontal cortex strengthens existing episodic memories and reduces forgetting in the elderly. Front. Aging Neurosci. 6:289. doi: 10.3389/fnagi.2014.00289 
Schag, C. C., Heinrich, R. L., and Ganz, P. A. (1984). Karnofsky performance status revisited: reliability, validity, and guidelines. J. Clin. Oncol. 2, 187-193. doi: 10.1200/JCO.1984.2.3.187

Seibt, O., Brunoni, A. R., Huang, Y., and Bikson, M. (2015). The pursuit of DLPFC: Non-neuronavigated methods to target the left dorsolateral pre-frontal cortex with symmetric bicephalic transcranial direct current stimulation (tDCS). Brain Stimul. 8, 590-602. doi: 10.1016/j.brs.2015.01.401

Stagg, C., Lin, R., Mezue, M., Segerdahl, A., Kong, Y., Xie, J., et al. (2013). Widespread modulation of cerebral perfusion induced during and after transcranial direct current stimulation applied to the left dorsolateral prefrontal cortex. J. Neurosci. 33, 11425-11431. doi: 10.1523/JNEUROSCI.388712.2013

Stefani, L. C., Torres, I. L., de Souza, I. C., Rozisky, J. R., Fregni, F., and Caumo, W. (2012). BDNF as an effect modifier for gender effects on pain thresholds in healthy subjects. Neurosci. Lett. 514, 62-66. doi: 10.1016/j.neulet.2012.02.057

Stock, V. M., Knotkova, H., and Nitsche, M. A. (2016). "Principles of neuromodulation," in Textbook of Neuromodulation, eds H. Knotkova and D. Rasche (New York, NY: Springer), 3-7.

Valle, A., Roizenblatt, S., Botte, S., Zaghi, S., Riberto, M., Tufik, S., et al. (2009). Efficacy of anodal transcranial direct current stimulation (tDCS) for the treatment of fibromyalgia: results of a randomized, sham-controlled longitudinal clinical trial. J. Pain Manag. 2, 353-362.
Weaver, A., Young, A. M., Rowntree, J., Townsend, N., Pearson, S., Smith, J., et al. (2007). Application of mobile phone technology for managing chemotherapyassociated side-effects. Ann. Oncol. 18, 1887-1892. doi: 10.1093/annonc/ $\operatorname{mdm} 354$

Woods, A. J., Antal, A., Bikson, M., Boggio, P. S., Brunoni, A. R., Celnik, P., et al. (2016). A technical guide to tDCS, and related non-invasive brain stimulation tools. Clin. Neurophysiol. 127, 1031-1048. doi: 10.1016/j.clinph.2015.11.012

Conflict of Interest Statement: MB has equity in Soterix Medical Inc. The City University of New York has patents on brain stimulation with MB as co-inventor.

The other authors declare that the research was conducted in the absence of any commercial or financial relationships that could be construed as a potential conflict of interest.

Copyright (c) 2018 Riggs, Patel, Paneri, Portenoy, Bikson and Knotkova. This is an open-access article distributed under the terms of the Creative Commons Attribution License (CC BY). The use, distribution or reproduction in other forums is permitted, provided the original author(s) and the copyright owner are credited and that the original publication in this journal is cited, in accordance with accepted academic practice. No use, distribution or reproduction is permitted which does not comply with these terms. 


\section{OPEN ACCESS}

Edited by:

Bahar Güntekin,

Istanbul Medipol University, Turkey

Reviewed by:

Mitchell Ryan Goldsworthy, University of Adelaide, Australia

Sébastien Hétu,

Université de Montréal, Canada

*Correspondence:

Sara Marceglia smarceglia@units.it

Received: 12 March 2018 Accepted: 20 September 2018 Published: 29 October 2018

Citation:

Ferrucci R, Mrakic-Sposta S, Gardini S, Ruggiero F, Vergari M, Mameli F, Arighi A, Spallazzi M, Barocco F, Michelini G, Pietroboni AM, Ghezzi L, Fumagalli GG, D'Urso G, Caffarra P, Scarpini E, Priori A and Marceglia S (2018) Behavioral and

Neurophysiological Effects of Transcranial Direct Current Stimulation (tDCS) in Fronto-Temporal Dementia.

Front. Behav. Neurosci. 12:235 doi: 10.3389/fnbeh.2018.00235

\section{Behavioral and Neurophysiological Effects of Transcranial Direct Current Stimulation (tDCS) in Fronto-Temporal Dementia}

\author{
Roberta Ferrucci ${ }^{1,2,3}$, Simona Mrakic-Sposta ${ }^{1,4}$, Simona Gardini ${ }^{5}$, Fabiana Ruggiero ${ }^{1}$, \\ Maurizio Vergari ${ }^{1}$, Francesca Mameli ${ }^{1}$, Andrea Arighi ${ }^{1,6}$, Marco Spallazzi ${ }^{7}$, \\ Federica Barocco ${ }^{7}$, Giovanni Michelini ${ }^{5}$, Anna Margherita Pietroboni ${ }^{1,6}$, Laura Ghezzi ${ }^{1,6}$, \\ Giorgio Giulio Fumagalli ${ }^{1,6,8}$, Giordano D'Urso ${ }^{9}$, Paolo Caffarra ${ }^{7,10}$, Elio Scarpini ${ }^{1,6}$, \\ Alberto Priori ${ }^{2,3}$ and Sara Marceglia ${ }^{1,11 *}$ \\ ${ }^{1}$ Fondazione Ca' Granda, IRCCS Ospedale Maggiore Policlinico, Milan, Italy, "2Aldo Ravelli" Research Center for \\ Neurotechnology and Experimental Brain Therapeutics, University of Milan Medical School, Milan, Italy, ${ }^{3}$ III Neurological \\ Clinic, San Paolo Hospital, Milan, Italy, ${ }^{4}$ National Council of Research, Institute of Bioimaging and Molecular Physiology, \\ Segrate, Italy, ${ }^{5}$ Department of Neuroscience, University of Parma, Parma, Italy, ${ }^{6}$ Department of Pathophysiology and \\ Transplantation, "Dino Ferrari" center, University of Milan, Milan, Italy, ${ }^{7}$ Dementia Unit, Azienda Ospedaliero-Universitaria of \\ Parma, Parma, Italy, ${ }^{8}$ Department of Neurosciences, Psychology, Drug Research and Child Health (NEUROFARBA), \\ University of Florence, Florence, Italy, ${ }^{9}$ University of Naples "Federico II"-AOU, Naples, Italy, ${ }^{10}$ Center for Cognitive Disorders \\ and Dementia, AUSL of Parma, Parma, Italy, " Department of Engineering and Architecture, University of Trieste, Trieste, Italy
}

Fronto-temporal dementia (FTD) is the clinical-diagnostic term that is now preferred to describe patients with a range of progressive dementia syndromes associated with focal atrophy of the frontal and anterior temporal cerebral regions. Currently available FTD medications have been used to control behavioral symptoms, even though they are ineffective in some patients, expensive and may induce adverse effects. Alternative therapeutic approaches are worth pursuing, such as non-invasive brain stimulation with transcranial direct current (tDCS). tDCS has been demonstrated to influence neuronal excitability and reported to enhance cognitive performance in dementia. The aim of this study was to investigate whether applying Anodal tDCS (2 mA intensity, 20 min) over the fronto-temporal cortex bilaterally in five consecutive daily sessions would improve cognitive performance and behavior symptoms in FTD patients, also considering the neuromodulatory effect of stimulation on cortical electrical activity measured through EEG. We recruited 13 patients with FTD and we tested the effect of Anodal and Sham (i.e., placebo) tDCS in two separate experimental sessions. In each session, at baseline (T0), after 5 consecutive days (T1), after 1 week (T2), and after 4 weeks (T3) from the end of the treatment, cognitive and behavioral functions were tested. EEG (21 electrodes, 10-20 international system) was recorded for 5 min with eyes closed at the same time points in nine patients. The present findings showed that Anodal tDCS applied bilaterally over the fronto-temporal cortex significantly improves (1) neuropsychiatric symptoms (as measured by the neuropsychiatric inventory, NPI) in FTD patients immediately after tDCS treatment, and (2) simple visual reaction times (sVRTs) up to 1 month after tDCS treatment. These cognitive improvements significantly correlate with the time course of 
the slow EEG oscillations (delta and theta bands) measured at the same time points. Even though further studies on larger samples are needed, these findings support the effectiveness of Anodal tDCS over the fronto-temporal regions in FTD on attentional processes that might be correlated to a normalized EEG low-frequency pattern.

Keywords: transcranial direct current stimulation (tDCS), neuromodulation, fronto-temporal dementia, EEG, reaction time, neuropsychiatric inventory

\section{INTRODUCTION}

Fronto-temporal dementia (FTD) is the clinical diagnostic term that is now preferred to describe patients with a range of progressive dementia syndromes associated with focal atrophy of the frontal and anterior temporal cerebral region (Piguet and Hodges, 2013). Epidemiological studies suggest that FTD is the second most common cause of young-onset dementia after Alzheimer's disease (AD) and accounts for 5-15\% of all types of dementia (Seltman and Matthews, 2012).

Currently, available FTD medications have been used to control behavioral symptoms, even though they are ineffective in some patients, expensive and may induce adverse effects (Allain et al., 2003). Given this paucity of pharmacological interventions, strategies for non-pharmacological enhancement are receiving increasing attention, including the use of noninvasive stimulation, such as transcranial Direct Current Stimulation (tDCS), a neuromodulatory technique that delivers low-intensity direct current to cortical areas that facilitates or inhibits cortical spontaneous neuronal activity (Woods et al., 2016). Interesting findings have emerged in healthy volunteers and in clinical populations (Floel, 2014; Summers et al., 2016). Collectively, these studies have shown that tDCS is a safe tool able to enhance memory, language, attention, and learning processes (Shin et al., 2015). In clinical studies, previous findings in $\mathrm{AD}$ patients demonstrated that Anodal tDCS, both after a single session and after five consecutive daily sessions of tDCS over the temporal and parietal cortices, produces significant improvements in verbal and visual recognition memory (Ferrucci et al., 2008; Boggio et al., 2009, 2012) Notably, the tDCS effect persisted for at least 4 weeks after intervention.

Only few studies have tested the effects of tDCS treatment in FTD and the results are controversial. tDCS, usually applied bilaterally over the left inferior parieto-temporal region, provided encouraging results in treating anomia and other cognitive disabilities in demented individuals (Roncero et al., 2017) and in improving behavioral disturbances predominantly characterized by apathy (Agarwal et al., 2016), but failed to produce any improvement in behavioral and language function immediately after a single session of stimulation (Huey et al., 2007). Only one case-study reported the successful application of tDCS over 5 consecutive days that substantially improved behavioral disturbances and socio-occupational functioning in a woman with FTD (Agarwal et al., 2016). These results suggest that repeated tDCS sessions may be useful to enhance long-lasting tDCS effects, but need to be tested in larger samples.
As well as in other applications of non-invasive neuromodulation, the heterogeneity of stimulation protocols and the type of outcomes measured are among the major challenges to obtain consistent and comparable results (Elder and Taylor, 2014; Lefaucheur et al., 2017).

Recently, the use of quantitative electroencephalography (qEEG) to study the neurophysiological effects of tDCS showed that $\mathrm{tDCS}$-induced modulations of EEG rhythms and coherences are consistent with the tDCS-induced effects on memory in patients with Alzheimer's Disease (Marceglia et al., 2016). The patients analyzed in Marceglia et al. (2016) were those described in a previous paper, in which the clinical effects of Anodal and cathodal tDCS applied bilaterally over temporo-parietal areas (P3-T5 and P4-T6 according to the international 10-20 EEG standard), with reference on the right shoulder, were studied (Ferrucci et al., 2008). Studying qEEG modifications in parallel with clinical and neuropsychological variables could hence strengthen the findings on the overall effects of tDCS. tDCS, in fact, could "normalize" the EEG pattern typical of the pathology under study (Koberda et al., 2013; Marceglia et al., 2016), thus providing both the neurophysiological basis of its positive effects on patients and a quantitative and repeatable outcome representative of the patient's state. Patients with cognitive decline are characterized by an increased power in the theta band $(4-7 \mathrm{~Hz})$ in fronto-temporal regions, and an overall decrease of beta power $(13-35 \mathrm{~Hz})$ with a focus in temporo-parietal areas (Koberda et al., 2013). In Alzheimer's disease, the abnormal beta pattern was reverted by Anodal tDCS, and tDCS-induced changes correlated well with the positive effects of the stimulation on working memory (Marceglia et al., 2016).

The purposes of this study were to investigate (1) whether applying Anodal tDCS over the frontal cortex in five consecutive daily sessions would improve cognitive performance and behavioral symptoms in FTD patients, and (2) whether these effects correlate with the neurophysiological pattern measured by EEG.

\section{MATERIALS AND METHODS Participants}

We enrolled 13 patients diagnosed with FTD according to published criteria (Brun et al., 1994). Eight had the predominantly behavioral variant (3 female; mean age \pm SD: 76.6 \pm 0.57 years; 5 male; $69.4 \pm 4.1$ years) and five had the language variant ( 2 female; mean age \pm SD: $73 \pm 1.4$ years; 3 male; $66.0 \pm$ 
TABLE 1 | Demographic and clinical features of the enrolled patients.

\begin{tabular}{|c|c|c|c|c|c|c|}
\hline & $\begin{array}{l}\text { Education } \\
\text { (Y) }\end{array}$ & MMSE & $\begin{array}{l}\text { FTD } \\
\text { variant }\end{array}$ & $\begin{array}{c}\text { TAU } \\
\text { protein } \mathrm{pg} / \mathrm{ml}\end{array}$ & $\begin{array}{l}\text { Phosphorylates } \\
\text { TAU protein pg/ml }\end{array}$ & Medication \\
\hline 1 & 13 & 24 & BV & 138 & 38 & Anti-hypertensive; Antidepressive \\
\hline 3 & 13 & 21 & BV & 1,119 & 89 & Anti-hypertensive \\
\hline 5 & 13 & 23 & BV & 586 & 71 & $\begin{array}{l}\text { Antipsicotic } \\
\text { Antidiabetic }\end{array}$ \\
\hline 6 & 18 & 22 & PPA & 1,005 & 95 & Anti-depressive \\
\hline 7 & 18 & 27 & PPA & 580 & 61 & $\begin{array}{l}\text { Anti-hypertensive; } \\
\text { Antiplatelet }\end{array}$ \\
\hline 10 & 13 & 25 & BV & 313 & 35 & Anti-hypertensive; Anti-depressive \\
\hline 11 & 8 & 28 & PPA & 211 & 81 & Antiplatelet; Antipsicotic \\
\hline 12 & 5 & 30 & BV & 237 & 128 & $\begin{array}{l}\text { Anti-hypertensive; Anti-depressive; } \\
\text { Statins }\end{array}$ \\
\hline 13 & 13 & 21 & PPA & 864 & 86 & Antipsicotic \\
\hline
\end{tabular}

MMSE, mini mental state examination; FTD, fronto-temporal dementia; BV, behavioral variant of FTD; PPA, primary progressive aphasia; SD, standard deviation; anti-hypertensive: nifedipine, candesartancilexetil, amlodipine, indapamide hemihydrates, hydrochlorothiazide/irbesartan, nebivolol; anti-depressive: citalopram; antipsicotic: quetiapine, promazine, haloperidol; cholinesterase inhibitor: rivastigmine; antiplatelet: cardioaspirine; statins: atorvastatin, antidiabetic: metformin; anxiolytic: hydroxyzine hydrochloride.

3.6 years). Of these, one was excluded because did not complete the full study protocol. We therefore analyzed 12 subjects.

All patients were screened and recruited in the Center for Neurodegenerative Diseases at the Fondazione IRCCS Ca' Granda Ospedale Maggiore Policlinico, Milan, and at the Dementia Unit, Azienda Ospedaliero-Universitaria of Parma, Italy, by a team of experienced neurologists and neuropsychologists through appropriate diagnostic tests.

Participants were included in the study if their Mini Mental State Examination (MMSE) score was above 20 (mean \pm SD: $24.4 \pm 3.3$ ) and if they had no other neuropsychiatric diseases. The demographic characteristics of the groups are summarized in Table 1. The patients were taking CNS-active medications and they maintained their medication regimen unchanged throughout the study (Table 1). Tau-protein measurements were collected (Table 1) and were in line with the available data for dementia patients (van Harten et al., 2011). CSF samples were obtained using a standardized protocol; lumbar punctures were performed in the mornings at L3/L4 or L4/L5 interspaced. About $1 \mathrm{ml}$ of CSF was immediately frozen and stored at $-80^{\circ} \mathrm{C}$ until biochemical assays for Tau-protein levels were performed. CSF levels of Tau-protein phosphorylated at threonine-181 were measured by ELISA, using a commercially available kit (Innotest PHOSPHO-TAU Antigen, Innogenetics, Belgium). The monoclonal antibodies which are coated on the ELISA plate recognize both the entire moiety and its fragments (Vanmechelen et al., 2000). Tau-protein values are expressed as $\mathrm{pg} / \mathrm{mL}$.
The study was performed according to the Declaration of Helsinki and approved by the local institutional review board. Patients and their caregivers provided their informed and written consent before participation.

\section{Experimental Protocol}

We tested the effect of Anodal and Sham (i.e., placebo) tDCS applied daily to fronto-temporal lobes for 5 consecutive days in two separate experimental sessions. All subjects received both types of stimulation in a randomized and counterbalanced order (1:1 ratio). To avoid carry-over effects, an average of $60 \pm 5$ days elapsed between sessions. The patients and the examiner who performed the neuropsychological assessment were blind to the type of tDCS delivered in each session.

Cognitive functions and behavior were tested four times: at baseline (T0), after 5 consecutive days (T1), at 1 week (T2), and at 4 weeks (T3) after the end of the treatment. In addition, in 9 out of 13 patients, EEG was recorded four times, at the same time points (T0, T1, T2, T3).

\section{tDCS Protocol}

According to the available literature, tDCS was delivered bilaterally through a battery-driven constant current stimulator (HDCStim, Newronika srl, Milan, Italy) using three surface saline-soaked sponge electrodes, two placed on the scalp and one placed over the right deltoid muscle (each scalp electrode measured $35 \mathrm{~cm}^{2}$; the deltoid electrode measured $64 \mathrm{~cm}^{2}$ ). 
The rationale of bilateral stimulation is based on the fact that no asymmetry is expected in the areas that are treated, and, therefore, a unilateral stimulation would introduce an unwanted asymmetry, whereas bilateral stimulation would provide a balanced effect on both sides. The same stimulation protocol was proposed by Ferrucci et al. (2008) to treat Alzheimer's patients, but with a different electrode location.

Anodal stimulation consisted of $20 \mathrm{~min}$ of $2 \mathrm{~mA}$ direct current per session (with $10 \mathrm{~s}$ for ramping up and down) with the anode placed over the fronto-temporal lobes bilaterally (F7 and F8, according to the 10-20 EEG International System) and the reference electrode above the right deltoid muscle. The same procedure was used for Sham stimulation, but current was applied only for the first $10 \mathrm{~s}$ (Figure 1). To verify whether the patients could distinguish between active and Sham stimulation, we asked them to refer any sensation felt during tDCS sessions. They confirmed that in both cases they felt only the initial itching sensation disappearing after 10-20 s, without differences perceived between active and Sham stimulations.

\section{Cognitive and Behavioral Assessment}

Cognitive functions were evaluated through five different tasks: the Phonemic Verbal Fluency Task (PFT) (Novelli et al., 1986), the Visual Recognition Task (VRT) (Boggio et al., 2012), the Picture Naming Task (PNT) (Viggiano et al., 2004), the Go no-Go
Task (GGT) (Barbarotto et al., 1998) and, to investigate whether the effects of tDCS of cognitive performance can reflect changes in arousal, the Simple Visual Reaction Times Task (sVRT) (Barbarotto et al., 1998). Behavioral changes were evaluated with the Neuropsychiatric Inventory (NPI) (Cummings et al., 1994) and the Frontal Behavioral Inventory (FBI) (Kertesz et al., 1997). Furthermore, to evaluate the caregiver's burden, the Zarit Burden Inventory (ZBI) (Zarit and Zarit, 1990) was administered.

All the tests including possible biases due to learning were developed using variants, in order to avoid habituation and improvements due to the test replication, as described below.

PFT: this task was performed to measure the number of words beginning with a target letter that could be generated in $60 \mathrm{~s}$, excluding proper nouns, numbers, and different forms of the same word. One letter for each condition was used, for a total of four letters (P, S, L, F) counterbalanced across stimulation conditions and order of presentation. The fluency score was the total number of words for each condition. Increased values indicate improvement.

VRT: in this task we specifically evaluated visual memory using a computer-controlled procedure.

The task comprised both encoding and recognition phases. It started with the encoding phase (two items), in which drawings of animals, persons, and objects were displayed on a computer screen for $10 \mathrm{~s}$, followed $1 \mathrm{~s}$ later by the recognition phase, when

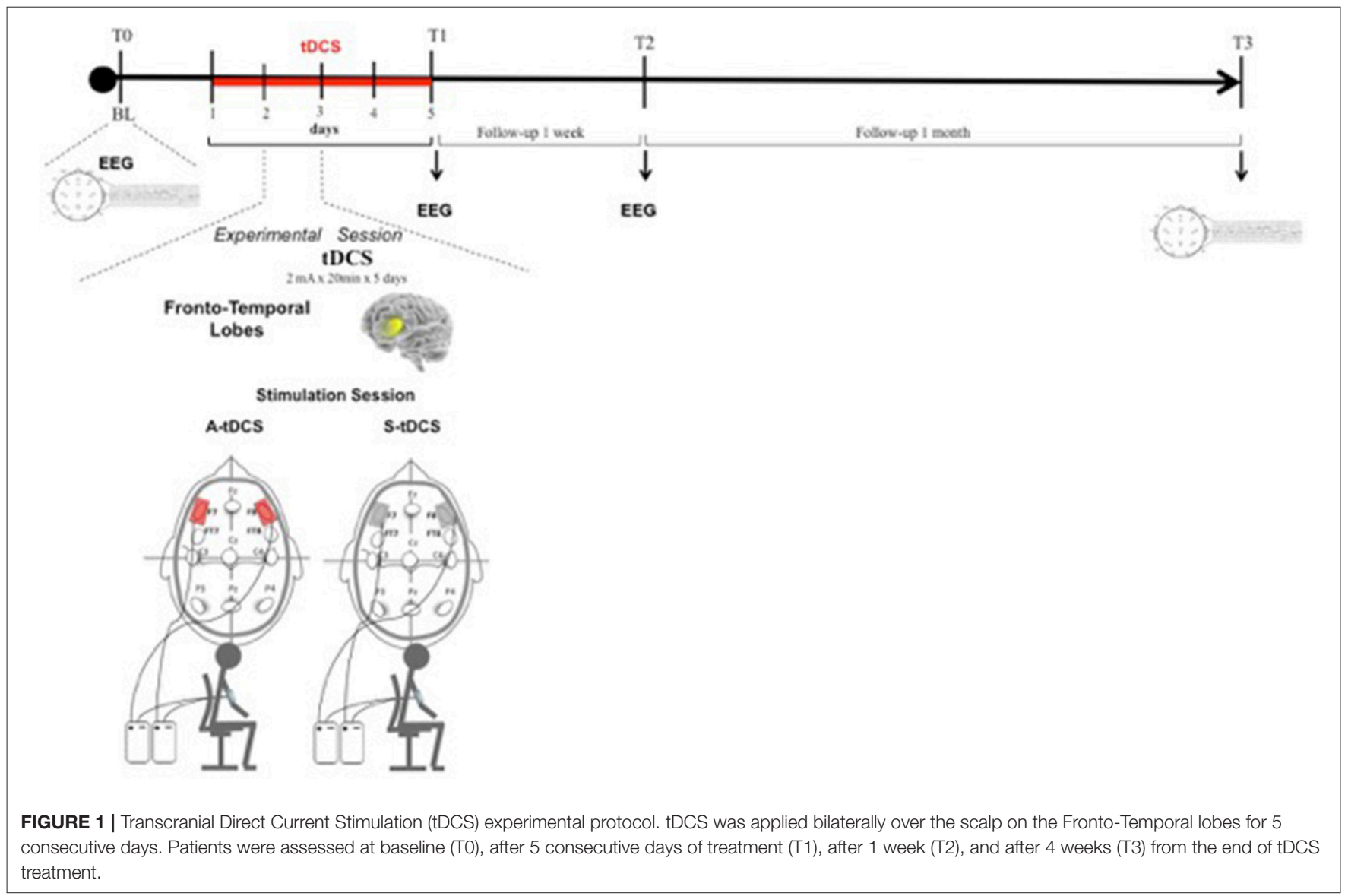


patients were shown a single picture (test trial) and asked to say whether the picture had been presented before. Patients underwent this procedure eight times during the test. These eight encoding/recognition sequences included two study trials of two, four, six, and eight stimuli. Patients therefore studied a total 40 drawings during the test. Each study trial included test trials (recognition phase). Three test trials were presented after each two-item study trial; six test trials after each four-item study trial; eight test trials after each six-item study trial; and 10 test trials after each eight-item study trial. To avoid learning, we used alternative versions of this task and randomized them between assessment sessions. The memory score was the total number of items recognized for each condition (Boggio et al., 2012). Increased scores indicate improvement.

PNT: Subjects were asked to name pictures presented on a personal computer screen from one out of four lists (A-D). The lists were homogeneous for difficulties and were controlled for frequency of use, familiarity, visual complexity, grammatical class (nouns), and length in syllables; each list contained two items from a variety of semantic categories (living and nonliving). Italian standardized norms for the name agreement and synonyms of the target word were accepted. The accuracy was the number of pictures correctly named in a 20-item list; we scored " 1 " for correct responses and " 0 " for errors. Increased values indicate improvement.

GGT: we administered this task to investigate response inhibition using a computer-controlled procedure (E-PrimePsychology Software Tools, Inc.). Participants were required to look at a series of geometric figures, which could be either "square" or "circle," randomly displayed on the screen and respond to a 35 target figure by pressing a button. The dependent variables measured to investigate response inhibition were RTs and accuracy (number of correct responses; Barbarotto et al., 1998). Decreased RTs and increased accuracy values indicate improvement.

sVRT: Thirty-five fully white squares appear one at a time on a PC screen at randomized intervals. The subject is asked to push down the space bar as quickly as possible after the stimulus appears. The median value of all the recorded time values is considered. The number of omissions is also registered (Barbarotto et al., 1998). Decreased values indicate improvement.

NPI: The NPI is a caregiver-based structured interview designed to briefly assess problematic behaviors and psychopathology in dementia. It evaluates 12 neuropsychiatric disturbances common in dementia: delusions, hallucinations, agitation, dysphoria, anxiety, apathy, irritability, euphoria, disinhibition, aberrant motor behavior, night-time behavior disturbances, and appetite and eating abnormalities. The severity and frequency of each neuropsychiatric symptom are rated on the basis of written questions administered to the patient's caregiver (Cummings et al., 1994). Decreased values indicate improvement.

ZBI: The caregiver's burden was evaluated using the 22-item ZBI (Zarit and Zarit, 1990). It consists of a semistructured questionnaire administered during the assessment interview and can be used to simultaneously evaluate both the material and emotional burden experienced by the caregiver. The scale is made up of 22 items evaluating disease impact on a caregiver's quality of life, psychological suffering, financial difficulties, Shame, guilt, and difficulties in social and family relationships. Scores range from 0 to 88 . Decreased values indicate improvement.

FBI: The Frontal Behavioral Inventory (FBI) (Kertesz et al., 1997 ) is a 24 -item caregiver questionnaire specifically developed to assess the behavioral disturbances of FTD. It has been shown to discriminate between different FTD phenotypes and between FTD and other forms of dementia. Decreased values indicate improvement.

\section{EEG Recordings and Analysis}

EEG was recorded in a quiet room, with the subject awake, seated on a comfortable high-backed chair, under healthcare personnel continuous control, immediately after the administration of cognitive and behavioral tests. 21 electrodes $(\mathrm{Ag} / \mathrm{AgCl})$ were positioned according to the 10-20 International System using the EBNeuro Mizar-Light system (EBNeuro, Florence, IT). The average reference was used. The sampling frequency was $1,024 \mathrm{~Hz}$ with a bandpass of $0.5-500 \mathrm{~Hz}$ and a sensibility of $7 \mathrm{uV} / \mathrm{mm}$. Signals were stored for further analysis. EEG was recorded for $5 \mathrm{~min}$ with eyes closed at the same time points used for neuropsychological and behavioral assessments: at baseline (T0), after 5 days of tDCS treatment (T1), after 1 week (T2), and after 4 weeks (T3) from the end of tDCS treatment.

The software toolbox EEGLAB, running under the crossplatform MATLAB environment (The Math-Works 7.0, Inc) was used for data processing. Preprocessing procedures included artifact rejection and filtering. EEG was analyzed in the frequency domain through parametric power spectrum estimation (Delorme and Makeig, 2004). Spectral power in the classical bands of EEG oscillatory activity, namely delta $(1-3 \mathrm{~Hz})$, $\theta(4-7 \mathrm{~Hz}), \alpha(8-12 \mathrm{~Hz})$, and $\beta(13-35 \mathrm{~Hz})$, was calculated for each subject below each electrode at each time point (T0, T1, T2, and T3).

We followed the same analysis methodology previously described in Marceglia et al. (2016) to assess EEG oscillatory activity in Alzheimer's disease. More specifically, as noted by Klimesch (1999), the exact definition of EEG frequency band can vary between subjects, and hence band powers should not be considered as fully independent variables. We therefore applied the same methodology as in Marceglia et al. (2016), and summed the contributions of delta and theta bands to cover the whole 2-7 Hz "low-frequency" range (i.e., the power for delta and theta was calculated separately and then summed), and summed the contributions of the alpha and beta bands to cover the whole $8-25 \mathrm{~Hz}$ "high-frequency" band, and focused our analysis on these two broad bands.

In addition, we divided the scalp into four regions of interest, namely frontal area (Fp1, Fp2, F3, F4, F7, F8), temporo-parietal area (T3, T4, T5, T6, P3, P4), central area (C3, C4), and occipital area $(\mathrm{O} 1, \mathrm{O} 2)$. To obtain the low- and high- frequency power in each region of interest, we averaged EEG oscillations measured below each electrode belonging to the region. The right and the left areas were averaged, according to the assumption that no asymmetry is expected. 


\section{Statistical Analysis}

To assess the neuropsychological and behavioral effects induced by tDCS, each test for cognitive functions (PFT, VRT, PNT, GGT), arousal (sVRT), behavioral changes (NPI and FBI) and caregiver's burden (ZBI) was analyzed independently. In addition, considering their non-continuous nature, we applied non-parametric statistics for clinical scales whereas parametric statistic was applied to continuous variables (such as reaction times). To account for the low number of subjects available, we ran two separate non-parametric one-way Friedman's ANOVAs with factor "time" (4 levels, T0-T3), one for the Anodal and one for the Sham tDCS session, and we corrected the overall result for these two comparisons (Bonferroni correction, $p<$ 0.025). Then, to verify the effect at the single time points (whether existing) we applied post-hoc Wilcoxon signed ranked test with Bonferroni correction to take into account the effect of multiple comparisons $(p<0.01)$. We adopted the same analysis approach for continuous variables, but we used standard Bonferroni corrected one-way ANOVAs $(p<0.025)$ and Tukey's honest post-hoc test $(p<0.05)$ that already takes into account the effects of multiple comparisons (Cramer et al., 2016).

Finally, to have a direct comparison of the Anodal tDCS and Sham tDCS effects, we applied a two-way ANOVA with factors stimulation (2 levels, Anodal and Sham) and time (3 levels, T1$\mathrm{T} 3$ ) on the changes from baseline of the clinical scales at T1, T2, and T3. For this analysis, to obtain the changes from baseline of clinical scales, we normalized the scale scores for the total of the scale as it follows:

$$
D_{\mathrm{Tx}-\mathrm{T} 0}=\left(S_{\mathrm{Tx}}-S_{\mathrm{T} 0}\right) / S_{\mathrm{Tot}}
$$

Where $\mathrm{D}_{\mathrm{Tx}-\mathrm{T} 0}$ is the change of the scale at the time point $\mathrm{Tx}$ with respect to $\mathrm{T} 0, \mathrm{~S}_{\mathrm{Tx}}$ is the score at $\mathrm{Tx}, \mathrm{S}_{\mathrm{T} 0}$ is the score at $\mathrm{T} 0$, and $S_{\text {Tot }}$ is the total value of the scale. Conversely, for continuous variables, such as reaction times, we calculated the percentage change from baseline as it follows:

$$
\operatorname{Dperc}_{\mathrm{Tx}}=\left(R T_{\mathrm{Tx}}-R T_{\mathrm{T} 0}\right) / R T_{\mathrm{T} 0}^{*} 100
$$

Where RTperc $\mathrm{Tx}_{\mathrm{x}}$ is the percentage change from baseline of the continuous variable at the selected time point $\mathrm{Tx}, \mathrm{RT}_{\mathrm{Tx}}$ is value at $\mathrm{Tx}$, and $\mathrm{RT}_{\mathrm{T} 0}$ is the value at $\mathrm{T} 0$.

Since we only wanted to verify whether there was any difference between the effects of Anodal and Sham tDCS on the clinical scales, regardless of the time, we considered this ANOVA as a planned comparison and only the factor stimulation was taken into account, thus allowing us not to correct the $p$-value (Cramer et al., 2016).

The analysis of EEG, considering that nine patients is a small sample size to obtain statistically relevant conclusions, is considered as an exploratory study and, therefore, only descriptive statistics are reported. We however wanted to verify whether there was a relationship between clinical outcomes significantly modulated by tDCS application and qEEG features. We considered as multiple predictors the values of the clinical scales that resulted significantly modulated by tDCS, and the qEEG power in each region of interest and in each band of interest as dependent variables.
We therefore applied separate multiple linear regression analyses between the power of each EEG band in each region of interest and the clinical scores at all time points (T0-T3), including only those scales identified as significant by the previous statistical analysis.

\section{RESULTS}

\section{Neuropsychological and Behavioral Effects of tDCS}

To evaluate the effects of Anodal and Sham tDCS on neuropsychological and behavioral variables, we first assessed whether their time course showed significant changes in the two sessions. We found that, whereas Anodal tDCS significantly improved NPI scores and sVRTs, Sham tDCS failed to induce changes in the outcomes of these tests after its application.

More specifically, the non-parametric Friedman's ANOVA showed significant differences across time in NPI scores (Figure 2A) after Anodal tDCS $(p=0.006)$ but not after Sham tDCS $(p=0.11)$. Post-hoc analysis highlighted a significant decrease of NPI scores at T1 as compared to T0 after Anodal tDCS (T0 vs. T1: $16.09 \pm 2.76$ vs. $9.27 \pm 2.50, p=0.0077$ ), and a tendency to decrease at T2 and T3 as compared to T0 (vs. T2: $10.55 \pm 3.48, p=0.047$; vs. T3 $10.91 \pm 2.84, p=0.075)$. This differential effect of tDCS was confirmed by the comparative analysis between changes from baseline after Anodal and Sham tDCS, that showed a significant effect of the "stimulation type" $(p=0.034)$. Because, as shown in Figure 2A, the NPI score at T0 in the Sham condition is less, on average, than in the Anodal condition, we ran a Wilcoxon signed rank test between baseline (T0) values in the two tDCS conditions, and found that there is no statistical difference (T0 Sham vs. T0 Anodal: $8.83 \pm 9.15$ vs. $16.05 \pm 9.59, p=0.075)$.

Also sVRTs (Figure 2B) were improved by Anodal tDCS (ANOVA $p=0.025$ ) but not by Sham tDCS (ANOVA $p=0.15$ ). Post-hoc analysis showed a significant decrease of sVRTs at T1, T2, and T3 as compared to T0 after Anodal tDCS (T0 vs. T1 $671.59 \pm 132.1$ vs. $488.46 \pm 65.32, p=0.002$; $\mathrm{T} 2: 501.62 \pm 57.22$, $p=0.003$; T3: $465.63 \pm 49.34, p=0.005)$. The comparative analysis of percentage changes after Anodal and Sham tDCS confirmed this observation (ANOVA factor "stimulation type," $p=0.046)$.

No significant changes were observed in the other neuropsychological measures for both stimulation types. Figure $2 \mathrm{C}$ reports the behavior of FBI-A scores in the two stimulation conditions, that showed a tendency toward improvement after Anodal tDCS (non-parametric ANOVA $p=0.057)$.

Finally, we found a relationship between TAU protein $(\mathrm{pg} / \mathrm{mL})$ and MMSE score (Spearman's correlation coefficient $R^{2}=0.32$, $p=0.05$, Figure 2D).

\section{Correlation Between Clinical and qEEG Effects}

Table 2 reports the detailed descriptive statistics of LF and HF band power in all the different regions of interest during the 


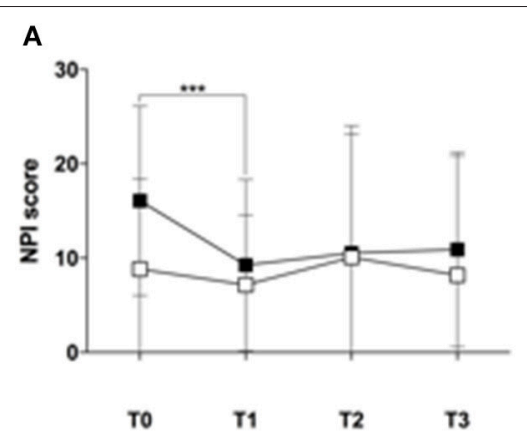

C

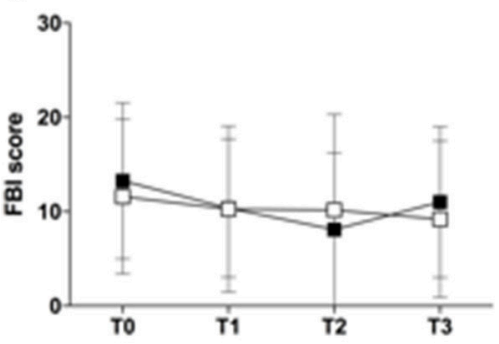

StDCS
B

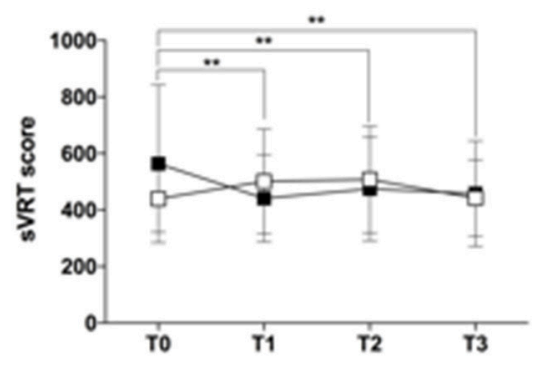

D

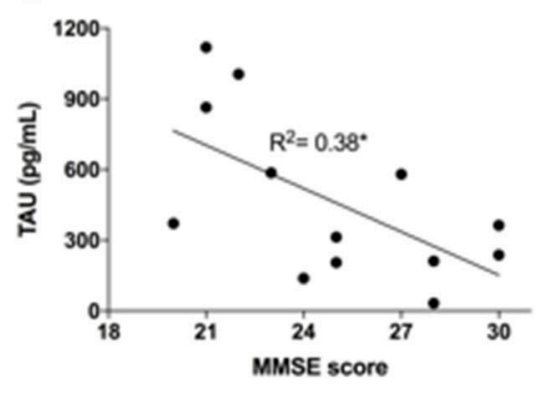

AtDCS

FIGURE 2 | Findings on clinical variables (A). Effect of Anodal (black squares) and Sham (white squares) tDCS on the Neuropsychiatric Inventory (NPI). Squares represent the average NPI score on the 12 subjects analyzed, at T0, T1, T2, and T3. Error bars are standard deviations. ${ }^{* \star *} p<0.01$ at the post-hoc Wilcoxon signed ranked test with Bonferroni correction (significant); (B). Effect of Anodal (black squares) and Sham (white squares) tDCS on the simple Visual Reaction Time (sVRT) test. Squares represent the average sVRT score on the 12 subjects analyzed, at T0, T1, T2, and T3. Error bars are standard deviations. ${ }^{\star \star} p<0.01$ at the post-hoc Wilcoxon signed ranked test with Bonferroni correction (significant) (C). Effect of Anodal (black squares) and Sham (white squares) tDCS on the Frontal Behavioral Inventory (FBI-A). Squares represent the average FBI-A score on the 12 subjects analyzed, at T0, T1, T2, and T3. Error bars are standard deviations (D). Scatter plot of the correlation between TAU protein $(\mathrm{pg} / \mathrm{mL})$ and MMSE score. The line represents the estimated linear regression. ${ }^{*} p<0.05$.

Anodal and Sham tDCS sessions. LF power shows a decreasing behavior more marked after Anodal than after Sham tDCS in the Frontal and Temporo-Parietal areas, thus supporting the hypothesis that Anodal tDCS improves the bioelectrical pattern of FTD patients. Conversely, it seems that the effect on HF band power is similar between Anodal and Sham tDCS, with a general decreasing behavior over time.

As shown in Figure 3, LF power in the Frontal area is significantly correlated to both NPI $(b=0.779, p=0.009)$ and sVRTs $(b=0.43, p=0.001)$, and LF power in the TemporoParietal area is correlated to sVRTs $(b=0.36, p=0.003)$ thus suggesting that the tendential improvement in the EEG pattern is consistent with the observed clinical improvement in these patients. Conversely, LF power in the Central and Occipital areas, as well as HF power in all the regions of interest did not significantly correlate with clinical outcomes.

\section{DISCUSSION}

Our findings showed that Anodal tDCS over the frontotemporal cortex improves both processing speed, as measured by simple reaction times, and the neuropsychiatric symptoms of dementia, as measured by the NPI scores, in FTD patients. The improvements observed were registered immediately after the end of the treatment and tended to be maintained after 1 week and 1 month. Also, the time course of the clinical measurements correlated with the time course of the neurophysiological qEEG pattern, showing a tendency toward normalization of LF activity which is known to be abnormally increased in dementia patients (Koberda et al., 2013).

The sVRT paradigm has been extensively used to measure processing speed and to evaluate attentional functions, and it is considered to be a suitable measure of dementia risk (Kochan et al., 2016). Indeed, people with AD present a slower reaction time, as well as prodromal individuals with Mild Cognitive Impairment (MCI) (Gorus et al., 2008). FTD patients tend to be slower than healthy controls in the RT paradigm, showing abnormal attentive processes related to frontal lesions. Manenti et al. (2015) found a reduction of vocal RTs during action naming after Anodal tDCS over the parietal cortex in a sample of patients with corticobasal syndrome, which is a neurodegenerative disorder that overlaps both clinically and neuropathologically with FTD (Manenti et al., 2015). Whereas, the choice of the tDCS target by Manenti et al. (2015) was based on the results on AD patients, which are usually stimulated over parietal or temporo-parietal areas, our tDCS target choice was based on the known characteristics of brain areas impairments in FTD patients. In fact, our sample 


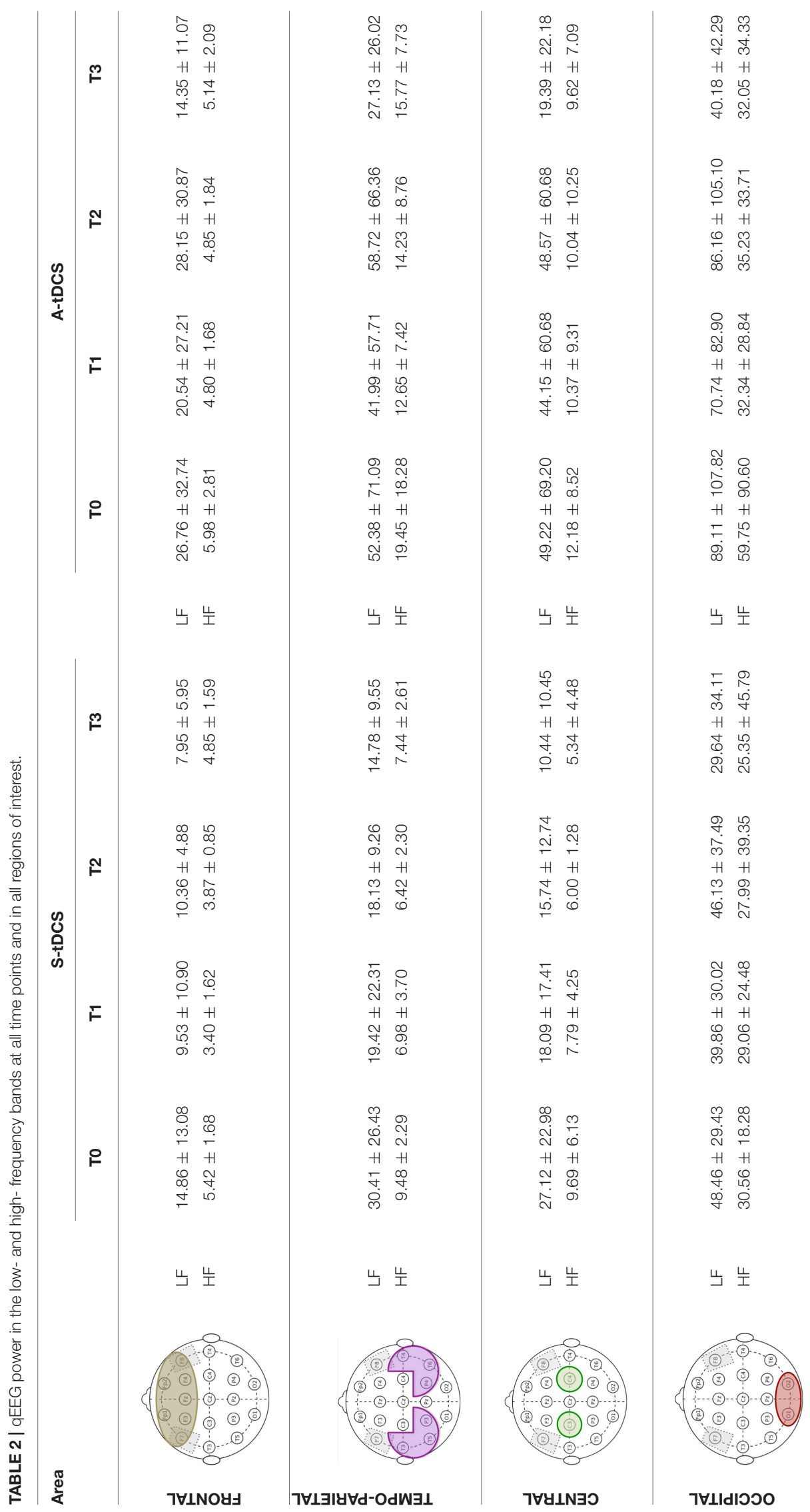



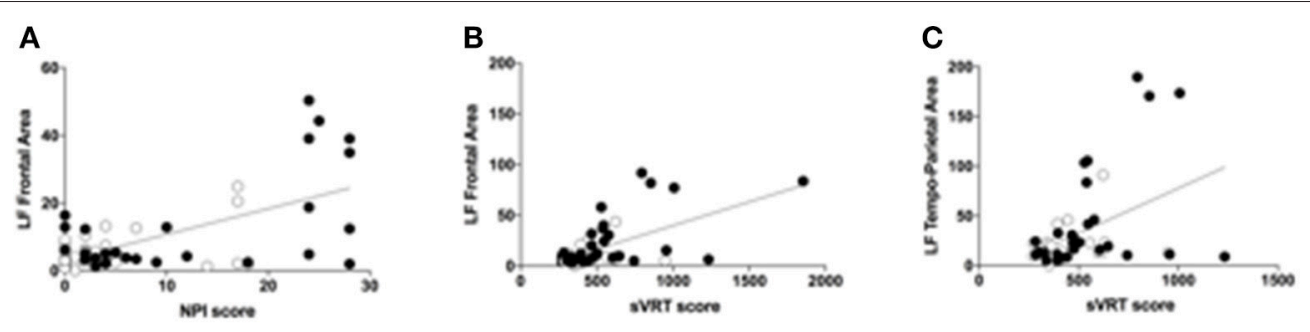

FIGURE 3 | Correlations between qEEG and clinical variables (A). Scatter plot of the correlation between the low-frequency (LB) band power calculated in the frontal area and the NPI scores. Black circles represent the values during the Anodal tDCS session, while the white circles represent the values during the Sham tDCS session. The scatter plot represents all the values at all time points (T0, T1, T2, and T3) (B). Scatter plot of the correlation between the low-frequency (LB) band power calculated in the frontal area and the sVRT scores. Black circles represent the values during the Anodal tDCS session, while the white circles represent the values during the Sham tDCS session. The scatter plot represents all the values at all time points (T0, T1, T2, and T3) (C). Scatter plot of the correlation between the low-frequency (LB) band power calculated in the temporo-parietal area and the SVRT scores. Black circles represent the values during the Anodal tDCS session, while the white circles represent the values during the Sham tDCS session. The scatter plot represents all the values at all time points (T0, T1, T2, and T3).

encompasses a heterogeneous group of FTD conditions and can be broadly divided into behavioral variant fronto-temporal dementia (bv-FTD) and primary progressive aphasia (PPA). BvFTD is associated with predominant atrophy in the frontal and paralimbic areas, while PPA is commonly associated with temporal atrophy. We therefore chose to stimulate the frontotemporal areas bilaterally. Furthermore, we used the Simple RTs task to measure general alertness and motor speed while Manenti et al. used a vocal reaction times to measure naming performance, and, despite different protocols, we obtained similar results. A reduction of RTs (perceptuo-motor vs. the verbal task) provide further evidence of the relationship between action and language. This fits in well with the perception-for-action-control theory (PACT) (Schwartz et al., 2002), stating that the perceptuomotor links contribute to co-structuring of perceptual and motor representations and to perceptual organization of speech (Basirat et al., 2012).

Reaction Time is an important factor in relation to the integrity and efficiency of brain functions, such as those involved in attention, cognition, and perception. It has been defined as a behavioral "marker" of neurophysiological integrity (Haworth et al., 2016) and it might provide a "real-life" indicator of changes to everyday functions. RTs studies allow measuring other parameters, such as fatigue, stimuli and threshold responses, processing load, resource availability and utilization, patterns of functional decline and integrity, and response to interventions.

The loss of white matter integrity is associated with a disproportionate slowing of RTs (Kerchner et al., 2012). In particular, cognitive processing speed is related to the integrity of the frontal lobe (Kochunov et al., 2010).

The reduction of RTs in FTD patients observed in this study after Anodal tDCS might represent a cognitive marker of increased functional integrity (i.e., normal functioning) (Phillips et al., 2013) in these patients. In fact, because excitability alterations have been shown to have a specific effect on RT task performance (Nitsche et al., 2003; Antal et al., 2004; Wade and Hammond, 2015), our findings suggest that the cortical excitability changes induced by tDCS can improve cerebral integrity. The improvement of this cognitive index was accompanied by a reduction of the neuropsychiatric symptoms of dementia (NPI scores). In contrast with our results, Huey et al. (2007), studying 10 FTD patients receiving single sessions of unilateral Anodal and Sham tDCS in the frontal areas (above F3 electrode in the international 10-20 system), found no effects of Anodal tDCS on NPI scores. The differences could depend on methodological issues, including the duration, type and site of stimulation. In fact, we applied tDCS bilaterally over the fronto-temporal areas for 5 consecutive days, thus suggesting that a longer exposure to tDCS might be more effective than the application of a single session (Lefaucheur et al., 2017)

The FTD patients involved in the present study displayed prominent apathy that is the most common neuropsychiatric symptom associated with FTD. The behavioral and biological mechanisms of apathy, however, are not well-understood. Massimo et al. (2015) hypothesized that goal-directed behavior is supported by a network of multiple frontal brain regions. Overall, data from studies on psychiatric disorders suggest that tDCS over the dorsolateral prefrontal cortex (DLPFC) (Brunoni et al., 2010, 2011; Kuo et al., 2014; D'Urso et al., 2017, 2018) has the potential to induce clinically relevant behavioral changes in difficult-totreat patient populations and could thus represent a valuable tool for intervention in a range of mental and neurological disorders.

Conversely, our findings of no tDCS-induced effects on language and verbal fluency confirms that of Huey et al. (2007). In fact, they did not find any effect of tDCS in improving verbal fluency. The authors proposed that this negative result may have been due to the fact that the stimulation session was not coupled with language therapy (Huey et al., 2007). Other studies that did not couple tDCS with language therapy have repeatedly yielded no improvement in both healthy and patient populations (Antal et al., 2007; Segrave et al., 2014). In contrast, Cotelli et al. (2014) found a beneficial effect of language training in combination with brain stimulation in PPA patients (Cotelli et al., 2014). Furthermore, the lack of language improvement could depend on sample characteristics (ceiling effect in linguistic tasks), given that most patients had predominantly behavioral symptoms.

Finally, the improvement in RT performance and NPI scores correlated with the qEEG pattern in the LF band that showed a tendency to decrease after Anodal tDCS. The abnormal increase of LF activity is suggested to be associated to Alzheimer's Disease 
(Duffy et al., 1984; Chiaramonti et al., 1997; Jelic et al., 2000; Kramer et al., 2007; Koberda et al., 2013; Fonseca et al., 2015), and, more specifically, to the slower information encording processes in these patients (Klimesch, 1999).

Despite exploratory, these results on the correlation between clinical and neurophysioogical variables suggests that studying qEEG features could help complementing clinical findings, especially in small groups of patients, by showing a tendency to improvements in the general brain state of the patients undergoing $\mathrm{tDCS}$ treatment.

This study was limited by the low sample size, which did not allow to run a full statistical comparison, especially for evaluating the effects of Active vs. Sham tDCS in time, and for comparing tDCS effects over the different variances of FTD. Further studies on a larger sample of FTD patients considering the different variants may be useful in understanding the maintenance effect of cognitive and behavioral improvement associated with fronto-temporal Anodal tDCS.

Altogether, these findings support the effectiveness of Anodal tDCS over the fronto-temporal regions in FTD on attentional processes, and suggest that tDCS-related improvements are

\section{REFERENCES}

Agarwal, S. M., Rajur, S., Bose, A., Shenoy, S., Miriyala, S., Shivakumar, V., et al. (2016). Use of transcranial direct current stimulation (tDCS) in a woman with behavioral variant fronto-temporal dementia. Asian J. Psychiatr. 21, 31-32. doi: 10.1016/j.ajp.2016.02.007

Allain, H., Bentue-Ferrer, D., Tribut, O., Merienne, M., and Belliard, S. (2003). Drug therapy of frontotemporal dementia. Hum. Psychopharmacol. 18, 221-225. doi: 10.1002/hup.472

Antal, A., Nitsche, M. A., Kincses, T. Z., Kruse, W., Hoffmann, K. P., and Paulus, W. (2004). Facilitation of visuo-motor learning by transcranial direct current stimulation of the motor and extrastriate visual areas in humans. Eur. J. Neurosci. 19, 2888-2892. doi: 10.1111/j.1460-9568.2004.03367.x

Antal, A., Terney, D., Poreisz, C., and Paulus, W. (2007). Towards unravelling task-related modulations of neuroplastic changes induced in the human motor cortex. Eur. J. Neurosci. 26, 2687-2691. doi: 10.1111/j.1460-9568.2007. 05896.x

Barbarotto, R., Laiacona, M., Frosio, R., Vecchio, M., Farinato, A., and Capitani, E. (1998). A normative study on visual reaction times and two Stroop colour-word tests. Ital. J. Neurol. Sci. 19, 161-170.

Basirat, A., Schwartz, J. L., and Sato, M. (2012). Perceptuo-motor interactions in the perceptual organization of speech: evidence from the verbal transformation effect. Philos. Trans. R. Soc. Lond. B Biol. Sci. 367, 965-976. doi: 10.1098/rstb.2011.0374

Boggio, P. S., Ferrucci, R., Mameli, F., Martins, D., Martins, O., Vergari, M., et al. (2012). Prolonged visual memory enhancement after direct current stimulation in Alzheimer's disease. Brain Stimul. 5, 223-230. doi: 10.1016/j.brs.2011.06.006

Boggio, P. S., Fregni, F., Valasek, C., Ellwood, S., Chi, R., Gallate, J., et al. (2009). Temporal lobe cortical electrical stimulation during the encoding and retrieval phase reduces false memories. PLoS ONE 4:e4959. doi: 10.1371/journal.pone.0004959

Brun, A., Englund, B., Gustafson, L., Passant, U., Mann, D. M. A., Neary, D., et al. (1994). Clinical and neuropathological criteria for frontotemporal dementia. J. Neurol. Neurosurg. Psychiatry 57, 416-418.

Brunoni, A. R., Nitsche, M. A., Bolognini, N., Bikson, M., Wagner, T., Merabet, L., et al. (2011). Clinical research with transcranial direct current stimulation (tDCS): challenges and future directions. Brain Stimul. 5, 175-195. doi: 10.1016/j.brs.2011.03.002

Brunoni, A. R., Teng, C. T., Correa, C., Imamura, M., Brasil-Neto, J. P., Boechat, R., et al. (2010). Neuromodulation approaches for the treatment of major depression: challenges and recommendations from a working group meeting. Arq. Neuropsiquiatr. 68, 433-451. doi: 10.1590/1414-431X20133115 related to a normalization of low frequency oscillations at the frontal and temporo-parietal levels.

\section{AUTHOR CONTRIBUTIONS}

RF, SM-S, ES, AP, and SM: design/conception; RF, SM-S, SG, FB, MV, FM, AA, MS, FB, GM, AP, LG, GF, GD, PC, ES, AMP, and SM: literature and database search; SM, SM-S, and RF: data analysis; RF, SM-S, SG, FB, MV, FM, AA, MS, FB, GM, AP, LG, GF, GD, PC, ES, AMP, and SM: writing the initial draft of the manuscript; All authors critically revised and approved the final manuscript.

\section{ACKNOWLEDGMENTS}

The study was partly supported by POR-FESR 2014-2010 (ID247367), by donation in memory of Aldo Ravelli, by the Italian Ministry of Health grant (RC-2017 and GR-2011- 02352807) and Roche Research grant 2017. GF was supported by Associazione Italiana Ricerca Alzheimer ONLUS (AIRAlzh Onlus)-COOP Italia.

Chiaramonti, R., Muscas, G. C., Paganini, M., Müller, T. J., Fallgatter, A. J., Versari, A., et al. (1997). Correlations of topographical EEG features with clinical severity in mild and moderate dementia of Alzheimer type. Neuropsychobiology 36, 153-158.

Cotelli, M., Manenti, R., Petesi, M., Brambilla, M., Cosseddu, M., Zanetti, O., et al. (2014). Treatment of primary progressive aphasias by transcranial direct current stimulation combined with language training. J. Alzheimers Dis. 39, 799-808. doi: 10.3233/JAD-131427

Cramer, A. O. J., van Ravenzwaaij, D., Matzke, D., Steingroever, H., Wetzels, R., Grasman, R. P. P. P., et al. (2016). Hidden multiplicity in exploratory multiway ANOVA: Prevalence and remedies. Psychon. Bull. Rev. 23:640. doi: 10.3758/s13423-015-0913-5

Cummings, J. L., Mega, M., Gray, K., Rosenberg-Thompson, S., Carusi, D. A., and Gornbein, J. (1994). The Neuropsychiatric Inventory: comprehensive assessment of psychopathology in dementia. Neurology 44, 2308-2314. doi: 10.1016/j.acn.2005.04.011

Delorme, A., and Makeig, S. (2004). EEGLAB: an open source toolbox for analysis of single-trial EEG dynamics including independent component analysis. J. Neurosci. Methods 134, 9-21. doi: 10.1016/j.jneumeth.2003.10.009

Duffy, F. H., Albert, M. S., and McAnulty, G. (1984). Brain electrical activity in patients with presenile and senile dementia of the Alzheimer type. Ann. Neurol. 16, 439-448. doi: 10.1002/ana.410160404

D’Urso, G., Dell'Osso, B., Rossi, R., Brunoni, A. R., Bortolomasi, M., Ferrucci, R., et al. (2017). Clinical predictors of acute response to transcranial direct current stimulation (tDCS) in major depression. J. Affect. Disord. 219, 25-30. doi: 10.1016/j.jad.2017.05.019

D’Urso, G., Mantovani, A., Patti, S., Toscano, E., and de Bartolomeis, A. (2018). Transcranial direct current stimulation in obsessive-compulsive disorder, posttraumatic stress disorder, and anxiety disorders. J. ECT 34, 172-181. doi: 10.1097/YCT.0000000000000538

Elder, G. J., and Taylor, J. P. (2014).Transcranial magnetic stimulation and transcranial direct current stimulation: treatments for cognitive and neuropsychiatric symptoms in the neurodegenerative dementias? Alzheimers. Res. Ther. 6:74. doi: 10.1186/s13195-014-0074-1

Ferrucci, R., Mameli, F., Guidi, I., Mrakic-Sposta, S., Vergari, M., Marceglia, S., et al. (2008). Transcranial direct current stimulation improves recognition memory in Alzheimer disease. Neurology 71, 493-498. doi: 10.1212/01.wnl.0000317060.43722.a3

Floel, A. (2014). tDCS-enhanced motor and cognitive function in neurological diseases. Neuroimage 85(Pt 3), 934-947. doi: 10.1016/j.neuroimage.2013.05.098 Fonseca, L. C., Tedrus, G. M. A. S., Rezende, A. L. R. A., and Giordano, H. F. (2015). Coherence of brain electrical activity: a quality of life indicator 
in Alzheimer's disease? Coerência da atividade elétrica cerebral: indicador da qualidade de vida na doença de Alzheimer? Arq. Neuropsiquiatr. 73, 396-401. doi: 10.1590/0004-282X20150035

Gorus, E., De Raedt, R., Lambert, M., Lemper, J. C., and Mets, T. (2008). Reaction times and performance variability in normal aging, mild cognitive impairment, and Alzheimer's disease. J. Geriatr. Psychiatry Neurol. 21, 204-218. doi: 10.1177/0891988708320973

Haworth, J., Phillips, M., Newson, M., Rogers, P. J., Torrens-Burton, A., and Tales, A. (2016). Measuring information processing speed in mild cognitive impairment: clinical versus research dichotomy. J. Alzheimers. Dis. 51, 263-275. doi: 10.3233/JAD-150791

Huey, E. D., Probasco, J. C., Moll, J., Stocking, J., Ko, M. H., Grafman, J., et al. (2007). No effect of DC brain polarization on verbal fluency in patients with advanced frontotemporal dementia. Clin. Neurophysiol. 118, 1417-1418. doi: 10.1016/j.clinph.2007.02.026

Jelic, V., Johansson, S.-E., Almkvist, O., Shigeta, M., Julin, P., Nordberg, A., et al. (2000). Quantitative electroencephalography in mild cognitive impairment: longitudinal changes and possible prediction of Alzheimer's disease. Neurobiol. Aging 21, 533-540. doi: 10.1016/S0197-4580(00)00153-6

Kerchner, G. A., Racine, C. A., Hale, S., Wilheim, R., Laluz, V., Miller, B. L., et al. (2012). Cognitive processing speed in older adults: relationship with white matter integrity. PLOS ONE 7:e50425. doi: 10.1371/journal.pone. 0050425

Kertesz, A., Davidson, W., and Fox, H. (1997). Frontal behavioral inventory: diagnostic criteria for frontal lobe dementia. Can. J. Neurol. Sci. 24, 29-36.

Klimesch, W. (1999). EEG alpha and theta oscillations reflect cognitive and memory performance: a review and analysis. Brain Res. Brain Res. Rev. 29, 169-195.

Koberda, J. L., Moses, A., Koberda, P., and Koberda, L. (2013). Clinical advantages of quantitative electroencephalogram (QEEG)-electrical neuroimaging application in general neurology practice. Clin. EEG Neurosci. 44, 273-285. doi: 10.1177/1550059412475291

Kochan, N. A., Bunce, D., Pont, S., Crawford, J. D., Brodaty, H., and Sachdev, P. S. (2016). Reaction time measures predict incident dementia in community-living older adults: the sydney memory and ageing study. Am. J. Geriatr. Psychiatry 24, 221-231. doi: 10.1016/j.jagp.2015.12.005

Kochunov, P., Coyle, T., Lancaster, J., Robin, D. A., Hardies, J., Kochunov, V., et al. (2010). Processing speed is correlated with cerebral health markers in the frontal lobes as quantified by neuroimaging. Neuroimage 49, 1190-1199. doi: 10.1016/j.neuroimage.2009.09.052

Kramer, M. A., Chang, F.-L., Cohen, M. E., Hudson, D., and Szeri, A. J. (2007). Synchronization measures of the scalp electroencephalogram can discriminate healthy from Alzheimer's subjects. Int. J. Neural Syst. 17, 61-69. doi: 10.1142/S0129065707000932

Kuo, M. F., Paulus, W., and Nitsche, M. A. (2014). Therapeutic effects of non-invasive brain stimulation with direct currents (tDCS) in neuropsychiatric diseases. Neuroimage 85 ( $\mathrm{Pt} \quad 3)$, 948-960. doi: 10.1016/j.neuroimage.2013.05.117

Lefaucheur, J. P., Antal, A., Ayache, S. S., Benninger, D. H., Brunelin, J., Cogiamanian, F., et al. (2017). Evidence-based guidelines on the therapeutic use of transcranial direct current stimulation (tDCS). Clin. Neurophysiol. 128, 56-92. doi: 10.1016/j.clinph.2016.10.087

Manenti, R., Bianchi, M., Cosseddu, M., Brambilla, M., Rizzetti, C., Padovani, A., et al. (2015). Anodal transcranial direct current stimulation of parietal cortex enhances action naming in Corticobasal Syndrome. Front. Aging Neurosci. 7:49. doi: 10.3389/fnagi.2015.00049

Marceglia, S., Mrakic-Sposta, S., Rosa, M., Ferrucci, R., Mameli, F., Vergari, M., et al. (2016). Transcranial direct current stimulation modulates cortical neuronal activity in Alzheimer's disease. Front. Neurosci. 10:134. doi: 10.3389/fnins.2016.00134

Massimo, L., Powers, J. P., Evans, L. K., McMillan, C. T., Rascovsky, K., Eslinger, P., et al. (2015). Apathy in frontotemporal degeneration: neuroanatomical evidence of impaired goal-directed behavior. Front. Hum. Neurosci. 9:611. doi: 10.3389/fnhum.2015.00611

Nitsche, M. A., Schauenburg, A., Lang, N., Liebetanz, D., Exner, C., Paulus, W., et al. (2003). Facilitation of implicit motor learning by weak transcranial direct current stimulation of the primary motor cortex in the human. Cogn. Neurosci. 15, 619-626. doi: 10.1162/089892903321662994
Novelli, G., Papagno, C., Capitani, E., Laiacona, M., Cappa, S. F., Vallar, G. (1986). Tre test clinici di ricerca e produzione lessicale. Taratura su soggetti normali. Arch. Psicol. Neurol. Psichiatr. 47, 477-506.

Phillips, M., Rogers, P., Haworth, J., Bayer, A., and Tales, A. (2013). Intraindividual reaction time variability in mild cognitive impairment and Alzheimer's disease: gender, processing load and speed factors. PLoS ONE 8:e65712. doi: 10.1371/journal.pone.0065712

Piguet, O., and Hodges, J. R. (2013). Behavioural-variant frontotemporal dementia: an update. Dement. Neuropsychol. 7, 10-18. doi: 10.1590/S1980-57642013DN70100003

Roncero, C., Kniefel, H., Service, E., Thiel, A., Probst, S., and Chertkow, H. (2017). Inferior parietal transcranial direct current stimulation with training improves cognition in anomic Alzheimer's disease and frontotemporal dementia. Alzheimers Dement (N Y). 3, 247-253. doi: 10.1016/j.trci.2017.03.003

Schwartz, J.-L., Abry, C., Boë L.-J., and Cathiard, M. (2002). "Phonology in a theory of perception-for-action-control," in Phonetics, Phonology and Cognition, eds J. Durand, and B. Laks (New York, NY: Oxford University Press), 244-280.

Segrave, R. A., Arnold, S., Hoy, K., and Fitzgerald, P. B. (2014). Concurrent cognitive control training augments the antidepressant efficacy of tDCS: a pilot study. Brain Stimul. 7, 325-331. doi: 10.1016/j.brs.2013.12.008

Seltman, R. E., and Matthews, B. R. (2012). Frontotemporal lobar degeneration: epidemiology, pathology, diagnosis and management. CNS Drugs 26, 841-870. doi: 10.2165/11640070-000000000-00000

Shin, Y. I., Foerster, A., and Nitsche, M. A. (2015). Transcranial direct current stimulation (tDCS)-application in neuropsychology. Neuropsychologia 69, 154-175. doi: 10.1016/j.neuropsychologia.2015.02.002

Summers, J. J., Kang,. N., and Cauraugh, J. H. (2016). Does transcranial direct current stimulation enhance cognitive and motor functions in the ageing brain? A systematic review and meta-analysis. Ageing Res. Rev. 25, 42-54. doi: 10.1016/j.arr.2015.11.004

van Harten, A. C., Kester, M. I., Visser, P. J., Blankenstein, M. A., Pijnenburg, Y. A., van der Flier, W. M., et al. (2011). Tau and p-tau as CSF biomarkers in dementia: a meta-analysis. Clin. Chem. Lab. Med. 49, 353-366. doi: 10.1515/CCLM.2011.086

Vanmechelen, E., Vanderstichele, H., Davidsson, P., Van Kerschaver, E., Van Der Perre, B., Sjögren, M., et al. (2000). Quantification of tau phosphorylated at threonine 181 in human cerebrospinal fluid: a sandwich ELISA with a synthetic phosphopeptide for standardization. Neurosci. Lett. 285, 49-52. doi: 10.1016/S0304-3940(00)01036-3

Viggiano, M. P., Vannucci, M., and Righi, S. (2004). A new standardized set of ecological pictures for experimental and clinical research on visual object processing. Cortex 40, 491-509. doi: 10.1016/S0010-9452(08)70142-4

Wade, S., and Hammond, G. (2015). Anodal transcranial direct current stimulation over premotor cortex facilitates observational learning of a motor sequence. Eur. J. Neurosci. 41, 1597-1602. doi: 10.1111/ejn.12916. Epub 2015

Woods, A. J., Antal, A., Bikson, M., Boggio, P. S., Brunoni, A. R., Celnik, P., et al. (2016). A technical guide to tDCS, and related non-invasive brain stimulation tools. Clin. Neurophysiol. 127, 1031-1048. doi: 10.1016/j.clinph.2015.11.012

Zarit, S. H., and Zarit, J. M. (1990). The Memory and Behavior Problems Checklist and the Burden Interview. University Park, PA: Pennsylvania State University, Gerontology Center.

Conflict of Interest Statement: RF, SM-S, MV, FM, AP, and SM are stakeholders in Newronika s.r.l., a spin-off company formed by the Fondazione IRCCS Ca' Granda Ospedale Maggiore Policlinico and Università degli Studi di Milano, Italy.

The remaining authors declare that the research was conducted in the absence of any commercial or financial relationships that could be construed as a potential conflict of interest.

Copyright (c) 2018 Ferrucci, Mrakic-Sposta, Gardini, Ruggiero, Vergari, Mameli, Arighi, Spallazzi, Barocco, Michelini, Pietroboni, Ghezzi, Fumagalli, D'Urso, Caffarra, Scarpini, Priori and Marceglia. This is an open-access article distributed under the terms of the Creative Commons Attribution License (CC BY). The use, distribution or reproduction in other forums is permitted, provided the original author(s) and the copyright owner(s) are credited and that the original publication in this journal is cited, in accordance with accepted academic practice. No use, distribution or reproduction is permitted which does not comply with these terms. 


\section{OPEN ACCESS}

Edited by:

Nicholas J. Kelley,

Northwestern University,

United States

Reviewed by:

Adriana Salatino,

Università degli Studi di Torino, Italy

Winston D. Byblow,

University of Auckland, New Zealand

*Correspondence:

Ferdinando Sartucci

ferdinando.sartucci@med.unipi.it

Received: 28 February 2018 Accepted: 09 May 2018

Published: 29 May 2018

Citation:

Bocci T, Nasini F, Caleo M, Restani L, Barloscio D, Ardolino G, Priori A Maffei L, Nardi $M$ and Sartucci $F$ (2018) Unilateral Application of Cathodal tDCS Reduces Transcallosal Inhibition and Improves Visual Acuity in Amblyopic Patients. Front. Behav. Neurosci. 12:109. doi: 10.3389/fnbeh.2018.00109

\section{Unilateral Application of Cathodal tDCS Reduces Transcallosal Inhibition and Improves Visual Acuity in Amblyopic Patients}

\author{
Tommaso Bocci 1,2, Francesco Nasini ${ }^{3}$, Matteo Caleo ${ }^{4}$, Laura Restani ${ }^{4}$, Davide Barloscio ${ }^{1}$, \\ Gianluca Ardolino ${ }^{2}$, Alberto Priori ${ }^{2,5}$, Lamberto Maffei $^{4}$, Marco Nardi ${ }^{3}$ \\ and Ferdinando Sartucci ${ }^{1,4 *}$

\begin{abstract}
'Section of Neurophysiopathology, Department of Clinical and Experimental Medicine, University of Pisa, Pisa, Italy, ${ }^{2}$ Clinical Center for Neurotechnologies, Neuromodulation, and Movement Disorders, Fondazione IRCCS Ca'Granda Ospedale Maggiore Policlinico, Milan, Italy, ${ }^{3}$ Department of Surgical, Medical, and Molecular Pathology and Critical Care, University of Pisa, Pisa, Italy, ${ }^{4} \mathrm{CNR}$ Institute of Neuroscience, University of Pisa, Pisa, Italy, ${ }^{5}$ Department of Health Sciences, University of Milan and Ospedale San Paolo, Milan, Italy
\end{abstract}

Objective: Amblyopia is a neurodevelopmental disorder characterized by visual acuity and contrast sensitivity loss, refractory to pharmacological and optical treatments in adulthood. In animals, the corpus callosum (CC) contributes to suppression of visual responses of the amblyopic eye. To investigate the role of interhemispheric pathways in amblyopic patients, we studied the response of the visual cortex to transcranial Direct Current Stimulation (tDCS) applied over the primary visual area (V1) contralateral to the "lazy eye."

Methods: Visual acuity (logMAR) was assessed before $\left(T_{0}\right)$, immediately after $\left(T_{1}\right)$ and 60 ' following the application of cathodal tDCS (2.0 mA, 20') in 12 amblyopic patients. At each time point, Visual Evoked Potentials (VEPs) triggered by grating stimuli of different contrasts (K90\%, K20\%) were recorded in both hemispheres and compared to those obtained in healthy volunteers.

Results: Cathodal tDCS improved visual acuity respect to baseline $(p<0.0001$ ), whereas sham polarization had no significant effect. At $T_{1}$, tDCS induced an inhibitory effect on VEPs amplitudes at all contrasts in the targeted side and a facilitation of responses in the hemisphere ipsilateral to the amblyopic eye; compared with controls, the facilitation persisted at $\mathrm{T}_{2}$ for high contrasts (K90\%; Holm-Sidak post hoc method, $p<0.001)$, while the stimulated hemisphere recovered more quickly from inhibition (Holm-Sidak post hoc method, $p<0.001$ ).

Conclusions: tDCS is a promising treatment for amblyopia in adults. The rapid recovery of excitability and the concurrent transcallosal disinhibition following perturbation of cortical activity may support a critical role of interhemispheric balance in the pathophysiology of amblyopia.

Keywords: amblyopia, tDCS, amblyopia treatment in adults, corpus callosum, ocular dominance, visual system plasticity 


\section{INTRODUCTION}

Amblyopia is a neurodevelopmental disorder clinically characterized by visual acuity and contrast sensitivity loss, refractory to pharmacological and mechanical treatments in adulthood (Holmes and Clarke, 2006): given the lack of any organic cause, it has been also defined as a disorder "in which the patient sees nothing and the doctor sees nothing" (Holmes and Clarke, 2006). Amblyopia results in an abnormal binocular experience due to a mismatch between the images perceived with each eye. Although the retina is generally spared, microscopic anatomical and structural abnormalities in lateral geniculate bodies and visual cortex can occur (von Noorden and Crawford, 1992; Davis et al., 2003); fMRI studies are consistent with the hypothesis of a selective involvement of the parvocellular stream at a precortical or early cortical site, thus leading to detection and processing deficit for high-contrast stimuli (Li et al., 2007; Hess et al., 2010).

Permanent monocular visual impairment is a risk for blindness, if the dominant eye is injured or becomes affected later in life (Williams et al., 2003). For this reason, the early treatment is critical. Eye-patching has been used for centuries, whereas the use of atropine has only recently emerged (Repka et al., 2005).

In the past few years, new approaches are being developed, such as dichoptic visual training aimed at stimulating the amblyopic eye, reducing the interocular suppression by balancing stimulus contrast between visual hemifields (Stewart et al., 2007; Vedamurthy et al., 2015b; Žiak et al., 2017). Nonetheless, all these treatments appear to be effective for up to 7 years of age (Holmes et al., 2011), showing transient and inconclusive results in older patients (Gao et al., 2018). Moreover, current treatments are often associated with residual monocular and binocular deficits (Pediatric Eye Disease Investigator Group et al., 2008), with a high rate of recurrence (Bhola et al., 2006).

In animal models, the corpus callosum (CC) plays a critical role in the suppression of deprived eye responses after a period of monocular occlusion (Restani et al., 2009; Cerri et al., 2010); in humans, callosal connections appear to inhibit the responsiveness of the neurons located in the opposite hemisphere (Bocci et al., 2014). Moreover, reduced visual cortex excitability has been observed in patients with amblyopia, possibly reflecting abnormally high levels of cortico-cortical inhibition (Thompson et al., 2008; Hess and Thompson, 2015). Thus, the reduced responses of the amblyopic eye may be due to active inhibition (suppression) within the primary visual cortex. Here we tested the contribution of interhemispheric pathways to such inhibition.

It has been recently proved that inter-hemispheric connections regulate cortical gain by dampening neural responses to high-contrast stimuli in the target hemisphere (Bocci et al., 2011). Concurrently, we have suggested that the rapid recovery of excitability and the persistent transcallosal disinhibition following perturbation of cortical activity may exert a key role in the pathophysiology of photosensitive epilepsy (Bocci et al., 2016). Altogether, we reasoned that changes in transcallosal inhibition may explain the unbalanced mechanisms of contrast gain control and ocular dominance in amblyopia. To this aim, we enrolled 12 patients and compared changes in visual acuity and Visual Evoked Potential (VEP) amplitudes induced by inhibitory cathodal transcranial Direct Current Stimulation (tDCS) applied to the occipital lobe contralateral to the amblyopic eye.

\section{MATERIALS AND METHODS}

\section{Participants and Experimental Protocol}

Twelve adult patients with unilateral amblyopia (Table 1) and 12 sex and age-matched healthy volunteers were enrolled in the study (mean age $26.1 \pm 6.0$ years; range 24-44, five females). Patients had an intraocular acuity difference of at least 0.2 LogMAR and were classified as strabismic, anisometropic or mixed amblyopia (both strabismus and anisometropia; see Table 1). Anisometropia was defined as a spherical equivalent difference of 1 dioptre or more between the eyes. Best refractive correction was worn during testing. Healthy volunteers did not have history of neurological or psychiatric disorders and they were all drug-free. Controls with normal vision had 0.1 LogMAR acuity or better in each eye and no history of visual disorders.

In patients, visual acuity was assessed at baseline (T0), immediately after (T1) and 60' following the completion of tDCS applied over the primary visual area (V1) contralateral to the amblyopic eye.

At same time points, VEPs were recorded both in amblyopic patients and controls, at two different luminance contrasts (K90\% and K20\%).

Patients were enrolled by a clinician (FN), whereas electrophysiological recordings were performed by a different neurologist (DB), both blinded to the tDCS condition.

Written informed consent was signed by all subjects prior to participation in the study, approved by the local ethical Committee in accordance with the tenets of Helsinki. The study was approved by the local ethical committee (registration number 3135), at the University of Pisa (formally named "Comitato Etico di Area Vasta Nord Ovest della Toscana").

\section{Transcranial Direct Current Stimulation (tDCS)}

We applied tDCS over the V1, using a battery-driven constant current stimulator (HDCStim, Newronika, Italy) and a pair of electrodes in two saline-soaked synthetic sponges with a surface area of $25 \mathrm{~cm}^{2}(5 \times 5 \mathrm{~cm})$. Amblyopic patients underwent both cathodal (real) and sham stimulation, while in healthy controls only the cathodal polarization was applied. For cathodal stimulation, the cathode was centered either on $\mathrm{O} 1$ or $\mathrm{O} 2$ (according to the 10-20 international EEG system) and the anode on the right shoulder.

Anatomical correspondence between the target region and V1 was confirmed by a navigated stimulation system 
TABLE 1 | Demographic and clinical assessment.

\begin{tabular}{|c|c|c|c|c|}
\hline Patient's number & Previous treatment & Type of Amblyopia & Visual acuity (logMAR) & Timeline of intervention \\
\hline \multirow[t]{2}{*}{1} & None & LE & 0.0 & cathodal/sham \\
\hline & & RE Aniso & $0.55(0.55)$ & \\
\hline 2 & None & LE & 0.0 & cathodal/sham \\
\hline \multirow[t]{2}{*}{3} & None & $\mathrm{RE}$ & 0.0 & sham/cathodal \\
\hline & & LE Aniso & $0.40(0.38)$ & \\
\hline 4 & None & $\mathrm{RE}$ & 0.0 & cathodal/sham \\
\hline 5 & & LE Aniso & $0.22(0.22)$ & \\
\hline \multirow[t]{2}{*}{6} & None & LE & 0.0 & cathodal/sham \\
\hline & & RE Strab & $0.55(0.58)$ & \\
\hline \multirow[t]{2}{*}{7} & Patching & LE & 0.0 & sham/cathodal \\
\hline & & RE Aniso & $0.38(0.42)$ & \\
\hline 8 & None & RE & 0.0 & sham/cathodal \\
\hline \multirow[t]{2}{*}{11} & None & LE & 0.0 & cathodal/sham \\
\hline & & RE Aniso & $0.92(0.84)$ & \\
\hline \multirow[t]{2}{*}{12} & Patching & $\mathrm{RE}$ & 0.0 & sham/cathodal \\
\hline & & LE Strab & $0.26(0.26)$ & \\
\hline
\end{tabular}

Each patient underwent both cathodal and sham tDCS, elapsed by at least 1 week. Visual acuity, expressed as LogMAR, refers to the first clinical evaluation, immediately after the enrollment, but it was checked again before the second treatment (either sham or cathodal; in this case, checked values of the "lazy eye" are expressed within brackets). LE, left eye; RE, right eye; Aniso, anisomoetropic amblyopia; Strab, strabismic amblyopia. LogMAR was calculated according to the formula: LogMAR = -log (decimal acuity).

(SofTaxic optically-tracked by EMS, Italy). Tridimensional space positions of the head and electrode were reproduced on the computer screen in relation to an average brain anatomy based on a 3D realistic MR-constructed brain model: in accordance with previous articles, the lower horizontal border of the electrode was marked by a scalp point superficial to the tentorium cerebelli, while the medial vertical one corresponded to a scalp point superficial to the brain location $1 \mathrm{~cm}$ lateral to the interhemispheric falx cerebri (Olma et al., 2013; Behrens et al., 2017).

Direct currents were applied for $20 \mathrm{~min}$ with an intensity of $1.5 \mathrm{~mA}$ (current density $0.06 \mathrm{~mA} / \mathrm{cm}^{2}$ ). The intensity and duration of stimulation were comparable to those used in previous studies (Antal et al., 2004, 2006; Lang et al., 2007), below the threshold for tissue damage (Nitsche et al., 2003). tDCS strength remained below the sensory threshold throughout the experimental session. At the offset of tDCS, the current was decreased in a ramp-like manner, a method shown to achieve a good level of blinding among sessions (Gandiga et al., 2006; Galea et al., 2009). In the sham condition, the current was turned on for $5 \mathrm{~s}$ and then turned off in a ramp-shaped fashion.

\section{Visual Acuity Assessment}

Each patient underwent a complete ophthalmologic examination to exclude other causes of poor vision, thus confirming that the patient's refractive correction (where applicable) was accurate in order to perform the The Best-Corrected Visual Acuity (BCVA) testing.
The BCVA was tested for both eyes by means of standard "Early Treatment Diabetic Retinopathy Study (ETDRS) Revised" translucent visual acuity charts (with the following features: same number of letters per line, equal spacing between lines on a $\log$ scale, equal spacing of letters on a log scale and balanced letter difficulty in the individual lines). Retro-illuminated ETDRS viewing cabinet was used.

Both eyes were separately tested at a distance of $4 \mathrm{~m}$ (about 13 feet). Chart 1 was used for visual acuity testing of the right eye and chart 2 for testing the left eye. The patient was asked to read slowly, beginning from the top line of the chart, from left to right. The patient was told that one chance is given to read each letter. If the patient changed a response (e.g., "that was a "C" not an "O"') before he/she has read the next letter, then the change was accepted.

- If a patient was able to read at least 20 letters on the chart, the visual acuity score of the tested eye was recorded as the number of letters read correctly at $4 \mathrm{~m}($ sum $=\mathrm{A})$ plus 30 ( credit of 30 score points $=B$ ).

- If a patient could not read at least 20 letters on the chart at $4 \mathrm{~m}$, the test was repeated at a distance of $1 \mathrm{~m}$. In this case, the visual acuity score for the tested eye was recorded as the number of letters read correctly at $1 \mathrm{~m}(\mathrm{sum}=\mathrm{C})$ plus the number of letters read correctly at $4 \mathrm{~m}($ sum $=\mathrm{A})$.

For each eye, the visual acuity score was the sum of A, B and C. If no letters were read correctly at either $4 \mathrm{~m}$ or $1 \mathrm{~m}$, the visual acuity score was recorded as "0". All procedures were done by an expert and certified ophthalmologist (FN). 


\section{Visual Evoked Potentials (VEPs)}

A detailed description of the protocol has been reported elsewhere (Bocci et al., 2011, 2016). VEPs were recorded in response to abrupt reversal $(3 \mathrm{~Hz})$ of a horizontal square wave grating (spatial frequency $2 \mathrm{c} / \mathrm{deg}$ ), generated by computer on a display (Sony; refresh rate $60 \mathrm{~Hz}$; subtending $20 \times 15^{\circ}$ of visual angle) by a VSG card (Cambridge Research Systems). The display was centered on the vertical meridian. VEPs were recorded simultaneously in both hemispheres, with $\mathrm{Ag} / \mathrm{AgCl}$ electrodes positioned $2 \mathrm{~cm}$ above the inion (active) and at the right mastoid (reference).

VEP amplitudes were defined as the difference between the N1 negative peak and the P1 positive peak amplitudes in microvolts (Ding et al., 2016). The N1 was defined as a negative peak $60-110 \mathrm{~ms}$ after the pattern reversal and the peak of the first positive wave after $\mathrm{N} 1$ was named as $\mathrm{P} 1$.

VEPs were recorded before $\left(\mathrm{T}_{0}\right)$, at the end $\left(\mathrm{T}_{1}\right)$ and $45^{\prime}$ $\left(\mathrm{T}_{2}\right)$ after tDCS. Grating stimuli were centered on the fixation point and tDCS was applied to V1. We analyzed 18 blocks of 100 averaged VEP responses ( 6 blocks at T0, 6 at T1 and 6 at T2), in terms of both mean amplitude (expressed as $\mu \mathrm{V}$ ) and latency (ms) for two contrast levels (K90\% and 20\%). Visual stimuli at different contrasts were presented randomly and the obtained electrophysiological responses for each contrast were then averaged.

\section{Statistical Analysis}

Parametric analyses were used, as all data sets successfully passed the Shapiro-Wilk test for normality $(p>0.05)$. A one-way analyses of variance (ANOVA) was used to compare baseline values for each subject between sham and cathodal condition. As VEP amplitudes are higher in healthy subjects and in the fellow eyes compared with the amblyopic ones, all values were normalized at baseline $\left(\mathrm{T}_{0}\right.$, i.e., before $\mathrm{tDCS}$ : $\left.(\mathrm{T} 1 / \mathrm{T} 0) \times 100 \%\right)$.

\section{Visual Acuity}

In each patient, changes in visual acuity (logMAR) were assessed by using a two-way repeated-measures (RM) ANOVA, with "stimulation" (two levels: cathodal and sham) and "time" (three levels: $\mathrm{T}_{0}, \mathrm{~T}_{1}$ and $\mathrm{T}_{2}$ ) as experimental factors, followed by
Holm-Sidak post hoc method. The Pearson's correlation was used to compare the average changes in visual acuity respect to the baseline values.

\section{Electrophysiological Measures (VEPs)}

A three-way repeated measures ANOVA assessed the effects of "time" $\times$ "stimulation" $\times$ "contrast" interaction in amblyopic patients. At each time interval, a two-way RM ANOVA compared peak-to-peak amplitudes between cathodal and sham polarization at different contrasts; significant effects were checked by post hoc Holm-Sidak test.

\section{Comparison Between Amblyopic Patients and Controls}

A three-way RM ANOVA analyzed the effects of "group" × "time" $\times$ "contrast" interaction between patients and healthy controls. At each time interval, a two-way ANOVA on ranks compared peak-to-peak amplitudes between amblyopic participants and healthy controls; significant effects were followed by post hoc Holm-Sidak test to compare VEP changes over time.

Statistical significance was set at $p<0.05$. Data were analyzed using SPSS v. 21.0 for Windows (SPSS Inc., Chicago, IL, USA) or SigmaPlot v. 12.0.

\section{RESULTS}

\section{Clinical Assessment: Visual Acuity}

Baseline $\left(\mathrm{T}_{0}\right) \log \mathrm{MAR}$ values did not change between real and sham sessions $(p=0.83)$.

A remarkable improvement occurred at $\mathrm{T} 1$ when cathodal polarization was delivered within the hemisphere contralateral to the amblyopic eye, with changes lasting up to $1 \mathrm{~h}$ after tDCS completion $\left(F_{(2,22)}=8.14, p=0.0023\right.$, two-way ANOVA, with "time" and "treatment" as factors). This reduction ranged from 0.11 to $0.88 \log$ MAR, with a mean of about $0.27 \log$ MAR (see Table 2 and Figure 1), and it was significant both at $\mathrm{T}_{1}\left(p=0.0029\right.$, Holm-Sidak post hoc comparison) and $\mathrm{T}_{2}$ $(p=0.0019)$ when compared to the sham group.

\begin{tabular}{|c|c|c|c|c|}
\hline \multirow[b]{2}{*}{ Patient's number } & \multicolumn{2}{|c|}{ Cathodal tDCS } & \multicolumn{2}{|c|}{ Sham tDCS } \\
\hline & $T_{1}$ & $T_{2}$ & $T_{1}$ & $\mathrm{~T}_{2}$ \\
\hline 2 & -0.33 & -0.47 & 0.03 & -0.09 \\
\hline 3 & -0.14 & -0.12 & -0.02 & -0.10 \\
\hline 4 & -0.61 & -0.27 & -0.19 & -0.10 \\
\hline 7 & -0.22 & -0.16 & 0.04 & 0.14 \\
\hline 8 & -0.25 & -0.20 & 0.02 & 0.00 \\
\hline 9 & -0.35 & -0.37 & 0.05 & -0.06 \\
\hline 10 & -0.41 & -0.08 & -0.05 & -0.07 \\
\hline 11 & -0.10 & -0.88 & 0.07 & 0.08 \\
\hline 12 & -0.02 & -0.08 & 0.02 & -0.04 \\
\hline
\end{tabular}

Changes in logMAR score compared to baseline values are shown for the amblyopic eyes; notably, cathodal tDCS improved visual function both at $T_{1}$ and $T_{2}$. 


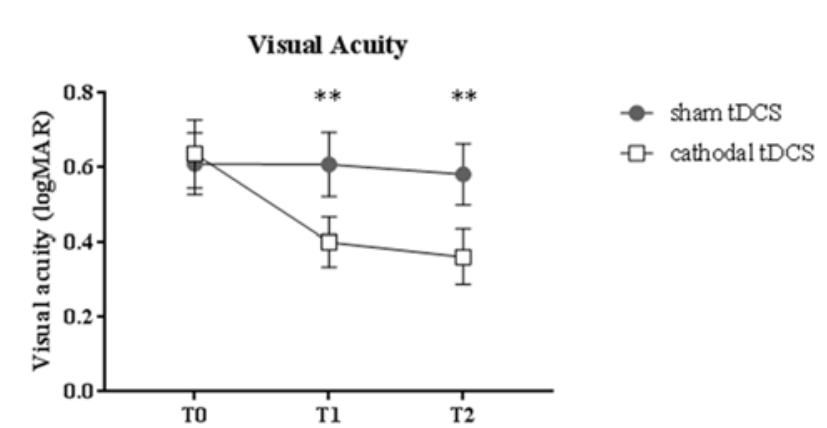

FIGURE 1 | Changes in visual acuity (LogMAR). Amblyopic patients showed a significant improvement of visual acuity following cathodal transcranial direct current stimulation (tDCS) compared to sham polarization, with effects lasting for up to $1 \mathrm{~h}$. Data are given as mean values \pm standard error (SE); ${ }^{* *} p<0.001$.

Changes in logMAR score linearly correlated with baseline values. Indeed, patients with greater impaired at baseline showed a more robust improvement in visual acuity $(p=0.0004$, Pearson's correlation; Figure 2).

\section{Visual Evoked Potentials (VEPs): Amblyopic Patients}

At baseline, in agreement with previous data reported elsewhere (Ding et al., 2016), mean VEP amplitudes for amblyopic eyes were significantly lower than those recorded by stimulating the fellow eyes $(6.54 \pm 0.91$ vs. $9.67 \pm 1.99 \mu \mathrm{V}$ at $\mathrm{K} 90 \%$ : $t=4.61$, $p<0.0001$, unpaired $t$-test).

Representative VEPs from one patient are shown in Figure 3A.

A three-way repeated measures ANOVA revealed significant effects of stimulation $\left(F_{(1,132)}=24.2, p<0.0001\right)$, contrast $\left(F_{(1,132)}=96.8, \quad p<0.0001\right)$, stimulation $\times$ contrast $\left(F_{(1,132)}=23.3, p<0.0001\right)$, time $\times$ stimulation $\left(F_{(2,132)}=8.7\right.$, $p=0.0003)$ and time $\times$ contrast interaction $\left(F_{(2,132)}=5.7\right.$, $p=0.0041)$. In particular, at high-contrast, VEP amplitudes recorded ipsilaterally to amblyopic eyes dramatically improved

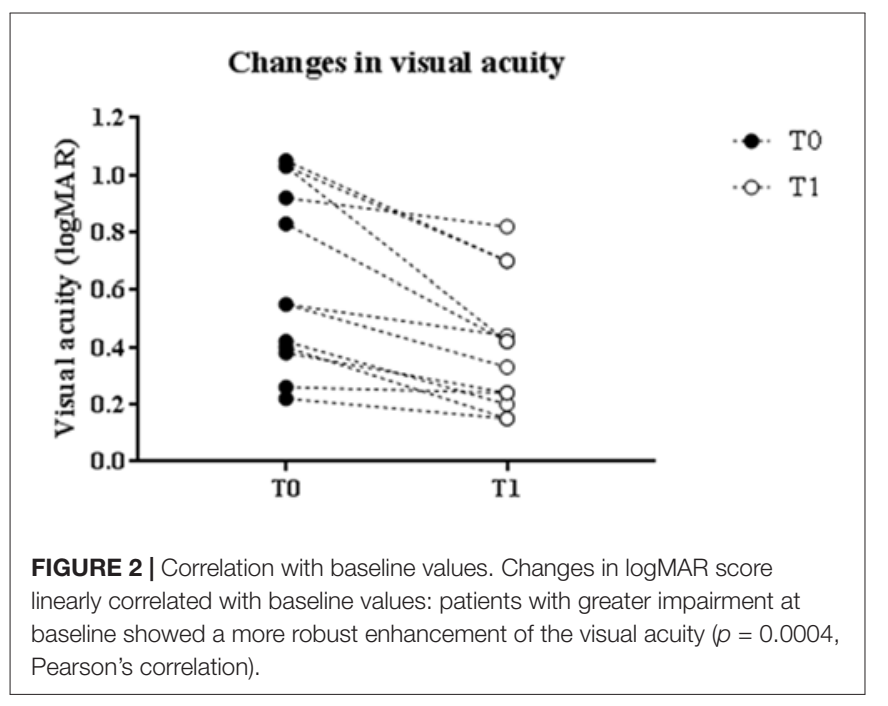

compared to low-contrast $\left(F_{(2,66)}=14.9, p<0.0001\right.$, two-way ANOVA on ranks) and sham stimulation $\left(F_{(2,66)}=35.9\right.$, $p<0.0001$, two-way RM ANOVA), remaining persistently elevated at $\mathrm{T}_{2}(p<0.0001$, Holm-Sidak test $)$.

A significant correlation between the enhancement of visual acuity and the relative increase of VEP amplitudes in the amblyopic side was found (Pearson's correlation: $p=0.002$ ).

On the opposite side, as expected due to the inhibitory effect of cathodal polarization, we observed a reduction of VEP amplitudes at $\mathrm{T}_{1}$, both at high and low contrasts. At $\mathrm{T}_{2}$ all values returned to baseline, both for high (Holm-Sidak test, $\mathrm{T}_{1}$ vs. $\mathrm{T}_{2}$ : $p<0.0001)$ and low contrasts $(p=0.001)$.

\section{Visual Evoked Potentials (VEPs): Comparison Between Patients and Controls}

When VEPs recorded from the side contralateral to tDCS were analyzed, a three-way ANOVA showed significant effects of time $\left(F_{(2,132)}=20.4, p<0.0001\right)$, contrast $\left(F_{(1,132)}=64.9, p<0.0001\right)$, time $\times$ contrast $\left(F_{(2,132)}=30.7, p<0.0001\right)$, group $\times$ contrast $\left(F_{(1,132)}=11.4, p=0.001\right)$ and contrast $\times$ group $\times$ time interaction $\left(F_{(2,132)}=8.0, p=0.0005\right)$. When analyzed separately, at high contrasts (K90\%), we found a persistent enhancement of VEP amplitude in amblyopic subjects but not controls at $\mathrm{T}_{2}$ (Holm-Sidak test, $p<0.0001$ ): thus, transcallosal disinhibition persisted in amblyopic patients, while it vanished in controls (compare Figures 3, 4, contralateral side).

On the hemisphere in which inhibitory cathodal polarization was applied, patients and controls showed a similar reduction in VEP amplitudes at T1; a three-way ANOVA showed significant effects of time $\left(F_{(2,132)}=104.3, p<0.0001\right)$, contrast $\left(F_{(2,132)}=3.9, p=0.049\right)$ and group $\times$ time interaction $\left(F_{(2,132)}=49.8, p<0.0001\right)$. At high contrasts, values returned to baseline in patients (Holm-Sidak test, $\mathrm{T}_{1}$ vs. $\mathrm{T}_{2}$ in patients, $p<0.0001$ ), remaining significantly reduced in controls (HolmSidak test, $\mathrm{T}_{1}$ vs. $\mathrm{T}_{2}$ in controls, $p=0.42$; compare Figures 3,4 ).

\section{DISCUSSION}

Our results suggest that cathodal tDCS applied over the V1 contralateral to the "lazy eye" improves visual acuity, supporting the use of non-invasive brain stimulation techniques (NIBS) for the treatment of adult patients with amblyopia. Inhibitory cathodal tDCS dampened VEP amplitudes in both healthy and amblyopic subjects; concurrently, facilitation of visual responses in the contralateral side occurred, possibly due to the removal of interhemispheric inhibitory influences (Restani et al., 2009; Bocci et al., 2011). Significant differences were found at $\mathrm{T}_{2}$, with a faster normalization of VEP amplitudes in the stimulated side and a persistent disinhibition in the opposite hemisphere in amblyopic patients. This disinhibition may be at the basis of the behavioral improvement of visual acuity, which was detected at T1 and persisted at T2 (Figure 1). This interpretation is supported by the significant correlation between VEP changes and the enhancement of clinical outcome (i.e., reduction of $\log M A R$ ). 


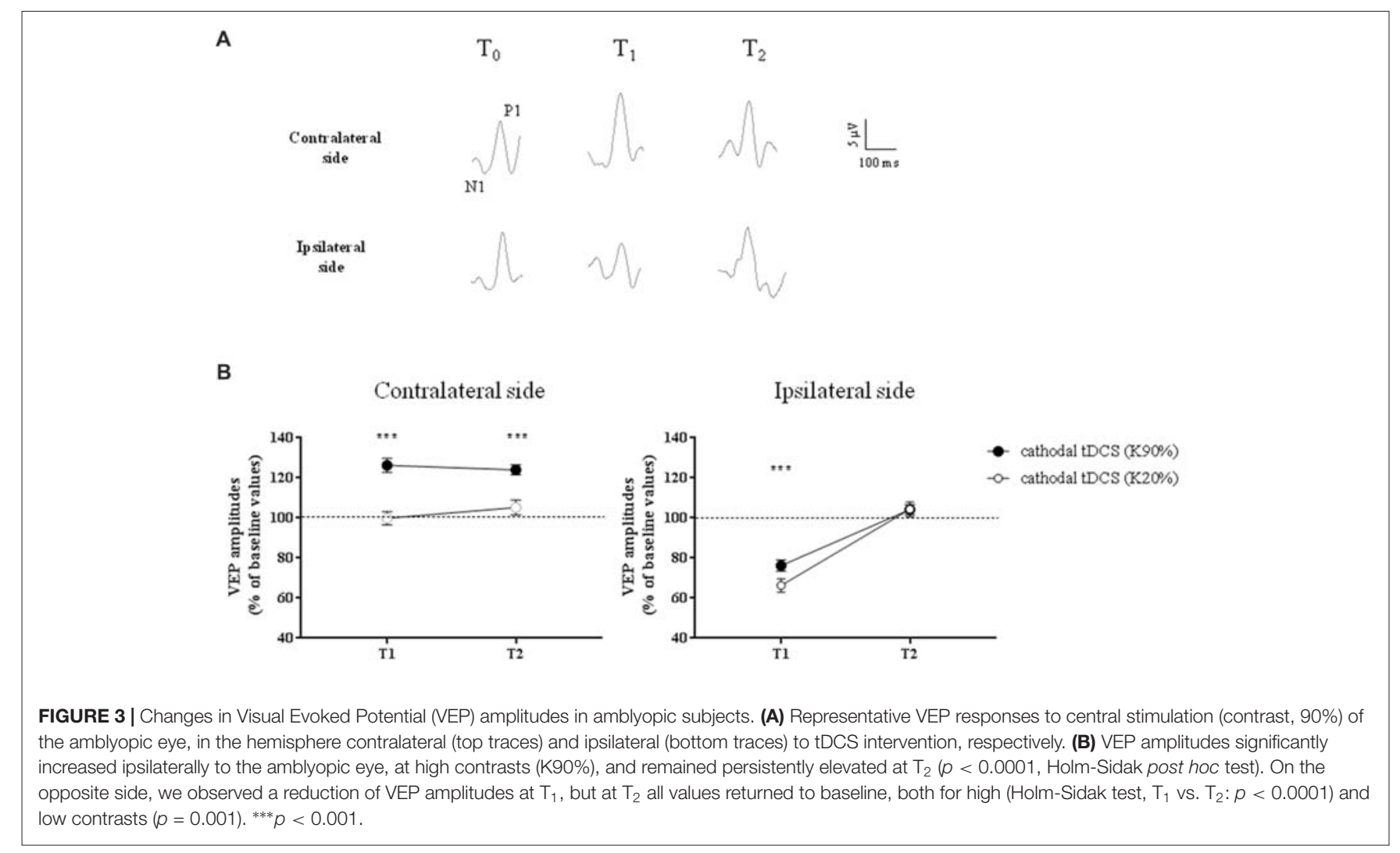

A growing bulk of literature suggests that the adult visual system retains a high degree of plasticity (Lunghi et al., 2010, 2011; Lo Verde et al., 2017), indicating that the excitatory/inhibitory balance that modulates gain control mechanisms could be particularly susceptible to NIBS interventions, even at short timescales (Reinhart et al., 2016). In humans, previous articles have reported a significant effect of tDCS for the recovery of contrast sensitivity and stereopsis in amblyopia, providing a novel and safe approach to improve outcome in adults (Spiegel et al., 2013a; Ding et al., 2016).
Authors demonstrated an enhancement of both monocular (visual acuity) and binocular (stereopsis) measures of visual function, especially when the polarization of the visual cortex was associated with dichoptic videogame-based treatment (Spiegel et al., 2013b). Nonetheless, these studies have used excitatory, anodal tDCS bilaterally applied over the V1. Here, we reasoned to dampen the excitability of the visual area contralateral to the "lazy eye", with the aim to restore the balance of transcallosal inhibitory influences between hemispheres.

\section{Contralateral Side}

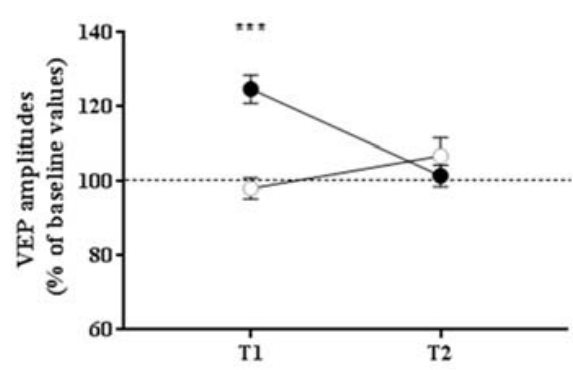

Ipsilateral Side

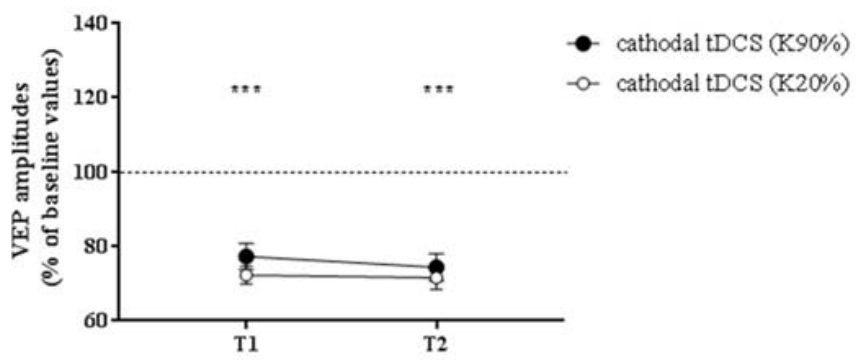

FIGURE 4 | VEP amplitudes in subjects with normal visual acuity. VEP amplitudes increased on the side contralateral to the application of cathodal tDCS, while they were dampened ipsilaterally. Nonetheless, different from amblyopic participants, at T2 there was a loss of the contralateral facilitation, paralleled by a persistent inhibition of the responses recorded from the polarized hemisphere. Data are given as mean values \pm standard error (SE); ${ }^{* * *} p<0.0001$. 
Our hypothesis is consistent with data in animals, showing that transcallosal connections are primarily involved in the weakening of deprived eye responses during monocular deprivation (Restani et al., 2009; Pietrasanta et al., 2014). Since transcallosal neurons are excitatory, interhemispheric inhibition depends upon the activation of GABAergic neurons in the target side, which contact local cortical pyramids via GABA-B receptors (Irlbacher et al., 2007; Palmer et al., 2012). Along this line, in primate models of amblyopia, the magnitude of side-to-side suppression seems to be closely related to the behavioral loss of contrast sensitivity in the amblyopic eye (Bi et al., 2011; Li et al., 2011, 2013; Tao et al., 2014).

Further support for a key role of interhemispheric pathways in amblyopia comes from recent data showing a higher vulnerability of the parvocellular pathway to the effects of visual deprivation, thus affecting the chromatic vision in humans (Hess et al., 2010; Lunghi et al., 2013). Notably, the callosum preferentially processes high-contrast stimuli and robustly transfers chromatic information related to the activation of the parvocellular stream (Berardi and Fiorentini, 1987; Berardi et al., 1987; Corballis, 1996; Roser and Corballis, 2003). Also in our sample, the persistent facilitation of visual responses has been observed for high contrasts only (see Figure 3).

\section{Limitations and Alternative Explanations}

The main limitation of our study is the small number of patients, due to the difficulty in recruiting a homogeneous group of subjects. High-powered studies, with largest samples, are needed in the future to confirm our data and assess the efficacy of unilateral cathodal tDCS as a valuable option for the long-term treatment of amblyopia. Despite the low number of cases, cathodal tDCS displayed a consistent effect on visual acuity (see Figure 2).

Although our results appear to fit an explanation based on imbalance of V1 cortical excitability between hemispheres, additional possibilities need to be considered. First, the rapid changes triggered by tDCS (in terms of both visual acuity and VEP amplitudes) strongly support alterations in the excitatory/inhibitory balance within the visual system rather than structural rearrangements of inputs from the lazy eye. In this context, there is evidence that responses of the weak eye are actively suppressed by GABAergic inhibition (Duffy et al., 1976), and tDCS may alter GABA concentrations in the cerebral cortex (Stagg et al., 2009).

Second, the reduction of cortical excitability mediated by cathodal tDCS in the stimulated hemisphere could potentiate weak responses from the lazy eye via homeostatic mechanisms (Turrigiano, 2012). For example, it has been previously shown that brief period of monocular deprivation in adult subjects strongly alters ocular balance, producing a

\section{REFERENCES}

Antal, A., Nitsche, M. A., Kincses, T. Z., Kruse, W., Hoffmann, K. P., and Paulus, W. (2004). Facilitation of visuo-motor learning by transcranial direct current stimulation of the motor and extrastriate visual areas in humans. Eur. J. Neurosci. 19, 2888-2892. doi: 10.1111/j.1460-9568.2004.03367.x perceptual boost of the deprived eye (Lunghi et al., 2011). Along this line, the binocular imbalance that characterizes amblyopia can be reduced by occluding the amblyopic eye with a translucent patch for a few hours (Zhou et al., 2013).

Another possibility is that tDCS affects brainstem nuclei or thalamic structures, such as the lateral geniculate nucleus. In this case, the effects of the manipulation on acuity and VEP responses could be at least partly due to an action at subcortical level. Although this hypothesis cannot be definitely ruled out, VEP changes following hemifield visual stimulation seem to be consistent with a selective modulation of the interhemispheric route, as described in more detail elsewhere (Bocci et al., 2011, 2016). Moreover, direct geniculocortical connections are mildly affected by monocular deprivation in animals, with effects requiring at least 20 days of ocular deprivation (Antonini et al., 1999).

\section{CONCLUSION}

Overall, our data support the use of unilateral cathodal tDCS for the treatment of amblyopia in adults, when pharmacological and mechanical therapies are completely ineffective; in order to improve and prolong the clinical outcome, both in adults and children, tDCS may be also combined with novel behavioral methods, comprising dichoptic training, perceptual learning and video gaming (Tsirlin et al., 2015; Vedamurthy et al., 2015a,b). Although promising, these therapies are currently influenced by visual attention, possibly narrowing their application in clinical practice.

\section{AUTHOR CONTRIBUTIONS}

TB: conception and design of research, data analysis, interpretation of the results, writing article; MC, LR, AP, $\mathrm{MN}$ and FS: conception and design of research, interpretation of the results, writing article; FN: data collection and analysis, patients' enrollment; GA and LM: conception and design of research, interpretation of the results; DB performed the experiments, writing the article.

\section{ACKNOWLEDGMENTS}

We gratefully acknowledge the participation of all subjects and Mr. C. Orsini for his excellent technical assistance. We thank Dr. Francesca Bovis (Institute Gaslini, Genova) for her valuable statistical revision. The article was supported in part by the Italian operating and development MIUR PRIN grant year 2006, n.2006062332_002.

Antal, A., Nitsche, M. A., and Paulus, W. (2006). Transcranial direct current stimulation and the visual cortex. Brain Res. Bull. 68, 459-463. doi: 10.1016/j. brainresbull.2005.10.006

Antonini, A., Fagiolini, M., and Stryker, M. P. (1999). Anatomical correlates of functional plasticity in mouse visual cortex. J. Neurosci. 19, 4388-4406. doi: 10.1523/JNEUROSCI.19-11-04388.1999 
Behrens, J. R., Kraft, A., Irlbacher, K., Gerhardt, H., Olma, M. C., and Brandt, S. A. (2017). Long-lasting enhancement of visual perception with repetitive noninvasive transcranial direct current stimulation. Front. Cell. Neurosci. 11:238. doi: 10.3389/fncel.2017.00238

Berardi, N., Bisti, S., and Maffei, L. (1987). The transfer of visual information across the corpus callosum: spatial and temporal properties in the cat. J. Physiol. 384, 619-632. doi: 10.1113/jphysiol.1987.sp016473

Berardi, N., and Fiorentini, A. (1987). Interhemispheric transfer of visual information in humans: spatial characteristics. J. Physiol. 384, 633-647. doi: 10.1113/jphysiol.1987.sp016474

Bhola, R., Keech, R. V., Kutschke, P., Pfeifer, W., and Scott, W. E. (2006). Recurrence of amblyopia after occlusion therapy. Ophthalmology 113, 2097-2100. doi: 10.1016/j.ophtha.2006.04.034

Bi, H., Zhang, B., Tao, X., Harwerth, R. S., Smith, E. L. III., and Chino, Y. M. (2011). Neuronal responses in visual area V2 (V2) of macaque monkeys with strabismic amblyopia. Cereb. Cortex 21, 2033-2045. doi: 10.1093/cercor/bhq272

Bocci, T., Caleo, M., Giorli, E., Barloscio, D., Maffei, L., Rossi, S., et al. (2011). Transcallosal inhibition dampens neural responses to high contrast stimuli in human visual cortex. Neuroscience 187, 43-51. doi: 10.1016/j.neuroscience. 2011.04.050

Bocci, T., Caleo, M., Restani, L., Barloscio, D., Rossi, S., and Sartucci, F. (2016). Altered recovery from inhibitory repetitive transcranial magnetic stimulation (rTMS) in subjects with photosensitive epilepsy. Clin. Neurophysiol. 127, 3353-3361. doi: 10.1016/j.clinph.2016.06.013

Bocci, T., Pietrasanta, M., Cerri, C., Restani, L., Caleo, M., and Sartucci, F. (2014). Visual callosal connections: role in visual processing in health and disease. Rev. Neurosci. 25, 113-127. doi: 10.1515/revneuro-2013-0025

Cerri, C., Restani, L., and Caleo, M. (2010). Callosal contribution to ocular dominance in rat primary visual cortex. Eur. J. Neurosci. 32, 1163-1169. doi: 10.1111/j.1460-9568.2010.07363.x

Corballis, M. C. (1996). A dissociation in naming digits and colors following commissurotomy. Cortex 32, 515-525. doi: 10.1016/s0010-9452(96)80008-6

Davis, A. R., Sloper, J. J., Neveu, M. M., Hogg, C. R., Morgan, M. J., and Holder, G. E. (2003). Electrophysiological and psychophysical differences between early- and late-onset strabismic amblyopia. Invest. Ophthalmol. Vis. Sci. 44, 610-617. doi: 10.1167/iovs.02-0240

Ding, Z., Li, J., Spiegel, D. P., Chen, Z., Chan, L., Luo, G., et al. (2016). The effect of transcranial direct current stimulation on contrast sensitivity and visual evoked potential amplitude in adults with amblyopia. Sci. Rep. 6:19280. doi: 10.1038/srep19280

Duffy, F. H., Burchfiel, J. L., and Conway, J. L. (1976). Bicuculline reversal of deprivation amblyopia in the cat. Nature 260, 256-257. doi: 10.1038/260256a0

Galea, J. M., Jayaram, G., Ajagbe, L., and Celnik, P. (2009). Modulation of cerebellar excitability by polarity-specific noninvasive direct current stimulation. J. Neurosci. 29, 9115-9122. doi: 10.1523/JNEUROSCI.2184 $-09.2009$

Gandiga, P. C., Hummel, F. C., and Cohen, L. G. (2006). Transcranial DC stimulation (tDCS): a tool for double-blind sham-controlled clinical studies in brain stimulation. Clin. Neurophysiol. 117, 845-850. doi: 10.1016/j.clinph.2005. 12.003

Gao, T. Y., Guo, C. X., Babu, R. J., Black, J. M., Bobier, W. R., Chakraborty, A., et al. (2018). Effectiveness of a binocular video game vs. placebo video game for improving visual functions in older children, teenagers, and adults with amblyopia: a randomized clinical trial. JAMA Ophthalmol. 136, 172-181. doi: 10.1001/jamaophthalmol.2017.6090

Hess, R. F., Li, X., Lu, G., Thompson, B., and Hansen, B. C. (2010). The contrast dependence of the cortical fMRI deficit in amblyopia; a selective loss at higher contrasts. Hum. Brain Mapp. 31, 1233-1248. doi: 10.1002/hbm.20931

Hess, R. F., and Thompson, B. (2015). Amblyopia and the binocular approach to its therapy. Vision Res. 114, 4-16. doi: 10.1016/j.visres.2015.02.009

Holmes, J. M., and Clarke, M. P. (2006). Amblyopia. Lancet 367, 1343-1351. doi: 10.1016/S0140-6736(06)68581-4

Holmes, J. M., Lazar, E. L., Melia, B. M., Astle, W. F., Dagi, L. R., Donahue, S. P., et al. (2011). Effect of age on response to amblyopia treatment in children. Arch. Ophthalmol. 129, 1451-1457. doi: 10.1001/archophthalmol.2011.179

Irlbacher, K., Brocke, J., Mechow, J. V., and Brandt, S. A. (2007). Effects of $\mathrm{GABA}_{A}$ and $\mathrm{GABA}_{\mathrm{B}}$ agonists on interhemispheric inhibition in man. Clin. Neurophysiol. 118, 308-316. doi: 10.1016/j.clinph.2006.09.023
Lang, N., Siebner, H. R., Chadaide, Z., Boros, K., Nitsche, M. A., Rothwell, J. C., et al. (2007). Bidirectional modulation of primary visual cortex excitability: a combined tDCS and rTMS study. Invest. Ophthalmol. Vis. Sci. 48, 5782-5787. doi: 10.1167/iovs.07-0706

Li, X., Dumoulin, S. O., Mansouri, B., and Hess, R. F. (2007). Cortical deficits in human amblyopia: their regional distribution and their relationship to the contrast detection deficit. Invest. Ophthalmol. Vis. Sci. 48, 1575-1591. doi: 10.1167/iovs.06-1021

Li, J., Hess, R. F., Chan, L. Y., Deng, D., Yang, X., Chen, X., et al. (2013). Quantitative measurement of interocular suppression in anisometropic amblyopia: a case-control study. Ophthalmology 120, 1672-1680. doi: 10.1016/j. ophtha.2013.01.048

Li, J., Thompson, B., Lam, C. S., Deng, D., Chan, L. Y., Maehara, G., et al. (2011). The role of suppression in amblyopia. Invest. Ophthalmol. Vis. Sci. 52, 4169-4176. doi: 10.1167/iovs.11-7233

Lo Verde, L., Morrone, M. C., and Lunghi, C. (2017). Early cross-modal plasticity in adults. J. Cogn. Neurosci. 29, 520-529. doi: 10.1162/jocn_a_01067

Lunghi, C., Binda, P., and Morrone, M. C. (2010). Touch disambiguates rivalrous perception at early stages of visual analysis. Curr. Biol. 20, R143-R144. doi: 10.1016/j.cub.2009.12.015

Lunghi, C., Burr, D. C., and Morrone, C. (2011). Brief periods of monocular deprivation disrupt ocular balance in human adult visual cortex. Curr. Biol. 21, R538-R539. doi: 10.1016/j.cub.2011.06.004

Lunghi, C., Burr, D. C., and Morrone, M. C. (2013). Long-term effects of monocular deprivation revealed with binocular rivalry gratings modulated in luminance and in color. J. Vis. 13:1. doi: 10.1167/13.6.1

Nitsche, M. A., Liebetanz, D., Lang, N., Antal, A., Tergau, F., and Paulus, W. (2003). Safety criteria for transcranial direct current stimulation (tDCS) in humans. Clin. Neurophysiol. 114, 2220-2222; author reply 2222-2223. doi: 10.1016/s1388-2457(03)00235-9

Olma, M. C., Dargie, R. A., Behrens, J. R., Kraft, A., Irlbacher, K., Fahle, M., et al. (2013). Long-term effects of serial anodal tDCS on motion perception in subjects with occipital stroke measured in the unaffected visual hemifield. Front. Hum. Neurosci. 7:314. doi: 10.3389/fnhum.2013.00314

Palmer, L. M., Schulz, J. M., Murphy, S. C., Ledergerber, D., Murayama, M., and Larkum, M. E. (2012). The cellular basis of $\mathrm{GABA}_{\mathrm{B}}$-mediated interhemispheric inhibition. Science 335, 989-993. doi: 10.1126/science.1217276

Pediatric Eye Disease Investigator Group, Repka, M. X., Kraker, R. T., Beck, R. W., Holmes, J. M., Cotter, S. A., et al. (2008). A randomized trial of atropine vs. patching for treatment of moderate amblyopia: follow-up at age 10 years. Arch. Ophthalmol. 126, 1039-1044. doi: 10.1001/archopht.126. 8.1039

Pietrasanta, M., Restani, L., Cerri, C., Olcese, U., Medini, P., and Caleo, M. (2014). A switch from inter-ocular to inter-hemispheric suppression following monocular deprivation in the rat visual cortex. Eur. J. Neurosci. 40, 2283-2292. doi: 10.1111/ejn.12573

Reinhart, R. M., Xiao, W., McClenahan, L. J., and Woodman, G. F. (2016). Electrical stimulation of visual cortex can immediately improve spatial vision. Curr. Biol. 26, 1867-1872. doi: 10.1016/j.cub.2016.05.019

Repka, M. X., Wallace, D. K., Beck, R. W., Kraker, R. T., Birch, E. E., Cotter, S. A., et al. (2005). Two-year follow-up of a 6-month randomized trial of atropine vs. patching for treatment of moderate amblyopia in children. Arch. Ophthalmol. 123, 149-157. doi: 10.1001/archopht.123.2.149

Restani, L., Cerri, C., Pietrasanta, M., Gianfranceschi, L., Maffei, L., and Caleo, M. (2009). Functional masking of deprived eye responses by callosal input during ocular dominance plasticity. Neuron 64, 707-718. doi: 10.1016/j.neuron.2009. 10.019

Roser, M., and Corballis, M. C. (2003). Interhemispheric neural summation in the split brain: effects of stimulus colour and task. Neuropsychologia 41, 830-846. doi: 10.1016/s0028-3932(02)00290-7

Spiegel, D. P., Byblow, W. D., Hess, R. F., and Thompson, B. (2013a). Anodal transcranial direct current stimulation transiently improves contrast sensitivity and normalizes visual cortex activation in individuals with amblyopia. Neurorehabil. Neural Repair 27, 760-769. doi: 10.1177/1545968313 491006

Spiegel, D. P., Li, J., Hess, R. F., Byblow, W. D., Deng, D., Yu, M., et al. (2013b). Transcranial direct current stimulation enhances recovery of stereopsis in 
adults with amblyopia. Neurotherapeutics 10, 831-839. doi: 10.1007/s13311013-0200-y

Stagg, C. J., Best, J. G., Stephenson, M. C., O’Shea, J., Wylezinska, M., Kincses, Z. T., et al. (2009). Polarity-sensitive modulation of cortical neurotransmitters by transcranial stimulation. J. Neurosci. 29, 5202-5206. doi: 10.1523/JNEUROSCI. 4432-08.2009

Stewart, C. E., Stephens, D. A., Fielder, A. R., Moseley, M. J., and MOTAS Cooperative (2007). Modeling dose-response in amblyopia: toward a childspecific treatment plan. Invest. Ophthalmol. Vis. Sci. 48, 2589-2594. doi: 10.1167/iovs.05-1243

Tao, X., Zhang, B., Shen, G., Wensveen, J., Smith, E. L. III., Nishimoto, S., et al. (2014). Early monocular defocus disrupts the normal development of receptive-field structure in V2 neurons of macaque monkeys. J. Neurosci. 34, 13840-13854. doi: 10.1523/JNEUROSCI.1992-14.2014

Thompson, B., Mansouri, B., Koski, L., and Hess, R. F. (2008). Brain plasticity in the adult: modulation of function in amblyopia with rTMS. Curr. Biol. 18, 1067-1071. doi: 10.1016/j.cub.2008.06.052

Tsirlin, I., Colpa, L., Goltz, H. C., and Wong, A. M. (2015). Behavioral training as new treatment for adult amblyopia: a meta-analysis and systematic review. Invest. Ophthalmol. Vis. Sci. 56, 4061-4075. doi: 10.1167/iovs.15 $-16583$

Turrigiano, G. (2012). Homeostatic synaptic plasticity: local and global mechanisms for stabilizing neuronal function. Cold Spring Harb. Perspect. Biol. 4:a005736. doi: 10.1101/cshperspect.a005736

Vedamurthy, I., Nahum, M., Bavelier, D., and Levi, D. M. (2015a). Mechanisms of recovery of visual function in adult amblyopia through a tailored action video game. Sci. Rep. 5:8482. doi: 10.1038/srep08482
Vedamurthy, I., Nahum, M., Huang, S. J., Zheng, F., Bayliss, J., Bavelier, D., et al. (2015b). A dichoptic custom-made action video game as a treatment for adult amblyopia. Vision Res. 114, 173-187. doi: 10.1016/j.visres.2015.04.008

von Noorden, G. K., and Crawford, M. L. (1992). The lateral geniculate nucleus in human strabismic amblyopia. Invest. Ophthalmol. Vis. Sci. 33, 2729-2732.

Williams, C., Northstone, K., Harrad, R. A., Sparrow, J. M., Harvey, I., and ALSPAC Study Team. (2003). Amblyopia treatment outcomes after preschool screening $\mathrm{v}$ school entry screening: observational data from a prospective cohort study. Br. J. Ophthalmol. 87, 988-993. doi: 10.1136/bjo.87.8.988

Zhou, J., Thompson, B., and Hess, R. F. (2013). A new form of rapid binocular plasticity in adult with amblyopia. Sci. Rep. 3:2638. doi: 10.1038/srep02638

Žiak, P., Holm, A., Halicka, J., Mojzis, P., and Piñero, D. P. (2017). Amblyopia treatment of adults with dichoptic training using the virtual reality oculus rift head mounted display: preliminary results. BMC Ophthalmol. 17:105. doi: 10.1186/s12886-017-0501-8

Conflict of Interest Statement: The authors declare that the research was conducted in the absence of any commercial or financial relationships that could be construed as a potential conflict of interest.

Copyright (C) 2018 Bocci, Nasini, Caleo, Restani, Barloscio, Ardolino, Priori, Maffei, Nardi and Sartucci. This is an open-access article distributed under the terms of the Creative Commons Attribution License (CC BY). The use, distribution or reproduction in other forums is permitted, provided the original author(s) and the copyright owner are credited and that the original publication in this journal is cited, in accordance with accepted academic practice. No use, distribution or reproduction is permitted which does not comply with these terms. 


\section{OPEN ACCESS}

Edited by:

Claudio Lucchiari,

Università degli Studi di Milano, Italy

Reviewed by:

Nico Sollmann,

Technische Universität München,

Germany

Martin Victor Sale,

The University of Queensland,

Australia

Davide Crivelli, Università Cattolica del Sacro Cuore,

Italy

*Correspondence:

Junhong Zhou

junhongzhou@hsl.harvard.edu

Tao Feng

happyft@sina.com

${ }^{+}$Co-last authors

Received: 01 February 2018 Accepted: 20 April 2018

Published: 09 May 2018

Citation:

Liu Z, Ma H, Poole V, Wang $X$, Wang $Z$, Yang $Y$, Meng $L$, Manor $B$,

Zhou $J$ and Feng $T$ (2018) Effects of Multi-Session Repetitive

Transcranial Magnetic Stimulation on

Motor Control and Spontaneous Brain Activity in Multiple System Atrophy: A Pilot Study.

Front. Behav. Neurosci. 12:90. doi: 10.3389/fnbeh.2018.00090

\section{Effects of Multi-Session Repetitive Transcranial Magnetic Stimulation on Motor Control and Spontaneous Brain Activity in Multiple System Atrophy: A Pilot Study}

\author{
Zhu Liu', Huizi Ma', Victoria Poole ${ }^{2}$, Xuemei Wang ${ }^{1}$, Zhan Wang', Yaqin Yang ${ }^{1}$, \\ Lanxi Meng' ${ }^{1}$, Brad Manor ${ }^{2}$, Junhong Zhou ${ }^{1,2 *+}$ and Tao Feng ${ }^{1 * t}$ \\ 'Department of Neurodegenerative Disease, Centre of Neurology, Beijing Tiantan Hospital, Capital Medical University,
Beijing, China, ${ }^{2}$ Harvard Medical School, Harvard University, Roslindale, MA, United States
}

Background: Impaired motor control is one of the most common symptoms of multiple system atrophy (MSA). It arises from dysfunction of the cerebellum and its connected neural networks, including the primary motor cortex (M1), and is associated with altered spontaneous (i.e., resting-state) brain network activity. Non-invasive repetitive transcranial magnetic stimulation (rTMS) selectively facilitates the excitability of supraspinal networks. Repeated rTMS sessions have been shown to induce long-term changes to both resting-state brain dynamics and behavior in several neurodegenerative diseases. Here, we hypothesized that a multi-session rTMS intervention would improve motor control in patients with MSA, and that such improvements would correlate with changes in resting-state brain activity.

Methods: Nine participants with MSA received daily sessions of $5 \mathrm{~Hz}$ rTMS for 5 days. rTMS targeted both the cerebellum and the bilateral M1. Before and within 3 days after the intervention, motor control was assessed by the motor item of the Unified Multiple System Atrophy Rating Scale (UMSARS). Resting-state brain activity was recorded by blood-oxygen-level dependency (BOLD) functional magnetic resonance imaging. The "complexity" of resting-state brain activity fluctuations was quantified within seven wellknown functional cortical networks using multiscale entropy, a technique that estimates the degree of irregularity of the BOLD time-series across multiple scales of time.

Results: The rTMS intervention was well-attended and was not associated with any adverse events. Average motor scores were lower (i.e., better performance) following the rTMS intervention as compared to baseline ( $t_{8}=2.3, p=0.003$ ). Seven of nine participants exhibited such pre-to-post intervention improvements. A trend toward an increase in resting-state complexity was observed within the motor network $\left(t_{8}=1.86\right.$, $p=0.07)$. Participants who exhibited greater increases in motor network resting-state complexity demonstrated greater improvement in motor control $\left(r^{2}=0.72, p=0.004\right)$. 
Conclusion: This pilot study demonstrated that a five-session rTMS intervention targeting the cerebellum and bilateral M1 is feasible and safe for those with MSA. More definitive, well-controlled trials are warranted to confirm our preliminary results that rTMS may alleviate the severity of motor dysfunction and modulate the multiscale dynamics of motor network brain activity.

Keywords: repetitive transcranial magnetic stimulation, multiple system atrophy, motor control, multiscale entropy, resting-state fMRI

\section{INTRODUCTION}

Multiple system atrophy (MSA) is a fatal neurodegenerative disorder marked by progressive parkinsonian symptoms and both cerebellar and autonomic dysfunction. Up to $87 \%$ of patients with MSA exhibit motor disturbances, which in turn increase morbidity and reduce quality of life (Fanciulli and Wenning, 2015). Current pharmacological therapies targeting rigidity and bradykinesia, which most commonly entail antiparkinsonism drugs (e.g., Levodopa) (Kollensperger et al., 2010), are largely ineffective (Krismer and Wenning, 2017). Only $31 \%$ of patients with MSA benefit from Levodopa, and such improvements tend to be short-lasting (Gilman et al., 2008; Maass et al., 2016; Rohrer et al., 2017). Moreover, these medications often cause side effects including hypotension, cognitive impairment, and hypersomnia in this population. There is thus an urgent need to develop new non-pharmacological therapeutic strategies to enhance motor function in those suffering from MSA.

Motor control impairments in MSA are believed to arise from dysfunction of the cerebellum and its connected neural networks (Payoux et al., 2010; Lu et al., 2013). MSA leads to atrophy in cerebellar regions, as well as altered plasticity and decreased neuronal excitability (e.g., prolonged central motor conduction time and cortical silent period) within the motor cortex (M1) (Fanciulli and Wenning, 2015). Burciu et al. (2016) observed in a longitudinal study of patients with MSA that the responsiveness [i.e., activation measured by functional magnetic resonance imaging (fMRI) signal] of the M1 and cerebellum to a motor task diminished over a 1 -year period. This observation suggests that therapeutic strategies designed to modulate activity in the cerebellum and M1 may be particularly well-suited to slow the progression of functional decline, or even enhance it, in this population.

Non-invasive repetitive transcranial magnetic stimulation (rTMS) is a safe technique that selectively modulates the excitability of neuronal populations and their connected neural networks via current induced by an electro-magnetic field. Yildiz et al. (2017), for example, observed that rTMS targeting the cerebellum reduced short-latency afferent inhibition of M1 and enhanced performance of a cognitive reaction time task in those with MSA-cerebellar subtype (MSA-C). The multisession rTMS intervention protocol has shown great promise to induce longer-term effects on the behavioral performance. Chang et al. (2010) observed that 10 sessions of daily rTMS induced improvement in motor function of subacute stroke patients and such improvement lasted for 3 months. Still, the effects of a multi-session rTMS intervention on motor control performance are largely unknown.

The functional regions of the brain are continuously interacting with each other over multiple temporal scales, even during "resting-state" (Khambhati et al., 2015). As such, the fluctuations of spontaneous brain activity, which can be recorded with blood-oxygen-level dependency (BOLD) fMRI, are "complex." Here, complexity refers to the presence of information-rich fractal-like patterns within the time-series. The degree of such resting-state complexity within largescale functional networks has been associated with functional performance. For example, Yang et al. (2013) reported that older adults who exhibited greater MSE-derived resting-state complexity within default mode network had better cognitive function.

In this study, we examined the effects of a five-session, daily rTMS intervention targeting both the bilateral cerebellum and M1 on motor control and the resting-state complexity of brain activity in a small sample of patients with MSA. We hypothesized that as compared to baseline, participants would demonstrate improved motor control, along with increased resting-state complexity, following the rTMS intervention. We further hypothesized that observed improvements in motor control would correlate with observed increases in resting-state complexity.

\section{MATERIALS AND METHODS}

\section{Participants}

Nine participants with de novo MSA [four men; age (mean \pm standard deviation): $58.0 \pm 7.0$ years, ranging from 50 to 66] were recruited from the Department of Neurodegenerative Disease, Center of Neurology, Beijing Tiantan Hospital, Capital Medical University (Beijing, China). The characteristics of each participant are summarized in Table 1. MSA was diagnosed according to Gilman et al. (2008) by two experienced clinicians. Three participants were diagnosed as MSA-parkinsonian subtype (MSA-P) and the other six presented with MSA-C. Exclusion criteria included contraindications to rTMS (i.e., significant medical or psychiatric illnesses, pregnancy, mental diseases, brain trauma, personal or family history of seizures or epilepsy, metallic or electrical bio-implants), contraindications to magnetic resonance imaging (MRI) (i.e., personal or family history of seizures or epilepsy, BMI $>40$, metallic or electrical bio-implants, claustrophobia), and cognitive impairment as defined by a Mini-Mental State Examination (MMSE) 
TABLE 1 | Participant demographics and clinical characteristics.

\begin{tabular}{ccccccc}
\hline ID & $\begin{array}{c}\text { MSA } \\
\text { subtype }\end{array}$ & $\begin{array}{c}\text { Age } \\
\text { (years) }\end{array}$ & Sex & $\begin{array}{c}\text { MSA } \\
\text { duration } \\
\text { (years) }\end{array}$ & IALT & $\begin{array}{c}\text { Baseline } \\
\text { motor score } \\
\text { of UMSARS }\end{array}$ \\
\hline 1 & P & 53 & M & 4 & 0 & 19 \\
2 & P & 57 & F & 3 & $22.58 \%$ & 22 \\
3 & P & 66 & F & 3 & $19.60 \%$ & 23 \\
4 & C & 51 & F & 2 & 0 & 19 \\
5 & C & 50 & F & 1.5 & 0 & 26 \\
6 & C & 66 & M & 2 & 0 & 12 \\
7 & C & 66 & M & 2 & 0 & 18 \\
8 & C & 62 & F & 2 & 0 & 22 \\
9 & C & 51 & M & 2 & 0 & 17 \\
\hline
\end{tabular}

P, MSA-P; C, MSA-C; M, male; F, female; IALT, improvement rate of acute stepwise levodopa challenge test; UMSARS, Unified Multiple System Atrophy Rating Scale.

score $\leq 24$. None of these participants had responded well to previous Levodopa treatment.

\section{Ethics Statement}

This study was approved by institutional review board of Beijing Tiantan Hospital, Capital Medical University and conducted according to the principles of the Declaration of Helsinki. The registration number of this study in Beijing Tiantan Hospital was KYSB2017-169-01. All participants provided written informed consent prior to screening and study participation.

\section{Study Protocol}

All participants completed one daily session of rTMS targeting both the cerebellum and bilateral M1 over five consecutive days. Motor control and resting-state brain activity were assessed at baseline and within 3 days of the last rTMS session. All participants stayed within the Tiantan Hospital Clinical Center throughout the entire study. Pre- and post-intervention assessments were conducted at approximately the same time of day and a clinician confirmed the absence of any acute medical condition that may have interfered with functional performance. Dopaminergic treatments, if used, were withheld throughout the course of the study.

\section{Repetitive Transcranial Magnetic Stimulation (rTMS)}

Participants received rTMS while seated in a chair with electromyography electrodes over the abductor pollicis brevis (APB) muscle to record motor evoked potentials (MEPs). A MagPro Compact stimulator (Dantec Medical, Copenhagen, Denmark) was connected to a 50-mm round coil (MCF-B65). The left and right targeting sites of M1 were determined as the location on the participant's scalp where TMS evoked maximal MEPs in the contralateral APB. The targeting sites of the cerebellum were $3 \mathrm{~cm}$ lateral to the left of the inion and $3 \mathrm{~cm}$ lateral to the right of the inion. The resting motor threshold (RMT) was measured with the left M1 and then the delivered intensity was set to at $100 \%$ of the RMT. The rTMS course consisted of daily sessions of 500 pulses for each M1 target and
500 pulses for each cerebellum target (i.e., 2000 pulses and 50 trains at $5 \mathrm{~Hz}$ for 5 days). In each session, rTMS was delivered over the left and right M1 and cerebellum sequentially.

\section{Motor Control Performance}

Motor control was assessed using the Motor Examination Scale within the Unified Multiple System Atrophy Rating Scale (UMSARS) (Wenning et al., 2004). This scale consists of 14 tests that measure multiple aspects of motor control (e.g., finger tapping, facial expression, posture, gait, etc.). Participant performance in each test was scored by an experienced clinician on a five-point scale ranging from 0 to 4 , where lower scores reflected better performance. Scores on the 14 tests were summed and used for analysis.

\section{Magnetic Resonance Imaging}

A Siemens Trio 3-Tesla scanner (Siemens, Erlangen, Germany) was used to acquire all MRI data. High-resolution brain structural images were acquired using T1-weighted, sagittal 3D magnetization-prepared rapid-gradient echo (MPRAGE) sequences with the following parameters: repetition time $(\mathrm{TR}) /$ echo time $(\mathrm{TE}) /$ inversion time $=2000 \mathrm{~ms} / 2.19 \mathrm{~ms} / 900 \mathrm{~ms}$, flip angle $(\mathrm{FA})=9^{\circ}$, field of view $(\mathrm{FOV})=224 \mathrm{~mm} \times 256 \mathrm{~mm}$, in-plane resolution $=224 \times 256$, slice thickness $=1 \mathrm{~mm}$, and 176 sagittal slices. Functional images were axially collected using an echo-planar imaging (EPI) sequence with the following settings: $\mathrm{TR} / \mathrm{TE}=2000 \mathrm{~ms} / 40 \mathrm{~ms}$, $\mathrm{FA}=90^{\circ}$, $\mathrm{FOV}=256 \mathrm{~mm} \times 256 \mathrm{~mm}$, resolution $=64 \times 64$, axial slices $=28$, thickness/gap $=4 \mathrm{~mm} / 1 \mathrm{~mm}$, bandwidth $=2230 \mathrm{~Hz} /$ pixel.

\section{Data Analysis}

\section{Resting-State fMRI}

Resting-state functional data were pre-processed using AFNI. The following steps were performed: volume registration, alignment to the T1 anatomy, warp into Talairach space, $8-\mathrm{mm}$ kernel smoothing, and scaling to a percentage of the mean. A band-pass filter was used to remove fluctuations below 0.01 and above $0.08 \mathrm{~Hz}$. Filtered data were enter into a general linear model to remove the effects of 6 degrees of motion, nuisance CSF, white matter, and global signal. The residual time series in each voxel from this deconvolve was then used to calculate multiscale entropy.

\section{Multiscale Entropy (MSE)}

Multiscale entropy (MSE) (Costa et al., 2002) was used to quantify the complexity of the spontaneous BOLD time series in each brain voxel (Figure 1A). MSE is a widely-used technique for quantifying the degree of re-occurrence of repetitive behavior (or patterns) across multiple time scales in a bio-physiological time-series, such that series with less pattern re-occurrence are more complex. Here, complexity was quantified using time scales one through five, as follows. The BOLD time series of each voxel was "coarse-grained" on these five scales by averaging point values using non-overlapping windows of length equaling to the scale factor $\tau$ (i.e., $\tau=1$ to 5 ). For example, the time series used for scale one was the original (filtered) time-series. The time-series at scale three was constructed by averaging every three points in the 
original time-series. The sample entropy of each coarse-grained time series was then calculated. Sample entropy is defined by the negative natural logarithm of the conditional probability that a time-series, having repeated itself within a tolerance $r$ for $\mathrm{m}$ points (defined pattern length), will also repeat itself for $m+1$ points without self-matches. Here, we chose $m=1$ and $r=0.35$, based upon previous studies that used MSE to analyze BOLD time-series (Yang et al., 2013, 2014, 2015).

The length of each coarse-grained time series was equal to the length of the raw time series divided by the scale factor. The number of data points in the coarse-grained time series at the maximum scale thus equaled to 23 (i.e., 117 divided by 5), greater than the $10^{\mathrm{m}}$ to $20^{\mathrm{m}}$ points (i.e., 10 to 20 as $m=1$ ) required for reliable estimation of sample entropy (Costa et al., 2002, 2005). The complexity index of each individual voxel BOLD time-series was then computed by averaging sample entropy across the five time scales (Yang et al., 2014) (see Figure 1B). Network-level resting-state complexity was calculated by parceling the brain into seven large functional networks (i.e., visual, motor, dorsal attention, ventral attention, limbic, executive, and default mode networks) according to Yeo et al. (2011). The parcellation was Talairach-normalized, resampled to $3 \times 3 \times 3$ voxels, and separated into individual networks. Resting-state complexity of each network was defined as the average complexity of all voxels within each network. As such, greater values reflected greater resting-state complexity of a given network.

\section{Statistical Analysis}

Analyses were performed with JMP Pro 12 software (SAS Institute, Cary, NC, United States). Primary outcomes were the UMSARS motor score and the resting-state complexity of the seven functional networks. Variable normality was examined with the Shapiro-Wilk $W$-test and homogeneity of variance was determined with the Levene test. Paired- $t$ tests were used to analyze the effects of tTMS on each outcome.

Linear regression analyses were used to determine the relationship between rTMS-induced percent changes in motor control and the resting-state complexity of each network at baseline, as well as the percent change in complexity from preto-post intervention. Significance level was set to $p<0.05$ for all analyses.

\section{RESULTS}

All participants completed the study and intervention compliance was $100 \%$. Stimulation was well-tolerated by all subjects and was not associated with any self-reported adverse event.

\section{The Effects of rTMS Intervention on Motor Control}

Motor scores at baseline and after intervention was normally distributed and exhibited homogeneity of variance. After the rTMS intervention, seven participants exhibited improved motor control (i.e., lower motor scores) as compared to baseline (Figure 2). At the group-level, the motor item score decreased from $19.8 \pm 4.1$ at baseline to $17.1 \pm 4.4$ after the intervention (averaged decrease: $2.7 \pm 1.9)\left(t_{8}=2.3, p=0.003\right)$.

\section{The Effect of rTMS Intervention on Resting-State Complexity}

Resting-state complexity values of the seven brain functional networks at baseline and after intervention were normally distributed and exhibited homogeneity of variance. After the rTMS intervention, six participants demonstrated an increase in resting-stated complexity within the motor cortex (Figure 3 ).
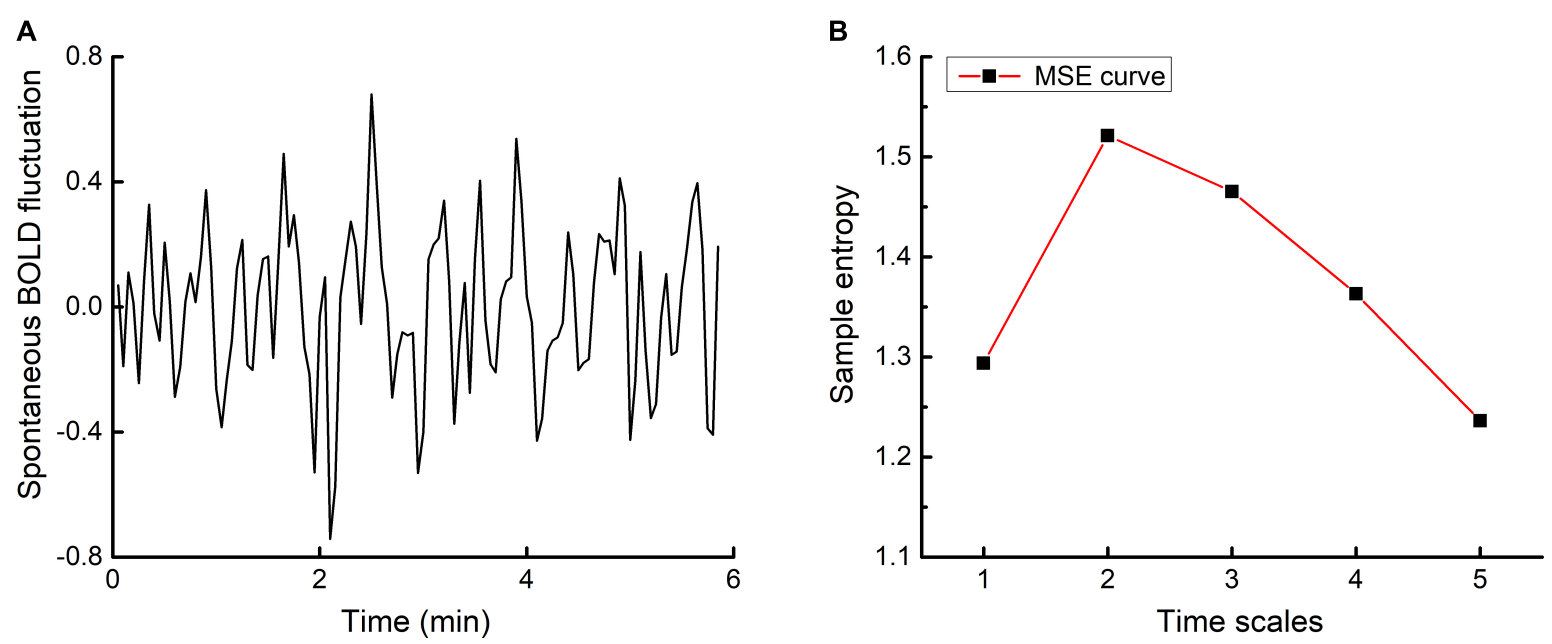

FIGURE 1 | An example time-series of resting-state BOLD fluctuations within one brain voxel (A) and its corresponding multiscale entropy (MSE) curve (B). The complexity of voxel-level BOLD fluctuations was determined by computing sample entropy across multiple time scales and averaging across said scales. Network-level resting-state complexity was then determined by parceling the brain into seven large-scale networks according to Yeo et al. (2011) and averaging the complexity values associated with all voxels within each network. 


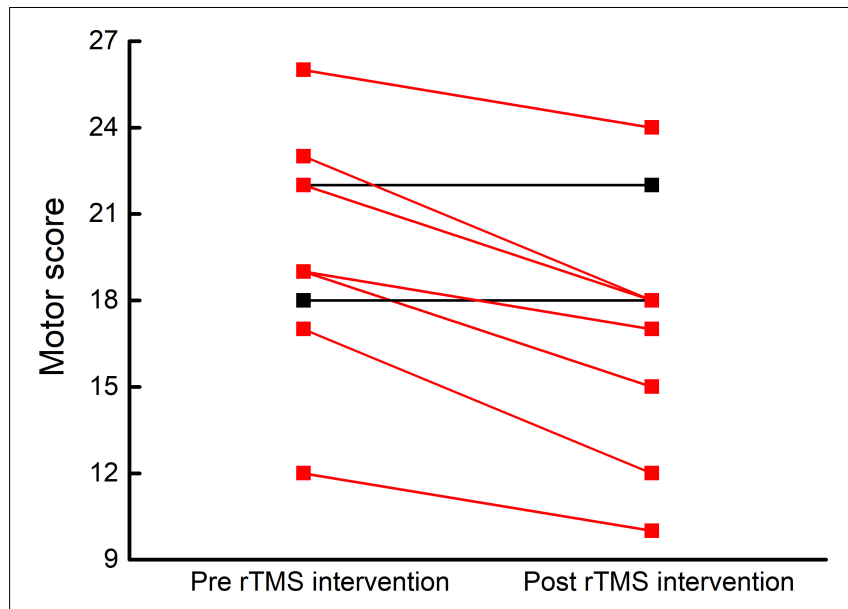

FIGURE 2 | Participant-level motor control before and after rTMS intervention. Motor control was assessed by the Motor Examination Scale within the Unified Multiple System Atrophy Rating Scale (UMSARS), where lower scores reflect better motor control. After five, once-daily sessions of rTMS targeting the cerebellum and bilateral M1, seven of nine participants exhibited lower motor scores (red lines).

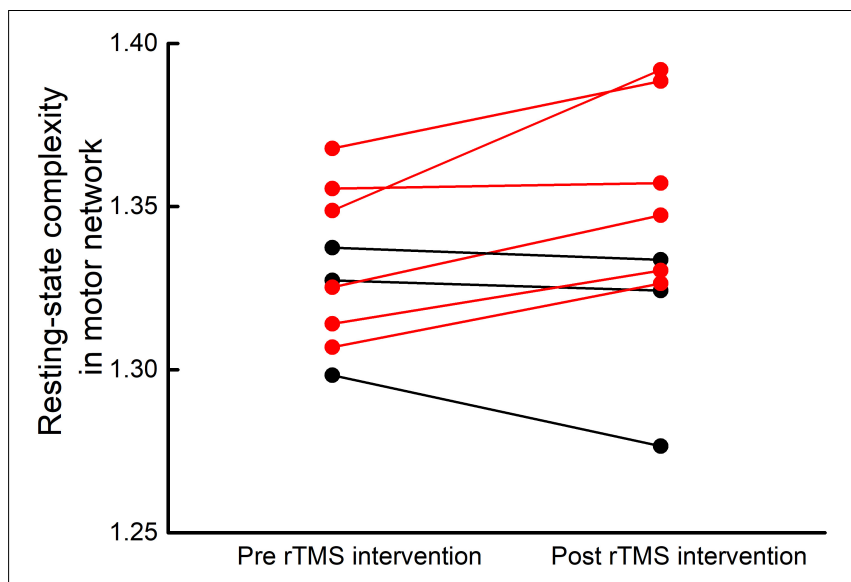

FIGURE 3 | Participant-level motor network resting-state complexity before and after rTMS intervention. After five, once-daily sessions of rTMS targeting the cerebellum and bilateral M1, six of nine participants exhibited increased resting-state complexity within the motor network (red lines).

A trend toward increased pre-to-post intervention motor network resting-state complexity was observed at the group level $\left(t_{8}=1.86, p=0.07\right)$. No changes were observed in the restingstate complexity of the other six networks $\left(t_{8}<1.7, p>0.13\right)$.

\section{Relationship Between Motor Function and Resting-State Complexity}

At baseline, motor scores did not correlate with the resting-state complexity of brain networks. However, linear regression analysis revealed that the percent change in motor score from pre-to-post intervention correlated with the pre-to-post percent change in motor network resting-state complexity $\left(r^{2}=0.72, p=0.004\right)$. Those who exhibited greater increases in motor control also

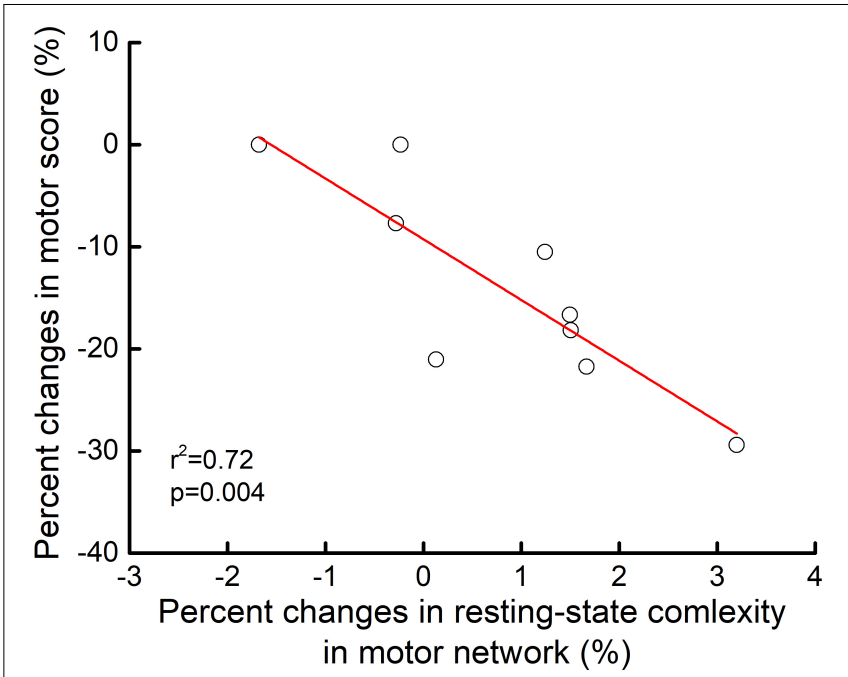

FIGURE 4 | Relationship between pre-to-post rTMS intervention changes in motor control and motor network resting-state complexity. From pre-to-post intervention, participants who exhibited greater percent decrease in motor item score (i.e., better performance) demonstrated greater percent increase in resting-state complexity within the motor network $\left(r^{2}=0.72, p=0.04\right)$. No other relationships between motor control and resting-state complexity reached significance.

experienced greater increases in resting-state complexity within the motor network (Figure 4).

\section{DISCUSSION}

Dysfunction of motor control is a severe pathological symptom of MSA and there are currently no effective strategies for alleviating such burden. This pilot study provided first-of-itskind preliminary evidence that a five-session rTMS intervention targeting both cerebellum and bilateral M1 is feasible and may improve motor control in patients with MSA. Moreover, such improvements appear to be linked to an increase in the physiologic complexity of resting-state brain activity dynamics, specifically within the motor network. Together, these initial observations warrant more definitive, well-controlled studies to establish the effectiveness of rTMS on motor control and underlying brain function in this vulnerable population.

The cerebellum and its connected neural network, including the bilateral M1, are closely involved in motor control. In the cerebellum-M1 circuit, the excitability (i.e., likelihood of neuronal firing) of $\mathrm{M} 1$ is modulated by the dentate nucleus and Purkinje cells within the cerebellum (Grimaldi et al., 2014). MSA impairs regulation of both the dentate nucleus and Purkinje cells, resulting in diminished excitability of M1 and ultimately, motor control dysfunction. High-frequency rTMS stimulation facilitates neuronal excitability within targeted regions (Rossi et al., 2009; Lefaucheur et al., 2014) and such facilitation can induce functional improvements. Recent metaanalyses and systematic reviews indicate that, for example, high frequency rTMS targeting M1 induces mild-to-moderate motor 
improvements (Chou et al., 2015; Wagle Shukla et al., 2016) in Parkinson disease (PD). Similarly, high frequency rTMS targeting the cerebellum may reduce cerebellar inhibition by modifying the activity of the dentate nucleus and Purkinje cells in the cerebellum (Cury et al., 2015), of which the underlying mechanism is worthwhile to be explored in future studies.

Recent non-invasive brain stimulation studies have demonstrated that "multi-focal" interventions (i.e., stimulating multiple regions within one session) may be particularly beneficial to motor control. Spagnolo et al. (2014) reported that a 12-session rTMS intervention targeting both the M1 and prefrontal regions induced significant improvement of motor function in those with Parkinson's disease, as evidenced by an 11 point average reduction in UPDRS scores. In the current study, rTMS targeted both the cerebellum and bilateral M1. This novel protocol may thus both directly increase the excitability of the M1 and reduce cerebellar inhibition of the M1. As the two regions are structurally and functionally connected, studies are needed to determine if targeting both regions have greater effects as compared to targeting either region separately.

Numerous studies have demonstrated that for a given physiological system, fluctuations within its spontaneous behavior are not random, but instead contain "meaningful" patterns over multiple scales of time (Lipsitz and Goldberger, 1992; Pikkujämsä et al., 1999; Manor and Lipsitz, 2013). The degree of complexity associated with these fluctuations reflects their "richness," or information content, and has been linked to system functionality (Manor et al., 2010; Zhou et al., 2017). Within the current small cohort, the rTMS intervention was associated with a trend toward increased resting-state complexity, specifically within the motor network. Moreover, those who exhibited greater increases in complexity also tended to make larger improvements in motor control. While appropriately-powered sham-controlled studies are needed to confirm these preliminary results, these observations suggest that observed motor control improvements were likely due to specific changes in brain function, rather than

\section{REFERENCES}

Ahdab, R., Ayache, S. S., Brugieres, P., Farhat, W. H., and Lefaucheur, J. P. (2016). The hand motor hotspot is not always located in the hand knob: a neuronavigated transcranial magnetic stimulation study. Brain Topogr. 29, 590-597. doi: 10.1007/s10548-016-0486-2

Burciu, R. G., Chung, J. W., Shukla, P., Ofori, E., Li, H., McFarland, N. R., et al. (2016). Functional MRI of disease progression in Parkinson disease and atypical parkinsonian syndromes. Neurology 87, 709-717. doi: 10.1212/WNL. 0000000000002985

Chang, W. H., Kim, Y. H., Bang, O. Y., Kim, S. T., Park, Y. H., and Lee, P. K. (2010). Long-term effects of rTMS on motor recovery in patients after subacute stroke. J. Rehabil. Med. 42, 758-764. doi: 10.2340/16501977-0590

Chou, Y.-h., Hickey, P. T., Sundman, M., Song, A. W., and Chen, N. K. (2015). Effects of repetitive transcranial magnetic stimulation on motor symptoms in Parkinson disease: a systematic review and meta-analysis. JAMA Neurol. 72, 432-440. doi: 10.1001/jamaneurol.2014.4380

Costa, M., Goldberger, A. L., and Peng, C. K. (2002). Multiscale entropy analysis of complex physiologic time series. Phys. Rev. Lett. 89:068102. doi: 10.1103/ PhysRevLett.89.068102 by a placebo effect or a general effect of rTMS on brain excitability.

In this small pilot study, rTMS targets were determined by anatomical landmarks. The use of neuro-navigated rTMS based upon structural brain images may improve the effects of intervention (Ruohonen and Karhu, 2010), especially within this population as brain anatomy varies considerably across individuals (Ahdab et al., 2016). Future studies should also consider examining additional clinically-meaningful aspects of cognitive-motor performance, such as complex reaction time and/or the kinematics of gait and postural control. Finally, this study focused only on the immediate effects of rTMS intervention. As several studies have demonstrated longer-lasting effects of similar intervention within other populations (Helmich et al., 2006; Chang et al., 2010), studies with longer follow-ups are warranted.

\section{AUTHOR CONTRIBUTIONS}

ZL, HM, JZ, and TF designed the study. XW, ZW, YY, and LM collected the data. ZL, JZ, and VP analyzed the data and performed statistical analyses. ZL, BM, JZ, and TF drafted the manuscript. All authors contributed and approved the final version.

\section{FUNDING}

This study was supported by the Golden Bridge Project Seed Funding (Beijing Association for Science and Technology). JZ was supported by the Irma and Paul Milstein Program for Senior Health Fellowship Award from the Milstein Medical Asian American Partnership (MMAAP) Foundation. BM was supported by the National Institute on Aging-funded Boston Claude D. Pepper Older Americans Independence Center (Grant No. 2 P30 AG031679).

Costa, M., Goldberger, A. L., and Peng, C. K. (2005). Multiscale entropy analysis of biological signals. Phys. Rev. E 71:021906. doi: 10.1103/PhysRevE.71.021906

Cury, R. G., Teixeira, M. J., Galhardoni, R., Barboza, V. R., Alho, E., Seixas, C. M., et al. (2015). Neuronavigation-guided transcranial magnetic stimulation of the dentate nucleus improves cerebellar ataxia: a sham-controlled, double-blind $\mathrm{n}=1$ study. Parkinsonism Relat. Disord. 21, 999-1001. doi: 10.1016/j.parkreldis. 2015.05.010

Fanciulli, A., and Wenning, G. K. (2015). Multiple-system atrophy. N. Engl. J. Med. 372, 249-263. doi: 10.1056/NEJMra1311488

Gilman, S., Wenning, G. K., Low, P. A., Brooks, D. J., Mathias, C. J., Trojanowski, J. Q., et al. (2008). Second consensus statement on the diagnosis of multiple system atrophy. Neurology 71, 670-676. doi: 10.1212/01.wnl.0000324625.004 04.15

Grimaldi, G., Argyropoulos, G. P., Boehringer, A., Celnik, P., Edwards, M. J., Ferrucci, R., et al. (2014). Non-invasive cerebellar stimulation-a consensus paper. Cerebellum 13, 121-138. doi: 10.1007/s12311-013-0514-7

Helmich, R. C., Siebner, H. R., Bakker, M., Munchau, A., and Bloem, B. R. (2006). Repetitive transcranial magnetic stimulation to improve mood and motor function in Parkinson's disease. J. Neurol. Sci. 248, 84-96. doi: 10.1016/j.jns. 2006.05.009 
Khambhati, A. N., Davis, K. A., Oommen, B. S., Chen, S. H., Lucas, T. H., Litt, B., et al. (2015). Dynamic network drivers of seizure generation, propagation and termination in human neocortical epilepsy. PLoS Comput. Biol. 11:e1004608. doi: 10.1371/journal.pcbi.1004608

Kollensperger, M., Geser, F., Ndayisaba, J. P., Boesch, S., Seppi, K., Ostergaard, K., et al. (2010). Presentation, diagnosis, and management of multiple system atrophy in Europe: final analysis of the European multiple system atrophy registry. Mov. Disord. 25, 2604-2612. doi: 10.1002/mds.23192

Krismer, F., and Wenning, G. K. (2017). Multiple system atrophy: insights into a rare and debilitating movement disorder. Nat. Rev. Neurol. 13, 232-243. doi: 10.1038/nrneurol.2017.26

Lefaucheur, J.-P., André-Obadia, N., Antal, A., Ayache, S. S., Baeken, C., Benninger, D. H., et al. (2014). Evidence-based guidelines on the therapeutic use of repetitive transcranial magnetic stimulation (rTMS). Clin. Neurophysiol. 125, 2150-2206. doi: 10.1016/j.clinph.2014.05.021

Lipsitz, L. A., and Goldberger, A. L. (1992). Loss of 'complexity' and aging. Potential applications of fractals and chaos theory to senescence. JAMA 267, 1806-1809. doi: 10.1001/jama.1992.03480130122036

Lu, C. F., Soong, B. W., Wu, H. M., Teng, S., Wang, P. S., and Wu, Y. T. (2013). Disrupted cerebellar connectivity reduces whole-brain network efficiency in multiple system atrophy. Mov. Disord. 28, 362-369. doi: 10.1002/mds. 25314

Maass, S., Levin, J., and Hoglinger, G. (2016). Current treatment of multiple system atrophy. Curr. Treat. Options Neurol. 18, 51.

Manor, B., Costa, M. D., Hu, K., Newton, E., Starobinets, O., Kang, H. G., et al. (2010). Physiological complexity and system adaptability: evidence from postural control dynamics of older adults. J. Appl. Physiol. 109, 1786-1791. doi: 10.1152/japplphysiol.00390.2010

Manor, B., and Lipsitz, L. A. (2013). Physiologic complexity and aging: implications for physical function and rehabilitation. Prog. Neuropsychopharmacol. Biol. Psychiatry 45, 287-293. doi: 10.1016/j.pnpbp.2012.08.020

Payoux, P., Brefel-Courbon, C., Ory-Magne, F., Regragui, W., Thalamas, C., Balduyck, S., et al. (2010). Motor activation in multiple system atrophy and Parkinson disease: a PET study. Neurology 75, 1174-1180. doi: 10.1212/WNL. 0b013e3181f4d78f

Pikkujämsä, S. M., Mäkikallio, T. H., Sourander, L. B., Räihä, I. J., Puukka, P., Skyttä, J., et al. (1999). Cardiac interbeat interval dynamics from childhood to senescence : comparison of conventional and new measures based on fractals and chaos theory. Circulation 100, 393-399. doi: 10.1161/01.CIR.100.4.393

Rohrer, G., Hoglinger, G. U., and Levin, J. (2017). Symptomatic therapy of multiple system atrophy. Auton. Neurosci. 211, 26-30. doi: 10.1016/j.autneu.2017.10.006

Rossi, S., Hallett, M., Rossini, P. M., Pascual-Leone, A., and The Safety of TMS Consensus Group (2009). Safety, ethical considerations, and application guidelines for the use of transcranial magnetic stimulation in clinical practice and research. Clin. Neurophysiol. 120, 2008-2039. doi: 10.1016/j.clinph.2009. 08.016

Ruohonen, J., and Karhu, J. (2010). Navigated transcranial magnetic stimulation. Neurophysiol. Clin. 40, 7-17. doi: 10.1016/j.neucli.2010.01.006
Spagnolo, F., Volonté, M. A., Fichera, M., Chieffo, R., Houdayer, E., Bianco, M., et al. (2014). Excitatory deep repetitive transcranial magnetic stimulation with H-coil as add-on treatment of motor symptoms in Parkinson's disease: an open label, pilot study. Brain Stimul. 7, 297-300. doi: 10.1016/j.brs.2013. 10.007

Wagle Shukla, A., Shuster, J. J., Chung, J. W., Vaillancourt, D. E., Patten, C., Ostrem, J., et al. (2016). Repetitive transcranial magnetic stimulation (rTMS) therapy in Parkinson disease: a meta-analysis. $P M R$ 8, 356-366.

Wenning, G. K., Tison, F., Seppi, K., Sampaio, C., Diem, A., Yekhlef, F., et al. (2004). Development and validation of the Unified Multiple System Atrophy Rating Scale (UMSARS). Mov. Disord. 19, 1391-1402. doi: 10.1002/mds.20255

Yang, A. C., Hong, C. J., Liou, Y. J., Huang, K. L., Huang, C. C., Liu, M. E., et al. (2015). Decreased resting-state brain activity complexity in schizophrenia characterized by both increased regularity and randomness. Hum. Brain Mapp. 36, 2174-2186. doi: 10.1002/hbm.22763

Yang, A. C., Huang, C. C., Liu, M. E., Liou, Y. J., Hong, C. J., Lo, M. T., et al. (2014). The APOE varepsilon4 allele affects complexity and functional connectivity of resting brain activity in healthy adults. Hum. Brain Mapp. 35, 3238-3248. doi: 10.1002/hbm.22398

Yang, A. C., Huang, C. C., Yeh, H. L., Liu, M. E., Hong, C. J., Tu, P. C., et al. (2013). Complexity of spontaneous BOLD activity in default mode network is correlated with cognitive function in normal male elderly: a multiscale entropy analysis. Neurobiol. Aging 34, 428-438. doi: 10.1016/j.neurobiolaging.2012.0 5.004

Yeo, B. T., Krienen, F. M., Sepulcre, J., Sabuncu, M. R., Lashkari, D., Hollinshead, M., et al. (2011). The organization of the human cerebral cortex estimated by intrinsic functional connectivity. J. Neurophysiol. 106, 1125-1165. doi: 10.1152/jn.00338.2011

Yildiz, F. G., Saka, E., Elibol, B., and Temucin, C. M. (2017). Modulation of cerebellar-cortical connections in multiple system atrophy type $\mathrm{C}$ by cerebellar repetitive transcranial magnetic stimulation. Neuromodulation doi: 10.1111/ ner.12589 [Epub ahead of print].

Zhou, J., Habtemariam, D., Iloputaife, I., Lipsitz, L. A., and Manor, B. (2017). The complexity of standing postural sway associates with future falls in communitydwelling older adults: the MOBILIZE Boston study. Sci. Rep. 7:2924. doi: $10.1038 /$ s41598-017-03422-4

Conflict of Interest Statement: The authors declare that the research was conducted in the absence of any commercial or financial relationships that could be construed as a potential conflict of interest.

Copyright (c) 2018 Liu, Ma, Poole, Wang, Wang, Yang, Meng, Manor, Zhou and Feng. This is an open-access article distributed under the terms of the Creative Commons Attribution License (CC BY). The use, distribution or reproduction in other forums is permitted, provided the original author(s) and the copyright owner are credited and that the original publication in this journal is cited, in accordance with accepted academic practice. No use, distribution or reproduction is permitted which does not comply with these terms. 


\section{OPEN ACCESS}

Edited by:

Roberta Ferrucci,

Fondazione IRCCS Ca' Granda Ospedale Maggiore Policlinico, Italy

Reviewed by:

Ali Yadollahpour

Ahvaz Jundishapur University of Medical Sciences, Iran

Filippo Brighina,

Università degli Studi di Palermo, Italy Simona Mrakic-Sposta, Istituto di Bioimmagini e Fisiologia Molecolare (IBFM), Italy

${ }^{*}$ Correspondence: Ulrich Palm ulrich.palm@med.uni-muenchen.de

Received: 01 February 2018 Accepted: 07 August 2018 Published: 24 August 2018

Citation:

Behler N, Leitner B, Mezger E, Weidinger E, Musil R, Blum B, Kirsch B, Wulf L, Löhrs L, Winter C, Padberg F and Palm U (2018) Cathodal tDCS Over Motor Cortex Does Not Improve Tourette Syndrome: Lessons Learned From a Case Series.

Front. Behav. Neurosci. 12:194. doi: 10.3389/fnbeh.2018.00194

\section{Cathodal tDCS Over Motor Cortex Does Not Improve Tourette Syndrome: Lessons Learned From a Case Series}

\author{
Nora Behler ${ }^{1}$, Bianka Leitner ${ }^{1}$, Eva Mezger ${ }^{1}$, Elif Weidinger ${ }^{1}$, Richard Musil ${ }^{1}$, \\ Bernhard Blum ${ }^{1,2}$, Beatrice Kirsch ${ }^{1}$, Linda Wulf ${ }^{1,3}$, Lisa Löhrs ${ }^{1}$, Christine Winter ${ }^{4}$, \\ Frank Padberg ${ }^{1}$ and Ulrich Palm ${ }^{1 *}$
}

'Department of Psychiatry and Psychotherapy, Ludwig-Maximilian University, Klinikum der Universität München, Munich, Germany, ${ }^{2}$ Department of Neurology, Ludwig-Maximilian University, Klinikum der Universität München, Munich, Germany, ${ }^{3}$ neuroCare Group, Munich, Germany, ${ }^{4}$ Department of Psychiatry and Psychotherapy, Charité Universitätsmedizin Berlin, Berlin, Germany

Introduction: Current pathophysiological hypotheses of Gilles de la Tourette Syndrome (GTS) refer to temporally abnormal neuronal activation in cortico-striato-thalamo-cortical (CSTC) networks. Modifying cortical activity by non-invasive brain-stimulation appears to be a new treatment option in GTS.

Background: Previous studies suggested therapeutic effects of cathodal transcranial direct current stimulation (tDCS) to pre-supplementary motor areas (SMA), however, treatment modalities concerning electrode placement, current intensity and stimulation-rate have not been systematically explored. Aim of this study was to assess efficacy of an alternative stimulation regime on GTS symptoms in a pilot study. To test a treatment protocol with tDCS twice a day, we administered 10 sessions over 5 days of bilateral cathodal tDCS (30 min, $2 \mathrm{~mA}$ ) over the pre-SMA in three patients with severe GTS. Tic severity as well as obsessive-compulsive $(\mathrm{OC})$ symptoms and affective scales were rated before and after tDCS treatment.

Discussion: Only one out of three patients showed a 34.5\% reduction in tic severity. The two other patients showed an increase in tic severity. All patients showed a mild increase in positive affect and a reduction in negative affect, OC symptom changes were heterogeneous. Our results do not support earlier findings of extensive therapeutic effects of cathodal tDCS on tics in patients with GTS and show that prediction of stimulation effects on a targeted brain area remains inaccurate.

Concluding Remarks: Future research will have to focus on the determination of most effective stimulation modes regarding site, polarity and frequency of tDCS in GTS patients.

Keywords: tDCS, transcranial direct current stimulation, GTS, tourette syndrome, supplementary motor areas, OCD, obsessive compulsive disorder 


\section{INTRODUCTION}

Gilles de la Tourette syndrome (GTS) is a neuropsychiatric disorder with chronic motor and vocal tics, manifesting in the course of childhood and early adolescence. Tics are usually preceded by premonitory urges and can be suppressed at will. The disorder is self-limiting in at least $44 \%$ of all cases and symptoms tend to significantly decrease in early adulthood. Still there are $22 \%$ of adult patients retaining clinically significant symptoms despite adequate medication (Leckman et al., 1998; Burd et al., 2001; Pappert et al., 2003; Evans et al., 2016). Approved pharmacological treatment consists of alpha-2-adreno-receptor-blockers, and distinct antipsychotics. Both groups of medications are associated with mild to severe side effects including sedation, cardiovascular dysregulation, extrapyramidal motor symptoms (EPMS), sexual dysfunction, weight gain or cardiac risks that are scarcely tolerated by patients. Moreover, depression and anxiety are common co-morbid disorders in adult GTS patients (Evans et al., 2016), and there is an additional need for specific treatment in these domains. Psychotherapeutic treatment such as habit reversal training or exposure with response prevention require sufficient adherence while also yielding only incomplete remission rates (Capriotti et al., 2014). Tic manifestation in GTS is usually referred to an organic etiology. Functional neuroimaging has revealed that tic symptoms originate from a dysregulation of corticostriato-thalamo-cortical (CSTC) networks. Results show a down regulation of movement inhibition in caudate and anterior cingulate cortex and an hyperactivation of motor pathways in putamen, pallidum and substania nigra, as well as in cortical regions of the sensorimotor cortex with cortical hyperactivation in pre-motor regions preceding basal ganglia activation (Wang et al., 2011). Therefore, modulation of basal ganglia function by deep brain stimulation (DBS) might offer a third track of treatment besides psychopharmacology and psychotherapy. DBS has shown promising results in GTS while resulting in high operative risk and diverse side effects, restricting treatment to a severely affected group of adult patients (Visser-Vandewalle et al., 2014; Schrock et al., 2015). In summary, treatment alternatives are sparse and results are dissatisfying due to lack in treatment response or extensive side effects. In view of the success in DBS and the implication of cortical dysregulation, non-invasive brain stimulation (NIBS) appears a viable choice without the risk of invasive treatments. Repetitive transcranial magnetic stimulation (rTMS), inducing an electric current in cortical regions via a pulsed magnetic field, has been shown to be efficient in tic reduction when applied at $1 \mathrm{~Hz}$ over the supplementary motor area (SMA; Mantovani et al., 2006). In children, positive results lasted at least 6 months (Le et al., 2013). However these studies were open label without sham control. Landeros-Weisenberger et al. (2015) presented the first sham controlled double-blind rTMS study in patients with severe GTS and could not show a significant difference in tic improvement after active rTMS compared to sham rTMS. Thus, results of rTMS in the treatment of GTS are controversial so far (Kious et al., 2016; Pedroarena-Leal and Ruge, 2017). Another emerging NIBS technique, transcranial direct current stimulation (tDCS) has been shown to modulate motor cortex excitability (Nitsche and Paulus, 2001) and frontal network activity (Keeser et al., 2011a). The modulation of large scale brain networks close to the stimulation site and in remote areas could serve as a surrogate for a hypothesized influence of tDCS on activation in basal ganglia. Altering activity in whole-brain networks by stimulation of specific nodes has been proposed as a mode of treatment in GTS, while the appropriate dosage and targets remain to be established (Pedroarena-Leal and Ruge, 2017). tDCS is considered safe, with very limited side effects, such as itching of the skin, light headaches or dizziness (Nitsche et al., 2003; Bikson et al., 2016). Commonly, daily treatment is performed in acute phases of disease and intermittent and even home treatment are being explored in chronic therapeutic settings (Charvet et al., 2015; Palm et al., 2018) and in phases of remission, treatment-free periods can be elongated according to individual patient needs. These options appear especially appealing in a usually young and mentally unimpaired patient group such as GTS patients.

In tDCS, polarity of stimulation over the affected cortical region is a determinant of treatment effects. Cathodal stimulation is thought to have inhibiting effects, while anodal stimulation inversely has increasing effects on cortical excitability (Nitsche and Paulus, 2001). As functional magnetic resonance imaging (fMRI) revealed increased activity preceding tic manifestation in cortical pre-motor and motor regions (Wang et al., 2011), cathodal tDCS over these areas may reduce excitability in these regions. Previous findings in single GTS patients suggested therapeutic effects of cathodal tDCS of pre-SMA both via monolateral treatment of the most affected side or bilateral treatment, with extracephalic anodal reference electrodes, respectively (Mrakic-Sposta et al., 2008; Carvalho et al., 2015). Though randomized controlled clinical trials (RCT) investigating tDCS in GTS are lacking, further data together with an RCT protocol have been published recently (Eapen et al., 2017). There, patients will be treated with 18 sessions of $1.4 \mathrm{~mA}$ tDCS with the cathode positioned over SMA area and the reference electrode over the right deltoid muscle. At least one of the two pilot patients showed relevant improvement of tic severity during treatment period. As optimal tDCS parameters have not been identified to date, we investigated twice-daily sessions of bilateral cathodal tDCS over the pre-SMA for 5 days in three patients with GTS. This approach aims to assess a different stimulation regime from previous studies to further knowledge on stimulation effects in patients with this condition.

\section{MATERIALS AND METHODS}

Three GTS patients from the Department of Psychiatry, University of Munich, underwent twice-daily tDCS treatment for 5 days. All patients gave their written informed consent for an individual treatment attempt by use of tDCS as an experimental compassionate use and agreed on their data being anonymously published after completion of the treatment. Pre-existing pharmacological treatment was not altered before or during tDCS treatment and, if receiving psychopharmacologic 
drugs, patients had a stable dosage for at least 3 weeks without change of motor symptoms.

\section{Transcranial Direct Current Stimulation}

Two milliampere tDCS was applied twice a day for $30 \mathrm{~min}$ (in total 10 stimulations in 5 days) following previous findings on safety of repeated twice-daily tDCS in depressive disorders (Palm et al., 2015). Both tDCS sessions were performed in the morning to avoid further circadian influences and were separated by an interval of approximately $3 \mathrm{~h}$ (e.g., 8:00, 11:00). Two independent stimulators were used parallel to stimulate both hemispheres. Cathodal stimulation was performed using rectangular $7 \times 5 \mathrm{~cm}=35 \mathrm{~cm}^{2}$ saline soaked sponge electrodes, placed bilaterally and longitudinally over motor areas of the cortex (C3, C4, according to 10-20 EEG system) with $1 \mathrm{~cm}$ space between them (Figure 1). With this montage, SMA and pre-SMA areas were also covered. Anodal electrodes were each fixed with adhesive tape ipsilateral over the sternocleidomastoid muscle (extracephalic electrodes). Direct current was applied with an eldith ${ }^{\circledR}$ DC-stimulator (neuroConn, Ilmenau, Germany). For better tolerance, stimulation was ramped up and ramped down over $15 \mathrm{~s}$

\section{Measurements}

Before and after tDCS series, the following questionnaires were administered: Yale Global Tic Severity Scale (YGTSS;
Leckman et al., 1989; Storch et al., 2005) to quantify tic severity, Yale-Brown Obsessive Compulsive Scale (Y-BOCS; Goodman et al., 1989) to assess obsessive and compulsive behavior and the Positive and Negative Affect Schedule (PANAS; Krohne et al., 1996) to evaluate affective symptoms. Tic frequency was assessed by counting tics during a 3 min interval while sitting at rest in a quiet room. To evaluate the effect of tDCS, percentage changes of data obtained at day five as compared to results at baseline were calculated.

\section{PATIENT CHARACTERISTICS AND CLINICAL HISTORY}

The first patient (P1), a 55 year old male, presented with simple motor and simple vocal tics (age at onset: 9/10 years) as well as obsessive compulsive disorder (OCD). Motor tics included grimacing, grinding of teeth, tensing of shoulder and nuchal muscles, increasing during times of rest and stressful situations. Vocal tics included loud exclamations, sniffing, harrumphing or grunting. Tic frequency was low. OCD symptoms included compulsive checking and writing. Current medication was limited to antihypertensive medication (candesartan $8 \mathrm{mg} /$ day), previous treatment with aripiprazole showed slight reduction in tic frequency with unacceptable side effects of extensive weight gain and was discontinued 4 years ago. Psychotherapeutic treatment was
A

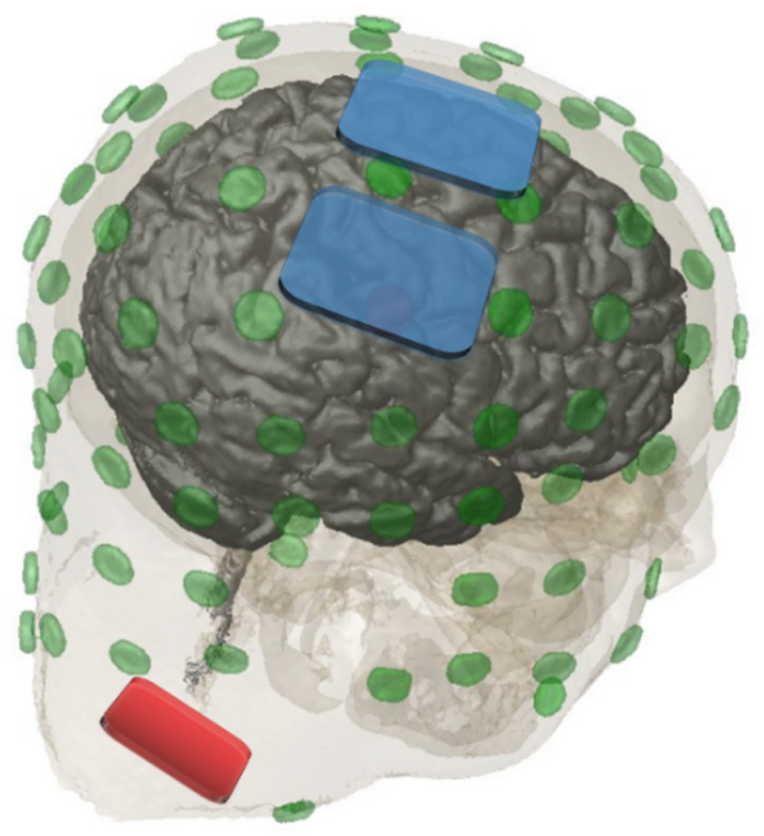

B

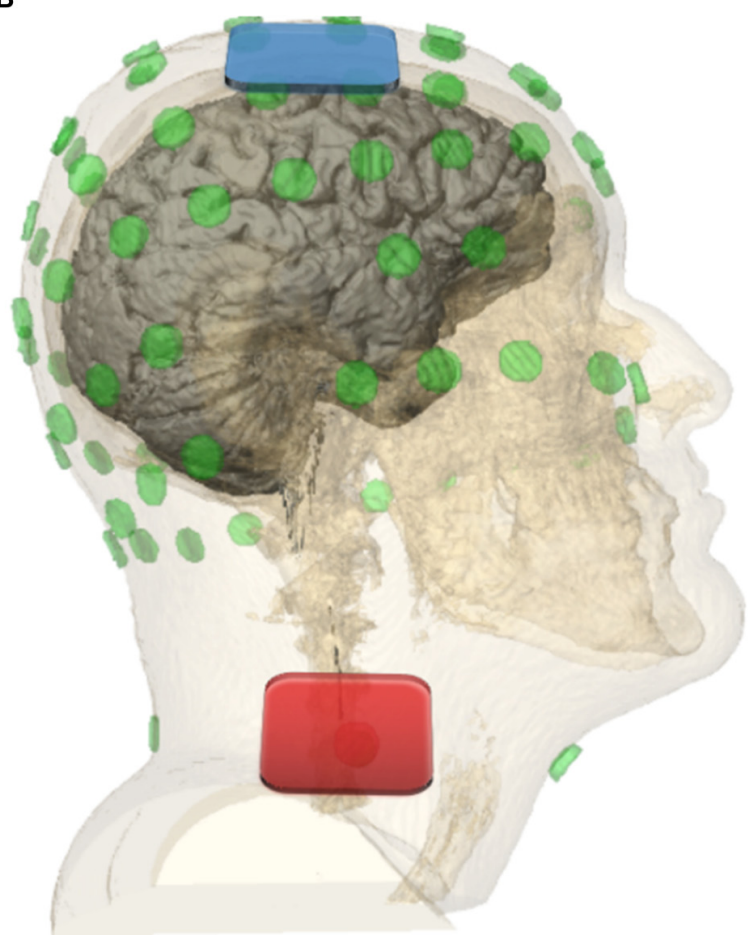

FIGURE 1 | Dorsal lateral view (A) and lateral view (B; Soterix Medical HD-Explore $\left.{ }^{\mathrm{TM}}\right)$. Cathodal electrodes $\left(7 \times 5 \mathrm{~cm}=35 \mathrm{~cm}^{2}\right)$ were placed bilaterally over (pre-)motor cortical areas (C3, C4) with $1 \mathrm{~cm}$ space in between. Anodal electrodes were placed ipsilateral over the sternocleidomastoid muscle. Current strength of $2 \mathrm{~mA}$ was applied for $30 \mathrm{~min}$. 
limited to treatment for OCD symptoms several months before undergoing tDCS.

The second patient (P2), a 20 year old male, presented with simple motor and simple vocal tics (age at onset: 16 years). P2 did not show any OCD symptoms. Motor tics included striking out of both arms (with ensuing self-harming effects), jerking of the head and facial muscles. Vocal tics included barking and uttering of syllables. Current treatment consisted of atypical antipsychotics (tiapride $600 \mathrm{mg} /$ day, olanzapine $15 \mathrm{mg} /$ day). Previous treatment also comprised atypical antipsychotics (risperidone, amisulpride, aripiprazole, olanzapine and quetiapine). The patient showed a history of substance-induced psychotic symptoms (tetrahydrocannabinol and "spice") without current use of illegal substances and was abstinent since 3 years. He also exhibited dissociative seizures during youth that have been treated successfully by an alternative practitioner by eye movement desensitization and reprocessing (EMDR) and hypnosis.

The third patient (P3), an 18 year old female, presented with frequent simple and complex motor as well as vocal tics (age at onset: 14 years). She was also suffering from OCD symptoms. Motor tics included hitting own thorax and pelvis with her fists, flipping, grimacing, jerking of the head, shoulders and hands, gesturing (Russian roulette), saluting and locking her feet while walking. Vocal tics included harrumphing, whistling, caterwauling, uttering syllables, words, limited sentences and echolalia. Tic-free sequences were short (max. $1 \mathrm{~min}$ ), urges to perform tics were rated very high by the patient. OCD symptoms included compulsive counting, repeating, checking and arranging/collocating. Current treatment consisted of risperidone $6 \mathrm{mg} /$ day and biperiden $4 \mathrm{mg} /$ day in a stable dose for 3 weeks. Previous treatment included antidepressants and atypical antipsychotics (fluoxetine $30 \mathrm{mg} /$ day, aripiprazole $10 \mathrm{mg} /$ day, tiapride $600 \mathrm{mg} / \mathrm{day}$, and quetiapine in unknown dosage) and was discontinued more than 1 year before due to symptom-alleviation (fluoxetine) or lack in positive therapeutic outcome or inacceptable side effects (aripiprazole, tiapride, quetiapine).

\section{RESULTS}

All patients completed twice daily sessions of tDCS for 5 days and stimulations were generally well tolerated. Side effects were mild skin irritation in P1 due to adhesive tape over the electrode, mild headache and metallic taste during stimulation in P2, none in P3.

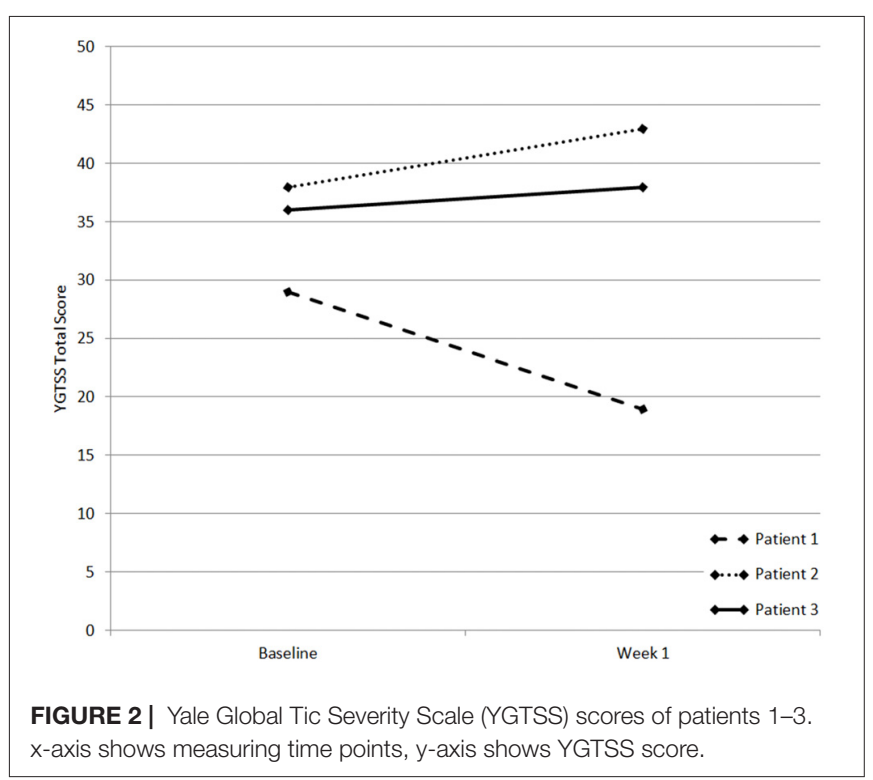

Prior to stimulation, global tic severity scores (YGTSS), not including general impairment scores, were rated at 29, 38 and 36 points for patients 1,2 and 3 , respectively. After tDCS for 5 days, tic-severity decreased in patient 1 by 10 points to finally 19 points. Both patients 2 and 3 showed an increase in tic-severity by 5 points to 43 points (P2) and 2 points to 38 points (P3, Figure 2). General impairment scores as measured by the YGTSS did not differ in comparison between pre and post tDCS measurements. P1 showed impairment of 10 points, P2 30 points and $\mathrm{P} 350$ points.

OCD symptoms were present only in patients 1 and 3, rated at 18 and 10 points, respectively. Patient 1 reported a reduction of $83.3 \%$ in OCD symptoms following stimulation. Patient 3 showed an increase of $20 \%$ in OCD symptoms.

Affective symptoms were self-rated and categorized into PANAS negative and positive subscores. All three patients expressed a decrease in negative and an increase in positive mood following stimulation. Most notable changes occurred in patient 2 with an increase of positive affect of $30.8 \%$ and a decrease of negative affect of $20 \%$.

The $3 \mathrm{~min}$ tic-count showed high inter-individual variability, patients 1 and 2 experienced 11 and 19 tics in $3 \mathrm{~min}$, whereas patient 3 experienced 218 tics in $3 \mathrm{~min}$. Surprisingly, contrary to the reduction in tic severity observed in patient 1, tic-counts showed an increase in all three subjects, following stimulation (increases by $18.2 \%$ in patient 1 , by $200 \%$ in patient 2 and by $70.2 \%$ in patient 3). Clinical data is reported in Table 1.

TABLE 1 | Clinical rating before (pre) and after (post) transcranial direct current stimulation (tDCS) treatment for 5 days.

\begin{tabular}{|c|c|c|c|c|c|c|c|c|c|}
\hline \multirow[t]{2}{*}{ Tests } & \multicolumn{3}{|c|}{ Patient 1} & \multicolumn{3}{|c|}{ Patient 2} & \multicolumn{3}{|c|}{ Patient 3} \\
\hline & pre & post & $\Delta[\%]$ & pre & post & $\Delta[\%]$ & pre & post & $\Delta[\%]$ \\
\hline YGTSS & 29 & 19 & -34.5 & 38 & 43 & 13.2 & 36 & 38 & 5.6 \\
\hline YBOCS & 18 & 3 & -83.3 & 0 & 0 & 0.0 & 10 & 12 & 20.0 \\
\hline PANAS neg. & 17 & 16 & -5.9 & 15 & 12 & -20.0 & 23 & 19 & -17.4 \\
\hline PANAS pos. & 34 & 35 & 2.9 & 26 & 34 & 30.8 & 26 & 27 & 3.8 \\
\hline tic count (3 min) & 11 & 13 & 18.2 & 19 & 57 & 200.0 & 218 & 371 & 70.2 \\
\hline
\end{tabular}




\section{DISCUSSION}

Here, we report three cases of GTS patients undergoing bilateral cathodal tDCS of SMA and pre-SMA areas. This intervention did not decrease tic severity in two out of three patients. Only one patient reported a reduction of tic severity, but even more marked of OCD symptoms. In contrast, an increase in tic severity was observed in the other two subjects. All subjects showed an increase in $3 \mathrm{~min}$ tic count and a decrease in negative emotions following stimulation as well as a slight increase in positive affect. Thus, the current tDCS protocol over SMA and pre-SMA areas was not successful in treating GTS core symptoms in contrast to previous studies (Mrakic-Sposta et al., 2008; Carvalho et al., 2015; Eapen et al., 2017).

There are clear limitations of our approach: first, the problem of natural waxing and waning of tics in GTS which is adding to the issue of the very small sample size of our observation. Second, the heterogeneous clinical characteristics of our patients and the variance of co-medication (Brunoni et al., 2012; Nitsche et al., 2012) may impact on stimulation efficacy, but also reflect a disorder with frequent and typical side diagnoses and insufficient efficacy of singular pharmacotherapy. Of note, regarding GTS epidemiology, P2 and P3 showed an untypically late onset of tics at the age of 16 and 14 years respectively and did not show any therapeutic efficacy, whereas P1 had a more typical tic onset at 9 years of age and showed a decrease in tic severity following tDCS. This difference in age at onset might constitute an indicator for efficacy of tDCS in GTS, eventually referring to differences in pathology and etiology. A major limitation is the lack of a sham tDCS condition, however, the application of tDCS was for compassionate use and not part of a study protocol. Thus, all effects observed may be due to placebo or nocebo effects of stimulation.

Concerning stimulation parameters, i.e., current strength, duration and number of sessions per day, we deviated from previously reported cases in GTS and this also might have contributed to our negative result. In this respect our case series was rather designed to empirically explore a new stimulation regime than to reproduce and validate earlier findings, since to this end, larger controlled trials would be needed rather than small case series. In comparison, Carvalho et al. (2015) applied a more focal stimulation, using smaller surface electrodes that presumably resulted in a more specific stimulation of the pre-SMA. Similarly, Mrakic-Sposta et al. (2008) used smaller electrodes but applied current more generally over the motor cortex, and Eapen et al. (2017) applied electrodes with a size of $25 \mathrm{~cm}^{2}$ over the anterior portion of the SMA. Although supported by evidence of the proposed parameters in the treatment of major depression (Palm et al., 2016) and taking into account a lack in large controlled studies on effective stimulation parameters in GTS, our less focal approach may not have been an optimal mode of stimulation. However positioning of reference electrodes is the same across cases with extracephalic placement over right deltoid muscle (MrakicSposta et al., 2008; Carvalho et al., 2015; Eapen et al., 2017) whereas we used an ipsilateral montage on sternocleidomastoid muscles.
A further difference was the use of $2 \mathrm{~mA}$ intensity here and in Mrakic-Sposta et al.'s (2008) study instead of $1.4 \mathrm{~mA}$ (Carvalho et al., 2015; Eapen et al., 2017). This intensity has been selected based on our experimental studies of prefrontal tDCS in humans (Keeser et al., 2011a,b) and treatment parameter for major depression (Brunoni et al., 2013). These empirically established parameters were adopted from recent findings in the treatment of major depression where a tDCS relationship between total dosage and efficacy of stimulation was found (Brunoni et al., 2016), but these results are restricted to anodal prefrontal stimulation. Therefore, our cathodal tDCS protocol was rather comparable to the protocol applied in a randomized controlled trial in OCD by D'Urso et al. (2016). Interestingly, the largest and clinically meaningful effect was not found on GTS, but on OCD symptoms, i.e., a reduction in YBOCS score in P1. However, nonlinear dose dependency has been proposed as a principle of action in tDCS. Research on motor cortex stimulation has shown that previously postulated inhibiting effects of cathodal stimulation as well as exciting effects of anodal stimulation might be inversed depending on ground activity of stimulated tissue, current intensity and frequentness of stimulation (Brunoni et al., 2012; Batsikadze et al., 2013). It is likely that the impact of the stimulation parameters, i.e., current strength, polarity, duration and interval between stimulations, strictly depends on the stimulated area and might have led to counter regulatory or homeostatic plasticity effects in motor cortical areas as previously shown in neurophysiology studies (Nitsche and Paulus, 2001; MonteSilva et al., 2010). Therefore, the mere transmission of those anodal stimulation parameters used to enhance dorsolateral prefrontal function in depressed patients to a hypothesized decrease of hyperactivity by cathodal motor cortex stimulation in GTS patients might induce unwanted non-linear, even inverse effects. Here, guidance on stimulation intensity, duration, and frequentness is still lacking and therapeutic options are restricted to empirical considerations. The issue of a critical dosage has been very recently emphasized in a rat model of GTS where different polarities and current intensities were applied (Edemann-Callesen et al., 2018). There, tDCS showed a polarity-specific (anodal) and non-linear intensity dependent (i.e., $200 \mu \mathrm{A}$ as the only effective tDCS intensity) reduction of repetitive behavior in DAT-tg rats with hyperresponsive dopaminergic system in comparison to wildtype rats. The authors hypothesize that tDCS restores previously imbalanced sensorimotor striatal-thalamo-cortical networks in DAT-tg rats.

Finally, GTS is a complex disorder with highly variable phenotypic manifestations and high frequency of comorbidities (Qi et al., 2017). It is possible that the effects of tDCS treatment depend on a variety of genomic variants and chromosomal aberrations making a linear tDCS effect in the whole patient sample unlikely and showing the need for a further characterization of genetic subtypes and their impact on tDCS efficacy.

Thus, development of tDCS under use of translational research options rather than single-case guided research may represent an alternative and promising strategy towards 
development of tDCS as potential treatment for GTS. Finally, there may be a need to define tDCS intensities based on electrical field modeling than applying fixed tDCS intensities (Lisanby, 2017).

\section{CONCLUSION}

Our case series shows that modulating cortical excitability in GTS has not yet been developed to a clinically applicable treatment. A proof-of-concept study in humans is still lacking. Due to the variety and complexity of cortical regions involved in GTS, there is a multitude of possible tDCS approaches but also interferences that inhibit prognoses of, or change expected results. Our results hint that future research will have to focus on the determination of most effective stimulation modes regarding site, polarity and

\section{REFERENCES}

Batsikadze, G., Moliadze, V., Paulus, W., Kuo, M.-F., and Nitsche, M. A. (2013). Partially non-linear stimulation intensity-dependent effects of direct current stimulation on motor cortex excitability in humans. J. Physiol. 591, 1987-2000. doi: 10.1113/jphysiol.2012.249730

Bikson, M., Grossman, P., Thomas, C., Zannou, A. L., Jiang, J., Adnan, T., et al. (2016). Safety of transcranial direct current stimulation: evidence based update 2016. Brain Stimul. 9, 641-661. doi: 10.1016/j.brs.2016.06.004

Brunoni, A. R., Moffa, A. H., Fregni, F., Palm, U., Padberg, F., Blumberger, D. M., et al. (2016). Transcranial direct current stimulation for acute major depressive episodes: meta-analysis of individual patient data. Br. J. Psychiatry 208, 522-531. doi: 10.1192/bjp.bp.115.164715

Brunoni, A. R., Nitsche, M. A., Bolognini, N., Bikson, M., Wagner, T., Merabet, L., et al. (2012). Clinical research with transcranial direct current stimulation (tDCS): challenges and future directions. Brain Stimul. 5, 175-195. doi: 10.1016/j.brs.2011.03.002

Brunoni, A. R., Valiengo, L., Baccaro, A., Zanão, T. A., de Oliveira, J. F., Goulart, A., et al. (2013). The sertraline vs. electrical current therapy for treating depression clinical study: results from a factorial, randomized, controlled trial. JAMA Psychiatry 70, 383-391. doi: 10.1001/2013.jamapsychiatry.32

Burd, L. K., Kerbeshian, P. J., Barth, A., Klug, M. G., Avery, P. K., and Benz, B. (2001). Long-term follow-up of an epidemiologically defined cohort of patients with Tourette syndrome. J. Child Neurol. 16, 431-437. doi: 10.1177/088307380101600609

Capriotti, M. R., Himle, M. B., and Woods, D. W. (2014). Behavioral treatments for Tourette syndrome. J. Obsessive Compuls. Relat. Disord. 3, 415-420. doi: 10.1016/j.jocrd.2014.03.007

Carvalho, S., Gonçalves, Ó. F., Soares, J. M., Sampaio, A., Macedo, F., Fregni, F., et al. (2015). Sustained effects of a neural-based intervention in a refractory case of tourette syndrome. Brain Stimul. 8, 657-659. doi: 10.1016/j.brs.2014. 12.008

Charvet, L. E., Kasschau, M., Datta, A., Knotkova, H., Stevens, M. C., Alonzo, A., et al. (2015). Remotely-supervised transcranial direct current stimulation (tDCS) for clinical trials: guidelines for technology and protocols. Front. Syst. Neurosci. 9:26. doi: 10.3389/fnsys.2015.00026

D’Urso, G., Brunoni, A. R., Mazzaferro, M. P., Anastasia, A., de Bartolomeis, A., and Mantovani, A. (2016). Transcranial direct current stimulation for obsessive-compulsive disorder: a randomized, controlled, partial crossover trial. Depress. Anxiety 33, 1132-1140. doi: 10.1002/da.22578

Eapen, V., Baker, R., Walter, A., Raghupathy, V., Wehrman, J. J., and Sowman, P. F. (2017). The role of transcranial direct current stimulation (tDCS) in tourette syndrome: a review and preliminary findings. Brain Sci. 7:E161. doi: 10.3390/brainsci7120161

Edemann-Callesen, H., Habelt, B., Wieske, F., Jackson, M., Khadka, N., Mattei, D., et al. (2018). Non-invasive modulation reduces repetitive behavior in a rat model through the sensorimotor cortico-striatal circuit. Transl. Psychiatry 8:11. doi: 10.1038/s41398-017-0059-5 frequentness in a first step before exploring tDCS in a larger clinical setting.

\section{AUTHOR CONTRIBUTIONS}

BL, EM, BK, LW, EW and LL helped with data preparation. NB and UP drafted the manuscript. FP, RM, BB and CW helped with proof reading.

\section{FUNDING}

This work was funded by the German Federal Ministry of Education and Research (Bundesministerium für Bildung und Forschung, BMBF), grant code 01EE1403E (German Center for Brain Stimulation, http://gcbs.network/gcbs).

Evans, J., Seri, S., and Cavanna, A. E. (2016). The effects of Gilles de la Tourette syndrome and other chronic tic disorders on quality of life across the lifespan: a systematic review. Eur. Child Adolesc. Psychiatry 25, 939-948. doi: 10.1007/s00787-016-0823-8

Goodman, W. K., Price, L. H., Rasmussen, S. A., Mazure, C., Fleischmann, R. L., Hill, C. L., et al. (1989). The Yale-Brown obsessive compulsive scale: I. Development, use, and reliability. Arch. Gen. Psychiatry 46, 1006-1011. doi: 10.1001/archpsyc.1989.01810110048007

Keeser, D., Meindl, T., Bor, J., Palm, U., Pogarell, O., Mulert, C., et al. (2011a). Prefrontal transcranial direct current stimulation changes connectivity of resting-state networks during fMRI. J. Neurosci. 31, 15284-15293. doi: 10.1523/JNEUROSCI.0542-11.2011

Keeser, D., Padberg, F., Reisinger, E., Pogarell, O., Kirsch, V., Palm, U., et al. (2011b). Prefrontal direct current stimulation modulates resting EEG and event-related potentials in healthy subjects: a standardized low resolution tomography (sLORETA) study. Neuroimage 55, 644-657. doi: 10.1016/j. neuroimage.2010.12.004

Kious, B. M., Jimenez-Shahed, J., and Shprecher, D. R. (2016). Treatmentrefractory tourette syndrome. Prog. Neuropsychopharmacol. Biol. Psychiatry 70 , 227-236. doi: 10.1016/j.pnpbp.2016.02.003

Krohne, H. W., Egloff, B., Kohlmann, C.-W., and Tausch, A. (1996). Untersuchungen mit einer deutschen Version der "Positive and Negative Affect Schedule" (PANAS). Diagnostica 42, 139-156.

Landeros-Weisenberger, A., Mantovani, A., Motlagh, M. G., de Alvarenga, P. G., Katsovich, L., Leckman, J. F., et al. (2015). Randomized sham controlled double-blind trial of repetitive transcranial magnetic stimulation for adults with severe Tourette syndrome. Brain Stimul. 8, 574-581. doi: 10.1016/j.brs.2014. 11.015

Le, K., Liu, L., Sun, M., Hu, L., and Xiao, N. (2013). Transcranial magnetic stimulation at 1 Hertz improves clinical symptoms in children with Tourette syndrome for at least 6 months. J. Clin. Neurosci. 20, 257-262. doi: 10.1016/j. jocn.2012.01.049

Leckman, J. F., Riddle, M. A., Hardin, M. T., Ort, S. I., Swartz, K. L., Stevenson, J., et al. (1989). The Yale Global Tic Severity Scale: initial testing of a clinicianrated scale of tic severity. J. Am. Acad. Child Adolesc. Psychiatry 28, 566-573. doi: 10.1097/00004583-198907000-00015

Leckman, J. F., Zhang, H., Vitale, A., Lahnin, F., Lynch, K., Bondi, C., et al. (1998). Course of tic severity in Tourette syndrome: the first two decades. Pediatrics 102, 14-19. doi: 10.1542/peds.102.1.14

Lisanby, S. H. (2017). Noninvasive brain stimulation for depression-the devil is in the dosing. N. Engl. J. Med. 376, 2593-2594. doi: 10.1056/NEJME1702492

Mantovani, A., Lisanby, S. H., Pieraccini, F., Ulivelli, M., Castrogiovanni, P., and Rossi, S. (2006). Repetitive transcranial magnetic stimulation (rTMS) in the treatment of obsessive-compulsive disorder (OCD) and Tourette's syndrome (TS). Int. J. Neuropsychopharmacol. 9, 95-100. doi: $10.1017 /$ s1461145705005729

Monte-Silva, K., Kuo, M. F., Liebetanz, D., Paulus, W., and Nitsche, M. A. (2010). Shaping the optimal repetition interval for cathodal transcranial direct 
current stimulation (tDCS). J. Neurophysiol. 103, 1735-1740. doi: 10.1152/jn.00 924.2009

Mrakic-Sposta, S., Marceglia, S., Mameli, F., Dilena, R., Tadini, L., and Priori, A. (2008). Transcranial direct current stimulation in two patients with tourette syndrome. Mov. Disord. 23, 2256-2257. doi: 10.1002/mds.22292

Nitsche, M. A., Liebetanz, D., Lang, N., Antal, A., Tergau, F., and Paulus, W. (2003). Safety criteria for transcranial direct current stimulation (tDCS) in humans. Clin. Neurophysiol. 114, 2220-2222. doi: 10.1016/s13882457(03)00235-9

Nitsche, M. A., Müller-Dahlhaus, F., Paulus, W., and Ziemann, U. (2012). The pharmacology of neuroplasticity induced by non-invasive brain stimulation: building models for the clinical use of CNS active drugs. J. Physiol. 590, 4641-4662. doi: 10.1113/jphysiol.2012.232975

Nitsche, M. A., and Paulus, W. (2001). Sustained excitability elevations induced by transcranial DC motor cortex stimulation in humans. Neurology 57, 1899-1901. doi: 10.1212/WNL.57.10.1899

Palm, U., Hasan, A., Strube, W., and Padberg, F. (2016). tDCS for the treatment of depression: a comprehensive review. Eur. Arch. Psychiatry Clin. Neurosci. 266, 681-694. doi: 10.1007/s00406-016-0674-9

Palm, U., Kumpf, U., Behler, N., Wulf, L., Kirsch, B., Wörsching, J., et al. (2018). Home use, remotely supervised, and remotely controlled transcranial direct current stimulation: a systematic review of the available evidence. Neuromodulation 21, 323-333. doi: 10.1111/ner.12686

Palm, U., Leitner, B., Strube, W., Hasan, A., and Padberg, F. (2015). Safety of repeated twice-daily 30 minutes of $2 \mathrm{~mA}$ tDCS in depressed patients. Int. Neuropsychiatr. Dis. J. 4, 168-171. doi: 10.9734/indj/2015/19719

Pappert, E. J., Goetz, C. G., Louis, E. D., Blasucci, L., and Leurgans, S. (2003). Objective assessments of longitudinal outcome in Gilles de la Tourette's syndrome. Neurology 61, 936-940. doi: 10.1212/01.WNL.0000086370.10186.7c

Pedroarena-Leal, N., and Ruge, D. (2017). Toward a symptom-guided neurostimulation for Gilles de la Tourette syndrome. Front. Psychiatry 8:29. doi: 10.3389/fpsyt.2017.00029

Qi, Y., Zheng, Y., Li, Z., and Xiong, L. (2017). Progress in genetic studies of Tourette's syndrome. Brain Sci. 7:E134. doi: 10.3390/brainsci7100134
Schrock, L. E., Mink, J. W., Woods, D. W., Porta, M., Servello, D., VisserVandewalle, V., et al. (2015). Tourette syndrome deep brain stimulation: a review and updated recommendations. Mov. Disord. 30, 448-471. doi: $10.1002 / \mathrm{mds} .26094$

Storch, E. A., Murphy, T. K., Geffken, G. R., Sajid, M., Allen, P., Roberti, J. W., et al. (2005). Reliability and validity of the Yale Global Tic Severity Scale. Psychol. Assess. 17, 486-491. doi: 10.1037/1040-3590.17.4.486

Visser-Vandewalle, V., Huys, D., Neuner, I., Zrinzo, L., Okun, M. S., and Kuhn, J. (2014). Deep brain stimulation for tourette syndrome: the current state of the field. J. Obsessive Compuls. Relat. Disord. 3, 401-406. doi: 10.1016/j.jocrd.2014. 06.005

Wang, Z., Maia, T. V., Marsh, R., Colibazzi, T., Gerber, A., and Peterson, B. S. (2011). The neural circuits that generate tics in Tourette's syndrome. Am. J. Psychiatry 168, 1326-1337. doi: 10.1176/appi.ajp.2011.09111692

Conflict of Interest Statement: UP received paid speakership from neuroCare Group and has a private practice with neuroCare Group, Munich, Germany. FP received research support from NeuroConn $\mathrm{GmbH}$, Ilmenau, Germany, and Brainsway Inc., Tel Aviv, Israel, as well as speaker's honorarium from Mag \& More $\mathrm{GmbH}$, Munich, Germany, and neuroCare Group. LW is part-time employee of neuroCare Group.

The remaining authors declare that the research was conducted in the absence of any commercial or financial relationships that could be construed as a potential conflict of interest.

Copyright (C) 2018 Behler, Leitner, Mezger, Weidinger, Musil, Blum, Kirsch, Wulf, Löhrs, Winter, Padberg and Palm. This is an open-access article distributed under the terms of the Creative Commons Attribution License (CC BY). The use, distribution or reproduction in other forums is permitted, provided the original author(s) and the copyright owner(s) are credited and that the original publication in this journal is cited, in accordance with accepted academic practice. No use, distribution or reproduction is permitted which does not comply with these terms. 


\section{OPEN ACCESS}

Edited by:

Andrea Antal,

University Medical Center Göttingen

Germany

Reviewed by:

Francesca Pacitti,

University of L'Aquila, Italy

Benjamin Straube,

Philipps University of Marburg,

Germany

*Correspondence:

Tina Gupta

tinagupta2021@u.northwestern.edu

Received: 01 March 2018

Accepted: 23 April 2018

Published: 28 May 2018

Citation:

Gupta T, Kelley NJ,

Pelletier-Baldelli A and Mittal VA (2018) Transcranial Direct Current

Stimulation, Symptomatology, and Cognition in Psychosis:

A Qualitative Review.

Front. Behav. Neurosci. 12:94.

doi: 10.3389/fnbeh.2018.00094

\section{Transcranial Direct Current Stimulation, Symptomatology, and Cognition in Psychosis: A Qualitative Review}

\author{
Tina Gupta ${ }^{1 *}$, Nicholas J. Kelley ${ }^{1}$, Andrea Pelletier-Baldelli² and Vijay A. Mittal ${ }^{1,3,4,5,6}$ \\ ${ }^{1}$ Department of Psychology, Northwestern University, Evanston, IL, United States, ${ }^{2}$ Department of Psychology and \\ Neuroscience, University of Colorado Boulder, Boulder, CO, United States, ${ }^{3}$ Department of Psychiatry, Northwestern \\ University, Chicago, IL, United States, ${ }^{4}$ Institute for Policy Research, Northwestern University, Evanston, IL, United States, \\ ${ }^{5}$ Medical Social Sciences, Northwestern University, Chicago, IL, United States, ${ }^{6}$ Institute for Innovations in Developmental \\ Sciences, Northwestern University, Chicago, IL, United States
}

Schizophrenia is a chronic, debilitating condition that affects approximately $1 \%$ of the population. Individuals diagnosed with schizophrenia typically exhibit positive (e.g., hallucinations) and negative symptoms (e.g., anhedonia) and impairments in cognitive function. Given the limitations of antipsychotic medication and psychotherapy in fully treating psychosis symptomatology, there has been increasing interest in other interventions such as transcranial direct current stimulation (tDCS). tDCS is a noninvasive neuromodulation technique, that is safe, cost-effective, and widely accessible. Here, we discuss treatment studies that seek to improve symptoms and cognitive performance in schizophrenia using tDCS. Currently within the literature, there is support for reductions in positive symptoms such as hallucinations after receiving tDCS. Further, studies indicate that tDCS can improve cognitive functioning, which is an area of investigation that is sorely needed, as it is unclear which types of interventions may be useful in ameliorating cognitive deficits among this group. Taken together, the evidence suggests that tDCS holds promise in improving symptoms and cognition. To that end, tDCS has critical clinical implications for this population.

Keywords: transcranial direct current stimulation, tDCS, cognition, neurocognition, symptoms, schizophrenia

\section{INTRODUCTION}

Schizophrenia is a heterogeneous, chronic condition that affects approximately $1 \%$ of the population (Saha et al., 2005). The disorder typically emerges in early adulthood and can have tremendous impacts on an individual's overall quality of life (Hutchinson et al., 1999; Mueser et al., 2001; Marwaha and Johnson, 2004). Schizophrenia is characterized by positive (e.g., hallucinations and delusions) and negative symptoms (e.g., anhedonia and avolition). In addition, there tends to impairments in cognitive function, particularly in working memory and attention (Schaefer et al., 2013; Fatouros-Bergman et al., 2014; Green and Harvey, 2014). 
Efforts to ameliorate symptomatology and cognitive impairments are ongoing, with antipsychotics representing the most commonly used treatment (Tandon et al., 2010). Although antipsychotic medications are effective in reducing symptomatology, they are expensive (Geddes et al., 2000), and can have side effects such as weight gain and fatigue (Young et al., 2015). As such, adherence is often an ongoing challenge and treatment with these medications is generally not considered effective for cognitive function (Young et al., 1986; Fenton et al., 1997; Lacro et al., 2002; Green and Harvey, 2014). Thus, there is a critical need for efficacious treatments that have favorable side-effect profiles, are cost-effective, and address additional impacted domains. Within the last decade, there has been interest in using transcranial direct current stimulation (tDCS), a neuromodulatory technique that releases a weak electrical current through the skull to reduce clinical symptoms and cognitive deficits (Bose et al., 2014; Hoy et al., 2014; Mondino et al., 2015, 2016; Rassovsky et al., 2015; Hasan et al., 2016; Palm et al., 2016). Overall, tDCS is promising, but results remain mixed and future work is needed to understand the efficacy of this technique.

To date, there are informative reviews investigating the impacts of tDCS on schizophrenia populations (e.g., Brunoni et al., 2014; Fregni et al., 2015; Fröhlich et al., 2015; Kadosh, 2015; Mondino et al., 2015) and more recent reviews discussing tDCS and symptoms (Osoegawa et al., 2018; Pontillo et al., 2018). However, there have been several new tDCS studies conducted which are summarized in the current review. The current review discusses (1) recent findings exploring the impacts of tDCS on symptoms and cognition among schizophrenia populations (Fröhlich et al., 2016; Palm et al., 2016), (2) tDCS and other neuromodulatory techniques (Hasan et al., 2016; Hopfinger et al., 2017; Mellin et al., 2018), (3) tDCS and neuroimaging (Mondino et al., 2016; Palm et al., 2016), (4) tDCS in conjunction with cognitive remediation (Orlov et al., 2017), and (5) tDCS and the impacts on antipsychotic medication use (Agarwal et al., 2016). Furthermore, other populations are also discussed including relevant studies in healthy individuals (Khalighinejad and Haggard, 2015; Khalighinejad et al., 2016; Mai et al., 2016; Schülke and Straube, 2017; Straube et al., 2017), childhood onset schizophrenia (Mattai et al., 2011), and non-clinical psychosis (Gupta et al., 2017). The following qualitative review summarizes the current literature and offers future directions, taking recent findings into account, for tDCS research in schizophrenia populations.

\section{TRANSCRANIAL DIRECT CURRENT STIMULATION}

Transcranial direct current stimulation is a non-invasive brain stimulation technique which modulates cortical excitability by means of a weak electrical current [typically less than 2 milliamps $(\mathrm{mA})$ ] traveling between two electrodes. The positively charged anode increases cortical excitability whereas the negatively charged change decreases cortical excitability. Researchers interested in increasing cortical excitability in a target brain region place the anode over that region with the cathode placed over another region irrelevant to the target behavior. This is known as a bilateral montage. In other cases, researchers may place the cathode on an extracephalic region (e.g., shoulder) and this is known as a unipolar montage. Likewise, a bilateral montage aimed at decreasing cortical excitability in a target brain region would place the cathode over the target region with the anode now placed over another region irrelevant to the target behavior. Finally, the positioning of a unipolar montage aimed at decreasing cortical excitability would be the same except for the positioning of the anode and cathode reversed. In addition to active tDCS conditions aimed at increasing or decreasing cortical excitability, most tDCS studies also include a sham condition. The sham condition is a placebo condition where participants receive brief stimulation (approximately $30 \mathrm{~s}$ ) while all other parameters are held constant. This is a reliable placebo control that does not result in any aftereffects (Gandiga et al., 2006). Sham conditions are increasingly used in study designs (Mattai et al., 2011; Brunelin et al., 2012; Gomes et al., 2015; Smith et al., 2015; Mondino et al., 2016; Palm et al., 2016), and one potential benefit of this condition is the ability to conduct double-blind studies. The ability to conduct sham-controlled double-blind studies is one key strength of tDCS over other brain stimulation techniques.

To date, the precise mechanism of action underlying tDCS remains uncertain. As noted earlier, tDCS involves the use of a weak electrical current (0.5-2 mA) traveling between the anode and cathode (Nitsche et al., 2008; Brunoni et al., 2012). This weak current does not directly cause action potentials as is the case with transcranial magnetic stimulation (TMS). Instead, the current seems to modulate the probability of cell firing without directly triggering an action potential with anodal stimulation increasing and cathodal stimulation decreasing that probability (Nitsche et al., 2008; Agarwal et al., 2013; Mondino et al., 2015). Several animal studies indicate that anodal tDCS causes depolarization by increasing resting membrane potentials, thus leading to spontaneous cell firing (Nitsche and Paulus, 2000; Kekic et al., 2016). In contrast, cathodal tDCS may be decreasing the resting membrane potential leading to a hyperpolarization of neurons.

It is unclear how long the effects resulting from tDCS remain present. Studies indicate that more than $10 \mathrm{~min}$ of tDCS can lead to effects lasting over an hour, while shorter durations of tDCS may not have longer after-effects; however, it can vary depending on the region of interest (Nitsche and Paulus, 2000). Furthermore, some studies implement a single session of tDCS, while others utilize multiple sessions (Agarwal et al., 2013; Kekic et al., 2016). Although both approaches are informative, the lasting effects, optimal number of length per session, and duration remain an empirical question (Agarwal et al., 2013; Kekic et al., 2016). While there are aspects of tDCS that have yet to be determined, it is evident that the affordable and portable nature of this neuromodulation technique can have the potential to reach individuals that are more difficult to access (e.g., individuals in more rural locations). Perhaps because of the affordability and portability of tDCS there has been sustained interest in implementing tDCS protocols 
in clinical populations (e.g., depression, see Brunoni et al., 2016).

\section{STUDIES EVALUATING THE EFFICACY OF tDCS ON INFLUENCING CLINICAL SYMPTOMS IN PSYCHOSIS}

Hallmark symptoms of schizophrenia include positive symptoms such as hallucinations (e.g., false perceptions such as auditory hallucinations like hearing voices or visual hallucinations such as seeing something that is not there) and delusions (e.g., irrational beliefs such as believing in conspiracy theories or someone is watching, without any reason to believe this). While the development of auditory hallucinations remains unclear, there is evidence indicating abnormal connectivity between the dorsal lateral prefrontal cortex (DLPFC) and temporal parietal junction (TPJ) are linked with auditory hallucinations (Jardri et al., 2011; Mondino et al., 2016). There is also some work indicating that patients diagnosed with schizophrenia exhibit over-activation in brain regions such as left-temporo-parietal areas and left inferior frontal areas (Jardri et al., 2011).

\section{tDCS and Auditory Hallucinations}

The most common montage used when applying tDCS in order to reduce treatment-resistant auditory hallucinations entails placing electrodes over the fronto-temporal network (Mondino et al., 2015). Brunelin et al. (2012) implemented the first study design that was double-blind, randomized, and also included a sham condition in a sample of 30 patients diagnosed with schizophrenia experiencing auditory verbal hallucinations. In this study, 15 patients were randomized to receive active tDCS and 15 patients were assigned to the sham condition. The cathode in this design was placed over the TPJ and the anode was placed over the left DLPFC in order to test whether tDCS could reduce auditory hallucinations. Specifically, active $2 \mathrm{~mA}$ tDCS or sham was administered for $20 \mathrm{~min}$, twice daily, for 5 days. Data from the Auditory Hallucination Rating Scale (AHRS; Hoffman et al., 2003) was gathered immediately following the 5 days of receiving tDCS, 1 month, and 3 months later. Findings from the study indicate that auditory verbal hallucinations were robustly reduced by tDCS in the active tDCS condition compared to the sham and importantly, effects lasted up to 3 months. These data are informative because they shed light on the interplay between the DLPFC, TPJ, and auditory hallucinations and provide evidence for the clinical utility of tDCS in both the short and long term and have important lasting effects.

Contrary to the work from Brunelin et al. (2012), null findings have also been reported. For example, in another study using a sample of 24 patients diagnosed with schizophrenia and schizoaffective disorder, the anode was applied over the DLPFC, and the cathode over the temporal parietal area in comparison with a sham condition (Fitzgerald et al., 2014). Active tDCS was applied for 15 daily sessions over three consecutive weeks and outcome measures consisted of the Positive and Negative Syndrome Scale (PANSS) to assess for auditory hallucinations. In contrast to Brunelin et al. (2012), this study did not exhibit changes in reports of auditory hallucinations after receiving tDCS. Differences in findings in the study conducted by Brunelin et al. (2012) and Fitzgerald et al. (2014) could be better explained by the amount of stimulation (twice daily for 5 days vs. once daily for 3 weeks). However, the study did indicate that tDCS was safely implemented and reported minimal side effects and adverse effects providing further evidence for the tolerability of tDCS with patients.

Given the contradictory findings, other groups have attempted to replicate results indicating that tDCS may reduce auditory hallucinations (Bose et al., 2014; Fröhlich et al., 2016). For example, in a study with 21 patients with schizophrenia experiencing persistent auditory hallucinations (even on antipsychotic medications), the anode was applied over the left DLPFC and the cathode over the left TPJ. This study found that tDCS contributed to decreased auditory hallucinations (Bose et al., 2014). Similarly, an exploratory study was conducted using daily tDCS to treat auditory hallucinations in a sample of 26 patients diagnosed with schizophrenia and schizoaffective disorder (Fröhlich et al., 2016). Following similar methods from Brunelin et al. (2012), the group used a randomized, double-blind, sham-controlled study design and applied active tDCS over the left frontal and temporal-parietal areas for 5 days, once a day (in contrast to Brunelin et al., 2012 in which tDCS was applied twice daily) due to difficulties with patient compliance. Results suggest a significant reduction in auditory hallucinations measured by the AHRS in the sham condition. These findings provide information regarding the importance of the design of the study (continuing to work on determining what is considered gold-standard regarding dose) and working toward teasing apart the mechanisms underlying the sham condition given the placebo response that was observed.

\section{tDCS and Negative Symptoms}

There is also research exploring the impacts of tDCS on negative symptoms (Osoegawa et al., 2018; Pontillo et al., 2018). Previous work has examined negative symptoms in conjunction with positive symptoms such as auditory hallucinations, also targeting the DLPFC (Brunelin et al., 2012; Fröhlich et al., 2016). Negative symptoms are a collection of experiences that have been described as a loss of regular functions such as having a lack of motivation to persist in goal-directed activities or emotional blunting (Fusar-Poli et al., 2015). While antipsychotic medications have been impactful in the reduction of positive symptoms, negative symptoms have been suggested to persist (Fusar-Poli et al., 2013). In a recent review, the authors concluded that there is evidence supporting the use of tDCS particularly over prefrontal areas to decrease negative symptoms (Aleman et al., 2018). Brunelin et al. (2012) (described above) also reported decreases in negative symptom scores based off of the PANSS. Similarly, Gomes et al. (2015) conducted a randomized, doubleblind study and applied the anode over the left DLPFC and placed the cathode over the right DLPFC. A total of 15 patients with schizophrenia were randomized to either active condition or the sham condition and received 20 min of tDCS once a day for 10 days and found reductions in negative symptom scores (also assessed by the PANSS). Together, there is evidence 
indicating that tDCS may improve negative symptoms. Future work will benefit from investigating other relevant brain regions and outcome measures in addition to determining the effects of tDCS on specific types of negative symptoms (e.g., anhedonia, avolition).

\section{Other Neuromodulation Techniques and Clinical Symptoms}

More recently, studies have expanded on the current work exploring the nature of tDCS and symptoms but now have integrated other stimulation techniques such as transcranial alternating current stimulation (tACS) (Paulus, 2011). This technique releases a weak electric current similar to tDCS, however, the current targets brain oscillations specific to frequency that is indicated to enhance naturally occurring brain oscillations. Repeated TMS (rTMS) is another stimulation method that has been used within the field, causing the release of new action potentials via activating axons (Aleman et al., 2007). Together, there is evidence for symptom reduction using these approaches within schizophrenia populations (Aleman et al., 2007; Paulus, 2011; Hasan et al., 2016; Hopfinger et al., 2017; Alexander et al., 2018; Mellin et al., 2018).

According to a meta-analysis conducted by Kennedy et al. (2018) (in which seven tDCS RCTs and 30 rTMS RCTs were identified), compared to sham, tDCS improved symptoms with effect sizes around 0.10-0.63. Most notably, higher cumulative stimulation was related to a reduction in auditory hallucinations, while rTMS was not related to cumulative dose, but rTMS was also found to be effective in the treatment of hallucinations. In another study, tDCS and tACS were compared (Mellin et al., 2018). In the first tACS clinical trial for the treatment of symptoms in a psychiatric population, a total of 22 participants with schizophrenia were randomized to receive (twice daily for $20 \mathrm{~min}$ for 5 days) sham, $2 \mathrm{~mA}$ peak-to-peak tACS or $2 \mathrm{~mA}$ tDCS. tACS had the largest reduction in auditory hallucinations scores (measured by the AHRS) compared to sham and tDCS. While the sample size was small in this study, this work provided important initial evidence for the utility of another stimulation approach (tACS). Future work is needed in order to understand similarities and differences of tACS and tDCS as it is unclear what the overlapping mechanisms are of these approaches, which may contribute to the differing effects and findings.

\section{tDCS and Neuroimaging}

There is a particular interest in integrating functional magnetic resonance imaging (fMRI) methodologies in order to understand the mechanism underlying multiple tDCS sessions, and the relationship with symptomatology (Mondino et al., 2016; Palm et al., 2016). For example, Palm et al. (2016) applied tDCS over the prefrontal cortex, where the anode was placed over the left DLPFC and the cathode was placed over the right supraorbital; a sham condition was also included. A total of 20 patients diagnosed with schizophrenia (endorsing predominately negative symptoms) were randomized to 10 sessions of either active or sham. In addition to applying tDCS, participants received fMRI scans pre-and-post the first tDCS session and pre-and-post the tenth tDCS session. Results indicate decreases in the Scale for Assessment of Negative Symptoms (SANS) and PANSS after active tDCS compared to sham. Furthermore, results indicate changes in subgenual cortex and DLPFC connectivity within frontal-thalamic-temporo-parietal networks - areas that have been identified to be related to negative symptoms.

Similarly, in a study conducted by Mondino et al. (2016), 23 patients with schizophrenia who also endorsed treatment resistant auditory hallucinations were randomly assigned to receive 10 sessions of active $(2 \mathrm{~mA}, 20 \mathrm{~min})$ or sham tDCS (two sessions a day for 5 days). Additionally, resting-state functional connectivity of the left TPJ was also examined. Active tDCS reduced auditory hallucinations and this was related to reductions in resting state connectivity between the left TPJ and the left anterior insula.

\section{Summary}

Overall, tDCS has shown some efficacy for symptoms, specifically refractory auditory hallucinations and negative symptoms. A major strength within the existing work is an effort to replicate previous tDCS findings, which is critical in order to truly comprehend the nature of tDCS. There is also promise in utilizing sham conditions in order to compare effects after being in the active condition. Although outcomes remain ambiguous and the impact of multiple versus single sessions is unclear, tDCS is a promising area of research. There is also promise in exploring the use of other neuromodulatory techniques. The fact that symptoms have been unsuccessfully treated in schizophrenia, and tDCS has shown some potential in targeting these domains, provides support for future work that is needed. In particular, further research is needed to target the potential for impact on other symptomatology (e.g., other aspects positive and negative symptoms). See Table 1 for a summary of noted studies that include information regarding the population, sample size, design of the study, montages/sites, mA, duration/frequency, variables of interest, and key findings.

\section{STUDIES EVALUATING THE EFFICACY OF tDCS ON INFLUENCING COGNITION IN PSYCHOSIS}

Cognitive deficits contribute vastly to an individual's every day functioning and can interfere with social and occupational aspects of daily living (Marwaha and Johnson, 2004; Insel, 2010). As noted, traditional approaches such as antipsychotic medication have been ineffective in improving these functions (Marwaha and Johnson, 2004; Green and Harvey, 2014). Some examples of neurocognitive deficits observed in schizophrenia include working memory, attention, inhibition, and executive function (Green et al., 2012; Barch and Sheffield, 2014; Green and Harvey, 2014).

According to Barch and Sheffield (2014), it has been argued that cognitive impairments may be a result of difficulties in representing goal information in working memory that is important for directing behavior; it is suggested that this deficit may be related to processes within the DLPFC. Overall, it may 
TABLE 1 | Summary of tDCS and clinical symptoms studies discussed.

\begin{tabular}{|c|c|c|c|c|c|c|c|c|}
\hline Author & Population & $N$ & Design & Montage/sites & $\mathbf{m A}$ & $\begin{array}{l}\text { Duration/ } \\
\text { frequency }\end{array}$ & $\begin{array}{l}\text { Variables of } \\
\text { interest }\end{array}$ & Key findings \\
\hline Brunelin et al., 2012 & Schizophrenia & 30 & $\begin{array}{l}\text { Double-blind, } \\
\text { randomized, } \\
\text { sham- } \\
\text { controlled }\end{array}$ & $\begin{array}{l}\text { Anode over the left } \\
\text { DLPFC; cathode over } \\
\text { the left TPJ }\end{array}$ & 2 & $\begin{array}{l}2 \times \text { a day, } \\
20 \text { min, } 5 \text { days }\end{array}$ & AHRS, PANSS & $\begin{array}{l}\text { Reductions in } \mathrm{AH} \\
\text { scores lasting for } \\
3 \text { months. } \\
\text { Decreases in } \\
\text { positive and } \\
\text { negative symptoms }\end{array}$ \\
\hline Bose et al., 2014 & Schizophrenia & 21 & Open-label & $\begin{array}{l}\text { Anode over the left } \\
\text { DLPFC; cathode over } \\
\text { the left TPJ }\end{array}$ & 2 & $\begin{array}{l}2 \times \text { a day, } \\
20 \text { min, } 5 \text { days }\end{array}$ & PSYRATS, SAI & $\begin{array}{l}\text { Reduction in } \mathrm{AHs} \\
\text { and insight scores }\end{array}$ \\
\hline Fitzgerald et al., 2014 & $\begin{array}{l}\text { Schizophrenia, } \\
\text { Schizoaffective }\end{array}$ & 24 & $\begin{array}{l}\text { Double-blind, } \\
\text { randomized } \\
\text { controlled trial }\end{array}$ & $\begin{array}{l}\text { Anode over the DLPFC } \\
\text { and cathode over the } \\
\text { temporoparietal area }\end{array}$ & 2 & $\begin{array}{l}1 \times \text { per day, } \\
20 \text { min, } \\
15 \text { days }\end{array}$ & PANSS, SANS & $\begin{array}{l}\text { No reductions in } \\
\text { AHs or other } \\
\text { symptoms }\end{array}$ \\
\hline Gomes et al., 2015 & Schizophrenia & 15 & $\begin{array}{l}\text { Double-blind, } \\
\text { randomized, } \\
\text { sham- } \\
\text { controlled }\end{array}$ & $\begin{array}{l}\text { Anode over the left } \\
\text { DLPFC and cathode } \\
\text { over the right DLPFC }\end{array}$ & 2 & $\begin{array}{l}1 \times \text { per } \\
\text { weekday, } \\
20 \text { min, } \\
10 \text { days }\end{array}$ & PANSS & $\begin{array}{l}\text { Improvement in } \\
\text { negative but not } \\
\text { positive symptoms }\end{array}$ \\
\hline Fröhlich et al., 2016 & $\begin{array}{l}\text { Schizophrenia, } \\
\text { Schizoaffective }\end{array}$ & 26 & $\begin{array}{l}\text { Double-blind, } \\
\text { randomized, } \\
\text { sham- } \\
\text { controlled }\end{array}$ & $\begin{array}{l}\text { An ode over the left } \\
\text { DLPFC and cathode } \\
\text { over the left TPJ }\end{array}$ & 2 & $\begin{array}{l}1 \times \text { per day, } \\
20 \text { min, } 5 \text { days }\end{array}$ & AHRS, PANSS & $\begin{array}{l}\text { Reduction in AHs } \\
\text { not specific to } \\
\text { treatment group. } \\
\text { No changes in } \\
\text { positive and } \\
\text { negative symptom } \\
\text { scores }\end{array}$ \\
\hline Palm et al., 2016 & Schizophrenia & 20 & $\begin{array}{l}\text { Double-blind, } \\
\text { randomized, } \\
\text { sham- } \\
\text { controlled }\end{array}$ & $\begin{array}{l}\text { Anode over the left } \\
\text { DLPFC and cathode } \\
\text { over the right } \\
\text { supraorbital }\end{array}$ & 2 & $\begin{array}{l}1 \times \text { per day, } \\
20 \text { min, } \\
10 \text { days }\end{array}$ & SANS, PANSS & $\begin{array}{l}\text { Decreases in SANS } \\
\text { and PANSS }\end{array}$ \\
\hline
\end{tabular}

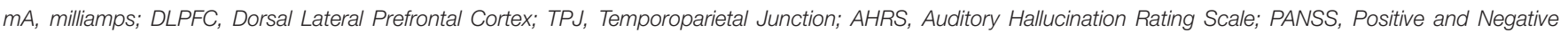
Syndrome Scale; SANS, Scale for Assessment of Negative Symptoms; PSYRATS, Auditory Hallucination Subscale of PSYRATS; AHs, Auditory Hallucinations.

be that there are impairments in context-processing, that is, the way individuals are able to take in prior information from working memory and engage in current processes (Barch and Sheffield, 2014). Within this context, there have been several recent studies using tDCS to target neurocognitive functioning in this population, particularly applying tDCS over the DLPFC (Vercammen et al., 2011; Hoy et al., 2014; Rassovsky et al., 2015; Smith et al., 2015).

\section{tDCS and Neurocognition}

Hoy et al. (2014), in a sample of 18 patients with schizophrenia, applied $20 \mathrm{~min}$ of active ( 1 and $2 \mathrm{~mA}$ ) tDCS and sham over the left DLPFC to improve working memory performance (measured by the $\mathrm{N}$-back task requiring participants to continuously maintain, update, and recall number sequences). Working memory performance was evaluated immediately following the active or sham treatment, $20 \mathrm{~min}$ after, and $40 \mathrm{~min}$ after tDCS. Results suggest that there were no improvements in working memory performance after sham or after receiving $1 \mathrm{~mA}$ of active tDCS over time. However, there was a significant improvement over time when receiving $2 \mathrm{~mA}$ of tDCS, but not immediately after receiving tDCS in contrast with previous work (Nitsche et al., 2008). These data bring to light the importance of considering the dose of stimulation in tDCS designs and the potential for $2 \mathrm{~mA}$ of $\mathrm{tDCS}$ to be impactful for working memory improvements.
In another study, a randomized double-blind, shamcontrolled design was used in order to test the effects of multiple sessions of tDCS (five sessions on consecutive days) in improving neurocognition in a sample of 37 individuals (19 randomized to active tDCS and 18 randomized to sham tDCS) with schizophrenia and schizoaffective disorder (Smith et al., 2015). The anode was placed over the DLPFC and the cathode was placed over the contralateral supraorbital ridge. Neurocognition was assessed using the MATRICS Consensus Cognitive Battery (MCCB; Nuechterlein et al., 2008), a commonly used battery of tasks used for schizophrenia populations. Findings indicate that after being in the active condition, improvements in the MCCB composite score were observed after the fifth tDCS session in addition to working memory and attention scores. These data provide further evidence for the efficacy of tDCS on cognitive performance and particularly show benefits of multiple, consecutive sessions of tDCS.

\section{tDCS and Social Cognition}

Social cognition is a particularly relevant domain of interest given connections with functioning and overall quality of life (Green et al., 2015). Much of what is known about the effects of tDCS on social cognition stems from studies of healthy individuals (Santiesteban et al., 2012; Khalighinejad and Haggard, 2015; Khalighinejad et al., 2016; Mai et al., 2016; Schülke and Straube, 2017; Straube et al., 2017). Social cognition, distinctly different 
from neurocognitive processes, has been described as the ability to develop mental representations about oneself, others, and interactions between the two (Adolphs, 2001; Fett et al., 2011). Research in this area using tDCS have found that the left angular gyrus plays a fundamental role in the perceptual experience of agency (being in control of an individual's own actions and the results of these actions) (Khalighinejad and Haggard, 2015). Furthermore, the DLPFC (which was indicated to have implications for individuals diagnosed with depression) has been found to be relevant (Khalighinejad et al., 2016). Studies among healthy groups have also focused efforts on the TPJ (which was discussed above in the context of auditory hallucinations), relevant for social cognition (Santiesteban et al., 2012; Mai et al., 2016). For example, Mai et al. (2016) found, in a sample of 68 healthy adults who received both anodal and cathodal tDCS over the right TPJ, decreases in theory of mind and cognitive empathy after cathodal stimulation. Similarly, in another study, social cognition was improved after receiving anodal stimulation in imitation and perspective-taking tasks (Santiesteban et al., 2012).

Studies from healthy populations, particularly relating to social cognition, can inform research in tDCS and clinical populations through methodology. This is particularly relevant as studies within schizophrenia populations have shown mixed findings for tDCS effficacy. For example, social cognition was assessed in a sample of 36 individuals with schizophrenia (Rassovsky et al., 2015). Specifically, anode $(N=12)$, cathodal $(N=12)$, or sham $(N=12)$ tDCS (for 20 min with tDCS electrodes placed bilaterally over the DLPFC), was administered and then social cognition performance was re-evaluated. Active tDCS improved performance on an emotion identification task; however, changes were not observed in the other three tasks related to managing emotions, social perception, and theory of mind. Furthermore, tolerability of tDCS was assessed and tDCS seemed to be well-tolerated, with only minimal side effects (e.g., itchiness) reported.

\section{tDCS and Cognitive Remediation}

Research has examined the efficacy of tDCS for targeting cognitive deficits in conjunction with other interventions, such as cognitive remediation. For example, in a study conducted by Orlov et al. (2017), a total of 49 participants were asked to complete a baseline assessment of working memory and implicit learning tasks and following, four cognitive remediation training days (days 1, 2, 14, and 56). In day 1 and day 14 , participants also received either active $(N=24)$ or sham $(N=25)$ tDCS for $30 \mathrm{~min}$ with the anode over the left DLPFC and the cathode over the right supraorbital area. In the active condition, participants showed improvements in working memory performance compared to individuals in the sham condition on day 2 and during follow up on day 56. This study showed the long-term impact of tDCS on working memory improvements.

\section{Summary}

Together, the literature examining tDCS and cognition is quickly growing. Given that cognition can impact functional outcome, it is an important area to target for intervention. The strengths of tDCS and cognition may lie in the design of the studies (e.g., double-blind, randomized, sham-controlled). Work understanding tDCS and social cognition is still mixed but tDCS has shown some efficacy. tDCS has shown promise in conjunction with other treatment approaches such as cognitive remediation, and further evaluation of tDCS as an adjunctive intervention may prove informative. Limitations include the small number of investigations, and to some extent, the exclusive focus on the DLPFC. While studies applying tDCS over the

TABLE 2 | Summary of tDCS and cognition studies discussed.

\begin{tabular}{|c|c|c|c|c|c|c|c|c|}
\hline Author & Population & $N$ & Design & Montage/sites & $\mathbf{m A}$ & $\begin{array}{l}\text { Duration/ } \\
\text { frequency }\end{array}$ & $\begin{array}{l}\text { Variables } \\
\text { of interest }\end{array}$ & Key findings \\
\hline Hoy et al., 2014 & Schizophrenia & 18 & $\begin{array}{l}\text { Double-blind, } \\
\text { randomized, } \\
\text { sham-controlled }\end{array}$ & $\begin{array}{l}\text { Anode over the left } \\
\text { DLPFC; cathode over } \\
\text { the right supraorbital } \\
\text { region }\end{array}$ & $\begin{array}{l}1 \\
2\end{array}$ & $\begin{array}{l}1 \times \text { a day } \\
20 \text { min }\end{array}$ & N-back task & $\begin{array}{l}\text { Improvements in } \\
\text { working memory } \\
40 \text { min later }\end{array}$ \\
\hline $\begin{array}{l}\text { Rassovsky } \\
\text { et al., } 2015\end{array}$ & Schizophrenia & 36 & $\begin{array}{l}\text { Randomized, } \\
\text { sham-controlled }\end{array}$ & $\begin{array}{l}\text { Anode and cathode } \\
\text { bilaterally over the } \\
\text { DLPFC }\end{array}$ & 2 & $\begin{array}{l}1 \times \text { a day } \\
20 \text { min }\end{array}$ & $\begin{array}{l}\text { MSCEIT } \\
\text { FEIT } \\
\text { PONS } \\
\text { TASIT } \\
\text { MCCB }\end{array}$ & $\begin{array}{l}\text { Improvements in } \\
\text { emotion } \\
\text { identification }\end{array}$ \\
\hline $\begin{array}{l}\text { Smith et al., } \\
2015\end{array}$ & $\begin{array}{l}\text { Schizophrenia } \\
\text { Schizoaffective }\end{array}$ & 37 & $\begin{array}{l}\text { Double-blind, } \\
\text { randomized, } \\
\text { sham-controlled }\end{array}$ & $\begin{array}{l}\text { Anode over the left } \\
\text { DLPFC and cathode } \\
\text { over the contralateral } \\
\text { supraorbital ridge }\end{array}$ & 2 & $\begin{array}{l}1 \times \text { a day } \\
20 \text { min } \\
5 \text { days }\end{array}$ & MCBB & $\begin{array}{l}\text { Improvements in } \\
\text { MCCB composite } \\
\text { score, working } \\
\text { memory, and } \\
\text { attention }\end{array}$ \\
\hline $\begin{array}{l}\text { Orlov et al., } \\
2017\end{array}$ & $\begin{array}{l}\text { Schizophrenia } \\
\text { Schizoaffective }\end{array}$ & 49 & $\begin{array}{l}\text { Double-blind, } \\
\text { randomized, } \\
\text { sham-controlled }\end{array}$ & $\begin{array}{l}\text { Anode over the left } \\
\text { DLPFC and cathode } \\
\text { over the right } \\
\text { supraorbital area }\end{array}$ & 2 & $\begin{array}{l}1 \times \text { on } \\
\text { day } 1 \text { and day } \\
14 \text { of cognitive } \\
\text { training }\end{array}$ & $\begin{array}{l}\text { N-back task; } \\
\text { implicit learning } \\
\text { task }\end{array}$ & $\begin{array}{l}\text { Improvements in } \\
\text { working memory, } \\
\text { but not implicit } \\
\text { learning }\end{array}$ \\
\hline
\end{tabular}

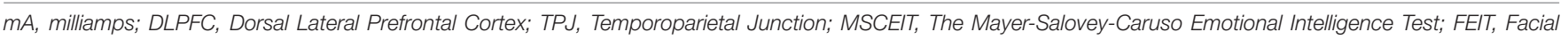
Emotion Identification Test; PONS, Profile of Nonverbal Sensitivity; TASIT, The Awareness of Social Inference Test; MCCB, MATRICS Consensus Cognitive Battery. 
DLPFC are useful, there are other regions of interest that may be relevant to the etiology of psychosis, which may expand our knowledge of tDCS, schizophrenia, and cognition. See Table 2 for a summary of noted studies that include information regarding the population, sample size, design of the study, montages/sites, $\mathrm{mA}$, duration/frequency, variables of interest, and key findings.

\section{FUTURE DIRECTIONS}

To date, studies have shown the potential for tDCS in reducing clinical symptoms and improving cognition, despite null findings that have also been reported. Overall, more work is needed to understand many aspects of tDCS. Presently in the literature, the sample sizes are small, and conducting studies with a larger number of participants is critical. Further, tDCS has been tested in other psychiatric populations such as childhood onset schizophrenia (Mattai et al., 2011), and research in this area could benefit from continuing to extend this work so we can fully understand the strengths and limitations of this neuromodulatory technique.

Research could benefit from additional probing around what the optimal number of sessions of tDCS are and period of time (e.g., days, weeks, months). More answers are needed to tease apart appropriate electrode montages and targets that are influenced such as symptoms and cognition. Further, although work indicates that the effects of tDCS may last up to $1 \mathrm{~h}$ after (Reis et al., 2009), more research could contribute to our current implementation. Questions still remain regarding the mechanisms of change which one way to determine this is using sham-controlled designs. Understanding the similarities and differences between tDCS and TMS and other techniques may offer perspective regarding strengths and limitations of this approach.

Other than enhancing study design and improving our understanding regarding the mechanism(s) underlying tDCS, future work could also benefit from examining relationships with medication and direct links with psychosis pathophysiology. There has also been some interesting work conducted to determine the influence of antipsychotic drug use on the impact of tDCS among schizophrenia groups (Agarwal et al., 2016) and determining the pathophysiology of psychosis through tDCS (Hasan et al., 2011) of which more work could continue to expand.

A direction in which tDCS may be advantageous is implementing this technique in at-risk populations such as individuals described as representing non-clinical psychosis (NCP). Psychosis, described as the loss of reality, falls on a continuum with some individuals endorsing psychotic disorders such as schizophrenia and individuals on the other endorse infrequent symptoms such as fleeting auditory hallucinations (e.g., hearing their name being called 1-2 times a year or seeing a shadow 1-2 times a year) (van Os, 2002). The distinguishing factors of this group with other psychosis populations are (1) symptoms are infrequent,
(2) symptoms are often accompanied by lower distress and impairment and, (3) functioning is more intact (Kelleher and Cannon, 2011). Although there are pronounced differences, NCP individuals tend to share similar vulnerability deficits observed in psychosis populations (e.g., schizophrenia) and clinical high-risk (CHR; individuals considered at imminent risk for developing psychosis) samples such as emotion recognition deficits (Pelletier et al., 2013), and procedural learning impairments (van Os, 2002; Kelleher and Cannon, 2011; Mittal et al., 2012). Our group investigated whether tDCS could improve procedural learning deficits given studies indicating impairments in these processes among NCP (Mittal et al., 2012; Lunsford-Avery et al., 2017) and CHR samples (Dean et al., 2014). Specifically, using a double-blind, randomized, sham-controlled design, we investigated, in a sample of 18 controls and $24 \mathrm{NCP}$ individuals, whether cerebellar tDCS could improve procedural learning (using a pursuit rotor task) in the NCP group and normalize performance to the level of controls (Gupta et al., 2017). Participants were randomized to receive $25 \mathrm{~min}$ of active cerebellar tDCS or sham on separate laboratory visits, 1 week apart. After being in the sham condition, NCP individuals had significantly lower procedural learning performance compared to the control group. However, after being in the active condition, the NCP group normalized procedural learning performance to the level of controls. These data highlight the possibility of using NCP as an analog sample within this work. Furthermore, these findings continue to disentangle the complexities regarding the pathogenesis of psychosis in efforts to develop targeted treatment interventions such as tDCS in order to prevent further symptom decline.

Additional research applying tDCS among individuals at CHR for developing psychotic disorders such as schizophrenia would be also beneficial. It is suggested that about $10-35 \%$ of individuals within this group may go on to develop the disorder (Fusar-Poli et al., 2012) and tDCS may be a way to intervene. Studies within this group can contribute to our knowledge regarding psychosis more broadly and also inform the development of targeted treatment interventions as such. Utilizing risk samples may be a first step in extending the present work among schizophrenia populations in order to determine if this technique can provide clinical utility as an intervention approach.

\section{AUTHOR CONTRIBUTIONS}

All authors contributed to the drafting and editing of the manuscript.

\section{FUNDING}

This work was supported by T32 NS047987, and in part by Brain \& Behavior Research Foundation, (NARSAD) Independent Investigator Award to VM. 


\section{REFERENCES}

Adolphs, R. (2001). The neurobiology of social cognition. Curr. Opin. Neurobiol. 11, 231-239. doi: 10.1016/S0959-4388(00)00202-6

Agarwal, S. M., Bose, A., Shivakumar, V., Narayanaswamy, C., Chhabra, H., Kalmady, S. V., et al. (2016). Impact of antipsychotic medication on transcranial direct current stimulation (tDCS) effects in schizophrenia patients. Psychiatry Res. 235, 97-103. doi: 10.1016/j.psychres.2015.11.042

Agarwal, S. M., Venkataram, S., Bose, A., Subramaniam, A., Nawani, H., Chhabra, H., et al. (2013). Transcranial direct current stimulation in schizophrenia. Clin. Psychopharmacol. Neurosci. 11, 118-125. doi: 10.9758/cpn.2013.11. 3.118

Aleman, A., Enriquez-Geppert, S., Knegtering, H., and Dlabac-de Lange, J. J. (2018). Moderate effects of non-invasive brain stimulation of the frontal cortex for improving negative symptoms in schizophrenia: meta-analysis of controlled trials. Neurosci. Biobehav. Rev. 89, 111-118. doi: 10.1016/j.neubiorev.2018. 02.009

Aleman, A., Sommer, I. E., and Kahn, R. S. (2007). Efficacy of slow repetitive transcranial magnetic stimulation in the treatment of resistant auditory hallucinations in schizophrenia: a meta-analysis. J. Clin. Psychiatry 68, 416-421. doi: 10.4088/JCP.v68n0310

Alexander, M. L., Gilmore, J. H., Jarskog, L. F., and Frohlich, F. (2018). Randomized trial of transcranial alternating current stimulation for treatment of auditory hallucinations in schizophrenia. Eur. Psychiatry 51, 25-53. doi: 10.1016/j. eurpsy.2018.01.004

Barch, D. B., and Sheffield, J. M. (2014). Cognitive impairments in psychotic disorders: common mechanisms and measurement. World Psychiatry 13, 224-232. doi: 10.1002/wps.20145

Bose, A., Shivakumar, V., Narayanaswamy, J. C., Nawani, H., Subramaniam, A., Agarwal, S. M., et al. (2014). Insight facilitation with add-on tDCS in schizophrenia. Schizophr. Res. 156, 63-65. doi: 10.1016/j.schres.2014. 03.029

Brunelin, J., Mondino, M., Gassab, L., Haesebaert, F., Gaha, L., Suaud-Chagny, M. F., et al. (2012). Examining transcranial direct-current stimulation (tDCS) as a treatment for hallucinations in schizophrenia. Am. J. Psychiatry 169, 719-724. doi: 10.1176/appi.ajp.2012.11071091

Brunoni, A. R., Moffa, A. H., Fregni, F., Palm, U., Padberg, F., Blumberger, D. M., et al. (2016). Transcranial direct current stimulation for acute major depressive episodes: meta-analysis of individual patient data. Br. J. Psychiatry 208, 522-531. doi: 10.1192/bjp.bp.115.164715

Brunoni, A. R., Nitsche, M. A., Bolognini, N., Bikson, M., Wagner, T., Merabet, L., et al. (2012). Clinical research with transcranial direct current stimulation (tDCS): challenges and future directions. Brain Stimul. 5, 175-195. doi: 10.1016/ j.brs.2011.03.002

Brunoni, A. R., Shiozawa, P., Truong, D., Javitt, D. C., Elkis, H., Fregni, F., et al. (2014). Understanding tDCS effects in schizophrenia: a systematic review of clinical data and an integrated computation modeling analysis. Expert Rev. Med. Devices 11, 383-394. doi: 10.1586/17434440.2014. 911082

Dean, D. J., Bernard, J. A., Orr, J. M., Pelletier-Baldelli, A., Gupta, T., Carol, E. E., et al. (2014). Cerebellar morphology and procedural learning impairment in neuroleptic-naïve youth at ultrahigh risk for psychosis. Clin. Psychol. Sci. 2, 152-164. doi: 10.1177/2167702613500039

Fatouros-Bergman, H., Cervenka, S., Flyckt, L., Edman, G., and Farde, L. (2014). Meta analysis of cognitive performance in drug-naive patients with schizophrenia. Schizophr. Res. 158, 156-162. doi: 10.1016/j.schres.2014. 06.034

Fenton, W. S., Blyler, C. R., and Heinssen, R. K. (1997). Determinants of medication compliance in schizophrenia: empirical and clinical findings. Schizophr. Bull. 23, 637-651. doi: 10.1093/schbul/23.4.637

Fett, A. J., Viechtbauer, W., Dominguez, M., Penn, D. L., van Os, J., and Krabbendam, L. (2011). The relationship between neurocognition and social cognition with functional outcomes in schizophrenia: a meta-analysis. Neurosci. Biobehav. Res. 35, 573-588. doi: 10.1016/j.neubiorev.2010.07.001

Fitzgerald, P. B., McQueen, S., Daskalakis, Z. J., and Hoy, K. E. (2014). A negative pilot study of daily bimodal transcranial direct current stimulation in schizophrenia. Brain Stimul. 7, 813-816. doi: 10.1016/j.brs.2014. 08.002
Fregni, F., Nitsche, M. A., Loo, C. K., Brunoni, A. R., Marangolo, P., Leite, J., et al. (2015). Regulatory considerations for the clinical and research use of transcranial direct current stimulation (tDCS): review and recommendations from an expert panel. Clin. Res. Regul. Aff. 32, 22-35. doi: 10.3109/10601333. 2015.980944

Fröhlich, F., Burrello, T. N., Mellin, J. M., Cordle, A. L., Lustenberger, C. M., Gilmore, J. H., et al. (2016). Exploratory study of once-daily transcranial direct current stimulation (tDCS) as a treatment for auditory hallucinations in schizophrenia. Eur. Psychiatry 33, 54-60. doi: 10.1016/j.eurpsy.2015. 11.005

Fröhlich, F., Sellers, K. K., and Cordle, A. L. (2015). Targeting the neurophysiology of cognitive systems with transcranial alternating current stimulation. Expert Rev. Neurother. 15, 145-167. doi: 10.1586/14737175.2015.99 2782

Fusar-Poli, P., Bonoldi, I., Yung, A. R., Borgwardt, S., Kempton, M. J., Valmaggia, L., et al. (2012). Predicting psychosis. Arch. Gen. Psychiatry 69, 220-229. doi: 10.1001/archgenpsychiatry.2011.1472

Fusar-Poli, P., Papanastasiou, E., Stahl, D., Rocchetti, M., Carpenter, W., Shergill, S., et al. (2015). Treatments of negative symptoms in schizophrenia: meta-analysis of 168 randomized placebo-controlled trials. Schizophr. Bull. 41, 892-899. doi: 10.1093/schbul/sbu170

Fusar-Poli, P., Smieskova, R., Kempton, M. J., Ho, B. C., Andreasen, N. C., and Borgwardt, S. (2013). Progressive brain changes in schizophrenia related to antipsychotic treatment? A meta-analysis of longitudinal MRI studies. Neurosci. Biobehav. Rev. 37, 1680-1691. doi: 10.1016/j.neubiorev.2013. 06.001

Gandiga, P. C., Friedhelm, C., Hummel, F. C., and Cohen, L. G. (2006). Transcranial DC stimulation (tDCS): a tool for double-blind sham-controlled clinical studies in brain stimulation. Clin. Neurophysiol. 117, 845-850. doi: 10.1016/j.clinph.2005.12.003

Geddes, J., Freemantle, N., Harrison, P., and Bebbington, P. (2000). Atypical antipsychotics in the treatment of schizophrenia: systematic overview and meta-regression analysis. BMJ 321, 1371-1376. doi: 10.1136/bmj.321.7273. 1371

Gomes, J. S., Shiozawa, P., Dias, A. M., Valverde Ducos, D., Akiba, H., Trevizol, A. P., et al. (2015). Left dorsolateral prefrontal cortex anodal tDCS effects on negative symptoms in schizophrenia. Brain Stimul. 8, 989-991. doi: 10.1016/j. brs.2015.07.033

Green, M. F., and Harvey, P. D. (2014). Cognition in schizophrenia: past, present, and future. Schizophr. Res. 1, e1-e9. doi: 10.1016/j.scog.2014.02.001

Green, M. F., Hellemann, G., Horan, W. P., Lee, J., and Wynn, J. K. (2012). From perception to functional outcomes in schizophrenia modeling the role of ability and motivation. Arch. Gen. Psychiatry 69, 1216-1224. doi: 10.1001/ archgenpsychiatry.2012.652

Green, M. F., Horan, W. P., and Lee, J. (2015). Social cognition in schizophrenia. Nat. Rev. Neurosci. 16, 620-631. doi: 10.1038/nrn4005

Gupta, T., Dean, D. J., Kelley, N. J., Bernard, J. A., Ristanovic, I., and Mittal, V. A. (2017). Cerebellar transcranial direct current stimulation improves procedural learning in nonclinical psychosis: a double-blind crossover study. Schizophr. Bull. doi: 10.1093/schbul/sbx179 [Epub ahead of print].

Hasan, A., Nitsche, M. A., Rein, B., Schneider-Axmann, T., Guse, B., Gruber, O., et al. (2011). Dysfunctional long-term potentiation-like plasticity in schizophrenia revealed by transcranial direct current stimulation. Behav. Brain Res. 224, 15-22. doi: 10.1016/j.bbr.2011.05.017

Hasan, A., Strube, W., Palm, U., and Wobrock, T. (2016). Repetitive noninvasive brain stimulation to modulate cognitive functions in schizophrenia: a systematic review of primary and secondary outcomes. Schizophr. Bull. 42, 95-109. doi: 10.1093/schbul/sbv158

Hoffman, R. E., Hawkins, K. A., Gueorguieva, R., Boutros, N. N., Rachid, F., Carroll, K., et al. (2003). Transcranial magnetic stimulation of left temporoparietal cortex and medication-resistant auditory hallucinations. Arch. Gen. Psychiatry 60, 49-56. doi: 10.1001/archpsyc.60.1.49

Hopfinger, J. B., Parsons, J., and Fröhlich, F. (2017). Differential effects of 10-Hz and $40-\mathrm{Hz}$ transcranial alternating current stimulation (tACS) on endogenous versus exogenous attention. Cogn. Neurosci. 8, 102-111. doi: 10.1080/17588928. 2016.1194261

Hoy, K. E., Arnold, S. L., Emonson, M. R., Daskalakis, Z. J., and Fitzgerald, P. B. (2014). An investigation into the effects of tDCS dose on cognitive performance 
over time in patients with schizophrenia. Schizophr. Res. 155, 96-100. doi: 10.1016/j.schres.2014.03.006

Hutchinson, G., Bhugra, D., Mallett, R., Burnett, R., Corridan, B., and Leff, J. (1999). Fertility and marital rates in first-onset schizophrenia. Soc. Psychiatry Psychiatr. Epidemiol. 34, 617-621. doi: 10.1007/s0012700 50183

Insel, T. (2010). Rethinking schizophrenia. Nature 468, 187-193. doi: 10.1038/ nature09552

Jardri, R., Pouchet, A., Pins, D., and Thomas, P. (2011). Cortical activations during auditory verbal hallucinations in schizophrenia: a coordinate-based meta-analysis. Am. J. Psychiatry 168, 73-81. doi: 10.1176/appi.ajp.2010.091 01522

Kadosh, R. C. (2015). Modulating and enhancing cognition using brain stimulation: science and fiction. J. Cogn. Psychol. 27, 141-163. doi: 10.1080/ 20445911.2014.996569

Kekic, M., Boysen, E., Campbell, I. C., and Schmidt, U. (2016). A systematic review of the clinical efficacy of transcranial direct current stimulation (tDCS) in psychiatric disorders. J. Psychiatr. Res. 74, 70-88. doi: 10.1016/j.jpsychires. 2015.12.018

Kelleher, I., and Cannon, M. (2011). Psychotic-like experiences in the general population: characterizing high-risk group for psychosis. Psychol. Med. 41, 1-6. doi: 10.1017/S0033291710001005

Kennedy, N. I., Lee, W. H., and Frangou, S. (2018). Efficacy of non-invasive brain stimulation on the symptom dimensions of schizophrenia: a meta-analysis of randomized controlled trials. Eur. Psychiatry 49, 69-77. doi: 10.1016/j.eurpsy. 2017.12.025

Khalighinejad, N., Di Costa, S., and Haggard, P. (2016). Endogenous action selection processes in dorsolateral prefrontal cortex contribute to sense of agency: a meta-analysis of tDCS studies of "intentional binding”. Brain Stimul. 9, 372-379. doi: 10.1016/j.brs.2016. 01.005

Khalighinejad, N., and Haggard, P. (2015). Modulating human sense of agency with non-invasive brain stimulation. Cortex 69, 93-103. doi: 10.1016/j.cortex.2015. 04.015

Lacro, J. P., Dunn, L. B., Dolder, C. R., Leckband, S. G., and Jeste, D. V. (2002). Prevalence of and risk factors for medication nonadherence in patients with schizophrenia: a comprehensive review of recent literature. J. Clin. Psychiatry 63, 892-909. doi: 10.4088/JCP.v63n1007

Lunsford-Avery, J. R., Dean, D. J., and Mittal, V. A. (2017). Self-reported sleep disturbances associated with procedural learning impairment in adolescents at ultra-high risk for psychosis. Schizophr. Res. 190, 160-163. doi: 10.1016/j.schres. 2017.03.025

Mai, X., Zhang, W., Hu, X., Zhen, Z., Xu, Z., Zhang, J., et al. (2016). Using tDCS to explore the role of the right temporo-parietal junction in theory of mind and cognitive empathy. Front. Psychol. 7:380. doi: 10.3389/fpsyg.2016. 00380

Marwaha, S., and Johnson, S. (2004). Schizophrenia and employment - a review. Soc. Psychiatry Psychiatr. Epidemiol. 39, 337-349. doi: 10.1007/s00127-0040762-4

Mattai, A., Miller, R., Weisinger, B., Greenstein, D., Bakalar, J., Tossell, J., et al. (2011). Tolerability of transcranial direct current stimulation in childhoodonset schizophrenia. Brain Stimul. 4, 275-280. doi: 10.1016/j.brs.2011. 01.001

Mellin, J. M., Alagapan, S., Lustenberger, C., Lugo, C. E., Alexander, M. L., Gilmore, J. H., et al. (2018). Randomized trial of transcranial alternating current stimulation for treatment of auditory hallucinations in schizophrenia. Eur. Psychiatry 51, 25-33. doi: 10.1016/j.eurpsy.2018.01.004

Mittal, V. A., Dean, D. J., and Pelletier, A. (2012). Dermatoglyphic asymmetries and fronto-striatal dysfunction in young-adults reporting non-clinical psychosis. Acta Psychiatr. Scand. 126, 290-297. doi: 10.1111/j.1600-0447.2012. 01869.x

Mondino, M., Brunelin, J., Palm, U., Brunoni, A. R., Poulet, E., and Fecteau, S. (2015). Transcranial direct current stimulation for the treatment of refractory symptoms of schizophrenia. Current evidence and future directions. Curr. Pharm. Des. 21, 3373-3383. doi: 10.2174/13816128216661506190 93648

Mondino, M., Jardri, R., Saoud, M., and Poulet, E. (2016). Effects of fronto-temporal transcranial direct current stimulation on auditory verbal hallucinations and resting-state functional connectivity of the left temporoparietal junction in patients with schizophrenia. Schizophr. Bull. 42, 318-326. doi: $10.1093 /$ schbul/sbv114

Mueser, K. T., Salyers, M. P., and Mueser, P. R. (2001). A prospective analysis of work in schizophrenia. Schizophr. Bull. 27, 281-296. doi: 10.1093/ oxfordjournals.schbul.a006874

Nitsche, M. A., Cohen, L. G., Wassermann, E. M., Priori, A., Lang, N., Antal, A., et al. (2008). Transcranial direct current stimulation: state of the art 2008. Brain Stimul. 1, 206-223. doi: 10.1016/j.brs.2008.06.004

Nitsche, M. A., and Paulus, W. (2000). Excitability changes induced in the human motor cortex by weak transcranial direct current stimulation. J. Physiol. 527, 633-639. doi: 10.1111/j.1469-7793.2000.t01-1-00633.x

Nuechterlein, K. H., Green, M. F., Kern, R. S., Baade, L. E., Barch, D. M., Cohen, J. D., et al. (2008). The MATRICS consensus cognitive battery, part 1: test selection, reliability, and validity. Am. J. Psychiatry 165, 203-213. doi: 10.1176/ appi.ajp.2007.07010042

Orlov, N. D., Tracy, D. K., Joyce, D., Patel, S., Rodzinka-Pasko, J., Dolan, H., et al. (2017). Stimulating cognition in schizophrenia: a controlled pilot study of the effects of prefrontal transcranial direct current stimulation upon memory and learning. Brain Stimul. 10, 560-566. doi: 10.1016/j.brs.2016. 12.013

Osoegawa, C., Gomes, J. S., Grigolon, R. B., Brietzke, E., Gadelha, A., Lacerda, A. L. T., et al. (2018). Non-invasive brain stimulation for negative symptoms in schizophrenia: an updated systematic review and meta-analysis. Schizophr. Res. doi: 10.1016/j.schres.2018.01.010 [Epub ahead of print].

Palm, U., Keeser, D., Hasan, A., Kupka, M. J., Blautzik, J., Sarubin, N., et al. (2016). Prefrontal transcranial direct current stimulation for treatment of schizophrenia with predominant negative symptoms: a double-blind, shamcontrolled proof-of-concept Study. Schizophr. Bull. 42, 1253-1261. doi: 10.1093/ schbul/sbw041

Paulus, W. (2011). Transcranial electrical stimulation (tES - tDCS; tRNS, tACS) methods. Neuropsychol. Rehabil. 21, 602-617. doi: 10.1080/09602011.2011. 557292

Pelletier, A. L., Dean, D. J., Lunsford-Avery, J. R., Smith, A. K., Orr, J. M., Gupta, T., et al. (2013). Emotion recognition and social/role dysfunction in non-clinical psychosis. Schizophr. Res. 143, 70-73. doi: 10.1016/j.schres.2012. 10.039

Pontillo, M., Costanzo, F., Menghini, D., Averna, R., Santonastaso, O., Tata, M. C., et al. (2018). Use of transcranial direct stimulation in the treatment of negative symptoms of schizophrenia. Clin. EEG Neurosci. 49, 18-26. doi: $10.1177 / 1550059417746531$

Rassovsky, Y., Dunn, W., Wynn, J., Wu, A. D., Iacoboni, M., Hellemann, G., et al. (2015). The effect of transcranial direct current stimulation on social cognition in schizophrenia: a preliminary study. Schizophr. Res. 165, 171-174. doi: 10.1016/j.schres.2015.04.016

Reis, J., Schambra, H. M., Cohen, L. G., Buch, E. R., Fritsch, B., Zarahn, E., et al. (2009). Noninvasive cortical stimulation enhances motor skill acquisition over multiple days through an effect on consolidation. Proc. Natl. Acad. Sci. U.S.A. 106, 1590-1595. doi: 10.1073/pnas.0805413106

Saha, S., Chant, D., Welham, J., and McGrath, J. (2005). A systematic review of the prevalence of schizophrenia. PLoS Med. 2:e141. doi: 10.1371/journal.pmed. 0020141

Santiesteban, I., Banissy, M. J., Catmur, C., and Bird, G. (2012). Enhancing social ability by stimulating right temporoparietal junction. Curr. Biol. 22, 2274-2277. doi: 10.1016/j.cub.2012.10.018

Schaefer, J., Giangrande, E., Weinberger, D. R., and Dickinson, D. (2013). The global cognitive impairment in schizophrenia: consistent over decades and around the world. Schizophr. Res. 150, 42-50. doi: 10.1016/j.schres.2013. 07.009

Schülke, R., and Straube, B. (2017). Modulating the assessment of semantic speech-gesture relatedness via transcranial direct current stimulation of the left frontal cortex. Brain Stimul. 10, 223-230. doi: 10.1016/j.brs.2016. 10.012

Smith, R. C., Boules, S., Mattiuz, S., Youssef, M., Tobe, R. H., Sershen, H., et al. (2015). Effects of transcranial direct current stimulation (tDCS) on cognition, symptoms, and smoking in schizophrenia: a randomized controlled study. Schizophr. Res. 168, 260-266. doi: 10.1016/j.schres.2015. 06.011 
Straube, B., Schülke, R., Drewing, K., Kircher, T., and van Kemenade, B. M. (2017). Hemispheric differences in the processing of visual consequences of active vs. passive movements: a transcranial direct current stimulation study. Exp. Brain Res. 235, 3207-3216. doi: 10.1007/s00221-017-5053-x

Tandon, R., Nasrallah, H. A., and Keshavan, M. S. (2010). Schizophrenia, “just the facts" 5. Treatment and prevention. Past, present, and future. Schizophr. Res. 122, 1-23. doi: 10.1016/j.schres.2010.05.025

van Os, J. (2002). Psychotic symptoms in non-clinical populations and the continuum of psychosis. Schizophr. Res. 54, 59-65. doi: 10.1016/S0920-9964(01) 00352-8

Vercammen, A., Rushby, J. A., Loo, C., Short, B., Weickert, C. S., and Weickert, T. W. (2011). Transcranial direct current stimulation influences probabilistic association learning in schizophrenia. Schizophr. Res. 131, 198-205. doi: 10.1016/j.schres.2011.06.021

Young, J. L., Zonana, H. V., and Shepler, L. (1986). Medication noncompliance in schizophrenia: codification and update. Bull. Am. Acad. Psychiatry Law 14, 105-122.
Young, S. L., Taylor, M., and Lawrie, S. M. (2015). “First do no harm.” A systematic review of the prevalence and management of antipsychotic adverse effects. $J$. Psychopharmacol. 29, 353-362. doi: 10.1177/0269881114562090

Conflict of Interest Statement: VM is a consultant to Takeda Pharmaceuticals.

The remaining authors declare that the research was conducted in the absence of any commercial or financial relationships that could be construed as a potential conflict of interest.

Copyright (C) 2018 Gupta, Kelley, Pelletier-Baldelli and Mittal. This is an open-access article distributed under the terms of the Creative Commons Attribution License (CC BY). The use, distribution or reproduction in other forums is permitted, provided the original author(s) and the copyright owner are credited and that the original publication in this journal is cited, in accordance with accepted academic practice. No use, distribution or reproduction is permitted which does not comply with these terms. 


\section{OPEN ACCESS}

Edited by:

Claudio Lucchiari,

Università degli Studi di Milano, Italy

Reviewed by:

Martin J. Herrmann,

Universität Würzburg, Germany

Junhong Zhou,

Harvard Medical School,

United States

*Correspondence:

Floriana Costanzo

floriana.costanzo@opbg.net

Received: 11 March 2018 Accepted: 13 June 2018

Published: 20 July 2018

Citation:

Costanzo F, Menghini D, Maritato $A$ Castiglioni MC, Mereu A, Varuzza C,

Zanna V and Vicari S (2018) New

Treatment Perspectives in

Adolescents With Anorexia Nervosa:

The Efficacy of Non-invasive

Brain-Directed Treatment.

Front. Behav. Neurosci. 12:133.

doi: 10.3389/fnbeh.2018.00133

\section{New Treatment Perspectives in Adolescents With Anorexia Nervosa: The Efficacy of Non-invasive Brain-Directed Treatment}

\author{
Floriana Costanzo *, Deny Menghini, Antonella Maritato, Maria C. Castiglioni, \\ Alberta Mereu, Cristiana Varuzza, Valeria Zanna and Stefano Vicari
}

Child Neuropsychiatric Unit, Department of Neuroscience, Bambino Gesù Children Hospital, Rome, Italy

Poor treatment outcomes are available for anorexia nervosa (AN) and treatment innovations are urgently needed. Recently, non-invasive neuromodulation tools have suggested to have potential for reducing an symptomatology targeting brain alterations. The objective of the study was to verify whether left anodal/right cathodal prefrontal cortex transcranial direct current stimulation (tDCS), may aid in altering/resetting inter-hemispheric balance in patients with $\mathrm{AN}$, re-establishing control over eating behaviors. Twenty-three adolescents with an underwent a treatment as usual (AU), including nutritional, pharmacological, and psychoeducational treatment, plus 18 sessions of tDCS (TDCS $+\mathrm{AU}=\mathrm{n} 11$; mean age $=13.9, S D=1.8$ years) or a family based therapy ( $\mathrm{FBT}+\mathrm{AU}=\mathrm{n} 12$, mean age $=15.1, \mathrm{SD}=1.5$ years). Psychopathological scales and the body mass index (BMI) were assessed before and after treatment. After 6 weeks of treatment, the BMl values increased only in the tDCS group, even at 1-month follow-up. Independently of the treatment, all participants improved in several psychopathological measures, included AN psychopathology and mood and anxiety symptoms. Our results demonstrated for the first time a specific effect of the left anodal/right cathodal tDCS treatment protocol on stable weight gain and a superiority compared to an active control treatment for adolescents with AN. Results were interpreted as a possible direct/indirect effect of tDCS in into some pathophysiological mechanisms of AN, involving the mesocortical dopaminergic pathways and the promotion of food intake. This pilot study opens new perspectives in the treatment of an in adolescence, supporting the targeted and beneficial effects of a brain-based treatment.

Keywords: tDCS, anorexia, prefrontal cortex, adolescents, BMI

\section{INTRODUCTION}

Eating disorders (ED) are highly distinctive disorders characterized by pathological eating behaviors and body image disturbance. according to the diagnostic and statistical manual of mental disorders, fifth edition (Dsm-5), ED shall be considered as a spectrum of over-eating and under-eating, associated with altered weight and with altered food reward that results in significant biologic, psychological, and social complications. Anorexia nervosa (AN) is a severe ED associated whit other important physical and psychological comorbidities. It is defined by an extremely low 
Body Mass Index (BMI $\left.<18.5 \mathrm{~kg} / \mathrm{m}^{2}\right)$ and concomitant anxiety and preoccupations related to weight and body image, and exerts a significant individual and societal impact. Levels of mortality and disability and lifetime prevalence in AN are high (Arcelus et al., 2011; Smink et al., 2012; Walsh, 2013). Onset is increasing in the early adolescence, mainly in the females, with a symptomatology most common in teen-ager and adults.

To data, available treatments for AN are only moderately effective. Pharmacological treatment plays a limited role (Aigner et al., 2011; Gustavsson et al., 2011; Dold et al., 2015; Garner et al., 2016). Selective serotonin reuptake inhibitors (SSRIs) and neuroleptics constitute the mainstay treatments, but the efficacy of these medications is fairly poor and up to $30 \%$ of patients with AN prove to be medically intractable (Steinhausen, 2002; Kontis and Theochari, 2012). In turn, no Food and Drug Administration indication for any pharmacological treatment for $\mathrm{AN}$ is given.

Psychological therapies, such as Cognitive Behavioral Therapy and Family Psychotherapy, are widely considered the treatment of choice. However, no single psychological intervention has shown clear superiority in treating adults with AN, while in adolescents with AN, the evidence base is strongest for the use of Family Psychotherapy over alternative individual psychotherapies (Lock et al., 2010; Herpertz-Dahlmann and de Zwaan, 2011; Le Grange et al., 2012; Agras et al., 2014; Rienecke, 2017). Recently, it was shown the efficacy of integrated treatment as opposed to single therapies, as suggested by the current guidelines for the treatment of ED in children and adolescents (National Institute for Clinical Excellence, 2004; American Psychiatric Association, 2006). In particular, the application of a Multifocal Integrated Treatment, based on both family and individualized psychotherapy, nutritional and pharmacological interventions, showed improvement in the eating psychopathology in adolescents with AN (Laghi et al., 2017). However, there is a need for continued efforts to develop novel interventions (Bodell and Keel, 2010), since evidence-base treatment for AN is still lacking.

Novel approaches to treatment of ED in general and specifically for AN have been called for by august bodies such as the National Institute for Health and Care Excellence (NICE) in the UK and the National Institutes of Health (NIH) in the USA. Recently, neuromodulation procedures, which are emerging techniques that can be used to stimulate or inhibit neural activity, has been postulated as a potential treatment for ED (for recent review see McClelland et al., 2013; Lee et al., 2018).

Marked increase in neuromodulation approaches for treatment of ED grounds on recent year's extensive neuroscience data related to $\mathrm{ED}$ and to the emergence of testable neurobiological models. Altered activity in the insula (Kaye et al., 2010; Mohr et al., 2010; Bär et al., 2013; Oberndorfer et al., 2013; Strigo et al., 2013), abnormalities in the processing of rewards (Bohon and Stice, 2011; Avena and Bocarsly, 2012; Ritschel et al., 2017) and alterations in frontal regions have been reported (Brooks et al., 2011; Celone et al., 2011; Strigo et al., 2013; Kullmann et al., 2014). Subsequently, neural models of ED have been developed (Steinglass and Walsh, 2006; Brooks et al., 2011; Friederich and Herzog, 2011; Riva, 2016; Steinglass et al., 2017).
In $\mathrm{AN}$, some studies considered abnormalities in dorsolateral prefrontal cortex (DLPFC) (Kaye et al., 2009; Van Kuyck et al., 2009; Brooks et al., 2011; Ehrlich et al., 2015; Hestad et al., 2016). Furthermore, fMRI studies, EEG measurements and PET scan in individuals with AN showed a hyperactivity of right hemisphere (Grunwald et al., 2004; Brooks et al., 2011; Bär et al., 2013; Phillipou et al., 2015) and clusters' increase of serotonergic and dopaminergic bindings (e.g., Kontis and Theochari, 2012; Kaye et al., 2013; Riva, 2016), particularly in right frontal-temporal regions (Bailer et al., 2007; Galusca et al., 2008).

Despite the marked increase in neurobiological data related to AN, there is still a lack of targeted brain-directed treatment interventions. Most convincing evidence comes from studies applying deep brain stimulation across a variety of brain targets involved in the hypothalamic-mesocorticolimbic pathways. Potential anatomical targets were those involved with reward, cognitive control, motivation, and the learning/memory circuits, such as the ventral tegmental area, the nucleus accumbens and caudate, the subgenual cingulate cortex, the amigdala, the hypppocampus, the insula, and the ventral striatum (for review see McClelland et al., 2013; Lee et al., 2018).

Conversely, the exiting studies that have investigated the efficacy of transcranial non-invasive brain stimulation for reducing AN symptomatology and related behaviors (e.g., Kamolz et al., 2008; McClelland et al., 2013, 2016a,b; Van den Eynde et al., 2013; Khedr et al., 2014), have typically targeted the pre-frontal cortex (PFC), mainly the DLPFC. The DLPFC has a key node of the brain's frontostriatal cognitive circuits, important for inhibitory cognitive control (Miller and Cohen, 2001; Hare et al., 2009), self-control in a dietary context (Wagner et al., 2010; Lowe et al., 2014) and for higher-order reward processing, as part of its involvement in the mesocortical dopaminergic pathway (Diana, 2011), linked to regulation of food intake (Doherty et al., 2016).

Two forms of non-invasive neuromodulatory techniques used in the treatment of AN were transcranial magnetic stimulation (TMS) and transcranial direct current stimulation (tDCS). Both TMS and tDCS have been proven to reduce AN-related behaviors and thoughts (McClelland et al., 2013; Lee et al., 2018). The TMS studies have mostly involved high-frequency (excitatory) TMS to the left PFC. For example, a reduction in levels of feeling full, feeling fat and anxiety has been reported when a single TMS session was applied to the left DLPFC in cases with AN (Van den Eynde et al., 2013; McClelland et al., 2016a,b). Moreover, significant improvement in depressive symptomology and BMI (Kamolz et al., 2008) was described in a case report of 41 sessions of left DLPFC excitatory rTMS, as well as improvement in AN symptomatology and affective symptoms has reported in three of five patients, after 20 sessions of excitatory left DLPFC rTMS. However, such improvement was not always translated into weight gain (McClelland et al., 2016a,b).

Despite recent findings, showing symptoms reduction following TMS excitation of the left DLPFC, one study directly investigated the effects of tDCS on the AN symptomatology (Khedr et al., 2014). Specifically, in this study, it was documented an improvement in AN and in associated depression following 10 
daily anodal tDCS (2 mA) sessions of 25 min over the left DLPFC (cathode in extracephalic montage) in five of seven patients immediately post-treatment and in three of seven patients at 1 month follow up.

Conversely, tDCS research has mostly focused on food cravings (Fregni et al., 2008; Goldman et al., 2011; Montenegro et al., 2012; Boggiano et al., 2017) and evidences have been provided that food cravings is reduced when excitatory (anodal) tDCS is applied over the right DLPFC (as opposite to reports on AN where excitatory stimulation is applied over the left DLPFC). For example, Fregni et al. (2008) compared both tDCS protocols, anode right/cathode left and anode left/cathode right, to sham stimulation, and found that food cravings reduced, remained stable or increased in these conditions, respectively. Moreover, both Goldman et al. (2011) and Montenegro et al. (2012) found reduced food cravings and desire to eat in overweight individuals following single session of right anodal/left cathodal tDCS compared to sham. Similarly, Ljubisavljevic et al. (2016) demonstrated that repeated sessions of right anodal/left cathodal tDCS increase the single sessions effect, byreducing the intensity of food craving and the habitual experiences of food craving in individuals with frequent food craving. These studies ground on the evidence that hyperphagia, and more in general craving and substance abuse, is associated with right frontal hypoactivation (Isern, 1987; Fisher et al., 1994; Miller and Cohen, 2001), an opposite pattern of inter-hemispheric imbalance found in $\mathrm{AN}-$ i.e., right frontal hyperactivation and dominance (Grunwald et al., 2004; Brooks et al., 2011; Bär et al., 2013; Phillipou et al., 2015).

Bilateral prefrontal cortex tDCS, aimed at rebalancing such inter-hemispheric imbalance by increasing excitability of right hemisphere and decreasing excitability of left hemisphere (the opposite of what is proposed for AN), showed positive findings in different disorders of craving and substance abuse. In particular, left cathodal/right anodal tDCS increased the abstinent rate (Klauss et al., 2014) and reduced the craving in alcoholic patients, with more negative processing of alcohol-related cues after treatment (Wietschorke et al., 2016).

However, in healthy individuals, Vierheilig et al. (2016) aimed at investigating the effects of bilateral tDCS with different electrode montages, on the interaction of attention and emotion processes, found that only left cathodal/right anodal tDCS leads to increase visual attention but neither left cathodal/right anodal or left anodal/right cathodal did influence emotional processing.

Increased knowledge on the role of hemispheric lateralization in $\mathrm{ED}$, together with improvements in the design of neuromodulation protocols, is likely to emerge from studies involving tDCS. tDCS applied on DLPFC should act on the right hyperactivity reported in $\mathrm{AN}$ to balancing the activity in both hemispheres.

Considering the paucity of effective treatments for $\mathrm{AN}$, and the increasing incidence rate of $\mathrm{AN}$ among children and adolescents, with common long-term physical and psychosocial disability outcomes as well as life risk (Arcelus et al., 2011; Smink et al., 2012; Walsh, 2013), the study was aiming at proving the effectiveness of a non-invasive neuromodulation treatment by tDCS in improving the outcome of traditional treatment in developmental populations with AN.

The safety and tolerability of tDCS in children and adolescents has been proven (Antal et al., 2017) so, in a single-blindcontrolled study, we hypothesized that excitatory tDCS over the left DLPFC and inhibitory tDCS over the right DLPFC (anode left/cathode right) may aid in altering/resetting interhemispheric balance in adolescents with $\mathrm{AN}$, re-establish their control over eating behaviors. This montage was based on the dominance hypothesis in $\mathrm{AN}$ and in accordance with previous studies, where opposite stimulation montage showed an improvement in "over-eating" for ED symptoms (Fregni et al., 2008; Goldman et al., 2011; Montenegro et al., 2012; Boggiano et al., 2017).

\section{MATERIALS AND METHODS}

\section{Participants}

Twenty-three adolescents with diagnosis of AN participated in the study. Eleven participants (10 females; 1 males; mean age $=13.9, S D=1.8$ years, range $10.3-15.6$; mean $\mathrm{IQ}=102.2$, $S D=4.9$, range 95-108; mean $\mathrm{BMI}=14.7, S D=2.2$, range 11.8-17.5) received an experimental treatment with tDCS additionally to the classical treatment called "as usual" (AU). Twelve participants ( 12 females; mean age $=15.1, S D=1.5$ years, range 13.1-17.8; mean $\mathrm{IQ}=100.5, S D=4.6$, range 92-106; mean $\mathrm{BMI}=15.5, S D=1.6$, range $12-17.9)$ received a Family Based Therapy (FBT) additionally to the AU. All participants received a diagnosis of AN based on the DSM-5 criteria (APA American Psychiatric Association, 2013). The principal eligibility criterions required were an age between 10 and 17 years, a BMI between 12 and $18 \mathrm{~kg} / \mathrm{m}^{2}$ and IQ $\geq 85$. None had a personal history of neurological disease or a family history of epilepsy and none had comorbidity with other clinically relevant disorders. Both groups did not differ for chronological age $\left[t_{(21)}=-1.71, p=0.10\right]$, IQ (Raven, 1994) $\left[t_{(21)}=0.85, p=0.41\right]$ and BMI $\left[t_{(21)}=-1.01\right.$, $p=0.32]$.

All participants received Atypical antipsychotics (AA) as pharmacological treatment, in particular Aripiprazole. Some of them also received SSRIs ( 5 in the tDCS group and 9 in the FBT group) or Benzodiazepines (Benz: 2 in the tDCS group and 1 in the FBT group). The two groups did not differ in the frequency of each pharmacological treatment $\left[\chi_{(2)}^{2}=2.10, p=0.35\right]$.

Written informed consent was obtained from all participants and their parents after the procedures had been fully explained.

\section{Materials \\ Outcome Measures}

All participants were evaluated through clinical tests assessing psychopathological conditions. The AN symptomatology assessment included: Eating Disorder Inventory (Giannini et al., 2008; EDI-3, Garner, 2004), Eating Attitudes Test (EAT-26, Garner et al., 1982), and Body Uneasiness Test (BUT, Cuzzolaro et al., 2006). The EDI-3 is a self-report questionnaire, including 91 items divided into 12 subscales rated on a $0-4$ point scoring system. It gives a measure of the basic characteristics of the eating disorder. The EAT-26 is a forced choice, self-report 
questionnaire, including 40 items, measuring anorexia nervosa symptoms. The BUT is a 71-item self-report questionnaire which measures body image concerns.

Moreover, anxiety and depressive symptoms were evaluated through the Multidimensional Anxiety Scale for Children (MASC, March et al., 1997) and the Children's Depression Inventory (CDI, Kovacs, 1982, 1992). The MASC is a quantitative self-report scale on anxiety symptoms, age range: $8-19$. The CDI is a self-report questionnaire with 27 items, which evaluates the mood symptoms in the last 2 weeks, age range: $8-17$. All clinical questionnaires were completed by the participants themselves. In addition, anthropometric measures, as BMI, were measured.

\section{Safety and Tolerability}

Side-effects of tDCS were assessed by a standard questionnaire (Brunoni et al., 2011) which was completed by participants after each stimulation session. The questionnaire lists adverse effects, such as headache, neck pain, scalp pain, tingling, itching, burning sensation, skin redness, sleepiness, trouble concentrating, and acute mood change. Participants quantify the intensity of the symptoms or side-effects related to tDCS (1, absent; 2 , mild; 3 , moderate; 4 , severe).

\section{Procedures}

All participants underwent the treatment $\mathrm{AU}$, including nutritional, pharmacological, and psychoeducational treatment. The experimental group received an add-on treatment with 18 sessions of tDCS (TDCS+AU) for 6 weeks, while the control group received the $\mathrm{FBT}(\mathrm{FBT}+\mathrm{AU})$.

All participants have not received the AU, the FBT, or the tDCS treatment previously. Outcome measures were assessed before (T0) and immediately after the end of treatments (T1), i.e., 6 weeks later. Nine of the eleven participants of the tDCS+AU group were followed-up 1 month after the end of treatment (T2).

\section{Treatments \\ $t D C S$}

In the tDCS condition all the participants received $1 \mathrm{~mA}$ tDCS. Direct current was applied for $20 \mathrm{~min}, 3$ times a week for 6 weeks (18 sessions). Anodal electrode was positioned over the left DLPFC and cathodal electrode over the right DLPFC according to the 10-20 EEG system and the sites corresponding respectively at F3 and F4 area. The selected montage (anodal/left_cathodal/right) was applied to re-balance the right frontal hyperactivity reported in literature in individuals with AN (Grunwald et al., 2004; Brooks et al., 2011; Bär et al., 2013; Phillipou et al., 2015). Direct current was generated by a BrainStim stimulator by E.M.S. s.r.l. (Bologna, Italy). It was delivered on the scalp via a pair of identical, rectangular, electrodes $(5 \times 5 \mathrm{~cm})$ covered with conductive rubber and saline soaked synthetic sponges. During the tDCS sessions, all participant have been sat in a comfortable chair.

\section{$A U$}

Each participant undergone the treatment AU treatment during the study. In this study, after a first assessment whit a psychiatric interview, an interview for family diagnosis and nutritional monitoring, participants and parents received a psychological support. Furthermore, meetings for the nutritional and psychiatric monitoring for patients were given (once every 2 weeks). Psychological support for patients were given by individual sessions (once every 2 weeks, 60 min duration) and group sessions (once every week, 60 min duration). Their parents received the psychoeducation in group sessions (once every 2 weeks, 60 min duration).

\section{FBT}

In this group, participants and parents received family psychotherapy provided by professionals (psychotherapists and psychiatrists), additionally to the AU treatment. Weekly sessions of participants' group therapy and parents' group therapy were delivered while, family meetings occurred every 15 days.

\section{Data Analysis}

To evaluate the effect of treatment, repeated measures ANOVAs were performed on each psychopathological measure (EDI-3; EAT-26; BUT; CDI; MASC) and on BMI values, with Group $(\mathrm{tDCS}+\mathrm{AU}$ vs. FBT $+\mathrm{AU})$ as between subjects factor and Time (T0, T1) as within subjects factor.

The demographic variables and the baseline measures were compared through the Student's $t$-Test for independent samples. Chi-squared test was used to value the non-parametric variables. Post hoc analyses were performed by the Tukey's test. In the tDCS group the follow-up data were analyzed through the Wilcoxon signed-rank test. Partial eta squares $\left(\eta_{p}^{2}\right)$ have been reported as effect size measures. A $p$-value less to 0.05 was considered as statistically significant. Sphericity was verified by Mauchly's sphericity test. The Pearson correlation was used to test the association between the outcome of improvement [(T1$\left.\mathrm{T} 0 / \mathrm{T} 0)^{*} 100\right]$ on BMI and the other psychopathological measures.

\section{Ethic Approval}

This study was performed in accordance with the World Medical Association's Declaration of Helsinki and The Research Ethical Committee of the Bambino Gesù Children's Hospital approved this study under process number 763-OPBG-2014.

\section{RESULTS}

\section{Baselines Measures}

$\mathrm{tDCS}+\mathrm{AU}$ and $\mathrm{FBT}+\mathrm{AU}$ groups did not differ for BMI as well as for baseline psychopathological measures: EDI-3 $\left[t_{(21)}=-1.14\right.$, $p=0.27]$, EAT-26 [ $\left.t_{(21)}=-0.70, p=0.49\right]$, BUT $\left[t_{(21)}=-1.33\right.$, $p=0.20]$, MASC $\left[t_{(21)}=1.11, p=0.28\right]$, CDI $\left[t_{(21)}=0.46, p=\right.$ 0.65]. Baseline psychopathological measures did not significantly differ between groups even on subscales of EDI-3 and MASC. As concerns EDI-3 subscales: Drive for Thinness $\left[t_{(21)}=-1.23\right.$, $p=0.23]$, Bulimia $\left[t_{(21)}=-0.66, p=0.52\right]$, Body Dissatisfaction $\left[t_{(21)}=-0.76, p=0.46\right]$, Eating Disorder Risk $\left[t_{(21)}=-1.0\right.$, $p=0.33$ ], Low Self-Esteem $\left[t_{(21)}=-0.61, p=0.55\right]$, Personal Alienation $\left[t_{(21)}=-0.51, p=0.62\right]$, Interpersonal Insecurity $\left[t_{(21)}=-0.70, p=0.50\right]$, Interpersonal Alienation $\left[t_{(21)}=-1.11\right.$, $p=0.28]$, Interoceptive Deficits, $\left[t_{(21)}=-0.98, p=0.34\right]$, Emotional Dysregulation $\left[t_{(21)}=-0.77, p=0.45\right]$, Perfectionism $\left[t_{(21)}=-1.94, p=0.07\right]$, Asceticism $\left[t_{(21)}=-0.17, p=\right.$ 
0.86], Maturity Fears [ $\left.t_{(21)}=-0.44, p=0.67\right]$, Ineffectiveness $\left[t_{(21)}=-0.73, p=0.47\right]$, Interpersonal Problems $\left[t_{(21)}=-1.05\right.$, $p=0.31]$, Affective Problems $\left[t_{(21)}=-1.24, p=0.23\right.$, Overcontrol $\left[t_{(21)}=-1.08, p=0.29\right]$, Global Psychological Maladjustment $\left[t_{(21)}=-1.55, p=0.14\right]$. As concerns MASC subscales: Physical Symptoms $\left[t_{(21)}=0.67, p=0.51\right]$, Harm Avoidance $\left[t_{(21)}=0.74, p=0.47\right]$, Social Anxiety $\left[t_{(21)}=0.58\right.$, $p=0.57]$, Separation/Panic $\left[t_{(21)}=0.03, p=0.97\right]$, Adi $\left[t_{(21)}=0.80, p=0.43\right]$.

Means and Standard Deviations per each measure are shown on Table 1.

\section{Post-treatment Measures BMI}

Results showed a significant interaction between Group and Time $\left[F_{(1,21)}=9.75 ; p<0.01, \eta_{p}^{2}=0.32\right]$. BMI improved significantly in the tDCS $+\mathrm{AU}(p<0.001)$, while in the FBT $+\mathrm{AU}$ no difference emerged in BMI after treatment compared to baseline $(p=$ 0.2 ). Figure 1A, shows the mean BMI values in both groups: at baseline (pre-treatment) and after 18th session (post-treatment). The mean percentage of BMI improvement [(T1-T0/T0 $\left.)^{*} 100\right]$ in the $\mathrm{tDCS}+\mathrm{AU}$ group amounted to $13.3 \%( \pm 9.4)$ while in the FBT + AU group it amounted to $4.2 \%( \pm 5.7)$. See Figure 1B.

In the tDCS group the BMI improvement persisted also 1 month later (T0 vs. T2: $Z=2.66, p<0.01$ ). See also Figure 2A.

A general Group effect did not emerged $\left[F_{(1,21)}=0.05\right.$; $\left.p=0.83, \eta_{p}^{2}=0.01\right]$ while emerged a main effect of Time $\left[F_{(1,21)}=35.78 ; p<0.001, \eta_{p}^{2}=0.42\right]$. See Table 1 .

\section{Psychopathological Assessment}

In the psychopathological scales there were no significant interaction between Group and Time (all comparisons $p>0.1$ ). No main Group effect emerged in each measure (all comparisons $p>0.1$ ). A Time effect was significant independently of the group, in most of the comparison: both groups improved in the clinical scales EDI-3, MASC, and CDI with the exception of BUT. Moreover, both groups improved in most subscales of EDI-3 and MASC. In particular, as concerns EDI3 subscales, independently of the group, a mean score reduction was observed after treatment on Drive for Thinness, Body Dissatisfaction, Eating Disorder Risk, Low Self-Esteem, Personal Alienation, Interpersonal Insecurity, Interpersonal Alienation, Asceticism, Ineffectiveness, Interpersonal Problems and Global Psychological Maladjustment (all $p<0.05$ ). However no improvement emerged on the subscales Bulimia, Interoceptive Deficits, Emotional Dysregulation, Perfectionism, Maturity Fears, Affective Problems, and Overcontrol (all $p>0.10$ ). As concerns MASC subscales, independently of the group, a mean score reduction was observed after treatment on Physical Symptoms and Adi (all $p<0.05$ ) while no improvement emerged on Harm Avoidance, Social Anxiety, and Separation/Panic (all $p>0.10)$.

Table 1 shows the total score on these scales and their subscales in both groups at baseline (pre-treatment) and after 18 session (post-treatment), as well as the statistics for the main effect of Time and for the interaction effect of Time per Group.
In the $\mathrm{tDCS}+\mathrm{AU}$ group, which was followed-up for 1 month, the effect on most of the psychological measures persisted 1 month after the end of treatment (EAT-26, T0 vs. T2: $Z=2.37$, $p=0.02$; MASC, T0 vs. T2: $Z=1.96, p=0.05$; CDI, T0 vs. T2: $Z=2.19, p=0.04$ ), but not on EDI-3 (T0 vs. T2: $Z=1.33$, $p=0.18)$. Again no improvement was observed on BUT even after 1 month (T0 vs. T2: $Z=0.56, p=0.57$ ). See Figures 2B,C.

\section{Correlation Between Outcomes}

In the $\mathrm{tDCS}+\mathrm{AU}$ group we found a medium negative correlation between the improvement on BMI and the score changes on EDI-3 $(r=-0.65, p=0.04)$, i.e., the higher the BMI increased (amelioration), the lower the EDI-3 was after treatment (amelioration). However, we failed to found such correlation in the FBT+AU group $(p=0.29)$.

By considering the EDI-3 subscales, in the tDCS+AU group we found a medium negative correlation between the improvement on BMI and the score changes on Bulimia $(r=-0.68, p=0.03)$ and on Global Psychological Maladjustment $(r=-0.67, p=0.03)$. Moreover we found a strong negative correlation between the improvement on BMI and the score changes on Interpersonal Problems $(r=-0.71$, $p=0.02$ ). However, we did not found any correlation between the BMI improvement and other EDI-3 subscales score changes (all $p>0.1$ ). Conversely, by considering the EDI-3 subscales, in the FBT+AU group we found a medium positive correlation between the improvement on BMI and the score changes on Low Self-Esteem $(r=0.60, p=0.04)$, i.e., the higher the BMI increased (amelioration), the higher the Low Self-Esteem score was after treatment (worsening).

Finally, we did not found any correlation between the improvement on BMI and the other psychopathologiocal scales or subscales, neither in the $\mathrm{tDCS}+\mathrm{AU}$ group nor in the $\mathrm{FBT}+\mathrm{AU}$ group (all $p>0.1)$.

\section{Safety and Tolerability}

Concerning safety and tolerability, no participant asked to stop the study or reported significant discomfort at the electrode sites. Participant tolerated tDCS well. The most frequent adverse effects were itching sensation, burning sensation (reported by 9 participant), especially in the first seconds of stimulation, which diminished rapidly with water's addition under the sponge and local redness (report by 8 participant). Others effects were headache (reported by 5 participants), tingling (reported by 5 participants) and, mostly in the mild intensity (see Table 2).

Neck pain, sleepiness, and trouble concentrating were not reported. No psychological symptoms, such as acute mood changes and irritability, nor other discomforts or adverse effects were reported.

\section{DISCUSSION}

The present study evaluated the efficacy of a tDCS treatment in a young population with AN.

Two groups were compared: a group received tDCS treatment in addition to "as usual" treatment; another group received an 


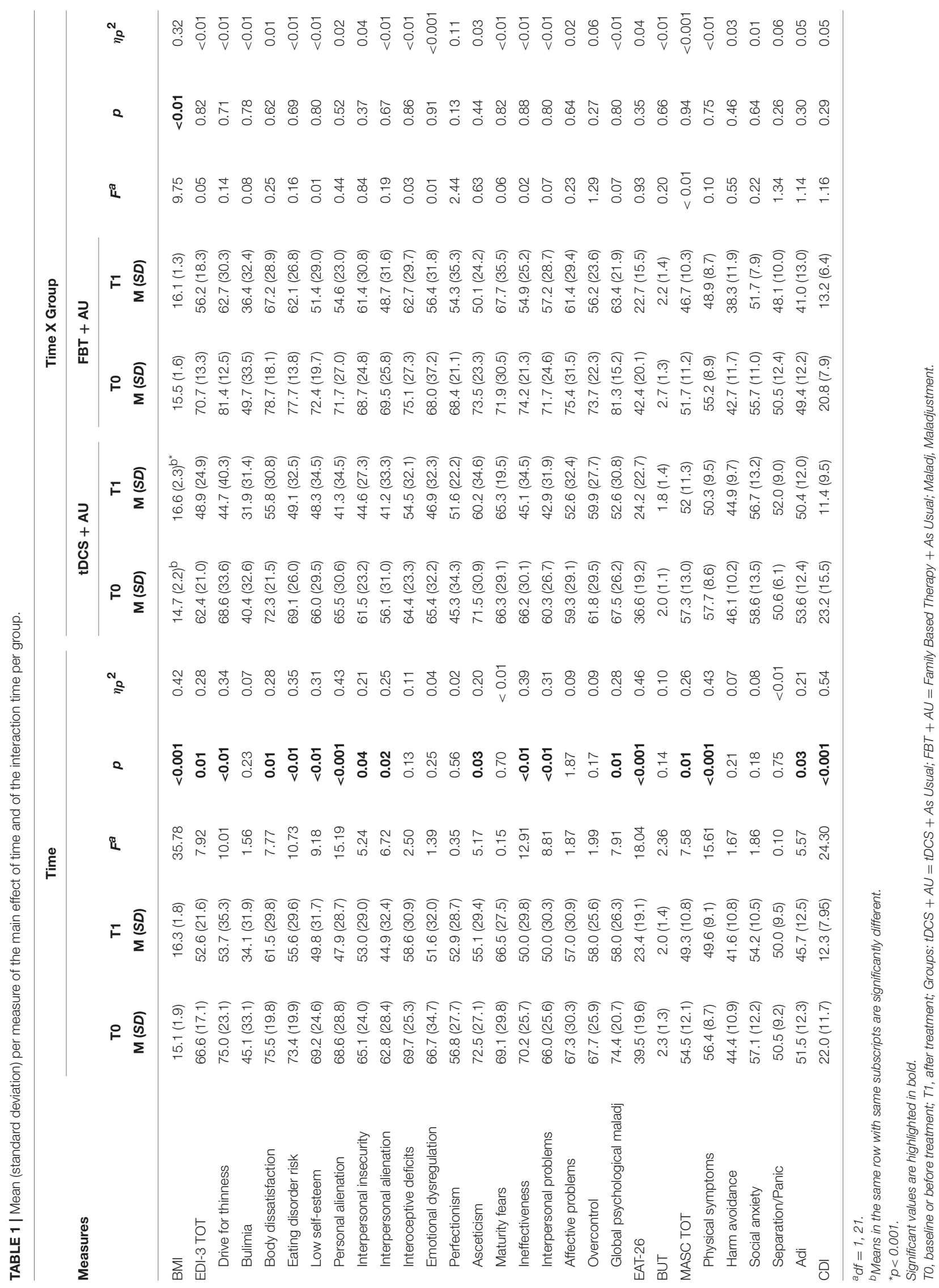




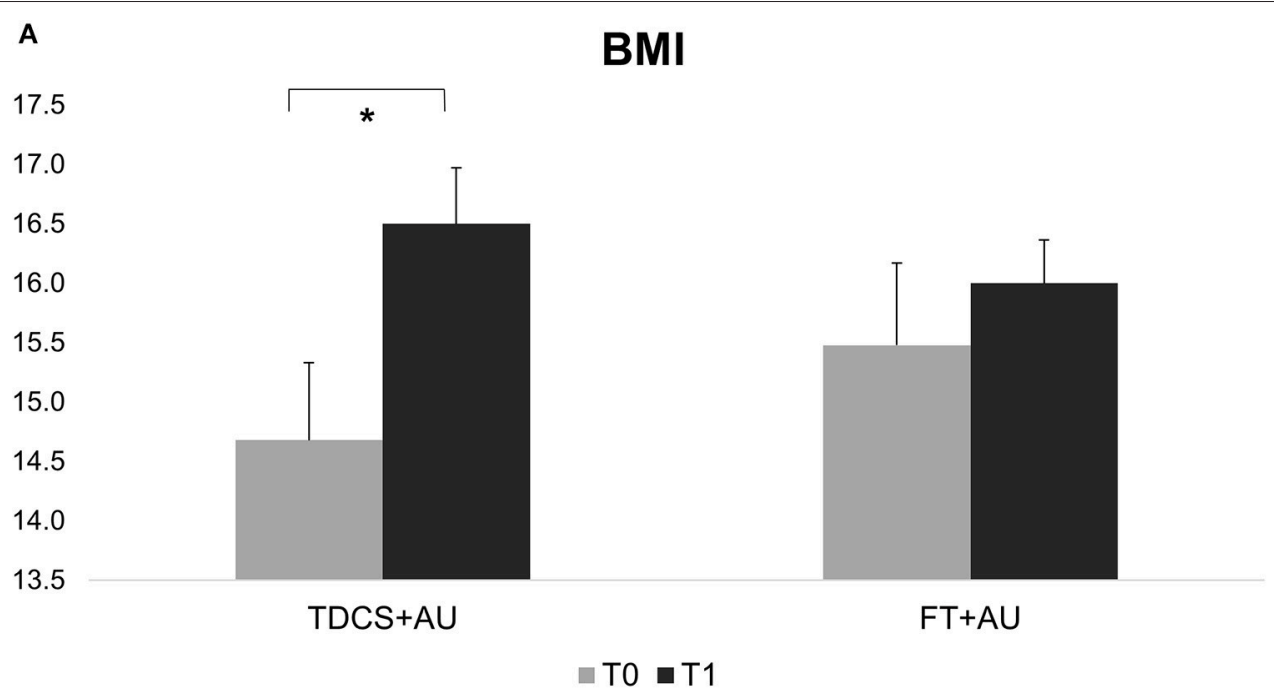

B

BMI improvement \%

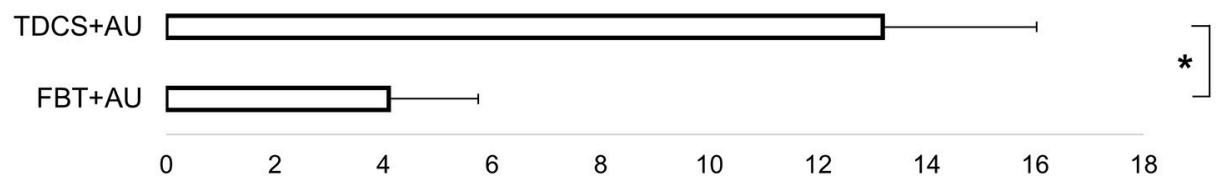

FIGURE 1 | (A) Shows BMI values expressed in mean at baseline (TO) and after the end of treatment (T1). In the tDCS+AU group BMI values increased significantly more than in the FBT+AU group. (B) Shows the percentage of increase [(T1-T0/T0)*100]: in the tDCS+AU group it amounted to 13.3\% ( \pm 9.4$)$ while in the FBT+AU group it amounted to $4.2 \%$ ( \pm 5.7$) .{ }^{*} p<0.05$.

active control treatment, i.e., a family based psychotherapy, in addition to "as usual" treatment.

In the experimental group, tDCS was applied to the DLPFC and, more specifically, anodal electrode was positioned over the left and cathodal electrode over the right area. According to literature, this montage, with concurrent left excitatory and right inhibitory stimulation, was applied to re-balance the right frontal hyperactivity reported in AN (Grunwald et al., 2004; Brooks et al., 2011; Bär et al., 2013; Phillipou et al., 2015). Furthermore, all participants received a nutritional and psychiatric monitoring as well as psychological support.

The main finding was that after 6 weeks of treatment, the tDCS+AU group, but not the control group, showed significant increase of BMI values. Indeed, the mean percentage of BMI increment in the group receiving tDCS amounted to $13.3 \%$ compared to $4.2 \%$ in the $\mathrm{FBT}+\mathrm{AU}$ group. Moreover, independently of the treatment, all participants improved in several psychopathological measures, included AN psychopathology and mood and anxiety symptoms, with the exception of the measure assessing body uneasiness (BUT). The psychopathological improvement profile seems to overlap in the two groups, indeed there are no differences in any subscales of each psychopathological measure. In the tDCS+AU group, the BMI increment as well as the improvement in some psychopathological measures persisted even at 1-month follow-up.

Our results confirmed previous findings on AN reporting positive effects in mood and AN symptoms after non-invasive brain stimulation, as described on case reports with tDCS (Khedr et al., 2014), and on group studies with TMS (Kamolz et al., 2008; McClelland et al., 2013, 2016a,b; Van den Eynde et al., 2013). However, present study firstly demonstrated a specific effect of a tDCS treatment on stable weight gain and a superiority compared to an active control treatment for adolescents with AN.

It is important to note that the two groups received similar nutritional, psychoeducational and pharmacological concurrent treatment (the AU treatment), which might have a main role in eating behavior and weight gain, while they differed for the specific add-on treatment (i.e., the experimental tDCS or the treatment of choice FBT). This means that the results obtained can be explained by the add-on treatment or by the interaction between each add-on treatment and the AU treatment.

Literature on clinical trials for $\mathrm{AN}$, strengthens the importance of obtaining BMI improvement, together with the psychopathological improvement, as the optimal outcome for AN remediation (Halmi, 1982; Guarda, 2008). Although 

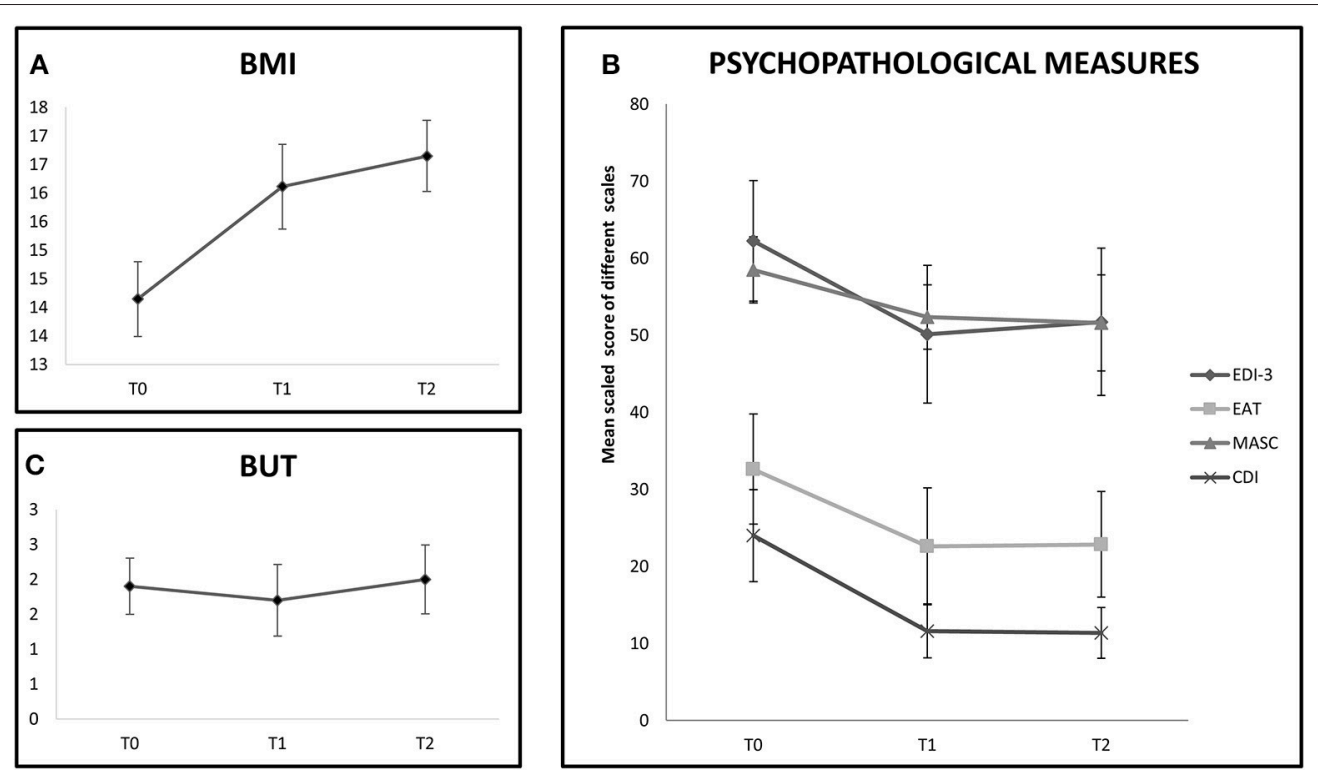

FIGURE 2 | The chart shows follow-up evalution of outcome measures in the tDCS+AU group (only 9 participants). (A) Shows BMI values expressed in mean at baseline (TO), at the end of treatment (T1) and at 1 month follow-up (T2). BMI values increased even at 1-month follow-up. (B) Shows mean scaled score of EDI-III, EAT-26, MASC, and CDI at baseline (TO), at the end of treatment (T1) and at 1 month follow-up (T2). Improvement was stable until 1-month follow-up in the EAT-26, MASC, and CDI scales, but not on EDI-III. (C) Shows mean score of BUT at baseline (TO), at the end of treatment (T1) and at 1 month follow-up (T2). No improvement was shown at any evaluation time.

TABLE 2 | Percentage of adverse effects reported by participants.

\begin{tabular}{lccc}
\hline Adverse effect & Mild (\%) & Moderate (\%) & Severe (\%) \\
\hline Headache & 18.1 & 27.2 & 0.0 \\
Neck pain & 0.0 & 0.0 & 0.0 \\
Scalp pain & 0.0 & 0.0 & 0.0 \\
Tingling & 45.4 & 0.0 & 0.0 \\
Itching & 54.5 & 27.2 & 0.0 \\
Burning sensation & 27.7 & 27.7 & 9.0 \\
Local redness & 36.3 & 45.4 & 0.0 \\
Sleepiness & 0.0 & 0.0 & 0.0 \\
Trouble concentrating & 0.0 & 0.0 & 0.0 \\
Acute mood changes & 0.0 & 0.0 & 0.0 \\
Irritability & 0.0 & 0.0 & 0.0 \\
\hline
\end{tabular}

weight gain has been consistently found using invasive neuromodulation approaches to AN, such as DBS, evidence from non-invasive brain stimulation indicated an impact on self-reported symptoms but not always translated to weight gain (Lee et al., 2018). Our tDCS study, instead, gives first evidence of the positive effect of a 6 weeks tDCS treatment in improving both the psychopathological symptoms and the MBI in adolescents with $\mathrm{AN}$, compared to a control active treatment, widely considered treatment of choice.

Moreover, our study shows a beneficial association between the improvement in BMI and the amelioration of psychopathological symptomats with the tDCS treatment but not with the control treatment. Specifically, it has been found that, within the experimental group, the increase in BMI was associated to a reduction of EDI-3 total scores (risk of eating disorder) and of Bulimia, Interpersonal Problems and Global Psychological Maladjustment subscales. On the other hand, in the control group, a relation was found between the increase in BMI and the worsening to the Low Self-Esteem subscale.

These results suggests that tDCS may have an action in the improvement of cognitive symptoms linked to incorrect food behavior, as already highlighted by other researchers (McClelland et al., 2013; Lee et al., 2018). In particular, the reduction of the Bulimia subscale score, with the increase of BMI, is particularly relevant because it indicates an improvement in cognitive control, i.e., the reduction of compensatory behaviors in order to prevent weight gain, which is often observed in the weight recovery phases in patients with AN (Bulik et al., 1997; Fairburn and Harrison, 2003). Moreover, the positive effect of tDCS on BMI seems to be accompanied by a more general positive effect on other behaviors, such as interpersonal relationships and psychological adaptation, resulting in an improvement of the risk of eating disorder (reduction of EDI-3 total score).

On the other hand, it is often reported in people with AN that an improvement on weight, but not at the same time on cognitive and behaviors symptoms, is negatively experienced and affects their self-esteem, characteristic trait of the AN disease (StriegelMoore et al., 2004). This seems the case of the control group, who showed a worsening of self-esteem score with BMI improvement.

All together these results seem to suggest a superior combination of psychological changes associated with weight gain in the experimental group, than in the control group. The combination of weight gain and psychological change is of crucial 
importance for eating disorders recovery (Bardone-Cone et al., 2010).

The reason why in our study there was a positive and specific effect on BMI, unlike what has been reported so far by non-invasive brain stimulation studies, may depend on the type of stimulation performed. Most of the non-invasive brain stimulation studies used TMS to facilitate left PFC. In comparison with TMS, the mechanism by which tDCS works enables multiple stimulation designs. Switching the position of the electrodes enables swapping of excitation/inhibition between the right and left hemispheres. This may act to balancing the activity in both hemispheres, thus being likely more efficient in resetting the inter-hemispheric balance alterated in AN.

The lone tDCS study (Khedr et al., 2014) was an open label, single-arm study, showing improvement in psychopathology scales, with important variation between seven patients, after 10 sessions of left Anodal tDCS on DLPFC. In particular, five patients improved in all scales, but just three maintained this scores 1 month later. This work included the total scores of the EAT, EDI, and the Beck Depression Inventory. However, the study did not report BMI measures, showing only positive correlations in the percent of improvement between eating disorder scales and depression scale.

Compared to our study, difference in some elements might be relevant. In the Khedr et al.'s study, tDCS was applied for $25 \mathrm{~min}(2 \mathrm{~mA})$ with an extracephalic cathodal electrode, in a monopolar montage to activate the left DLPC. The study ground on previous TMS literature, using a facilitatory stimulation to the left DLPC to improve AN. Conversely, we used a bilateral montage of electrodes on DLPFC, anodal/left and cathodal/right, with the aim to induce a simultaneously increasing in the left DLPFC activation and reducing in the right DLPFC activation (Nasseri et al., 2015).

We hypothesize that, the relevant BMI increase in the tDCS group may be due to the concurrent delivering of the cathodal stimulation to the right DLPFC, rather than just to the anodal stimulation to the left PFC, for a possible direct/indirect action into some pathophysiological mechanisms of $\mathrm{AN}$, involving the mesocortical dopaminergic pathways and the promotion of food intake.

Cortical tDCS stimulation has been shown to modulate dopamine (DA) release in striatum (Tanaka et al., 2013). In particular, following the application of cathodal, but not anodal tDCS for 10 min, extracellular DA levels, measured with in vivo microdialysis, increased for more than $400 \mathrm{~min}$ in the in rat striatum. This result suggested that $\mathrm{tDCS}$ has a direct and/or indirect effect on the dopaminergic system in the subcortical area. However, there is concern if a low intensity current can go that deep in human brain. Very recently, the first evidence in humans has been published (Fonteneau et al., 2018) that bifrontal tDCS induces neurotransmitter release in subcortical areas. Specifically, left anodal/right cathodal tDCS (our study montage) induced a significant increase in extracellular dopamine in a part of the striatum involved in the reward-motivation network.

Mesolimbic dopaminergic projections into striatum are hypothesized to play a key role in governing eating behavior, by modulating appetitive motivational processes. A perturbations in the dopaminergic reward pathways has been hypothesized to play a role in the AN pathogenesis (Casper, 2006; Alcaro et al., 2007). Those alteration included reduced Cerebro Spinal Fluid levels of DA metabolites (Kontis and Theochari, 2012), functional DA D2 receptor gene polymorphisms (Bergen et al., 2005), and increased D2/D3 receptor binding in the striatum (Kaye et al., 2013; Riva, 2016), indicating an increased D2/D3 densities and a decreased extracellular DA in this region. Moreover, the evidence of a DA imbalance in the ventral striatum in patients with $\mathrm{AN}$, is considered to contribute to anhedonia of feeding behavior, ascetic and anhedonic temperament (Frank et al., 2017), as well as dysphoric mood and anxiety (Kaye et al., 2009). Although these findings on the role of DA in AN is still a matter of debate (Gillman and Lichtigfeld, 1986; Kontis and Theochari, 2012).

A possible interpretation of such contrasting results is in the dissociation in the pattern of tonic and phasic firing of dopaminergic signaling (Kontis and Theochari, 2012). Preclinical data show that average extracellular DA in the nucleus accumbens is low in AN and largely arises from phasic DA transients (Owesson-White et al., 2012). Response to behaviorally relevant stimuli triggers the phasic component of DA release onto postsynaptic targets, while the tonic DA levels are proposed to regulate the amplitude of the phasic DA response.

It is believed that glutamate mainly from prefrontal projections (which is usually increased by tDCS) promotes tonic release of DA in the dorsal striatum and in the nucleus accumbens (Grace, 1991; Södersten et al., 2016). Grace (1991) proposed a model for which a pathological alteration in the PFC may cause a reduction in the tonic levels of DA, resulting in excess response of the phasic dopaminergic system and to an increase in the responsiveness of the dopaminergic system.

An increase in the responsiveness of the dopaminergic system has been indeed reported in AN (for a review see Södersten et al., 2016). One might argue that if the dopaminergic system is hypersensitive in $\mathrm{AN}$, it should promote food seeking instead of avoidance. Research that investigated those phenomena has suggested that dopaminregic hypersensitivity in $\mathrm{AN}$ is not meant to imply that the so called "reward stimuli" (such as sugar solution) are necessarily a reward in the sense of positive reinforcer or pleasant experience for individuals with anorexia nervosa (Frank et al., 2017). Rather brain dopaminergic circuits in AN could be hypersensitive to salient stimuli in general, including both rewarding or punishing stimuli (Frank et al., 2017). Such failure to appropriately bind, modulate or discriminate responses to stimuli has been taken into account to explain the role of starvation as a reward stimulus and the negative emotional activity in front of food stimuli (Berridge and Robinson, 1998; Frank et al., 2017).

It is therefore possible to hypothesize, albeit only speculatively, that our left anodal/right cathodal tDCS treatment, aimed at rebalancing the hyperactivity of right DLPC, may have aid in restoring the cortical glutamatergic system regulating DA tonic relies in striatum, thus in turn acting in rebalancing the dopaminergic alterations seen in the reward brain network in AN, crucial for regulate the food intake behavior.

A possible confound in our study is the interaction of tDCS with the concurrent pharmacological treatment (Nitsche and 
Paulus, 2000; Nitsche et al., 2003, 2006, 2012; Monte-Silva et al., 2009; Ridding and Ziemann, 2010; Fresnoza et al., 2014). In particular, there is evidence that SSRIs may themselves increase the response to tDCS (Normann et al., 2007).

Conversely, medications that interfere with dopaminergic signaling, such as antipsychotics, are likely to have a negative impact on tDCS plasticity (Monte-Silva et al., 2009). However, such impact is related to the pharmacological profile of the antipsychotic medication. In particular, the availability of D2 receptors is vital for potentially adaptive neuroplastic effects of tDCS (Nitsche et al., 2009). Aripiprazole, the medication received by all participants, differently from other antipsychotic agents, it is not a pure antagonist at the D2 receptor but a partial agonist (Ziadi Trives et al., 2013). Hence, the drug molecule allows the physiological activity of these receptors, which in turn allows for the beneficial neuromodulatory effects of tDCS. Indeed, tDCS in conjunction with Aripiprazole (but not with other antipsychotics) have proven to improve schizophrenic symptoms (Agarwal et al., 2015).

These considerations lead us to suppose that, the interaction of tDCS with the concurrent pharmacological treatment may have increased, rather than reduced, the plasticity induced effect of our stimulation protocol. Moreover, Aripiprazole may have specifically improved and amplifying the hypothesized tDCS action in regulating the DA tonic component in striatum, given that the drug acts suppressing the phasic component, while relatively preserving the release of the tonic component of DA (Hamamura and Harada, 2007). A positive effect of Aripiprazole on the dopaminergic reward circuit alterations has been recently reported in patients with AN, resulting in an increasing in BMI values (Frank et al., 2017).

So that, it is plausible that the positive effects found on both BMI and psychopathological symptoms, may arise by a synergistic action between a cortical stimulation and a medical stimulation in regulating the imbalance between the tonic and phasic component of DA in AN.

In summary, it is possible to speculate that tDCS treatment may have a direct/indirect action to one of the etiopathogenetic mechanism of AN and may represent a more targeted perspective treatment for AN, especially in adolescence age. Indeed, tDCS has the potential to timely target brain abnormalities through brain plasticity mechanisms, essential in development. Actually,

\section{REFERENCES}

Agarwal, S. M., Bose, A., Shivakumar, V., Narayanaswamy, J. C., Chhabra, H., Kalmady, S. V., et al. (2015). Impact of antipsychotic medication on transcranial direct current stimulation (tDCS) effects in schizophrenia patients. Psychiatry Res. 235, 97-103. doi: 10.1016/j.psychres.2015.11.042

Agras, W. S., Lock, J., Brandt, H., Bryson, S. W., Dodge, E., Halmi, K. A., et al. (2014). Comparison of 2 family therapies for adolescent anorexia nervosa: a randomized parallel trial. JAMA Psychiatry 71, 1279-1286. doi: 10.1001/jamapsychiatry.2014.1025

Aigner, M., Treasure, J., Kaye, W., and Kasper, S. (2011). World Federation of Societies of Biological Psychiatry (WFSBP) guidelines for the pharmacological treatment of eating disorders. World J. Biol. Psychiatry 12, 400-443. doi: $10.3109 / 15622975.2011 .602720$ although "malleable" during the early stages, once established, AN are remarkably persistent (Walsh, 2013), therefore it is essential to timely treat the disorder.

Our results are promising, since participants in the tDCS group have increased by almost tri-times their BMI values compared to the participants receiving the AU treatment (both pharmacological, nutritional and psychoeducational) and the psychological treatment of choice. Moreover, our participants did not experience any notable symptoms or side effects (for Brunoni's standard questionnaire, Brunoni et al., 2011) after any stimulation session, thus confirming a high tolerability and feasibility of a tDCS treatment in children and adolescents with AN.

However, an important limitation to these data is that this study was an open label study with an active control group but not a sham control group, therefore any effect cannot be distinguished from a possible tDCS placebo effect. However, given the difficult in enrolling adolescents with AN, which are usually reluctant to receive any kind of treatment, this study serve as a pilot, with preliminary results, to lay the groundwork for a clinical trial.

Undoubtedly, new studies are required with double blind randomized clinical trials, larger sample, and longer follow-ups to confirm our results. Nevertheless, our promising results deserve to be followed by future studies with more advanced analysis, with insight from functional neuroimaging and animal studies, to deeper understand the role of left anodal/right cathodal tDCS to the DLPFC in potentially contrasting some etiopathogenetic mechanisms of AN. If the tDCS treatment will be confirmed to be effective, it may lead to important changes in the treatment of AN which could be translated into novel and effective rehabilitation strategies, especially in developmental age.

\section{AUTHOR CONTRIBUTIONS}

All authors provided substantial contributions to the work. FC, DM, and SV conceived and designed the study. FC and AnM conducted experimental treatment.VZ, MC, and AlM performed the measurements, contributed to sample preparation and to follow up. FC, DM, and AnM drafted the manuscript. SV, AlM, $\mathrm{DM}$, and VZ revised the manuscript. After revisions and editing by all authors, the article was submitted.
Alcaro, A., Huber, R., and Panksepp, J. (2007). Behavioral functions of the mesolimbic dopaminergic system: an affective neuroethological perspective. Brain Res. Rev. 56, 283-321. doi: 10.1016/j.brainresrev.2007. 07.014

Antal, A., Alekseichuk, I., Bikson, M., Brockmöller, J., Brunoni, A. R., Chen, R., et al. (2017). Low intensity transcranial electric stimulation: safety, ethical, legal regulatory and application guidelines. Clin. Neurophysiol. 19:30212. doi: 10.1016/j.clinph.2017.06.001

American Psychiatric Association (2006). Practice guideline for the treatment of patients with eating disorders (revision). Am. J. Psychiatry 163, 1-54.

APA American Psychiatric Association (2013). Diagnostic and Statistical Manual of Mental Disorders, 5th edn. Arlington, VA: American Psychiatric Publishing.

Arcelus, J., Mitchell, A. J., Wales, J., and Nielsen, S. (2011). Mortality rates in patients with anorexia nervosa and other eating disorders. 
A meta-analysis of 36 studies. Arch. Gen. Psychiatry 68, 724-731. doi: 10.1001/archgenpsychiatry.2011.74

Avena, N. M., and Bocarsly, M. E. (2012). Dysregulation of brain reward systems in eating disorders: neurochemical information from animal models of binge eating, bulimia nervosa, and anorexia nervosa. Neuropharmacology 63, 87-96. doi: 10.1016/j.neuropharm.2011.11.010

Bailer, U. F., Frank, G. K., Henry, S. E., Price, J. C., Meltzer, C. C., Becker, C., et al. (2007). Serotonin transporter binding after recovery from eating disorders. Psychopharmacology 195, 315-324. doi: 10.1007/s00213-007-0896-7

Bär, K. J., Berger, S., Schwier, C., Wutzler,U., and Beissner, F. (2013). Insular dysfunction and descending pain inhibition in anorexia nervosa. Acta Psychiatr. Scand. 127, 269-278. doi: 10.1111/j.1600-0447.2012.01896.x

Bardone-Cone, A. M., Harney, M. B., Maldonado, C. R., Lawson, M. A., Robinson, D. P., Smith, R., et al. (2010). Defining recovery from an eating disorder: conceptualization, validation, and examination of psychosocial functioning and psychiatric comorbidity. Behav. Res. Ther. 48, 194-202. doi: 10.1016/j.brat.2009.11.001

Bergen, A. W., Yeager, M., Welch, R. A., Haque, K., Ganjei, J. K., van den Bree, M. B., et al. (2005). Association of multiple DRD2 polymorphisms with anorexia nervosa. Neuropsychopharmacology 30, 1703-1710. doi: $10.1038 /$ s..npp. 1300719

Berridge, K., and Robinson, T. (1998). What is the role of dopamine in reward: hedonic impact, reward learning, or incentive salience? Brain Res. 28, 309-369. doi: 10.1016/S0165-0173(98)00019-8

Bodell, L. P., and Keel, P. K. (2010). Current treatment for anorexia nervosa: efficacy, safety, and adherence. Psychol. Res. Behav. Manag. 3, 91-108. doi: 10.2147/PRBM.S13814

Boggiano, M. M., Wenger, L. E., Burgess, E. E., Tatum, M. M., Sylvester, M. D., Morgan, P. R., et al. (2017). Eating tasty foods to cope, enhance reward, socialize or conform: what other psychological characteristics describe each of these motives. J. Health Psychol. 22, 280-289. doi: 10.1177/1359105315600240

Bohon, C., and Stice, E. (2011). Reward abnormalities among women with full and subthreshold bulimia nervosa: a functional magnetic resonance imaging study. Int. J. Eat. Disord. 44, 585-595. doi: 10.1002/eat.20869

Brooks, S. J., O'Daly, OG., Uher, R., Friederich, H. C., Giampietro, V., Brammer, M., et al. (2011). Differential neural responses to food images in women with bulimia versus anorexia nervosa. PLOS ONE 6:22259. doi: 10.1371/journal.pone.0022259

Brunoni, A. R., Amadera, J., Berbel, B., Volz, M. S., Rizzerio, B. G., and Fregni, F. (2011). A systematic review on reporting and assessment of adverse effects associated with transcranial direct current stimulation. Int. J. Neuropsychopharmacol. 14, 1133-1145. doi: 10.1017/S1461145710001690

Bulik, CM., Sullivan, P. F., Fear, J., and Pickering, A. (1997). Predictors of the development of bulimia nervosa in women with anorexia nervosa. J. Nerv. Ment. Dis. 185, 704-707. doi: 10.1097/00005053-199711000-00009

Casper, R. C. (2006). The "drive for activity" and "restlessness" in anorexia nervosa: potential pathways. J. Affect. Disord. 92, 99-107. doi: 10.1016/j.jad.2005.12.039

Celone, K. A., Thompson-Brenner, H., Ross, R. S., Pratt, E. M., and Stern, C. E. (2011). An fMRI investigation of the fronto-striatal learning system in women who Exhibit eating disorder behaviors. Neuroimage 56, 1749-1757. doi: 10.1016/j.neuroimage.2011.03.02

Cuzzolaro, M., Vetrone, G., Marano, G., and Garfinkel, P. E. (2006). The Body Uneasiness Test (BUT): development and validation of a new body image assessment scale. Eat. Weight Disord. 11, 1-13. doi: 10.1007/BF03327738

Diana, M. (2011). The dopamine hypothesis of drug addiction and its potential therapeutic value. Front. Psychiatry 2:64. doi: 10.3389/fpsyt.2011.00064

Doherty, J. M., Schier, C. J., Vena, A. A., Geoffrey, A. D., and Rueben, A. G. (2016). Medial prefrontal cortical dopamine responses during operant selfadministration of sweetened ethanol. Alcohol. Clin. Exp. Res. 40, 1662-1670. doi: 10.1111 acer.13141

Dold, M., Aigner, M., Klabunde, M., Treasure, J., and Kasper, S. (2015). Second-generation antipsychotic drugs in anorexia nervosa: a meta-analysis of randomized controlled trials. Psychother. Psychosom. 21, 110-116. doi: $10.1159 / 000369978$

Ehrlich, S., Geisler, D., Ritschel, F., King, J. A., Seidel, M., Boehm, I., et al. (2015). Elevated cognitive control over reward processing in recovered female patients with anorexia nervosa. J. Psychiatry Neurosci. 40, 307-315. doi: $10.1503 /$ jpn. 140249
Fairburn, C. G., and Harrison, P. J. (2003). Eating disorders. Lancet 361, 407-416. doi: 10.1016/S0140-6736(03)12378-1

Fisher, W. W., Piazza, C. C., Bowman, L. G., Kurtz, P. F., Sherer, M. R., and Lachman, S. R. (1994). A preliminary evaluation of empirically derived consequences for the treatment of pica. J. Appl. Behav. Anal. 27, 447-457. doi: 10.1901/jaba.1994.27-447

Fonteneau, C., Redoute, J., Haesebaert, F., Le Bars, D., Costes, N., Suaud-Chagny, M. F., et al. (2018). Frontal transcranial direct current stimulation induces dopamine release in the ventral striatum in human. Cereb. Cortex 28, 2636-2646. doi: 10.1093/cercor/bhy093

Frank, G. K., Shott, M. E., Hagman, J. O., Schiel, M. A., DeGuzman, M. C., and Rossi, B. (2017). The partial dopamine D2 receptor agonist aripiprazole is associated with weight gain in adolescent anorexia nervosa. Int. J. Eat. Disord. 50, 447-450. doi: 10.1002/eat.22704

Fregni, F., Orsati, F., Pedrosa, W., Fecteau, S., Tome, F. A., Nitsche, M. A., et al. (2008). Transcranial direct current stimulation of the prefrontal cortex modulates the desire for specific foods. Appetite 51, 34-41. doi: 10.1016/j.appet.2007.09.016

Fresnoza, S., Stiksrud, E., Klinker, F., Liebetanz, D., Paulus, W., Kuo, M. F., et al. (2014). Dosage-dependent effect of dopamine D2 receptor activation on motor cortex plasticity in humans. J. Neurosci. 6, 10701-10709. doi: 10.1523/JNEUROSCI.0832-14.2014

Friederich, H. C., and Herzog, W. (2011). Cognitive-behavioral flexibility in anorexia nervosa. Curr. Top. Behav. Neurosci. 6, 111-123. doi: $10.1007 / 7854 \_2010 \_83$

Galusca, B., Costes, N., Zito, N. G., Peyron, R., Bossu, C., Lang, F., et al. (2008). Organic background of restrictive-type anorexia nervosa suggested by increased serotonin $1 \mathrm{~A}$ receptor binding in right frontotemporal cortex of both lean and recovered patients. Biol. Psychiatry 64, 1009-1013. doi: 10.1016/j.biopsych.2008.06.006

Garner, D. M. (2004). Eating Disorder Inventory-3. Professional Manual. Lutz, FL: Psychological Assessment Resources.

Garner, D. M., Olmsted, M. P., Bohr, Y., and Garfinkel, P. E. (1982). The eating attitudes test: psychometric features and clinical correlates. Psychol. Med. 12, 871-878. doi: 10.1017/S0033291700049163

Garner, D., Desmond, M., Desai, J., and Lockert, J. (2016). The disconnect between treatment outcome data and reimbursement for the treatment of anorexia nervosa. Int J. Physiatry 2, 1-6. doi: 10.23937/2572-4215.1510006

Giannini, M., Pannocchia, L., Dalle Grave, R., and Muratori, F. (2008). Adattamento Italiano dell'EDI-3. Eating Disorder Inventory-3. Firenze: Giunti O.S. Organizzazioni Speciali.

Gillman, M. A., and Lichtigfeld, F. J. (1986). The opioids, dopamine, cholecystokinin, and eating disorders. Clin. Neuropharmacol. 9, 91-97. doi: 10.1097/00002826-198602000-00011

Goldman, R. L., Borckardt, J. J., Frohman, H. A., O’Neil, P. M., Madan, A., Campbell, L. K., et al. (2011). Prefrontal cortex transcranial direct current stimulation (tDCS) temporarily reduces food cravings and increases the selfreported ability to resist food in adults with frequent food craving. Appetite 56, 741-746. doi: 10.1016/j.appet.2011.02.013

Grace, A. A. (1991). Phasic versus tonic dopamine release and the modulation of dopamine system responsivity: a hypothesis for the etiology of schizophrenia. Neuroscience 41, 1-24. doi: 10.1016/0306-4522(91)90196-U

Grunwald, M., Weiss, T., Assmann, B., and Ettrich, C. (2004). Stable asymmetric interhemispheric theta power in patients with anorexia nervosa during haptic perception even after weight gain: a longitudinal study. J. Clin. Exp. Neuropsychol. 26, 608-620. doi: 10.1080/138033904096 09785

Guarda, A. (2008). Treatment of anorexia nervosa: insights and obstacles. Physiol. Behav. 94, 113-120. doi: 10.1016/j.physbeh.2007.11.020

Gustavsson, A., Svensson, M., Jacobi, F., Allgulander, C., Alonso, J., Beghi, E., et al. (2011). Cost of disorders of the brain in Europe 2010. Eur. Neuropsychopharmacol. 21, 718-779. doi: 10.1016/j.euroneuro.2011. 08.008

Halmi, K. (1982). Pragmatic information on the eating disorders. Psychiatr. Clin. North Am. 5, 371-377. doi: 10.1016/S0193-953X(18)30873-6

Hamamura, T., and Harada, T. (2007). Unique pharmacological profile of aripiprazole as the phasic component buster. Psychopharmacology 191, 741-743. doi: 10.1007/s00213-006-0654-2 
Hare, T. A., Camerer, C. F., and Rangel, A. (2009). Selfcontrol in decision-making involves modulation of the vmPFC valuation system. Science 324, 646-648. doi: $10.1126 /$ science. 1168450

Herpertz-Dahlmann, B., and de Zwaan, M. (2011). Eating disorders: new findings for diagnosis and treatment. Nervenarzt 82, 1091-1092. doi: 10.1007/s00115-010-3226-y

Hestad, K. A., Weider, S., Nilsen, K. B., Indredavik, M. S., and Sand, T. (2016). Increased frontal electroencephalogram theta amplitude in patients with anorexia nervosa compared to healthy controls. Neuropsychiatr. Dis. Treat. 21, 2419-2423. doi: 10.2147/NDT.S1 13586

Isern, R. D. (1987). Family violence and the Klüver-Bucy syndrome. South Med. J. 80, 373-377.

Kamolz, S., Richter, M. M., Schmidtke, A., and Fallgatter, A. J. (2008). Transcranial magnetic stimulation for comorbid depression in anorexia. Nervenarzt 79, 1071-1073. doi: 10.1007/s00115-008-2537-8

Kaye, W. H., Fudge, J. L., and Paulus, M. (2009). New insights into symptoms and neurocircuit function of anorexia nervosa. Nat. Rev. Neurosci. 10, 573-584 doi: $10.1038 / \mathrm{nrn} 2682$

Kaye, W. H., Wagner, A., Fudge, J. L., and Paulus, M. (2010). Neurocircuity of eating disorders. Curr. Top. Behav. Neurosci. 6, 37-57. doi: 10.1007/7854_2010_85

Kaye, W. H., Wierenga, C. E., Bailer, U. F., Simmons, A. N., and Bischoff-Grethe, A. (2013). Nothing tastes as good as skinny feels: the neurobiology of anorexia nervosa. Trends Neurosci. 36, 110-120. doi: 10.1016/j.tins.2013.01.003

Khedr, E. M., Elfetoh, N. A., Ali, A. M., and Noamany, M. (2014). Anodal transcranial direct current stimulation over the dorsolateral prefrontal cortex improves anorexia nervosa: a pilot study. Restor. Neurol. Neurosci. 32, 789-797. doi: $10.3233 / \mathrm{RNN}-140392$

Klauss, J., Penido Pinheiro, L. C., Silva Merlo, B. L., de Almeida Correia Santos, G., Fregni, F., Nitsche,. M. A., et al. (2014). A randomized controlled trial of targeted prefrontal cortex modulation with tDCS in patients with alcohol dependence. Int. J. Neuropsychopharmacol. 17, 1793-1803. doi: $10.1017 /$ S1461145714000984

Kontis, D., and Theochari, E. (2012). Dopamine in anorexia nervosa: a systematic review. Behav. Pharmacol. 23, 496-515. doi: 10.1097/FBP.0b013e328357e115

Kovacs, M. (1982). C.D.I.: Children's Depression Inventory. Questionario di Autovalutazione. Adattamento Italiano a Cura di Camuffo M., Cerutti R., Lucarelli L., Mayer R. (1988). Firenze: Organizzazioni Speciali.

Kovacs, M. (1992). Children's Depression Inventory (CDI) Manual. New York, NY: Multi Health Systems.

Kullmann, S., Giel, K. E., Hu, X., Bischoff, S. C., Teufel, M., Thiel, A., et al. (2014). Impaired inhibitory control in anorexia nervosa elicited by physical activity stimuli. Soc. Cogn. Affect. Neurosci. 9, 917-923. doi: 10.1093/scan/nst070

Laghi, F., Pompili, S., Zanna, V., Castiglioni, M. C., Criscuolo, M., Chianello, I., et al. (2017). How adolescents with anorexia nervosa and their parents perceive family functioning? J. Health Psychol. 22, 197-207. doi: $10.1177 / 1359105315597055$

Le Grange, D., Lock, J., Agras, W. S., Moye, A., Bryson, S. W., Jo, B., et al. (2012). Moderators and mediators of remission in family-based treatment and adolescent focused therapy for anorexia nervosa. Behav. Res. Ther. 50, 85-92. doi: 10.1016/j.brat.2011.11.003

Lee, D. J., Elias, G. J. B., and Lozano, A. M. (2018). Neuromodulation for the treatment of eating disorders and obesity. Ther. Adv. Psychopharmacol. 8, 73-92. doi: 10.1177/2045125317743435

Ljubisavljevic, M., Maxood, K., Bjekic, J., Oommen, J., and Nagelkerke, N. (2016). Long-term effects of repeated prefrontal cortex Transcranial Direct Current Stimulation (tDCS) on food craving in normal and overweight young adults. Brain Stimul. 9, 826-833. doi: 10.1016/j.brs.2016.07.002

Lock, J., Le Grange, D., Agras, W. S., Moye, A., Bryson, S. W., and Jo, B. (2010). Randomized clinical trial comparing family-based treatment with adolescentfocused individual therapy for adolescents with anorexia nervosa. Arch. Gen. Psychiatry 67, 1025-1032. doi: 10.1001/archgenpsychiatry.2010.128

Lowe, C. J., Hall, P. A., and Staines, W. R. (2014). The effects of continuous theta burst stimulation to the left dorsolateral prefrontal cortex on executive function, food cravings, and snack food consumption. Psychosom. Med. 76, 503-511. doi: 10.1097/PSY.00000000000 00090
March, J. S., Parker, J. D., Sullivan, K., Stallings, P., and Conners, C. K. (1997) The Multidimensional Anxiety Scale for Children (MASC): factor structure, reliability, and validity. J. Am. Acad. Child Adolesc. Psychiatry 36, 554-565. doi: 10.1097/00004583-199704000-00019

McClelland, J., Bozhilova, N., Campbell, L., and Schmidt, U. (2013). A Systematic review of the effects of neuromodulation on eating and body weight: evidence from human and animal studies. Eur. Eat. Disord. Rev. 21, 436-455. doi: 10.1002/erv.2256

McClelland, J., Kekic, M., Bozhilova, N., Nestler, S., Dew, T., Van den Eynde, F., et al. (2016a). A randomised controlled trial of neuronavigated repetitive transcranial magnetic stimulation (rTMS) in anorexia nervosa. PLoS ONE 11:148606. doi: 10.1371/journal.pone.0148606

McClelland, J., Kekic, M., Campbell, I. C., and Schmidt, U. (2016b). Repetitive transcranial magnetic stimulation (rTMS) treatment in enduring anorexia nervosa: a case series. Eur. Eat. Disord. Rev. 24, 157-163. doi: 10.1002/erv.2414

Miller, E. K., and Cohen, J. D. (2001). An integrative theory of prefrontal cortex function. Annu. Rev. Neurosci. 24, 167-202. doi: 10.1146/annurev.neuro.24.1.167

Mohr, H. M., Zimmermann, J., Röder, C., Lenz, C., Overbeck, G., and Grabhorn, R. (2010). Separating two components of body image in anorexia nervosa using fMRI. Psychol. Med. 40, 1519-1529. doi: 10.1017/S0033291709991826

Montenegro, R. A., Okano, A. H., Cunha, F. A., Gurgel, J. L., Fontes, E. B., and Farinatti, P. T. (2012). Prefrontal cortex transcranial direct current stimulation associated with aerobic exercise change aspects of appetite sensation in overweight adults. Appetite 58, 333-338. doi: 10.1016/j.appet.2011.11.008

Monte-Silva, K., Kuo, M. F., Thirugnanasambandam, N., Liebetanz, D., Paulus, W., and Nitsche, M. A. (2009). Dose-dependent inverted U-shaped effect of dopamine (D2-like) receptor activation on focal and nonfocal plasticity in humans. J. Neurosci. 29, 6124-6131. doi: 10.1523/JNEUROSCI.0728-09.2009

Nasseri, P., Nitsche, M. A., and Ekhtiari, H. (2015). A framework for categorizing electrode montages in transcranial direct current stimulation. Front. Hum. Neurosci. 9:54. doi: 10.3389/fnhum.2015.00054

National Institute for Clinical Excellence (2004). National Collaborating Centre for Mental Healt, Eating Disorders. (NICE) Core Interventions in the Treatment and Management of Anorexia Nervosa, Bulimia Nervosa, and Related Eating Disorders. Leicester, UK: British Psychological Society.

Nitsche, M. A., and Paulus, W. (2000). Excitability changes induced in the human motor cortex by weak transcranial direct current stimulation. J. Physiol. 527(Pt 3), 633-639. doi: 10.1111/j.1469-7793.2000.t01-1-00633.x

Nitsche, M. A., Boggio, P. S., Fregni, F., and Pascual-Leone, A. (2009). Treatment of depression with transcranial direct current stimulation (tDCS): a review. Exp. Neurol. 219, 14-19. doi: 10.1016/j.expneurol.2009.03.038

Nitsche, M. A., Lampe, C., Antal, A., Liebetanz, D., Lang, N., Tergau, F., et al. (2006). Dopaminergic modulation of long-lasting direct current-induced cortical excitability changes in the human motor cortex. Eur. J. Neurosci. 23, 1651-1657. doi: 10.1111/j.1460-9568.2006.04676.x

Nitsche, M. A., Liebetanz, D., Antal, A., Lang, N., Tergau, F., and Paulus, W (2003). Modulation of cortical excitability by weak direct current stimulation technical, safety and functional aspects. Suppl. Clin. Neurophysiol. 56, 255-276. doi: 10.1016/S1567-424X(09)70230-2

Nitsche, M. A., Muller-Dahlhaus, F., Paulus, W., and Ziemann, U. (2012). The pharmacology of neuroplasticity induced by non-invasive brain stimulation: building models for the clinical use of CNS active drugs. J. Physiol. 590, 4641-4662. doi: 10.1113/jphysiol.2012.232975

Normann, C., Schmitz, D., Fürmaier, A., Döing, C., and Bach, M. (2007). Longterm plasticity of visually evoked potentials in humans is altered in major depression. Biol. Psychiatry 62, 373-380. doi: 10.1016/j.biopsych.2006.10.006

Oberndorfer,T., Simmons, A., McCurdy, D., Strigo, I., Matthews, S., Yang, T., et al. (2013). Greater anterior insula activation during anticipation of food images in women recovered from anorexia nervosa versus controls. Psychiatry Res. 214, 132-141. doi: 10.1016/j.pscychresns.2013.06.010

Owesson-White, C. A., Roitman, M. F., Sombers, L. A., Belle, A. M., Keithley, R. B., Peele, J. L., et al. (2012). Sources contributing to the average extracellular concentration of dopamine in the nucleus accumbens. J. Neurochem. 121, 252-262. doi: 10.1111/j.1471-4159.2012.07677.x

Phillipou, A., Gurvich, C., Castle, D. J., Abel, L. A., and Rossell, S. L. (2015). Comprehensive neurocognitive assessment of patients with anorexia nervosa. World J. Psychiatry 22, 404-411. doi: 10.5498/wjp.v5.i4.404 
Raven, J. C. (1994). CPM, Coloured Progressive Matrices. Firenze: Giunti OrganizzazioniSpeciali. (Italian Adaptation, 2008).

Ridding, M. C., and Ziemann, U. (2010). Determinants of the induction of cortical plasticity by non-invasive brain stimulation in healthy subjects. J. Physiol. 588(Pt 13) 2291-2304. doi: 10.1113/jphysiol.2010.190314

Rienecke, R. D. (2017). Family-based treatment of eating disorders in adolescents: current insights. Adolesc. Health Med. Ther. 1, 69-79. doi: 10.2147/AHMT.S115775

Ritschel, F., Geisler, D., King, J. A., Bernardoni, F., Seidel, M., Boehm, I., et al. (2017). Neural correlates of altered feedback learning in women recovered from anorexia nervosa. Sci. Rep. 7:5421. doi: 10.1038/s41598-017-04761-y

Riva, G. (2016). Neurobiology of anorexia nervosa: serotonin dysfunctions link self-starvation with body image disturbances through an impaired body memory. Hum Neurosci. 24:10. doi: 10.3389/fnhum.2016.00600

Smink, F. R., van Hoeken, D., and Hoek, H. W. (2012). Epidemiology of eating disorders: incidence, prevalence and mortality rates. Curr. Psychiatry Rep. 14, 406-414. doi: 10.1007/s11920-012-0282-y

Södersten, P., Bergh, C., and Leon, M. (2016). Commentary: new insights in anorexia nervosa. Front. Neurosci. 10:483. doi: 10.3389/fnins.2016.00483

Steinglass, J. E., Lempert, K. M., Choo, T. H., Kimeldorf, M. B., Wall, M., Walsh, B. T., et al. (2017). Temporal discounting across three psychiatric disorders: anorexia nervosa, obsessive compulsive disorder, and social anxiety disorder. Depress. Anxiety 34, 463-470. doi: 10.1002/da.22586

Steinglass, J., and Walsh, B. T. (2006). Habit learning and anorexia nervosa: a cognitive neuroscience hypothesis. Int. J. Eat. Disord. 39, 267-275. doi: $10.1002 /$ eat.20244

Steinhausen, H. C. (2002). The outcome of anorexia nervosa in the 20th century. Am. J. Psychiatry 159, 1284-1293. doi: 10.1176/appi.ajp.159.8.1284

Striegel-Moore, R. H., Franko, D. L., Thompson, D., Barton, B., Schreiber, G. B., and Daniels, S. R. (2004). Changes in weight and body image over time in women with eating disorders. Int. J. Eat. Disord. 36, 315-327. doi: 10.1002/eat.20053

Strigo, I. A., Matthews, S. C., Simmons, A. N., Oberndorfer, T., Klabunde, M., Reinhardt, L. E., et al. (2013). Altered insula activation during pain anticipation in individuals recovered from anorexia nervosa: evidence of interoceptive dysregulation. Int. J. Eat. Disord. 46, 23-33. doi: 10.1002/eat.22045

Tanaka, T., Takano, Y., Tanaka, S., Hironaka, N., Kobayashi, K., Hanakawa, T., et al. (2013). Transcranial direct-current stimulation increasesextracellular dopamine levels in the rat striatum. Front. Syst. Neurosci. 7:6. doi: 10.3389/fnsys.2013.00006

Van den Eynde, F., Guillaume, S., Broadbent, H., Campbell, I. C., and Schmidt, U. (2013). Repetitive transcranial magnetic stimulation in anorexia nervosa: a pilot study. Eur. Psychiatry 28, 98-101. doi: 10.1016/j.eurpsy.2011.06.002

Van Kuyck, K., Gérard, N., Van Laere, K., Casteels, C., Pieters, G., Gabriëls, L., et al. (2009). Towards a neurocircuitry in anorexia nervosa: evidence from functional neuroimaging studies. J. Psychiatr. Res. 43, 1133-1145. doi: 10.1016/j.jpsychires.2009.04.005

Vierheilig, N., Mühlberger, A., Polak, T., and Herrmann, M. J. (2016). Transcranial direct current stimulation of the prefrontal cortex increases attention to visual target stimuli. J. Neural. Transm. 123, 1195-1203. doi: 10.1007/s00702-016-1542-5

Wagner, A., Aizenstein, H., Venkatraman, V. K., Bischoff-Grethe, A., Fudge, J., May, J. C., et al. (2010). Altered striatal response to reward in bulimia nervosa after recovery. Int. J. Eat. Disord. 43, 289-294. doi: 10.1002/eat.20699

Walsh, B. T. (2013). The enigmatic persistence of anorexia nervosa. Am. J. Psychiatry 170, 477-484. doi: 10.1176/appi.ajp.2012.12081074

Wietschorke, K., Lippold, J., Jacob, C., Polak, T., and Herrmann, M. J. (2016). Transcranial direct current stimulation of the prefrontal cortex reduces cuereactivity in alcohol-dependent patients. J. Neural Transm. 123, 1173-1178. doi: 10.1007/s00702-016-1541-6

Ziadi Trives, M., Bonete Llácer, J. M., García Escudero, M. A., and Martínez Pastor, C. J. (2013). Effect of the addition of aripiprazole on hyperprolactinemia associated with risperidone long-acting injection. J. Clin. Psychopharmacol. 33, 538-541. doi: 10.1097/JCP.0b013e3182970431

Conflict of Interest Statement: The authors declare that the research was conducted in the absence of any commercial or financial relationships that could be construed as a potential conflict of interest.

Copyright (C) 2018 Costanzo, Menghini, Maritato, Castiglioni, Mereu, Varuzza, Zanna and Vicari. This is an open-access article distributed under the terms of the Creative Commons Attribution License (CC BY). The use, distribution or reproduction in other forums is permitted, provided the original author(s) and the copyright owner(s) are credited and that the original publication in this journal is cited, in accordance with accepted academic practice. No use, distribution or reproduction is permitted which does not comply with these terms. 


\section{OPEN ACCESS}

Edited by:

Maria Elide Vanutelli,

Università degli Studi di Milano, Italy

Reviewed by:

Seth Davin Norrholm,

Emory University School of Medicine,

United States

Mascha Van'T. Wout,

Brown University, United States

Rany Abend,

National Institute of Mental Health

(NIMH), United States

*Correspondence:

Martin J. Herrmann

Herrmann_m@ukw.de

Received: 30 January 2018 Accepted: 06 April 2018

Published: 25 April 2018

Citation:

Dittert N, Hüttner S, Polak T and Herrmann MJ (2018) Augmentation of Fear Extinction by Transcranial Direct Current Stimulation (tDCS).

Front. Behav. Neurosci. 12:76.

doi: 10.3389/fnbeh.2018.00076

\section{Augmentation of Fear Extinction by Transcranial Direct Current Stimulation (tDCS)}

\author{
Natalie Dittert, Sandrina Hüttner, Thomas Polak and Martin J. Herrmann* \\ Department of Psychiatry, Psychosomatics, and Psychotherapy, Center of Mental Health, University Hospital Würzburg, \\ Würzburg, Germany
}

Although posttraumatic stress disorder (PTSD; DSM-V 309.82) and anxiety disorders (DSM-V 300.xx) are widely spread mental disorders, the effectiveness of their therapy is still unsatisfying. Non-invasive brain-stimulation techniques like transcranial direct current stimulation (tDCS) might be an option to improve extinction learning, which is a main functional factor of exposure-based therapy for anxiety disorders. To examine this hypothesis, we used a fear conditioning paradigm with female faces as conditioned stimuli (CS) and a 95-dB female scream as unconditioned stimulus (UCS). We aimed to perform a tDCS of the ventromedial prefrontal cortex (vmPFC), which is mainly involved in the control of extinction-processes. Therefore, we applied two $4 \times 4 \mathrm{~cm}$ electrodes approximately at the EEG-positions F7 and F8 and used a direct current of $1.5 \mathrm{~mA}$. The 20-min stimulation was started during a 10-min break between acquisition and extinction and went on overall extinction-trials. The healthy participants were randomly assigned in two double-blinded process into two sham stimulation and two verum stimulation groups with opposite current flow directions. To measure the fear reactions, we used skin conductance responses (SCR) and subjective ratings. We performed a generalized estimating equations model for the SCR to assess the impact of tDCS and current flow direction on extinction processes for all subjects that showed a successful conditioning $(N=84)$. The results indicate that tDCS accelerates early extinction processes with a significantly faster loss of CS+/CS- discrimination. The discrimination loss was driven by a significant decrease in reaction toward the CS+ as well as an increase in reaction toward the CS- in the tDCS verum groups, whereas the sham groups showed no significant reaction changes during this period. Therefore, we assume that tDCS of the vmPFC can be used to enhance early extinction processes successfully. But before it should be tested in a clinical context further investigation is needed to assess the reason for the reaction increase on CS-. If this negative side effect can be avoided, tDCS may be a tool to improve exposure-based anxiety therapies.

Keywords: fear conditioning, brain stimulation, tDCS, skin conduction response, ventromedial prefrontal cortex 


\section{INTRODUCTION}

Posttraumatic stress disorder (PTSD; DSM-V 309.81) and anxiety disorders (DSM-V 300.xx) have a high 12-monthprevalence of $16 \%$ (PTSD 2\%, anxiety disorder 14\%) in Europe and belong thereby to the most common psychiatric diseases (Wittchen et al., 2011). Cognitive behavioral therapy with exposure elements is, amongst others, the recommended treatment form for PTSD and many entities of anxiety disorders, namely specific phobia (DSM-V 300.29), social anxiety disorder (DSM-V 300.23), generalized anxiety disorder (DSM$\mathrm{V}$ 300.xx), panic disorder (DSM-V 300.01), and agoraphobia (DSM-V 300.22; Rauch et al., 2012; Bandelow et al., 2014). The development of this therapy is based on an explanatory cognitive and learning model of anxiety. Such models assume that processes of classical conditioning result in the development of anxiety. Classical fear conditioning is an associative learning process, which links harmless stimuli with fearful experiences. Anxiety toward these former harmless stimuli is then preserved by processes of operant conditioning, which lead to avoidance behavior. This avoidance behavior prevents the ability for other associations to be made, leaving the fear intact (Mowrer, 1956). Therefore, new neutral experiences with the former harmless stimuli lead to a reduction of the fearful association (Myers and Davis, 2002). According to classical conditioning, this process is called extinction learning. As extinction learning can lower anxiety through the diminishing of fearful associations it was used as a basis for the development of exposure therapies for anxiety disorders (McNally, 2007). Hence, finding a method that improves extinction processes is a good starting point to discover a targeted modulation procedure for enhancing exposure therapies. One option could be the use of non-invasive brain-stimulation techniques (Bajbouj and Padberg, 2014; Marin et al., 2014).

Non-invasive brain-stimulation works through changing the activity of several brain areas by application of magnetic fields or currents with devices that are placed on the exterior of the head. Thereby non-invasive brain-stimulation can support the effect of psychotherapy (Bajbouj and Padberg, 2014) as psychotherapy also changes several neuronal structures and their activity like Beauregard (2014) has proven for anxiety disorders. One non-invasive stimulation technique, that is already recommended for the treatment of unipolar depression in Germany, is repetitive transcranial magnetic stimulation (rTMS; DGPPN, November 2015) ${ }^{1}$. Besides depression, recent studies showed that rTMS could successfully improve extinction processes and lower anxiety as well. Guhn et al. (2014) stimulated the ventromedial prefrontal cortex (vmPFC) with rTMS prior to extinction learning and could achieve a successful improvement of early extinction learning and extinction recall. Further investigation revealed the effects of Guhn's stimulation protocol for the improvement of exposure therapy

${ }^{1}$ DGPPN, B., KBV, AWMF, AkdÄ, BPtK, BApK, DAGSHG, DEGAM, DGPM, DGPs, DGRW (Hrsg.) für die Leitliniengruppe Unipolare Depression (November 2015). "S3-Leitlinie/Nationale VersorgungsLeitlinie Unipolare Depression Langfassung, 2. Auflage, Version 1."). of acrophobic patients (Herrmann et al., 2017). Raij et al. (2017) tried a temporally specific rTMS of the prefrontal cortex according to a prior study, which showed, that stimulation of the infralimbic cortex of rats-the equivalent to the human vmPFC-only improves extinction when it is applied $100 \mathrm{~ms}$ after the stimulus onset (Milad et al., 2004). Raij et al. (2017) used an incomplete extinction paradigm with just four trials and could achieve an improvement of extinction recall on the next day. They made no measurements during extinction learning, thus, rTMS could have affected extinction recall or the four trials of early extinction learning equally.

Transcranial direct current stimulation (tDCS), which we used in this study, is another important non-invasive brain stimulation technique that receives increasing attention in psychiatric research. In comparison to rTMS, tDCS is more pleasant, has less aversive side effects and its application is easier and cheaper (Poreisz et al., 2007). tDCS is applied using two electrodes placed around the stimulation area on the scalp. The current flows from anode to cathode and passes through all brain areas that are located between the electrodes, thus, the focal specification is not very high. Overall, a subthreshold activation of brain areas near the anode and a deactivation near the cathode is generated (Nitsche and Paulus, 2000). To be more precise the orientation of the cell axes in relation to the current flow is important for the outcoming activation. Cell parts nearer the anode become hyperpolarized, those nearer the cathode depolarized (Bikson et al., 2004). A successful increase in brain activation can usually be obtained with a somatic depolarization and a terminal hyperpolarization. So, pyramidal cells in cortical regions that lie parallel to the scalp near the anode get thereby activated by these so-called radial current proportions (Rahman et al., 2013). On the contrary, tangential current proportions, that flow parallel and not vertical to the cortical surface, do not activate entire cortical areas, but rather several corticocortical afferent nerve pathways or single axon terminals (Rahman et al., 2013). Especially the effect of tangential current flow proportions cannot be predicted precisely. The angle in which the current meets the cortex and individual anatomical factors such as cortical folding must be considered as well (Bikson et al., 2013). Additionally, there are activity- and input-selective mechanisms (Bikson et al., 2013). So, the task, which is done during stimulation, influences the brain activity, too (Reato et al., 2010). Further, tDCS improves the processing of some contents but this goes usually at the expense of other contents, whose reception decreases parallelly (Bikson et al., 2004). In addition to the direct activation of several brain areas, tDCS seems to modulate the dopamine secretion (Tanaka et al., 2013; Broeder et al., 2015; Agarwal et al., 2016).

Even though the focal specification of tDCS is rather low, a specific electrode placement can get several brain areas into the focus of stimulation. So, the underlying neuronal processes that are associated with a specific psychiatric disease determine a therapeutic reasonable stimulation aim in the brain and lead thereby the decision for the electrode positions. Hence, for the alleviation of anxiety disorders, adequate stimulation aims can be 
derived from the underlying neuronal mechanism of exposure therapies, which is extinction learning. One important brain area for extinction processes is the vmPFC. Results of functional resonance imaging (fMRI) proved a heightened activation of the vmPFC during extinction learning (Gottfried and Dolan, 2004) and a decrease in vmPFC depression during progressive extinction learning as well as a correlation of vmPFC activation and extinction retention (Phelps et al., 2004). Consistent with these findings the vmPFC activity increased during late extinction learning in near-infrared spectroscopy (NIRS; Guhn et al., 2012). Furthermore, lesions or pharmacological deactivation of this brain area led to an impairment of extinction consolidation and recall in rats (Quirk et al., 2000; Morgan et al., 2003; Sierra-Mercado et al., 2006). Apart from the vmPFC, the amygdala plays a vital role according to extinction processes (Knight et al., 2004; Sotres-Bayon et al., 2007; Herry et al., 2008). Herry et al. (2008) found special extinction neurons in the amygdala that were activated during extinction learning. To close the circle, these neurons showed also strong bidirectional connections with the vmPFC. Based on these data we aimed-like many other researchers, who tried to modulate extinction processes via brain stimulationto activate the vmPFC (Guhn et al., 2014; Abend et al., 2016; Van't Wout et al., 2016, 2017; Raij et al., 2017). On the neurobiological level, especially the neurotransmission of dopamine seems to be a crucial factor for functioning extinction (Hikind and Maroun, 2008; Raczka et al., 2011; Abraham et al., 2016).

About the effects of tDCS on extinction mainly two researchers, R. Abend and M. van't Wout, have published so far, though none of their studies could substantially improve extinction processes. Abend et al. (2016), who placed the anode on the forehead and the cathode on the back of the head and stimulated parallel to extinction learning, did a 3-day fear conditioning paradigm with extinction learning and recall on different days. They found no improvement of extinction but anxiety generalization effects with increased reactions on CSin their tDCS condition and a fear potentiation toward the $\mathrm{CS}+$ in their alternating current condition. As probable causes for these effects the authors considered on the one hand the unintentional stimulation of dorsomedial brain areas and on the other hand that the stimulation was not temporally specific, which seemed to be important in Milad et al. (2004) as mentioned above. Van't Wout published twice exploring a cross-over-design with the anode on the left forehead on EEG-Position AF3 and the cathode on the contralateral mastoid. She used a 2-day paradigm with conditioning and extinction learning on the first and extinction recall on the second day. In 2016 she found a slight improvement of late extinction learning in healthy participants when the stimulation took place during early extinction learning, but this effect could not be distinguished from anxiolytic tDCSaftereffects with certainty (Van't Wout et al., 2016). In 2017 she stimulated PTSD patients during or after extinction learning and found only a trend-significant improvement of extinction recall in the after extinction learning condition (Van't Wout et al., 2017). Both authors aimed to stimulate the vmPFC but used different electrode positions to do so. As mentioned above the angle in which the current meets the cortical surface affects the stimulation effects, too. On the one hand this could be a reason for their distinct results, but on the other hand, it leaves hope that the investigation of further electrode positions could finally lead to a successful improvement of extinction processes. Therefore, we want to go on finding a tDCS-protocol that can substantially improve extinction processes by increasing the activation of the vmPFC.

Furthermore, we wanted to assess the effect of the current flow direction. Opposite current flow directions in a bitemporal electrode placement do not determinedly lead to opposite tDCS effects. The outcome can rather be distinct because of the complex tDCS mechanisms, which include e.g., parameters of cortical folding and changes in the dopamine secretion as already said. Additionally, lateral cortical areas get affected by right or left anodal tDCS in diverse ways, whereas our actual stimulation aim, the vmPFC, was equidistantly located between anode and cathode. Thus, we expected that both current flow directions would lead to similar activation patterns in the vmPFC region but have different effects on other prefrontal cortical areas, which may affect our outcome measures. According to the functional diversity of the two hemispheres, both current flow direction can have advantages and disadvantages. The right lateral prefrontal cortex seems to be important for emotional regulation processes (Klumpers et al., 2010; Herrmann et al., 2016a). Therefore, right anodal stimulation, which rather activates the right hemisphere, could lead to stronger extinction learning. But on the other hand, patients with anxiety disorders show a decreased left cortical activation (Thibodeau et al., 2006), thus, it is conceivable that the increase of left cortical activity by left anodal tDCS might reduce anxiety and improve extinction learning as well. Investigating the effects of the current flow direction in this context seemed to be very interesting but difficult to predict at the same time. Therefore, we could not make any certain predictions about its effect in advance, but we expected that right and left anodal stimulation would not result in similar effects.

As the main effect of our tDCS-protocol we expectedaccording to the successful improvement of early extinction learning by rTMS by Guhn et al. (2014) —an improvement in extinction learning most notably in early extinction processes. To avoid anxiety generalization effects, as in Abend et al. (2016), we chose a bitemporal electrode positioning which ensured a recess of fear-generating dorsomedial brain areas.

\section{MATERIALS AND METHODS}

\section{Participants}

One hundred and thirty one healthy participants were recruited through online displays and randomly and double-blinded assigned into four groups: two real-stimulation groups with right vs. left anodal stimulation and two sham-stimulation groups. Blinding worked through a random assignment of codes to each participant via code lists, which were separated by sex and current flow direction. The investigator keyed in these codes into the stimulation device, which then decided if the participants received sham- or real-stimulation. Therefore, it was necessary to collect data from two separate sham groups (right or left anodal) 
to uphold the blinding of the investigator until the end of data collection.

All subjects gave self-disclosure about the main inclusion criteria, which were no psychiatric or neurological diseases (especially no epilepsy or elevated brain pressure) now or earlier, no current heart disease or hearing loss, age between 18 and 35 years, right-handedness, no metal or cochlear head-implants and no recent consumption of psychotropic drugs. To control hormonal levels, an additional inclusion criterion for women was being in the intake-phase of hormonal contraceptives (cf. Guhn et al., 2012), whereas pregnancy or current breastfeeding were exclusion criteria. In total 47 participants were excluded, most of them (36) because of insufficient fear conditioning. Sufficient fear conditioning was defined by a higher skin conductance response (SCR) toward the conditioned stimulus (CS+) that was paired with the unconditioned stimulus (UCS), compared to the unpaired conditioned stimulus (CS-) during the last two acquisition trials. Other reasons for exclusion were high depression scores (Allgemeine Depressionsskala in Kurzform; ADS-K; Hautzinger and Bailer, 1993 ADS-K $>16 ; N=8$ ), technical problems $(N=1)$, early termination at own request $(N=1)$, and undetectable SCR response after a deep breath at the beginning of measurements $(N=1)$. Finally, 84 participants remained for the analysis. For these remaining subjects, no significant group differences for age, gender, body size (weight, height, and head size), drug use (caffeine, nicotine, cannabis), trait-anxiety (state and trait anxiety inventory, form X2; STAI-X2; Laux et al., 1981), anxiety sensitivity (anxiety sensitivity index 3; ASI-3; Taylor et al., 2007), depression (ADS-K), and negative and positive affect (positive and negative affect scale; PANAS; Watson et al., 1988) could be found in statistical group comparisons with generalized estimating equation models (GEEs) (see Table $\mathbf{1}$ ). After participants were given a complete description of the study and its procedures, written informed consent was obtained in accordance with the Declaration of Helsinki in its latest version. All procedures were approved by the ethics committee of the medical faculty of the University of Würzburg. All subjects participated voluntary and received an expense allowance of 15 euros.

\section{Stimulation}

tDCS was applied by a battery powered stimulator (Eldith DC-Stimulator, NeuroConn, Ilmenau, Germany) using two approximately $4 \times 4 \mathrm{~cm}$ rubber electrodes coated with electrode gel (TEN20 conductive neurodiagnostic electrode paste). The stimulation started during a 10-min break between acquisition and extinction and went on until the end of extinction. The real-stimulation protocol had a duration of $1,200 \mathrm{~s}$ on a constant level of $1.5 \mathrm{~mA}$ and a fade-in and fade-out phase of $10 \mathrm{~s}$ each during which the current was slowly turned on in the beginning and off in the end. The sham-stimulation protocol had the same fade-in and fade-out phases, but the constant current phase was shortened to $40 \mathrm{~s}$. The electrode positions were selected by the support of the computer program HD explore by Soterix Medical 3.2 (Kempe et al., 2014), which simulates the brain activation of different stimulation protocols. Our aim was to achieve an intense stimulation of the vmPFC, whereas fear generating dorsomedial brain areas like the dorsal anterior cingulate cortex should be spared out. We chose the positions M20, M21, I20, I21, J13, and J14 for the left and M9, M10, I9, $\mathrm{I10}, \mathrm{J} 6$, and $\mathrm{J} 7$ for the right electrode pad in the $332+4$ electrodes model of the HD explore software (see Figure 1). To simplify the electrode application, we calculated the distance of these electrode positions from easily measurable points of the EEG10-20-system for each participant's head circumference. The final positions were slightly below the EEG-10-20 positions F7 and F8.

The two experimental groups were treated with the same electrode positions but opposite current flow directions. Figure 1 shows the HD explore modeling only for the left anodal stimulation because the activity in the vmPFC, our main stimulation aim, looked similar in modeling for right or left anodal current flow. As mentioned in the introduction, the background of the current flow direction effects is complex and escapes thereby activity modeling.

\section{Fear Conditioning}

\section{Stimuli}

According to Lau et al. (2008), two neutral looking female phases of the NimStim Face Stimulus Set (03F_NE_C and 10F_NE_C; Tottenham et al., 2009) were used as CS. One of these faces was randomly selected as a CS + and followed by the UCS in the acquisition phase, the other one functioned as a CS- and was never paired with the UCS. As UCS a 95-dB loud female scream simultaneously presented with a fearful expression of the CS+ face was used (sound: FemScream2, no. 276 of the International Affective Digitized Sounds; Bradley and Lang, 1999; pictures: 03F_FE_O or 10_FE_O of the NimStim Face Stimulus Set; Tottenham et al., 2009). The sound was applied via inear-headphones (3M E-A-RTONE ${ }^{\mathrm{TM}}$ GOLD 3A Insert Earphone with natus ${ }^{\circledR}$ neurology attachment; Natus Europe GmbH).

\section{Task}

The experiment was designed with Presentation ${ }^{\circledR}$ software (version 16.5, Neurobehavioral Systems, Inc., Berkeley, CA, www. neurobs.com) and consisted of a habituation phase, two blocks of acquisition and two blocks of extinction, that all took place on the same day (see Figure 2). We separated the extinction phase into two blocks to differentiate between early and late extinction learning and to perform a subjective rating about arousal and valence of both CSs after early extinction learning. The acquisition was split into similar blocks to ensure a regular experimental schedule for the subjects. Both CSs were presented for $6 \mathrm{~s}$ in a pseudo-randomized order ensuring a maximum of 2 consecutive presentations of the same stimulus. The intertrial interval had a randomized duration of 9 to $12 \mathrm{~s}$. Whereas, the habituation phase consisted of four presentations of each CS, in every block of the acquisition and extinction phase each CS was shown six times. During the acquisition phase the CS+ was followed by the UCS in five of the six trials per block, so the reinforcement rate was about $80 \%$ similar to Abend et al. (2016). We chose a partial reinforcement as it prolongs the process of extinction learning compared to a continuous CS-UCS-pairing (Hilton, 1969; Schurr and Runquist, 1973). Thus, hoping to have more time to detect effects during extinction learning. 
TABLE 1 | Sample description.

\begin{tabular}{|c|c|c|c|c|c|c|c|c|}
\hline & \multicolumn{4}{|c|}{ Sham } & \multicolumn{4}{|c|}{ Real } \\
\hline & \multicolumn{2}{|c|}{ Right anodal $(N=17,10$ ㅇ) } & \multicolumn{2}{|c|}{ Left anodal $(N=17,9$ ㅇ) } & \multicolumn{2}{|c|}{ Right anodal ( $N=26,14$ ㅇ) } & \multicolumn{2}{|c|}{ Left anodal $(N=24,13$ $)$} \\
\hline & $M$ & $S D$ & $M$ & $S D$ & $M$ & $S D$ & $M$ & $S D$ \\
\hline Age (years) & 24.0 & 3.9 & 25.3 & 4.1 & 24.4 & 4.4 & 23.3 & 3.6 \\
\hline Height (cm) & 171.7 & 10.2 & 174.5 & 9.0 & 175.7 & 7.7 & 171.7 & 8.8 \\
\hline Weight (kg) & 66.2 & 13.7 & 67.6 & 11.2 & 67.5 & 10.5 & 67.3 & 10.6 \\
\hline $\mathrm{BMI}(\mathrm{kg} / \mathrm{cm} 2)$ & 22.2 & 2.9 & 22.1 & 2.2 & 21.8 & 2.2 & 22.8 & 3.0 \\
\hline Head size (cm) & 55.8 & 2.0 & 55.9 & 2.0 & 56.0 & 1.7 & 55.9 & 1.7 \\
\hline $\mathrm{EIH}$ & 0.8 & 0.2 & 0.8 & 0.2 & 0.9 & 0.1 & 0.8 & 0.2 \\
\hline ASI-3 & 18.1 & 8.8 & 14.2 & 11.9 & 15.6 & 8.7 & 18.0 & 6.7 \\
\hline ADS-K & 7.8 & 4.1 & 5.2 & 4.1 & 6.6 & 3.5 & 6.7 & 4.2 \\
\hline \multicolumn{9}{|l|}{ STAI } \\
\hline Trait & 33.9 & 6.3 & 32.3 & 6.9 & 31.7 & 6.4 & 32.7 & 6.7 \\
\hline State $\mathrm{t} 1$ & 41.4 & 8.1 & 37.7 & 7.1 & 38.5 & 8.2 & 39.0 & 9.9 \\
\hline State t2 & 33.8 & 4.5 & 33.7 & 6.1 & 35.0 & 5.5 & 31.1 & 5.7 \\
\hline \multicolumn{9}{|l|}{ PANAS } \\
\hline PA baseline & 35.2 & 6.0 & 35.8 & 5.7 & 36.8 & 5.5 & 37.3 & 5.7 \\
\hline PA t1 & 26.9 & 4.7 & 28.2 & 5.8 & 28.7 & 7.3 & 29.6 & 5.7 \\
\hline PA t2 & 27.6 & 6.3 & 29.3 & 5.7 & 27.5 & 5.5 & 30.4 & 6.3 \\
\hline NA baseline & 17.9 & 5.1 & 16.4 & 5.0 & 16.6 & 4.2 & 16.0 & 3.9 \\
\hline NA t1 & 15.1 & 4.4 & 14.0 & 5.8 & 14.5 & 3.9 & 14.3 & 4.0 \\
\hline NA t2 & 11.1 & 1.5 & 11.6 & 3.5 & 11.8 & 3.5 & 10.7 & 1.0 \\
\hline
\end{tabular}

Displayed are means and standard deviations of sample characteristics and questionnaire scores for sham and real stimulation groups separated for the current flow direction. $M$, mean; SD, standard deviation; EIH, Edinburgh Inventory of Handedness; ASI-3, Anxiety Sensitivity Index 3; ADS-K, depression score; STAl, State Trait Anxiety Inventory with trait-scale and

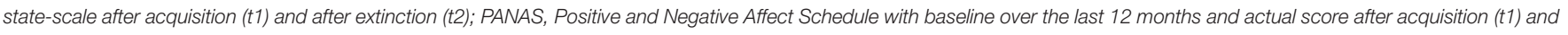
after extinction (t2); PA, positive affect; $N A$, negative affect.

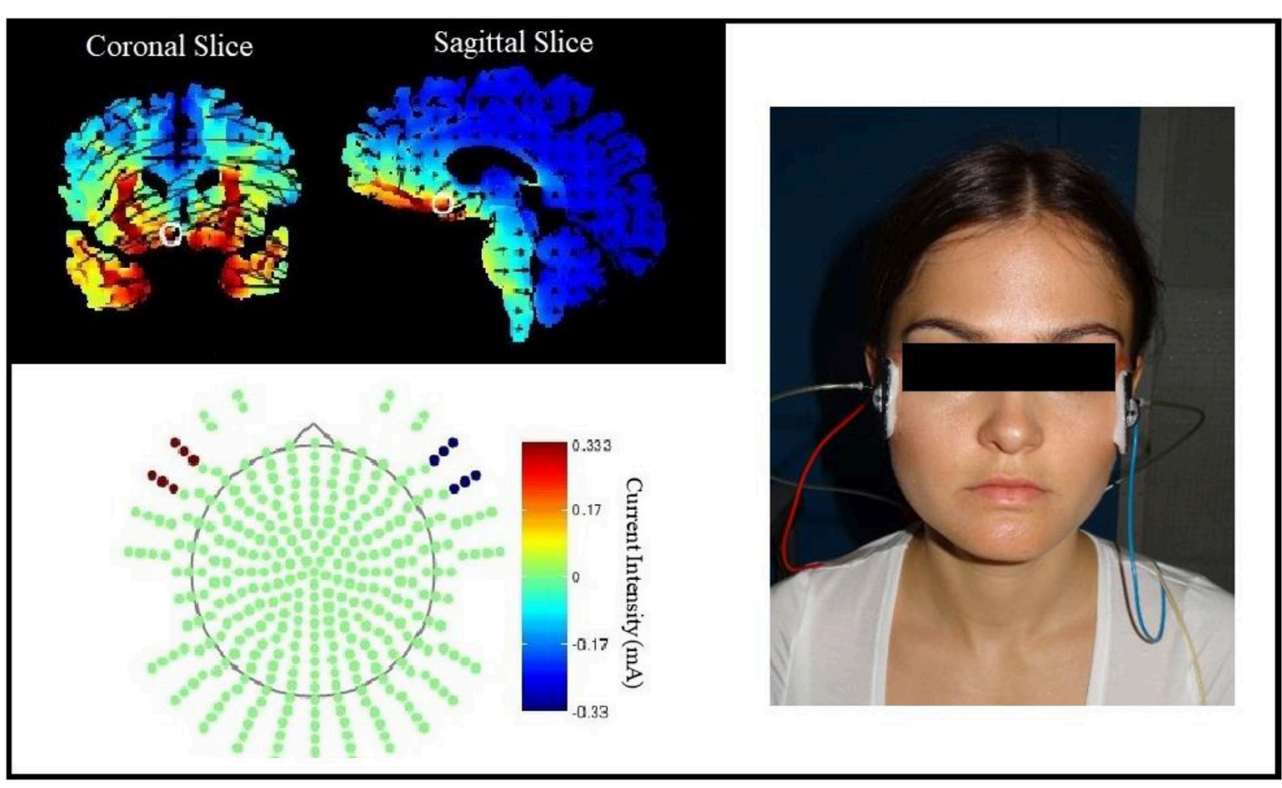

FIGURE 1 | Electrode position selection. The current intensity modeling with HD Explore by Soterix Medical led to the selection of electrode positions near EEG-positions F7 and F8. The figure on the left side shows the modeling for left anodal current flow, the picture on the right shows the actual electrode placement on a participant for right anodal current flow. 


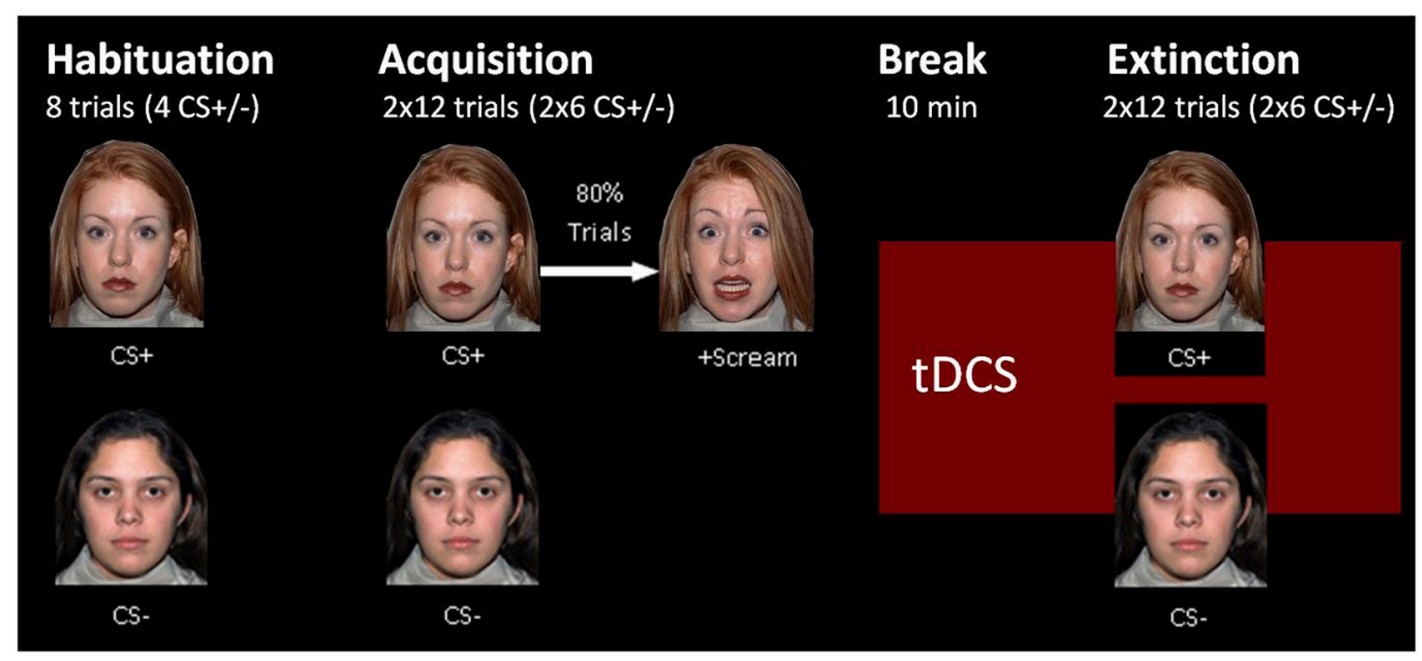

FIGURE 2 | Fear conditioning paradigm

\section{Measurements Questionnaires}

To assess the baseline criteria, we measured handedness (Edinburgh inventory of handedness; EIH; Oldfield, 1971), depression (ADS-K), trait anxiety, anxiety sensitivity, and positive and negative affect during the last 12 months and asked for sociodemographic data before the experiment started (for a specification of these questionnaires see section Participants). Additionally, we wanted to assess the change of state anxiety and affect in the course of extinction and questioned them after acquisition and extinction using the STAI-X1 (state and trait anxiety inventory, form X1; Laux et al., 1981) and PANAS forms.

\section{Subjective Ratings}

Arousal, valence, and CS-UCS-contingency were repeatedly rated during the experiment for both CSs. The ratings for arousal and valence were performed on a 9-step visual analog Likert-scale (arousal: from very calm to very exciting, valence: from very pleasant to very unpleasant) after each experimental block. Because extinction learning should not be influenced by artificially induced contingency-attention within extinction processes, the ratings for the CS-UCS-contingency took place after every block except the first extinction block. The contingency was rated on an 11-step visual analog Likert-scale, that ranged from 0 to $100 \%$. Participants were asked how likely they would expect a sound after the CSpictures.

\section{SCR}

SCR was recorded with two $5 \mathrm{~mm} \mathrm{Ag} / \mathrm{AgCl}$ surface electrodes placed on thenar and hypothenar of the left hand. The electrodes were connected to an amplifier and recorded via BrainVision Recorder (version 1.20.0701, Brain Products GmbH) in DC mode at a sampling rate of $500 \mathrm{~Hz}$, a range of $\pm 5,000 \mathrm{mV}$, and a high cutoff filter of $1,000 \mathrm{~Hz}$. A gradient of $25 \mathrm{mv} / \mu \mathrm{S}$ was used.

SCR analysis was performed with the program PsPM 3.1.1 (http://pspm.sourceforge.net/) using its general linear model (GLM) for SCR, which was designed for evoked responses, but is appropriate for event-related SCR with short inter stimuli intervals as well (Bach et al., 2009). The GLM has a high predictive validity for trial-by-trial analysis of SCR data (Bach et al., 2013) and a higher predictive validity than other SCRanalysis methods like the continuous decomposition analysis by Ledalab or conventional peak-scoring (Bach, 2014; Staib et al., 2015). PsPM uses a linear model based approach like fMRI models and calculates beta-estimates for the sympathetic arousal of different experimental conditions, which are defined as regressors. We used one regressor for all UCSs together and built single regressors for each following pair of CS + or CS - trials, thus, e.g., SCR data for the first and the second $\mathrm{CS}+$, which were presented during extinction learning, built together one regressor. The summation of two following trials to one regressor heightened the predictive validity of our model, which is claimed to be lower for single trials (Bach et al., 2013). For the model calculations, we chose PsPM's skin conductance response function ("number 1"), which includes the SCR and its temporal invariants. As PsPM filters data during its processing, we decided to keep the default filter settings (downsampling to $10 \mathrm{~Hz}$, unidirectional first order Butterworth high and low pass filter on a cut-off-frequency of 0.05 and $5 \mathrm{~Hz}$ ). After model calculation, the statistics of all regressors were exported for statistical analyses. Because SCR data usually show large individual differences, a standardization is useful for the performance of interindividual comparisons (Boucsein et al., 2012). We z-normalized our data as zstandardization seems to be more advantageous in comparison to other standardization methods for SCR data (Ben-Shakhar, 1985). 


\section{Procedure}

After completion of written informed consent, the participants filled out questionnaires for baseline measurements and the electrodes for tDCS and SCR were applied. The experiment took place in a darkened and soundproof investigation chamber, in which the subjects were placed alone. As a task description, participants were told that photographs and sounds would be presented and that they had to rate valence and arousal of the presented pictures. After the acquisition, the investigator entered the chamber to start the tDCS. The extinction phase began automatically $10 \mathrm{~min}$ after stimulation onset. In the end, the participants received their allowance expense and were discharged.

\section{Statistical Analysis}

For statistical analysis of SCR data, GEEs were performed with SPSS (IBM Corp. Released 2017. IBM SPSS Statistics for Windows, Version 24.0. Armonk, NY: IBM Corp). GEEs are particularly recommended for analyses with correlated residuals and thus appropriate for longitudinal analyses with repeated measures (Liang and Zeger, 1986). We wanted to compare our two experimental groups with each other and with our control subjects in one statistical model, thus, we chose a two-factorial between-subject design and used two experimental and two sham groups. Stimulus (CS+ vs. CS-) and time (different factor steps for each model) were used as repeated within-subject factors and current flow direction (right vs. left anodal) and stimulation group (sham- vs. real-stimulation) as between-subject factors. To assess conditioning, the factor time consisted of two steps, one for the last two habituation trials and one for the last two acquisition trials. For analysis of early extinction processes, an initial model with the above-listed factors was done. The factor time included the last two acquisition trials as intercept and all regressors of the first extinction block (trial $1+2,3+4,5+6$ ) as comparative values were created. To examine more precisely when the effect took place, further GEEs the same factors were built. These compared the regressor of the last two acquisition trials to every early extinction regressor in single models. We started with the first two extinction trials and moved on with the following trialpairs in chronological order. After our adoption that tDCS will influence most notably early extinction learning, we stopped the model calculation as soon as one of the models did not show a significant stimulation effect anymore. Our hypotheses were limited to early extinction learning but because data for late extinction learning had been collected, we explored these late extinction trials, too. This explorative analysis was performed analogically to the analysis of early extinction processes, but with the last regressor of the first extinction block as intercept and all regressors from the second extinction block (trial $7+8,9+10$, $11+12$ ) as comparative values.

Subjective ratings, state anxiety and affect were analyzed using GEEs again with stimulus (CS+ vs. CS-) and time (different factor steps for each model) as repeated withinsubject factors and current flow direction (right vs. left anodal) and stimulation group (sham- vs. real-stimulation) as betweensubject factors. To assess conditioning and CS-US-contingency awareness, the valence-, arousal-, and CS-UCS-contingency ratings after habituation and after the second acquisition block were compared. Analysis of early extinction processes was done by comparing the rating after the second acquisition block with the rating after the first extinction block. This was only possible for valence and arousal because CS-UCS-contingency. State anxiety and affect were only questioned after the acquisition and the second extinction block, so we could only analyze the reaction changes over the whole extinction course. Similar to the SCR analysis, we again did an explorative analysis of late extinction processes for valence and arousal ratings by comparing the ratings after the first and second extinction block in further GEEs as well.

Significant effects were defined with $\alpha \leq 0.05$, all tests were two-sided. The post-hoc analysis of significant GEEs outcomes was done with $t$-tests for independent or paired samples.

\section{RESULTS}

\section{Conditioning}

Successful conditioning was reflected in a significant time $\mathrm{x}$ stimulus interaction for the last habituation regressor (trial $3+4)$ and acquisition regressor [trial $11+12$; wald- $\chi^{2}{ }_{(1,336)}=43.60$, $p<0.001]$. For the between subject factors stimulation group and current flow direction no significant main effects or interactions could be found, suggesting that conditioning processes ran equal in all groups. Post-hoc paired-sample $t$-tests revealed a significant increase of reaction on CS + [difference $=0.64$, $\left.t_{(83)}=5.11, p=<0.001\right]$ while the reaction on CSdecreased [difference $=-0.30, t_{(83)}=-3.27, p=0.002$ ]. The increase of the CS+/CS- discrimination during acquisition [difference $=0.94, t_{(83)}=6.43, p<0.001$ ] led to significant reaction differences for CS + and CS- in the end of the acquisition phase [difference $=0.91, t_{(83)}=9.18, p<0.001$ ]

In subjective ratings all evaluation modalities showed successful conditioning and awareness of the CS-UCScontingency with significant time $\mathrm{x}$ stimulus interactions [valence: wald $-\chi^{2}(1,336)=94.24, p<0.001$; arousal: wald $-\chi^{2}{ }_{(1,336)}=93.88, p<0.001$, contingency: wald$\left.\chi^{2}(1,336)=206.48, p<0.001\right]$. Post-hoc $t$-tests revealed a valence decrease and an arousal increase for the CS+ [valence: difference $=-1.70, t_{(83)}=-8.44, p<0.001$; arousal: difference $=2.24$, $\left.t_{(83)}=8.96, p<0.001\right]$ and opposite rating changes for the CS- [valence: difference $=1.06, t_{(83)}=6.15, p<0.001$; arousal: difference $\left.=-0.86 t_{(83)}=-4.09, p<0.001\right]$. Therefore, for valence and arousal a crucial increase of discrimination learning took place during acquisition [valence: difference $=2.76, t_{(83)}=$ 9.67, $p<0.001$; arousal: difference $\left.=3.10, t_{(83)}=9.61, p<0.001\right]$ and led to a significantly different rating for CS+ and CS- in the end of the acquisition phase [valence: difference $=2.63$, $t_{(83)}=9.91, p=0.002$; arousal: difference $=3.05, t_{(83)}=11.72$, $p<0.001]$. Further, participants became aware of the CS-UCScontingency during acquisition with an increase of CS+ rating from 39.05 to $78.69 \%\left[t_{(83)}=12.67, p<0.001\right]$ and a decrease of CS- rating from 38.21 to $18.21 \%$ [ $\left.t_{(83)}=-6.62, p<0.001\right]$. There were no group differences for valence and contingency ratings, only for arousal ratings a significant main factor for the current flow direction appeared $\left[\right.$ wald $-\chi^{2}(1,336)=4.37$, 
$p=0.037]$. The following data inspection revealed that both right anodal stimulated groups rated CS + and CS- in average 0.5 points more arousing than the other groups.

\section{Extinction}

The GEE for the whole early extinction (see Figure 3) with the last acquisition regressor and all regressors of the first extinction block showed a significant time $\mathrm{x}$ stimulus $\mathrm{x}$ stimulation group interaction [ wald $-\chi^{2}{ }_{(3,672)}=8.12, p=0.044$ ] while no effects on the current flow direction could be found. To narrow down the exact time point of the effect, the last acquisition regressor was then compared to each early extinction regressor in single GEEs starting with the regressor for trials $1+2$ and moving on with the other regressors until the effect remained non-significant. Here, only the model with the first two extinction trials showed a significant time $\mathrm{x}$ stimulus $\mathrm{x}$ stimulation group interaction [wald$\left.\chi^{2}(1,336)=6.40, p=0.011\right]$ with again no effects for the current flow direction.

Post-hoc t-tests then revealed a significantly stronger decrease of CS+/CS- discrimination in both real-stimulation groups [difference $=-0.82, t_{(82)}=-2.39, p=0.019$ ], but no significant group differences for reaction changes on $\mathrm{CS}+$ and CS- separately. For a more precise background assessment of the discrimination loss, we performed paired-sample $t$-tests for the reaction changes on CS+ and CS- in real- and sham-stimulation groups individually. Here, the real-stimulation groups showed a significant decrease of CS + reaction [difference $=-0.47$, $\left.t_{(49)}=-2.59, p=0.013\right]$, but also an increase of CS- reaction [difference $=0.73, t_{(49)}=3.99, p<0.001$ ]. Because of these reaction changes, the $\mathrm{CS}+/ \mathrm{CS}$ - discrimination diminished to a non-significant level after the first two extinction trials. In comparison there were no significant reaction changes in both sham-stimulation groups at all, so sham-stimulated participants still showed a relevant CS+/CS- discrimination after the first two extinction trials [difference $=0.40, t_{(33)}=2.12, p=0.041$ ]. Thus, the CS+ reaction loss started earlier in both real-stimulation groups (see Figure 4).

The explorative analysis of the late extinction processes showed a significant stimulation group $\mathrm{x}$ stimulus $\mathrm{x}$ time interaction for the whole second extinction block [wald$\left.\chi^{2}{ }_{(3,672)}=8.58, p=0.035\right]$, which could be narrowed down temporally between the last regressor of the first and the first regressor of the second extinction block $\left[\right.$ wald $-\chi^{2}(1,336)=5.03$, $p=0.025]$. Post-hoc $t$-tests resulted in a short initial increase of CS- in both real-stimulation groups compared to the shamstimulation groups [difference $=0.65, t_{(82)}=2.59, p=0.011$ ] (see Figure 5).

Additionally, we had a significant stimulation group $\mathrm{x}$ current flow direction $\mathrm{x}$ time interaction between the last regressor of the first and the first regressor of the second extinction block $\left[\right.$ wald $\left.-\chi_{(1,336)}^{2}=4.16, p=0.041\right]$. This was caused by a stronger increase of the averaged reaction over CS + and CS- for the left anodal real-stimulation group compared to the right anodal realstimulation group [difference $=0.50, t_{(48)}=2.15, p=0.037$ ] and left anodal sham-stimulation group [difference $=0.67$, $t_{(39)}=2.72, p=0.010$ ] (see Figure 6).

For valence and arousal ratings no significant interactions for the stimulation group or current flow direction could be found, but both rating modalities showed significant stimulus $\mathrm{x}$ trials interactions [valence: wald $-\chi^{2}{ }_{(1,336)}=32.78, p \leq 0.001$; arousal: wald $\left.-\chi^{2}(1,336)=10.71, p=0.001\right]$, which revealed successful extinction processes in all groups alike. Post-hoc ttests showed a significant increase in valence and decrease in arousal for the CS + [valence: difference $=1.04, t_{(83)}=6.86, p$ $\leq 0.001$; arousal: difference $\left.=-0.89, t_{(83)}=-4.21, p<0.001\right]$ and no significant rating changes for the CS-. Although the discrimination loss for both rating modalities was significant [valence: difference $=-1.26, t_{(83)}=-5.91, p<0.001$; arousal: difference $\left.=0.96, t_{(83)}=3.44, p<0.001\right]$, there were still substantial rating differences after the first extinction block [valence: difference $=1.37, t_{(83)}=7.65, p \leq 0.001$; arousal: difference $\left.=-2.08, t_{(83)}=-10.07, p<0.001\right]$. Neither the explorative analysis of the second extinction block nor the analysis of the CS-UCS-contingency for the whole extinction yielded significant effects for the stimulation group or current flow direction.

The analysis of the questionnaires (see Table 1) revealed no positive affect changes, but an equal decrease of negative affect in all groups during extinction [difference $=-3.18, t_{(82)}=-7.88, p$ $<0.001]$. Furthermore, a significant stimulation group $\mathrm{x}$ current flow direction $\mathrm{x}$ time interaction for the state anxiety [wald$\chi^{2}(1,167)=8.58, p=0.003$ ] was found. A following breakdown of this three-way-interaction into a two-way-interaction for left and right anodal stimulated subjects separately revealed a significant stimulation group $\mathrm{x}$ time interaction for both current flow directions [right anodal: wald- $\chi^{2}(1,86)=4.41, p=0.036$; left anodal: wald- $\left.\chi^{2}(1,81)=4.15, p=0.042\right]$. Post-hoc pairedsample $t$-tests showed a significant decrease of state anxiety in all 4 groups [right anodal real: difference $=-3.58, t_{(25)}$ $=-3.21, p=0.004$; right anodal sham: difference $=-7.65$, $t_{(16)}=-4.64, p<0.001$; left anodal real: difference $=-7.92$, $t_{(23)}=-5.18, p<0.001$; left anodal sham: difference $=$ $\left.-4.38, t_{(15)}=-4.26, p=0.001\right]$. Further independent-sample $t$-tests compared the change of state anxiety from the rating before to the rating after extinction learning between real- and sham-stimulated subjects again for both current flow directions separately. These tests showed a significant lower decrease of state anxiety in real- compared to sham-stimulated subjects in the right anodal stimulated group [difference $=-4.07, t_{(41)}=$ $-2.12, p=0.040]$. In contrary, in the left anodal stimulated group real-stimulated subjects had compared to their shamstimulated control group a trend-significant higher decrease of state anxiety during extinction [difference $=3.54, t_{(38)}$ $=1.92, p=0.062$; see Figure 7 ). Thus, right anodal tDCS attenuated the reduction of state anxiety during extinction learning. For an overview about the SCR data of all time points that were used for statistical analysis see Supplementary Figure 1.

\section{DISCUSSION}

The results of this study indicated a successful improvement of early extinction learning with a faster loss of CS+/CSdiscrimination and an earlier decrease of reaction on $\mathrm{CS}+$ in both real-stimulation groups. But a crucial limitation to this result is, that the faster CS+/CS- discrimination loss is not only driven 
A Sham-Stimulation, Right Anodal

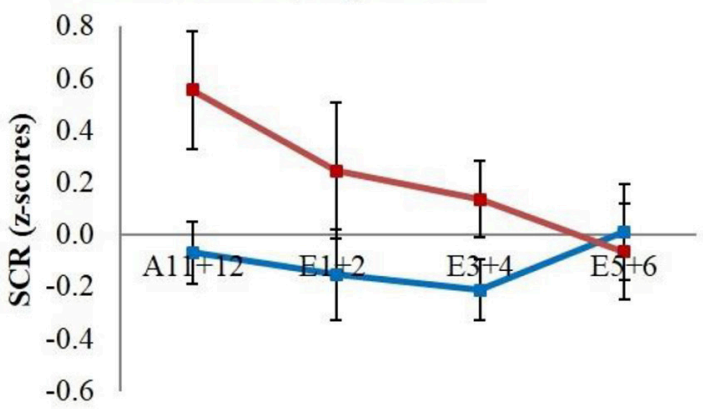

C Real-Stimulation, Right Anodal

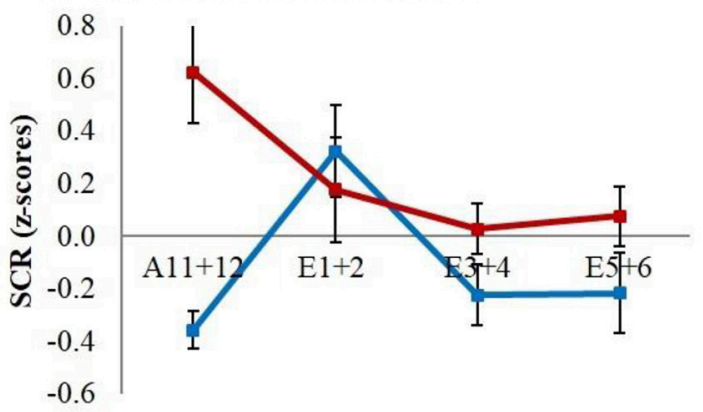

B Sham-Stimulation, Left Anodal

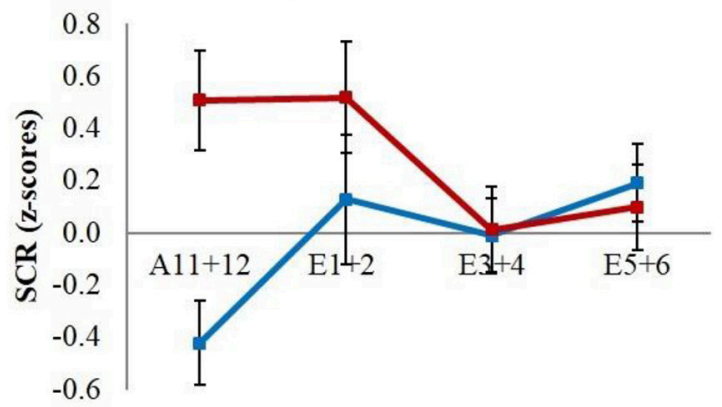

D Real-Stimulation, Left Anodal

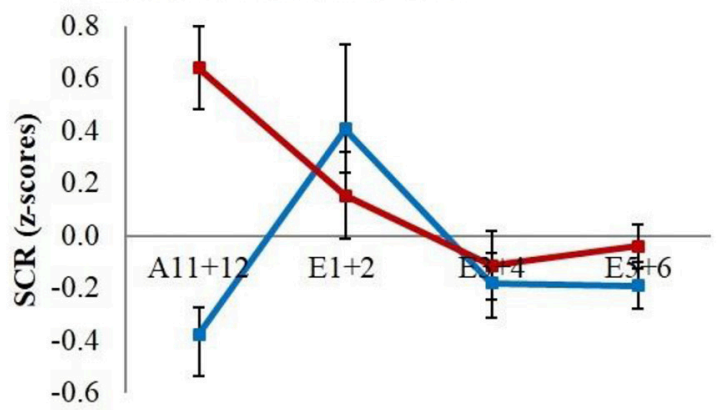

$=\mathrm{CS}-\mathrm{CS}+$

FIGURE 3 | SCR during early extinction. Displayed are z-scored SCR values with their standard errors for early extinction learning separated for sham- and real-stimulated groups and both current flow directions. A11 $12=$ acquisition trials 11 and $12, E 1+2=$ extinction trials 1 and $2, E 3+4=$ extinction trials 3 and 4 , E5+6 $=$ extinction trials 5 and 6.

\section{A Sham-Stimulation}

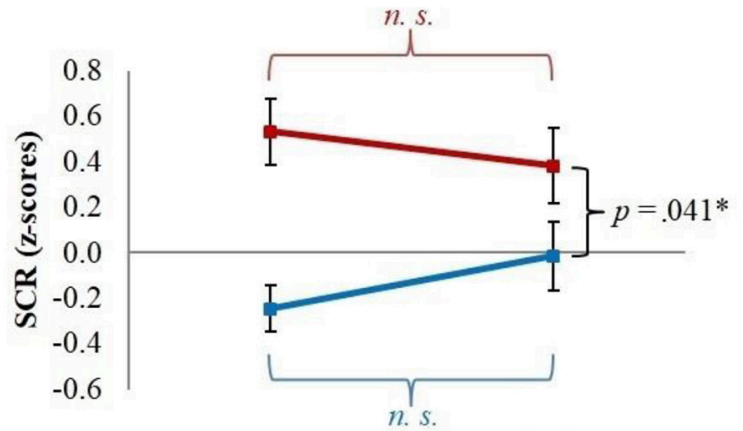

$\mathrm{A} 11+12$
B Real-Stimulation

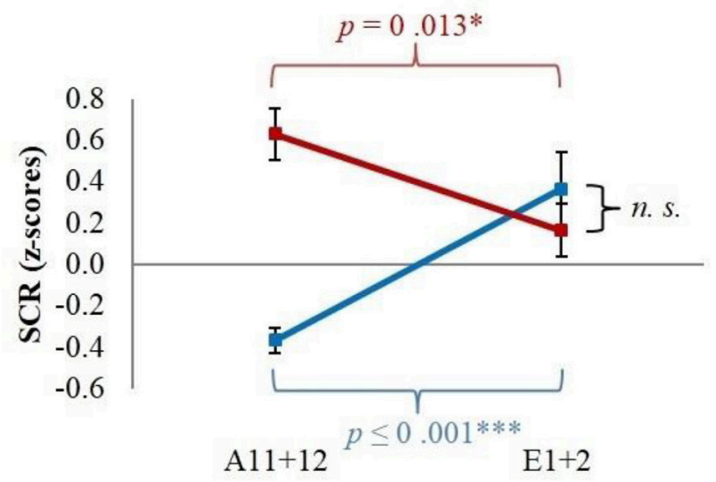

$=-\mathrm{CS}-$

$-\mathrm{CS}+$

FIGURE 4 | Improvement of early extinction learning. Displayed are z-scored SCR values with their standard errors for the last two acquisition (A11+12) and first two extinction trials $(\mathrm{E} 1+2)$ for sham- and real-stimulated groups. Both real-stimulation groups showed a significant reaction decrease on CS+ and increase on CS- and a diminishing of CS+/CS- discrimination, whereas no significant reaction changes occurred in the sham-stimulation groups.

by the reaction loss on CS+ but also by an unexpected initial increase of reaction on CS-. The additional explorative analysis of late extinction learning revealed a short initial increase of CS- reaction again at the beginning of the second extinction block in both stimulation groups. Contrary to our hypotheses, we found no differences in the current flow direction during early extinction learning. Only the explorative analysis of late extinction showed that the averaged reaction over CS+ and 


\section{A Sham-Stimulation}

B Real-Stimulation

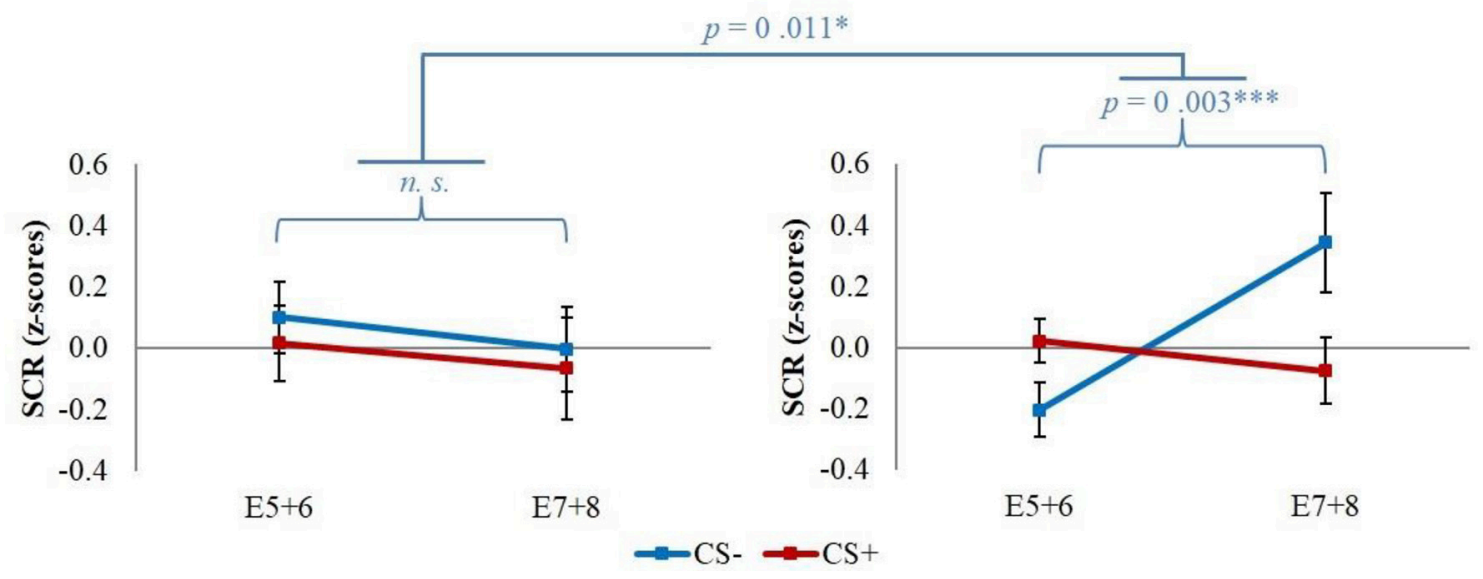

FIGURE 5 | Initial CS- increase during late extinction in both real-stimulated groups. Displayed are z-scored SCR values with their standard errors for CS+ and CSduring the last two trials of the first extinction block $(E 5+6)$ and the first two trials of the second extinction block (E7+8). The real-stimulated groups showed a significantly higher increase of CS- reaction.

CS- had a higher increase between the end of the first and the beginning of the second extinction block in the left anodal compared to the right anodal real-stimulation group. Thus, according to this explorative analysis, right anodal current flow seemed to be the preferred direction at first glance. But on the contrary, questionnaires revealed a lower loss of state anxiety during extinction in exactly this right anodal real-stimulation group compared to its control group.

Surprisingly, extinction learning took place very fast and the participants needed only 4 extinction trials for a diminishing of their conditioned reaction. Thus, we had to correct our definition of "early extinction," which was meant to take place during the whole first extinction block. As a temporal narrowing analysis was performed, we, however, recognized that our effect took place during the first half of actual extinction learning. Some other studies, that used similar conditioning paradigms with neutral looking faces as CSs and a scream as UCS, showed similarly short extinction learning phases. E. g. in Abend et al. (2016) CS+/CSdiscrimination diminished during the first 4 and in Guhn et al. (2014) during the first 6 extinction trials (Guhn et al., 2014; Abend et al., 2016).

\section{Comparison to Prior Studies}

The improvement of early extinction learning in this study resembles the effects that Guhn et al. (2014) could achieve by rTMS of the prefrontal cortex prior to extinction learning. Guhn's work did indeed lead our decision to expect tDCS effects notably during early learning processes. Another rTMS study by Raij et al. (2017) showed again a possible improvement of early extinction learning, but because of methodological manners, they could not state with certainty if their effect took place during early extinction learning or extinction recall.

So far, no study, that tried to modulate extinction via tDCS, had effects during early extinction learning. We cannot compare

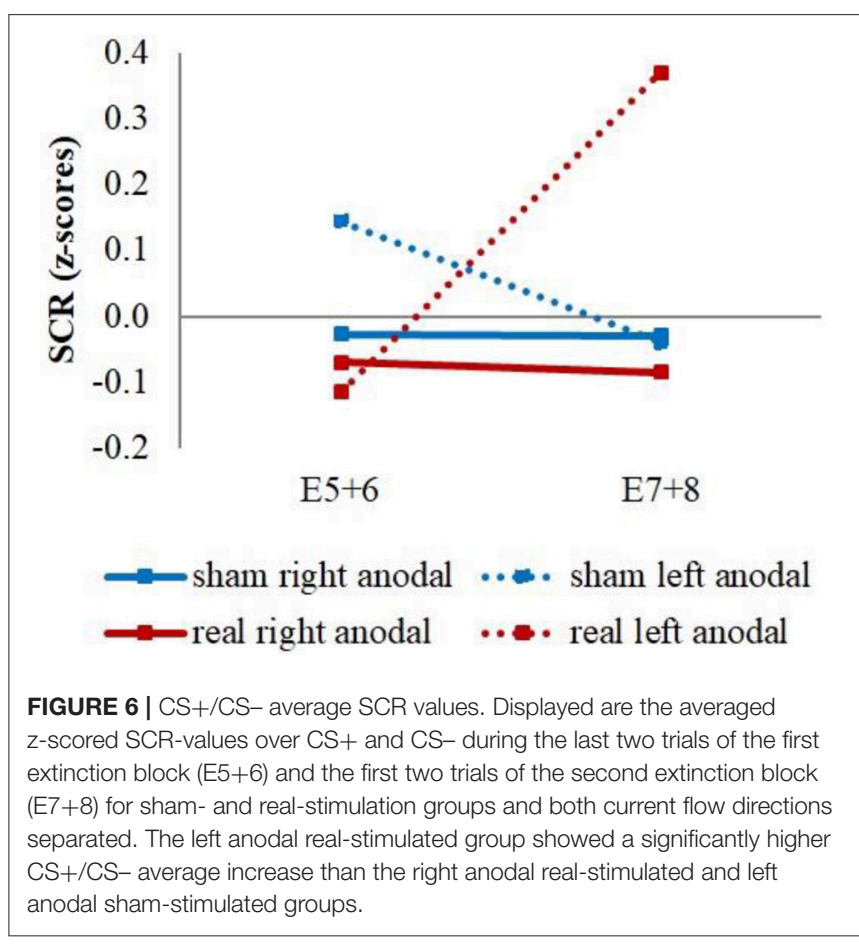

our effects to the extinction recall findings of Van't Wout et al. (2017) because we performed no extinction recall testing in our study. Between our effects and van't Wout's study in 2016, that indicated an improvement of late extinction learning, we do not see any parallels. But like Abend et al. (2016) we had a reaction increase on CS-. Compared to Abend's work, which showed a CS- increase during extinction recall, in our study short CS- increases at the beginning of every extinction learning block occurred. Because we did no extinction recall 


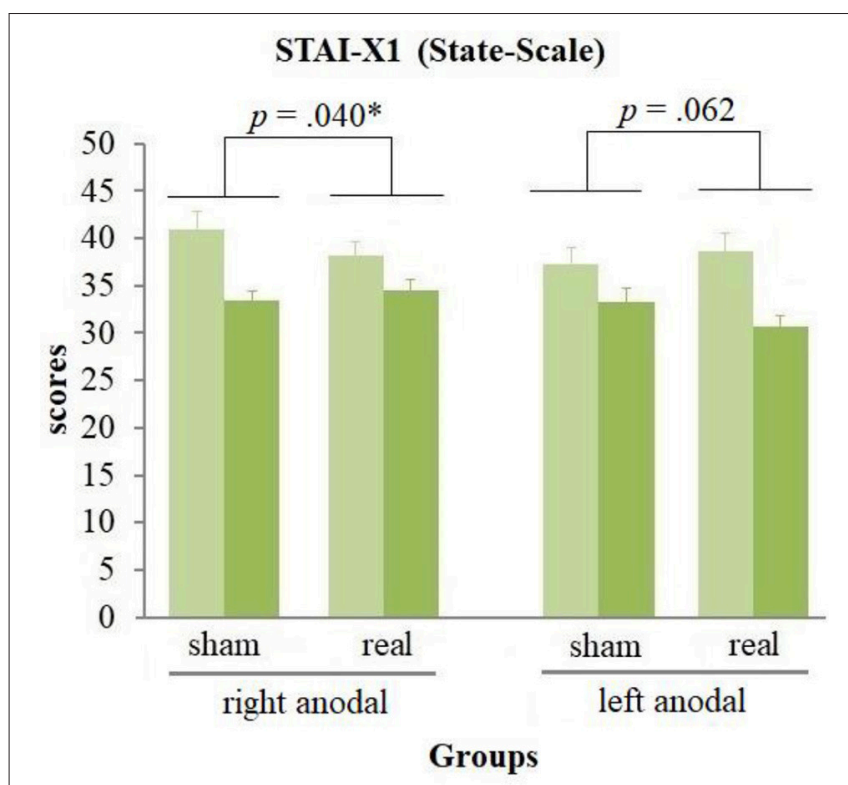

a before extinktion $\square$ after extinktion

FIGURE 7 | State anxiety before and after extinction. Displayed are the scores of the STAI-X1 questionnaires with their standard errors before and after extinction separated for both current flow directions and sham- and real-stimulation. The decrease of state anxiety was in right anodal stimulated participants significantly lower, in left anodal stimulated participants trend-significantly higher for real- compared to sham-stimulated subjects.

testing, we cannot make any statements about how the reaction on CS- could have developed during recall. As mentioned in the introduction, Abend et al. (2016) saw the unintentional stimulation of dorsomedial brain areas and their not temporally specific stimulation protocol as probable reasons for their results. We tried to prevent a CS- reaction increase by avoiding the stimulation of fear-generating dorsomedial brain areas, therefore, this does not seem to have caused the CS- increase. A temporally specific stimulation, in which the stimulation was started $100 \mathrm{~ms}$ after CS onset, could successfully improve extinction in rats (Milad et al., 2004) and in the rTMS approach of Raij et al. (2017). tDCS needs a fade-in and fade-out phase during which the current gets slightly ramped up and down, thus, it is not possible to perform such an exact timed stimulation protocol with tDCS. Guhn et al. (2014) did not use a temporally specific stimulation but could, however, improve extinction learning without a CS- increase. Therefore, other reasons might have caused this undesired side effect (see section Initial Reaction Increase on CS-).

With respect to these prior studies, there is some evidence that the modulation of extinction processes with brain stimulation may especially effect early extinction learning. Further, an impact of tDCS on CS- has occurred twice so far, thus, tDCS seems to enhance fear reactions on safety cues.

\section{Initial Reaction Increase on CS-}

Besides the above-discussed causes for the unexpected short initial increase of reaction on CS-, another possible explanation is that our stimulation interfered with the safety information of the CS-, which is usually acquired during fear conditioning (Pavlov and Anrep, 1927; Rescorla, 1969). There is further evidence that supports this hypothesis. Firstly, our tDCS may have reached the amygdala, which is usually deactivated during safety learning (Schiller et al., 2008; Pollak et al., 2010). Secondly, tDCS did not affect the dorsolateral prefrontal cortex, which happens to be an important control area for safety learning processes (Pollak et al., 2010). Thus, maybe the stimulation led to a primary processing of extinction learning at the expense of safety information processing. This is compliant with the abovedescribed input selective tDCS effect mechanisms. Thirdly, tDCS might have increased the dopaminergic secretion, which is beneficial for extinction learning (see Introduction), but affects safety learning unfavorably, as safety learning was increased under the use of dopamine D2 receptor antagonists (Pollak et al., 2008).

Another possible explanation for the CS- increase is fear generalization. This assumption was supported by a study of Kaczkurkin et al. (2017) who examined PTSD patients. PTSD patients usually generalize fear on an elevated level, thus, they show heightened fear responses to CS+ and to CS- in fear conditioning paradigms (Jovanovic et al., 2010; Norrholm et al., 2011). Kaczkurkin et al. (2017) found a flatter brain activity gradient between CS+ and CS- presentations in these patients in several brain areas. Some of these brain areas like the insula, the hippocampus, and the amygdala were located around the stimulated area in our study and could thereby be affected by the current flow as well. Our tDCS ran during CS+ and CSpresentations equally, thereby it could have led to a reduction of activity gradients between these two stimuli and to anxiety generalization processes.

A third explanation for this phenomenon is the fact that tDCS might have elevated the amount of sustained fear. Several fMRI studies demonstrated that sustained fear is associated with increased brain activity in the bed nucleus of the stria terminalis (BNST) and the insula (Munsterkotter et al., 2015; Herrmann et al., 2016b; Brinkmann et al., 2017). These brain areas were located around the area of our current flow as well, therefore, their affection could have elevated the level of sustained fear and created the reaction increase on CS-. The higher reaction increase on averaged CS+ and CS- in the left anodal realstimulation group at the beginning of the second extinction block is compliant with this hypothesis, too. It has already been proven that right anodal tDCS of the lateral inferior frontal gyrus enhances emotional regulation processes and decreases anxiety reactions during sustained fear phases (Herrmann et al., 2016a). So, in our study, the right anodal stimulation could have led to a better control of sustained fear and thus countered the reaction increase.

\section{Effects of the Current Flow Direction}

Against our expectations, the current flow direction had no influence on the tDCS effects during early extinction learning. Only evidence from late extinction and state anxiety questionnaires revealed current flow direction effects. 
Left anodal stimulated participants showed a higher initial increase on averaged CS+/CS- reaction in the second extinction block, so right anodal seemed to be advantageous at first glance. A possible explanation for this phenomenon could be that one brain area, which lies definitively nearer the activating effect of the anode during right anodal stimulation, namely the right lateral prefrontal cortex, is especially involved in emotional regulation processes as already said. Klumpers et al. (2010) revealed that the activity of the right lateral prefrontal cortex was correlated with downregulation of anxiety in a sustained fear paradigm. The activation of the right vmPFC, which is involved in the processing of negative emotions, was decreased simultaneously. Klumpers et al. (2010) concluded that the right lateral prefrontal cortex downregulates the right vmPFC and controls anxiety thereby. Herrmann et al. (2016a) could use the evidence from Klumpers's paper by increasing emotional regulation during a sustained fear phase through tDCS of the right inferior frontal gyrus. Thus, right anodal stimulation in our study could have increased emotional regulation, too.

But right anodal stimulation had some disadvantages as well. State anxiety decreased significantly less in the right anodal realstimulated group and trend-significantly more in the left anodal real-stimulated group compared to their respective control groups, indicating that left anodal stimulation was the better choice. As there is some evidence that the right vmPFC is highly involved in the processing of negative emotions, one could imagine that a left-sided vmPFC activation could reduce anxiety better. In rat studies, a high rate of dopamine turnover, as well as a lesion of the right vmPFC, led to an increase in anxiety and stress. This indicates that dopamine reduces the activity of the right vmPFC and that the activity the right vmPFC increases the stress level (Thiel and Schwarting, 2001; Sullivan and Gratton, 2002). Consistent with this data from rats, human patients with rightsided vmPFC lesions showed in contrast to left lesioned patients, no anticipatory SCR in the Iowa gambling task, had an abnormal social behavior and problems with emotional processing (Tranel et al., 2002). Furthermore, a meta-analysis revealed a decreased left cortical activation in patients with an anxiety disorder (Thibodeau et al., 2006).

Therefore, the current flow direction can have different advantages or disadvantages. The decision which direction to prefer should be made with regard to the desired effects of the stimulation. Especially the use of right anodal tDCS should be taken with caution in anxiety patients in a clinical context as it seems to lower the loss of state anxiety compared to sham-stimulation during extinction learning.

\section{The Mechanism for Extinction Improvement}

We cannot say with certainty which of the above-presented tDCS effect mechanism was the main reason for the improvement of extinction learning in our study. The direct activation of the vmPFC, especially its left side, is a feasible option, but the elevation of the dopamine secretion, which is an important neurotransmitter for extinction processes, is a possible explanation, too. Furthermore, the activation of corticocortical pathways through tangential currents and thus an altered communication between several brain areas is worth considering as well.

In addition to the enhancement of extinction learning, tDCS could have elicited a more sensitive reaction to prediction errors and supported extinction learning thereby, too. The principle behind the concept of prediction errors is that learning arrives from the violation of expectations. Referring to extinction learning, this means that extinction is-at least partially-driven by the violation of the expectation that the UCS will occur after the CS+, which is a negative aversive prediction error. Thus, the main learning through prediction errors takes place at the beginning of the extinction phase, when the expectancy of the UCS is still strong (Rescorla and Wagner, 1972; Schultz and Dickinson, 2000; Niv and Schoenbaum, 2008). On the neuronal level, especially the transmitter dopamine is suspected to establish the link between extinction learning and prediction errors (Raczka et al., 2011; Berg et al., 2014).

There is some evidence which makes an involvement of enhanced prediction error processing in our study considerable. Firstly, our effect occurred only during very early extinction learning and thus during the period, in which the greatest prediction errors take place. Secondly, there was not only a very fast reaction loss on $\mathrm{CS}+$ but also on $\mathrm{CS}-$ (after its initial increase). Classical extinction is generally understood as a relearning process of the CS-UCS-association (Bouton, 2002), thereby, it does not respond to reaction changes on the CS-, which was never paired with the UCS and thus acquired no association that could be relearned. However, through several possible mechanisms declared above, the CSacquired a negative connotation in our study and-referring to prediction errors-this expectation of a negative outcome was then disappointed during the CS- presentations in the extinction phase. A feasible background mechanism of the enhanced prediction error processing in our study is that some brain areas, namely the vmPFC, middle temporal gyri and left lateral orbital gyrus, whose activity was also associated with prediction errors during extinction learning in a fMRI study by Spoormaker et al. (2011), were located around the current flow area of our tDCS as well. Thus, a tDCS induced activity increase in these areas could have mediated the effect on prediction error processing.

\section{Limitations}

This study has some important limitations that require further research to state the effects of tDCS on extinction processes more clearly.

A first limitation is the sample size. Overall, we had 84 subjects for our analysis, which seems to be adequate at first glance, but considering the splitting into 4 groups made it rather modest. Further, about one-quarter of our participants had to be excluded due to insufficient conditioning. Exclusion rates in other studies, which used similar exclusion criteria, varied. E. g. Asthana et al. (2013) had to exclude only $14 \%$ of their subjects because of insufficient conditioning, whereas it was 
nearly 20\% in Phelps et al. (2004) and Van't Wout et al. (2017). Lonsdorf et al. (2017) stated in her review that performancebased exclusion can easily lead to exclusion rates of over $50 \%$. Additionally, despite blinding around $70 \%$ of our participants were able to evaluate their group assignment right. Poreisz et al. (2007), who investigated methodological manners regarding tDCS, declared a far lower detection rate with less than $20 \%$. Our electrodes were applied on the face near the eye, where the skin is rather sensitive. This could be a reason for our high detection rate.

Secondly, the main stimulation target of this study, the vmPFC, plays a key role for extinction learning, consolidation, and recall (see Background), but it also seems to be important for the suppression of fear reactions. Several studies indicated that stimulation of the vmPFC led to a suppression of conditioned fear expression in rats (Milad and Quirk, 2002; Vidal-Gonzalez et al., 2006). The background mechanism of these reduced fear reactions can be explained by an improvement of extinction learning, but another explanation could be the simple reduction of fear expressions. Equally, we cannot definitively decide whether the tDCS in our study improved extinction learning or just suppressed fear expressions.

Another limitation is that tDCS started directly after the acquisition phase, so, it took place before the consolidation process of acquisition learning was completed. Some data suggest that extinction learning which takes place directly after conditioning has distinct neuronal mechanisms compared to extinction learning that is started after the completion of the consolidation of fear acquisition. For immediate extinction learning deleting processes seem to play a crucial role, whereas delayed extinction learning is rather a new associative learning process (Myers et al., 2006). But some of these data could not be replicated completely, thus, the data situation regarding this topic is still inconsistent (Herry et al., 2010; Lueken and Maslowski, 2012). Nevertheless, to prove the validity of our effects this study's tDCS protocol has to be replicated in a paradigm with fear conditioning and extinction learning on different days like it was e.g., in Abend et al. (2016) performed.

A further major limitation is that we did not implement an extinction recall testing. Thus, we cannot state if our effect is long-lasting, but the improvement of extinction learning can only have a positive effect on exposure therapies if the effect maintains between the therapy sessions. To make any clinical implications a testing of our tDCS protocol on extinction recall, like e.g., Abend et al. (2016) and Van't Wout et al. $(2016,2017)$, did, is necessary first.

Additionally, the effects of tDCS on extinction in our study were rather short and involved only the first two trials. But relatively speaking, the first two extinction trials represented the first half of the extinction learning process because after trial 4 the CS+/CS- discrimination vanished in our sham groups, too. But to ensure that our tDCS effects are strong and long-lasting enough to improve extinction learning in anxiety patients, which is typically a more complicated and protracted procedure than it is in healthy persons (Robinson et al., 2012), our stimulation protocol should be tested in these patients, too. Therefore, a direct testing during exposure settings would be useful because the long-term profit of this study was to find a tDCS protocol that can boost exposure therapy effects. Thus, the transfer of our effects to exposure therapy needs to be explicitly tested to assess whether a clinically relevant therapy improvement can be achieved.

But before this study's stimulation protocol should be tested in anxiety patients, further research is still needed. Besides our positive effects, we had a reaction increase on CS-, which is a crucial limitation for the results of this study. On the one hand it was jointly responsible for our main effect, the stronger CS+/CS- discrimination increase and on the other hand it could cause negative effects like a sustained fear increase, disruption of safety learning or anxiety generalization in patients. Therefore, the reason for the reaction increase on CS- needs to be explored. Additionally, some of our results indicate that the reduction of state anxiety during extinction learning is attenuated by right anodal tDCS. Subsequently, the tDCS protocol should be modified to prevent negative consequences for anxiety patients and ensure the safety of the stimulation.

\section{CONCLUSION}

The current study has shown that tDCS with a bitemporal electrode positioning around the EEG positions F7 and F8 aimed to stimulate the vmPFC can improve extinction learning through a stronger CS+/CS- discrimination loss and a faster reaction decrease on CS+. But a crucial negative side effect, which also drove the CS+/CS- discrimination loss jointly, was an unexpected initial reaction increase on CS-. Such a reaction increase on CS- did not only occur in our study, it has already been observed by Abend et al. (2016). Thus, the background of this aspect should be investigated further. We assume that the interference with safety learning, fear generalization effects or the elevation of sustained fear are feasible reasons. Further, we discovered that the current flow direction had no effect during early extinction, but distinct advantages and disadvantages for the whole course of extinction. Left anodal stimulation led to a greater loss of subjectively rated state anxiety, whereas right anodal stimulation seemed to enhance emotional regulation. The intended stimulation effects and the anxiety extent of the stimulated population should thereby influence the decision which current flow direction to prefer.

Overall, the results of this study provide an important basis for the improvement of exposure therapies with tDCS. But for convincing remarks on the therapy success, the CS- increase and the transfer of our effects on anxiety patients in exposure situations must be explored first.

\section{AUTHOR CONTRIBUTIONS}

ND and SH collected and analyzed data, ND drafted the paper. $\mathrm{TP}$ and $\mathrm{MH}$ designed the study, supervised the data collection 
and analyzes and revised the paper. All authors approved the final version of the paper.

\section{FUNDING}

This study was funded by SFB TRR 58, C06, and C07. The funding sources had no role in study design, data collection, and analyses, decision to publish, or preparation of the manuscript.

\section{REFERENCES}

Abend, R., Jalon, I., Gurevitch, G., Sar-El, R., Shechner, T., Pine, D. S., et al. (2016). Modulation of fear extinction processes using transcranial electrical stimulation. Transl. Psychiatry 6:e913. doi: 10.1038/tp.2016.197

Abraham, A. D., Neve, K. A., and Lattal, K. M. (2016). Activation of D1/5 dopamine receptors: a common mechanism for enhancing extinction of fear and reward-seeking behaviors. Neuropsychopharmacology 41, 2072-2081. doi: $10.1038 /$ npp. 2016.5

Agarwal, S. M., Bose, A., Shivakumar, V., Narayanaswamy, J. C., Chhabra, H., Kalmady, S. V., et al. (2016). Impact of antipsychotic medication on transcranial direct current stimulation (tDCS) effects in schizophrenia patients. Psychiatry Res. 235, 97-103. doi: 10.1016/j.psychres.2015.11.042

Asthana, M., Nueckel, K., Mühlberger, A., Neueder, D., Polak, T., Domschke, K., et al. (2013). Effects of transcranial direct current stimulation on consolidation of fear memory. Front. Psychiatry 4:107. doi: 10.3389/fpsyt.2013.00107

Bach, D. R. (2014). A head-to-head comparison of SCRalyze and Ledalab, two model-based methods for skin conductance analysis. Biol. Psychol. 103, 63-68. doi: 10.1016/j.biopsycho.2014.08.006

Bach, D. R., Flandin, G., Friston, K. J., and Dolan, R. J. (2009). Time-series analysis for rapid event-related skin conductance responses. J. Neurosci. Methods 184, 224-234. doi: 10.1016/j.jneumeth.2009.08.005

Bach, D. R., Friston, K. J., and Dolan, R. J. (2013). An improved algorithm for model-based analysis of evoked skin conductance responses. Biol. Psychol. 94, 490-497. doi: 10.1016/j.biopsycho.2013.09.010

Bajbouj, M., and Padberg, F. (2014). A perfect match: noninvasive brain stimulation and psychotherapy. Eur. Arch. Psychiatry Clin. Neurosci. 264 (Suppl. 1), S27-S33. doi: 10.1007/s00406-014-0540-6

Bandelow, B., Wiltink, J., Alpers, G. W., Benecke, C., Deckert, J., Eckhardt-Henn, A., et al. (2014). Deutsche S3-Leitlinie Behandlung von Angststörungen.

Beauregard, M. (2014). Functional neuroimaging studies of the effects of psychotherapy. Dialogues Clin. Neurosci. 16, 75-81.

Ben-Shakhar, G. (1985). Standardization within individuals: a simple method to neutralize individual differences in skin conductance. Psychophysiology 22, 292-299. doi: 10.1111/j.1469-8986.1985.tb01603.x

Berg, B. A., Schoenbaum, G., and McDannald, M. A. (2014). The dorsal raphe nucleus is integral to negative prediction errors in Pavlovian fear. Eur. J. Neurosci. 40, 3096-3101. doi: 10.1111/ejn.12676

Bikson, M., Inoue, M., Akiyama, H., Deans, J. K., Fox, J. E., Miyakawa, H., et al. (2004). Effects of uniform extracellular DC electric fields on excitability in rat hippocampal slices in vitro. J. Physiol. 557(Pt 1), 175-190. doi: 10.1113/jphysiol.2003.055772

Bikson, M., Name, A., and Rahman, A. (2013). Origins of specificity during tDCS: anatomical, activity-selective, and input-bias mechanisms. Front. Hum. Neurosci. 7:688. doi: 10.3389/fnhum.2013.00688

Boucsein, W., Fowles, D. C., Grimnes, S., Ben-Shakhar, G., Roth, W. T., Dawson, M. E., et al. (2012). Publication recommendations for electrodermal measurements. Psychophysiology 49, 1017-1034. doi: $10.1111 /$ j.1469-8986.2012.01384.x

Bouton, M. E. (2002). Context, ambiguity, and unlearning: sources of relapse after behavioral extinction. Biol. Psychiatry 52, 976-986. doi: 10.1016/S0006-3223(02)01546-9

Bradley, M. M., and Lang, P. J. (1999). International Affective Digitized Sounds (IADS): Stimuli, Instruction Manual and Affective Ratings (Tech. Rep. No. $B-2)$. The Center for Research in Psychophysiology; University of Florida, Gainesville, FL.
This publication was supported by the Open Access Publication Fund of the University of Wuerzburg.

\section{SUPPLEMENTARY MATERIAL}

The Supplementary Material for this article can be found online at: https://www.frontiersin.org/articles/10.3389/fnbeh. 2018.00076/full\#supplementary-material

Brinkmann, L., Buff, C., Feldker, K., Tupak, S. V., Becker, M. P. I., Herrmann, M. J., et al. (2017). Distinct phasic and sustained brain responses and connectivity of amygdala and bed nucleus of the stria terminalis during threat anticipation in panic disorder. Psychol. Med. 47, 2675-2688. doi: 10.1017/S0033291717001192

Broeder, S., Nackaerts, E., Heremans, E., Vervoort, G., Meesen, R., Verheyden, G., et al. (2015). Transcranial direct current stimulation in Parkinson's disease: neurophysiological mechanisms and behavioral effects. Neurosci. Biobehav. Rev. 57, 105-117. doi: 10.1016/j.neubiorev.2015.08.010

Gottfried, J. A., and Dolan, R. J. (2004). Human orbitofrontal cortex mediates extinction learning while accessing conditioned representations of value. Nat. Neurosci. 7, 1144-1152. doi: 10.1038/nn1314

Guhn, A., Dresler, T., Andreatta, M., Müller, L. D., Hahn, T., Tupak, S. V., et al. (2014). Medial prefrontal cortex stimulation modulates the processing of conditioned fear. Front. Behav. Neurosci. 8:44. doi: 10.3389/fnbeh.2014.00044

Guhn, A., Dresler, T., Hahn, T., Mühlberger, A., Ströhle, A., Deckert, J., et al. (2012). Medial prefrontal cortex activity during the extinction of conditioned fear: an investigation using functional near-infrared spectroscopy. Neuropsychobiology 65, 173-182. doi: 10.1159/000337002

Hautzinger, M., and Bailer, M. (1993). Allgemeine Depressions Skala. Manual. Gottingen; Deutschland: Beltz Test GmbH.

Herrmann, M. J., Beier, J. S., Simons, B., and Polak, T. (2016a). Transcranial Direct Current Stimulation (tDCS) of the right inferior frontal gyrus attenuates skin conductance responses to unpredictable threat conditions. Front. Hum. Neurosci. 10:352. doi: 10.3389/fnhum. 2016.00352

Herrmann, M. J., Boehme, S., Becker, M. P., Tupak, S. V., Guhn, A., Schmidt, B., et al. (2016b). Phasic and sustained brain responses in the amygdala and the bed nucleus of the stria terminalis during threat anticipation. Hum. Brain Mapp. 37, 1091-1102. doi: 10.1002/hbm.23088

Herrmann, M. J., Katzorke, A., Busch, Y., Gromer, D., Polak, T., Pauli, P., et al. (2017). Medial prefrontal cortex stimulation accelerates therapy response of exposure therapy in acrophobia. Brain Stimul. 10, 291-297. doi: 10.1016/j.brs.2016.11.007

Herry, C., Ciocchi, S., Senn, V., Demmou, L., Müller, C., and Lüthi, A. (2008). Switching on and off fear by distinct neuronal circuits. Nature 454, 600-606. doi: 10.1038/nature07166

Herry, C., Ferraguti, F., Singewald, N., Letzkus, J. J., Ehrlich, I., and Lüthi, A. (2010). Neuronal circuits of fear extinction. Eur. J. Neurosci. 31, 599-612. doi: 10.1111/j.1460-9568.2010.07101.x

Hikind, N., and Maroun, M. (2008). Microinfusion of the D1 receptor antagonist, $\mathrm{SCH} 23390$ into the IL but not the BLA impairs consolidation of extinction of auditory fear conditioning. Neurobiol. Learn. Mem. 90, 217-222. doi: 10.1016/j.nlm.2008. 03.003

Hilton, A. (1969). Partial reinforcement of a conditioned emotional response in rats. J. Comp. Physiol. Psychol. 69, 253-260. doi: 10.1037/h0028235

Jovanovic, T., Norrholm, S. D., Blanding, N. Q., Davis, M., Duncan, E., Bradley, B., et al. (2010). Impaired fear inhibition is a biomarker of PTSD but not depression. Depress. Anxiety 27, 244-251. doi: 10.1002/da.20663

Kaczkurkin, A. N., Burton, P. C., Chazin, S. M., Manbeck, A. B., Espensen-Sturges, T., Cooper, S. E., et al. (2017). Neural substrates of overgeneralized conditioned fear in PTSD. Am. J. Psychiatry 174, 125-134. doi: 10.1176/appi.ajp.2016.15121549

Kempe, R., Huang, Y., and Parra, L. C. (2014). Simulating pad-electrodes with high-definition arrays in transcranial electric stimulation. J. Neural Eng. 11:026003. doi: 10.1088/1741-2560/11/2/026003 
Klumpers, F., Raemaekers, M. A., Ruigrok, A. N., Hermans, E. J., Kenemans, J. L., and Baas, J. M. (2010). Prefrontal mechanisms of fear reduction after threat offset. Biol. Psychiatry 68, 1031-1038. doi: 10.1016/j.biopsych.2010.09.006

Knight, D. C., Smith, C. N., Cheng, D. T., Stein, E. A., and Helmstetter, F. J. (2004). Amygdala and hippocampal activity during acquisition and extinction of human fear conditioning. Cogn. Affect. Behav. Neurosci. 4, 317-325. doi: 10.3758/CABN.4.3.317

Lau, J. Y., Lissek, S., Nelson, E. E., Lee, Y., Roberson-Nay, R., Poeth, K., et al. (2008). Fear conditioning in adolescents with anxiety disorders: results from a novel experimental paradigm. J. Am. Acad. Child Adolesc. Psychiatry 47, 94-102. doi: 10.1097/chi.0b01e31815a5f01

Laux, L., Glanzmann, P., Schaffner, P., and Spielberger, C. D. (1981). Das StateTrait-Angstinventar (Testmappe mit Handanweisung, Fragebogen STAI-G Form $X 1$ und Fragebogen STAI-G Form X2). W. Beltz.

Liang, K. Y., and Zeger, S. L. (1986). Longitudinal data-analysis using generalized linear-models. Biometrika 73, 13-22. doi: 10.1093/biomet/73.1.13

Lonsdorf, T. B., Menz, M. M., Andreatta, M., Fullana, M. A., Golkar, A., Haaker, J., et al. (2017). Don't fear 'fear conditioning': methodological considerations for the design and analysis of studies on human fear acquisition, extinction, and return of fear. Neurosci. Biobehav. Rev. 77, 247-285. doi: 10.1016/j.neubiorev.2017.02.026

Lueken, U., and Maslowski, N. I. (2012). "The neural substrates of fear extinction," in Exposure Therapy: Rethinking the Model - Refining the Method, eds P. Neudeck and H.-U. Wittchen (New York, NY: Springer-Verlag), 65-88.

Marin, M. F., Camprodon, J. A., Dougherty, D. D., and Milad, M. R. (2014). Device-based brain stimulation to augment fear extinction: implications for PTSD treatment and beyond. Depress. Anxiety 31, 269-278. doi: $10.1002 /$ da.22252

McNally, R. J. (2007). Mechanisms of exposure therapy: how neuroscience can improve psychological treatments for anxiety disorders. Clin. Psychol. Rev. 27, 750-759. doi: 10.1016/j.cpr.2007.01.003

Milad, M. R., and Quirk, G. J. (2002). Neurons in medial prefrontal cortex signal memory for fear extinction. Nature 420, 70-74. doi: 10.1038/nature 01138

Milad, M. R., Vidal-Gonzalez, I., and Quirk, G. J. (2004). Electrical stimulation of medial prefrontal cortex reduces conditioned fear in a temporally specific manner. Behav. Neurosci. 118, 389-394. doi: 10.1037/0735-7044.118.2.389

Morgan, M. A., Schulkin, J., and LeDoux, J. E. (2003). Ventral medial prefrontal cortex and emotional perseveration: the memory for prior extinction training. Behav. Brain Res. 146, 121-130. doi: 10.1016/j.bbr.2003.09.021

Mowrer, O. H. (1956). Two-factor learning theory reconsidered, with special reference to secondary reinforcement and the concept of habit. Psychol. Rev. 63, 114-128. doi: 10.1037/h0040613

Munsterkotter, A. L., Notzon, S., Redlich, R., Grotegerd, D., Dohm, K., Arolt, V., et al. (2015). Spider or no spider? Neural correlates of sustained and phasic fear in spider phobia. Depress. Anxiety 32, 656-663. doi: 10.1002/da.22382

Myers, K. M., and Davis, M. (2002). Behavioral and neural analysis of extinction. Neuron 36, 567-584. doi: 10.1016/S0896-6273(02)01064-4

Myers, K. M., Ressler, K. J., and Davis, M. (2006). Different mechanisms of fear extinction dependent on length of time since fear acquisition. Learn. Mem. 13, 216-223. doi: 10.1101/lm.119806

Nitsche, M. A., and Paulus, W. (2000). Excitability changes induced in the human motor cortex by weak transcranial direct current stimulation. J. Physiol. $527(\mathrm{Pt}$ 3), 633-639. doi: 10.1111/j.1469-7793.2000.t01-1-00633.x

Niv, Y., and Schoenbaum, G. (2008). Dialogues on prediction errors. Trends Cogn. Sci. 12, 265-272. doi: 10.1016/j.tics.2008.03.006

Norrholm, S. D., Jovanovic, T., Olin, I. W., Sands, L. A., Karapanou, I., Bradley, B., et al. (2011). Fear extinction in traumatized civilians with posttraumatic stress disorder: relation to symptom severity. Biol. Psychiatry 69, 556-563. doi: $10.1016 /$ j.biopsych.2010.09.013

Oldfield, R. C. (1971). The assessment and analysis of handedness: the Edinburgh inventory. Neuropsychologia 9, 97-113. doi: 10.1016/0028-3932(71)90067-4

Pavlov, I. P., and Anrep, G. V. (1927). Conditioned Reflexes; An Investigation of the Physiological Activity of the Cerebral Cortex. London: Oxford University Press; Humphrey Milford.

Phelps, E. A., Delgado, M. R., Nearing, K. I., and LeDoux, J. E. (2004). Extinction learning in humans: role of the amygdala and vmPFC. Neuron 43, 897-905. doi: $10.1016 /$ j.neuron.2004.08.042
Pollak, D. D., Monje, F. J., Zuckerman, L., Denny, C. A., Drew, M. R., and Kandel, E. R. (2008). An animal model of a behavioral intervention for depression. Neuron 60, 149-161. doi: 10.1016/j.neuron.2008.07.041

Pollak, D. D., Rogan, M. T., Egner, T., Perez, D. L., Yanagihara, T. K., and Hirsch, J. (2010). A translational bridge between mouse and human models of learned safety. Ann. Med. 42, 115-122. doi: 10.3109/07853890903583666

Poreisz, C., Boros, K., Antal, A., and Paulus, W. (2007). Safety aspects of transcranial direct current stimulation concerning healthy subjects and patients. Brain Res. Bull. 72, 208-214. doi: 10.1016/j.brainresbull.2007.01.004

Quirk, G. J., Russo, G. K., Barron, J. L., and Lebron, K. (2000). The role of ventromedial prefrontal cortex in the recovery of extinguished fear. J. Neurosci. 20, 6225-6231. doi: 10.1523/JNEUROSCI.20-16-06225.2000

Raczka, K. A., Mechias, M. L., Gartmann, N., Reif, A., Deckert, J., Pessiglione, M., et al. (2011). Empirical support for an involvement of the mesostriatal dopamine system in human fear extinction. Transl. Psychiatry 1:e12. doi: $10.1038 /$ tp.2011.10

Rahman, A., Reato, D., Arlotti, M., Gasca, F., Datta, A., Parra, L. C., et al. (2013). Cellular effects of acute direct current stimulation: somatic and synaptic terminal effects. J. Physiol. 591, 2563-2578. doi: 10.1113/jphysiol.2012.247171

Raij, T., Nummenmaa, A., Marin, M. F., Porter, D., Furtak, S., Setsompop, K., et al. (2017). Prefrontal cortex stimulation enhances fear extinction memory in humans. Biol. Psychiatry. doi: 10.1016/j.biopsych.2017.10.022. [Epub ahead of print].

Rauch, S. A., Eftekhari, A., and Ruzek, J. I. (2012). Review of exposure therapy: a gold standard for PTSD treatment. J. Rehabil. Res. Dev. 49, 679-687. doi: 10.1682/JRRD.2011.08.0152

Reato, D., Rahman, A., Bikson, M., and Parra, L. C. (2010). Low-intensity electrical stimulation affects network dynamics by modulating population rate and spike timing. J. Neurosci. 30, 15067-15079. doi: 10.1523/JNEUROSCI.2059-10.2010

Rescorla, R. A. (1969). Conditioned inhibition of fear resulting from negative CS-US contingencies. J. Comp. Physiol. Psychol. 67, 504-509. doi: $10.1037 /$ h0027313

Rescorla, R. A., and Wagner, A. R. (1972). "A theory of pavlovian conditioning: variations in the effectiveness of reinforcement and nonreinforcement," in Classical Conditioning II: Current Research and Theory, ed W.F.P. A. H. Black (New York, NY: Appleton- Century-Crofts), 64-99.

Robinson, O. J., Charney, D. R., Overstreet, C., Vytal, K., and Grillon, C. (2012). The adaptive threat bias in anxiety: amygdala-dorsomedial prefrontal cortex coupling and aversive amplification. Neuroimage 60, 523-529. doi: 10.1016/j.neuroimage.2011.11.096

Schiller, D., Levy, I., Niv, Y., LeDoux, J. E., and Phelps, E. A. (2008). From fear to safety and back: reversal of fear in the human brain. J. Neurosci. 28, 11517-11525. doi: 10.1523/JNEUROSCI.2265-08.2008

Schultz, W., and Dickinson, A. (2000). Neuronal coding of prediction errors. Annu. Rev. Neurosci. 23, 473-500. doi: 10.1146/annurev.neuro.23.1.473

Schurr, B. C., and Runquist, W. N. (1973). Acquisition and extinction of human eyelid conditioned-response as a function of schedule of reinforcement and unconditioned stimulus intensity under 2 masked conditioning procedures. J. Exp. Psychol. 101, 398-401. doi: 10.1037/h0035234

Sierra-Mercado, D. Jr., Corcoran, K. A., Lebrón-Milad, K., and Quirk, G. J. (2006). Inactivation of the ventromedial prefrontal cortex reduces expression of conditioned fear and impairs subsequent recall of extinction. Eur. J. Neurosci. 24, 1751-1758. doi: 10.1111/j.1460-9568.2006.05014.x

Sotres-Bayon, F., Bush, D. E., and LeDoux, J. E. (2007). Acquisition of fear extinction requires activation of NR2B-containing NMDA receptors in the lateral amygdala. Neuropsychopharmacology 32, 1929-1940. doi: $10.1038 /$ sj.npp.1301316

Spoormaker, V. I., Andrade, K. C., Schröter, M. S., Sturm, A., Goya-Maldonado, R., Sämann, P. G., et al. (2011). The neural correlates of negative prediction error signaling in human fear conditioning. Neuroimage 54, 2250-2256. doi: 10.1016/j.neuroimage.2010.09.042

Staib, M., Castegnetti, G., and Bach, D. R. (2015). Optimising a modelbased approach to inferring fear learning from skin conductance responses. J. Neurosci. Methods 255, 131-138. doi: 10.1016/j.jneumeth.2015. 08.009

Sullivan, R. M., and Gratton, A. (2002). Behavioral effects of excitotoxic lesions of ventral medial prefrontal cortex in the rat are hemisphere-dependent. Brain Res. 927, 69-79. doi: 10.1016/S0006-8993(01)03328-5 
Tanaka, T., Takano, Y., Tanaka, S., Hironaka, N., Kobayashi, K., Hanakawa, T., et al. (2013). Transcranial direct-current stimulation increases extracellular dopamine levels in the rat striatum. Front. Syst. Neurosci. 7:6. doi: 10.3389/fnsys.2013.00006

Taylor, S., Zvolensky, M. J., Cox, B. J., Deacon, B., Heimberg, R. G., Ledley, D. R., et al. (2007). Robust dimensions of anxiety sensitivity: development and initial validation of the Anxiety Sensitivity Index-3. Psychol. Assess. 19, 176-188. doi: 10.1037/1040-3590.19.2.176

Thibodeau, R., Jorgensen, R. S., and Kim, S. (2006). Depression, anxiety, and resting frontal EEG asymmetry: a meta-analytic review. J. Abnorm. Psychol. 115, 715-729. doi: 10.1037/0021-843X. 115.4.715

Thiel, C. M., and Schwarting, R. K. (2001). Dopaminergic lateralisation in the forebrain: relations to behavioural asymmetries and anxiety in male Wistar rats. Neuropsychobiology 43, 192-199. doi: 10.1159/000054889

Tottenham, N., Tanaka, J. W., Leon, A. C., McCarry, T., Nurse, M., Hare, T. A., et al. (2009). The NimStim set of facial expressions: judgments from untrained research participants. Psychiatry Res. 168, 242-249. doi: 10.1016/j.psychres.2008.05.006

Tranel, D., Bechara, A., and Denburg, N. L. (2002). Asymmetric functional roles of right and left ventromedial prefrontal cortices in social conduct, decision-making, and emotional processing. Cortex 38, 589-612. doi: 10.1016/S0010-9452(08)70024-8

Van't Wout, M., Mariano, T. Y., Garnaat, S. L., Reddy, M. K., Rasmussen, S. A., and Greenberg, B. D. (2016). Can transcranial direct current stimulation augment extinction of conditioned fear? Brain Stimul. 9, 529-536. doi: 10.1016/j.brs.2016.03.004
Van't Wout, M., Longo, S. M., Reddy, M. K., Philip, N. S., Bowker, M. T., and Greenberg, B. D. (2017). Transcranial direct current stimulation may modulate extinction memory in posttraumatic stress disorder. Brain Behav. 7:e00681. doi: 10.1002/brb3.681

Vidal-Gonzalez, I., Vidal-Gonzalez, B., Rauch, S. L., and Quirk, G. J. (2006). Microstimulation reveals opposing influences of prelimbic and infralimbic cortex on the expression of conditioned fear. Learn. Mem. 13, 728-733. doi: 10.1101/lm.306106

Watson, D., Clark, L. A., and Tellegen, A. (1988). Development and validation of brief measures of positive and negative affect: the PANAS scales. J. Pers. Soc. Psychol. 54, 1063-1070. doi: 10.1037/0022-3514.54.6.1063

Wittchen, H. U., Jacobi, F., Rehm, J., Gustavsson, A., Svensson, M., Jönsson, B. et al. (2011). The size and burden of mental disorders and other disorders of the brain in Europe 2010. Eur. Neuropsychopharmacol. 21, 655-679. doi: 10.1016/j.euroneuro.2011.07.018

Conflict of Interest Statement: The authors declare that the research was conducted in the absence of any commercial or financial relationships that could be construed as a potential conflict of interest.

Copyright (C) 2018 Dittert, Hüttner, Polak and Herrmann. This is an open-access article distributed under the terms of the Creative Commons Attribution License (CC $B Y)$. The use, distribution or reproduction in other forums is permitted, provided the original author(s) and the copyright owner are credited and that the original publication in this journal is cited, in accordance with accepted academic practice. No use, distribution or reproduction is permitted which does not comply with these terms. 
OPEN ACCESS

Edited by:

Roberta Ferrucci,

Fondazione IRCCS Ca' Granda

Ospedale Maggiore Policlinico

(IRCCS), Italy

Reviewed by:

Christoph S. Herrmann,

Carl von Ossietzky University

of Oldenburg, Germany

Thomas Dresler,

Eberhard Karls Universität Tübingen,

Germany

*Correspondence:

Michael Doppelmayr doppelma@uni-mainz.de

Received: 29 November 2017 Accepted: 23 March 2018 Published: 18 April 2018

Citation:

Berger A, Pixa NH, Steinberg F and Doppelmayr M (2018) Brain

Oscillatory and Hemodynamic Activity in a Bimanual Coordination Task Following Transcranial Alternating Current Stimulation (tACS): A Combined EEG-fNIRS Study. Front. Behav. Neurosci. 12:67. doi: 10.3389/fnbeh.2018.00067

\section{Brain Oscillatory and Hemodynamic Activity in a Bimanual Coordination Task Following Transcranial Alternating Current Stimulation (tACS): A Combined EEG-fNIRS Study}

\author{
Alisa Berger ${ }^{1}$, Nils H. Pixa ${ }^{1}$, Fabian Steinberg ${ }^{1}$ and Michael Doppelmayr ${ }^{1,2 *}$ \\ 'Department of Sports Psychology, Institute of Sport Science, Johannes Gutenberg-University Mainz, Mainz, Germany, \\ ${ }^{2}$ Centre for Cognitive Neuroscience, Paris Lodron University of Salzburg, Salzburg, Austria
}

Motor control is associated with synchronized oscillatory activity at alpha (8-12 Hz) and beta $(12-30 \mathrm{~Hz})$ frequencies in a cerebello-thalamo-cortical network. Previous studies demonstrated that transcranial alternating current stimulation (tACS) is capable of entraining ongoing oscillatory activity while also modulating motor control. However, the modulatory effects of tACS on both motor control and its underlying electro- and neurophysiological mechanisms remain ambiguous. Thus, the purpose of this study was to contribute to gathering neurophysiological knowledge regarding tACS effects by investigating the after-effects of $10 \mathrm{~Hz}$ tACS and $20 \mathrm{~Hz}$ tACS at parietal brain areas on bimanual coordination and its concurrent oscillatory and hemodynamic activity. Twenty-four right-handed healthy volunteers (12 females) aged between 18 and 30 ( $M=22.35 \pm 3.62)$ participated in the study and performed a coordination task requiring bimanual movements. Concurrent to bimanual motor training, participants received either $10 \mathrm{~Hz}$ tACS, $20 \mathrm{~Hz}$ tACS or a sham stimulation over the parietal cortex (at P3/P4 electrode positions) for $20 \mathrm{~min}$ via small gel electrodes $\left(3,14 \mathrm{~cm}^{2}\right.$ $\mathrm{Ag} / \mathrm{AgCl}$, amperage $=1 \mathrm{~mA}$ ). Before and three time-points after tACS (immediately, $30 \mathrm{~min}$ and 1 day), bimanual coordination performance was assessed. Oscillatory activities were measured by electroencephalography (EEG) and hemodynamic changes were examined using functional near-infrared spectroscopy (fNIRS). Improvements of bimanual coordination performance were not differently between groups, thus, no tACS-specific effect on bimanual coordination performance emerged. However, physiological measures during the task revealed significant increases in parietal alpha activity immediately following $10 \mathrm{~Hz}$ tACS and $20 \mathrm{~Hz}$ tACS which were accompanied by significant decreases of Hboxy concentration in the right hemispheric motor cortex compared to the sham group. Based on the physiological responses, we conclude that tACS applied at parietal brain areas provoked electrophysiological and hemodynamic changes at brain regions of the motor network which are relevant for bimanual motor behavior. The existence of neurophysiological alterations immediately following 
tACS, especially in the absence of behavioral effects, are elementary for a profound understanding of the mechanisms underlying tACS. The lack of behavioral modifications strengthens the need for further research on tACS effects on neurophysiology and behavior using combined electrophysiological and neuroimaging methods.

Keywords: high-definition tACS, after-effects, alpha oscillations, beta oscillations, Hboxy, bimanual movements

\section{INTRODUCTION}

Neurons in the human brain are interconnected and form functionally specialized networks. It is the temporally precise coordinated communication between and within these networks that enables the brain's complex processing capabilities (Schnitzler and Gross, 2005; Sauseng and Klimesch, 2008; Wach et al., 2013). It is thought that synchronous oscillatory activity represents the basic mechanism for functional communication (Wach et al., 2013; Pollok et al., 2015). The communication underlying motor control is represented in a dynamically functional interaction in a cerebello-thalamo-cortical network with synchronized brain oscillations in the alpha $(8-12 \mathrm{~Hz})$ and beta (13-30 Hz) range (Davis et al., 2012; Wach et al., 2013). In complex movements such as bimanual coordination, the oscillatory activity at $20 \mathrm{~Hz}$ dominates within and between the left and right hemispheric motor networks (Serrien et al., 2003; Rjosk et al., 2016). Swinnen and Wenderoth (2004) revealed that the respective network formation is dependent on the way in which the movements are internally generated or externally (visually) driven. If movements are internally generated, a basal ganglia-supplementary motor area circuit dominates, and a parietal-premotor pathway exerts control when movements are externally driven (Swinnen and Wenderoth, 2004). In the latter case, the posterior parietal cortex (PPC) integrates multi-sensory signals and is responsible for the spatial-temporal coordination of visually controlled movements. The premotor cortex (PMC) prepares and plans movements, whereas, the primary motor cortex (M1) controls the execution and is relevant for motor consolidation. If a given planned movement differs from the executed movements, the cerebellum and the PPC generate error signals to regulate movement impulses (Della-Maggiore et al., 2004). The corpus callosum plays a pivotal role in interhemispheric interaction in bimanually coordinated movements. By connecting the left and right hemispheres it enables the transfer of information and the maintenance of independent processing of one hemisphere (Takeuchi et al., 2012). The electro- and neurophysiological mechanisms underlying bimanual coordination are still controversially debated and far from being fully understood. Evidence exists supporting the functional role of interhemispheric synchronization in the organization of bimanual movements since bimanual movement disorders are accompanied by pathological oscillatory activity, reduced functional coupling of primary sensorimotor regions (Serrien and Brown, 2002) and altered interregional brain synchronization in patients suffering from neurological diseases (Stam et al., 2003; Schnitzler and Gross, 2005; Uhlhaas and Singer, 2006). Nevertheless, unambiguous evidence is missing, thereby preventing the suggestion that altered oscillatory activity is associated with movement disorders. One possibility to investigate the causality between brain oscillations and behavior is the modulation of oscillatory activity in a frequency-specific manner with transcranial alternating current stimulation (tACS) (Zaehle et al., 2010; Polanía et al., 2012). By applying a weak current in the form of sinusoidal waves, tACS is a promising tool to investigate causal relationships between synchronized oscillations, brain activity and cognitive/motor functions.

Numerous studies investigated the after-effects of tACS on perception (Strüber et al., 2014), cognitive functions (Vosskuhl et al., 2015a; Chander et al., 2016; Kasten and Herrmann, 2017), multitasking (Hsu et al., 2017) or motor control and learning (Pogosyan et al., 2009; Pollok et al., 2015; Cappon et al., 2016; Krause et al., 2016; Leunissen et al., 2017). Regarding tACS effects on motor learning, studies demonstrated heterogeneous results because of different system parameters, various motor tasks and high variabilities in tACS responders and non-responders. Whereas improvements in motor learning were demonstrated at $10 \mathrm{~Hz}$ tACS (Antal et al., 2008) and $20 \mathrm{~Hz}$ tACS (Krause et al., 2016), Pogosyan et al. (2009) reported that $20 \mathrm{~Hz}$ tACS slows voluntary movements during a bimanual tracking task (Pogosyan et al., 2009). Investigating tACS effects on interhemispheric interaction, Heise et al. (2017) applied a bilateral tACS montage on the motor cortex concurrently to a bimanual coordination task. While beta synchronization reduced bimanual coordination performance, alpha synchronization promotion was associated with behavioral improvements (Heise et al., 2017).

Concurrent to behavioral studies, additional and important physiological investigations rely mostly on resting-state measurements after tACS (Kasten and Herrmann, 2017). Zaehle et al. (2010) elevated EEG alpha power with tACS at the individual alpha frequency (IAF) and reported a direct electrophysiological evidence for the feasibility of tACS to modulate ongoing oscillatory activity in the human cortex (Zaehle et al., 2010). Others observed enhanced alpha power after $20 \mathrm{~min}$ of tACS at the IAF which lasted $90 \mathrm{~min}$ (Kasten et al., 2016). The difference between the stimulation group and the sham group was found to be diminished after $70 \mathrm{~min}$ due to a natural alpha increase of the sham group (Kasten et al., 2016) indicating that alpha-tACS can enhance the amplitude of the IAF. However, it remains unclear, which physiological mechanisms underlying tACS are responsible for the after-effects, how long potential after-effects may last and whether the effects can be elicited only during rest or also during complex motor behavior.

One approach explaining the mechanisms of tACS is that stimulation impacts intrinsic oscillatory brain activity through entrainment. Entraining a brain oscillation implies that phase and frequency of brain oscillations are modulated to follow 
the external stimulation (Antal and Paulus, 2013; Herrmann et al., 2013; Helfrich et al., 2014; Vosskuhl et al., 2015b; Ruhnau et al., 2016). Whether a neural oscillator follows the external induced tACS frequency depends on the frequency range around its intrinsic frequency. The closer the intrinsic frequency range of the neural oscillator is to the applied tACS frequency or the higher the intensity of tACS is, then the higher the probability of entrainment. Additionally, many other ratios between stimulation frequency and frequency of the neural oscillator (e.g., 1:2, 1:1, 2:1) are thought to cause an entrainment (Herrmann and Strüber, 2017). If altered oscillatory brain activity remains for some time after stimulation, plastic-related changes evoked through spike-timing dependent plasticity (Feldman, 2012) are believed to be the underlying mechanisms responsible for the after-effects (Zaehle et al., 2010; Polanía et al., 2012; Vossen et al., 2015).

However, the exact mechanism of tACS induced after-effects remains controversial due to varying stimulation parameters across the studies such as duration, intensity, frequency and electrode montage and the fragmentary knowledge regarding the underlying neurophysiological mechanisms of tACS. Therefore, we argue that combining EEG and fNIRS (Chen et al., 2015) to examine tACS is essential and fundamental for the understanding of its effects and clinical implementation as it might allow a multidimensional perspective on basic neural mechanisms (Bergmann et al., 2016; Choe et al., 2016).

Several EEG/fMRI studies revealed how the manipulated oscillatory activity of tACS is represented in the brain's metabolism while demonstrating that increased alpha and beta amplitudes correlate with deactivation in occipital areas measured by reduced blood oxygenation level dependent (BOLD) contrasts (Cabral-Calderin et al., 2016; Williams et al., 2017). Moreover, Vosskuhl et al. (2016) confirmed that entrainment of EEG alpha oscillations by tACS at the IAF reduces the BOLD response to visual stimuli (Vosskuhl et al., 2016). Cabral-Calderin et al. (2016) observed the strongest effects on BOLD activity following alpha/beta-tACS, but also specified that tACS affects the BOLD signal in a frequency and taskdependent manner. Furthermore, they emphasize that the strongest effects of tACS might exist in regions not necessarily situated below the stimulation electrodes (Cabral-Calderin et al., 2016). In two further neuroimaging studies, an application with fNIRS and transcranial direct stimulation (tDCS) was used (McKendrick et al., 2015; Muthalib et al., 2017), but to our knowledge, combined electro-/neurophysiological investigations of transcranial electrical stimulation (tES) during complex motor behaviors are rare. Only one approach applied simultaneously EEG and fNIRS to a complex bimanual motor task after tDCS (Choe et al., 2016). To investigate the modulatory effects of tDCS on neural functions and bimanual motor learning, 32 participants received either sham or anodal tDCS to the dorsolateral prefrontal cortex (DLPFC) or the left motor cortex (M1) during pilot training in four consecutive daily sessions. Results from days 1 to 4 demonstrated for M1 stimulated subjects that increased parietal alpha power correlated strongly with reduced fNIRS beta-values in M1 channels (Choe et al., 2016). A simultaneous EEG/fNIRS measure of tACS effects on complex motor performance was not carried out prior to this experimentation even though simultaneous recordings of EEG and neuroimaging that directly reflect the tACS effects are the keys for a better understanding of how tACS impacts brain activity (Herrmann and Strüber, 2017).

To summarize, previous studies investigated the effects of tACS on motor processes and brain oscillations. However, the functional role of synchronized oscillatory activity in interhemispheric communication during bimanual coordination, their modulation by tACS and the underlying neurophysiological mechanisms remain ambiguous. Therefore, further research concerning the mechanisms of tACS and its modulatory effect on complex movements is required giving the fact that such knowledge is of clinical relevance for the rehabilitation of bimanual coordination.

Consequently, the central purpose of this study was to investigate the modulatory effects of parietal $10 \mathrm{~Hz}$ tACS and $20 \mathrm{~Hz}$ tACS on brain oscillations, hemodynamic changes and bimanual coordination using a combined tACS/EEG/fNIRS approach. Assuming that alpha and beta frequencies represent different functions and that tACS provides the possibility to interact with oscillatory activity, we hypothesized that tACS after-effects vary in a frequency dependent manner. Owing to previous work, we hypothesized that parietal $10 \mathrm{~Hz}$ tACS evoke synchronized oscillatory activity in the alpha range that lead to reduced interhemispheric interaction and deterioration in bimanual coordination performance (Serrien and Brown, 2002) whereas $20 \mathrm{~Hz}$ tACS would promote the natural beta oscillation of bimanual coordination (Rjosk et al., 2016) that leads to an enhanced performance.

\section{MATERIALS AND METHODS}

The study was performed in accordance with the latest revision of the Declaration of Helsinki. Experimental procedures were performed along the recommendations of the Deutsche Gesellschaft für Psychologie (DGPs) and approved by the local ethics committee of the Johannes Gutenberg-Universität Mainz. Participants were informed about all relevant issues of the study and gave their written informed consent prior to the initiation of the experiment.

\section{Participants}

Twenty-four healthy subjects (12 females) aged between 18 and $30(M=22.35 ; S D=3.62)$ were recruited to participate in this double-blind study. All participants were right-handed according to the Edinburg handedness-scale (Oldfield, 1971), without any

TABLE 1 | Subject characteristics by group.

\begin{tabular}{lccc}
\hline & \multicolumn{3}{c}{ Group } \\
\cline { 2 - 4 } Characteristics & Sham & $\mathbf{1 0 ~ H z ~ t A C S}$ & $\mathbf{2 0 ~ H z ~ t A C S}$ \\
\hline Sex $($ female/male $)$ & $(5 / 3)$ & $(2 / 6)$ & $(5 / 3)$ \\
Age $(M \pm S D)$ & $23 \pm 3$ & $22 \pm 2$ & $22 \pm 3$
\end{tabular}


neurological or psychological disorders and with normal or corrected-to-normal vision. All participants were requested to disclose pre-existing neurological and psychological conditions, medical conditions, drug intake and alcohol or caffeine intake during the past week. Prior to initiating the study, participants were randomly assigned to a group receiving either $10 \mathrm{~Hz}$ tACS, $20 \mathrm{~Hz}$ tACS or sham stimulation (see Table 1).

\section{Bimanual Coordination Task}

The so-called 2HAND task is a well-known psychological diagnostic tool used in the examination of bimanual coordination and eye-hand coordination (Vienna Test System, Schuhfried, Austria). As depicted in Figure 1, it consists of a monitor, a keyboard, and two joysticks with fixed steering plates. This fixed steering plate restricts the participant's control of the left and right joysticks in a horizontal and a vertical direction, respectively. The participants were instructed to operate the two joysticks in order to direct a virtual dot as fast and as accurately as possible from the starting point $(\Delta)$ to the endpoint $(\square)$ of a predefined route which is presented on the screen while trying to avoid making mistakes. That is, a mistake is defined as the leaving of the shape (see Figure 1). This task requires the subject to guide the dot as smoothly as possible along the shape, which necessitates precisely timed and visually guided coordination between the left and right joysticks simultaneously.

\section{tACS/EEG/fNIRS Approach}

To realize the tACS/EEG/fNIRS approach, two devices were combined providing transcranial current stimulation (tCS) and EEG measures in one device (StarStim, Neuroelectrics, Spain) and a 20-channel fNIRS device (NIRSport, nirx, Germany) (see Figure 2A). For tACS and EEG, small, round gel electrodes

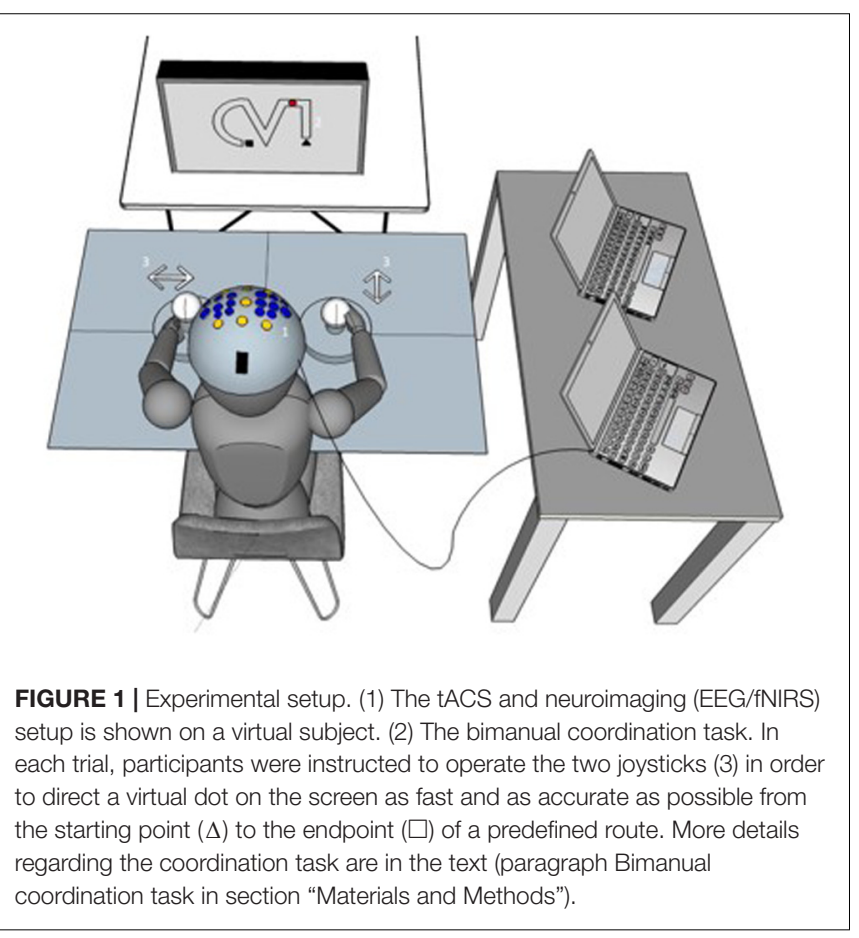

(Ag/AgCl, $3.14 \mathrm{~cm}^{2}$ ) were used (Pi-electrodes, Neuroelectrics) and realized a so-called high-definition tACS (HD-tACS). Eight electrodes were positioned in a non-conductive neoprene cap following the international 10-10 EEG system (Jurcak et al., 2007). Following the calculation of the electrical field of HD-tACS, electrode positions were selected based on the computational model by Ruffini et al. (2014). Based on previous studies which elicited and demonstrated physiological aftereffects of IAF tACS in alpha oscillations during rest (Zaehle et al., 2010; Vossen et al., 2015), the stimulation electrodes were placed at P3 (stimulation electrode) and P4 (return electrode) to cover the parietal cortices (see Figure 2B). These cortices play a functional role in visually guided bimanual coordination (Swinnen and Wenderoth, 2004; Buneo and Andersen, 2006; Culham et al., 2006).

The alternating current was delivered at an intensity of $1 \mathrm{~mA}$ resulting in a current density of $0.32 \mathrm{~mA} / \mathrm{cm}^{2}$ in the skin under the stimulation electrodes. The stimulation groups received 20 min of tACS either at $10 \mathrm{~Hz}$ or $20 \mathrm{~Hz}$ with 15 seconds (s) ramp-up and ramp-down at the beginning and at the end of stimulation. The control group (sham) received only $60 \mathrm{~s}$ of tACS (including $15 \mathrm{~s}$ ramp-up and ramp-down) to induce the physical sensation associated with tACS (e.g., tingling or itching). Thereby, the impedance values are limited to $10 \mathrm{k} \Omega$ during tACS and impedance values were controlled throughout the duration of the whole experiment. To ensure that participants and the investigator are blind to the stimulation condition, the password-protected double-blind mode of the control software (StarStim, Instrument Controller v 1.4, Neuroelectrics, Spain) was used. Further five electrodes were positioned at F3, Fz, F4, $\mathrm{Cz}$, and $\mathrm{Pz}$ and resulted in the provision of EEG measurements also during tACS. Before and after tACS, the actual stimulation electrodes (P3 and P4) were also used for EEG, resulting in seven EEG-channels before and after tACS and five EEGchannels during tACS. Additionally, a vertical electro-oculogram (EOG) was recorded from an electrode over the right eye. The common mode sense (CMS) and driven right leg (DRL) connections were placed on the right mastoid and corresponded to the electrical reference. Overall, the sampling rate was set at $500 \mathrm{~Hz}$.

For fNIRS measurement, a standard configuration for motor tasks was used to accommodate the fNIRS sources and detectors with tACS-EEG-electrodes. 20 channels (source-detector pairs) were positioned at an interval of $3 \mathrm{~cm}$ over the left motor cortex (10 channels) and the right motor cortex (10 channels) covering the arm and hand area of both M1 (see Figure 2A). fNIRS data were recorded at a sampling rate of $7.81 \mathrm{~Hz}$. Near-infrared light at the wavelengths of 760 and $850 \mathrm{~nm}$ which are thought to be optimal for measuring both chromophores (Hboxy/Hbdeoxy) were predefined.

\section{Debriefing}

After completing the experiment, participants rated their feelings and their state of arousal. They filled out a translated version of an adverse effects questionnaire where they had to rate the type and intensity of adverse effects including factors such as 
tiredness, concentration level, cognitive and motor enhancement, pains ( 1 - none, 5 - moderate, 9 - severe) and how much they were related to the stimulation (1 - none, 5 - uncertain, 9 - definite). In addition, they were requested to guess their experimental condition to ensure that they were naive toward real or sham stimulation. Therefore, a questionnaire asked whether they believe to have received real or sham stimulation or whether they are not sure. A chi-square test was used to assess whether subjects were able to correctly identify stimulation or sham.

\section{Experimental Design and Procedure}

A pre-/post-test design with retention-tests was utilized to investigate tACS effects on online (within day) and offline motor learning (across days) (Wessel et al., 2015) as well as its modulatory effects which were measured by EEG and fNIRS (see Figure 3). At the beginning of the 1st day, participants completed all forms and received a standardized written instruction along a video-based demonstration of the required bimanual coordination task. To familiarize participants with the equipment and the bimanual coordination task, an acquisition phase with two practice trials followed.

Before tACS (T0) and at three time-points after tACS (immediately $=\mathrm{T} 1,30 \mathrm{~min}=\mathrm{T} 2,1$ day $=\mathrm{T} 3$ ), participants performed trials of the bimanual coordination task and resting state measurements. The bimanual coordination task contained two blocks with five trials. Before, between and after these two bimanual coordination blocks, resting state measurements with eyes closed and eyes open were performed for 1 min each (see Figure 3). Following T0, training started with thirty trials of the bimanual coordination task which lasted $20 \mathrm{~min}$. Concurrent to this training, participants received either $10 \mathrm{~Hz}$ tACS, $20 \mathrm{~Hz}$ tACS or sham stimulation.

Whereas electrophysiological EEG data were collected at all points of time, fNIRS was only used at T0, training and T1, thereby, focusing the effects immediately after tACS while avoiding well-known side-effects of fNIRS (e.g., pressure pain on the head). Before the experiment started, resting state brain activity were collected for $30 \mathrm{~s}$ and served as baseline for NIRS analyses. In the following, groups are referred to as (1) $10 \mathrm{~Hz}$ tACS, (2) $20 \mathrm{~Hz}$ tACS and (3) sham.

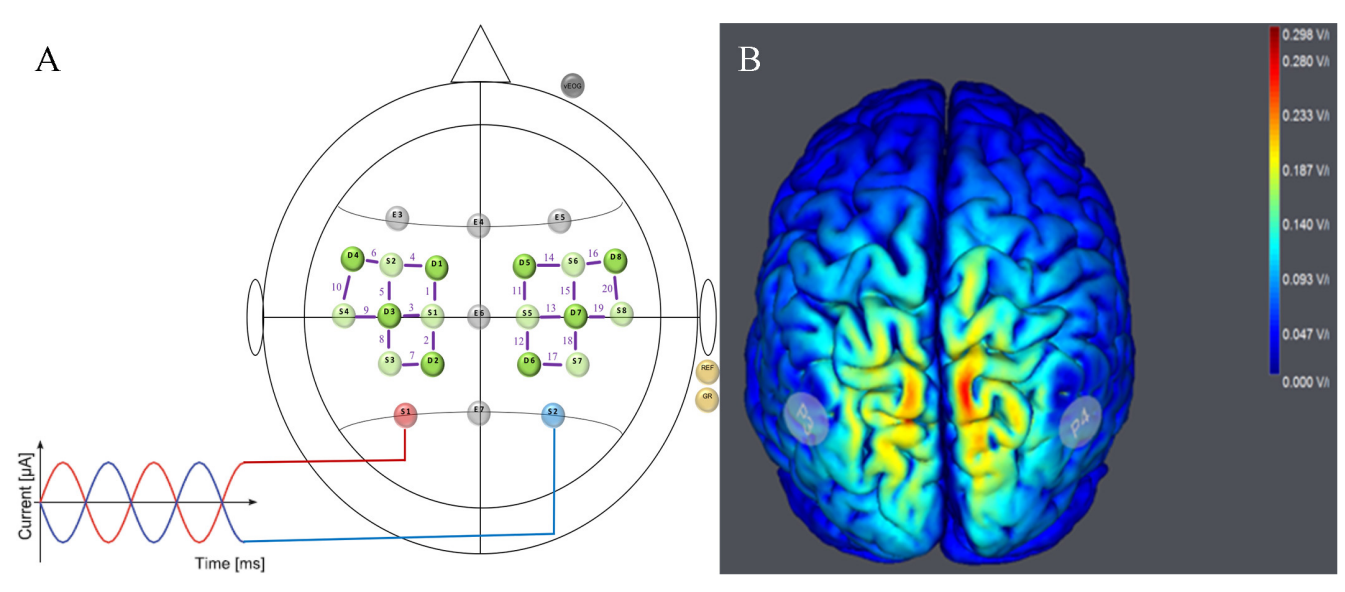

FIGURE 2 | Stimulation and neuroimaging setup. (A) tACS/EEG/fNIRS Montage for Parietal Stimulation. HD-tACS electrodes were positioned at P3 (red electrode = stimulation) and P4 (blue electrode = return). EEG was measured from five positions (F3, Fz, F4, Cz, and Pz) following the international 10-10 system (gray electrodes). fNIRS sources (light green) and detectors (dark green) are shown over the left and right motor cortices with channels (Source-Detector) depicted as purple lines (FC3-FC5, FC3-FC1, FC3-C3, C1-FC1, C1-C3, C1-CP1, C5-C3, C5-FC5, CP3-C3, CP3-CP1, FC4-FC2, FC4-FC6, FC4-C4, C2-FC2, C2-C4, C2-CP2, C6-FC6, C6-C4, CP4-CP2, und CP4-C4). (B) Simulated Electrical Field. Predicted electrical field intensities with a tACS application on P3/P4 (StartStim, Neuroelectrics, Spain). Full details regarding stimulation and brain activity measures are in the text (paragraph tACS/EEG/fNIRS approach).

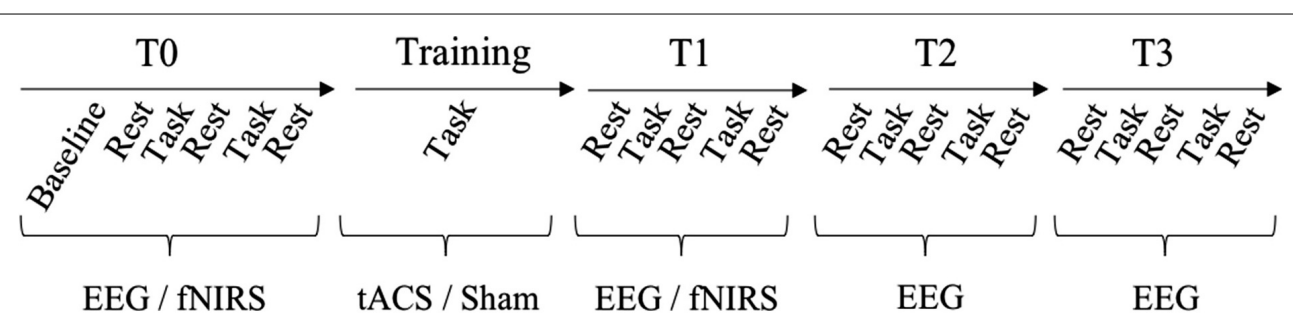

FIGURE 3 | Experimental design. Upper line depicts the timeline of test and training sessions. During the test session, participants performed two blocks of the bimanual coordination task (five trials each) and three blocks of rest with eyes closed and eyes open before tACS (TO) and three times after tACS (immediately = T1, $30 \mathrm{~min}=\mathrm{T} 2,1$ day = T3). During tACS/training (20 min), participants performed three blocks with ten trials of the bimanual coordination task each and resting states in between. Lower line presents when tACS, EEG and fNIRS were recorded. 


\section{Data Analyses}

Statistical analyses were performed using IBM SPSS 23. All variables were normally distributed as assessed by the ShapiroWilk test $(p>0.05)$. Examining statistical requirements, Levene's test was used to check for homogeneity and Mauchly's test was used to check for a violation of sphericity. Several factorial mixed ANOVAs were calculated to examine learning and tACS related changes in bimanual coordination performance and in alpha/beta activity. The exact definition of factors and factor levels of each ANOVA are specified below. For clarity, the ANOVA factors were presented once again at the respective positions in the result section. The overall level of significance was set at $p \leq 0.05$. If a violation of sphericity was detected $(p<0.05)$ and a GreenhouseGeisser epsilon $\varepsilon>0.75$ existed, Huynh-Feldt corrected $p$-values are reported. Otherwise (epsilon $\varepsilon<0.75$ ), a Greenhouse-Geisser correction is applied. Effects sizes (Es) of ANOVAs are given in partial eta-squared $\left(\eta_{\mathrm{p}}^{2}\right)$, where $\eta_{\mathrm{p}}^{2}=0.01$ indicates a small effect, $\eta_{\mathrm{p}}^{2}=0.06$ indicates a medium effect and $\eta_{\mathrm{p}}^{2}=0.14$ indicates a large effect.

\section{Bimanual Coordination Task}

Movement time (s) across 10 trials was calculated once before tACS (baseline $=\mathrm{T} 0)$, immediately after tACS $(=\mathrm{T} 1), 30 \mathrm{~min}$ after tACS $(=\mathrm{T} 2)$ and 1 day after tACS $(=\mathrm{T} 3)$. Next, for each task completion the differences from the pre-stimulation baseline were computed for further analysis. A two-way analysis of variance (ANOVA) with repeated measures (mixed-design) for TIME (T0, T1, T2, and T3) and GROUP (10 Hz tACS, $20 \mathrm{~Hz}$ tACS, Sham) was conducted to evaluate any performance changes in bimanual coordination over time and between the groups. Bonferroni-adjusted post hoc analysis was used to identify significant differences in bimanual coordination performance between days and groups in case of significant effects.

\section{Electrophysiological Data (EEG)}

Electrophysiological data were pre-processed using the Brain Vision Analyzer 2.0 (Brain Products, Gilching, Germany) by applying a $0.5 \mathrm{~Hz}$ high-pass and a $50 \mathrm{~Hz}$ low-pass filter. Horizontal and vertical eye movements were corrected (Gratton and Coles) and epochs containing further artifacts were visually identified and manually rejected. For the evaluation of sustained changes in alpha activity over time, three different conditions (1) eyes closed, (2) eyes open, and (3) bimanual coordination were subdivided into $1 \mathrm{~min}$ blocks in the sessions of task execution before (T0) and after stimulation (T1, T2, and T3). Each block was segmented in $1 \mathrm{~s}$ epochs, wherefore, a fast fourier transformation (FFT) was subsequently computed. Frequency spectra of the artifact-free segments were averaged for each block and the mean activity $(\mu \mathrm{V})$ in the alpha range $(8-12 \mathrm{~Hz})$ and beta range $(18-22 \mathrm{~Hz})$ was calculated and exported. For statistical comparisons, changes to $\mathrm{T} 0(\mu \mathrm{V})$ in alpha and beta activity were analyzed for eyes closed, eyes open and task and were fed separately into different ANOVAs. Firstly, a threeway repeated measure ANOVA with the within factors TIME (T0, T1, T2, and T3) and LOCATION (P3, P4, F3, Fz, F4, Cz, and $\mathrm{Pz})$ and the between factor GROUP $(10 \mathrm{~Hz}$ tACS, $20 \mathrm{~Hz}$
tACS, Sham) was calculated to check for global after-effects of tACS. Secondly, a two-way repeated measure ANOVA with the within factor TIME (T0, T1, T2, and T3) and the between factor GROUP (10 Hz tACS, $20 \mathrm{~Hz}$ tACS, Sham) was calculated for the stimulation electrodes P3 and P4 to check location-specific after-effects of tACS in the stimulated area. Changes in alpha and beta activity over time (pre to post tACS) or differences between groups were evaluated by using the Bonferroni-corrected $t$-tests separately for the resting states and the bimanual coordination task. Furthermore, in order to visually demonstrate statistical significant effects, frequency spectra $(1-30 \mathrm{~Hz})$ of P3 and P4 and alpha/beta topographies for all conditions and groups were presented in addition to the activity changes over time. Electrophysiological data collected during tACS were not further analyzed due to large tACS induced artifacts.

\section{Brain Oxygenation (fNIRS)}

Brain oxygenation raw data were preprocessed and analyzed within the MATLAB-based nirsLAB analysis package (Nirx Medical Technologies, Glen Head, NY, United States, "Biomedical Optics"). Concentration changes of Hboxy, Hbdeoxy, and Hbtot were (1) corrected for discontinuities, steps and spikes and (2) band-pass filtered from 0.01 to $0.2 \mathrm{~Hz}$ to remove slow drifts in the signal related to respiratory or cardiac rhythm. For calculating the hemodynamic response function based on the modified Beer-Lambert law, the distance between the source-detector pairs were computed within nirsLAB in accordance with the montage of the headcap.

Then, $30 \mathrm{~s}$ of the resting states (eyes closed/eyes open) and one min from each bimanual coordination block were used to specify the segment eyes closed, eyes open and task. The averaged baseline concentration values were subtracted from these rest-evoked and task-evoked concentration measurements. The averaged concentration value of $\Delta$ Hboxy was computed separately for each subject, time (T0/T1), channel, resting state (eyes closed/eyes open) and task. Statistical analyses focused on the increases in $\Delta$ Hboxy, because these appear to reflect task-related cortical activation more directly than decreases in $\Delta$ Hbdeoxy as evidenced by the stronger correlation between the former and the BOLD signal measured by fMRI (Strangman et al., 2002). To compute statistical significances of group-averaged Hboxy concentration changes ( $\Delta$ Hboxy) caused by tACS, Statistical Parametric Mapping (SPM) was applied. SPM values were further used to estimate beta-values for the subtraction of $\triangle$ Hboxy before tACS from $\triangle$ Hboxy after tACS (SPM, nirsLAB, v2016.05). For each participant, a $t$-statistic-threshold $(p<0.05)$ beta-image was computed for baseline-subtracted restor task-evoked $\Delta$ Hboxy before (T0) and immediately after tACS (T1). For group analysis, t-statistic maps (t-contrast: difference between two groups' hemodynamic responses to T0 and T1) were generated from the averaged beta-values. If the $t$-statistic exceeded the $p$-value threshold of 0.05 ( $t$-value $<-2.4$ and $>2.4$ ), the concentration values were determined to be significant and marked colored in the respective map. Furthermore, time courses of $\Delta$ Hboxy concentrations were calculated for all conditions, groups and channels. 


\section{RESULTS}

\section{Resting Alpha Activity (8-12 Hz) With Eyes Closed \\ Global Effect of tACS}

To analyze the global after-effect of tACS on alpha activity, the three-way rmANOVA with all EEG positions revealed a significant main effect for GROUP $[F(2,21)=3.466$, $\left.p<0.05, \eta_{\mathrm{p}}^{2}=0.25\right]$, a significant main effect for TIME $\left[F(3,63)=3.665, p<0.05, \eta_{\mathrm{p}}^{2}=0.15\right]$, a significant main effect for LOCATION $\left[F(4,91)=4.769, p<0.01, \eta_{\mathrm{p}}^{2}=0.19\right]$ and a significant TIME $\times$ LOCATION interaction $[F(9,181)=2.353$, $\left.p<0.05, \eta_{\mathrm{p}}^{2}=0.10\right]$. The TIME $\times$ LOCATION interaction demonstrates that there are significant differences between alpha activity on the eight EEG positions and the four time-points, independently of the GROUP. Neither TIME $\times$ GROUP interaction $\left[F(6,63)=1.250, p=0.29, \eta_{\mathrm{p}}^{2}=0.11\right]$ nor LOCATION $\times$ GROUP interaction $[F(9,90)=1.120$, $\left.p=0.35, \quad \eta_{\mathrm{p}}^{2}=0.11\right]$ nor the three-way interaction TIME $\times$ LOCATION $\times$ GROUP $[F(17,181)=0.774, p=0.83$, $\left.\eta_{\mathrm{p}}^{2}=0.07\right]$ revealed significance. The topographies in Figure 6A show changes in alpha activity over time for the groups Sham, $10 \mathrm{~Hz}$ tACS and $20 \mathrm{~Hz}$ tACS.

\section{Location-Specific Effect of tACS}

Analyzing the location-specific effects of tACS on alpha oscillatory activity in the stimulated parietal area, an ANOVA was computed for P3 and P4 separately. The two-way rmANOVA for P3 demonstrated a significant main effect for TIME $\left[F(3,63)=5.470, p<0.05, \eta_{\mathrm{p}}^{2}=0.22\right]$ and a significant main effect for GROUP $\left[F(2,21)=3.125, p<0.05, \eta_{\mathrm{p}}^{2}=0.23\right]$. The interaction TIME $\times$ GROUP did not reach significance $\left[F(6,63)=1.358, p=0.24, \eta_{\mathrm{p}}^{2}=0.12\right]$ (see Figure 4A). The two-way rmANOVA for P4 showed a significant main effect for TIME $\left[F(3,63)=4.209, p<0.05, \eta_{\mathrm{p}}^{2}=0.17\right]$ and a significant main effect for GROUP $\left[F(2,21)=3.361, p<0.05, \eta_{\mathrm{p}}^{2}=0.24\right]$ (see Figure 4B). The interaction TIME $x$ GROUP did not reach significance $\left[F(6,61)=1.259, p=0.29, \eta_{\mathrm{p}}^{2}=0.11\right]$. Frequency spectra of P3 and P4 for the groups Sham, $10 \mathrm{~Hz}$ tACS and $20 \mathrm{~Hz}$ tACS are presented in Figure 5.

\section{Resting Beta Activity (18-22 Hz) With Eyes Closed}

Statistical analysis of beta-band activity $(18-22 \mathrm{~Hz})$ using a three-way rmANOVA with the factors TIME, LOCATION, and GROUP revealed neither significant main effects nor significant interactions. As beta-band was completely unaffected by the stimulation protocol, those values will not be further visually or statistically reported. The topographies for beta activity are presented in Figure 6B, which additionally demonstrate that the beta-band was completely unaffected by the stimulation protocol.

\section{Resting fNIRS Activity ( $\Delta$ Hboxy) With Eyes Closed}

The fNIRS results demonstrated significant decreases in Hboxy concentration on the left and right hemisphere $(p<0.05)$ before and after training/tACS (see Supplementary Tables S1A,B). According to that, resting state with eyes closed is accompanied by decreased oxygenated hemoglobin in channels covering areas relevant for motor behavior. In addition, Hboxy $t$-values during EC did not differ significantly from $\mathrm{T} 0$ and $\mathrm{T} 1$ indicating that $\Delta$ Hboxy concentrations were not influenced by training or tACS (see Supplementary Tables S1C, S2, S3).

\section{Resting Alpha Activity (8-12 Hz) With Eyes Open \\ Global Effect of tACS}

For all electrodes, the three-way rmANOVA revealed a significant main effect for TIME $\left[F(3,63)=12,443, p<0.01, \eta_{\mathrm{p}}^{2}=0.37\right]$, a significant main effect for LOCATION $[F(3,66)=6,036$, $\left.p<0.01, \eta_{\mathrm{p}}^{2}=0.22\right]$ and a significant TIME $\times$ LOCATION interaction $\left[F(11,235)=2,829, p<0.05, \eta_{p}^{2}=0.12\right]$. The TIME $\times$ LOCATION interaction indicates that significant differences of alpha activity on the eight EEG positions to the four time-points exist, and that these are independent of the stimulation condition. Bonferroni-corrected post hoc $t$-tests revealed a significantly different alpha activity between $\mathrm{P} 4$ and F4 immediately after tACS, independent of tACS $(p<0.05)$. This indicates a location-specific response of frontal and parietal areas on the right hemisphere to training. The different alpha activity evolvement over time is visually reflected in the topographies for the three groups (see Figure 8A) by demonstrating stronger alpha activity changes in parietal areas compared to frontal areas.

\section{Location-Specific Effect of tACS}

For the resting condition with eyes open on P3, a twoway rmANOVA depicted a significant main effect of TIME $\left[F(3,63)=13.294, p<0.01, \eta_{\mathrm{p}}^{2}=0.38\right]$ indicating that alpha activity is different over time in the stimulated parietal area left hemispheric, independent of GROUP. Additionally, a significant tendency of GROUP $[F(2,21)=3.172, p=0.06$, $\left.\eta_{\mathrm{p}}^{2}=0.23\right]$ and a GROUP $\times$ TIME interaction $[F(3,63)=2.765$, $\left.p<0.05, \eta_{\mathrm{p}}^{2}=0.21\right]$ emerged, which shows that alpha activity is different over time in the three groups. Bonferroni post hoc comparisons clarify a significant increase of alpha activity following $10 \mathrm{~Hz}$ tACS up to $30 \mathrm{~min}$ (T2-T0; $p<0.05$ ) and after $20 \mathrm{~Hz}$ tACS up to 1 day after tACS (T3-T0; $p<0.05)$. Furthermore, change in alpha activity from T1 to T0 differs significantly $(p<0.05)$ between $20 \mathrm{~Hz}$ tACS and sham controls and from T3 to T0 between $20 \mathrm{~Hz}$ tACS and $10 \mathrm{~Hz}$ tACS (see Figure 4C). For eyes open on P4, a twoway rmANOVA revealed a significant main effect of TIME $\left[F(3,63)=14.135, p<0.01, \eta_{\mathrm{p}}^{2}=0.40\right]$ indicating that alpha activity is different over time in the stimulated parietal area right hemispheric, independent of GROUP. Additionally, a significant tendency of GROUP $[F(2,21)=3.159, p=0.06$, $\left.\eta_{\mathrm{p}}^{2}=0.23\right]$ emerged. The GROUP $\times$ TIME interaction did not reach significance $\left[F(3,63)=1.550, p<0.17, \eta_{\mathrm{p}}^{2}=0.13\right]$ (see Figure 4D). Frequency spectra of $\mathrm{P} 3$ and $\mathrm{P} 4$ for the groups Sham, $10 \mathrm{~Hz}$ tACS and $20 \mathrm{~Hz}$ tACS are presented in Figure 7. 


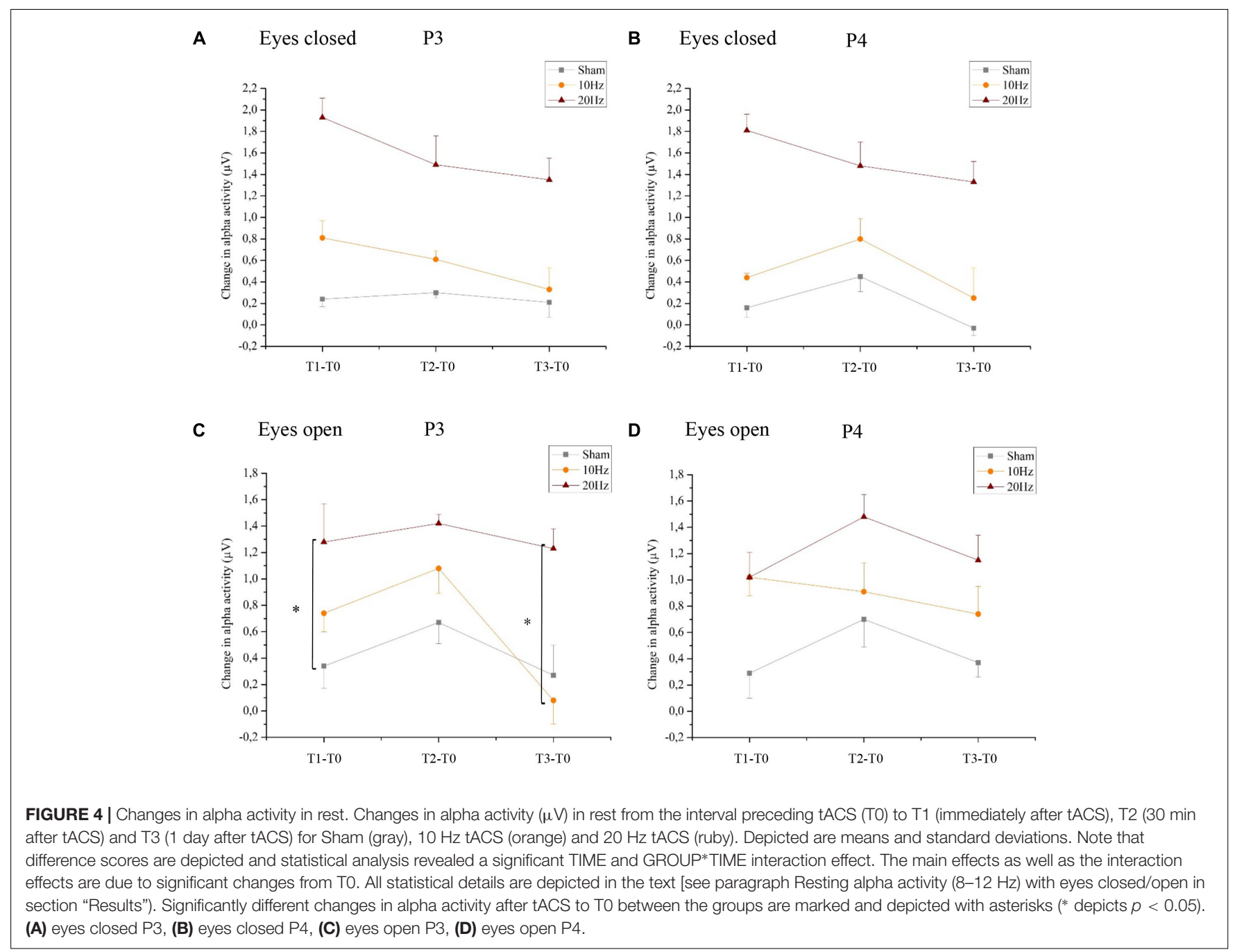

\section{Resting Beta Activity (18-22 Hz) With Eyes Open}

Statistical analysis of beta-band activity (18-22 Hz) using a threeway rmANOVA with the factors TIME, EEG POSITION, and GROUP revealed neither main effects nor interaction effects. As beta-band was completely unaffected by the stimulation protocol, those values will not be further visually or statistically reported. The topographies for beta activity are presented in Figure 8B and depicted that the beta-band was completely unaffected by the stimulation protocol.

\section{Resting fNIRS Activity ( $\Delta$ Hboxy) With Eyes Open}

Relative changes of Hboxy concentration decreased during rest with eyes open, but reached no significance before and after tACS (see Supplementary Tables S4A,B). Additionally, Hboxy $t$-values did not differ from T0 to T1 (see Supplementary Table S4C) indicating that hemodynamic responses during rest with eyes open are not significantly influenced by training or tACS.

\section{Bimanual Coordination Performance During Task Execution}

For the mean duration of the bimanual coordination task, the two-way rmANOVA showed a statistically significant main effect for TIME $\left[F(3,63)=35.338, p<0.001, \eta_{\mathrm{p}}^{2}=0.63\right]$. This result indicates that all participants significantly improved performance in bimanual coordination due to training, independent of tACS. Neither a main effect of group nor a significant TIME $\times$ GROUP interaction $\left[F(6,63)=0.405, p=0.087, \eta_{p}^{2}=0.04\right]$ was found (see Figure 9).

\section{Alpha Activity (8-12 Hz) During Task Execution \\ Global Effect of tACS}

Statistical analysis of alpha activity for all EEG electrodes using a three-way rmANOVA revealed a significant main effect for TIME $\left[F(3,63)=10,701, p<0.01, \eta_{\mathrm{p}}^{2}=0.34\right]$ and a significant main effect for LOCATION $[F(3,68)=3,090$, $\left.p<0.05, \eta_{\mathrm{p}}^{2}=0.13\right]$. The significant main effect for GROUP $(p<0.05)$ was due to a significant difference between the 


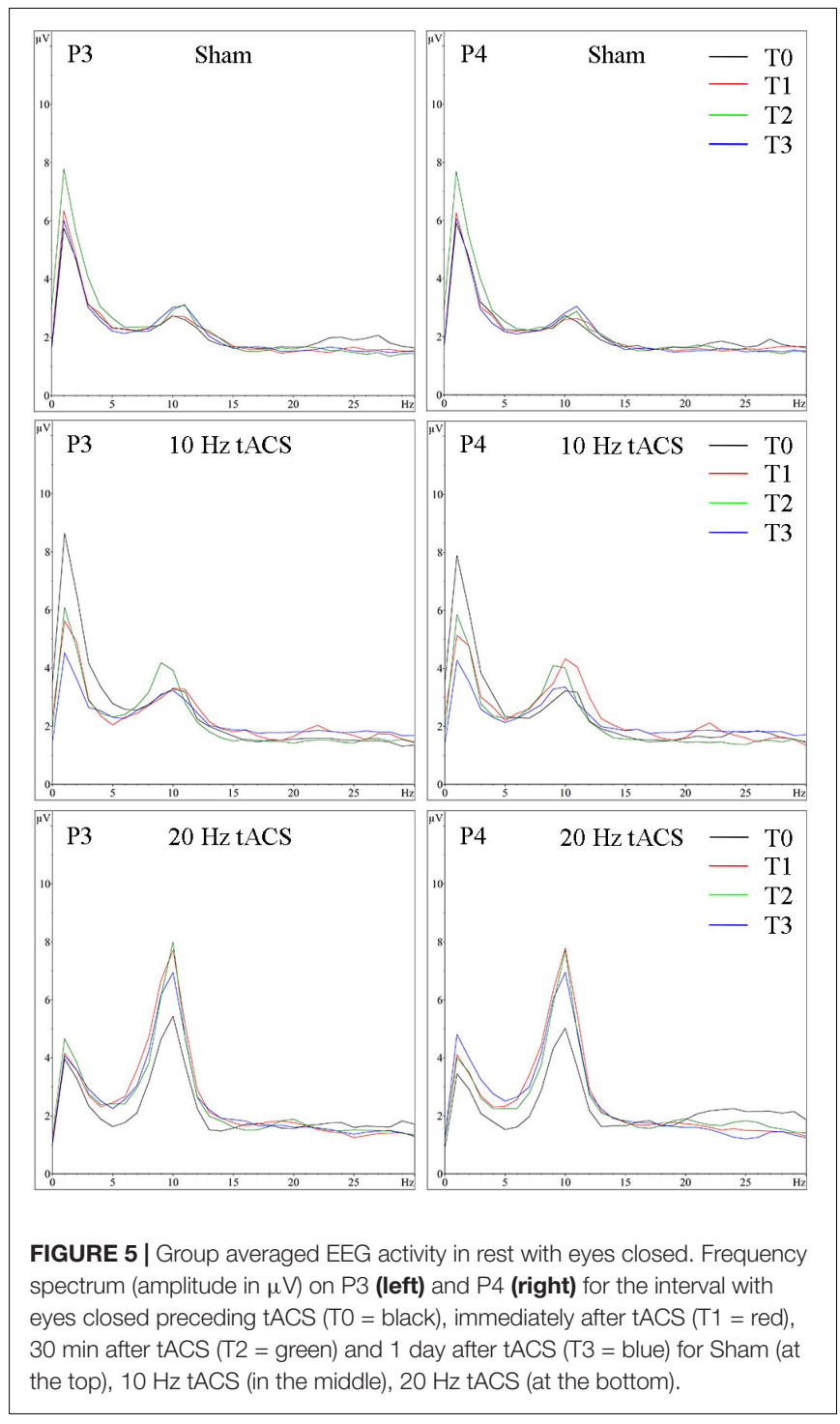

$20 \mathrm{~Hz}$ tACS group and the control group. Additionally, both stimulation groups had a significantly increased alpha activity during bimanual coordination; the $10 \mathrm{~Hz}$ tACS group up to $30 \mathrm{~min}$, the $20 \mathrm{~Hz}$ tACS group up to 1 day after tACS compared to baseline $(p<0.05)$. A significant interaction of TIME $\mathrm{x}$ GROUP did not emerge $(p>0.05)$ possibly due to an increasing alpha in the sham stimulated control group. Concerning the statistically significant interaction of TIME $\times$ LOCATION $\left[F(10,217)=1,957, p<0.05, \eta_{\mathrm{p}}^{2}=0.09\right]$, Bonferroni-corrected post hoc tests revealed significant differences in alpha activity between P4 and F4 immediately after stimulation and between $\mathrm{P} 4$ and $\mathrm{Fz} 30 \mathrm{~min}$ after tACS $(p<0.01)$. These results confirm the location-specific differences following training, and even during motor execution. The different alpha activity evolvement over time for the three groups is visually reflected in the topographies (see Figure 12A) by demonstrating stronger alpha activity changes in parietal areas compared to frontal areas.
A Change in alpha activity $(\mu \mathrm{V})$ in rest with eyes closed
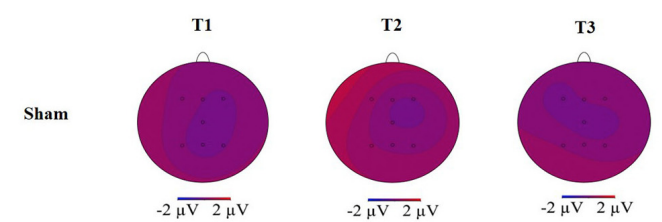

$10 \mathrm{~Hz}$ tACS
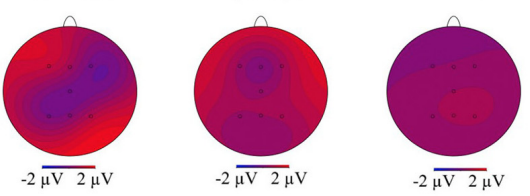

$20 \mathrm{~Hz}$ tACS
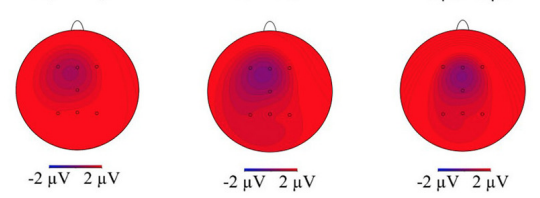

B

Change in beta activity $(\mu \mathrm{V})$ in rest with eyes closed

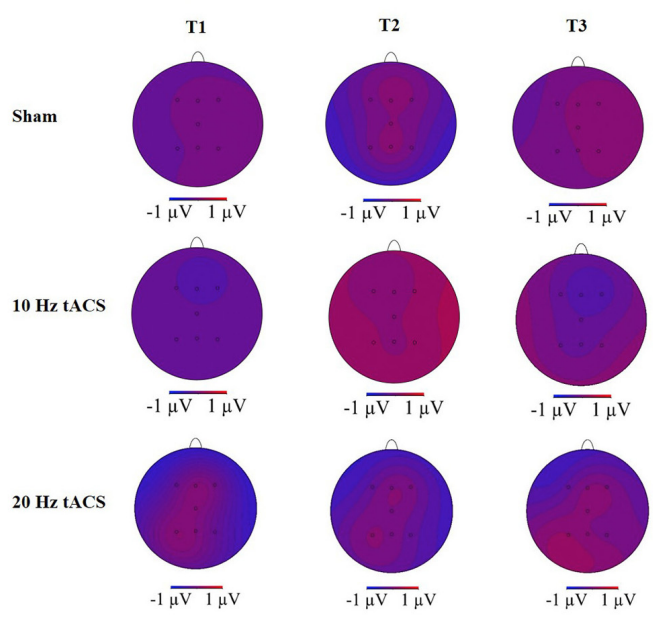

FIGURE 6 | Topographies of group averaged alpha and beta activity changes. (A) Changes in alpha activity $(\mu \mathrm{V})$ in rest from the interval with eyes closed preceding tACS (TO) to T1 (immediately after tACS), T2 (30 min after tACS), and T3 (1 day after tACS) for Sham (at the top), $10 \mathrm{~Hz}$ tACS (in the middle), $20 \mathrm{~Hz}$ tACS (at the bottom). (B) Changes in beta activity $(\mu \mathrm{V})$ in rest from the interval with eyes closed preceding tACS to T1 (immediately after tACS), T2 (30 min after tACS) and T3 (1 day after tACS) for Sham (at the top), $10 \mathrm{~Hz}$ tACS (in the middle), $20 \mathrm{~Hz}$ tACS (at the bottom). Note that these topographies are based on seven electrode positions only.

\section{Location-Specific Effect of tACS}

Analyzing the location-specific effect of tACS on alpha oscillatory activity above P3 during the bimanual coordination task, the two-way rmANOVA revealed a significant main effect for TIME $\left[F(3,63)=9.208, p<0.05, \eta_{\mathrm{p}}^{2}=0.31\right]$. Neither main effect GROUP $\left[F(2,21)=1,567, p=0.13, \eta_{\mathrm{p}}^{2}=0.09\right]$ nor interaction effect of TIME $\times$ GROUP $\left[F(2,21)=1,567, p=0.19, \eta_{\mathrm{p}}^{2}=0.13\right]$ was significant (see Figure 10A). In contrast, the two-way rmANOVA for P4 yielded a significant main effect for TIME $\left[F(3,63)=12.987, p<0.01, \eta_{\mathrm{p}}^{2}=0.38\right]$, a significant main effect for GROUP $\left[F(2,21)=4.400, p<0.05, \eta_{\mathrm{p}}^{2}=0.29\right]$ 

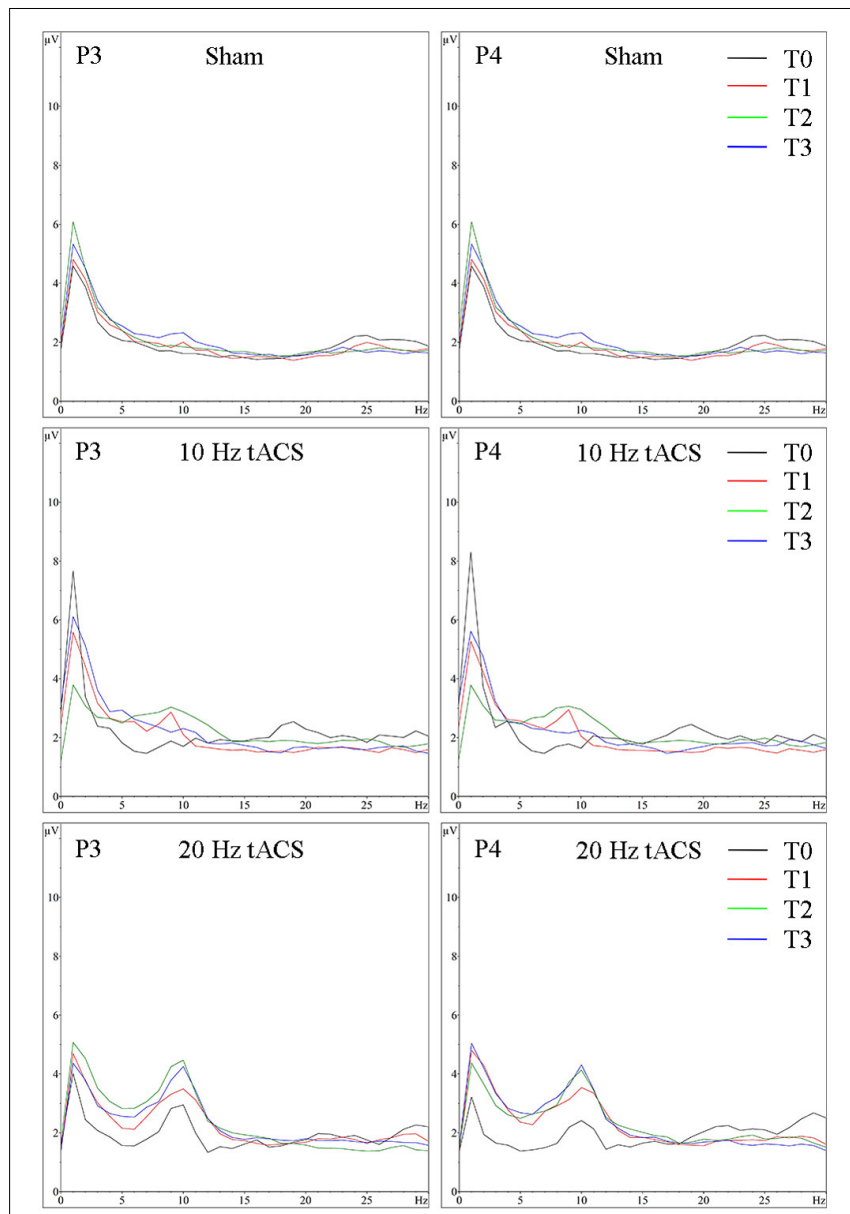

FIGURE 7 | Group averaged EEG activity in rest with eyes open. Frequency spectrum (amplitude in $\mu \mathrm{V}$ ) on P3 (left) and P4 (right) for the 1 min interval with eyes open preceding (TO = black), immediately after tACS (T1 = red), 30 min after tACS (T2 = green) and 1 day after tACS (T3 = blue) for Sham (at the top), $10 \mathrm{~Hz}$ tACS (in the middle), $20 \mathrm{~Hz}$ tACS (at the bottom).

as well as a TIME $\times$ GROUP interaction $[F(6,63)=2.222$, $\left.p<0.05, \eta_{\mathrm{p}}^{2}=0.18\right]$. Thus, alpha activity is different over time in the different groups. Bonferroni post hoc analysis exhibited significant differences in alpha activity between $20 \mathrm{~Hz}$ tACS and sham controls immediately after tACS compared to T0 (T1-T0) and between $10 \mathrm{~Hz}$ tACS and sham controls 30 min after tACS compared to T0 (T2-T0) (see Figure 10B). Frequency spectra of P3 and P4 for the groups Sham, $10 \mathrm{~Hz}$ tACS and $20 \mathrm{~Hz}$ tACS are presented in Figure 11.

\section{Beta-Activity (18-22 Hz) During Task Execution}

Statistical analysis of beta-band activity (18-22 Hz) using a threeway rmANOVA with the factors TIME, EEG POSITION, and GROUP revealed neither main effects nor interaction effects. As beta-band was completely unaffected by the stimulation protocol, those values will not be further visually or statistically reported. The topographies for beta activity are presented in Figure 12B
A Change in alpha activity $(\mu \mathrm{V})$ in rest with eyes open
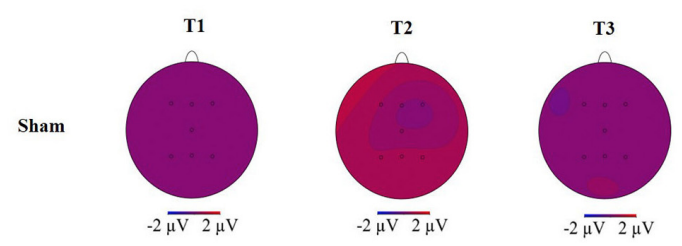

$10 \mathrm{~Hz}$ taCs
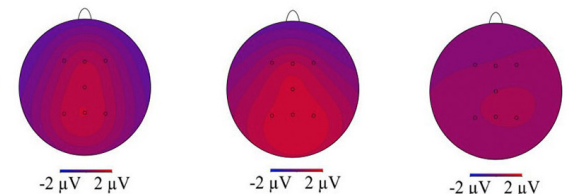

$20 \mathrm{~Hz}$ tACS
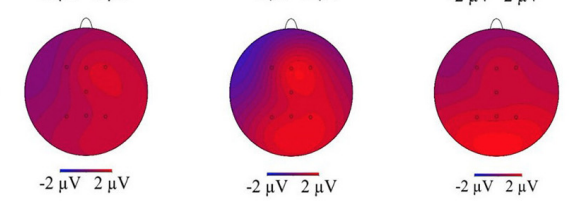

B Change in beta activity $(\mu \mathrm{V})$ in rest with eyes open
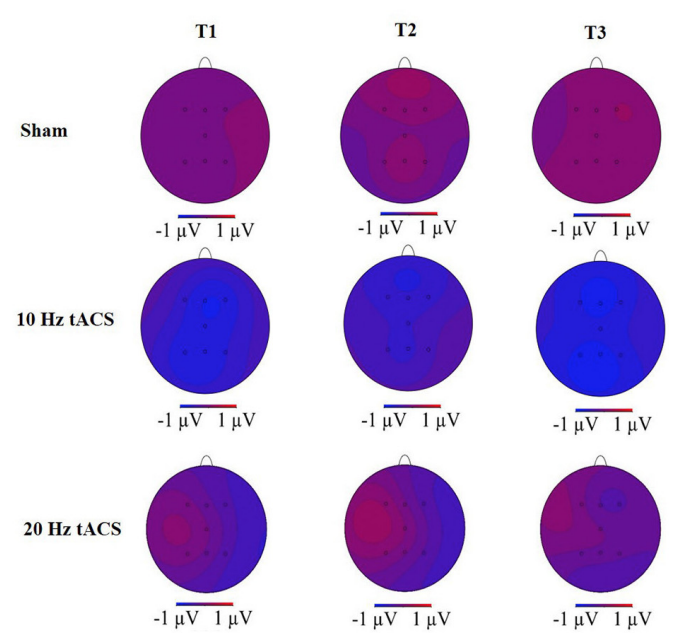

FIGURE 8 | Topographies of group averaged alpha and beta activity changes. (A) Changes in alpha activity $(\mu \mathrm{V})$ in rest from the interval with eyes open preceding tACS (TO) to T1 (immediately after tACS), T2 (30 min after tACS), and T3 (1 day after tACS) for Sham (at the top), $10 \mathrm{~Hz}$ tACS (in the middle), $20 \mathrm{~Hz}$ tACS (at the bottom). (B) Changes of beta activity $(\mu V)$ in rest from the interval with eyes open preceding tACS to T1 (immediately after tACS), T2 (30 min after tACS), and T3 (1 day after tACS) for Sham (at the top), $10 \mathrm{~Hz}$ tACS (in the middle), $20 \mathrm{~Hz}$ tACS (at the bottom). Note that these topographies are based on seven electrode positions only.

and demonstrate that the beta-band was completely unaffected by the stimulation protocol.

\section{fNIRS Activity ( $\Delta$ Hboxy) During Task}

\section{Execution}

As depicted in Figure 13, t-contrasts of the group averaged oxygenated hemoglobin using SPM analysis from the sham control group showed an increase of Hboxy concentration in 17 of 20 channels from T0 to T1. Analysis of $\Delta$ Hboxy 


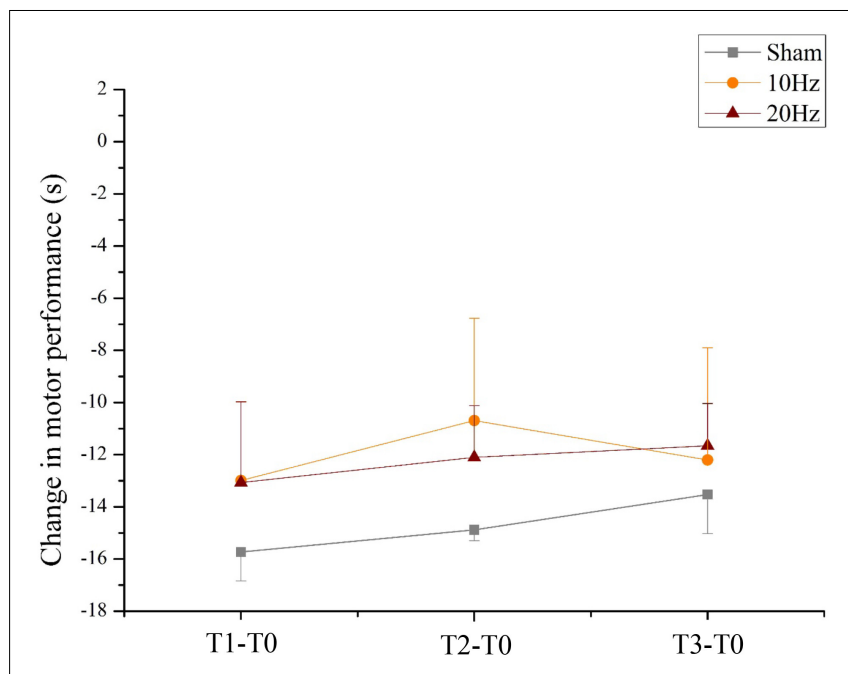

FIGURE 9 | Changes in motor performance. Changes in motor performance (s) from the bimanual coordination task preceding tACS (TO) to T1 (immediately after tACS), T2 (30 min after tACS), and T3 (1 day after tACS) for Sham (gray), $10 \mathrm{~Hz}$ tACS (orange) and $20 \mathrm{~Hz}$ tACS (ruby). Depicted are means and standard deviations. Note that difference scores are depicted and statistical analysis revealed a significant TIME effect. The main effect is due to significant changes from TO. All statistical details are depicted in the text (see paragraph Bimanual coordination performance during task execution in section "Results"). following $10 \mathrm{~Hz}$ tACS demonstrated increases in 13 of 20 channels and in 14 of 20 channels following $20 \mathrm{~Hz}$ tACS (see Supplementary Table S5). Whereas no group differences were observable at $\mathrm{T} 0$, significantly different $\Delta$ Hboxy concentrations were found between tACS groups and sham controls at T1 (see Supplementary Table S6). During the bimanual coordination task, $\Delta$ Hboxy concentration decreased significantly in three channels following $10 \mathrm{~Hz}$ tACS. These were right hemispheric (Channel 12, Channel 19, and Channel 20) covering regions of the premotor area (BA6), the primary motor cortex (BA4) and the primary somatosensory cortex $(B A 3 / 2 / 1)$ compared to Sham (see Figures 2, 13A,B). After $20 \mathrm{~Hz}$ tACS, one channel of the right hemisphere (Channel 16) covering the premotor area (BA6) revealed a significant decrease in $\Delta$ Hboxy concentration compared to the control group (see Figures 2, 13C,D, 14). These significant decreases of $\triangle$ Hboxy concentrations following tACS demonstrate a significant effect of stimulation on the brain's hemodynamic processes.

\section{Debriefing}

After stimulation, 10 of the 24 participants believed that tACS had a positive effect on motor performance in general. Two participants associated their acute motor enhancement in this study with after-effects of tACS. Beyond that, four of the 24 participants felt certain that they were stimulated by tACS, five participants indicated that they were not stimulated and the others had doubts whether they were stimulated or not. For only five participants, the reported answer is consistent with the applied stimulation suggesting that participants were naïve toward their experimental condition. Results showed no significant relation between suspected and real applied tACS, $\chi^{2}(2)=4.375, p=0.112, \varphi=0.12$.

\section{DISCUSSION}

In this study, we combined a complex motor task with simultaneous EEG-fNIRS measures to analyze the after-effects of $10 \mathrm{~Hz}$ tACS and $20 \mathrm{~Hz}$ tACS on oscillatory activity, hemodynamic changes and bimanual coordination. As expected,

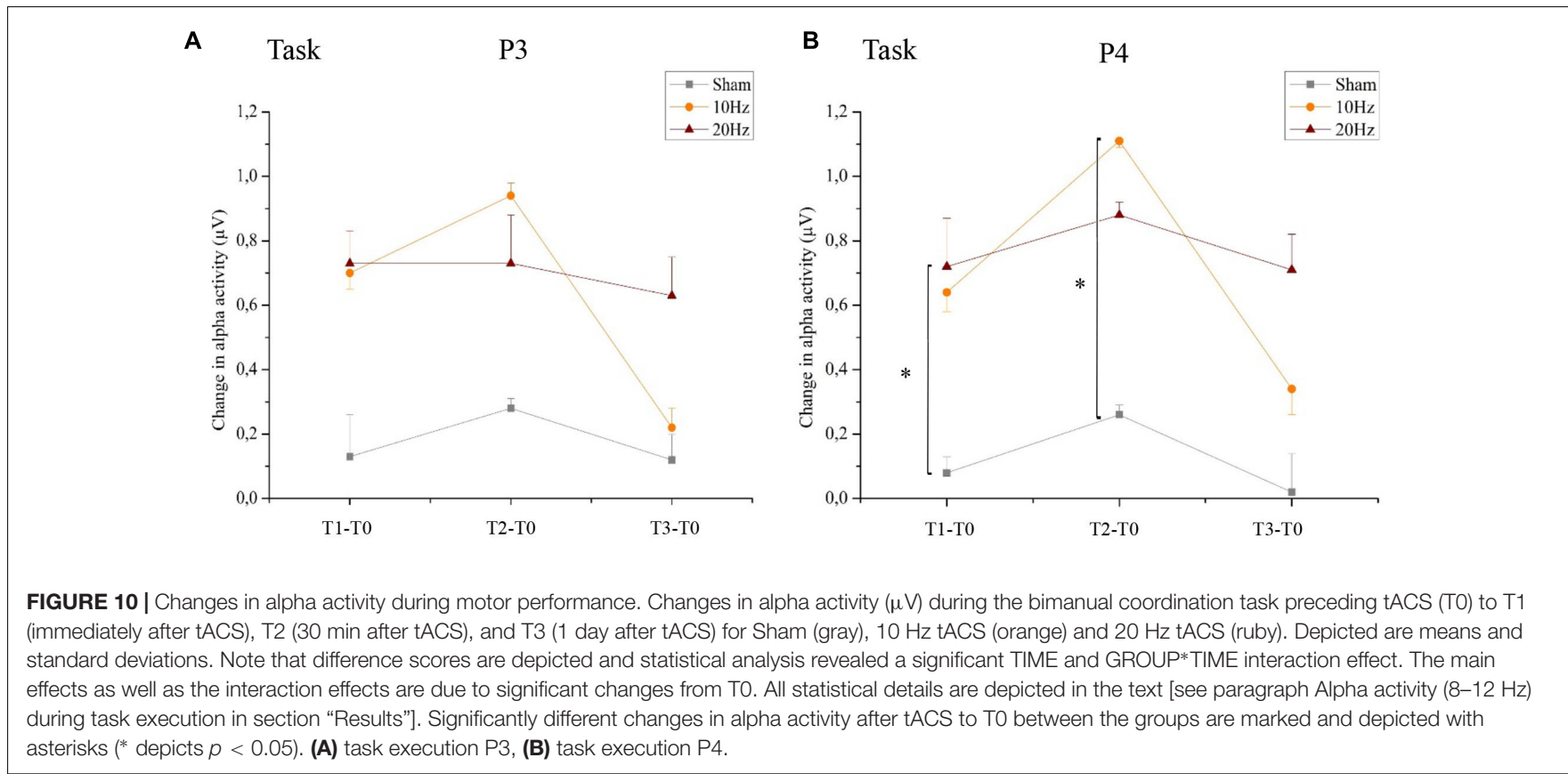




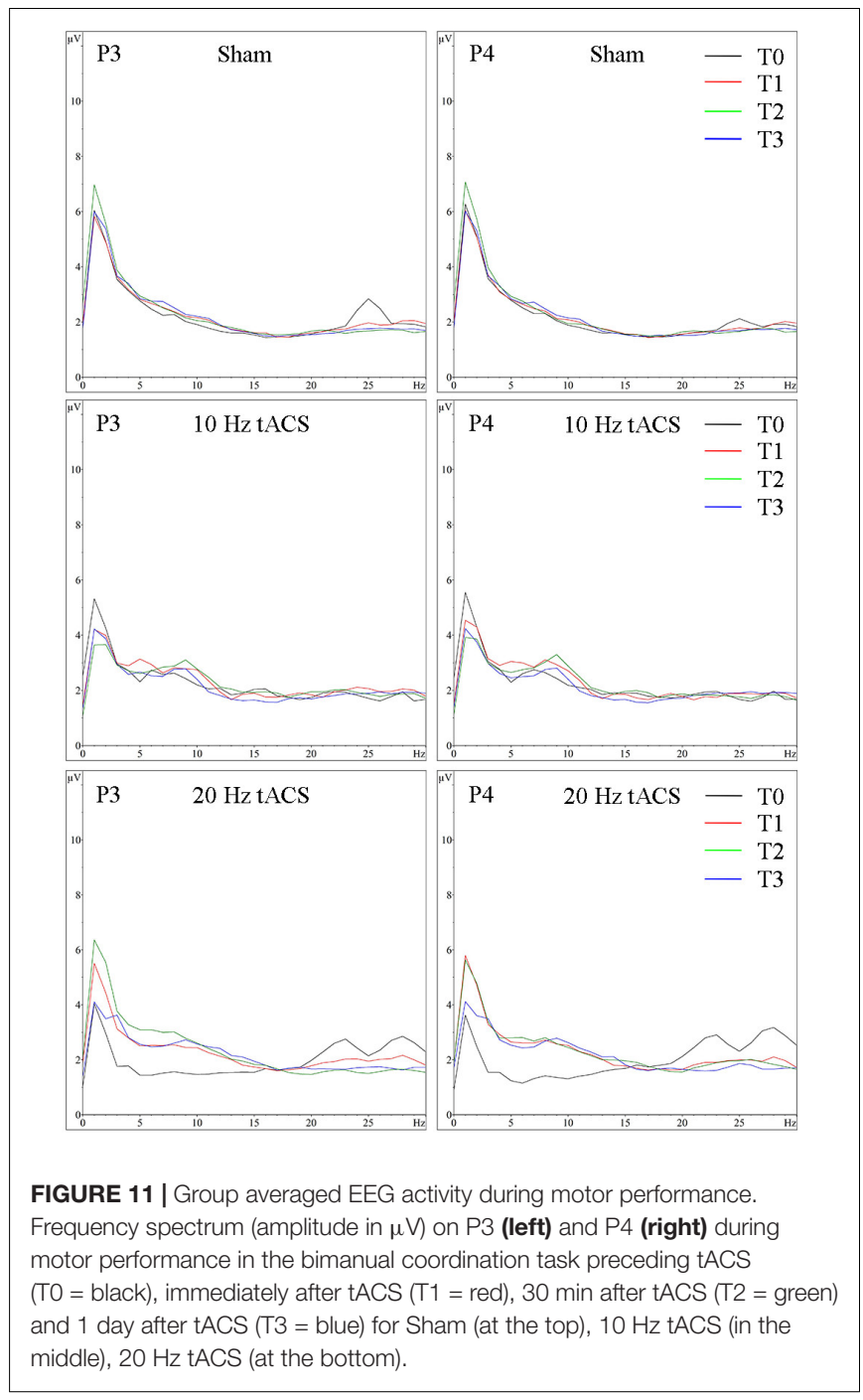

high alpha activity during rest with eyes closed was accompanied by decreases in $\Delta$ Hboxy concentrations, whereby it was not influenced by $10 \mathrm{~Hz}$ tACS. During rest with eyes open, alpha activity was significantly increased after $10 \mathrm{~Hz}$ tACS and $20 \mathrm{~Hz}$ tACS as we have gathered based on the literature (Zaehle et al., 2010) whereas beta band activity stayed unaffected. Furthermore, we hypothesized that parietal $10 \mathrm{~Hz}$ tACS evoke synchronized oscillatory activity in the alpha range that lead to reduced interhemispheric interaction and deterioration in bimanual coordination performance (Serrien and Brown, 2002) whereas $20 \mathrm{~Hz}$ tACS would promote the natural beta oscillation of bimanual coordination (Rjosk et al., 2016) that leads to an enhanced performance. Contrary to our expectations, parietal alpha activity increased significantly after both stimulation frequencies which was accompanied by significant decreases in $\Delta$ Hboxy concentrations right hemispheric whereas bimanual motor performance and beta band activity stayed unaffected.

So far, research regarding the effects of tACS focused mostly on either electrophysiological online effects (Helfrich
A

Change in alpha activity $(\mu \mathrm{V})$ during the task
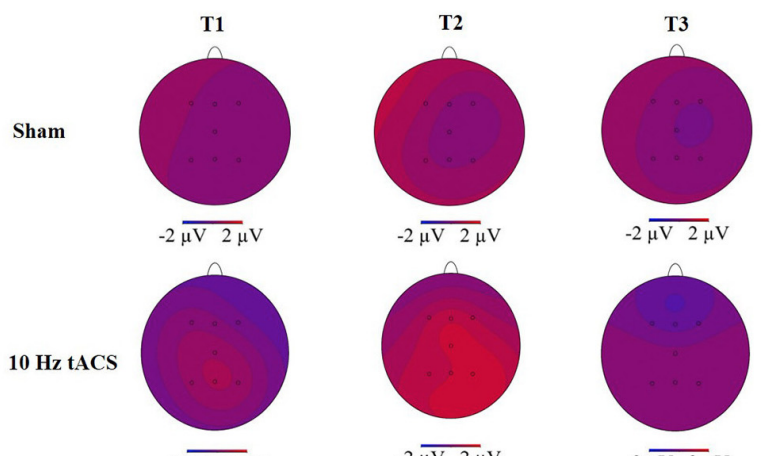

$-2 \overline{\mu \mathrm{V} 2 \mu \mathrm{V}}$

$-2 \overline{\mu \mathrm{V} 2 \mu \mathrm{V}}$

$20 \mathrm{~Hz}$ tACS
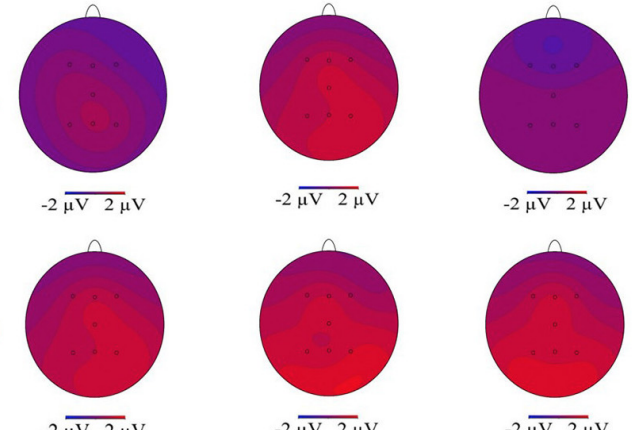

$-2 \overline{\mu \mathrm{V} 2 \mu \mathrm{V}}$

$-2 \overline{\mu \mathrm{V} 2 \mu \mathrm{V}}$

$-2 \overline{\mu \mathrm{V} 2 \mu \mathrm{V}}$
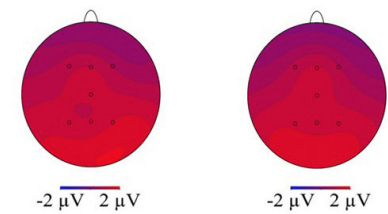

B

Change in beta activity $(\mu \mathrm{V})$ during the task

Sham
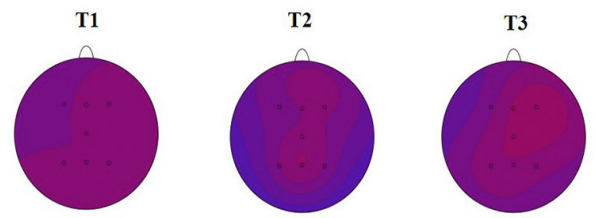

$-1 \overline{\mu \mathrm{V} 1 \mu \mathrm{V}}$

$-1 \overline{\mu \mathrm{V} 1 \mu \mathrm{V}}$

$-1 \overline{\mu \mathrm{V} 1 \mu \mathrm{V}}$

$10 \mathrm{~Hz}$ tACS
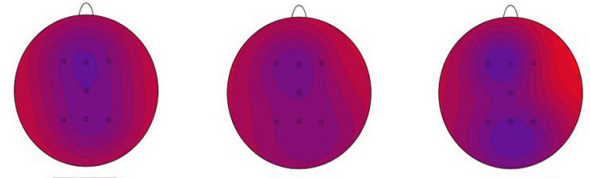

$-1 \overline{\mu \mathrm{V} 1 \mu \mathrm{V}}$

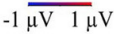

$20 \mathrm{~Hz}$ tACS
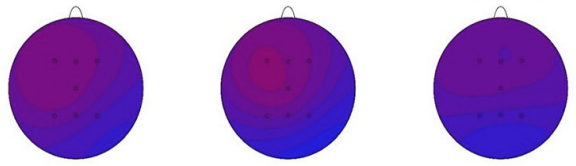

$-1 \overline{\mu \mathrm{V} 1 \mu \mathrm{V}}$

$-1 \overline{\mu \mathrm{V} 1 \mu \mathrm{V}}$

FIGURE 12 | Topographies of group averaged alpha and beta activity changes. (A) Changes in alpha activity $(\mu \mathrm{V})$ during motor performance in the bimanual coordination task preceding tACS (T0) to T1 (immediately after tACS), T2 (30 min after tACS), and T3 (1 day after tACS) for Sham (at the top), $10 \mathrm{~Hz}$ tACS (in the middle), $20 \mathrm{~Hz}$ tACS (at the bottom). (B) Changes in beta activity $(\mu \mathrm{V})$ during motor performance in the bimanual coordination task preceding tACS to T1 (immediately after tACS), T2 (30 min after tACS), and T3 (1 day after tACS) for Sham (at the top), $10 \mathrm{~Hz}$ tACS (in the middle), $20 \mathrm{~Hz}$ tACS (at the bottom). Note that these topographies are based on seven electrode positions only.

et al., 2014; Neuling et al., 2015; Ruhnau et al., 2016) or electrophysiological after-effects during rest (Zaehle et al., 2010; Neuling et al., 2013; Vossen et al., 2015; Kasten et al., 2016). This is the first study provides insights into tACS induced oscillatory and hemodynamic modulations during a 

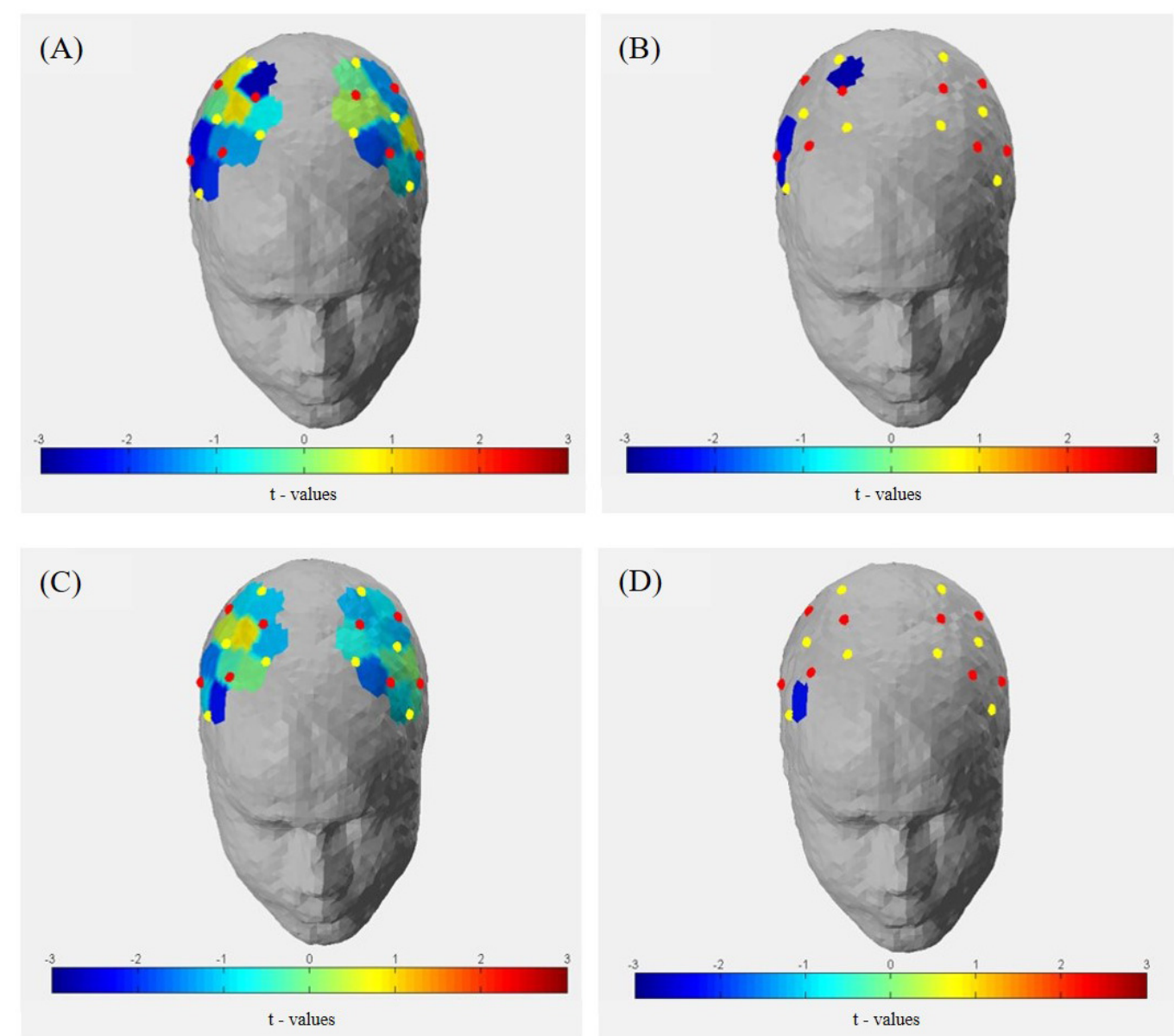

FIGURE 13 | Group averaged t-statistic beta maps. (A) t-contrast of $10 \mathrm{~Hz}$ tACS vs. Sham for Hboxy concentrations in all channels during the bimanual coordination task at T1. (B) Significant channels ( $t$-value $<-2.4$ and $>2.4, p<0.05$ ) were depicted colored in the brain map: channel 12 (S-D) covering the primary somatosensory cortex, channel 19 (S-D) covering the primary motor cortex (BA 4) and channel 20 covering the premotor area (BA 6) of the right hemisphere.

(C) t-contrast of $20 \mathrm{~Hz}$ tACS vs. sham for Hboxy concentrations in all channels during the bimanual coordination task at T1. (D) Significant channels ( $t$-value $<-2.4$ and $>2.4, p<0.05$ ) were depicted colored in the brain map: channel 16 (S6-D8) covering the premotor area (BA 6) of the right hemisphere.

complex motor task using combined electrophysiological and neuroimaging methods.

\section{tACS Effect on Resting State With Eyes Closed}

One of the most interesting findings was that during rest with eyes closed, alpha mean activity significantly increased after application of $20 \mathrm{~Hz}$ tACS compared to sham stimulation that persists even until 1 day after stimulation. Whereas Zaehle et al. (2010) or Kasten et al. (2016) demonstrated sustained physiological IAF tACS after-effects (Zaehle et al., 2010) lasting up to $70 \mathrm{~min}$ (Kasten et al., 2016), we did not observe any significant after-effects for the $10 \mathrm{~Hz}$ tACS on alpha activity. This controversial pattern, however, might be attributed to the performance of the bimanual coordination task concurrently to tACS whereas in the two studies aforementioned, participants were at rest during tACS (Zaehle et al., 2010; Kasten et al., 2016). Consequently, brain states during tACS might be one of the decisive factors for the cause of modulatory tACS after-effects on oscillatory brain activity.

Considering the mechanism of entrainment, both $10 \mathrm{~Hz}$ tACS and $20 \mathrm{~Hz}$ tACS had ratios with the intrinsic frequency that should have principally caused entrainment effects (Herrmann and Strüber, 2017). As a result of frequency-specific aftereffects on rest with eyes closed, it is discussible which role the entrainment mechanism has for plastic-related changes evoked through spike-timing dependent plasticity (Feldman, 2012) which are supposed to be the underlying mechanisms for after-effects (Zaehle et al., 2010; Polanía et al., 2012; Vossen et al., 2015). Regarding the hemodynamic changes during rest with eyes closed, $\Delta$ Hboxy concentrations decreased both before and after tACS. They did not differ significantly which is in line with previous work, where down-regulations of the BOLD signal were observed during rest with eyes closed and which were not modulated by brain stimulation (Vosskuhl et al., 2016). 


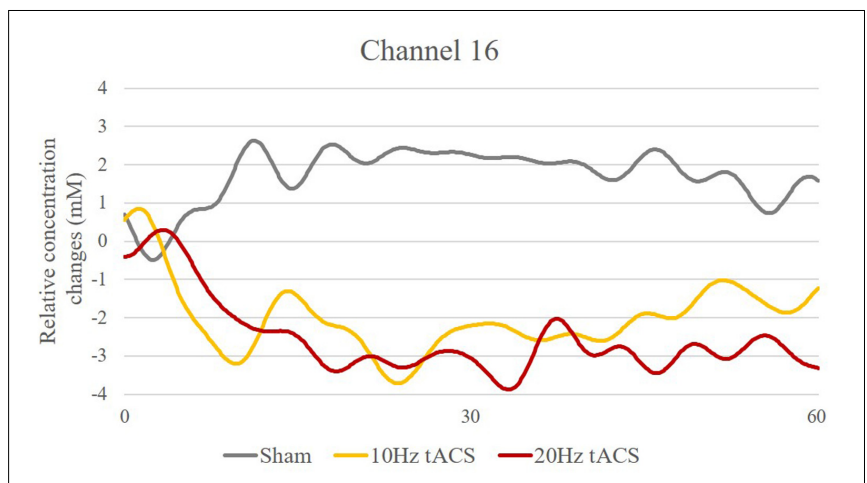

FIGURE 14 | Time-series of Hboxy concentration changes in Channel 16 (Source 6 - Detector 8). Exemplarily, one channel covering the premotor area with significantly different Hboxy concentration time-series for Sham (gray), $10 \mathrm{~Hz}$ tACS (orange) and $20 \mathrm{~Hz}$ tACS (ruby).

\section{tACS Effect on Resting State With Eyes Open}

In rest with eyes open, alpha activity significantly increased following $10 \mathrm{~Hz}$ tACS and $20 \mathrm{~Hz}$ tACS up to $30 \mathrm{~min}$ after stimulation compared to Sham. These results confirm previous work from Neuling et al. (2013) who detected significant effects of IAF tACS during rest with eyes open. However, this pattern was not observed in rest with the eyes closed where the natural high amplitude is already too high to be further elevated. This indicates that tACS in the alpha range is more effective when the power of alpha oscillations is lower (Neuling et al., 2013). As opposed to significant changes recently observed in the BOLD signal following $10 \mathrm{~Hz}$ tACS (Cabral-Calderin et al., 2016), our fNIRS data do not indicate any significant changes of Hboxy concentrations before and after tACS.

\section{tACS Effect on Bimanual Coordination Performance}

All participants improved their bimanual coordination performance significantly following training. However, immediately after training, the bimanual coordination performance leveled off at a performance of about $20 \mathrm{~s}$ for each trial without further improvements across the post measures which indicate a ceiling effect. Moreover, the non-significance of both tACS stimulation effects on bimanual coordination may be attributed to the complexity of the bimanual coordination task. Thus, the frequency-specific effects of $10 \mathrm{~Hz}$ tACS and $20 \mathrm{~Hz}$ tACS on motor learning (Pogosyan et al., 2009; Pollok et al., 2015; Krause et al., 2016) could not be confirmed due to a possible ceiling effect occurring during training. Pogosyan et al. (2009) demonstrated significant lowered movement times after one session of a bimanual tracking task while participants received concurrently $20 \mathrm{~Hz}$ tACS (Pogosyan et al., 2009). As opposed to this, Choe et al. (2016) used both an easy landing task for $10 \mathrm{~min}$ and six blocks of 20 trials from the n-back task each day on four consecutive daily sessions. The task performances of both the easy landing task and the n-back task did not change significantly over time possibly due to a ceiling effect (Choe et al., 2016).

\section{tACS Effect on Neurophysiological Activity During Task Execution}

Our electro- and neurophysiological data indicated for the sham group that alpha activity did not increase significantly after training whereas increased $\Delta$ Hboxy concentrations were observed in most fNIRS-channels covering the motor cortex as it was also reported by Della-Maggiore et al. (2004). This indicates physiological mechanisms underlying training where pre-existing coordination patterns must be suppressed to make space for new patterns (Neva et al., 2012). However, compared to Sham, significantly enhanced alpha activity was observed following $10 \mathrm{~Hz}$ tACS and $20 \mathrm{~Hz}$ tACS which was accompanied by significant decreases in $\Delta$ Hboxy concentrations in the right hemisphere. These findings fit well with our expectations, that increased alpha activity is accompanied by decreased hemodynamical activity which is also in line with previous work from Choe et al. (2016) where increased parietal alpha activity correlates with reduced fNIRS beta-values (Choe et al., 2016). Nevertheless, we had hypothesized that $20 \mathrm{~Hz}$ tACS does not elicit oscillatory activity in the alpha range which is associated with improved bimanual coordination performance, whereas $10 \mathrm{~Hz}$ tACS enhances alpha activity that slows bimanual movements. Contrary to this, the present experiment suggests that $20 \mathrm{~Hz}$ tACS enhanced alpha mean activity significantly up to 1 day after stimulation. However, as depicted in Figure 9, performance improvements in the bimanual coordination task were lower compared to the improvements of the Sham group, even though they did not achieve significance. Therefore, the interplay between oscillatory activity as well as hemodynamic processes and coordinated bimanual behavior remain an open question since tACS modulate oscillatory activity and hemodynamic changes significantly (Zaehle et al., 2010; Polanía et al., 2012) without significant effects on behavioral outcome. One explanation, however, might be due to the chosen stimulation location: We positioned the HD-tACS electrodes bilaterally on the parietal cortex (P3 and P4) because of its functional role in integrating multi-sensory signals and spatial-temporal coordination of visually controlled movements (Swinnen and Wenderoth, 2004). However, previous studies stimulated the primary motor cortex (M1) and revealed significant effects of tACS on motor learning (Krause et al., 2016; Heise et al., 2017).

Furthermore, based on our data pattern, two difficult and not yet easily answered questions occurred which should be mentioned, and which require further research. On the one hand, we found lateralized effects of tACS. The increased alpha activity between the $20 \mathrm{~Hz}$ tACS group and Sham at T1 was significant for the parietal area on the right hemisphere (P4). This was accompanied by significantly decreased Hboxy concentration changes in motor areas also in the right hemisphere. Thus, bilateral induced tACS might evoke changes in oscillatory and hemodynamic activity in intra-hemispheric motor networks. Considering the neural 
dynamics of hemispheric functions in bimanual coordination, the question arises of how the hemispheric specializations and integrations are organized in bimanual movements? The functional participation of both hemispheres in motor regulation is dynamical and versatile (Serrien et al., 2006). Evidences from callosal patients with bimanual coordination deficits indicate that bimanual patterns rely on interhemispheric couplings (Kennerley et al., 2002) whereby the dominant hemisphere (i.e., the left hemisphere in our population) controls the functional coupling between the motor cortices (Serrien et al., 2003). Both hemispheric asymmetries and an optimal balance between the left and right hemisphere are vital (Serrien et al., 2006). Although the right hemisphere also plays a crucial role for closed-loop aspects of movements dependent on sensory feedback (Haaland and Harrington, 1989) which is indispensable for the realization of goal-directed behavior (Serrien et al., 2006). Various studies highlight the responsibility of the dominant hemisphere for bimanual coordination (Serrien et al., 2003). However, whether this might explain the lateralized physiological effects of tACS without changes in bimanual coordination performance is speculative and requires further research.

On the other hand, we did not find any effects of tACS on brain oscillations in beta frequency. Both stimulation frequencies were applied during bimanual coordination training where entrainment (Antal and Paulus, 2013; Herrmann et al., 2013) and enhanced beta oscillations particularly following $20 \mathrm{~Hz}$ tACS were supposed. Whereas beta activity remained unaltered, increased alpha activity evoked by $20 \mathrm{~Hz}$ tACS lasted until 1 day after stimulation. One approach might be the ambiguous mechanisms underlying bimanual coordination. Rjosk et al. (2016) demonstrated that beta-band activity plays a crucial role in interhemispheric coordination of movements (Rjosk et al., 2016). Additionally, Davis et al. (2012) suppose that motor control is associated with synchronized oscillatory activity at beta frequency, whereby voluntary movements are associated with suppressed beta band activity (desynchronization) (Davis et al., 2012). Thus, alpha oscillations might have dominated the interhemispheric communication during training which resulted in enhanced alpha band activity during bimanual coordination after both $10 \mathrm{~Hz}$ tACS and $20 \mathrm{~Hz}$ tACS.

\section{Limitations and Future Work}

The present study's purpose was to extend the multimodal investigations of tACS effects performed so far by focusing on the modulatory effects of tACS on bimanual coordination and the underlying electro- and neurophysiological mechanisms. This knowledge from a basic research point of view is essential for possible transfers into interventional studies for rehabilitation of motor disorders in patients suffering from neurological diseases. While our results demonstrate a significant modulation of brain oscillatory and hemodynamic activity by tACS, no group differences in bimanual motor learning were observed. Because of the expected learning effect in the bimanual coordination task and the consecutive sessions, no within-subject design was chosen. Future studies should either consider within-subject designs regarding inter-individual variability of tACS effects or they should ascertain the initial skill levels in advance to categorize participants in homogenous groups before prior to alignment. However, the main challenge here and in other studies is the existence of both tACS responders and nonresponders with the same tACS protocol due to neuroanatomical and neurophysiological differences on the one side and different tACS effects within an individual over time due to neural plastic changes on the other side. One approach to avoid the "one size fits all" approach could be a closed-loop tACS based on EEG or neuroimaging techniques (Choe et al., 2016). The individualized tACS application may allow for a deeper insight in the mechanisms and effects of tACS which is elementary for future research and the practical transfer although, it will be a big challenge to implement individualized or closed-loop approaches in neurorehabilitation.

Additionally, three other limitations could be mentioned: firstly, the statistical power is relatively low because of $n=8$ subjects per group. Referring to Choe et al. (2016) where tDCS effects on bimanual motor learning were investigated with EEG and ANIRS in four different groups with comparable sample sizes per group ( $n=7-10)$, we focused on the methodological challenge as well. In further studies, the after-effects of tACS could be investigated either at consecutive days or with electrophysiological and neuroimaging methods to consider the complexity and to increase the sample size for higher statistical power. Secondly, no further stimulation conditions in terms of an active control group (e.g., receiving tACS on other brain regions like frontal areas) were included. Thirdly, only two stimulation electrodes were used to rely on previous studies demonstrating tACS effects with larger sponge-electrodes. In future, the eight HD-tACS electrodes could be used to design a montage covering left and right hemispherical motor networks for investigating the causality of altered interregional brain synchronization in patients with bimanual coordination disorders. Additionally, the subject's baseline performances are crucial for motor skill development and baseline measures of the differing initial skill levels for homogeneous classification were not considered in this study. Thus, subject's diverse experiences may be relevant in the interpretation of our behavioral findings.

\section{CONCLUSION}

Based on previous studies which determined either physiological or behavioral effects of tACS, we were able to demonstrate a selective enhancement in (1) brain oscillatory activity in the alpha range and (2) decreases in $\Delta$ Hboxy concentrations in regions of the premotor area (BA6), the primary motor cortex (BA4) and the primary somatosensory cortex $(\mathrm{BA} 3 / 2 / 1)$ of the right hemisphere following $10 \mathrm{~Hz}$ tACS and $20 \mathrm{~Hz}$ tACS compared to sham stimulation. The present findings of the simultaneous EEG/fNIRS application represent a valid starting point to close the gap in the tACS literature concerning stimulation effects on bimanual coordination and the underlying electro- and neurophysiological mechanisms. This tACS knowledge is of high importance for basic research and clinical transfer to improve treatments in 
neurorehabilitation for patients with pathological oscillations which are accompanied by bimanual coordination disorders.

\section{ETHICS STATEMENT}

This study was carried out in accordance with the recommendations of 'Ethical Principles of Psychologists and Code of Conduct, APA, Ethikkommission des FB02 Johannes GutenbergUniversität Mainz' with written informed consent from all subjects in accordance with the Declaration of Helsinki. The protocol was approved by the 'Ethikkommission des FB02 Johannes Gutenberg-Universität Mainz'.

\section{AUTHOR CONTRIBUTIONS}

$\mathrm{AB}$ contributed to the conceptualization and realization of the study, performed the data acquisition and the analysis and interpretation, and wrote the manuscript. NP and FS

\section{REFERENCES}

Antal, A., Boros, K., Poreisz, C., Chaieb, L., Terney, D., and Paulus, W. (2008). Comparatively weak after-effects of transcranial alternating current stimulation (tACS) on cortical excitability in humans. Brain Stimul. 1, 97-105. doi: 10.1016/ j.brs.2007.10.001

Antal, A., and Paulus, W. (2013). Transcranial alternating current stimulation (tACS). Front. Hum. Neurosci. 7:317. doi: 10.3389/fnhum.2013.00317

Bergmann, T. O., Karabanov, A., Hartwigsen, G., Thielscher, A., and Siebner, H. R. (2016). Combining non-invasive transcranial brain stimulation with neuroimaging and electrophysiology: current approaches and future perspectives. Neuroimage 140, 4-19. doi: 10.1016/j.neuroimage.2016.02.012

Buneo, C. A., and Andersen, R. A. (2006). The posterior parietal cortex: sensorimotor interface for the planning and online control of visually guided movements. Neuropsychologia 44, 2594-2606. doi: 10.1016/j.neuropsychologia. 2005.10.011

Cabral-Calderin, Y., Anne Weinrich, C., Schmidt-Samoa, C., Poland, E., Dechent, P., Bahr, M., et al. (2016). Transcranial alternating current stimulation affects the BOLD signal in a frequency and task-dependent manner. Hum. Brain Mapp. 37, 94-121. doi: 10.1002/hbm.23016

Cappon, D., D’Ostilio, K., Garraux, G., Rothwell, J., and Bisiacchi, P. (2016). Effects of $10 \mathrm{~Hz}$ and $20 \mathrm{~Hz}$ transcranial alternating current stimulation on automatic motor control. Brain Stimul. 9, 518-524. doi: 10.1016/j.brs.2016.01.001

Chander, B. S., Witkowski, M., Braun, C., Robinson, S. E., Born, J., Cohen, L. G., et al. (2016). tACS phase locking of frontal midline theta oscillations disrupts working memory performance. Front. Cell. Neurosci. 10:120. doi: 10.3389/fncel. 2016.00120

Chen, L.-C., Sandmann, P., Thorne, J. D., Herrmann, C. S., and Debener, S. (2015). Association of concurrent fNIRS and EEG signatures in response to auditory and visual stimuli. Brain Topogr. 28, 710-725. doi: 10.1007/s10548-015-0424-8

Choe, J., Coffman, B. A., Bergstedt, D. T., Ziegler, M. D., and Phillips, M. E. (2016). Transcranial direct current stimulation modulates neuronal activity and learning in pilot training. Front. Hum. Neurosci. 10:34. doi: 10.3389/fnhum. 2016.00034

Culham, J. C., Cavina-Pratesi, C., and Singhal, A. (2006). The role of parietal cortex in visuomotor control: what have we learned from neuroimaging? Neuropsychologia 44, 2668-2684. doi: 10.1016/j.neuropsychologia.2005.11.003

Davis, N. J., Tomlinson, S. P., and Morgan, H. M. (2012). The role of betafrequency neural oscillations in motor control. J. Neurosci. 32, 403-404. doi: 10.1523/JNEUROSCI.5106-11.2012

Della-Maggiore, V., Malfait, N., Ostry, D. J., and Paus, T. (2004). Stimulation of the posterior parietal cortex interferes with arm trajectory adjustments substantially contributed to the data analysis and interpretation and critically revised the manuscript. MD contributed to the conceptualization and design of the study and was involved in the data analysis and interpretation. He critically revised the manuscript, approved the final version and its content, and acted as corresponding author.

\section{ACKNOWLEDGMENTS}

We thank M. Klug, A. Bessling, and F. Czech for their support during data acquisition.

\section{SUPPLEMENTARY MATERIAL}

The Supplementary Material for this article can be found online at: https://www.frontiersin.org/articles/10.3389/fnbeh. 2018.00067/full\#supplementary-material

during the learning of new dynamics. J. Neurosci. 24, 9971-9976. doi: 10.1523/ JNEUROSCI.2833-04.2004

Feldman, D. E. (2012). The spike-timing dependence of plasticity. Neuron 75, 556-571. doi: 10.1016/j.neuron.2012.08.001

Haaland, K. Y., and Harrington, D. L. (1989). Hemispheric control of the initial and corrective components of aiming movements. Neuropsychologia 27, 961-969.

Heise, K.-F., Monteiro, T. S., Gijbels, V., Leunissen, I. H., Mantni, D., and Swinnen, S. P. (2017). Modulation of interhemispheric connectivity by alternating current stimulation and its impact on transitions between bimanual movements of varying stability. Brain Stimul. 10:452. doi: 10.1016/j.brs.2017.01.328

Helfrich, R. F., Schneider, T. R., Rach, S., Trautmann-Lengsfeld, S. A., Engel, A. K., and Herrmann, C. S. (2014). Entrainment of brain oscillations by transcranial alternating current stimulation. Curr. Biol. 24, 333-339. doi: 10.1016/j.cub.2013. 12.041

Herrmann, C. S., Rach, S., Neuling, T., and Struber, D. (2013). Transcranial alternating current stimulation: a review of the underlying mechanisms and modulation of cognitive processes. Front. Hum. Neurosci. 7:279. doi: 10.3389/ fnhum.2013.00279

Herrmann, C. S., and Strüber, D. (2017). What can transcranial alternating current stimulation tell us about brain oscillations? Curr. Behav. Neurosci. Rep. 4, 128-137. doi: 10.1007/s40473-017-0114-9

Hsu, W.-Y., Zanto, T. P., van Schouwenburg, M. R., and Gazzaley, A. (2017). Enhancement of multitasking performance and neural oscillations by transcranial alternating current stimulation. PLoS One 12:e0178579. doi: 10.1371/journal.pone.0178579

Jurcak, V., Tsuzuki, D., and Dan, I. (2007). 10/20, 10/10, and 10/5 systems revisited: their validity as relative head-surface-based positioning systems. Neuroimage 34, 1600-1611. doi: 10.1016/j.neuroimage.2006.09.024

Kasten, F. H., Dowsett, J., and Herrmann, C. S. (2016). Sustained aftereffect of alpha-tACS lasts up to $70 \mathrm{~min}$ after stimulation. Front. Hum. Neurosci. 10:245. doi: 10.3389/fnhum.2016.00245

Kasten, F. H., and Herrmann, C. S. (2017). Transcranial alternating current stimulation (tACS) enhances mental rotation performance during and after stimulation. Front. Hum. Neurosci. 11:2. doi: 10.3389/fnhum.2017. 00002

Kennerley, S. W., Diedrichsen, J., Hazeltine, E., Semjen, A., and Ivry, R. B. (2002). Callosotomy patients exhibit temporal uncoupling during continuous bimanual movements. Nat. Neurosci. 5, 376-381. doi: 10.1038/nn822

Krause, V., Meier, A., Dinkelbach, L., and Pollok, B. (2016). Beta band transcranial alternating (tACS) and direct current stimulation (tDCS) applied after initial learning facilitate retrieval of a motor sequence. Front. Behav. Neurosci. 10:4. doi: 10.3389/fnbeh.2016.00004 
Leunissen, I., Coxon, J. P., and Swinnen, S. P. (2017). Transcranial alternating current stimulation in the beta frequency promotes motor inhibition. Brain Stimul. 10, 440-441. doi: 10.1016/j.brs.2017.01.313

McKendrick, R., Parasuraman, R., and Ayaz, H. (2015). Wearable functional near infrared spectroscopy (fNIRS) and transcranial direct current stimulation (tDCS): expanding vistas for neurocognitive augmentation. Front. Syst. Neurosci. 9:27. doi: 10.3389/fnsys.2015.00027

Muthalib, M., Besson, P., Rothwell, J., and Perrey, S. (2017). Focal hemodynamic responses in the stimulated hemisphere during high-definition transcranial direct current stimulation. Neuromodulation doi: 10.1111/ner.12632 [Epub ahead of print].

Neuling, T., Rach, S., and Herrmann, C. S. (2013). Orchestrating neuronal networks: sustained after-effects of transcranial alternating current stimulation depend upon brain states. Front. Hum. Neurosci. 7:161. doi: 10.3389/fnhum. 2013.00161

Neuling, T., Ruhnau, P., Fuscà, M., Demarchi, G., Herrmann, C. S., and Weisz, N. (2015). Friends, not foes: magnetoencephalography as a tool to uncover brain dynamics during transcranial alternating current stimulation. Neuroimage 118, 406-413. doi: 10.1016/j.neuroimage.2015.06.026

Neva, J. L., Legon, W., and Staines, W. R. (2012). Primary motor cortex excitability is modulated with bimanual training. Neurosci. Lett. 514, 147-151. doi: 10.1016/ j.neulet.2012.02.075

Oldfield, R. C. (1971). The assessment and analysis of handedness: the Edinburgh inventory. Neuropsychologia 9, 97-113. doi: 10.1016/0028-3932(71)90067-4

Pogosyan, A., Gaynor, L. D., Eusebio, A., and Brown, P. (2009). Boosting cortical activity at Beta-band frequencies slows movement in humans. Curr. Biol. 19, 1637-1641. doi: 10.1016/j.cub.2009.07.074

Polanía, R., Nitsche, M. A., Korman, C., Batsikadze, G., and Paulus, W. (2012). The importance of timing in segregated theta phase-coupling for cognitive performance. Curr. Biol. 22, 1314-1318. doi: 10.1016/j.cub.2012.05.021

Pollok, B., Boysen, A.-C., and Krause, V. (2015). The effect of transcranial alternating current stimulation (tACS) at alpha and beta frequency on motor learning. Behav. Brain Res. 293, 234-240. doi: 10.1016/j.bbr.2015.07.049

Rjosk, V., Kaminski, E., Hoff, M., Gundlach, C., Villringer, A., Sehm, B., et al. (2016). Transcranial alternating current stimulation at beta frequency: lack of immediate effects on excitation and interhemispheric inhibition of the human motor cortex. Front. Hum. Neurosci. 10:560. doi: 10.3389/fnhum.2016. 00560

Ruffini, G., Fox, M. D., Ripolles, O., Miranda, P. C., and Pascual-Leone, A. (2014). Optimization of multifocal transcranial current stimulation for weighted cortical pattern targeting from realistic modeling of electric fields. Neuroimage 89, 216-225. doi: 10.1016/j.neuroimage.2013.12.002

Ruhnau, P., Neuling, T., Fuscá, M., Herrmann, C. S., Demarchi, G., and Weisz, N. (2016). Eyes wide shut: transcranial alternating current stimulation drives alpha rhythm in a state dependent manner. Sci. Rep. 6:27138. doi: 10.1038/srep 27138

Sauseng, P., and Klimesch, W. (2008). What does phase information of oscillatory brain activity tell us about cognitive processes? Neurosci. Biobehav. Rev. 32, 1001-1013. doi: 10.1016/j.neubiorev.2008.03.014

Schnitzler, A., and Gross, J. (2005). Normal and pathological oscillatory communication in the brain. Nat. Rev. Neurosci. 6, 285-296. doi: 10.1038/ nrn 1650

Serrien, D. J., and Brown, P. (2002). The functional role of interhemispheric synchronization in the control of bimanual timing tasks. Exp. Brain Res. 147, 268-272. doi: 10.1007/s00221-002-1253-z

Serrien, D. J., Cassidy, M. J., and Brown, P. (2003). The importance of the dominant hemisphere in the organization of bimanual movements. Hum. Brain Mapp. 18, 296-305. doi: 10.1002/hbm.10086

Serrien, D. J., Ivry, R. B., and Swinnen, S. P. (2006). Dynamics of hemispheric specialization and integration in the context of motor control. Nat. Rev. Neurosci. 7, 160-166. doi: 10.1038/nrn1849
Stam, C. J., van der Made, Y., Pijnenburg, Y. A. L., and Scheltens, P. (2003). EEG synchronization in mild cognitive impairment and Alzheimer's disease. Acta Neurol. Scand. 108, 90-96. doi: 10.1034/j.1600-0404.2003.02067.x

Strangman, G., Culver, J. P., Thompson, J. H., and Boas, D. A. (2002). A quantitative comparison of simultaneous BOLD fMRI and NIRS recordings during functional brain activation. Neuroimage 17, 719-731.

Strüber, D., Rach, S., Trautmann-Lengsfeld, S. A., Engel, A. K., and Herrmann, C. S. (2014). Antiphasic $40 \mathrm{~Hz}$ oscillatory current stimulation affects bistable motion perception. Brain Topogr. 27, 158-171. doi: 10.1007/s10548-013-0294-x

Swinnen, S. P., and Wenderoth, N. (2004). Two hands, one brain: cognitive neuroscience of bimanual skill. Trends Cogn. Sci. 8, 18-25. doi: 10.1016/j.tics. 2003.10.017

Takeuchi, N., Oouchida, Y., and Izumi, S.-I. (2012). Motor control and neural plasticity through interhemispheric interactions. Neural Plast. 2012:823285. doi: $10.1155 / 2012 / 823285$

Uhlhaas, P. J., and Singer, W. (2006). Neural synchrony in brain disorders: relevance for cognitive dysfunctions and pathophysiology. Neuron 52, 155-168. doi: 10.1016/j.neuron.2006.09.020

Vossen, A., Gross, J., and Thut, G. (2015). Alpha power increase after transcranial alternating current stimulation at alpha frequency (alpha-tACS) reflects plastic changes rather than entrainment. Brain Stimul. 8, 499-508. doi: 10.1016/j.brs. 2014.12.004

Vosskuhl, J., Huster, R. J., and Herrmann, C. S. (2016). BOLD signal effects of transcranial alternating current stimulation (tACS) in the alpha range: a concurrent tACS-fMRI study. Neuroimage 140, 118-125. doi: 10.1016/j. neuroimage.2015.10.003

Vosskuhl, J., Huster, R. J., and Herrmann, C. S. (2015a). Increase in shortterm memory capacity induced by down-regulating individual theta frequency via transcranial alternating current stimulation. Front. Hum. Neurosci. 9:257. doi: 10.3389/fnhum.2015.00257

Vosskuhl, J., Struber, D., and Herrmann, C. S. (2015b). Transkranielle wechselstromstimulation. Entrainment und funktionssteuerung neuronaler netzwerke [Transcranial alternating current stimulation. Entrainment and function control of neuronal networks]. Nervenarzt 86, 1516-1522. doi: 10.1007/s00115-015-4317-6

Wach, C., Krause, V., Moliadze, V., Paulus, W., Schnitzler, A., and Pollok, B. (2013). The effect of $10 \mathrm{~Hz}$ transcranial alternating current stimulation (tACS) on corticomuscular coherence. Front. Hum. Neurosci. 7:511. doi: 10.3389/fnhum. 2013.00511

Wessel, M. J., Zimerman, M., and Hummel, F. C. (2015). Non-invasive brain stimulation: an interventional tool for enhancing behavioral training after stroke. Front. Hum. Neurosci. 9:265. doi: 10.3389/fnhum.2015.00265

Williams, K. A., Cabral-Calderin, Y., Schmidt-Samoa, C., Weinrich, C. A., Dechent, P., and Wilke, M. (2017). Simultaneous transcranial alternating current stimulation and functional magnetic resonance imaging. J. Vis. Exp. 124:55866. doi: 10.3791/55866

Zaehle, T., Rach, S., and Herrmann, C. S. (2010). Transcranial alternating current stimulation enhances individual alpha activity in human EEG. PLoS One 5:e13766. doi: 10.1371/journal.pone.0013766

Conflict of Interest Statement: The authors declare that the research was conducted in the absence of any commercial or financial relationships that could be construed as a potential conflict of interest.

Copyright (c) 2018 Berger, Pixa, Steinberg and Doppelmayr. This is an open-access article distributed under the terms of the Creative Commons Attribution License (CC BY). The use, distribution or reproduction in other forums is permitted, provided the original author(s) and the copyright owner are credited and that the original publication in this journal is cited, in accordance with accepted academic practice. No use, distribution or reproduction is permitted which does not comply with these terms. 


\title{
Effects of tDCS on Bimanual Motor Skills: A Brief Review
}

\author{
Nils H. Pixa ${ }^{1 *}$ and Bettina Pollok ${ }^{2}$ \\ ${ }^{1}$ Department of Sport Psychology, Institute of Sports Science, Johannes Gutenberg-University Mainz, Mainz, Germany, \\ 2Institute of Clinical Neuroscience and Medical Psychology, Medical Faculty, Heinrich-Heine-University Düsseldorf, \\ Düsseldorf, Germany
}

\section{OPEN ACCESS}

Edited by:

Roberta Ferrucci, Fondazione IRCCS Ca' Granda Ospedale Maggiore Policlinico (IRCCS), Italy

Reviewed by: Wei Peng Teo, Deakin University, Australia Sean Kevin Meehan, University of Michigan, United States

*Correspondence:

Nils H. Pixa

pixa@uni-mainz.de

Received: 08 January 2018 Accepted: 16 March 2018 Published: 04 April 2018

Citation: Pixa NH and Pollok B (2018) Effects of tDCS on Bimanual Motor Skills: A Brief Review.

Front. Behav. Neurosci. 12:63. doi: 10.3389/fnbeh.2018.00063
Transcranial direct current stimulation (tDCS) is a non-invasive brain stimulation technique that allows the modulation of cortical excitability as well as neuroplastic reorganization using a weak constant current applied through the skull on the cerebral cortex. TDCS has been found to improve motor performance in general and motor learning in particular. However, these effects have been reported almost exclusively for unimanual motor tasks such as serial reaction time tasks, adaptation tasks, or visuomotor tracking. Despite the importance of bimanual actions in most activities of daily living, only few studies have investigated the effects of tDCS on bimanual motor skills. The objectives of this review article are: (i) to provide a concise overview of the few existing studies in this area; and (ii) to discuss the effects of tDCS on bimanual motor skills in healthy volunteers and patients suffering from neurological diseases. Despite considerable variations in stimulation protocols, the bimanual tasks employed, and study designs, the data suggest that tDCS has the potential to enhance bimanual motor skills. The findings imply that the effects of tDCS vary with task demands, such as complexity and the level of expertise of the participating volunteers. Nevertheless, optimized stimulation protocols tailored to bimanual tasks and individual performance considering the underlying neural substrates of task execution are required in order to probe the effectiveness of tDCS in greater detail, thus creating an opportunity to support motor recovery in neuro-rehabilitation.

Keywords: non-invasive brain stimulation, transcranial direct current stimulation, bimanual movements, bimanual coordination, motor learning and performance

\section{INTRODUCTION}

The non-invasive brain stimulation technique of transcranial direct current stimulation (tDCS) is a suitable method for modulating cortical excitability (Bindman et al., 1964; Nitsche and Paulus, 2001) as well as neuro-plastic reorganization (Fritsch et al., 2010; Hunter et al., 2013; Karabanov et al., 2015). Previous studies suggest that tDCS can be used to facilitate motor performance such as motor learning in healthy volunteers (Reis and Fritsch, 2011; Buch et al., 2017) and in patients suffering from neurological disorders (Bastani and Jaberzadeh, 2012; Flöel, 2014). Notably, the effects of tDCS on motor performance are almost exclusively evidenced by studies employing unimanual tasks. However, it is well known that in a large variety of daily activities both hands are required to accomplish required actions. Impaired bimanual skills due to neurological disease or age-related decline (for an overview see Maes et al., 2017) present a challenge to independent living. Despite the abundance of daily activities that require bimanual skills, only few studies have thus 
far investigated the effects of tDCS on bimanual performance. Considering the increasing number of tDCS studies and the high relevance of bimanual motor skills the purpose of this review article is to provide a preliminary systematic characterization of tDCS effects on bimanual actions.

\section{REVIEW CRITERIA}

The present review article focuses on studies addressing tDCS effects explicitly on bimanual motor performance, thus investigating bimanual motor outcome was the decisive criterion. To this end, a computer-based search of PubMed and Science Direct for articles from 2000 to 2017 was carried out in August 2017 using the following keywords: "bimanual" AND "transcranial direct current stimulation" OR "tDCS" OR "transcranial electrical stimulation" OR "non-invasive brain stimulation" OR "transcranial stimulation" and "coordination" AND "transcranial direct current stimulation" OR "tDCS" OR "transcranial electrical stimulation" OR "non-invasive brain stimulation" OR "transcranial stimulation". A total of 18 articles matched the criteria. Five articles did not directly investigate bimanual motor skills and were therefore excluded.

\section{TRANSCRANIAL DIRECT CURRENT STIMULATION}

TDCS allows the modulation of neural excitability (Bindman et al., 1962, 1964; Nitsche and Paulus, 2001). Using intensities of 0.5-2 milliampere $(\mathrm{mA})$, a constant current is applied to the cerebral cortex via saline soaked sponges or gel-electrodes through the skull. Two electrodes with a surface area of $15-35 \mathrm{~cm}^{2}$ are used mostly, resulting in a current density of 0.014-0.133 mA/cm² (Ho et al., 2016; Woods et al., 2016). In high-definition tDCS (HD-tDCS), smaller (1-5 $\mathrm{cm}^{2}$ ) and often more than two electrodes (e.g., $4 \times 1$ configuration) are used to increase the current density and focality of the stimulated area (Villamar et al., 2013; Alam et al., 2016). Although its exact physiological mechanisms are still under debate, evidence exists that anodal tDCS increases the neural excitability of the stimulated area, while cathodal tDCS decreases it. Anodal stimulation shifts the resting membrane potential closer to the critical depolarization threshold, resulting in a higher excitability and spiking rate, while the opposite effect of tonic hyperpolarization is associated with cathodal stimulation (Bindman et al., 1962; Nitsche and Paulus, 2000; Romero Lauro et al., 2014). These effects have mainly been derived from the stimulation of the primary motor cortex (M1), although comparable effects have also been demonstrated in visual (Antal et al., 2004; Accornero et al., 2007), somatosensory (Dieckhöfer et al., 2006), and auditory cortices (Zaehle et al., 2011). Nevertheless, anatomical differences_in particular, the spatial orientation of neurons - need to be considered in order to understand the effects of tDCS on other brain areas (Accornero et al., 2007). Moreover, the effects of tDCS depend on several factors like current intensity, duration and timing of tDCS relative to the specific task (prior to vs. during; Stagg et al.,
2011; Batsikadze et al., 2013; Monte-Silva et al., 2013). The shape, size, number, and positions of the electrodes also determine the characteristics of tDCS such as distribution of the induced electrical field (Ho et al., 2016; Naros et al., 2016; Woods et al., 2016). Individual attributes of the participants (e.g., head anatomy, age) but also the excitability of the stimulated area are thought to influence the effects of tDCS yielding intraand inter-individual variability on physiological and behavioral measures (Ridding and Ziemann, 2010; Li et al., 2015; Opitz et al., 2015). Besides local effects on the stimulated brain area, tDCS is presumed to affect the excitability of functionally connected areas as well. Such remote effects can be in the same (Antal et al., 2011) or opposite (Stagg et al., 2009) direction as the effects in the stimulated area.

Changes of M1 excitability associated with tDCS have been found to persist after cessation of stimulation (Nitsche and Paulus, 2000, 2001). Such after-effects are associated with alterations in $\mathrm{N}$-methyl-d-aspartate (NMDA) receptors (Liebetanz et al., 2002)—at least within M1-yielding long-term potentiation (LTP)-like mechanisms. In motor learning, these effects seem to be strongest when tDCS is co-applied with motor training (Reis and Fritsch, 2011; Stagg et al., 2011) and applied over multiple days (Reis et al., 2009; Alonzo et al., 2012; Saucedo Marquez et al., 2013).

\section{tDCS AND BIMANUAL MOTOR SKILLS IN HEALTHY VOLUNTEERS}

Bimanual motor skills require a well-coordinated interplay between the upper limbs. Multiple brain areas are involved in orchestrating bimanual movements in space and time. Because the supplementary motor area (SMA) plays a pivotal role in such tasks (Swinnen, 2002; Debaere et al., 2004; Swinnen and Wenderoth, 2004; Swinnen and Gooijers, 2015), Carter et al. (2015) examined the effects of tDCS applied over the SMA on the stability, consistency, and transition of metronome-paced bimanual forearm movements from in- to anti-phase movements and vice versa. They reported improved stability and consistency as well as a delayed spontaneous transition from the anti- to the more stable in-phase pattern following anodal tDCS, while cathodal tDCS did not affect task performance (Carter et al., 2015). In line with this observation, anodal tDCS of the SMA was found to be associated with faster intentional switches from anti- to in-phase movements (Carter et al., 2017). These findings underline a central role of the SMA in the control of bimanual movements and suggest that tDCS represents a suitable method for the transient modulation of this process.

Typing a text on a keyboard requires bilateral wellcoordinated, skilled finger movements, and particularly practice to achieve successful task performance. Gomes-Osman and Field-Fote (2013) investigated the effects of anodal tDCS over bilateral M1 on a bimanual typing task. Task performance was assessed immediately before and after stimulation. After five consecutive training days, participants showed a larger improvement in the number of correctly-typed bimanual sequences following anodal tDCS as compared to sham 
stimulation. However, this effect vanished after a 1-week retention interval. Ciechanski and Kirton (2017) examined the effects of tDCS on dexterity using the Purdue Pegboard Test (PPT; Tiffin and Asher, 1948). The PPT measures uni- and bimanual hand functions such as placing the maximum number of pegs in the pegboard within $30 \mathrm{~s}$. While the bimanual task requires symmetrical movements of both hands, a second bimanual task (assembly) requires the asymmetrical (alternate) use of both hands to build a maximum number of small assemblies consisting of pins, collars and washers within $60 \mathrm{~s}$ (Desrosiers et al., 1995). Ciechanski and Kirton (2017) reported improved bimanual motor performance in healthy school children after training of the left hand with concurrent anodal tDCS applied to the contralateral M1 corresponding to C4. Interestingly, this effect was also present when cathodal tDCS was applied to the ipsilateral M1 (C3), but not after sham tDCS. While the symmetrical bimanual performance was facilitated, the more complex (asymmetrical) "assembly test" was not affected by either stimulation. After a retention interval of 6 weeks, improved task performance remained stable, suggesting enhanced motor consolidation associated with tDCS. Pixa et al. (2017b) also used the PPT to investigate the effect of multichannel HD-tDCS targeting bilateral M1 (see Table 1 for electrode positions). After a training period of 3 days with concurrent stimulation, larger cumulative performance gains in participants receiving anodal HD-tDCS compared to the sham-stimulated control group were found. Improved performance was indicated for the unimanual task of the dominant right hand and the bimanual PPT. Performance gains remained stable over a 1-week retention interval and further improvement was found for right-hand performance. As compared to sham tDCS, no significant differences were found for the unimanual left-hand task and-again-the more complex bimanual "assembly" task was not affected by tDCS (Pixa et al., 2017b). The same stimulation protocol was used to investigate effects of bilateral anodal HD-tDCS on the performance of a complex sequential bimanual stacking task (Pixa et al., 2017a). After 3 days of training in sport stacking (cup stacking) with concurrent anodal HD-tDCS, faster stacking performance compared to the sham group was found. This effect occurred for only one of the two required stacking formations (3-6-3), while only a statistical trend emerged for the more complex task-version (1-10-1). Re-testing after 1 week revealed sustained superior performance in the 3-6-3 stack for the anodal HD-tDCS group.

In two studies, Furuya et al. $(2013,2014 a)$ demonstrated that the effects of tDCS on bimanual motor performance depend on the subjects' level of expertise. While highly-trained expert pianists did not benefit from bilateral tDCS over M1 (anode right $\mathrm{M} 1$ and cathode left $\mathrm{M} 1$ and vice versa) in a bimanual finger typing task, the analysis revealed that only pianists who commenced piano training at an advanced age showed selectively improved performance following tDCS, irrespective of the stimulation protocol (Furuya et al., 2013). A second study using the same bimanual task, suggests that musically untrained volunteers (novices) significantly improved bimanual performance following tDCS, whereas expert pianists did not. Rather, the skilled finger movements of the pianists were found to be slightly deteriorated after verum stimulation compared to sham stimulation (Furuya et al., 2014a).

It should also be stressed that other studies have failed to provide evidence of significant stimulation effects on bimanual task performance. McCambridge et al. (2016) applied bilateral tDCS with the anode over the right M1 and the cathode over the left M1 and assessed the effects on left-hand and bimanual circle tracing performance. The results indicated a marginal effect on the bimanual task in low performers who received sham stimulation, while no significant effect was found in participants who received active tDCS. Vancleef et al. (2016) examined the effects on task performance of anodal tDCS applied to the left M1 or the left dorsolateral prefrontal cortex (DLPFC) during training on a complex bimanual tracking task (BTT). The BTT requires tracking of a moving dot by rotating two dials with the left (vertical direction) and right (horizontal direction) hands in different frequency-ratios. The authors reported improved motor performance for all participants, independent of tDCS.

Despite the heterogeneity in findings, the data begin to reveal that tDCS may affect relatively easy rather than complex bimanual tasks in particular and that it appears to be more effective in novice volunteers rather than in experts.

\section{tDCS AND BIMANUAL MOTOR PERFORMANCE IN PATIENTS}

Impairment of bimanual skills can occur due to a wide variety of neurological diseases, like stroke or Parkinson's disease, and task performance might be influenced by altered cortical excitability, such as in focal dystonia (FD). In FD, the affected M1 shows pathologically increased activity that is associated with involuntary movements (e.g., tremor) and muscle spasms of the contralateral effector (Cohen and Hallett, 1988; Stinear and Byblow, 2004). Since tDCS likely modulates motor cortical excitability, Furuya et al. (2014b) investigated the effects of five different stimulation configurations (see Table 2 for an overview of different electrode montages) on bimanual task performance in pianists suffering from FD of the right hand and in healthy controls. The study revealed that bilateral tDCS over M1 (affected left M1; cathode over C3, anode over C4) led to increased accuracy of the dystonic hand, both during and after tDCS, while performing metronome-paced symmetrical bimanual finger movements. This effect was not found for bilateral tDCS with the anode over C3, unilateral tDCS (anode over C4), sham stimulation or-noteworthy for bilateral tDCS - with the cathode over C3 and anode over C4 without concurrent bimanual training. Interestingly, performance gains were positively correlated with FD severity.

Traumatic brain injuries (TBI) can also impair bimanual motor task performance. In a pilot study, Middleton et al. (2014) combined bihemispheric tDCS with physical therapy of the upper extremities in patients suffering from stroke and TBI. Bihemispheric tDCS was applied concurrently with physical therapy for $15 \mathrm{~min}$, with the anode over the ipsilesional M1. The intervention was implemented over 24 sessions, with three sessions per week. The results revealed improved function of the upper extremities in clinical (Fugl-Meyer Assessment 


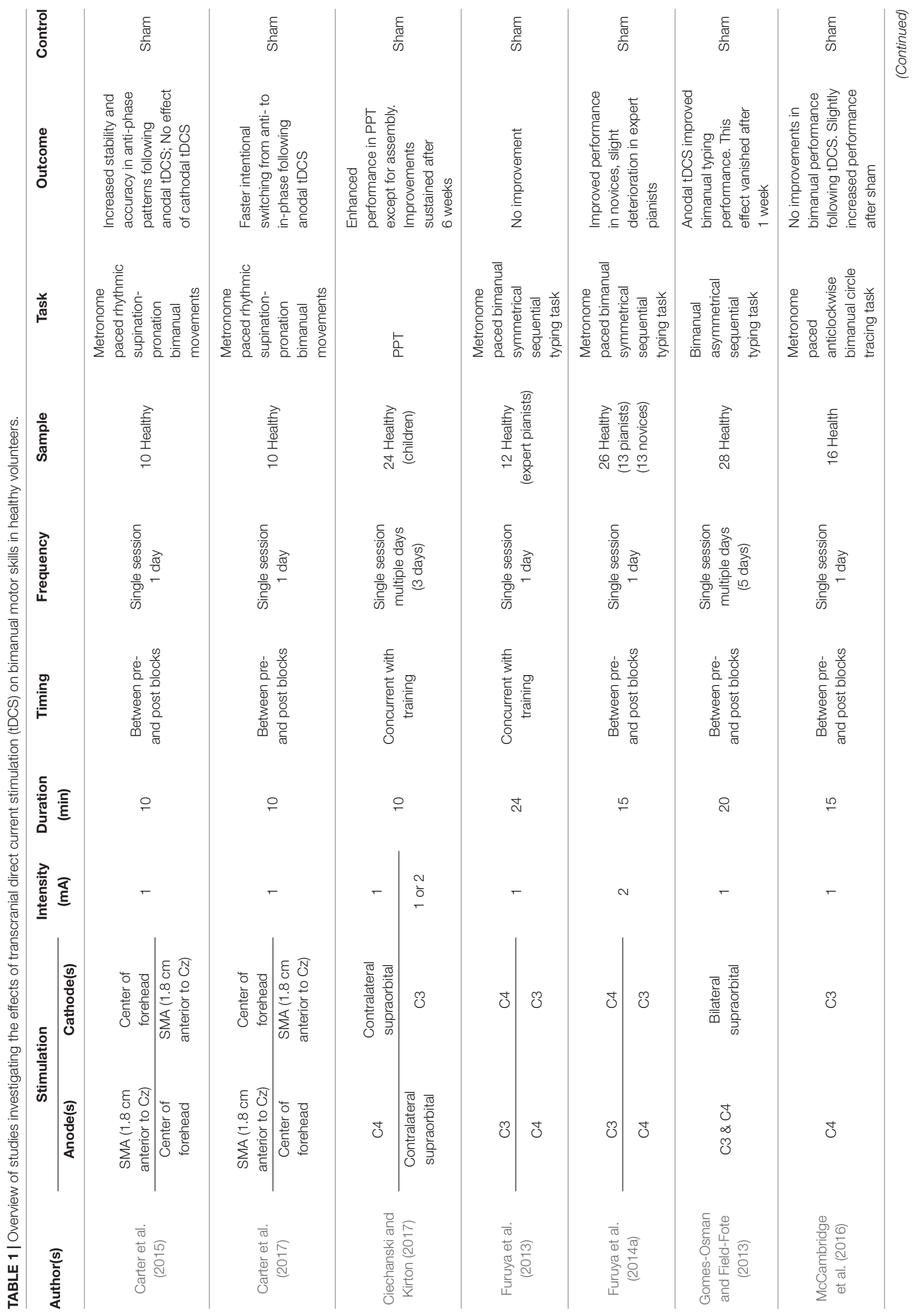




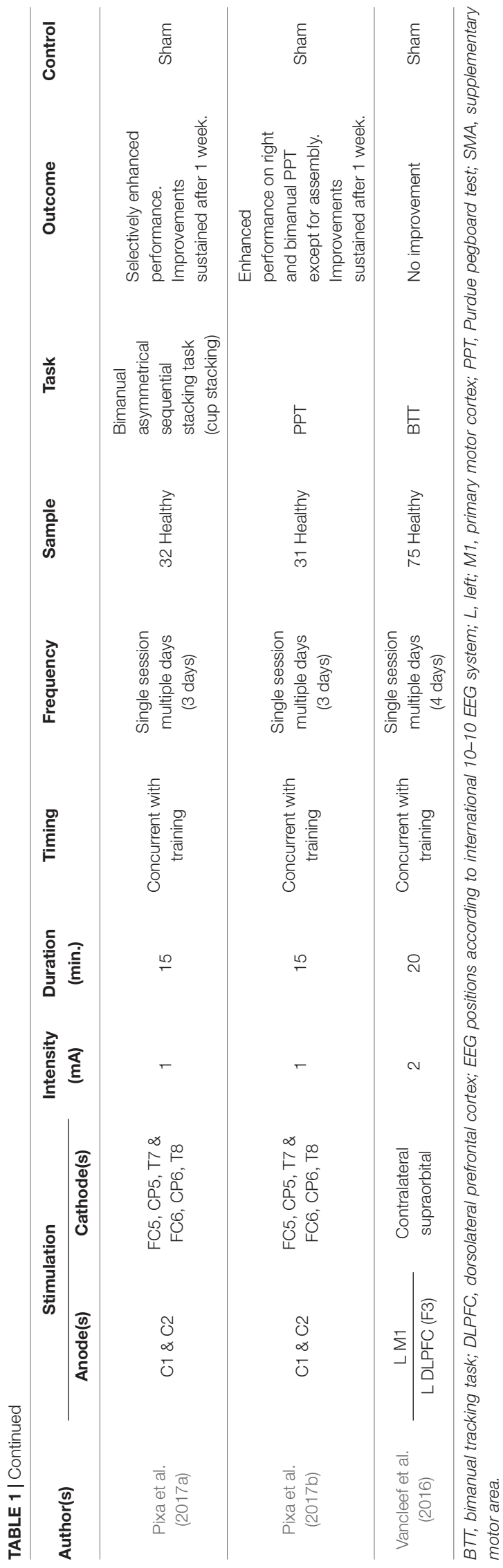

UE, Box and Block Test, PPT, Stroke Impact Scale) and robotic measures (Visually Guided Reaching Task, Object hit task (OHT)). Focusing on bimanual measures, performance in the OHT showed improved bimanual coordination, indicating superior gross motor function. However, bimanual fine motor control in the PPT did not benefit from stimulation. The observed gains persisted up to 6 months. In line with Middleton et al. (2014) the data support the notion that tDCS represents a feasible approach facilitating the effects of physical therapy of the upper extremities. Takeuchi et al. (2012) combined low-frequency, single-pulse, repetitive transcranial magnetic stimulation (rTMS) over the unaffected hemisphere of stroke patients with simultaneous application of anodal tDCS over the affected M1. The combination of rTMS and anodal tDCS was found to reduce motor impairment in these patients (Takeuchi et al., 2012).

\section{DISCUSSION}

In the light of the abundance of everyday tasks requiring bimanual actions and their importance in independent living, the purpose of this review article was to summarize the few existing studies investigating the effects of tDCS on bimanual motor skills. The data suggest that-although some studies have failed to show tDCS-specific effects on bimanual tasks-tDCS has the potential to enhance bimanual motor performance in healthy volunteers as well as in patients suffering from neurological diseases. The overview reveals that-besides the well-known inter- and intra-individual variability of outcome-measures, conflicting study results may be attributed to substantial variations in stimulation protocols, bimanual tasks, and study designs (see Tables 1, 2). Nevertheless, this review article provides a starting point for a systematic evaluation of tDCS effects on bimanual task performance.

Taken together, the data indicate that M1 was the preferred brain area for tDCS application to modulate bimanual performance, in line with the majority of tDCS studies addressing unimanual tasks (for an overview see Buch et al., 2017). However, it must be stressed that the use of stimulation protocols commonly applied for unimanual tasks might represent an oversimplification of the brain processes subserving bimanual task performance since brain areas involved and-more importantly-the temporally precise functional interaction between these areas are assumed to differ between bi- and unimanual tasks (Debaere et al., 2004; Swinnen and Wenderoth, 2004; Wenderoth et al., 2005; Pollok et al., 2007). Several brain regions which are involved in bimanual motor performance are accessible by means of tDCS. The parietal cortex (PC) is suggested to play a pivotal role in bimanual performance through multisensory integration and guidance of movements (Battaglia-Mayer et al., 2006; Buneo and Andersen, 2006), and, learning of bimanual skills (Debaere et al., 2004). Additionally, the right superior temporal gyrus (STG) is proposed to be causally involved in monitoring bimanual spatio-temporal goals (Duque et al., 2010). Furthermore, until now no study had investigated effects of premotor cortex (PMC) or cerebellar tDCS on bimanual motor skills. Moreover, 


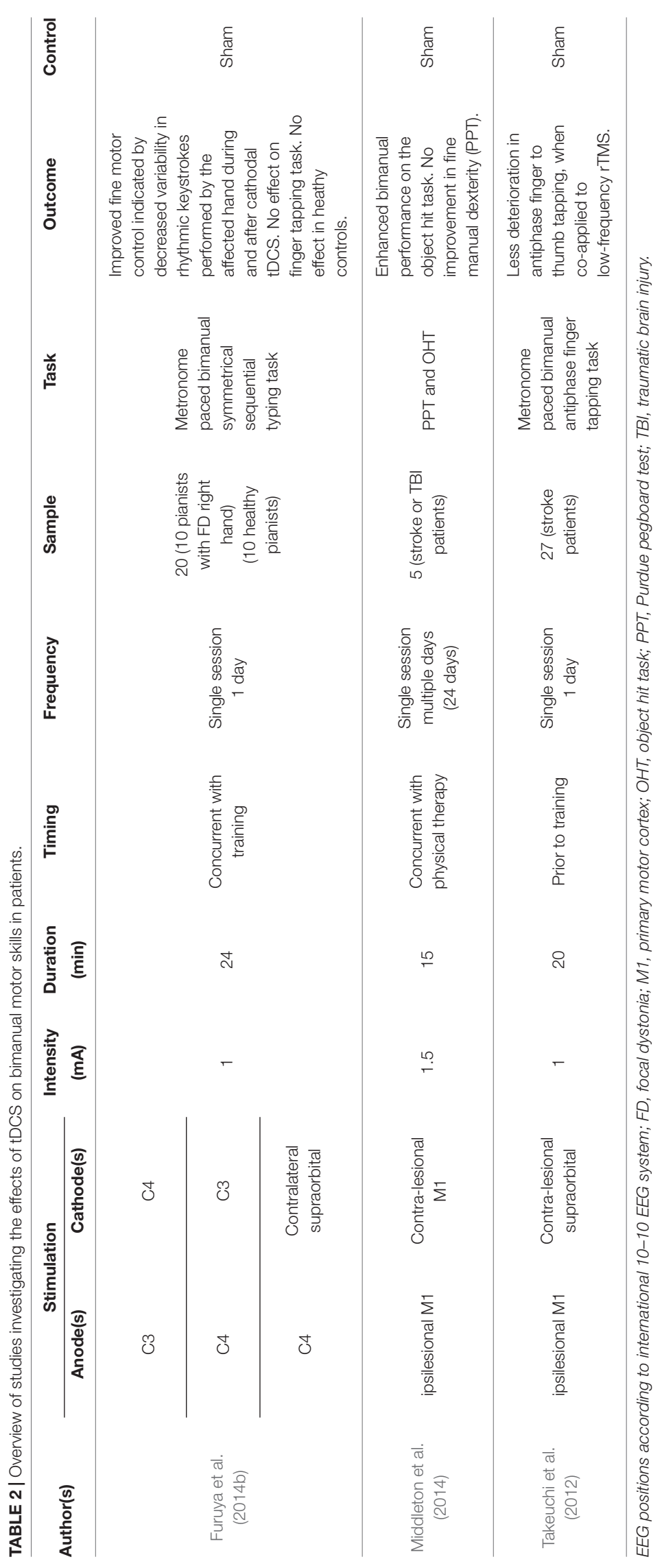


different task demands such as the required type of bimanual action (e.g., symmetric or asymmetric) to achieve a specific task-goal, as well as individual expertise (Furuya et al., 2014a), are likely related to distinct activation patterns within the motor network of bimanual actions (Puttemans et al., 2005; Jantzen et al., 2008; Duque et al., 2010; Whitall et al., 2011). Since complex bimanual tasks are associated with brain activation extending towards the prefrontal, parietal and temporal areas (Gross et al., 2002; Debaere et al., 2004; Hardwick et al., 2013; Swinnen and Gooijers, 2015), the stimulation of a particular brain area might not be effective in modulating complex bimanual skills. This hypothesis fits well with the observation that more complex bimanual tasks remain unaffected by tDCS (Vancleef et al., 2016; Ciechanski and Kirton, 2017; Pixa et al., $2017 a, b)$. Since the neural mechanisms of the wide variety of bimanual actions, as well as the neurophysiological mechanisms underlying tDCS, are not completely understood, future studies need to consider neurophysiological measures using, e.g., TMS, electroencephalography (EEG), magnetoencephalography (MEG) or functional near-infrared spectroscopy (fNIRS). This is particularly important since intra- and inter-individual variability in responses to tDCS is suggested to highly influence study outcomes (Li et al., 2015).

So far, only three studies were identified that have investigated the effects of tDCS on bimanual skills in patients. Although these studies widely differ in terms of the respective stimulation protocol and-even more important-the underlying disease, the findings suggest facilitating effects of tDCS on bimanual task performance. Although sparse, the data imply that tDCS in combination with motor training represents a suitable method for neuro-rehabilitation.

\section{REFERENCES}

Accornero, N., Li Voti, P., La Riccia, M., and Gregori, B. (2007). Visual evoked potentials modulation during direct current cortical polarization. Exp. Brain Res. 178, 261-266. doi: 10.1007/s00221-006-0733-y

Alam, M., Truong, D. Q., Khadka, N., and Bikson, M. (2016). Spatial and polarity precision of concentric high-definition transcranial direct current stimulation (HD-tDCS). Phys. Med. Biol. 61, 4506-4521. doi: 10.1088/00319155/61/12/4506

Alonzo, A., Brassil, J., Taylor, J. L., Martin, D., and Loo, C. K. (2012). Daily transcranial direct current stimulation (tDCS) leads to greater increases in cortical excitability than second daily transcranial direct current stimulation. Brain Stimul. 5, 208-213. doi: 10.1016/j.brs.2011.04.006

Antal, A., Kincses, T. Z., Nitsche, M. A., Bartfai, O., and Paulus, W. (2004). Excitability changes induced in the human primary visual cortex by transcranial direct current stimulation: direct electrophysiological evidence. Invest. Ophthalmol. Vis. Sci. 45, 702-707. doi: 10.1167/iovs. 03-0688

Antal, A., Polania, R., Schmidt-Samoa, C., Dechent, P., and Paulus, W. (2011). Transcranial direct current stimulation over the primary motor cortex during fMRI. Neuroimage 55, 590-596. doi: 10.1016/j.neuroimage.2010. 11.085

Bastani, A., and Jaberzadeh, S. (2012). Does anodal transcranial direct current stimulation enhance excitability of the motor cortex and motor function in healthy individuals and subjects with stroke: a systematic review and meta-analysis. Clin. Neurophysiol. 123, 644-657. doi: 10.1016/j.clinph.2011. 08.029

Batsikadze, G., Moliadze, V., Paulus, W., Kuo, M.-F., and Nitsche, M. A. (2013). Partially non-linear stimulation intensity-dependent effects of direct current
Finally, besides tDCS other techniques like transcranial alternate current stimulation (tACS) or transcranial random noise stimulation (tRNS) are suitable methods for the non-invasive modulation of brain processes subserving motor learning (Prichard et al., 2014; Pollok et al., 2015). However, to the best of our knowledge, no study that had adopted one of these methods in bimanual tasks has been published until yet.

\section{CONCLUSION}

Up until now, knowledge about the effects of tDCS on bimanual performance has remained limited due to a relatively small number of studies with mixed results. However, despite the heterogeneity in stimulation protocols, study designs, and paradigms, the data suggest that tDCS has the potential to enhance bimanual motor performance in healthy volunteers as well as in patients suffering from a variety of neurological diseases. Noteworthy, the data do not allow the identification of specific tDCS parameters as most effective to modulate bimanual motor skills. Therefore, tailoring tDCS protocols to bimanual motor tasks will critically challenge future studies.

\section{AUTHOR CONTRIBUTIONS}

NHP contributed to the conceptualization of the review, performed the literature research, interpretation of the data, wrote the manuscript, approved the final version of the manuscript and acted as corresponding author. BP substantially contributed to the data interpretation, wrote parts of the manuscript and critically revised the manuscript.

stimulation on motor cortex excitability in humans. J. Physiol. 591, 1987-2000. doi: 10.1113/jphysiol.2012.249730

Battaglia-Mayer, A., Archambault, P. S., and Caminiti, R. (2006). The cortical network for eye-hand coordination and its relevance to understanding motor disorders of parietal patients. Neuropsychologia 44, 2607-2620. doi: 10.1016/j. neuropsychologia.2005.11.021

Bindman, L. J., Lippold, O. C. J., and Redfearn, J. W. T. (1962). Long-lasting changes in the level of the electrical activity of the cerebral cortex produced by polarizing currents. Nature 196, 584-585. doi: 10.1038/196584a0

Bindman, L. J., Lippold, O. C. J., and Redfearn, J. W. T. (1964). The action of brief polarizing currents on the cerebral cortex of the rat (1) during current flow and (2) in the production of long-lasting after-effects. J. Physiol. 172, 369-382. doi: 10.1113/jphysiol.1964.sp007425

Buch, E. R., Santarnecchi, E., Antal, A., Born, J., Celnik, P. A., lassen, J., et al. (2017). Effects of tDCS on motor learning and memory formation: a consensus and critical position paper. Clin. Neurophysiol. 128, 589-603. doi: 10.1016/j. clinph.2017.01.004

Buneo, C. A., and Andersen, R. A. (2006). The posterior parietal cortex: sensorimotor interface for the planning and online control of visually guided movements. Neuropsychologia 44, 2594-2606. doi: 10.1016/j.neuropsychologia. 2005.10.011

Carter, M. J., Maslovat, D., and Carlsen, A. N. (2015). Anodal transcranial direct current stimulation applied over the supplementary motor area delays spontaneous antiphase-to-in-phase transitions. J. Neurophysiol. 113, 780-785. doi: 10.1152/jn.00662.2014

Carter, M. J., Maslovat, D., and Carlsen, A. N. (2017). Intentional switches between coordination patterns are faster following anodal-tDCS applied over the supplementary motor area. Brain Stimul. 10, 162-164. doi: 10.1016/j.brs. 2016.11.002 
Ciechanski, P., and Kirton, A. (2017). Transcranial direct-current stimulation can enhance motor learning in children. Cereb. Cortex 27, 2758-2767. doi: 10.1093/cercor/bhw114

Cohen, L. G., and Hallett, M. (1988). Hand cramps: clinical features and electromyographic patterns in a focal dystonia. Neurology 38, 1005-1012. doi: 10.1212/WNL.38.7.1005

Debaere, F., Wenderoth, N., Sunaert, S., van Hecke, P., and Swinnen, S. P. (2004). Changes in brain activation during the acquisition of a new bimanual coodination task. Neuropsychologia 42, 855-867. doi: 10.1016/j. neuropsychologia.2003.12.010

Desrosiers, J., Hébert, R., Bravo, G., and Dutil, E. (1995). The Purdue Pegboard Test: normative data for people aged 60 and over. Disabil. Rehabil. 17, 217-224. doi: 10.3109/09638289509166638

Dieckhöfer, A., Waberski, T. D., Nitsche, M., Paulus, W., Buchner, H., and Gobbelé, R. (2006). Transcranial direct current stimulation applied over the somatosensory cortex-differential effect on low and high frequency SEPs. Clin. Neurophysiol. 117, 2221-2227. doi: 10.1016/j.clinph.2006.06.415

Duque, J., Davare, M., Delaunay, L., Jacob, B., Saur, R., Hummel, F., et al. (2010). Monitoring coordination during bimanual movements: where is the mastermind? J. Cogn. Neurosci. 22, 526-542. doi: 10.1162/jocn.2009.21213

Flöel, A. (2014). tDCS-enhanced motor and cognitive function in neurological diseases. Neuroimage 85, 934-947. doi: 10.1016/j.neuroimage.2013.05.098

Fritsch, B., Reis, J., Martinowich, K., Schambra, H. M., Ji, Y., Cohen, L. G., et al. (2010). Direct current stimulation promotes BDNF-dependent synaptic plasticity: potential implications for motor learning. Neuron 66, 198-204. doi: 10.1016/j.neuron.2010.03.035

Furuya, S., Klaus, M., Nitsche, M. A., Paulus, W., and Altenmuller, E. (2014a). Ceiling effects prevent further improvement of transcranial stimulation in skilled musicians. J. Neurosci. 34, 13834-13839. doi: 10.1523/JNEUROSCI. 1170-14.2014

Furuya, S., Nitsche, M. A., Paulus, W., and Altenmüller, E. (2014b). Surmounting retraining limits in musicians' dystonia by transcranial stimulation. Ann. Neurol. 75, 700-707. doi: 10.1002/ana.24151

Furuya, S., Nitsche, M. A., Paulus, W., and Altenmüller, E. (2013). Early optimization in finger dexterity of skilled pianists: implication of transcranial stimulation. BMC Neurosci. 14:35. doi: 10.1186/1471-2202-14-35

Gomes-Osman, J., and Field-Fote, E. C. (2013). Bihemispheric anodal corticomotor stimulation using transcranial direct current stimulation improves bimanual typing task performance. J. Mot. Behav. 45, 361-367. doi: 10.1080/00222895.2013.808604

Gross, J., Timmermann, L., Kujala, J., Dirks, M., Schmitz, F., Salmelin, R., et al. (2002). The neural basis of intermittent motor control in humans. Proc. Natl. Acad. Sci. U S A 99, 2299-2302. doi: 10.1073/pnas.032682099

Hardwick, R. M., Rottschy, C., Miall, R. C., and Eickhoff, S. B. (2013). A quantitative meta-analysis and review of motor learning in the human brain. Neuroimage 67, 283-297. doi: 10.1016/j.neuroimage.2012.11.020

Ho, K.-A., Taylor, J. L., Chew, T., Gálvez, V., Alonzo, A., Bai, S., et al. (2016). The effect of transcranial direct current stimulation (tDCS) electrode size and current intensity on motor cortical excitability: evidence from single and repeated sessions. Brain Stimul. 9, 1-7. doi: 10.1016/j.brs.2015.08.003

Hunter, M. A., Coffman, B. A., Trumbo, M. C., and Clark, V. P. (2013). Tracking the neuroplastic changes associated with transcranial direct current stimulation: a push for multimodal imaging. Front. Hum. Neurosci. 7:495. doi: 10.3389/fnhum.2013.00495

Jantzen, K. J., Oullier, O., and Scott Kelso, J. A. (2008). Neuroimaging coordination dynamics in the sport sciences. Methods 45, 325-335. doi: 10.1016/j.ymeth. 2008.06.001

Karabanov, A., Ziemann, U., Hamada, M., George, M. S., Quartarone, A., Classen, J., et al. (2015). Consensus paper: probing homeostatic plasticity of human cortex with non-invasive transcranial brain stimulation. Brain Stimul. 8, 442-454. doi: 10.1016/j.brs.2015.01.404

Li, L. M., Uehara, K., and Hanakawa, T. (2015). The contribution of interindividual factors to variability of response in transcranial direct current stimulation studies. Front. Cell. Neurosci. 9:898. doi: 10.3389/fncel.2015.00181

Liebetanz, D., Nitsche, M. A., Tergau, F., and Paulus, W. (2002). Pharmacological approach to the mechanisms of transcranial DC-stimulation-induced after-effects of human motor cortex excitability. Brain 125, 2238-2247. doi: 10.1093/brain/awf238
Maes, C., Gooijers, J., Orban de Xivry, J.-J., Swinnen, S. P., and Boisgontier, M. P. (2017). Two hands, one brain, and aging. Neurosci. Biobehav. Rev. 75, 234-256. doi: 10.1016/j.neubiorev.2017.01.052

McCambridge, A. B., Stinear, J. W., and Byblow, W. D. (2016). Neurophysiological and behavioural effects of dual-hemisphere transcranial direct current stimulation on the proximal upper limb. Exp. Brain Res. 234, 1419-1428. doi: 10.1007/s00221-015-4547-7

Middleton, A., Fritz, S. L., Liuzzo, D. M., Newman-Norlund, R., and Herter, T. M. (2014). Using clinical and robotic assessment tools to examine the feasibility of pairing tDCS with upper extremity physical therapy in patients with stroke and TBI: a consideration-of-concept pilot study. NeuroRehabilitation 35, 741-754. doi: 10.3233/NRE-141178

Monte-Silva, K., Kuo, M.-F., Hessenthaler, S., Fresnoza, S., Liebetanz, D., Paulus, W., et al. (2013). Induction of late LTP-like plasticity in the human motor cortex by repeated non-invasive brain stimulation. Brain Stimul. 6, 424-432. doi: 10.1016/j.brs.2012.04.011

Naros, G., Geyer, M., Koch, S., Mayr, L., Ellinger, T., Grimm, F., et al. (2016). Enhanced motor learning with bilateral transcranial direct current stimulation: impact of polarity or current flow direction? Clin. Neurophysiol. 127, 2119-2126. doi: 10.1016/j.clinph.2015.12.020

Nitsche, M. A., and Paulus, W. (2000). Excitability changes induced in the human motor cortex by weak transcranial direct current stimulation. J. Physiol. 527, 633-639. doi: 10.1111/j.1469-7793.2000.t01-1-00633.x

Nitsche, M. A., and Paulus, W. (2001). Sustained excitability elevations induced by transcranial DC motor cortex stimulation in humans. Neurology 57, 1899-1901. doi: 10.1212/WNL.57.10.1899

Opitz, A., Paulus, W., Will, S., Antunes, A., and Thielscher, A. (2015). Determinants of the electric field during transcranial direct current stimulation. Neuroimage 109, 140-150. doi: 10.1016/j.neuroimage.2015.01.033

Pixa, N. H., Steinberg, F., and Doppelmayr, M. (2017a). Effects of high-definition anodal transcranial direct current stimulation applied simultaneously to both primary motor cortices on bimanual sensorimotor performance. Front. Behav. Neurosci. 11:4506. doi: 10.3389/fnbeh.2017.00130

Pixa, N. H., Steinberg, F., and Doppelmayr, M. (2017b). High-definition transcranial direct current stimulation to both primary motor cortices improves unimanual and bimanual dexterity. Neurosci. Lett. 643, 84-88. doi: 10.1016/j.neulet.2017.02.033

Pollok, B., Boysen, A.-C., and Krause, V. (2015). The effect of transcranial alternating current stimulation (tACS) at $\alpha$ and $\beta$ frequency on motor learning. Behav. Brain Res. 293, 234-240. doi: 10.1016/j.bbr.2015.07.049

Pollok, B., Butz, M., Gross, J., and Schnitzler, A. (2007). Intercerebellar coupling contributes to bimanual coordination. J. Cogn. Neurosci. 19, 704-719. doi: 10.1162/jocn.2007.19.4.704

Prichard, G., Weiller, C., Fritsch, B., and Reis, J. (2014). Effects of different electrical brain stimulation protocols on subcomponents of motor skill learning. Brain Stimul. 7, 532-540. doi: 10.1016/j.brs.2014.04.005

Puttemans, V., Wenderoth, N., and Swinnen, S. P. (2005). Changes in brain activation during the acquisition of a multifrequency bimanual coordination task: from the cognitive stage to advanced levels of automaticity. J. Neurosci. 25, 4270-4278. doi: 10.1523/JNEUROSCI.3866-04.2005

Reis, J., and Fritsch, B. (2011). Modulation of motor performance and motor learning by transcranial direct current stimulation. Curr. Opin. Neurol. 24 , 590-596. doi: 10.1097/WCO.0b013e32834c3db0

Reis, J., Schambra, H. M., Cohen, L. G., Buch, E. R., Fritsch, B., Zarahn, E., et al. (2009). Noninvasive cortical stimulation enhances motor skill acquisition over multiple days through an effect on consolidation. Proc. Natl. Acad. Sci. U S A 106, 1590-1595. doi: 10.1073/pnas.0805413106

Ridding, M. C., and Ziemann, U. (2010). Determinants of the induction of cortical plasticity by non-invasive brain stimulation in healthy subjects. J. Physiol. 588, 2291-2304. doi: 10.1113/jphysiol.2010.190314

Romero Lauro, L. J., Rosanova, M., Mattavelli, G., Convento, S., Pisoni, A., Opitz, A., et al. (2014). TDCS increases cortical excitability: direct evidence from TMS-EEG. Cortex 58, 99-111. doi: 10.1016/j.cortex.2014.05.003

Saucedo Marquez, C. M., Zhang, X., Swinnen, S. P., Meesen, R., and Wenderoth, N. (2013). Task-specific effect of transcranial direct current stimulation on motor learning. Front. Hum. Neurosci. 7:333. doi: 10.3389/fnhum.2013.00333

Stagg, C. J., Best, J. G., Stephenson, M. C., O’Shea, J., Wylezinska, M., Kincses, Z. T., et al. (2009). Polarity-sensitive modulation of cortical neurotransmitters by 
transcranial stimulation. J. Neurosci. 29, 5202-5206. doi: 10.1523/JNEUROSCI. 4432-08.2009

Stagg, C. J., Jayaram, G., Pastor, D., Kincses, Z. T., Matthews, P. M., and JohansenBerg, H. (2011). Polarity and timing-dependent effects of transcranial direct current stimulation in explicit motor learning. Neuropsychologia 49, 800-804. doi: 10.1016/j.neuropsychologia.2011.02.009

Stinear, C. M., and Byblow, W. D. (2004). Impaired modulation of intracortical inhibition in focal hand dystonia. Cereb. Cortex 14, 555-561. doi: 10.1093/cercor/bhh017

Swinnen, S. P. (2002). Intermanual coordination: from behavioural principles to neural-network interactions. Nat. Rev. Neurosci. 3, 348-359. doi: $10.1038 / \mathrm{nrn} 807$

Swinnen, S. P., and Gooijers, J. (2015). "Bimanual coordination," in Brain Mapping: An Encyclopedic Reference, ed. A. W. Toga (Burlington: Elsevier Science), 475-482.

Swinnen, S. P., and Wenderoth, N. (2004). Two hands, one brain: cognitive neuroscience of bimanual skill. Trends Cogn. Sci. 8, 18-25. doi: 10.1016/j.tics. 2003.10.017

Takeuchi, N., Tada, T., Matsuo, Y., and Ikoma, K. (2012). Low-frequency repetitive TMS plus anodal transcranial DCS prevents transient decline in bimanual movement induced by contralesional inhibitory rTMS after stroke. Neurorehabil. Neural Repair 26, 988-998. doi: 10.1177/1545968311433295

Tiffin, J., and Asher, E. J. (1948). The Purdue pegboard; norms and studies of reliability and validity. J. Appl. Psychol. 32, 234-247. doi: 10.1037/h0061266

Vancleef, K., Meesen, R., Swinnen, S. P., and Fujiyama, H. (2016). tDCS over left M1 or DLPFC does not improve learning of a bimanual coordination task. Sci. Rep. 6:35739. doi: 10.1038/srep35739

Villamar, M. F., Volz, M. S., Bikson, M., Datta, A., DaSilva, A. F., and Fregni, F. (2013). Technique and considerations in the use of $4 \mathrm{x} 1$ ring high-definition transcranial direct current stimulation (HD-tDCS). J. Vis. Exp. 77:e50309. doi: $10.3791 / 50309$

Wenderoth, N., Debaere, F., Sunaert, S., and Swinnen, S. P. (2005). Spatial interference during bimanual coordination: differential brain networks associated with control of movement amplitude and direction. Hum. Brain Mapp. 26, 286-300. doi: 10.1002/hbm.20151

Whitall, J., Waller, S. M., Sorkin, J. D., Forrester, L. W., Macko, R. F. Hanley, D. F., et al. (2011). Bilateral and unilateral arm training improve motor function through differing neuroplastic mechanisms: a single-blinded randomized controlled trial. Neurorehabil. Neural Repair 25, 118-129. doi: $10.1177 / 1545968310380685$

Woods, A. J., Antal, A., Bikson, M., Boggio, P. S., Brunoni, A. R., Celnik, P., et al. (2016). A technical guide to tDCS, and related non-invasive brain stimulation tools. Clin. Neurophysiol. 127, 1031-1048. doi: 10.1016/j.clinph.2015.11.012

Zaehle, T., Beretta, M., Jäncke, L., Herrmann, C. S., and Sandmann, P. (2011). Excitability changes induced in the human auditory cortex by transcranial direct current stimulation: direct electrophysiological evidence. Exp. Brain Res. 215, 135-140. doi: 10.1007/s00221-011-2879-5

Conflict of Interest Statement: The authors declare that the research was conducted in the absence of any commercial or financial relationships that could be construed as a potential conflict of interest.

Copyright (c) 2018 Pixa and Pollok. This is an open-access article distributed under the terms of the Creative Commons Attribution License (CC BY). The use, distribution or reproduction in other forums is permitted, provided the original author(s) and the copyright owner are credited and that the original publication in this journal is cited, in accordance with accepted academic practice. No use, distribution or reproduction is permitted which does not comply with these terms. 


\section{OPEN ACCESS}

Edited by:

Roberta Ferrucci,

Fondazione IRCCS Ca' Granda Ospedale Maggiore Policlinico

(IRCCS), Italy

Reviewed by:

Filippo Brighina,

Università degli Studi di Palermo, Italy

Claudio Lucchiari,

Università degli Studi di Milano, Italy Adriana Salatino,

Università degli Studi di Torino, Italy

*Correspondence:

Ulrich Palm

ulrich.palm@med.uni-muenchen.de

Received: 14 August 2017 Accepted: 18 January 2018 Published: 31 January 2018

Citation:

Brunnauer A, Segmiller FM, Löschner S, Grun V, Padberg F and Palm U (2018) The Effects

of Transcranial Direct Current Stimulation (tDCS) on Psychomotor and Visual Perception Functions Related to Driving Skills. Front. Behav. Neurosci. 12:16. doi: 10.3389/fnbeh.2018.00016

\section{The Effects of Transcranial Direct Current Stimulation (tDCS) on Psychomotor and Visual Perception Functions Related to Driving Skills}

\author{
Alexander Brunnauer, ${ }^{1,2}$, Felix M. Segmiller ${ }^{1}$, Sabine Löschner ${ }^{1}$, Valérie Grun ${ }^{1}$, \\ Frank Padberg ${ }^{1}$ and Ulrich Palm ${ }^{*}$ \\ 1 Department of Psychiatry and Psychotherapy, Klinikum der Universität München, Ludwig-Maximilians-University, Munich, \\ Germany, ${ }^{2}$ Psychiatric Clinic, kbo-Inn-Salzach-Klinikum, Wasserburg am Inn, Germany
}

Objective: It could be demonstrated that anodal transcranial direct current stimulation (tDCS) of the left dorsolateral prefrontal cortex (DLPFC) enhances accuracy in working memory tasks and reaction time in healthy adults and thus may also have an influence on complex everyday tasks like driving a car. However, no studies have applied tDCS to psychomotor skills related to a standard driving test so far.

Methods: 10 female and 5 male healthy adults without any medication and history of psychiatric or neurological illness were randomly assigned to two groups receiving active and sham stimulation in a double blind, cross-over study design. Standardized computerized psychomotor tests according to the German guidelines for road and traffic safety were administered at baseline. Then they performed the same tests during an anodal or sham tDCS of the left DLPFC in two separated sessions.

Results: No significant improvements in skills related to driving performance like visual perception, stress tolerance, concentration, and vigilance could be shown after left anodal prefrontal tDCS. Side effects were low and did not differ between active and sham stimulation.

Conclusions: The findings of our study indicate that left prefrontal tDCS may not alter driving skills affording more automated action patterns but as shown in previous studies may have an influence on driving behavior requiring executive control processes. This however has to be proved in future studies and within greater samples.

Keywords: tDCS, brain stimulation, dorsolateral prefrontal cortex, DLPFC, driving skills, driving performance

\section{INTRODUCTION}

Driving a car is considered an important part of daily life that embodies a complex and goaldirected task. This engages multiple interacting cognitive processes in different regions of our brain to maintain attention to traffic environment, focus on emerging information and threats, select and perform adequate reactions in terms of safety and traffic laws.

Most cognitive processes are modulated by the right and left dorsolateral prefrontal cortex (DLPFC), e.g., sustained attention (Pardo et al., 1991; Coull et al., 1998), error processing (Dosenbach et al., 2006), and planning (Unterrainer et al., 2005). However, left and right 
hemispheres seem to be responsible for different cognitive abilities. Thus, several neuroimaging studies addressed the specific functions of both hemispheres. The right DLPFC is involved in spatial tasks such as car-following and distancekeeping (Uchiyama et al., 2012), while the left DLPFC seems to be involved in tasks requiring sustained vigilance such as driving on a curved rural road with the need to pay attention to changing situations (Just et al., 2008).

In recent years, non-invasive brain stimulation methods such as transcranial direct current stimulation (tDCS) have been applied to focally change neuronal activation and its relevance in cognitive and behavioral performance. tDCS has been proven to change large-scale neuronal network function by application of weak direct current to the brain via a large electrode placed over the targeted brain regions (Keeser et al., 2011a,b) and has been shown to ameliorate symptoms in psychiatric disorders depending on its polarity (anodal and cathodal), e.g., depressive disorders (Palm et al., 2016). In neuropsychological studies, anodal and cathodal tDCS usually are applied to prefrontal and frontotemporal brain regions to assess the effects of inhibitory and excitatory stimulation on distinct neuropsychological functions and test performance. For example, in healthy adults it could be shown that anodal tDCS of the left DLPFC enhances accuracy in working memory tasks (Fregni et al., 2005; Zaehle et al., 2011), reaction time (Mulquiney et al., 2011; Teo et al., 2011), and declarative memory (Javadi and Walsh, 2012).

Only a few studies addressed the impact of tDCS on driving ability. Beeli et al. (2008) evaluated the impact of anodal and cathodal stimulation of the prefrontal cortex on risky driving behavior. They found that anodal stimulation of each right and left DLPFC but not cathodal stimulation resulted in a less risky driving style during a driving simulator test. The authors concluded that excitation of the right and left DLPFC caused stronger executive control and a more careful driving style. Sakai et al. (2014) found a better performance in car-following and lane-keeping after right-anodal/left-cathodal compared to left-anodal/right-cathodal tDCS. The authors conclude that this improvement is mediated by the enhancement of the right DLPFC where those spatial tasks are processed.

According to Michon (1989), driving behavior can be subdivided in three interacting hierarchical levels. A strategical level - e.g., choosing a route or consideration of road traffic rules, a tactical level - e.g., planning actions and maneuvre control, and an operational level with perceptual processing and action execution under high time pressure. Above mentioned studies (Beeli et al., 2008; Sakai et al., 2014) predominately investigated a more strategical and/or tactical level of driving behavior. The aim of the present study was to investigate the influence of left prefrontal tDCS on an operational level of driving behavior - i.e., visual perception, stress-tolerance, concentration, and vigilance. According to regulations of the German guidelines for road and traffic safety we focused on psychomotor- and visual perceptionfunctions that are thought to be critical for an assessment of driving ability. The validity of these tests has been confirmed in large samples of both healthy controls and clinical samples. It could be demonstrated that a $83.3 \%$ correct classification for adjusted and unadjusted driving behaviors could be obtained with these tests (Bukasa et al., 1990; Karner and Biehl, 2001).

\section{MATERIALS AND METHODS}

\section{Subjects}

This study was conducted at the Department of Psychiatry and Psychotherapy, Klinikum der Universität München, Germany, in accordance with the ethical standards laid down in the 1964 Declaration of Helsinki and its later amendments, and has been approved by the ethics committee of the Ludwig Maximilian University of Munich (No. 299-12). Written informed consent was obtained from all participants before enrolment. 15 healthy adults (10 female, 5 male) without any medication and any history of psychiatric and neurological illness were randomized to two groups receiving active and sham stimulation in a double blind, cross-over study design. Demographic variables and driving history were obtained from all subjects (see Table 1).

\section{Study Procedure}

After given informed consent, a baseline assessment was carried out which included demographic variables and driving history. All subjects were tested in individual sessions at $\sim 9$ am with standardized computerized psychomotor tests, according to the German guidelines for road and traffic safety. Complete testing lasted about 45-60 min depending on pace of work and was administered in the same sequence (visual perception - ATAVT, concentration - COG, stress-tolerance - DT, vigilance - VIGIL). To become familiar with the tests a training procedure $\left(\mathrm{t}_{0}\right)$ was first conducted. To disentangle retest effects from stimulation effects, one group got active tDCS first, followed by sham (early intervention [EI]), the other in reversed order (late intervention [LI]). Psychomotor tests were performed parallel to the beginning

TABLE 1 | Demographic variables and driving history, separated for early and late intervention group.

\begin{tabular}{|c|c|c|c|}
\hline & $\begin{array}{l}\text { Early } \\
\text { intervention } \\
(n=8)\end{array}$ & $\begin{array}{l}\text { Late } \\
\text { intervention } \\
(n=7)\end{array}$ & $\begin{array}{c}\text { Statistical } \\
\text { significance* }\end{array}$ \\
\hline Age, mean (SD), y & $33.1(6.4)$ & $31.0(4.5)$ & NS \\
\hline $\begin{array}{l}\text { Gender, } n \\
\text { (male/female) }\end{array}$ & $3 / 5$ & $2 / 5$ & NS \\
\hline \multicolumn{4}{|l|}{ Civil status, $n$} \\
\hline Unmarried & 5 & 5 & NS \\
\hline Married & 3 & 2 & \\
\hline $\begin{array}{l}\text { Grammar or middle } \\
\text { school }\end{array}$ & 1 & 0 & NS \\
\hline $\begin{array}{l}\text { High school or } \\
\text { university diploma }\end{array}$ & 7 & 7 & \\
\hline $\begin{array}{l}\text { Driving license, } \\
\text { mean }(S D), y\end{array}$ & $14.5(5.7)$ & $14.9(4.1)$ & NS \\
\hline $\begin{array}{l}\text { Yearly driven } \\
\text { kilometers, mean } \\
(S D)\end{array}$ & $4500.0(6546.5)$ & 4428.6 (4961.7) & NS \\
\hline
\end{tabular}


of the stimulation $\left(t_{1}, t_{2}\right)$. The training session and both interventional sessions were separated by an interval of at least $24 \mathrm{~h}$ to avoid carry-over effects (see Figure 1).

\section{Psychomotor and Visual Perception Tests}

Several domains were assessed according to the German guidelines for road and traffic safety (Gräcmann and Albrecht, 2016), including visual perception, stress tolerance, concentration, and vigilance. According to these guidelines, a test has to be considered as failed if a participant falls short of the threshold of one standard deviation below the mean of normative data, derived from a representative sample of car drivers. The procedure has been described in detail elsewhere (Brunnauer et al., 2006). Data was collected using the computerized Wiener Testsystem (Vienna test system, WTS). It has been verified that more than $80 \%$ of subjects can be correctly classified according to adequate/inadequate driving behavior using results from this test system (Bukasa et al., 1990, 2003; Karner and Biehl, 2001).

Visual perception was assessed using the Tachistoscope Test (TAVT-MB; test-version S1). It measures the capability to perceive visual input quickly. Typical traffic situations are presented on 20 color slides for $1 \mathrm{~s}$ each followed by a multiplechoice question, containing five possible answers. The variable analyzed was the number of correct items; dependent on speed of operation the test lasted $10 \mathrm{~min}$ on average. The critical Stress tolerance was examined with the Wiener Determinationstest (Vienna determination test, DT; test-version S1). In three test phases the participant is presented with color, tone and light stimuli, 180 signals each. The interstimulus intervals vary within the three test phases. Subjects have to react by pressing corresponding buttons, bars and pedals using both their hands and feet; omissions in this test procedure were the critical variables; test procedure lasted $6 \mathrm{~min}$. Concentration was measured with the attention and concentration test (COG; testversion S2). The task requires subjects to match simple figures with respect to similarity and dissimilarity. The test procedure lasted $8 \mathrm{~min}$; the percentage of errors was the critical variable analyzed. The Vigilance Test (VIGIL; test version S1) requires the participant to remain attentive under monotonous conditions. A dot of light moving along a circle in fixed steps has to be observed over a period of $25 \mathrm{~min}$. Irregularities - i.e., the dot skips over a circle - have to be identified by a keystroke. The variable analyzed was the number of correct items (i.e., number of stimuli minus omissions and errors).

\section{Transcranial Direct Current Stimulation}

Transcranial direct current stimulation was applied with a CEcertified Eldith DC-Stimulator PLUS (NeuroConn, Ilmenau, Germany). This device delivers active or sham tDCS after entering a number code to achieve blinding of both operator and participant. The sham function mimics active stimulation by a short fade-in and fade-out phase (each $15 \mathrm{~s}$ ) at the beginning and the end of the stimulation period (Palm et al., 2013). Current strength was set to $2 \mathrm{~mA}$, duration of stimulation was $20 \mathrm{~min}$ with $15 \mathrm{~s}$ fade-in and fade-out. Saline-soaked sponge electrodes $\left(35 \mathrm{~cm}^{2}\right)$ were placed over the left DLPFC (anode, F3) and the contralateral supraorbital area (cathode, Fp2-Af8). Positioning of the electrodes was performed with a standard EEG cap according to the 10-20 international EEG system.

\section{Measurement of Side Effects}

To control for potential side effects of the stimulation that could lead to unblinding of the participants, the Comfort Rating Questionnaire (CRQ) was used (Palm et al., 2014). This selfrating questionnaire assesses side effects (pain, tingling, burning, fatigue, nervousness, disturbed concentration, disturbed visual perception, headache) during and immediately after stimulation (sum scores) and general discomfort on a 10-point Likert scale ranging from "not at all" to "extremely." Furthermore occurrence of visual flashes (phosphenes) and sleep disturbances after stimulation are assessed in a dichotomous question. As the test procedure outlasted the duration of stimulation, participants were advised to report their sensations in the first minutes and after the end of the test sequence.

\section{Data Analysis}

Statistical analyses were performed using SPSS software (Statistical package for Social Sciences, Version 22, SPSS, Inc., Chicago, IL, United States). Demographic and clinical characteristics were analyzed using parametric and nonparametric tests (Chi-Square, Mann-Whitney $U$-test, $t$-tests). Due to different distributions and small sample size data from psychomotor assessments were z-transformed. A repeated measures analysis of variance was carried out separately for each functional domain (visual perception, concentration, stress tolerance, vigilance). Significant simple effects were localized with univariate $F$-tests. An alpha level of 0.05 was accepted as nominal level. To keep the type I error below this level, all post hoc tests were carried using the Sheffé test.

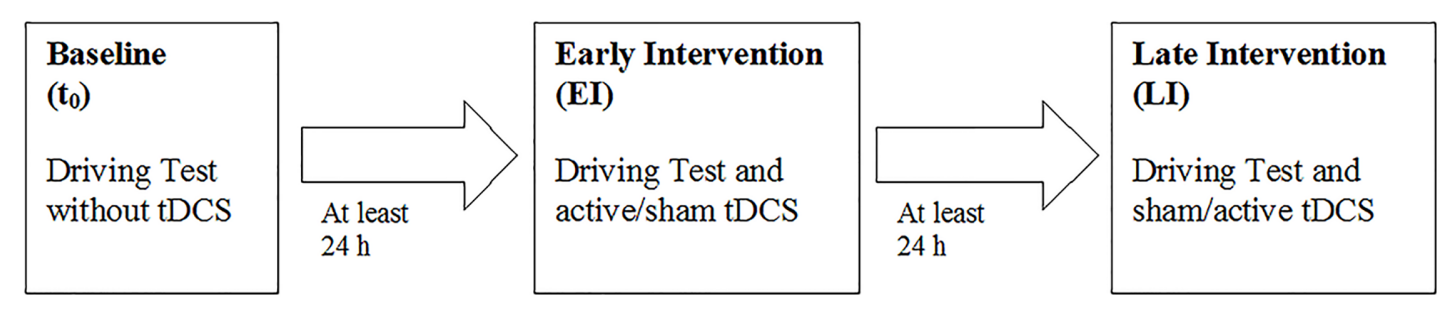

FIGURE 1 | Study flow. 


\section{RESULTS}

\section{Demographic Data}

Demographic characteristics and driving history are provided in Table 1. There were no differences in gender, age, education, years of driving experience and driven kilometers between the EI and the LI group.

\section{Psychomotor and Visual Perception Tests}

Multivariate analysis of variance was performed to assess effects of anodal prefrontal tDCS on functional domains relevant for driving behavior. With exception of the concentration test $[F(1,13)=6.4, p<0.05]$ in the EI group, no significant alterations over time could be demonstrated in driving skills, nor in the EI group neither in the LI group. Post hoc Sheffé tests revealed, that significant time effects in the EI-group with respect to the concentration task could be seen in the sham and in the verum condition (all $p<0.05$ ). Significant time-by-group effects, indicating specific stimulation effects were not found in both intervention groups (Table 2).

To sum up, no alterations on psychomotor skills relevant for driving could be demonstrated via anodal prefrontal tDCS stimulation in our sample.

\section{CRQ Results}

During sham stimulation, mean sum score of side effects was $12.1 \pm 3.4$, after sham stimulation $9.0 \pm 2.2$. During active stimulation, mean sum score of side effects was $15.7 \pm 9.5$, after active stimulation $10.7 \pm 3.9$. Paired $t$-tests showed no significant difference in sum scores during active and sham and after active and sham stimulation (all $p>0.05$ ).

Side effects were significantly lower after stimulation compared to during stimulation in the active $(p<0.05)$ and in the sham condition $(p<0.01)$. General discomfort showed no statistical significant difference between active (mean: 1.9) and sham (mean: 1.8$)$ stimulation $(p=$ n.s.). Sleep disturbances or phosphenes were not reported by any participant.

\section{DISCUSSION}

The aim of this study was to investigate the influence of left prefrontal tDCS on driving skills in a standardized driving test according to the German guidelines for road and traffic safety. 15 healthy adults without any medication and any history of psychiatric illness were randomized to two groups receiving active and sham stimulation in a double blind, cross-over study design.

This is - to our best knowledge - the first study investigating the influence of prefrontal tDCS on computerized psychomotor tests according to the German guidelines for traffic medicine and traffic psychology assessment. The main findings of our study are that no consistent improvements of driving skills like visual perception, stress tolerance, concentration, and vigilance could be found after active tDCS of the left DLPFC. tDCS was well-tolerated and there were low rates of discomfort after

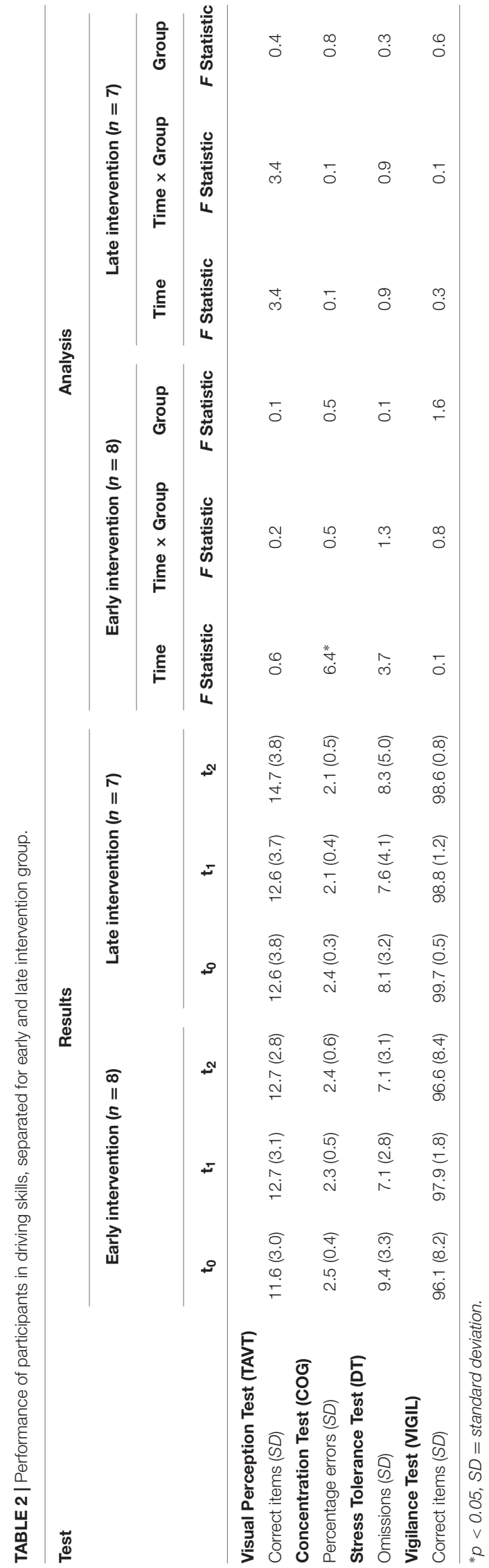


active and sham stimulation. It is therefore unlikely that side effects might have influenced the test performance.

According to Michon (1989), driving processes can be grouped into three interacting hierarchical levels: a strategical level and tactical level affording more executive control processes and an operational level with predominately automatic action patterns like action execution and perceptual processing. Albeit there is no conclusive model of neural substrates of driving behavior till now, there is however evidence, that these driving processes are associated with activations in specific brain regions (Spiers and Maguire, 2007). In contrast to other studies focusing on driving performance, we investigated the influence of left prefrontal tDCS on an operational level of driving behavior. Beeli et al. (2008) and Sakai et al. (2014) investigated a more strategic and tactical level of driving processes like risk behavior or longitudinal control. The study of Sakai reasoned that an upregulation of the right DLPFC (F4 anodal, F3 cathodal) improves vehicle control abilities in car-following and lane-keeping due to the processing of spatial tasks in the right hemisphere (Sakai et al., 2014). In a cross-over study with right and left anodal and cathodal tDCS, Beeli showed that anodal stimulation of both left and right DLPFC directly influences risky driving behavior when driving through a virtual environment in a driving simulator (anode F3 or F4, cathode on the ipsilateral mastoid) (Beeli et al., 2008). We investigated a more basic, operational level of driving processes requiring a high level of arousal to pass the test without failure. It is likely that because of high time pressure in these assessments more automatic response patterns are afforded compared to the studies of Beeli et al. (2008) and Sakai et al. (2014) where risky driving (speed, speed violation, distance, revolutions per minute) respectively fundamental vehicle control (car-following) were assessed, affording more anticipatory control processes. As Gill et al. (2015) outlined, the effects of a tDCS program on the DLPFC may be influenced by the cognitive demands of a task performed during stimulation.

There are some limitations to be considered in the interpretation of our study results. First of all, the number of investigated patients is, despite using cross-over design, rather small and a larger number could have brought different results. Second, healthy participants predominately were in an upper performance level and the speed tests used in our sample required a high level of arousal which probably could not have been increased by anodal stimulation because of ceiling effects. Moreover, prolonged stimulation at high intensity, particularly

\section{REFERENCES}

Batsikadze, G., Moliadze, V., Paulus, W., Kuo, M. F., and Nitsche, M. A. (2013). Partially non-linear stimulation intensity-dependent effects of direct current stimulation on motor cortex excitability in humans. J. Physiol. 591, 1987-2000. doi: 10.1113/jphysiol.2012.249730

Beeli, G., Koeneke, S., Gasser, K., and Jancke, L. (2008). Brain stimulation modulates driving behavior. Behav. Brain Funct. 4:34. doi: 10.1186/1744-90 81-4-34 in a condition of activated cortex, can induce paradoxical homeostatic effects (e.g., Batsikadze et al., 2013), however the chosen parameters are sound to modulate prefrontal function. Third, the operational level explored includes psychomotor and cognitive functions, that are not under the strict competence of the cortical areas stimulated. Besides, electrode placement could have contributed to the negative results as the cathode was placed over right anterior-frontal regions and could have interfered in right DLPFC function. Another limiting factor could have been the duration of tests outlasting of about $25 \mathrm{~min}$ that of stimulation, although other studies reported positive results with neuropsychological test outlasting the stimulation for the same amount of time (e.g., Teo et al., 2011).

\section{CONCLUSION}

Up to now, the relevance of different brain regions in driving performance and behavior remains unclear and the role of the interplay between both cortical hemispheres is not yet elucidated. Study designs and aims are heterogeneous and hamper comparability. Concerning tDCS studies on driving behavior, anodal tDCS of both hemispheres seems to improve risky driving whereas anodal tDCS of the right DLPFC seems to improve spatial functions. In our study we could not show an improvement in psychomotor and visual perception tests after anodal tDCS of the left DLPFC in psychomotor and visual perception functions related to driving skills, although improvement of cognitive functions by left-anodal tDCS has been shown in previous studies. Not least, with respect to rehabilitation efforts, there is a need for further studies on the effects of unilateral or bilateral tDCS on both hemispheres to elucidate the interplay of different neural substrates on specific processes of driving performance in clinical samples.

\section{AUTHOR CONTRIBUTIONS}

FS, AB, and UP designed the study and drafted the manuscript. VG performed tests and helped with manuscript preparation. SL and FP helped with scientific advice and proof reading.

\section{ACKNOWLEDGMENTS}

This work was part of the Medical Doctor Thesis of VG.

Brunnauer, A., Laux, G., Geiger, E., Soyka, M., and Möller, H. J. (2006). Antidepressants and driving ability: results from a clinical study. J. Clin. Psychiatry 67, 1776-1781. doi: 10.4088/JCP.v67n1116

Bukasa, B., Christ, R., and Pocorny-Seliger, E. (2003). Validitätsprüfung verkehrspsychologischer leistungstests für die fahreignungsbeurteilung. Z. Verkehrssicherheit 49, 191-197.

Bukasa, B., Kisser, R., and Wenninger, U. (1990). Computergestützte leistungsdiagnostik bei verkehrspsychologischen eignungsuntersuchungen. Diagnostica 36, 148-165. 
Coull, J. T., Frackowiak, R. S., and Frith, C. D. (1998). Monitoring for target objects: activation of right frontal and parietal cortices with increasing time on task. Neuropsychologia 36, 1325-1334. doi: 10.1016/S0028-3932(98)00035-9

Dosenbach, N. U., Visscher, K. M., Palmer, E. D., Miezin, F. M., Wenger, K. K., Kang, H. C., et al. (2006). A core system for the implementation of task sets. Neuron 50, 799-812. doi: 10.1016/j.neuron.2006.04.031

Fregni, F., Boggio, P. S., Nitsche, M., Bermpohl, F., Antal, A., Feredoes, E., et al. (2005). Anodal transcranial direct current stimulation of prefrontal cortex enhances working memory. Exp. Brain Res. 166, 23-30. doi: 10.1007/s00221005-2334-6

Gill, J., Shah-Basak, P. P., and Hamilton, R. (2015). It's the thought that counts: examining the task-dependent effects of transcranial direct current stimulation on executive function. Brain Stimul. 8, 253-259. doi: 10.1016/j.brs.2014. 10.018

Gräcmann, N., and Albrecht, M. (2016). Begutachtungsleitlinien zur Kraftfahreignung. Bundesanstalt für Straßenwesen. Bergisch Gladbach: Berichte der Bundesanstalt für Straßenwesen, Heft M115. Bremen: Carl Schunemann Verlag.

Javadi, A. H., and Walsh, V. (2012). Transcranial direct current stimulation (tDCS) of the left dorsolateral prefrontal cortex modulates declarative memory. Brain Stimul. 5, 231-241. doi: 10.1016/j.brs.2011.06.007

Just, M. A., Keller, T. A., and Cynkar, J. (2008). A decrease in brain activation associated with driving when listening to someone speak. Brain Res. 1205, 70-80. doi: 10.1016/j.brainres.2007.12.075

Karner, T., and Biehl, B. (2001). Über die zusammenhänge verschiedener versionen von leistungstests im rahmen der verkehrspsychologischen diagnostik. Z. Verkehrssicherheit 42, 53-63.

Keeser, D., Padberg, F., Reisinger, E., Pogarell, O., Kirsch, V., Palm, U., et al. (2011a). Prefrontal direct current stimulation modulates resting EEG and event-related potentials in healthy subjects: a standardized low resolution tomography (sLORETA) study. Neuroimage 55, 644-657. doi: 10.1016/j. neuroimage.2010.12.004

Keeser, D., Meindl, T., Bor, J., Palm, U., Pogarell, O., Mulert, C., et al. (2011b). Prefrontal transcranial direct current stimulation changes connectivity of resting-state networks during fMRI. J. Neurosci. 31, 15284-15293. doi: 10.1523/ JNEUROSCI.0542-11.2011

Michon, J. A. (1989). Explanatory pitfalls and rule-based driver models. Accid. Anal. Prev. 21, 341-353. doi: 10.1016/0001-4575(89)90025-0

Mulquiney, P. G., Hoy, K. E., Daskalakis, Z. J., and Fitzgerald, P. B. (2011). Improving working memory: exploring the effect of transcranial random noise stimulation and transcranial direct current stimulation on the dorsolateral prefrontal cortex. Clin. Neurophysiol. 122, 2384-2389. doi: 10.1016/j.clinph. 2011.05.009

Palm, U., Feichtner, K. B., Hasan, A., Gauglitz, G., Langguth, B., Nitsche, M. A., et al. (2014). The role of contact media at the skin-electrode interface during transcranial direct current stimulation (tDCS). Brain Stimul. 7, 762-764. doi: 10.1016/j.brs.2014.06.006

Palm, U., Hasan, A., Strube, W., and Padberg, F. (2016). tDCS for the treatment of depression: a comprehensive review. Eur. Arch. Psychiatry Clin. Neurosci. 266, 681-694. doi: 10.1007/s00406-016-0674-9

Palm, U., Reisinger, E., Keeser, D., Kuo, M. F., Pogarell, O., Leicht, G., et al. (2013). Evaluation of sham transcranial direct current stimulation for randomized, placebo-controlled clinical trials. Brain Stimul. 6, 690-695. doi: 10.1016/j.brs. 2013.01.005

Pardo, J. V., Fox, P. T., and Raichle, M. E. (1991). Localization of a human system for sustained attention by positron emission tomography. Nature 349, 61-64. doi: $10.1038 / 349061 \mathrm{a} 0$

Sakai, H., Uchiyama, Y., Tanaka, S., Sugawara, S. K., and Sadato, N. (2014). Prefrontal transcranial direct current stimulation improves fundamental vehicle control abilities. Behav. Brain Res. 273, 57-62. doi: 10.1016/j.bbr.2014. 07.036

Spiers, H. J., and Maguire, E. A. (2007). Neural substrates of driving behaviour. Neuroimage 36, 245-255. doi: 10.1016/j.neuroimage.2007.02.032

Teo, F., Hoy, K. E., Daskalakis, Z. J., and Fitzgerald, P. B. (2011). Investigating the role of current strength in tdcs modulation of working memory performance in healthy controls. Front. Psychiatry 2:45. doi: 10.3389/fpsyt.2011.00045

Uchiyama, Y., Toyoda, H., Sakai, H., Shin, D., Ebe, K., and Sadato, N. (2012). Suppression of brain activity related to a car-following task with an auditory task: an fMRI study. Transport Res. Part F Traffic Psychol. Behav. 15, 25-37. doi: 10.1016/j.trf.2011.11.002

Unterrainer, J. M., Ruff, C. C., Rahm, B., Kaller, C. P., Spreer, J., Schwarzwald, R., et al. (2005). The influence of sex differences and individual task performance on brain activation during planning. Neuroimage 24, 586-590. doi: 10.1016/j. neuroimage.2004.09.020

Zaehle, T., Sandmann, P., Thorne, J. D., Jäncke, L., and Herrmann, C. S. (2011). Transcranial direct current stimulation of the prefrontal cortex modulates working memory performance: combined behavioural and electrophysiological evidence. BMC Neurosci. 12:2. doi: 10.1186/1471-2202-12-2

Conflict of Interest Statement: The authors declare that the research was conducted in the absence of any commercial or financial relationships that could be construed as a potential conflict of interest.

Copyright (c) 2018 Brunnauer, Segmiller, Löschner, Grun, Padberg and Palm. This is an open-access article distributed under the terms of the Creative Commons Attribution License (CC BY). The use, distribution or reproduction in other forums is permitted, provided the original author(s) and the copyright owner are credited and that the original publication in this journal is cited, in accordance with accepted academic practice. No use, distribution or reproduction is permitted which does not comply with these terms. 


\section{OPEN ACCESS}

Edited by:

Maria Elide Vanutelli,

Università degli Studi di Milano, Italy

Reviewed by:

Ali Yadollahpour

Ahvaz Jundishapur University of

Medical Sciences, Iran Ilaria Cutica,

Università degli Studi di Milano, Italy

*Correspondence: Alice Cancer alice.cancer@unicatt.it

Received: 05 March 2018 Accepted: 11 July 2018 Published: 31 July 2018

Citation:

Cancer A and Antonietti A (2018) tDCS Modulatory Effect on Reading Processes: A Review of Studies on Typical Readers and Individuals With

Dyslexia.

Front. Behav. Neurosci. 12:162 doi: 10.3389/fnbeh.2018.00162

\section{tDCS Modulatory Effect on Reading Processes: A Review of Studies on Typical Readers and Individuals With Dyslexia}

\author{
Alice Cancer ${ }^{*}$ and Alessandro Antonietti \\ Department of Psychology, Università Cattolica del Sacro Cuore, Milan, Italy
}

The possibility to use non-invasive brain stimulation to modulate reading performance in individuals with developmental dyslexia (DD) has been recently explored by few empirical investigations. The present systematic review includes nine studies which have employed transcranial direct current stimulation (tDCS) aiming at improving reading abilities in both typical readers and individuals with DD. Anodal tDCS over the left temporo-parietal cortex - a region which is typically involved in phonological and orthographic processing during reading tasks and underactive in individuals with DD-was the most frequently used montage. The majority of studies employing such stimulation protocol showed significant improvement in differential reading subprocesses. More precisely, word decoding was improved in adult readers, whereas non-word and low-frequency word reading in younger individuals. Furthermore, tDCS was found to be specifically effective in poor readers and individuals with DD rather than typical readers, in spite of the specific brain region targeted by the stimulation; Left frontal, left temporo-parietal, and right cerebellar tDCS failed to modulate reading in already proficient readers. Overall, tDCS appears to be a promising remedial tool for reading difficulties, even when applied to younger populations with reading problems. Further empirical evidence is needed to confirm the potential of neuromodulation as a successful intervention method for DD.

Keywords: tDCS, neuromodulation, reading, dyslexia, intervention

\section{INTRODUCTION}

Developmental dyslexia (DD) is a neuropsychological disorder affecting the ability of reading. More precisely, the behavioral manifestations of DD include an inaccurate and/or slow decoding of written language, resulting in a hesitant and effortful reading. Such difficulties are not the consequence of intellectual deficit, sensory dysfunction, socioeconomic disadvantage, or lack of educational opportunities (Snowling and Hulme, 2012; American Psychiatric Association, 2013).

The majority of the intervention methods for DD which have been studied to be effective in overcoming dyslexia-related difficulties are behavioral and comprise activities aimed at improving reading by adopting process-based approaches. More precisely, such interventions are inspired by theoretical frameworks focusing on specific reading-related cognitive mechanisms, such as phonological processing (e.g., Shaywitz et al., 2004), temporal-auditory perceptual abilities (e.g., Gaab et al., 2007; Thomson et al., 2013), visuo-spatial attentional abilities (e.g., Franceschini et al., 2012), and grapheme-phoneme association (Saine et al., 2011). 
Although the outcome measures employed for assessing the effectiveness of remedial methods for DD are in most cases behavioral (e.g., standardized test measuring reading speed and accuracy, phonological awareness, verbal working memory, rapid automatized naming, school proficiency), few studies measured the neurobiological changes associated with DD intervention. Findings from a meta-analysis by Barquero et al. (2014), which considered studies investigating differences in functional activation following reading intervention, are convergent with neurofunctional models of DD (Pugh et al., 2000; Maisog et al., 2008; Richlan et al., 2011) in identifying a central role of left-lateralized inferior frontal, temporo-parietal, and occipitotemporal dysfunctions. In typically-reading adults, the reading system is dominated by a left-sided network, comprising three circuits (Shaywitz and Shaywitz, 2008): (a) A posterior ventral pathway centered in the inferior occipital-temporal area, engaged in the visual processing and recognition of words (Dehaene and Cohen, 2011); (b) A dorsal posterior region comprising the posterior superior temporal, supramarginal, and angular gyri, which is involved in phonological, orthographic, and semantic processing, and grapheme-phoneme conversion (Price, 2012); (c) An anterior component, located in the inferiorfrontal gyrus (IFG), involved in phonological processing and articulatory output (Levy et al., 2008). In DD, an underactivation of both temporo-parietal and occipito-temporal regions have been reported (Richlan, 2012).

The correspondence between the improved behavioral outcomes and the neurofunctional reorganization following treatment has, quite recently, led to the hypothesis of a neuromodulatory remedial intervention for DD (Krause and Cohen Kadosh, 2013; Vicario and Nitsche, 2013). To date, few experimental studies have explored the possibility to modulate reading performance using non-invasive brain stimulation (NIBS) by inducing excitability alterations in the brain regions shown to be underactivated in poor readers and individuals with DD.

The present review includes a collection of the studies which have employed transcranial electrical stimulation (tES) aiming at improving reading abilities in both typical readers and individuals with DD. In order to draw preliminary conclusions on the efficacy of neuromodulation as a potential remedial tool for reading difficulties, this review focuses on $\mathrm{tES}$, and specifically transcranial direct current stimulation (tDCS), thus excluding studies employing transcranial magnetic stimulation (TMS) (e.g., Costanzo et al., 2012, 2013). In tDCS, weak direct electrical currents, ranging from 1 to $2 \mathrm{~mA}$, are applied for a short duration (up to $20 \mathrm{~min}$ ) via two or more electrodes placed on the scalp (Priori et al., 1998; Nitsche and Paulus, 2000). Such transcranial application of current induces alterations of resting membrane potential and thus variation in the response threshold of the stimulated neurons (see Fertonani and Miniussi, 2017). Unlike TMS, the modifications induced by tES are insufficient to induce action potentials (Creutzfeldt et al., 1962; Bindman et al., 1964). However, the alteration in the threshold response via tES can induce long-lasting cognitive changes (Zaghi et al., 2010). Moreover, relative to TMS, tDCS is associated with fewer and minor adverse side effects (i.e., tingling, itching, burning sensation of the skin under the electrode, and in rare cases nausea and headaches) (Fertonani et al., 2015). Such features make tDCS more suitable for a cognitive enhancement program, which require multiple training sessions in order to be effective. For all the aforementioned reasons, the present review focuses on tDCS studies only.

\section{AIMS AND METHODS}

The aim of the present review is to explore the effects of different neuromodulation protocols on reading, specifically considering the variability in targeted cortical areas (electrode montages), number of sessions (single-session vs. multiple applications to the same cortical site with the same stimulation polarity), simultaneous application of behavioral interventions (tDCS only vs. tDCS combined with a behavioral intervention), and population targeted (typical readers vs. individuals with DD; children vs. adults). To do so, we performed a systematic review following the PRISMA guidelines (Liberati et al., 2009).

A literature search was conducted on studies published between 2008 and 2018 on Science Direct database using the search string "tDCS" OR "trascranial direct current stimulation" AND “reading” AND/OR "dyslexia.” Furthermore, we searched for additional references in retrieved articles and reviews and checked each article according to our inclusion criteria.

Only the articles meeting the following eligibility criteria have been selected: (a) peer-reviewed publications written in English; (b) studies including reading outcomes; (c) papers providing details of the protocol implemented; (e) presence of a control group or control condition (sham or opposite stimulation polarity).

\section{RESULTS}

One hundred fifty-five records were obtained through database and retrieval articles and reviews searching. However, 135 references were excluded after duplicate removal and title and abstract screening, and another 15 references were further excluded following a full-text assessment (Figure 1). The remaining 9 articles were included in the review (for an overview, see Table 1).

\section{Effect of tDCS on Reading in Typical-Readers}

In order to investigate the role of the left posterior temporal cortex (pTC) in reading ability, Turkeltaub et al. (2012) carried out an empirical investigation on healthy adults. The authors designed a tDCS intervention procedure based on functional neuroimaging evidence suggesting reduced left pTC activity in individuals with DD (Maisog et al., 2008) and increased left pTC lateralization in children with DD after successful remedial training (Simos et al., 2002). They hypothesized that enhancing left lateralization of pTC would facilitate lexical access and phonological processing, thus ultimately improving reading efficiency. In a within-subject design, 25 right-handed typicallyreading adults underwent two tDCS sessions, on different days, 


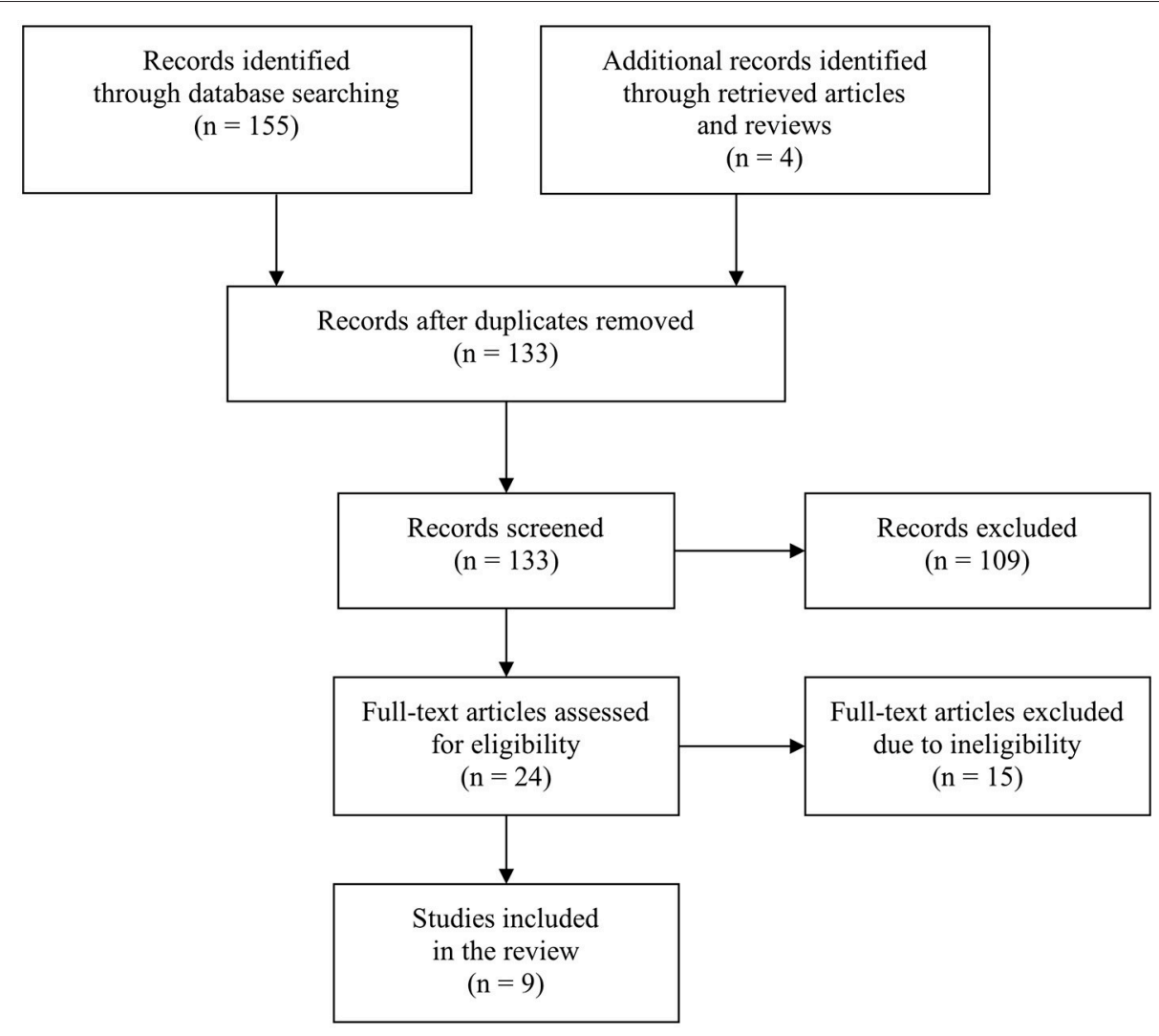

FIGURE 1 | PRISMA flowchart of database search strategy.

to compare a single-session tDCS intervention with a sham control condition. Criteria for participant's selection included: at least 12 years of education, no history of neurologic, psychiatric disorder, significant head trauma, hearing loss, or personal or family history of learning disorder (including DD). In real tDCS, a constant current of $1.5 \mathrm{~mA}$ was applied for $20 \mathrm{~min}$ via a pair of $25 \mathrm{~cm}^{2}$ electrodes. Such stimulation parameters, resulting in a current density of $0.06 \mathrm{~mA} / \mathrm{cm}^{2}$, were employed in the majority of the studies included in the present review. As for the electrode montage, the anodal electrode was positioned over the left pTC (midway between T7 and TP7) whereas the cathodal electrode over the contralateral homolog site (midway between T8 and TP8). During the last $15 \mathrm{~min}$ of tDCS, participants performed either a phoneme perception task or a color perception task to maintain attention and arousal. Word and non-word reading efficiency was assessed offline, immediately after each session, using the Test of Word Reading Efficiency - TOWRE (Torgesen et al., 1999), in which participants are asked to read aloud lists of words (Sight Word Efficiency subtest) and non-words (Phonetic Decoding Efficiency subtest) as quickly as possible. For both subtests (words and non-words reading), standard score is determined by the number of verbal stimuli read correctly within $45 \mathrm{~s}$. Authors found a significant effect of real tDCS on word reading efficiency performance, compared to the sham condition, and thus confirmed the short-term efficacy of the enhancement of left pTC lateralization via tDCS after just one session. The beneficial effect was specifically driven by a below-average reading subgroup of participants $(N=12)$, namely participants who scored below average (i.e., $<100)$ in the word reading test in the post-sham assessment. Conversely, the authors did not find a significant effect of real-tDCS in aboveaverage participants (i.e., TOWRE word reading score $>100$ ), thereby supporting the possibility to successfully address this intervention to individuals with reading difficulties.

A later tDCS study by Thomson et al. (2015) failed to replicate the findings of Turkeltaub and colleagues, despite testing a similar intervention protocol. The researchers used tDCS to stimulate an overlapping but slightly superior region, namely the left temporo-parietal junction (TPJ) (CP5). The aim of the study was to further investigate whether the effect found by Turkeltaub and colleagues was the result of the left anodal stimulation or of the conjunction of left anodal and right cathodal stimulation. To do so, Thomson and colleagues implemented a mixed factorial design, which included both a withinsubject comparison of the stimulation polarity (i.e., anodal vs. cathodal) and a between-subject comparison of the stimulation hemispheric lateralization (i.e., left CP5 vs. right CP6). No sham condition was included. Thirty-nine right-handed healthy adults were assigned to either a left or right stimulation condition and received, during two separate sessions, anodal 
Cancer and Antonietti

tDCS Modulatory Effect on Reading Processes

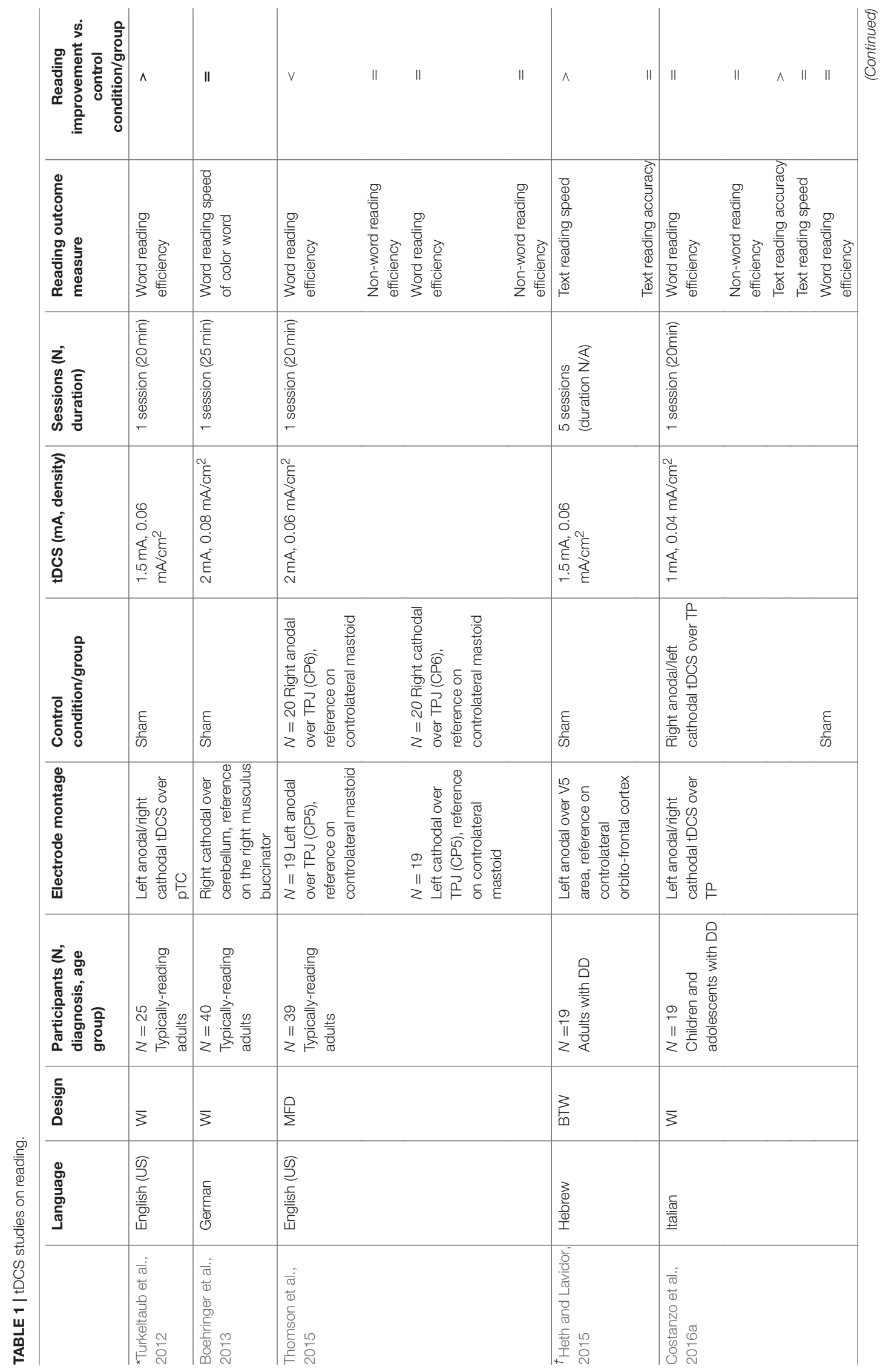

Frontiers in Behavioral Neuroscience | www.frontiersin.org

127

July 2018 | Volume 12 | Article 162 


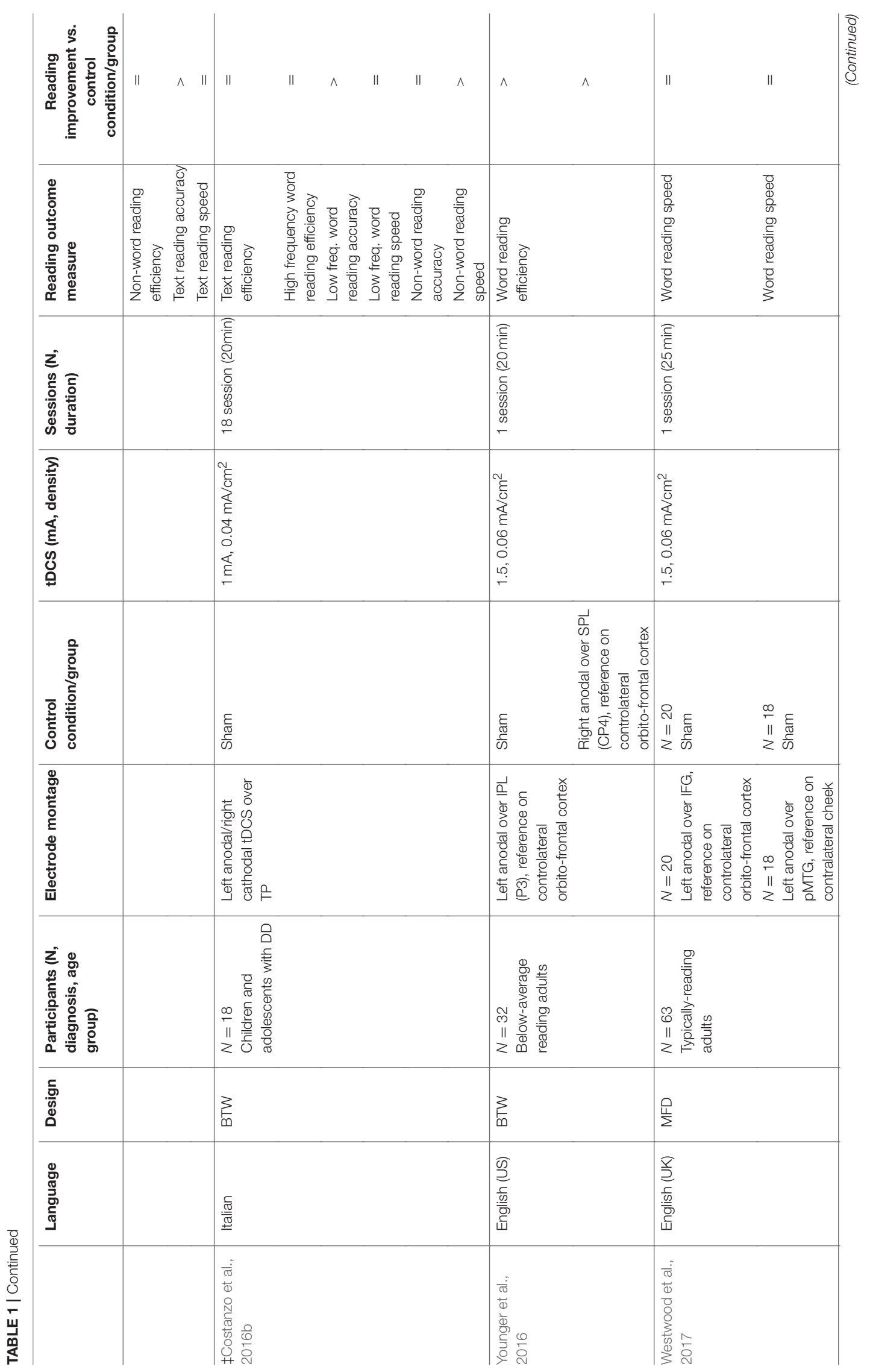




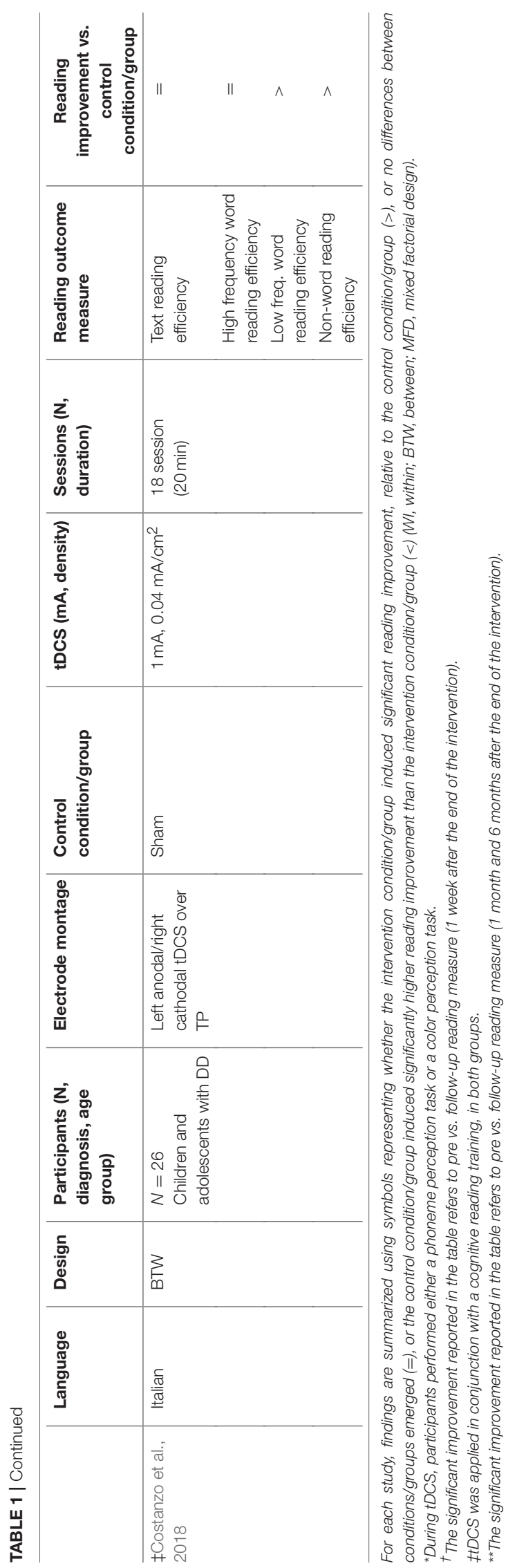

or cathodal stimulation ( $2 \mathrm{~mA}$ for $20 \mathrm{~min})$ over TPJ, while the reference electrode was positioned on the contralateral mastoid. Participants had no history or presence of reading disability or any neurological or psychiatric disorder and were not taking any central nervous system-active drugs or medications. Word and non-word reading efficiency measures (Sight Word Efficiency and Phonetic Decoding efficiency subtests of the Test of Word Reading Efficiency - 2nd edition: Torgesen et al., 2011) were administered before each tDCS session and after $10 \mathrm{~min}$ from the beginning of the stimulation. Differently than expected by the authors, results showed a mild but significant increase of word reading efficiency following right hemisphere anodal stimulation, compared to left hemisphere anodal stimulation. Moreover, word reading performance decrement was induced by left anodal stimulation. Thomson and colleagues speculated that, since participants were typical readers, no further improvement could yield from the enhancement of the already functioning left hemispheric phonological system. Conversely, they suggested that the activation of the normally less involved right temporoparietal region could have led to reading improvement, similarly to what was observed in other processing domains (i.e., motor functioning: Boggio et al., 2006). Finally, the absence of a cathodal flow directly at the right temporal parietal junction was proposed as a further explanation for the inconsistent results compared to Turkeltaub et al. (2012).

In their experimental investigation, Younger et al. (2016) targeted a more superior portion of the left temporo-parietal cortex, namely the inferior parietal lobe (IPL), based on its role in smaller-grained grapheme-to-phoneme mapping and its involvement in initial development of the reading network (Pugh et al., 2000). Word reading efficiency of 32 righthanded, low-to-average reading skilled adults (baseline: 80100 standard score in the Sight Word Efficiency subtest of the Test of Word Reading Efficiency: Torgesen et al., 1999) was measured before and immediately after a single-session tDCS intervention. Inclusion criteria, along with a below-average word reading performance, included no history of neurological disorder, psychiatric disorder, significant head trauma, hearing loss, substance abuse, seizure or migraine, metal implants, and current pregnancy. Participants have been assigned to one of three conditions: anodal tDCS over left IPL, right tDCS over right SPL, or sham. In all conditions the cathode electrode was positioned over the contralateral supraorbital frontal region, so to selectively measure the effect of the anodal stimulation on the target region. The real tDCS parameters replicated the ones used by Turkeltaub et al. (2012) $1.5 \mathrm{~mA}$ for $20 \mathrm{~min}$. Results supported the initial hypothesis: Participants who received the anodal stimulation over left IPL showed significantly greater improvement in word reading efficiency, relative to the participants assigned to sham condition and the right anodal over SPL condition (for the latter, the difference trended toward significance). The effect of the montage employed in Younger and colleagues' study resulted in a greater effect size compared to Turkeltaub and colleagues' (Cohen's d: 1.57 vs. $0.46)$.

More recently, Westwood et al. (2017) used naming and reading tasks to assess the effect of tDCS on the semantic 
interference effect in word retrieval (e.g., Belke, 2013), namely, the slower and less accurate responses in retrieving a target word when semantically related words are presented. More precisely, authors contrasted stimulation of frontal and temporal areas hypothesizing that frontal stimulation would boost selection mechanisms, thereby reducing the interference effects, whereas temporal stimulation would increase the activation of competing items resulting in a stronger interference, as suggested by Pisoni et al., 2012). Word reading served as a control task, to verify the specificity of semantic interference effect on naming. According to the authors, reading should not be affected by lexical-semantic selection, since orthographic processing is primarily involved (see Belke, 2013). Sixty-three right-handed healthy undergraduate students took part in two 25-min sessions one week apart, during which they completed both reading and picture naming tasks (the order of task presentation was counterbalanced). As for the reading task, 165 semantically related and unrelated words, corresponding to the stimuli of the picture naming task, were presented on a computer screen. Speed and accuracy performance were recorded. However, authors did not analyze error rates for word reading, since they were $<5 \%$. Participants with language impairments, history of migraine, headaches, skin disorders, any adverse experience to previous tDCS, any history of epilepsy or stroke, head/metal implants, any neurological disorders, as well as any volunteers who had participated in a tDCS or TMS study in the previous 6 months, were excluded. Authors implemented a mixed-factorial design, in which participants were assigned to either a frontal stimulation condition $(N=20)$ or a temporal stimulation condition $(N=18)$. In both experimental conditions participants received real and sham tDCS, each in one of the two sessions. Stimulation was delivered for $25 \mathrm{~min}$ at $1.5 \mathrm{~mA}$ using $25 \mathrm{~cm}^{2}$ electrodes. Both picture naming and word reading tasks were completed during the stimulation. In the frontal stimulation condition, the active electrode was placed over the left inferior frontal gyrus (IFG) and the reference electrode $\left(35 \mathrm{~cm}^{2}\right)$ was placed over the contralateral supraorbital area, whereas in the temporal stimulation condition the active electrode was placed over the left mid-posterior temporal lobe area (pMTG), and the reference was placed over the contralateral cheek, so to avoid current flow through frontal areas. To control for random variability between sessions, a control group $(N=25)$ was tested using the same protocol, however without receiving any stimulation. No significant effect of tDCS on either reading or naming was found in any condition, as well as no effect of stimulation site (frontal vs. temporal). In light of such findings, authors questioned the reliability of tDCS in inducing cognitive effects in healthy participants using single-session stimulation, in accordance with a quantitative review on a broad spectrum of cognitive outcome measures (including executive functions, language, and memory) by Horvath et al., 2015).

In a study aimed at investigating cerebellar contributions to verbal working memory, Boehringer et al. (2013) tested the effect of cathodal tDCS over the right cerebellum on forward and backward digit spans and other control tasks, among which word reading speed. In such reading task, the time needed to read aloud 42 color words was measured. Fourty right-handed, native German speaking, healthy participants were invited to participate in two tDCS sessions, at least 5 days apart; In each one, they received either cathodal ( $2 \mathrm{~mA}$ for $20 \mathrm{~min}$, using $25 \mathrm{~cm}^{2}$ electrodes) or sham tDCS over the right cerebellum $(2 \mathrm{~cm}$ below the inion and $1 \mathrm{~cm}$ posterior to the right mastoid), with the anode placed over the right musculus buccinator. Outcome measures (i.e., forward and backward digit spans, reading of color words, a visually cued sensory-motor task, and finger tapping) were collected before and immediately after tDCS. Authors found that whilst real cathodal tDCS significantly reduced verbal working memory performance, it did not affect word reading.

\section{Effect of tDCS on Reading in Individuals With DD}

The first tDCS study involving adults with a diagnosis of DD was carried out by Heth and Lavidor (2015). The authors targeted the visual extrastriate area V5/MT, whose activity has been reported to be reduced in individuals with DD (Demb et al., 1998; Eden and Zeffiro, 1998). To identify such stimulation site, the authors adopted a visuo-attention approach and specifically referred to the magnocellular deficit theory of DD (Stein, 2012). According to this theory, DD is associated with an abnormal visual motion processing, due to a dysfunction of the magnocellular system, a perceptual pathway projecting from the lateral geniculate nucleus to primary visual areas, responsible for detecting contrast, motion, and rapid changes in the visual field. As the hypothesis of causal role of the magnocellular system in DD is highly controversial due to insufficient empirical support and contrasting findings (Amitay et al., 2002; Ramus et al., 2003), the visual magnocellular dysfunction has been interpreted, instead, as a consequence of impoverished reading (Olulade et al., 2013). To examine the magnocellular involvement in the reading process, Heth and Lavidor designed an intervention comprising five $\mathrm{tDCS}$ sessions over 2 weeks, in which anodal stimulation was applied over the V5 area ( $1.5 \mathrm{~mA}$ for $20 \mathrm{~min}$ ), with the right orbito-frontal cortex as a reference site. Nineteen right-handed, native Hebrew speaking adults who had previously received a diagnosis of DD, without a comorbidity with attention deficit and hyperactive disorder (ADHD) nor neurological or psychiatric conditions, were randomly assigned to either the anodal or a sham condition. Text reading speed and accuracy were assessed before, immediately after and a week after the end of the 5-session intervention. Three one-page-long passages at 9th grade level, which are routinely included in the DD diagnostic procedure in Israel (Tov-Li, 1999), were used to assess participants' text reading speed and accuracy. The anodal tDCS group showed a significant improvement in text reading speed at follow-up assessment, compared to the sham group, whereas no difference between condition occurred immediately after the end of the intervention. Reading accuracy did not improve at any time point. These findings were interpreted by the researchers as indication of the involvement of the $\mathrm{V} 5$ area in reading.

Costanzo et al. (2016a), Costanzo et al. (2016b), and Costanzo et al. (2018) were the first to study the effect of tDCS on young populations with DD. Several researchers called for caution in 
the application of NIBS to children, pointing out the unknown consequences and the possible side effects of stimulating a developing brain (Kadosh et al., 2012; Krause and Cohen Kadosh, 2013). The major concern regards the potential deterioration of certain abilities as a consequence of the enhancement of specific learning skills. To date, empirical evidence from the application of tDCS to developmental samples is still limited (Mattai et al., 2011; Schneider and Hopp, 2011; Auvichayapat et al., 2013; Amatachaya et al., 2015) and no safety guidelines for children as been yet established. Despite these concerns, Costanzo and colleagues stressed the importance of the exploration of such potentially effective intervention for DD in developmental age, which could be critical to foster school learning and, consequentially, broaden future occupational opportunities.

In the light of the contrasting findings from the previous studies (Turkeltaub et al., 2012; Thomson et al., 2015), Costanzo and colleagues explored the optimal polarity of the stimulation for children and adolescents with DD in a single-session intervention study (Costanzo et al., 2016b). A within-subject design was implemented to compare left and right anodal stimulation over the temporo-parietal region: Midway between P7 and TP7 and midway between P8 and TP8, respectively. The reference electrode was placed on the contralateral homologs site in both conditions, in order to exclude brain regions involved in the reading process, such as the prefrontal and the occipital cortices (Eckert, 2004; Richlan, 2014) and thus focus on the role of temporo-parietal regions. Slightly lower current intensity was used $(1 \mathrm{~mA})$ and the stimulation was delivered for $20 \mathrm{~min}$. A sample of 19 right-handed, native Italian speaking children and adolescents with a diagnosis of DD, aged 10-18 years, participated in three tDCS sessions (i.e., left anodal/right cathodal, right anodal/left cathodal, and sham) on different days. Measures of word reading (20 high-frequency words and 20 low-frequency words), non-word reading (20 non-words), and text reading (a 400-syllable long passage) were collected before and immediately after each tDCS session. Reading accuracy was expressed by number of errors (1 point was assigned for each letter substitution and 0.5 point for every self-correction or hesitation), whereas reading speed by total reading time (in seconds). Results showed a significant text reading accuracy improvement following left anodal/right cathodal tDCS and an increase in errors after left cathodal/right anodal tDCS, relative to the other conditions. These findings, which are consistent with those of Turkeltaub and colleagues, support the efficacy of the simultaneous action of left anodal and right cathodal tDCS in inducing reading improvement in children and adolescents with DD.

In a second study by the same authors (Costanzo et al., 2016a), the tDCS protocol, which was found to be effective in the previous exploration (Costanzo et al., 2016b) (i.e., left anodal/right cathodal over the temporo-parietal regions), was applied to a group of children and adolescent with DD. To further improve reading abilities, and induce medium-term positive effects, a multiple-session intervention protocol was designed in which tDCS was paired with a remedial cognitive training, comprising tachistoscopic and phonic (training on phoneme awareness and grapheme-phoneme conversion) reading exercises. Eighteen right-handed, native Italian speaking participants were randomly assigned to either a real tDCS (1 $\mathrm{mA}$ for $20 \mathrm{~min}$ ) or sham condition. Participants had no history of neurological disease, nor a family history of epilepsy, nor comorbidity with ADHD. Both groups participated in an 18 -session intervention including the cognitive training over 6 weeks. The same reading measures as the previous study (Costanzo et al., 2016b) were collected before, immediately after, and 1 month after the end of the treatment. Consistently with the previous study, the active tDCS groups showed significant improvements in low frequency reading accuracy and non-word reading speed, compared to the sham group. Furthermore, the improvements persisted a month after the end of the intervention. Performance increases were specifically found in reading tasks involving phonological processing and letter-sound mapping (i.e., low frequency word and non-word reading).

In a more recent study, the same authors (Costanzo et al., 2018) replicated the same protocol on a larger group of children and adolescents with DD, including a further followup assessment 6 months after the end of the intervention, so to measure its long-term efficacy. Twenty-six right-handed children and adolescents with a diagnosis of DD were selected on the basis of the same inclusion criteria as the previous investigation. Differently than the previous study, results were reported considering a reading efficiency index, thus representing speed and accuracy together, for each task (i.e., high-frequency word, low-frequency word, non-word, and text reading). Whereas the participants who received sham tDCS $(N=13)$ did not show reading changes at any time point, the experimental group $(N=13)$ showed significant improvements in low-frequency word reading (1- and 6-month after the end of the treatment) and in non-word reading (immediately after, 1 month after, and 6 months after the end of the treatment). Costanzo and colleagues interpreted such findings as an evidence of tDCS delayed but long-lasting beneficial effect. Consistently with the previous study, no effects on high-frequency word nor text emerged.

Finally, the protocol of an ongoing study testing the effect of a multiple-session tDCS intervention combined with a cognitive training for DD has been recently reported (Cancer and Antonietti, 2017). A sample of undergraduate students with a diagnosis of DD has been involved in an intervention comprising a novel rhythm-based reading training (Bonacina et al., 2015; Cancer et al., 2016) paired with tDCS for 10 daily sessions over 2 weeks. The left temporo-parietal region was stimulated at a constant current of $1.5 \mathrm{~mA}$ for $20 \mathrm{~min}$, and the electrode montage replicated the one used by Costanzo et al. (2016a). Preliminary results from three single cases who took part in the real tDCS condition provided encouraging evidence about the efficacy of the combined intervention on undergraduate students with DD (Cancer and Antonietti, 2017). However, the pattern of reading sub-components improvement seemed to depend greatly on individual reading profile at baseline. Since no conclusion about the role of tDCS on reading can be draw from such preliminary single-case data, the study was not included in the overall comparison. 


\section{DISCUSSION}

Despite the limited number of studies included in the present review, and the procedural and methodological dissimilarities between them, a descriptive and critical analysis of their findings could provide some insights into tDCS modulation of reading processes. Characteristics and main results of the nine reviewed studies have been summarized in Table 1 .

Considering the different cortical areas targeted by anodal stimulation, the majority of the studies focused on the left temporo-parietal cortex (i.e., pTC, TPJ, pMTG, IPL). Such regions are typically involved in phonological, orthographic, and semantic processing during reading tasks (Price, 2012) and underactive in individuals with DD (see Richlan, 2012). Significant effects on reading were observed following left anodal temporo-parietal montages in 5 out of 7 studies (Turkeltaub et al., 2012; Costanzo et al., 2016a,b, 2018; Younger et al., 2016).

However, the type of population targeted, specifically typical readers vs. below-average readers and individuals with DD, appeared to significantly account for the outcomes of temporoparietal anodal tDCS interventions. As suggested by Thomson et al. (2015), whose findings on healthy participants showed an opposite trend relative to the other interventions, individuals with poor reading skills are more suitable to benefit from a neuromodulatory intervention enhancing left temporo-parietal lateralization, due to anomalies in their cortical activity, whereas similar beneficial effects cannot be replicated in already proficient readers. Null effect of a stimulation protocol similar to Thomson and colleagues' on healthy adults were also reported by Westwood et al. (2017). Consistently, the ameliorative effects of a similar protocol reported by Turkeltaub et al. (2012) were driven by a below-average reading subgroup of participants, whilst no significant effect of tDCS was found in aboveaverage participants. As suggested by Westwood et al. (2017), tDCS modulatory mechanisms are most likely to induce effect on cognition in brains with low or dysfunctional neuronal excitability, rather than already close to an optimal level of excitability.

The association between the precise electrode placement (i.e., anodal tDCS over superior vs. inferior portion of the left TP cortex; unilateral vs. bilateral montages) and the modulation of a specific reading sub-processes (i.e., grapheme-to-phoneme mapping vs. lexical representation access) was not consistent across studies. Similar left anodal/right cathodal montages (Turkeltaub et al., 2012; Costanzo et al., 2016a,b, 2018; Younger et al., 2016) led to improvement in different reading outcomes (either word efficiency, text accuracy, or low frequency and non-word reading speed). Costanzo et al. (2016a) suggested that, in order to enhance word and text reading, the medium and inferior temporal gyri, which are specifically involved in whole-word recognition (Dehaene et al., 2005; Vinckier et al., 2007), should be targeted. Consistently, the left temporo-parietal dysfunction in adults with DD has been observed during the performance of phonological tasks, such as non-word reading, phonological lexical decision, and word rhyme judgment (for a meta-analysis, see Richlan et al., 2011), whilst underactivation of the left fusiform gyrus (Brambati et al., 2006) and of occipito-temporal regions (McCrory et al., 2005) was specifically associated with word reading. Whereas this suggestion would explain why Costanzo and colleagues' left temporo-parietal anodal stimulation protocols did not induce changes in word reading, it would not explain why in other studies on belowaverage-reading adults word reading was improved using similar tDCS interventions. Specifically, word reading efficiency was successfully modulated by anodal stimulation of left temporoparietal regions in below-average readers, as seen in Turkeltaub et al. (2012) and Younger et al. (2016) studies.

We suggest that compensatory rather than "normalizing" functional changes could have enhanced reading ability in adult with poor reading skills via the recruitment of alternate circuits for word reading. According to such hypothesis, below-average adult readers, after tDCS modulation of the temporo-parietal areas, would rely on grapheme-to-phoneme mappings for word reading, instead of increasing the functionality of the circuits normally activated in adult proficient readers. However, only older populations with reading difficulties would exhibit such compensatory changes following intervention, whereas children with DD would exhibit normalization changes. The hypothesis is consistent with neuroimaging evidence showing an increased activation in both left middle temporal and posterior superior temporal areas after a successful behavioral treatment in children with DD, which improved both word and non-word reading (Simos et al., 2002; Barquero et al., 2014). On the other hand, a study measuring the neurofunctional and behavioral changes in adults with DD after an intensive phonologybased intervention program found significant increases in left hemisphere inferior parietal lobule and intraparietal sulcus in correspondence of both non-word and text reading, thus showing that improved phonological processing was transferred to other aspects of reading ability as well (Eden et al., 2004).

As for the electrode position differences in studies targeting difference portions of the temporo-parietal cortex, we argue that no major outcome variability was accounted for by it. Due to its limited spatial resolution, tDCS is not suitable for stimulating focal portions of the cortical tissues and the current will most likely flow outwards the targeted site, thus affecting the surrounding areas.

The ameliorative effects of bilateral tDCS montages reported by Turkeltaub et al. (2012), Costanzo et al. (2016a), Costanzo et al. (2016b), and Costanzo et al. (2018), in which cathodal stimulation was applied to right temporo-parietal regions, are consistent with literature on children with DD showing a reduction of right temporal activation after a successful reading intervention (Shaywitz et al., 2004) and a greater activation of the same area in children with DD who did not show reading improvements after a behavioral intervention (Odegard et al., 2008). Conversely, such effects were not replicated in typical readers, as shown by Thomson et al. (2015), who found a positive effect of right temporo-parietal anodal stimulation on word reading.

Only three studies included in the present review measured the effect of anodal tDCS outside the temporo-parietal cortex. Among these, null effect of tDCS intervention were found in 
studies on typical readers (Boehringer et al., 2013; Westwood et al., 2017), in spite of the site of anodal stimulation (i.e., cerebellum or inferior frontal gyrus). In contrast, Heth and Lavidor (2015) found positive effect of anodal stimulation over an occipital visual area (V5/MT) in adults with DD, as measured by text reading speed improvement 1 week after the end of the intervention. Such findings are consistent with the underactivation of occipital and occipito-temporal regions, specifically involved in visual processing and recognition of word, which were found in adults with DD (Richlan, 2012). Therefore, it will be of interest to further investigate its role on word reading as well.

Interestingly, the comparison between single- (Turkeltaub et al., 2012; Thomson et al., 2015; Costanzo et al., 2016b; Younger et al., 2016) and multiple-session tDCS interventions (Heth and Lavidor, 2015; Costanzo et al., 2016a, 2018) did not appear to give reason for major variability in the results. However, the small number of studies testing repeated tDCS methodologies for improving reading does not allow to draw any general conclusion.

Only two studies, by the same authors and with the same protocol (Costanzo et al., 2016a, 2018), tested the efficacy of the simultaneous application of behavioral intervention targeting reading during tDCS. Such approach, associated with a multiple-session protocol, was the only one to be effective in inducing significant improvements in reading measures involving phonological processing and grapheme-to-phoneme mapping. Furthermore, these studies also provided evidence of mid-term (up to 1 month) and long-term (up to 6 months) efficacy of left temporo-parietal tDCS on reading.

Finally, three studies conducted on younger populations with DD (Costanzo et al., 2016a,b, 2018) confirmed the possibility of successfully employing tDCS as a remedial intervention for $\mathrm{DD}$, especially when combined with a cognitive training targeting reading. These pioneering investigations showed that repeated tDCS applications can be tolerated by children and adolescents, without significant discomfort or adverse effects reported up to 3 months after the end of the intervention.

\section{REFERENCES}

Amatachaya, A., Jensen, M. P., Patjanasoontorn, N., Auvichayapat, N., Suphakunpinyo, C., Janjarasjitt, S., et al. (2015). The short-term effects of transcranial direct current stimulation on electroencephalography in children with autism: a randomized crossover controlled trial. Behav. Neurol. 2015:928631 doi: 10.1155/2015/928631

American Psychiatric Association (2013). Diagnostic and Statistical Manual of Mental Disorders (DSM-5®). Washington, DC: American Psychiatric Pub.

Amitay, S., Ben-Yehudah, G., Banai, K., and Ahissar, M. (2002). Disabled readers suffer from visual and auditory impairments but not from a specific magnocellular deficit. Brain 125, 2272-2285. doi: 10.1093/brain/ awf231

Auvichayapat, N., Rotenberg, A., Gersner, R., Ngodklang, S., Tiamkao, S., Tassaneeyakul, W., et al. (2013). Transcranial direct current stimulation for treatment of refractory childhood focal epilepsy. Brain Stimul. 6, 696-700. doi: 10.1016/j.brs.2013.01.009

\section{CONCLUSIONS}

The collection of studies included in the present descriptive and critical review supported the hypothesis to use neuromodulation for improving reading skills in individuals with DD. Positive effects have been reported in the majority of the tDCS studies reviewed. Anodal tDCS over left temporo-parietal region was the most frequently investigated montage, which was shown to be successful in improving reading, when compared to alternative tDCS montages. However, the exact nature of the observed reading improvements was rather controversial. Enhancement of differential sub-processes of the reading ability (i.e., graphemeto-phoneme mapping or lexical access; reading speed vs. reading efficiency) yielded from similar stimulation procedures. Furthermore, the outcome of the intervention varied according to the population targeted: tDCS was found to be specifically effective in poor readers and individuals with DD rather than typical readers.

Overall, tDCS appears to be a promising remedial tool for reading difficulties, even when applied to younger populations. However, further empirical evidence is needed to confirm its potential as a successful intervention method for DD.

As a future direction, reading performance gains should be maximized by combining specific approaches to reading remediation with cortical neuromodulatory techniques, so to engage specific reading sub-processes via neuroplasticity increase (Vicario and Nitsche, 2013). Furthermore, learning paradigms comprising repeated cortical stimulation applications resulting in cumulative effects could provide a medium- to long-term efficacy of the intervention.

\section{AUTHOR CONTRIBUTIONS}

$\mathrm{AC}$ and $\mathrm{AA}$ contributed to the conception of the work. AC was responsible for the literature search, selection of the articles, and manuscript drafting, the critical revision of the article was shared by both authors. AC and AA gave their final approval of the version to be published.
Barquero, L. A., Davis, N., and Cutting, L. E. (2014). Neuroimaging of reading intervention: a systematic review and activation likelihood estimate metaanalysis. PLoS ONE 9:e83668. doi: 10.1371/journal.pone.0083668

Belke, E. (2013). Long-lasting inhibitory semantic context effects on object naming are necessarily conceptually mediated: implications for models of lexicalsemantic encoding. J. Mem. Lang. 69, 228-256. doi: 10.1016/j.jml.2013.05.008

Bindman, L. J., Lippold, O. C. J., and Redfearn, J. W. T. (1964). The action of brief polarizing currents on the cerebral cortex of the rat (1) during current flow and (2) in the production of long-lasting after-effects. J. Physiol. (Lond). 172, 369-382. doi: 10.1113/jphysiol.1964.sp007425

Boehringer, A., Macher, K., Dukart, J., Villringer, A., and Pleger, B. (2013). Cerebellar transcranial direct current stimulation modulates verbal working memory. Brain Stimul. 6, 649-653. doi: 10.1016/j.brs.2012.10.001

Boggio, P. S., Castro, L. O., Savagim, E. A., Braite, R., Cruz, V. C., Rocha, R. R., et al. (2006). Enhancement of non-dominant hand motor function by anodal transcranial direct current stimulation. Neurosci. Lett. 404, 232-236. doi: 10.1016/j.neulet.2006.05.051 
Bonacina, S., Cancer, A., Lanzi, P. L., Lorusso, M. L., and Antonietti, A. (2015). Improving reading skills in students with dyslexia: the efficacy of a sublexical training with rhythmic background. Front. Psychol. 6:1510. doi: 10.3389/fpsyg.2015.01510

Brambati, S. M., Termine, C., Ruffino, M., Danna, M., Lanzi, G., Stella, G., et al. (2006). Neuropsychological deficits and neural dysfunction in familial dyslexia. Brain Res. 1113, 174-185. doi: 10.1016/j.brainres.2006.06.099

Cancer, A., and Antonietti, A. (2017). Remedial interventions for developmental dyslexia: how neuropsychological evidence can inspire and support a rehabilitation training. Neuropsychol. Trends 22, 73-95. doi: 10.7358/neur-2017-022-canc

Cancer, A., Bonacina, S., Lorusso, M. L., Lanzi, P. L., and Antonietti, A. (2016). "Rhythmic reading training (RRT)," in Pervasive Computing Paradigms for Mental Health, eds S. Serino, A. Matic, D. Giakoumis, G. Lopez, and P. Cipresso (Cham: Springer International Publishing), 249-258.

Costanzo, F., Menghini, D., Caltagirone, C., Oliveri, M., and Vicari, S. (2012). High frequency rTMS over the left parietal lobule increases non-word reading accuracy. Neuropsychologia 50, 2645-2651. doi: 10.1016/j.neuropsychologia.2012.07.017

Costanzo, F., Menghini, D., Caltagirone, C., Oliveri, M., and Vicari, S. (2013). How to improve reading skills in dyslexics: the effect of high frequency rTMS. Neuropsychologia 51, 2953-2959. doi: 10.1016/j.neuropsychologia.2013.10.018

Costanzo, F., Rossi, S., Varuzza, C., Varvara, P., Vicari, S., and Menghini, D. (2018). Long-lasting improvement following tDCS treatment combined with a training for reading in children and adolescents with dyslexia. Neuropsychologia doi: 10.1016/j.neuropsychologia.2018.03.016. [Epub ahead of print].

Costanzo, F., Varuzza, C., Rossi, S., Sdoia, S., Varvara, P., Oliveri, M., et al. (2016a). Evidence for reading improvement following tDCS treatment in children and adolescents with dyslexia. Restor. Neurol. Neurosci. 34, 215-226. doi: 10.3233/RNN-150561

Costanzo, F., Varuzza, C., Rossi, S., Sdoia, S., Varvara, P., Oliveri, M., et al. (2016b). Reading changes in children and adolescents with dyslexia after transcranial direct current stimulation. Neuroreport 27, 295-300. doi: 10.1097/WNR.0000000000000536

Creutzfeldt, O. D., Fromm, G. H., and Kapp, H. (1962). Influence of transcortical d-c currents on cortical neuronal activity. Exp. Neurol. 5, 436-452. doi: 10.1016/0014-4886(62)90056-0

Dehaene, S., and Cohen, L. (2011). The unique role of the visual word form area in reading. Trends Cogn. Sci. (Regul. Ed). 15, 254-262. doi: 10.1016/j.tics.2011.04.003

Dehaene, S., Cohen, L., Sigman, M., and Vinckier, F. (2005). The neural code for written words: a proposal. Trends Cogn. Sci. (Regul. Ed). 9, 335-341. doi: 10.1016/j.tics.2005.05.004

Demb, J. B., Boynton, G. M., and Heeger, D. J. (1998). Functional magnetic resonance imaging of early visual pathways in dyslexia. J. Neurosci. 18, 6939-6951. doi: 10.1523/JNEUROSCI.18-17-06939.1998

Eckert, M. (2004). Neuroanatomical markers for dyslexia: a review of dyslexia structural imaging studies. Neuroscientist 10, 362-371. doi: $10.1177 / 1073858404263596$

Eden, G. F., Jones, K. M., Cappell, K., Gareau, L., Wood, F. B., Zeffiro, T. A., et al. (2004). Neural changes following remediation in adult developmental dyslexia. Neuron 44, 411-422. doi: 10.1016/j.neuron.2004.10.019

Eden, G. F., and Zeffiro, T. A. (1998). Neural systems affected in developmental dyslexia revealed by functional neuroimaging. Neuron 21, 279-282. doi: 10.1016/S0896-6273(00)80537-1

Fertonani, A., Ferrari, C., and Miniussi, C. (2015). What do you feel if I apply transcranial electric stimulation? Safety, sensations and secondary induced effects. Clin. Neurophysiol. Off. J. Int. Fed. Clin. Neurophysiol. 126, 2181-2188. doi: 10.1016/j.clinph.2015.03.015

Fertonani, A., and Miniussi, C. (2017). Transcranial electrical stimulation: what we know and do not know about mechanisms. Neuroscientist 23, 109-123. doi: $10.1177 / 1073858416631966$

Franceschini, S., Gori, S., Ruffino, M., Pedrolli, K., and Facoetti, A. (2012). A causal link between visual spatial attention and reading acquisition. Curr. Biol. 22, 814-819. doi: 10.1016/j.cub.2012.03.013

Gaab, N., Gabrieli, J. D., Deutsch, G. K., Tallal, P., and Temple, E. (2007). Neural correlates of rapid auditory processing are disrupted in children with developmental dyslexia and ameliorated with training: an fMRI study. Restor. Neurol. Neurosci. 25, 295-310.

Heth, I., and Lavidor, M. (2015). Improved reading measures in adults with dyslexia following transcranial direct current stimulation treatment. Neuropsychologia 70, 107-113. doi: 10.1016/j.neuropsychologia.2015.02.022

Horvath, J. C., Forte, J. D., and Carter, O. (2015). Quantitative review finds no evidence of cognitive effects in healthy populations from single-session transcranial direct current stimulation (tDCS). Brain Stimulat. 8, 535-550. doi: 10.1016/j.brs.2015.01.400

Kadosh, R., Levy, N., O'Shea, J., Shea, N., and Savulescu, J. (2012). The neuroethics of non-invasive brain stimulation. Curr. Biol. 22, R108-R111. doi: 10.1016/j.cub.2012.01.013

Krause, B., and Cohen Kadosh, R. (2013). Can transcranial electrical stimulation improve learning difficulties in atypical brain development? a future possibility for cognitive training. Dev. Cogn. Neurosci. 6, 176-194. doi: 10.1016/j.dcn.2013.04.001

Levy, J., Pernet, C., Treserras, S., Boulanouar, K., Berry, I., Aubry, F., et al. (2008). Piecemeal recruitment of left-lateralized brain areas during reading: a spatio-functional account. Neuroimage 43, 581-591. doi: 10.1016/j.neuroimage.2008.08.008

Liberati, A., Altman, D. G., Tetzlaff, J., Mulrow, C., Gøtzsche, P. C., Ioannidis, J. P. A., et al. (2009). The PRISMA statement for reporting systematic reviews and meta-analyses of studies that evaluate health care interventions: explanation and elaboration. PLoS Med. 6:e1000100. doi: 10.1371/journal.pmed.1000100

Maisog, J. M., Einbinder, E. R., Flowers, D. L., Turkeltaub, P. E., and Eden, G. F. (2008). A meta-analysis of functional neuroimaging studies of dyslexia. Ann. N.Y. Acad. Sci. 1145, 237-259. doi: 10.1196/annals.1416.024

Mattai, A., Miller, R., Weisinger, B., Greenstein, D., Bakalar, J., Tossell, J., et al. (2011). Tolerability of transcranial direct current stimulation in childhoodonset schizophrenia. Brain Stimul. 4, 275-280. doi: 10.1016/j.brs.2011.01.001

McCrory, E. J., Mechelli, A., Frith, U., and Price, C. J. (2005). More than words: a common neural basis for reading and naming deficits in developmental dyslexia? Brain 128, 261-267. doi: 10.1093/brain/awh340

Nitsche, M. A., and Paulus, W. (2000). Excitability changes induced in the human motor cortex by weak transcranial direct current stimulation. J. Physiol. (Lond). 527, 633-639. doi: 10.1111/j.1469-7793.2000.t01-1-00633.x

Odegard, T. N., Ring, J., Smith, S., Biggan, J., and Black, J. (2008). Differentiating the neural response to intervention in children with developmental dyslexia. Ann. Dyslexia 58, 1-14. doi: 10.1007/s11881-008-0014-5

Olulade, O. A., Napoliello, E. M., and Eden, G. F. (2013). Abnormal visual motion processing is not a cause of dyslexia. Neuron 79, 180-190. doi: 10.1016/j.neuron.2013.05.002

Pisoni, A., Papagno, C., and Cattaneo, Z. (2012). Neural correlates of the semantic interference effect: new evidence from transcranial direct current stimulation. Neuroscience 223, 56-67. doi: 10.1016/j.neuroscience.2012.07.046

Price, C. J. (2012). A review and synthesis of the first 20years of PET and fMRI studies of heard speech, spoken language and reading. Neuroimage 62, 816-847. doi: 10.1016/j.neuroimage.2012.04.062

Priori, A., Berardelli, A., Rona, S., Accornero, N., and Manfredi, M. (1998). Polarization of the human motor cortex through the scalp. Neuroreport 9:2257 doi: 10.1097/00001756-199807130-00020

Pugh, K. R., Mencl, W. E., Jenner, A. R., Katz, L., Frost, S. J., Lee, J. R., et al. (2000). Functional neuroimaging studies of reading and reading disability(developmental dyslexia). Ment. Retard. Dev. Disabil. Res. Rev. 6, 207-213. doi: 10.1002/1098-2779(2000)6:3\%3C207::AID-MRDD8\%3E3.0. $\mathrm{CO} ; 2-\mathrm{P}$

Ramus, F., Rosen, S., Dakin, S. C., Day, B. L., Castellote, J. M., White, S., et al. (2003). Theories of developmental dyslexia: insights from a multiple case study of dyslexic adults. Brain 126, 841-865. doi: 10.1093/brain/awg076

Richlan, F. (2012). Developmental dyslexia: dysfunction of a left hemisphere reading network. Front. Hum. Neurosci. 6:120. doi: 10.3389/fnhum.2012.00120

Richlan, F. (2014). Functional neuroanatomy of developmental dyslexia: the role of orthographic depth. Front. Hum. Neurosci. 8:347. doi: 10.3389/fnhum.2014.00347

Richlan, F., Kronbichler, M., and Wimmer, H. (2011). Meta-analyzing brain dysfunctions in dyslexic children and adults. Neuroimage 56, 1735-1742. doi: 10.1016/j.neuroimage.2011.02.040 
Saine, N. L., Lerkkanen, M. K., Ahonen, T., Tolvanen, A., and Lyytinen, H. (2011). Computer-assisted remedial reading intervention for school beginners at risk for reading disability. Child Dev. 82, 1013-1028. doi: 10.1111/j.1467-8624.2011.01580.x

Schneider, H. D., and Hopp, J. P. (2011). The use of the bilingual aphasia test for assessment and transcranial direct current stimulation to modulate language acquisition in minimally verbal children with autism. Clin. Linguist. Phon. 25, 640-654. doi: 10.3109/02699206.2011.570852

Shaywitz, B. A., Shaywitz, S. E., Blachman, B. A., Pugh, K. R., Fulbright, R. K., Skudlarski, P., et al. (2004). Development of left occipitotemporal systems for skilled reading in children after a phonologically- based intervention. Biol. Psychiatry 55, 926-933. doi: 10.1016/j.biopsych.2003.12.019

Shaywitz, S. E., and Shaywitz, B. A. (2008). Paying attention to reading: the neurobiology of reading and dyslexia. Dev. Psychopathol. 20, 1329-1349. doi: 10.1017/S0954579408000631

Simos, P. G., Fletcher, J. M., Bergman, E., Breier, J. I., Foorman, B. R., Castillo, E. M., et al. (2002). Dyslexia-specific brain activation profile becomes normal following successful remedial training. Neurology 58, 1203-1213. doi: 10.1212/WNL.58.8.1203

Snowling, M. J., and Hulme, C. (2012). Annual research review: the nature and classification of reading disorders - a commentary on proposals for DSM-5. J. Child Psychol. Psychiatry 53, 593-607. doi: 10.1111/j.1469-7610.2011.02495.x

Stein, J. (2012). "The Neurobiological basis of dyslexia: the magnocellular theory," in The Handbook of the Neuropsychology of Language, ed M. Faust (Hoboken, NJ: Wiley-Blackwell), 938-962. doi: 10.1002/9781118432501.ch45

Thomson, J. M., Doruk, D., Mascio, B., Fregni, F., and Cerruti, C. (2015). Transcranial direct current stimulation modulates efficiency of reading processes. Front. Hum. Neurosci. 9:114. doi: 10.3389/fnhum.2015.00114

Thomson, J. M., Leong, V., and Goswami, U. (2013). Auditory processing interventions and developmental dyslexia: a comparison of phonemic and rhythmic approaches. Read. Writ. 26, 139-161. doi: $10.1007 /$ s11145-012-9359-6

Torgesen, J. K., Rashotte, C. A., and Wagner, R. K. (1999). TOWRE: Test of Word Reading Efficiency. Austin, TX: Pro-Ed.

Torgesen, J., Wagner, R., and Rashotte, C. (2011). Test of Word Reading Efficiency TOWRE-2, 2nd Edn. Austin, TX: Pro-Ed.
Tov-Li, A. (1999). Dyslexia and Disgraphia Diagnosis Battery. Kiriat Bialik: Ach Publishers.

Turkeltaub, P. E., Benson, J., Hamilton, R. H., Datta, A., Bikson, M., and Coslett, H. B. (2012). Left lateralizing transcranial direct current stimulation improves reading efficiency. Brain Stimulat. 5, 201-207. doi: 10.1016/j.brs.2011.04.002

Vicario, C. M., and Nitsche, M. A. (2013). Transcranial direct current stimulation: a remediation tool for the treatment of childhood congenital dyslexia? Front. Hum. Neurosci. 7:139. doi: 10.3389/fnhum.2013.00139

Vinckier, F., Dehaene, S., Jobert, A., Dubus, J. P., Sigman, M., and Cohen, L. (2007). Hierarchical coding of letter strings in the ventral stream: dissecting the inner organization of the visual word-form system. Neuron 55, 143-156. doi: 10.1016/j.neuron.2007.05.031

Westwood, S. J., Olson, A., Miall, R. C., Nappo, R., and Romani, C. (2017). Limits to tDCS effects in language: failures to modulate word production in healthy participants with frontal or temporal tDCS. Cortex J. Devoted Study Nerv. Syst. Behav. 86, 64-82. doi: 10.1016/j.cortex.2016.10.016

Younger, J. W., Randazzo Wagner, M., and Booth, J. R. (2016). Weighing the cost and benefit of transcranial direct current stimulation on different reading subskills. Front. Neurosci. 10:262. doi: 10.3389/fnins.2016.00262

Zaghi, S., Acar, M., Hultgren, B., Boggio, P. S., and Fregni, F. (2010). Noninvasive brain stimulation with low-intensity electrical currents: putative mechanisms of action for direct and alternating current stimulation. Neuroscientist 16 285-307. doi: 10.1177/1073858409336227

Conflict of Interest Statement: The authors declare that the research was conducted in the absence of any commercial or financial relationships that could be construed as a potential conflict of interest.

The reviewer IC and handling Editor declared their shared affiliation, at the time of the review.

Copyright (C) 2018 Cancer and Antonietti. This is an open-access article distributed under the terms of the Creative Commons Attribution License (CC BY). The use, distribution or reproduction in other forums is permitted, provided the original author(s) and the copyright owner(s) are credited and that the original publication in this journal is cited, in accordance with accepted academic practice. No use, distribution or reproduction is permitted which does not comply with these terms. 


\section{OPEN ACCESS}

Edited by:

Claudio Lucchiari, Università degli Studi di Milano, Italy

Reviewed by: Satoru Otani, Ryotokuji University, Japan Alice Cancer, Università Cattolica del Sacro Cuore,

*Correspondence: Jianbiao Li biaoj|@126.com

Received: 10 June 2017 Accepted: 19 October 2017 Published: 03 November 2017

Citation: Wang P, Wang G, Niu X, Shang $H$ and Li J (2017) Effect of Transcranial Direct Current Stimulation of the Medial Prefrontal Cortex on the Gratitude of Individuals with Heterogeneous Ability in an Experimental Labor Market. Front. Behav. Neurosci. 11:217. doi: 10.3389/fnbeh.2017.00217

\section{Effect of Transcranial Direct Current Stimulation of the Medial Prefrontal Cortex on the Gratitude of Individuals with Heterogeneous Ability in an Experimental Labor Market}

\author{
Pengcheng Wang ${ }^{1,2}$, Guangrong Wang ${ }^{2,3}$, Xiaofei $\mathrm{Niu}^{2,4}$, Huiliang Shang ${ }^{2}$ \\ and Jianbiao $L i^{2,4 *}$
}

\begin{abstract}
${ }^{1}$ Business School, Tianjin University of Finance and Economics, Tianjin, China, ${ }^{2}$ Reinhard Selten Laboratory, Nankai University, Tianjin, China, ${ }^{3}$ Neural Decision Science Laboratory, Weifang University, Weifang, China, ${ }^{4}$ China Academy of Corporate Governance, Business School, Nankai University, Tianjin, China
\end{abstract}

Gratitude is an important aspect of human sociality, which benefits mental health and interpersonal relationships. Thus, elucidating the neural mechanism of gratitude, which is only now beginning to be investigated, is important. To this end, this study specifies the medial prefrontal cortex (mPFC) involved in the gratitude of heterogeneous individuals using the transcranial direct current stimulation (tDCS) technique. Previous neural studies have shown the involvement of mPFC in social cognition and value evaluation, which are closely related to gratitude. However, the causal relationship between this neural area and gratitude has not been fully examined and the effect of individual social heterogeneity has been ignored. Meanwhile, behavioral economics studies have proposed that the abilities of employees in the labor market would affect their gratitude and emotional response. Thus, we designed an experiment based on gift exchange game to investigate the relationship between $\mathrm{mPFC}$ and gratitude of heterogeneous employees. Before the experiment, participants were asked to perform self-cognition of their abilities through an appropriately difficult task. We then used the effort of participants to imply their gratitude and analyzed the effort levels of employees with different abilities under anodal, cathodal, and sham stimulations. The results showed that employees under anodal stimulation were significantly likely to increase their effort than those under sham stimulation, and employees under cathodal stimulation ranked at the bottom of the list. Moreover, the effort levels of low-ability employees were obviously higher than those of high-ability employees. The cathodal stimulation of mPFC significantly reduced the effort levels of low-ability employees, whereas its anodal tDCS stimulation increased the effort levels of high-ability employees. These outcomes verify the relationship between mPFC and gratitude using tDCS and provided one of the first instances of neural evidence for the incentive mechanism design in the labor market to a certain extent.

Keywords: tDCS, mPFC, gratitude, social heterogeneity, gift exchange game 


\section{INTRODUCTION}

Gratitude, an important part of societal orientation, is a person's positive emotion when another person has intentionally given, or attempted to give, something of value (Bartlett and DeSteno, 2006). Cicero regarded gratitude as the parent of all other virtues (Cicero, 1851, p. 139), and Roman stoic Seneca conceived of gratitude as a fundamental motivational drive critical for building interpersonal relationships (McCullough et al., 2001; Fox et al., 2015). In line with such assertions of early writers, several theorists have believed that gratitude nurtured social relationships through its encouragement of reciprocal, prosocial behavior between a benefactor and a recipient (Emmons and McCullough, 2003).

Reciprocal behavior is one of the research focuses of experimental and behavioral economics, and behavioral economists usually use the gift exchange model to analyze this behavior (Fehr et al., 1993, 1998). Fehr et al. (1993) constructed a labor market environment in laboratory and found that worker effort increased with wage. They interpreted this as evidence of fairness or reciprocity effects, given that workers could (anonymously and with impunity) have simply selected the minimum effort level after accepting a wage offer, which was what conventional economic theory predicted that self-interested and effort averse workers would do.

From the perspective of gratitude, Baron (2013) investigated the gratitude-based employment system by drawing upon recent works on reciprocity and gift exchange. He proposed the notion of "empathy wages," in which the effect of the premium paid depended on the extent to which it elicited gratitude from recipients. He argued that prospects for eliciting gratitude were potentially greater in relative terms toward the bottom of the talent distribution, whereas creating equivalent feelings at the top was more difficult and costly. A field experiment among Canadian tree planters provided substantially informative data (Baron, 2013). Bellemare and Shearer (2009) showed that employees responded to gifts through discretionary effort, and this response appeared to have been markedly stronger among the least productive tree planters relative to other workers.

Psychological studies on gratitude have provided insights into its benefits. McCullough et al. (2001) proposed that gratitude was a moral affect with a moral motive function, which motivates a grateful person to behave prosocially toward a benefactor. Wood et al. (2008a) argued that gratitude was significantly related to the cognitive process of benefit appraisal. Despite recent findings on the effectiveness of gratitude intervention, the basic neural mechanisms involved in gratitude are relatively unknown (Kini et al., 2016). The investigation of the neural basis of gratitude would extend affective neuroscience beyond the study of basic emotions into complex social emotions essential for well-being.

The investigation of the experience and expression of gratitude is only the beginning at brain level (Fox et al., 2015). Zahn et al. (2014) determined that individual differences in gratitude tendencies correlated with gray matter volume in the right inferior temporal gyrus and posteromedial cortices.
Algoe and Way (2014) found the correlation between the genotype for oxytocin function and the behavioral expressions of gratitude. Kini et al. (2016) examined the neural bases of gratitude expression and how gratitude expression might lead to long-term effects on brain activity. Their cross-sectional study indicated that a simple gratitude letter writing intervention improved lasting neural sensitivity to gratitude. Gratitude letter writing participants exhibited a high degree of behavioral gratitude and a significantly high neural modulation of gratitude in the medial prefrontal cortex (mPFC) 3 months later. Fox et al. (2015) conducted an experiment and induced gratitude in participants who underwent functional magnetic resonance imaging (fMRI). They suggested that gratitude ratings would correlate with activities in brain regions associated with moral cognition (mPFC and anterior cingulate cortex), reward (vmPFC) and theory of mind (dorsal mPFC).

Gratitude is a social emotion that signals our recognition of the things others have done for us (Emmons and McNamara, 2006). This emotion correlates with brain activity in circuits associated with social cognitive processes, such as perspective taking and theory of mind (Fox et al., 2015). Ortony et al. (1988) suggested that emotion was the product of cognitive systems. Social neuroscience findings showed that activity in the mPFC was linked to social cognitive, reward (Amodio and Frith, 2006), decision-making and evaluation processes (Tabibnia and Lieberman, 2007; Weber and Huettel, 2008), as well as emotion (Damasio et al., 1996). Thus, we hypothesized that gratitude would relate to changes in activity in the mPFC.

However, most studies on the neural correlates of gratitude have typically categorized individuals into homogeneous categories and have ignored the role of individual social heterogeneity (such as the ability of employees in the labor market). Results of behavioral studies, such as that of Baron (2013), have indicated that relatively disadvantaged and/or low performing individuals do appear more grateful (or inclined to reciprocate gifts) than high performers. Wood et al. (2008b) reported that cognitive and assessment processes were crucial to enable an individual to experience gratitude, and an individual with high trait gratitude would feel more state gratitude. Moreover, Markus et al. (1985) suggested that high masculinity was associated with bias in information processing that emphasizes the masculine characteristics of others, even when their behavior was irrelevant to the issue of masculinity. Individual social heterogeneity (e.g., status, endowment, ability and masculinity) and reference point played pivotal roles in individual value judgments (Kahneman and Tversky, 1979). Among such individual social heterogeneities, Baron (2013) showed that employee ability was closely correlated with gratitude. In the labor market, ability determined the status of employees in the employment relationship and the reference point of their expected salaries to some extent. High offers would more likely exceed the expectations of low-ability individuals and consequently induce feelings of gratitude, that is, individual social heterogeneity, particularly employee ability, vitally influences their experience of gratitude.

In this study, we constructed a labor market context and used the transcranial direct current stimulation (tDCS) technique 
to elucidate the correlation between mPFC and gratitude specifically and to explore the effect of the heterogeneous ability of employees; this ability is proven to be closely related to the gratitude of employees (Baron, 2013). We hypothesized that modulating $\mathrm{mPFC}$ activity will change the experience and expression of gratitude and that individual social heterogeneity (e.g., heterogeneous ability of employees in the labor market) will affect the feeling of gratitude and the correlation between MPFC and gratitude.

Most studies on gratitude have used stories or vignette methods (Fox et al., 2015; Simão and Seibt, 2015), where participants were asked to place themselves in a specific context and imagine what they would feel. However, text-based approach might have a ceiling or floor effect problem while triggering emotional responses (Tsang, 2007). Thus, the accuracy of this approach is susceptible to social praise effects (Pedregon et al., 2012). Other scholars have utilized gratitude letter writing or keeping a gratitude diary to analyze gratitude (Algoe et al., 2010), which are difficult to quantify. Moreover, Algoe et al. (2008) examined the role of gratitude in actual ongoing relationships. In addition to the previously presented methods, several studies have induced gratitude toward a stranger through a laboratory experiment to obtain a high degree of experimental control (Leung, 2012). Behavioral game experiment places participants in a specific interactive environment with monetary incentive, wherein they behave according to their will. We believe that the gratitude of participants could be well investigated with a reasonable experimental design.

Thus, we designed a variant of gift exchange game, based on the studies of Fehr et al. (1993) and Baron (2013), to verify our hypotheses. Before the experiment, we divided participants into high- and low-ability groups using a task with certain difficulty. Participants were asked to join the experiment by playing the role of employee. Empirical literature on gratitude provided substantial findings on whether grateful individuals would repay a benefactor or a fortunate bystander (Bartlett and DeSteno, 2006). The link was relatively strong that repayment behavior was sometimes considered to imply feelings of gratitude (Algoe et al., 2008). Therefore, in our study, we used the efforts of participants to represent their levels of gratitude. Neuroimaging studies of Fox et al. (2015) and Kini et al. (2016) suggested that the $\mathrm{mPFC}$ is an important brain region for experiencing and expressing gratitude. Prior to the experiment, we used the tDCS technique to stimulate the $\mathrm{mPFC}$. Each participant randomly received one of anodal, cathodal, or sham stimulation. This approach allows us to measure the different effects of modulating the $\mathrm{mPFC}$ on low- and high-ability participants. We obtained two main results: First, the gratitude levels of low-ability employees were significantly higher than those of high-ability employees in the sham stimulation group. Second, compared with the sham stimulation, the anodal tDCS of the mPFC significantly increased the gratitude levels of high-ability employees, whereas the cathodal tDCS of the mPFC decreased the gratitude levels of low-ability employees. We investigated the relationship between $\mathrm{mPFC}$ and gratitude using the tDCS technique and provided neural evidence for the incentive mechanism design in the labor market.

\section{MATERIALS AND METHODS}

\section{Participants}

A total of 89 healthy young people (mean age of 20.3 years old, ranging from 17 to $24 ; 49$ females, 40 males) were recruited from the undergraduate and graduate student population of Nankai University. Participants were right-handed with normal or corrected normal vision. They have no previous knowledge of the tDCS technique or experience in a gift exchange game experiment. None of the participants reported a history of neurological or psychiatric problems. The experiment lasted approximately $60 \mathrm{~min}$. Each participant received a payment of approximately 65 Chinese Yuan (approximately 10 US dollars). Participants did not report any adverse side effects, such as scalp pain or headaches, after the experiment. All of them provided written informed consent, and the research was approved by the Ethics Committee of Nankai University. The experiment was performed in accordance with the Declaration of Helsinki and was approved by the Ethics Committee of Business School of Nankai university.

\section{tDCS}

In the tDCS technique, a direct current of low-level intensity (1-2 mA) is applied for a few minutes via electrodes placed on the subject's scalp. This current reaches the cortex and modulates the membrane polarity of neurons within a region of underlying neural tissue. tDCS-induced changes during stimulation are caused by changes in the permeability of the neural membrane, which is depolarized by anodal stimulation and hyperpolarized by cathodal stimulation (Nitsche and Paulus, 2000; Stagg and Nitsche, 2011). Therefore, tDCS can transiently influence behavior by altering neuronal activity, which may have facilitatory or inhibitory behavioral effects.

Transcranial direct current is generated by a batterydriven constant current stimulator (DC-Stimulator, NeuroConn, Germany), whereas anodal and cathodal stimulation electrodes are inserted into a $5 \mathrm{~cm}^{2} \times 7 \mathrm{~cm}^{2}$ physiological saline-soaked sponge. In our study, participants were blinded to the stimulation (single-blinded design) and randomly assigned to one of three groups, namely, mPFC anodal ( $n=29 ; 16$ females), mPFC cathodal ( $n=27 ; 15$ females), and sham ( $n=33 ; 18$ females). None of the participants reported any previous knowledge of the tDCS technique and any experience in stimulation. Moreover, none of the participants were aware of the type of stimulation they received, whereas the experimenter was fully informed. According to the International 10-20 EEG System, in the anodal stimulation group, the anode was placed at Fpz (Civai et al., 2015) and the cathode was placed at $\mathrm{Cz}$ (see Figure 1). In the cathodal stimulation group, the cathode was placed at Fpz and the anode at $\mathrm{Cz}$. The sham stimulation group was similar to the anodal stimulation group, except for the stimulation current that lasted only $30 \mathrm{~s}$. Participants may experience the initial micro itch, but differentiating sham stimuli from real stimuli is difficult (Gandiga et al., 2006). According to Civai et al. (2015), the two stimulus currents were fixed at $2 \mathrm{~mA}$ for $20 \mathrm{~min}$ with a $15 \mathrm{~s}$ rise and fall time. Previous studies have shown that the intensity of $0.057 \mathrm{~mA} / \mathrm{cm}^{2}$ and total charge of approximately $0.0063 \mathrm{C} / \mathrm{cm}^{2}$ 


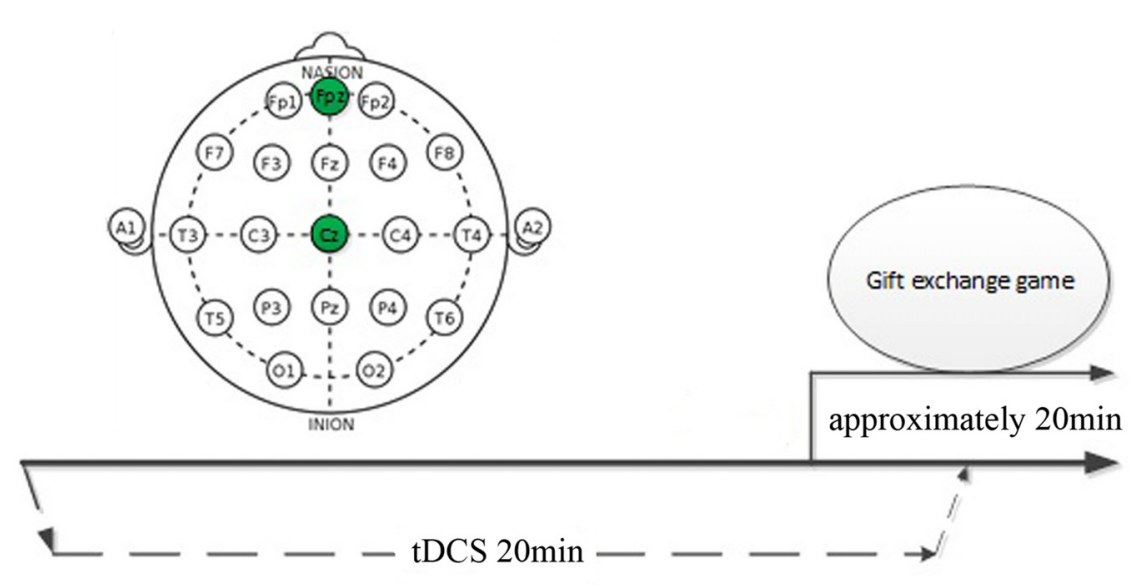

FIGURE 1 | Schematic representation of the experimental design.

were safe and well tolerated (Minhas et al., 2010; Borckardt et al., 2012). Moreover, specifying that the tDCS was not focal was important; thus, the simulation effects were more widespread and unclearly confined to the area identified by an imaging study; however, the area under the electrode could be assumed to be most affected by the stimulation (Civai et al., 2015).

\section{Task and Procedure}

The experiment is a revised gift exchange game. Classical gift exchange game has two roles, namely, employer and employee. One employer and one employee constitute a group and interact with each other through the entire experiment. First, the employer has a certain amount of initial endowment $g$ and decides to give a certain amount of wage $w$ to the employee. The employee then selects the degree of effort $e$, which generates a certain cost $c(e)$ to him/her. The employer obtains an income of $g-100 e$ from the employee's effort, whereas the employee earns $w-c(e)$.

In our experiment, all participants played the role of employees. Employers acted through computers, which was unknown to the participants. Baron (2013) suggested that the following factors would affect the gratitude of employees: (1) ability level: low-ability employees were tended to be grateful; (2) comparison between expected and real wage: unexpected wage could make employees more grateful; and (3) initial reference point: high wages would induce the gratitude of employees when their initial wages were low. Therefore, we classified participants into two types (i.e., low and high ability) by asking them to finish a task, i.e., answer nine questions (selected from the civil service exam test question bank, including three semantic, mathematical, and inference questions each) in $18 \mathrm{~min}$ before the experiment. Each question was worth 10 points. When the results were obtained, the participants were asked to answer two other questions, as follows: What do you think about your performance? How much would you like the employer to pay you? According to the results, employees whose scores were between 0 and 30 were classified as low ability, whereas employees with scores of 40 and 60 were categorized as high ability. No participants' scores were more than 70 . The expected wages of the two types of employees were significantly different.

In our experiment, employers (computers) received $100 \mathrm{G} \$$ at the start of each trial. Employees received no initial endowment. The test score of the employee was displayed on the screen. The employer selected wage $w$ (an integer from 0 to 100 , an arithmetic sequence with the interval of 10) after seeing the score of the employee. When the employee saw the wage given by the employer, he/she selected the degree of effort $e$ (a decimal number between 0 and 1), and the effort would generate a certain cost $c(e)$ to him/her (see Table 1). The employer could see the effort of the employee and obtain an income of $x e$, with $x$ determined by the score of the employee ( $[0,30], x=30$; [40, $60], x=60$; $[70,90], x=90)$. The final incomes of the employer and employee were $100-w+x e$ and $w-c(e)$, respectively. The wages given by the employers (computers) were gradually increased (see Table 2). The pretest showed that this setup could successfully induce the gratitude of participants and that repeated wages could verify the stability of the participants' behavior.

\section{RESULTS}

\section{Behavioral Data}

Behavioral data were statistically evaluated using the SPSS software (version 22; SPSS Inc., Chicago, IL, USA). The significance level was set at 0.05 for all analyses. We considered the effort levels of employees to represent degrees of gratitude. We analyzed the mean effort levels of participants with different abilities between three stimulation groups. A post hoc one-way analysis of variance (ANOVA) at stimulation type exhibited significant difference across any stimulation type $(F=3.45, p=0.036)$. In the case of significant

TABLE 1 C Cost of the effort of employees.
\begin{tabular}{lllllllllll}
\hline$E$ & 0.1 & 0.2 & 0.3 & 0.4 & 0.5 & 0.6 & 0.7 & 0.8 & 0.9 & 1 \\
c(e) & 0 & 1 & 2 & 4 & 6 & 8 & 11 & 14 & 17 & 20
\end{tabular}


TABLE 2 | Wages in 15 periods.

\begin{tabular}{|c|c|c|c|c|c|c|c|c|c|c|c|c|c|c|c|}
\hline Period & 1 & 2 & 3 & 4 & 5 & 6 & 7 & 8 & 9 & 10 & 11 & 12 & 13 & 14 & 15 \\
\hline W & 10 & 10 & 20 & 20 & 30 & 30 & 40 & 40 & 50 & 50 & 70 & 60 & 50 & 60 & 50 \\
\hline
\end{tabular}

Note: wages were exogenous and the same for all employees for comparison.

effects, post hoc Student's paired $t$ tests were conducted to examine whether an active intensity resulted in a significant difference relative to the sham stimulation in subsequent analyses.

\section{General tDCS Effect on the MPFC of Employees' Gratitude}

According to Baron (2013), employees would feel gratitude when the wage paid by employers exceeded their expectation. The current data showed that the mean expected wage of low-ability participants was $38.83(\mathrm{SD}=16.73, \max =70, \min =10)$, whereas that of high-ability participants was 53.89 ( $\mathrm{SD}=15.10$, $\max =80, \min =30$ ). Thus, we only analyzed scores greater than 30 (i.e., from periods 7-15) in the subsequent part. The mean effort levels of the anodal, sham, and cathodal stimulation groups were $0.581(\mathrm{SD}=0.25, \max =1, \min =0.2), 0.537$ $(\mathrm{SD}=0.24, \max =1, \min =0.1)$ and $0.478(\mathrm{SD}=0.27, \max =1$, min $=0.1$, respectively. The mean effort level of the anodal stimulation group was significantly higher than that of the sham ( $t=4.28, p=0.0013$, paired $t$ test $)$ and cathodal stimulation groups ( $t=8.2466, p=0.0000$, paired $t$ test). The mean effort level of the cathodal stimulation group was significantly lower than that of the sham stimulation group $(t=-3.164, p=0.0067$, paired $t$ test; see Figure 2).

\section{Effect of tDCS on the MPFC of Homogeneous Employees' Gratitude}

The study included 44 low- (15 anodal, 12 cathodal, and 17 sham) and 45 high-ability participants (14 anodal, 15 cathodal and 16 sham). We compared the mean effort levels of participants with different abilities. First, we analyzed the effect of mPFC stimulation on the gratitude of low-ability employees. Their

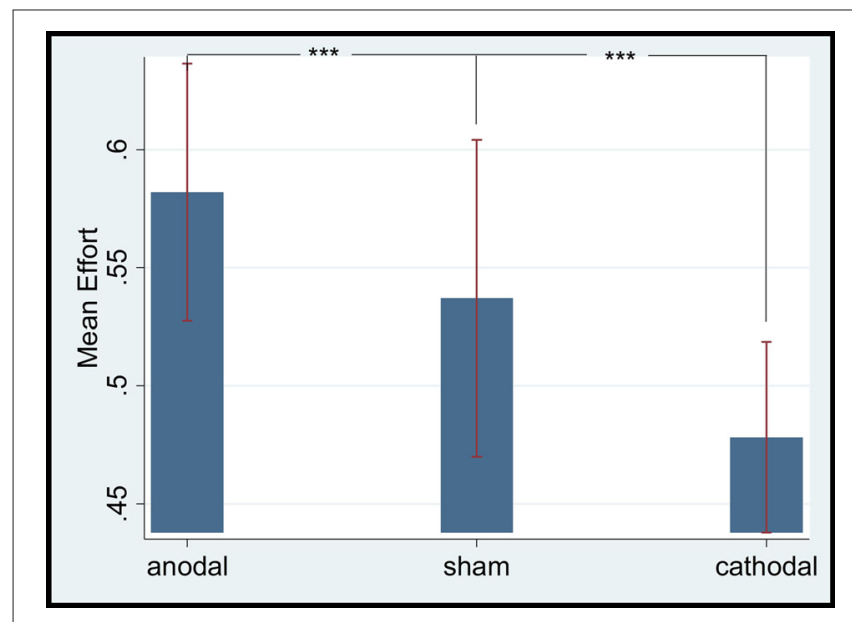

FIGURE 2 | Mean effort levels of different stimulation groups. ${ }^{* * *}$ Means significant difference at $1 \%$ level $(P<0.01)$. mean efforts in the anodal, sham, and cathodal groups were $0.574(\mathrm{SD}=0.28, \max =1, \min =0.1), 0.572(\mathrm{SD}=0.20$, $\max =1, \min =0.1)$ and $0.456(\mathrm{SD}=0.23, \max =0.8, \min =0.1)$, respectively. The mean effort level of the anodal stimulation group was higher than that of the cathodal stimulation group ( $t=5.5156, p=0.0003$, paired $t$ test), whereas the mean effort level of the cathodal stimulation group was significantly lower than that of the sham stimulation group $(t=6.2499, p=0.0001$, paired $t$ test). No significant difference in effort levels between anodal and sham stimulation groups was observed $(t=0.1105$, $p=0.4574$, paired $t$ test; see Figure 3).

Second, the effect of mPFC stimulation on the gratitude of high-ability employees was analyzed. Their mean efforts in the anodal, sham, and cathodal groups were $0.567(\mathrm{SD}=0.25$, $\max =1, \min =0.1), 0.47(\mathrm{SD}=0.26 \max =1 \mathrm{~min}=0.1)$, and 0.45 $(\mathrm{SD}=0.24, \max =1, \min =0.1)$, respectively. The mean effort level of the anodal stimulation group was significantly higher than that of the cathodal $(t=7.2737, p=0.0000$, paired $t$ test $)$ and sham stimulation groups $(t=4.3040, p=0.0013$, paired $t$ test $)$. No significant difference in effort levels between cathodal and sham stimulation groups was observed $(t=1.7264, p=0.0613$, paired $t$ test; see Figure 4).

\section{tDCS Effect on the MPFC of the Gratitude of Employees with Heterogeneous Ability}

We compared the effort levels between low- and high-ability employees under the same stimulation. No significant difference in the effort level between low-and high-ability employees was observed in the cathodal $(t=1.0934, p=0.1530$, paired $t$ test $)$ and anodal stimulation groups $(t=0.4456, p=0.3338$, paired $t$ test). In the sham stimulation group, the mean effort level of low-ability employees was significantly higher than that of

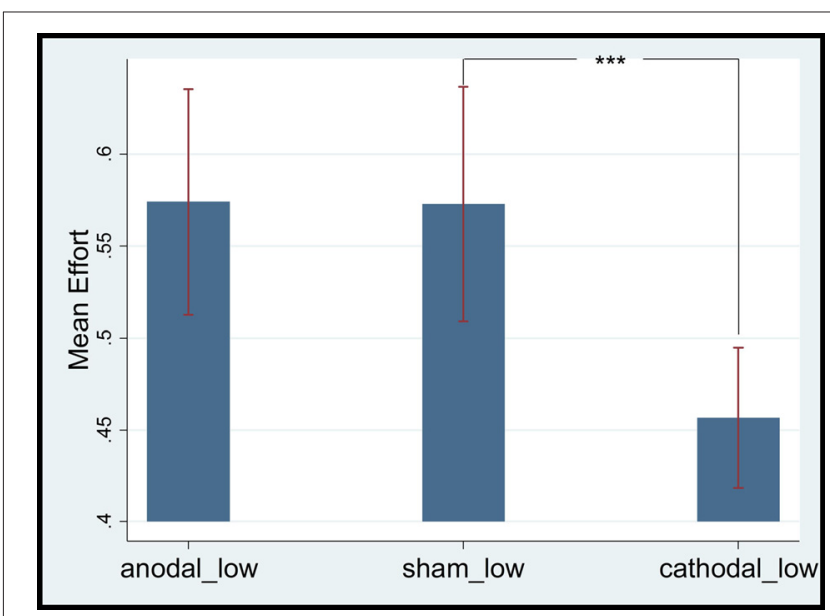

FIGURE 3 | Mean effort levels of low ability employees. *** Means significant difference at $1 \%$ level $(P<0.01)$. 


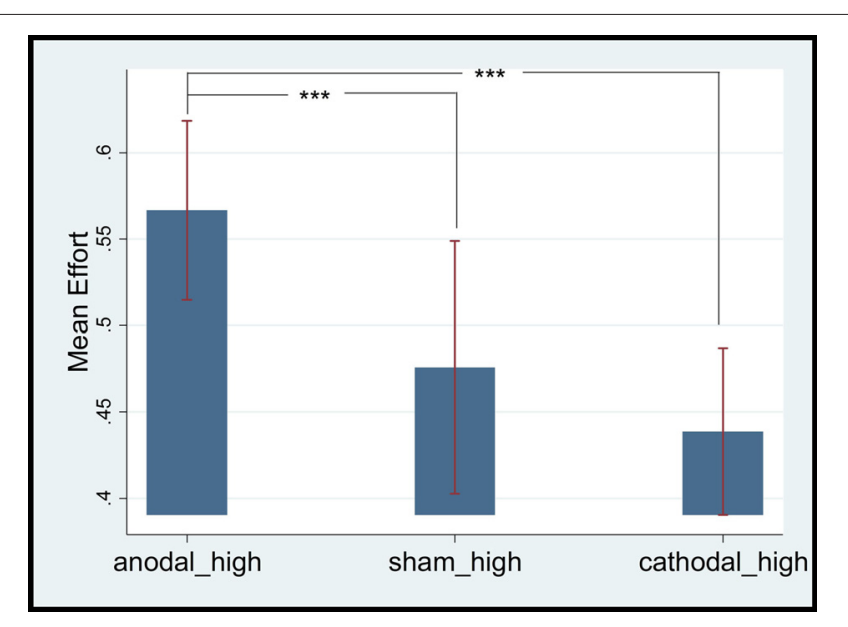

FIGURE 4 | Mean effort levels of high ability employees. *** Means significant difference at $1 \%$ level $(P<0.01)$.

high-ability employees ( $t=6.9353, p=0.0001$, paired $t$ test; see Figure 5).

Combing the results above, we suggested: (1) the gratitude degree of low-ability employees was significantly higher than that of high-ability employees; (2) the anodal stimulation of mPFC significantly improved the gratitude degree of high-ability employees, but indicated no significant effect on the gratitude of low-ability employees; and (3) the cathodal stimulation of $\mathrm{mPFC}$ decreased the gratitude degree of low-ability employees, but failed to significantly affect the gratitude of high-ability employees (see Table 3).

\section{DISCUSSION}

Previous fMRI studies (Fox et al., 2015; Kini et al., 2016) have shown that an increase in mPFC activation was specifically associated with the experience and expression of gratitude. In this work, we obtained converging evidence using a complementary

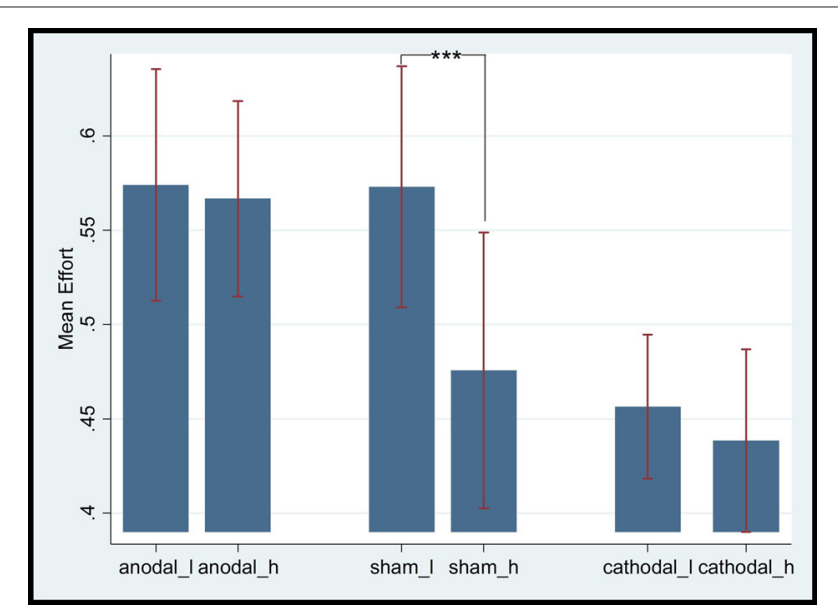

FIGURE 5 | Mean effort levels of two type employees in three simulation group. ${ }^{* * *}$ Means significant difference at $1 \%$ level $(P<0.01)$. technique (i.e., tDCS), in which we modulated the gratitude level of employees through a stimulus applied to the mPFC for $20 \mathrm{~min}$.

On the effects of tDCS, although the concept of tDCS changing performance seemed well established for tDCS in the motor system (Stagg and Nitsche, 2011), the same concept was not so directly applicable in the cognitive neuroscience field; furthermore, the relationship between type of stimulation and final behavior was often quite complex (e.g., Jacobson et al., 2012; Miniussi et al., 2013). The current data showed that anodal (cathodal) stimulation of the mPFC increased (decreased) the effort level of employees compared with sham stimulation, which might imply that anodal (cathodal) stimulation could facilitate (inhibit) the excitability of mPFC. In our study, the stimulation current was fixed at $2.0 \mathrm{~mA}$, based on the study of Civai et al. (2015). Batsikadze et al. (2013) proposed that enhanced tDCS current intensity did not necessarily increase the efficacy of cathodal stimulation, but might shift the direction of excitability alterations. However, Jamil et al. (2017) showed that the effect of $2.0 \mathrm{~mA}$ cathodal stimulation for 20 min differed with sham stimulation, although not significant and did not shift the excitability direction. They proposed that intensities of approximately $1.0 \mathrm{~mA}$ might be optimal in inducing the strongest inhibition of motor cortical excitability in healthy adults. In a study of adolescents, $10 \mathrm{~min}$ of $0.5 \mathrm{~mA}$ cathodal tDCS ( $35 \mathrm{~cm}^{2}$ electrodes) significantly decreased cortical excitability, but $1.0 \mathrm{~mA}$ cathodal tDCS increased cortical excitability (Moliadze et al., 2015). Civai et al. (2015) used $2.0 \mathrm{~mA}$ cathodal stimulation over mPFC and found that cathodal stimulation decreases the probability of rejecting unfair offers compared with the baseline. Shen et al. (2016) applied $2.0 \mathrm{~mA}$ cathodal stimulation to the left dlPFC using HD-tDCS and obtained a significant treatment effect. No consistent conclusion about the effect of tDCS intensity has yet been achieved. Thus, the only way to accurately determine what happens to $\mathrm{mPFC}$ functionality under a certain stimulation involves the collection of imaging data during, or soon after, stimulation. Further studies should combine these techniques to obtain detailed answers. The low spatial focality of tDCS due to heterogeneous tissue conductivities should also be considered (Nitsche and Paulus, 2011). We believe that the effects of the tDCS in this study should be interpreted in terms of effects on $\mathrm{mPFC}$, which was the area under the electrode.

Emotional cognitive theory accounts for the involvement of $\mathrm{mPFC}$ in gratitude, indicating emotion as the product of cognitive systems (Ortony et al., 1988). An increasing number of neuroimaging studies have proposed emotional evaluation as one of the important functions of mPFC (Knutson et al., 2001; McClure et al., 2004; Harris et al., 2007). Arnold (1968) argued that the nature of emotions was indirectly determined by stimulus scenarios and that subjective evaluation played a mediating role between stimuli and emotions. The basic process was as follows: stimulating scenarios $\rightarrow$ assessment $\rightarrow$ emotions. Gratitude is a social emotion that signals our recognition on what others have done for us (Emmons and McNamara, 2006). In the cognitive process of gratitude, recipients evaluate the value 
TABLE 3 | Summary of the results.

\begin{tabular}{|c|c|c|c|}
\hline Ability type & All & Low & High \\
\hline Gratitude level & Anodal $\succ$ sham $\succ$ cathodal & Anodal $\approx$ sham $\succ$ cathodal & Anodal $\succ$ sham $\approx$ cathodal \\
\hline Stimulation & Anodal & Sham & Cathodal \\
\hline Gratitude level & Low $\approx$ high & Low $\succ$ high & Low $\approx$ high \\
\hline
\end{tabular}

Note: this table summarizes the effects of mPFC stimulation on gratitude; " $\succ$ " represents "higher than," " $\approx$ " represents "no significant difference between".

of benevolence and the motivation of the benefactors (Algoe, 2012). Such evaluation is also affected by the characteristics of the beneficiaries and the relationship between benefactors and beneficiaries. Thus, different assessments of the same stimulus scenario would result in different emotional responses. Harris et al. (2007) suggests that affective evaluation may be a general function of mPFC. Using the tDCS technique to modulate mPFC activity would affect cognition and evaluation processes, ultimately influencing the feeling of gratitude, which could explain the conclusion of our study to a certain extent.

In this work, we verified the role of individual social heterogeneity (e.g., ability in the labor market) in the experience of gratitude and the correlation between $\mathrm{mPFC}$ and gratitude, which has been ignored by most neurological studies on emotion. The results of one-way ANOVA on behavioral data indicated no significant difference in the effort level between low- and high-ability employees. However, subsequent analysis showed that a significant difference in the effort level between low-ability and high-ability employees was observed in the sham stimulation group, which was consistent with the findings of Baron (2013). The cathodal stimulation of mPFC significantly reduced the gratitude level of low-ability employees, but has no significant effect on high-ability employees. By contrast, the anodal stimulation of the mPFC significantly increased the gratitude level of high-ability employees, but has no evident effect on low-ability employees. These findings indicated that the effect of modulating mPFC activity on gratitude might be different for heterogeneous individuals. Thus, different effects of tDCS on the $\mathrm{mPFC}$ of different employee types diminished the difference of gratitude between them.

Psychological research on counterfactual reasoning has accounted for the expected disproportionate response from less advantaged workers (Medvec et al., 1995; Medvec and Savitsky, 1997). Related literature has demonstrated how gratitude or satisfaction reflected not only one's absolute outcomes but also how those outcomes compared with what the person might have otherwise plausibly expected (Baron, 2013). In our experiment, participants were asked to recognize the classification and perform cognition about the relationship between ability and wage. We then let them write down their expected wages. Results showed that the mean expected wage of high-ability employees was significantly higher than that of low-ability employees (mean $H=53.89$, mean $L=38.83, t=4.20, p=0.000$, independent sample $t$ test). Differences in expected wage would affect feeling of gratitude. Findings indicated that low-ability employees would be more sensitive to feeling gratitude. Tesser et al. (1968) found that the higher the assessment of the cost of assistance and the value of favor, which were determined by the endowment of beneficiaries, the higher the gratitude degree that the employees felt. Baron (2013) suggested that ability would influence the gratitude of employees and affect their behavior in turn. Results showed that unexpected wages would make employees more grateful and motivate them to exert stronger efforts, whereas high wages could not induce the gratitude of high-ability employees successfully and the effect of incentive was obscure. Our behavioral results confirmed the findings of Baron (2013). The findings on the stimulation effects on employees with heterogeneous ability provided neural evidence for the incentive mechanism design in the labor market.

We obtained interesting and unexpected findings. No difference in effort levels of low-ability participants between anodal and sham stimulations and of high-ability participants between cathodal and sham stimulation were observed. Jacobson et al. (2012) assumed that the direct current might have different effects depending on the neuronal state and initial activation level in the stimulated regions. This finding may be attributed to the activation level in the mPFC of low-ability (high-ability) participants which might be sufficiently high (low) when facing the stimulus scenarios in our experiment. Stimulation effects might be affected by the ceiling or floor effect. However, exploring more detailed answers by combining fMRI and tDCS techniques in further studies is necessary.

Our study included several limitations. Given the use of a multistage repeated experimental framework, reputation and signal transmission might have affected the behavior of participants. de Quervain et al. (2004) argued that gratitude expression could be used to convey reciprocal promises to prevent social punishment as a free rider and to signal that they were fair partners to others (Sigmund, 2007). The significant treatment effects of stimulation under the same experimental design would keep the results robust. Further studies should consider the potential effect of these factors. Meanwhile, exploring the neural mechanisms of emotion- and strategydriven behaviors would be interesting.

\section{CONCLUSION}

In summary, our findings provided important information about the effect of tDCS on healthy participants, particularly with respect to the gratitude degrees of individuals with different abilities. Activating mPFC by tDCS could affect the gratitude degree in a gift exchange game. The gratitude degree of participants under anodal stimulation was higher than that under cathodal stimulation. Moreover, anodal stimulation could increase the gratitude degree of high-ability participants, whereas cathodal stimulation could decrease the gratitude degree of low-ability participants. 


\section{AUTHOR CONTRIBUTIONS}

PW, JL, GW and XN designed experiment; PW, JL and XN performed experiment; PW and HS analyzed data and wrote the manuscript.

\section{REFERENCES}

Algoe, S. B. (2012). Find, remind, and bind: the functions of gratitude in everyday relationships. Soc. Personal. Psychol. Compass 6, 455-469. doi: 10.1111/j.17519004.2012.00439.x

Algoe, S. B., Gable, S. L., and Maisel, N. C. (2010). It's the little things: everyday gratitude as a booster shot for romantic relationships. Pers. Relatsh. 17, 217-233. doi: 10.1111/j.1475-6811.2010.01273.x

Algoe, S. B., Haidt, J., and Gable, S. L. (2008). Beyond reciprocity: gratitude and relationships in everyday life. Emotion 8, 425-429. doi: 10.1037/1528-3542. 8.3.425

Algoe, S. B., and Way, B. (2014). Evidence for a role of the oxytocin system, indexed by genetic variation in $\mathrm{cd} 38$, in the social bonding effects of expressed gratitude. Soc. Cogn. Affect. Neurosci. 9, 1855-1861. doi: 10.1093/scan/nst182

Amodio, D. M., and Frith, C. D. (2006). Meeting of minds: the medial frontal cortex and social cognition. Nat. Rev. Neurosci. 7, 268-277. doi: 10.1038/nrn 1884

Arnold, M. B. (1968). The Nature of Emotion. Baltimore: Penguin.

Baron, J. N. (2013). Empathy wages? gratitude and gift exchange in employment relationships. Res. Org. Behav. 33, 113-134. doi: 10.1016/j.riob.2013. 10.006

Bartlett, M. Y., and DeSteno, D. (2006). Gratitude and prosocial behavior: helping when it costs you. Psychol. Sci. 17, 319-325. doi: 10.1111/j.1467-9280.2006. 01705.x

Batsikadze, G., Moliadze, V., Paulus, W., Kuo, M., and Nitsche, M. A. (2013). Partially non-linear stimulation intensity-dependent effects of direct current stimulation on motor cortex excitability in humans. J. Physiol. 591, 1987-2000. doi: 10.1113/jphysiol.2012.249730

Bellemare, C., and Shearer, B. (2009). Gift giving and worker productivity: evidence from a firm-level experiment. Games Econ. Behav. 67, 233-244. doi: 10.1016/j.geb.2008.12.001

Borckardt, J. J., Bikson, M., Frohman, H., Reeves, S. T., Datta, A., Bansal, V., et al. (2012). A pilot study of the tolerability and effects of high-definition transcranial direct current stimulation (hd-tdcs) on pain perception. J. Pain 13, 112-120. doi: 10.1016/j.jpain.2011.07.001

Cicero, M. T. (1851). The orations of Marcus Tullius Cicero, Vol. III (C.D. Younge, Trans.). London: George Bell and Sons.

Civai, C., Miniussi, C., and Rumiati, R. I. (2015). Medial prefrontal cortex reacts to unfairness if this damages the self: a tdcs study. Soc. Cogn. Affect. Neurosci. 10, 1054-1060. doi: 10.1093/scan/nsu154

Damasio, A. R., Damasio, H., and Christen, Y. (1996). Neurobiology of DecisionMaking. Berlin Heidelberg: Springer.

de Quervain, D. J. F., Fischbacher, U., Treyer, V., Schellhammer, M., Schnyder, U., Buck, A., et al. (2004). The neural basis of altruistic punishment. Science 305, 1254-1258. doi: 10.1126/science.1100735

Emmons, R. A., and McCullough, M. E. (2003). Counting blessings versus burdens: an experimental investigation of gratitude and subjective well-being in daily life. J. Pers. Soc. Psychol. 84, 377-389. doi: 10.1037//0022-3514. 84.2.377

Emmons, R. A., and McNamara, P. (2006). "Sacred emotions and affective neuroscience: gratitude, costly signaling, and the brain," in Where God and Science Meet: How Brain and Evolutionary Studies Alter Our Understanding of Religion, (Vol. 1) ed. P. McNamara (Westport, CT: Praeger Publishers), $11-31$.

Fehr, E., Kirchsteiger, G., and Riedl, A. (1993). Does fairness prevent market clearing? An experimental investigation. Q. J. Econ. 108, 437-459. doi: $10.2307 / 2118338$

Fehr, E., Kirchsteiger, G., and Riedl, A. (1998). Gift exchange and reciprocity in competitive experimental markets. Eur. Econ. Rev. 42, 1-34. doi: 10.1016/s0014-2921(96)00051-7

\section{ACKNOWLEDGMENTS}

This work was supported by the National Natural Science Fund of China (Grant Nos. 71602138, 71673152, 71372094 and 71533002).

Fox, G. R., Kaplan, J., Damasio, H., and Damasio, A. (2015). Neural correlates of gratitude. Front. Psychol. 6:1491. doi: 10.3389/fpsyg.2015.01491

Gandiga, P. C., Hummel, F. C., and Cohen, L. G. (2006). Transcranial dc stimulation (tdcs): a tool for double-blind sham-controlled clinical studies in brain stimulation. Clin. Neurophysiol. 117, 845-850. doi: 10.1016/j.clinph.2005. 12.003

Harris, L. T., McClure, S. M., van den Bos, W., Cohen, J. D., and Fiske, S. T. (2007). Regions of the mpfc differentially tuned to social and nonsocial affective evaluation. Cogn. Affect. Behav. Neurosci. 7, 309-316. doi: 10.3758/cabn.7. 4.309

Jacobson, L., Koslowsky, M., and Lavidor, M. (2012). Tdcs polarity effects in motor and cognitive domains: a meta-analytical review. Exp. Brain Res. 216, 1-10. doi: 10.1007/s00221-011-2891-9

Jamil, A., Batsikadze, G., Kuo, H., Labruna, L., Hasan, A., Paulus, W., et al. (2017). Systematic evaluation of the impact of stimulation intensity on neuroplastic after-effects induced by transcranial direct current stimulation. J. Physiol. 595, 1273-1288. doi: 10.1113/JP272738

Kahneman, D., and Tversky, A. (1979). Prospect theory: an analysis of decision under risk. Econometrica 47, 263-291. doi: 10.2307/1914185

Kini, P., Wong, J., McInnis, S., Gabana, N., and Brown, J. W. (2016). The effects of gratitude expression on neural activity. Neuroimage 128, 1-10. doi: 10.1016/j. neuroimage.2015.12.040

Knutson, B., Adams, C. M., Fong, G. W., and Hommer, D. (2001). Anticipation of increasing monetary reward selectively recruits nucleus accumbens. J. Neurosci. 21:RC159.

Leung, H. (2012). An Endogenous-Induction of Gratitude and its Effect on Cooperation in a Public Good Game. Chinese University of Hong Kong.

Markus, H., Smith, J., and Moreland, R. L. (1985). Role of the self-concept in the perception of others. J. Pers. Soc. Psychol. 49, 1494-1512. doi: 10.1037//00223514.49.6.1494

McClure, S. M., Laibson, D. I., Loewenstein, G., and Cohen, J. D. (2004). Separate neural systems value immediate and delayed monetary rewards. Science 306, 503-507. doi: 10.1126/science.1100907

McCullough, M. E., Kilpatrick, S. D., Emmons, R. A., and Larson, D. B. (2001). Is gratitude a moral affect? Psychol. Bull. 127, 249-266. doi: 10.1037/0033-2909. 127.2.249

Medvec, V. H., Madey, S. F., and Gilovich, T. (1995). When less is more: counterfactual thinking and satisfaction among olympic medalists. J. Pers. Soc. Psychol. 69, 603-610. doi: 10.1037//0022-3514.69.4.603

Medvec, V. H., and Savitsky, K. (1997). When doing better means feeling worse: the effects of categorical cutoff points on counterfactual thinking and satisfaction. J. Pers. Soc. Psychol. 72, 1284-1296. doi: 10.1037//0022-3514.72.6. 1284

Miniussi, C., Harris, J. A., and Ruzzoli, M. (2013). Modelling non-invasive brain stimulation in cognitive neuroscience. Neurosci. Biobehav. Rev. 37, 1702-1712. doi: 10.1016/j.neubiorev.2013.06.014

Minhas, P., Bansal, V., Patel, J., Ho, J. S., Diaz, J., Datta, A., et al. (2010). Electrodes for high-definition transcutaneous de stimulation for applications in drug delivery and electrotherapy, including tdcs. J. Neurosci. Methods 190, 188-197. doi: 10.1016/j.jneumeth.2010.05.007

Moliadze, V., Schmanke, T., Andreas, S., Lyzhko, E., Freitag, C. M., and Siniatchkin, M. (2015). Stimulation intensities of transcranial direct current stimulation have to be adjusted in children and adolescents. Clin. Neurophysiol. 126, 1392-1399. doi: 10.1016/j.clinph.2014.10.142

Nitsche, M. A., and Paulus, W. (2000). Excitability changes induced in the human motor cortex by weak transcranial direct current stimulation. J. Physiol. 527, 633-639. doi: 10.1111/j.1469-7793.2000.t01-1-00633.x

Nitsche, M. A., and Paulus, W. (2011). Transcranial direct current stimulation. Restor. Neurol. Neurosci. 29, 463-492. doi: 10.3233/RNN-2011 $-0618$ 
Ortony, A., Clore, G. L., and Collins, A. (1988). Cognitive structure of emotions. Contemp. Soc. 18, 2147-2153.

Pedregon, C. A., Farley, R. L., Davis, A., Wood, J. M., and Clark, R. D. (2012). Social desirability, personality questionnaires and the "better than average" effect. Pers. Individ. Dif. 52, 213-217. doi: 10.1016/j.paid.2011. 10.022

Shen, B., Yin, Y., Wang, J., Zhou, X., McClure, S. M., and Li, L. (2016). Highdefinition tdcs alters impulsivity in a baseline-dependent manner. Neuroimage 143, 343-352. doi: 10.1016/j.neuroimage.2016.09.006

Sigmund, K. (2007). Punish or perish? Retaliation and collaboration among humans. Trends Ecol. Evol. 22, 593-600. doi: 10.1016/j.tree.2007 .06 .012

Simão, C., and Seibt, B. (2015). Friendly touch increases gratitude by inducing communal feelings. Front. Psychol. 6:815. doi: 10.3389/fpsyg.2015. 00815

Stagg, C. J., and Nitsche, M. A. (2011). Physiological basis of transcranial direct current stimulation. Neuroscientist 17, 37-53. doi: 10.1177/1073858410386614

Tabibnia, G., and Lieberman, M. D. (2007). Fairness and cooperation are rewarding: evidence from social cognitive neuroscience. Ann. N Y Acad. Sci. 1118, 90-101. doi: 10.1196/annals.1412.001

Tesser, A., Gatewood, R., and Driver, M. (1968). Some determinants of gratitude. J. Pers. Soc. Psychol. 9, 233-236. doi: 10.1037/h0025905

Tsang, J. A. (2007). Gratitude for small and large favors: a behavioral test. J. Posit. Psychol. 2, 157-167. doi: 10.1080/17439760701229019
Weber, B. J., and Huettel, S. A. (2008). Neural substrates of probabilistic and intertemporal decision-making. Brain Res. 1234, 104-115. doi: 10.1016/j. brainres.2008.07.105

Wood, A. M., Joseph, S., and Maltby, J. (2008a). Gratitude uniquely predicts satisfaction with life: incremental validity above the domains and facets of the five factor model. Pers. Individ. Dif. 45, 49-54. doi: 10.1016/j.paid.2008. 02.019

Wood, A. M., Maltby, J., Stewart, N., Linley, P. A., and Joseph, S. (2008b). A social-cognitive model of trait and state levels of gratitude. Emotion 8, 281-290. doi: 10.1037/1528-3542.8.2.281

Zahn, R., Garrido, G., Moll, J., and Grafman, J. (2014). Individual differences in posterior cortical volume correlate with proneness to pride and gratitude. Soc. Cogn. Affect. Neurosci. 9, 1676-1683. doi: 10.1093/scan/nst158

Conflict of Interest Statement: The authors declare that the research was conducted in the absence of any commercial or financial relationships that could be construed as a potential conflict of interest.

Copyright (c) 2017 Wang, Wang, Niu, Shang and Li. This is an open-access article distributed under the terms of the Creative Commons Attribution License (CC BY). The use, distribution or reproduction in other forums is permitted, provided the original author(s) or licensor are credited and that the original publication in this journal is cited, in accordance with accepted academic practice. No use, distribution or reproduction is permitted which does not comply with these terms. 


\title{
Promoting Creativity Through Transcranial Direct Current Stimulation (tDCS). A Critical Review
}

\author{
Claudio Lucchiari*, Paola Maria Sala and Maria Elide Vanutelli \\ Department of Philosophy, Università degli Studi di Milano, Milan, Italy
}

Creativity, meant as the ability to produce novel, original and suitable ideas, has received increased attention by research in the last years, especially from neuroaesthetics and social neuroscience. Besides the research conducted on the neural correlates of such capacities, previous work tried to answer the question of whether it is possible to enhance creativity through cognitive and neural stimulation. In particular, transcranial direct current stimulation (tDCS) has been applied to increase neuronal excitability in those areas related to creativity. However, being a complex construct that applies to a huge variety of situations, available results are often confusing and inconsistent. Thus, in the present critical review, after selecting original research articles investigating creativity with tDCS, results will be reviewed and framed according to the different effects of tDCS and its underlying mechanisms, which can be defined as follows: the promotion of self-focused attention; the disruption of inhibiting mechanisms; the enhancement of creative thinking; the promotion of artistic enactment. Finally, a theoretical perspective,

OPEN ACCESS

Edited by:

Andrea Antal,

University Medical Center

Goettingen, Germany

Reviewed by:

Leila Chaieb,

Universität Bonn, Germany Barbara Colombo,

Champlain College, United States

*Correspondence:

Claudio Lucchiari claudio.Iucchiari@unimi.it

Received: 01 March 2018 Accepted: 16 July 2018 Published: 02 August 2018

Citation:

Lucchiari C, Sala PM and Vanutelli ME (2018) Promoting Creativity Through Transcranial Direct Current Stimulation (tDCS). A Critical

Review.

Front. Behav. Neurosci. 12:167. doi: 10.3389/fnbeh.2018.00167 the creative on/off model, will be provided to integrate the reported evidence with respect to both anatomical and functional issues and propose a cognitive explanation of the emergence of creative thinking.

Keywords: creativity, divergent thinking, convergent thinking, brain stimulation, tDCS, DMN

\section{CREATIVITY: A THEORETICAL FRAMEWORK}

Creativity has been defined by some scholars as humankind's ultimate resource (Toynbee, 1964). It is traditionally considered a mere outcome of an individual's genius, a gift, and a personality trait. Consequently, the interpretation that emerged in relation to this topic tended to consider creativity as something given without a reason, even genetically predetermined, and thus far from objective and systematic comprehension (Batey and Furnham, 2006). However, such ideas deal only with the first of the four points that Rhodes (1987) suggested for a complete framing of creativity. According to his proposal, it is possible to conceptualize creativity on the importance given to: (a) the person who creates; (b) the cognitive processes involved in the creation of ideas; (c) the environment in which creativity occurs; and (d) the outcome of the creative activity. Besides the specific focus on these different contributions to the phenomenon, it is also important to consider the multifaceted expressions of creativity, from artistic enactment, to scientific progress, and to problem-solving. Therefore, although there is a great interest dedicated to this issue, it is still difficult to define and study creativity in a comprehensive way. The most accepted definition that can be easily applied to all contexts proposes a double requirement for creativity: originality and usefulness (Barron, 1955). Moreover, different perspectives proposed creativity 
as being the result of a complex interaction between people and environment (Csikszentmihalyi, 1988; Mumford and Gustafson, 1988), revealing the importance of cognitive, social and cultural factors in creative studies (Amabile, 1983, 1996).

In the present article, we meant to emphasize the dynamic nature of creativity and the possibility of enhancing it. Starting by the Guilford (1957) model, which collocates the creative thinking within the interaction of specific cognitive processes (see Figure 1), we may describe the cognitive system as a substantially stable arrangement of components involved in many purposed tasks, which may be disrupted by a perturbation that produces a destabilization of the whole system, resulting in creative functioning.

For example, a problem that cannot be solved using the already available cognitive algorithms may produce an attention shift toward inner processes, the search for alternative solutions, and the subsequent activation of new neurocognitive paths. The same effect may be observed in artistic creativity. In this case, it is not important to reach a definitive solution, but there is anyhow an implicit need to express an indistinct thought in a synthetic form. In fact, we propose the presence of a specific need-forenactment, which aims at associating a widespread cognitive network with a motor program that can provide a behavioral output.

The aim of the present study is to review the most eminent work on the effects of transcranial direct current stimulation (tDCS) on creativity. Here, the neurocognitive underpinnings and empirical research are considered with special interest, since we aimed at demonstrating that it is possible to target this complexity by using tDCS. This noninvasive brain stimulation technique can modulate cortical excitability by influencing neuronal membrane potential within

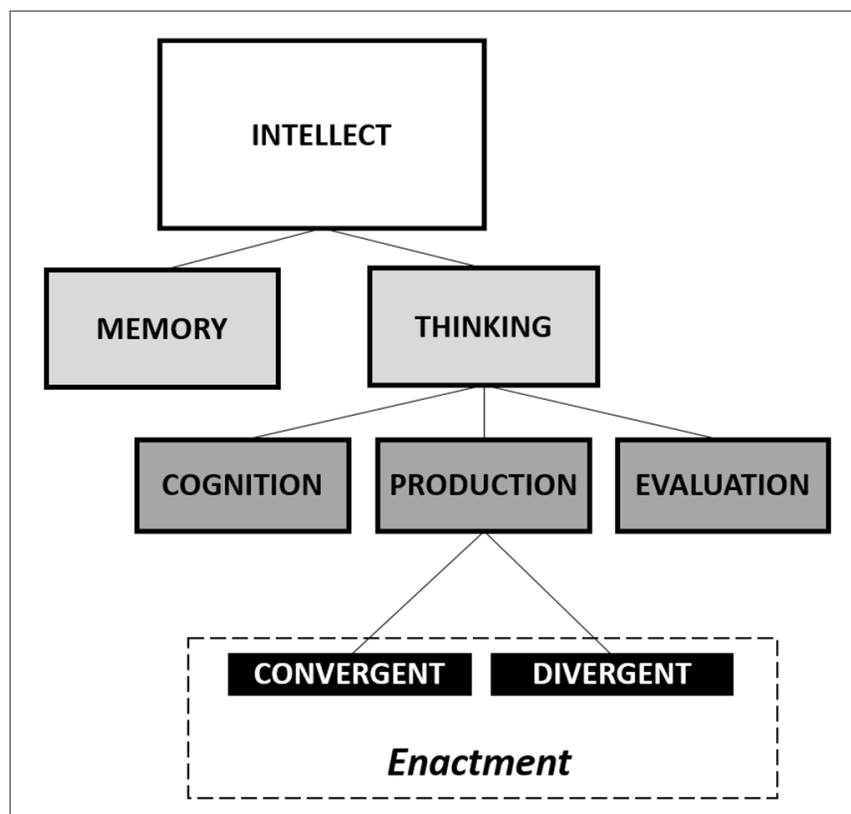

FIGURE 1 | Guilford's diagram of the intellectual factors. Modified from Guilford (1957). different neural networks. Anodal stimulation can increase excitability and result in a depolarization, while cathodal stimulation decreases it and leads to a hyperpolarization of the resting membrane potential (Sehm et al., 2012). Interestingly, previous research highlighted that anodal tDCS is associated with locally reduced GABA neurotransmitter (Stagg et al., 2009; Kim et al., 2014), while cathodal stimulation causes reduced glutamatergic neuronal activity (Stagg et al., 2009). In this view, brain modulation may be extremely useful both in understanding the functional neurophysiology of creativity and in developing tailored programs aimed to elicit a targeted creative experience. Since creativity cannot be captured by a single concept, being multifaceted and complex in nature, it could be considered as a meta-construct made by an aggregate of processes. Thus, we need a process-driven approach (Dietrich, 2004) to test if and how we can enhance it by tDCS. More specifically, we argue that brain modulation may be effective in: (1) promoting self-focused attention; (2) decreasing the effect of perceptual filters and inhibiting mechanisms; (3) enhancing creative thinking by facilitating the emergence of imagination and analogical mechanisms; and (4) favoring the way toward the enactment. Following this schema, we have grouped the available work and framed it accordingly.

\section{METHOD}

This critical review is composed of two main sections: the first one describes evidence related to each of the key processes implied in creativity studies by describing the main published articles in the area. In the second part, we will provide a general model that integrates the evidence discussed thus proposing an explanation of the effect of tDCS on creativity. Searches were conducted through PubMed, SCOPUS, PsycINFO and Google Scholar. The following keywords or combinations were used: neuromodulation, neurostimulation, tDCS, creativity, divergent thinking and artistic expression. We considered articles without any time limits, excluding previous reviews. Articles in languages other than English, letters and editorials were excluded. We considered as eligible for inclusion all original research articles reporting data about the use of tDCS to study any creativity-related cognitive process. Both studies on healthy and pathological subjects were considered. The first search identified 48 articles. An initial review of the abstracts of these articles by authors identified 22 articles that were potentially relevant to the current review. The abstracts of these articles were then evaluated by authors, resulting in 14 articles being identified as eligible for inclusion, with an additional four articles identified during manuscript preparation, for a total of 18 articles. This review provides a synthesis of the findings from previous key reviews and empirical studies identified in the literature search (see Table 1).

\section{NEUROMODULATION TO PROMOTE SELF-FOCUSED ATTENTION}

In this paragraph, we focus on the ability of subjects to detach attention from external stimuli. Indeed, creative thinking often 


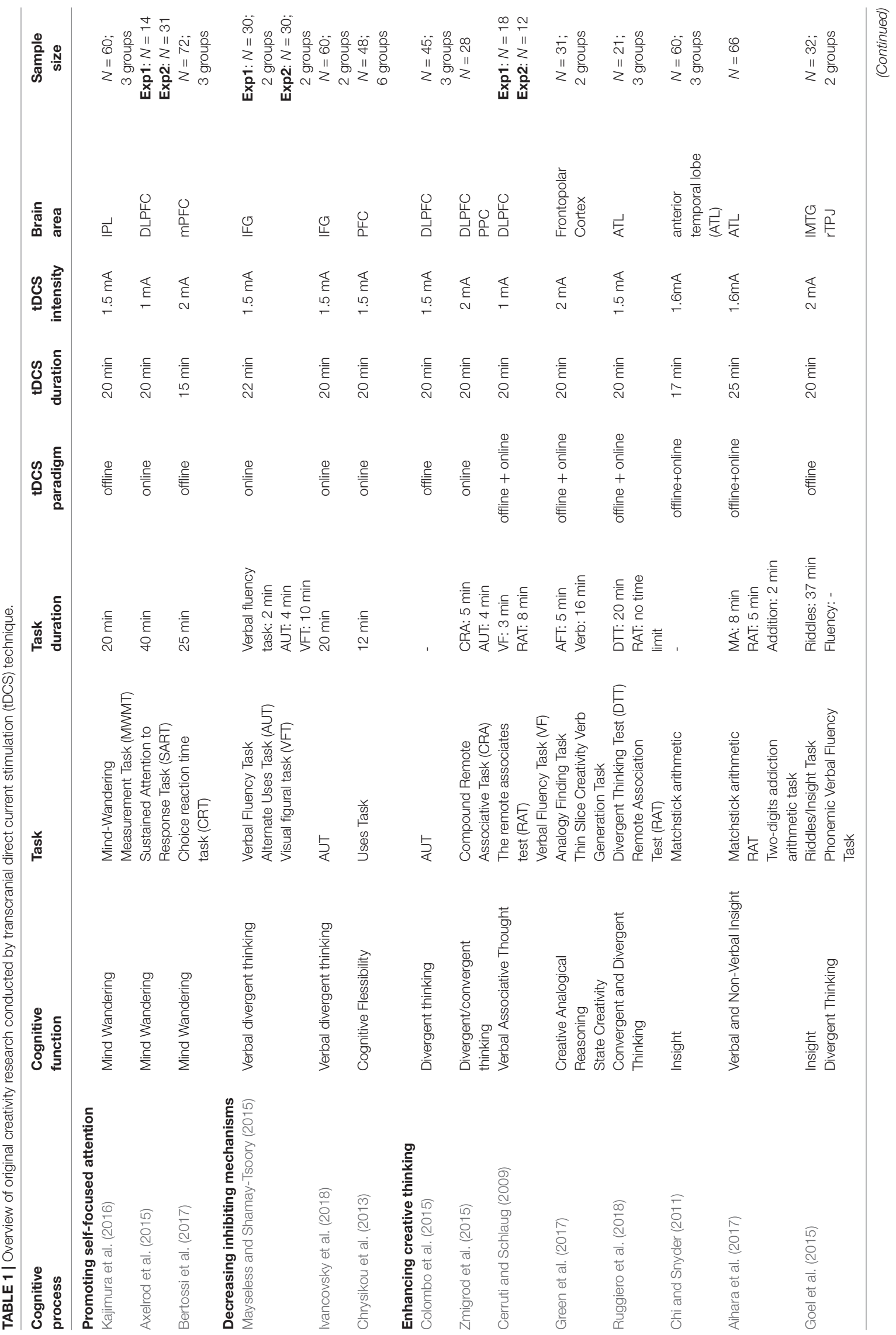




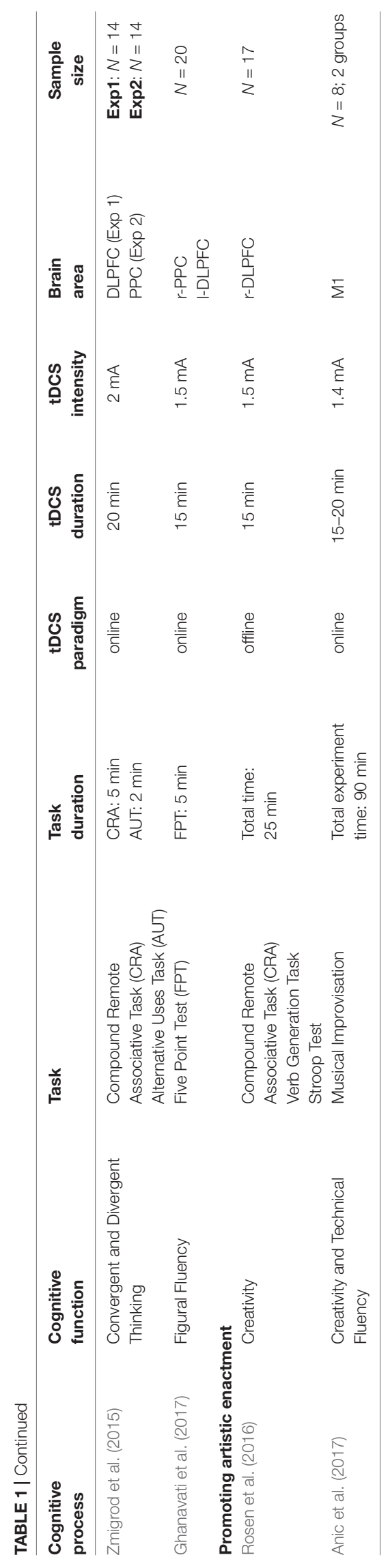

requires individuals to focus their attention on cognition, shifting from the external environment to the internal imaginative processes. We start from the idea that this task needs a mechanism able to support and actively maintain it, thus allowing the brain to activate creative processes while still remaining in touch with the environment. Here, we believe the default mode network (DMN) might play a fundamental role. The DMN comprises hubs and subsystems in interplay, which have been related to "internal mentation" (AndrewsHanna, 2012). The interaction between creativity and the DNM refers to spontaneous imagination and self-generated thought (Andrews-Hanna, 2012; Andrews-Hanna et al., 2014). The interaction between creativity and the DNM refers to spontaneous imagination and self-generated thought (AndrewsHanna, 2012; Andrews-Hanna et al., 2014). Interestingly, different regions of the DMN were found to be associated with creativity. For instance, divergent thinking is associated with a resting-state functional connectivity (RSFC) between the medial prefrontal cortex (mPFC) and the posterior cingulate cortex (PCC; Takeuchi et al., 2012). Similarly, Beaty et al. (2014) found greater RSFC between the left inferior frontal gyrus (IFG) and the entire default mode network in a high creativity group. Furthermore, research also suggests that increased RSFC between the mPFC and the middle temporal gyrus (mTG) might be crucial to creativity, defining a potential target for brain modulation with the aim of promoting creativity (Wei et al., 2014). Also, it seems that the topological properties of brain networks are highly associated with creative ability. For example, the small-world organization of the DMN allows information integration and segregation, thereby addressing the work to subnetworks within the global cognitive system. This result is a particularly desirable one when facing standard situations. However, it limits the increase in creative solutions. Indeed, it has been shown that high-creativity subjects have shorter links within local nodes and stronger connections with distal functional networks, which lead to a facilitated spread of information within the whole system (Hermundstad et al., 2012). This activity diffusion supports a flexible generation of original ideas, a task that requires the wide distribution of attentional resources (Jung et al., 2013), and the retrieval of potentially useful information (Andrews-Hanna et al., 2014). Here, the DMN proved to play a key role in producing interindividual variation during the creative performance. Finally, interesting associations have been found between personality traits, DMN functioning, and creativity. For example, a study by Beaty et al. (2014) highlighted that the openness personality trait, which is positively linked to creative performance, is related to the global efficiency of the DMN.

In this framework, we may hypothesize that $\mathrm{tDCS}$ training could be effective in promoting and enhancing DMN flexibility. In particular, research by Kajimura et al. (2016) explored the relationship between the activity of some areas belonging to the DMN-more specifically, the right inferior parietal lobe (rIPL) - and mind-wandering. They applied anodal tDCS over the right rIPL and cathode over the left lateral prefrontal cortex (ILPFC) as well as the opposite montage. Behavioral 
results showed reduced mind-wandering after applying the first montage when compared to the opposite one, while at the neural level some decreased functional connections from the right IPL were found. In fact, as discussed by the authors, it seems that the decrease in $\mathrm{mPFC}$-PCC connectivity mediated the decrease in mind wandering, thus suggesting a facilitation from their afferent connections to this mind functioning. These results may suggest that the modulation of specific regions of DMN may actually change the whole system balance, changing the attention focus and thus promoting or inhibiting imaginative processes.

Another interesting relationship is the one between the $\mathrm{DMN}$ and the executive functions modulated by the dorsolateral prefrontal cortex (DLPFC). Axelrod et al. (2015) applied the anode electrode to the left DLPFC and the cathode electrode to the right supraorbital area and found that the depolarization of the left DLPFC increased mind wondering propensity during a sustained attention task. During this task, subjects responded to relatively rare items within a long and boring list of repetitive trials. This situation is known to produce task-unrelated thoughts. In their study, authors found that the propensity to produce unrelated thoughts was increased during anodal tDCS of the left DLPFC without impairing attentional performance. Authors suggested that this effect might be linked to an indirect effect of the stimulation on the default mode network. Furthermore, since the mind-wandering evoked by tDCS did not impair the task performance, it is possible that the specific montage enhanced the cognitive resources of subjects, allowing both proper responses and unrelated thoughts. Generally speaking, the stimulation of prefrontal regions may influence the balance of the default network and modulate executive functions at the same time, an effect that might be desirable in creative tasks. Another recent study performed on a mind-wandering task (Bertossi et al., 2017) also highlighted the crucial role of the mPFC, since the administration of cathodal tDCS decreased the attitude to mind-wander. Moreover, the effect was also accompanied by a change in the content perspective, which became more related to other people rather than self-related issues. The idea that tDCS may produce an attention modulation to favor creative processes seems then to be supported by evidence.

\section{DECREASING THE EFFECT OF FILTERS AND INHIBITING MECHANISMS}

Another possible way to promote creative answers is by disrupting the inhibiting mechanisms that try to maintain individual thinking inside-the-box. In this way, the usual cognitive algorithm may be efficiently applied to incoming stimuli, inhibiting the search for further algorithms and filtering out less salient information. The left IFG (IIFG) and the anterior insula were reported to play key roles in these mechanisms (Abraham et al., 2012; Uddin, 2015). Since the insula may not be directly modulated by tDCS, studies targeted the IFG.

For example, a study by Mayseless and Shamay-Tsoory (2015) stimulated the IIFG to test the effect of tDCS on a divergent task. Authors moved from the neurofunctional assumption that creativity relies on a balance between right and left hemispheric activation (The Balance Hypothesis). Thus, the aim of their study was to test this model by altering this balance through tDCS at the IIFG during a divergent thinking performance. Researchers applied a bilateral stimulation with the cathode over the right IFG, and the anode over the left, and compared this condition with the opposite one. Results showed increased divergent scorings when the left IFG was deactivated by the cathodal stimulation, while the opposite condition did not affect the creative performance. To better explore these issues, in a second experiment authors administered unilateral stimulation with either the anode or the cathode over the left and right IFG alone. The stimulation of each area alone was not sufficient to modify the creative process Results supported the Balance Hypothesis since neither of the two conditions resulted in an enhanced or impaired creative performance.

Another study by Ivancovsky et al. (2018) targeted the same area (the IIFG) during the alternate uses task (AUT; Guilford et al., 1978). In this task, common objects are shown and participants are asked to report as many alternate uses as possible within a 4-min period. In their study, researchers applied a 20-min cathodal stimulation to the IIFG with the anode at the right supraorbital region as well as the opposite montage. Results showed an increase in creativity when the left IFG was hyperpolarized, while the depolarization of the same area decreased creativity scores. Considering that the IIFG was previously associated with inhibitory processes (Aron et al., 2003; Swick et al., 2008), we may suggest that the application of tDCS over the lIFG produces a lowered inhibition mechanism, thus allowing more flexible and creative thinking.

The notion that cognition may be modulated by disrupting top-down cognitive control, allowing the brain to process unfiltered bottom-up information, is also supported by a number of studies targeting the prefrontal cortices. For instance, Leite et al. (2011) found that the anodal stimulation of the DLPFC coupled with the cathodal stimulation of the primary motor area increases the efficiency in task shifting. Similarly, Chrysikou et al. (2013) measured the performance results of the uncommon use (UU) task, contrasted with the common use (CU) task, during the cathodal stimulation of the left or the right prefrontal cortex (F7/F8), with the cathode on the contralateral mastoid. They obtained that the hyperpolarization of the left prefrontal cortex led to an increase in the number of the UUs reported as well as in the speed of responses. Interestingly, the number of common uses reported was not affected by tDCS. The study supports the idea that the disruption of the prefrontal control system facilitates cognitive flexibility.

\section{ENHANCING CREATIVE THINKING}

A third fundamental strategy to affect creativity through neuromodulation is to directly target thinking processes. Traditionally, creativity has been associated with divergent thinking, which is the ability to follow unconventional ways of reasoning, thus producing new ideas and solutions. It is obvious that creativity also implies convergent thinking, i.e., the 
ability to select ideas for their appropriateness in relation to a given task (Finke et al., 1992; Amabile, 1996; Sternberg, 1999). To affect thinking processes different strategies are possible, from modulating attention to favor semantic connections and information integration. For the sake of clarity, in this section we will provide evidence categorized by the targeted cortical area.

\section{Prefrontal Areas}

As discussed above, neuromodulation may be effective in promoting both ideas generation (by enhancing analogical associations), and idea selection. Since these processes require high order information processing, studies often targeted the DLPFC. Colombo et al. (2015) investigated the role of the DLPFC in a task that required subjects to find original uses for common objects (so-called AU task). Twenty minutes of anodal tDCS over the left DLPFC increased creative responses, but only when the task was primed by a condition able to promote divergent thinking. This means that tDCS stimulation had an effect only when interacting with a creative primer. These results may be related to the role of the DLPFC in coordinating attentional shifts since attentional focusing was associated with a worsened performance of the AU task in previous studies (Friedman et al., 2003). However, it is likely that when the cognitive system is called to work on a similar task, the DLPFC also plays a role at a higher level. To use a metaphor, the DLPFC might be responsible for modulating a kind of cognitive search engine aimed at associating an object with possible uses. Normally, the first reference that comes to mind is related to the frequency of use and/or prototypical features of the probe, something like the sponsored links displayed by Web search engines. Sure, we can be aware of many other links (e.g., uses), but generally, we are "forced" to stop searching the list after having read the top of it, leaving uncommon and probably more creative uses out of mind. However, when the DLPFC is impaired, the search engine is free to search deeper and propose a randomly ordered list for frequency and/or prototypical features, thus allowing creative cognitive processes. The DLPFC might then be linked to the maintaining of a given view of the world by searching for a confirmation of previous expectations more than trying new, original cognitive solutions. It would implement a sort of cognitive inhibition on a largescale. Actually, a study by Zmigrod et al. (2015) reported increased performances on the Compound Remote Association task (CRA) when anodal tDCS stimulation was applied at the left DLPFC with the cathode at the right DLPFC. Since the CRA is intended to measure convergent thinking (asking responders to find a link between three unrelated words), authors suggest that the DLPFC plays a key role in analytical information processing. Similar results were found in a previous study by Cerruti and Schlaug (2009) who used a unilateral montage over the IDLPFC to modulate the performance of the Remote Association Task. Consequently, the role of the DLPFC appears to be complex and linked to highly demanding tasks, e.g., the integration of semantically distant information, creative idea selection, and convergent thinking. All of these processes require the brain to search in depth for higher-level connections.

\section{Temporal Areas}

A study by Chi and Snyder (2011) reported increased problemsolving abilities by insight in subjects who received cathodal stimulation over the left anterior temporal lobe (ATL) together with anodal stimulation of the contralateral region. The authors refer this increase in performance to a general modulator role of the left ATL, which is involved in a top-down inhibiting system that facilitates the use of routinized solutions. Authors suggest that the contemporary stimulation of the left ATL and DLPFC could induce even greater and/or generalized effects on creativity. The basic idea is that many neural circuits compete during a cognitive task. The increase of one over the others depends on both experience and anatomical (genetically driven) features. Thus, people with a middle to strong left dominance (in their study Chi and Snyder, 2011 controlled this variable by selecting only right-handed people with 50 or more on the Edinburgh Scale) generally show less hypothesis switching than less lateralized people due to the strong role played by the left ATL and frontal areas in top-down inhibiting processes. These mechanisms are tuned by experience and generally allow people to obtain good (or even excellent) results in their routinized activities. Actually, in the same study, people who received left cathodal and right anodal stimulation did not worsen their performance, since the left ATL seemed to be already optimized. Thus, a one-shot stimulation (20 min of tDCS) cannot obtain a substantial change in performance. In less lateralized people, it is possible that a lower top-down modulation allows for easier perspective shifting, thus facilitating insight solutions and ideas generation. At the opposite, an excessive imbalance toward right ATL functioning might impede or hinder the acquisition and/or the tuning of cognitive algorithms useful in repetitive daily or professional situations. However, a study by Aihara et al. (2017) on a sample of 66 Japanese subjects did not find any effect on insight after tDCS applied over ATL. In fact, neither the right ATL anodal/left ATL cathode nor the reversed montage affected the subjects' performance at the remote associates test (RAT; verbal insight) and at the matchstick task (nonverbal insight). Authors suggested that their results might be due to the specific experimental conditions adopted. This underlines the fundamental role of the experimental setting when testing the efficacy of tDCS programs. Actually, another study (Ruggiero et al., 2018) targeted the same areas obtained different results. Authors used a verbal task (the RAT) and a graphical task (the Divergent Thinking Test, DTT) and they found a positive effect of the anodal stimulation on the ATL with the cathode on the right ATL at RAT performance. However, results showed that this effect was significant only for reducing the time needed by subjects to produce a verbal insight (RAT), while accuracy was not affected. Furthermore, the DTT performances were not affected by tDCS, supporting the idea that the left ATL is involved in verbal creativity. Taken together, such evidence suggests that suppressing the left anterior temporal region could disengage the correspondent right region from the control of the left inhibiting system that normally 
limits access to cognitive resources, thus improving creative thinking skills. The left frontotemporal region was also shown to be linked to creative analogical reasoning and information integration since its activation correlates with semantic distance in an analogy generation task (Green et al., 2010, 2015). Finally, Goel et al. (2015) proposed different creative tasks in both divergent and convergent thinking domains to English speakers and 16 non-native English speakers. They wanted to test the effect of a neuromodulation protocol on the ability to solve linguistic riddles. The tDCS was administered over the left mTG and the right temporoparietal junction (TPJ). The stimulation was able to modulate the performance of the task in specific ways: while performing the insight task (convergent thinking), the stimulation of the right TPJ and the deactivation of the MTG improved results; conversely, during the divergent thinking task, the same stimulation resulted in decreased performances. Authors explained their results, considering the role of the MTG, which is involved in routine semantic processing. Instead, the TPJ is associated with the search for unusual and/or metaphorical meanings of the language. Consequently, the hyperpolarization of the left MTG may have facilitated a shift in perspective, allowing for the activation of an active search for alternative meanings. This effect may be particularly acute in non-native speakers since they lack the knowledge of idiomatic expressions and their poor language expertise does not easily allow them to go beyond the literal meaning. Favoring the work of the right TPJ and partially inhibiting the top-down supervision of the left MGT, non-English speakers gained a freer, creative interpretation of phrases.

\section{Parietal Areas}

The study by Zmigrod et al. (2015) investigated the influence of tDCS on performances in verbal tasks targeting the posterior parietal cortex (PPC). Authors found that the anodal stimulation of both the left and right PPC increased insight and decreased analytical answers when compared to the control condition. Considering that the PPC is related to goal-directed attentional processes (Behrmann et al., 2004), it could be hypothesized that the depolarization of this region might increase the ability of the person to disengage bottom-up automatic mechanisms. This disengagement may favor divergent thinking and insight solutions. Ghanavati et al. (2017) applied tDCS over the same brain site (both right and left PPC) during a figural fluency task. Results showed that participants produced more unique designs under anodal rPPC tDCS. Such findings support the idea that the PPC plays an important role in both verbal and visual creativity by modulating attention mechanisms.

\section{PROMOTING ARTISTIC ENACTMENT}

The last important issue related to creativity deals with enactment, which is the ability to finalize the creative course in a tangible and concrete way. Enactment may relate to any sphere of life, but it is particularly interesting when referred to artistic production since it merges different cognitive and motor processes in a unique and appreciable shape. At this regard, a case study provided original suggestions: Simis et al.
(2014) from the Harvard Medical School had the opportunity to study a man affected by a stroke of the left middle cerebral artery. After the stroke, this person, without any art education, reported the urge to draw and started training and perfecting his drawing and painting skills using his left hand (previously he was right-handed, but the right hand was impaired). Four years after the stroke, the patient underwent a tDCS protocol consisting of the anodal stimulation of the right (unaffected) frontotemporal region. Here, we have the effect of the stroke over the left hemisphere and the neuromodulation of the right frontotemporal region. The former produced the urge to draw and express the patient's new perspective of the world. The neuromodulation increased his drawing abilities and creativity. In particular, $30 \mathrm{~min}$ of anodal stimulation produced increased graphical abilities in a drawing task. Though it is difficult to provide a complete explanation of this effect based only on the interplay between the two hemispheres, it is possible that the impairment of the left frontotemporal region may disinhibit artistic motivation, which might partially substitute the communication functions affected by the stroke. At the same time, the anodal stimulation of the right frontotemporal area probably enhanced the function of the frontoparietal connectivity, which is supposed to play a fundamental role in perspective elaboration and is, consequently, vital in graphical representation. Results revealed in this work are in line with recent evidence acquired from the application of tDCS for improving behavioral performance and functional connectivity after stroke in both motor (Chen and Schlaug, 2016) and cognitive domains (Marangolo et al., 2016). For example, a recent work by Marangolo et al. (2016) about the effects of tDCS on language recovery and resting state functional connectivity found a positive effect of real vs. sham stimulation on behavioral performance, as well as on the interconnectedness of several regions of motor, default and control network.

Moving to music enactment, Rosen et al. (2016) proposed a dual process model for creative production in jazz improvisers: Type-1 would be based on automatic, associative processes, while Type-2 on executive, controlled ones. The use of either Type 1 or 2 is thought to be associated with domain expertise: novices would rely more on top-down, controlled mechanisms, while expert musicians to bottom-up, implicit processing. To test their hypothesis, authors performed a tDCS protocol by targeting the right DLPFC with anodal, cathodal, or sham stimulation. Results showed a significant interaction between the musicians' expertise and tDCS: after anodal stimulation, novices performed better in terms of originality as assessed by expert musician judges, while the opposite effect was obtained for the most experienced musicians, who reported poorer performance. Researchers interpreted their findings as a confirmation of the dual model since different modes of creative expertise were found between experts and novices. Also, it was proven that anodal stimulation may effectively modulate rDLPFC activity, which is recruited during improvisation. Remaining within the theme of musical improvisation, Anic et al. (2017) explored the role of another brain area, the Motor Cortex (M1), for the creative process. In particular, starting from the idea that it subserves the acquisition and consolidation of novel 
movements, they applied a bilateral tDCS montage (left and right M1) to two different groups of expert pianists while requiring them to improvise some musical sequences. The left and right $\mathrm{M} 1$ areas received either anodal or cathodal stimulation according to the group (anodal-left/cathodal-right; cathodal-left/anodal-right). Although this was a pilot study (only eight musicians took part in the experiment, four for each group), preliminary analyses showed that left excitatory stimulation increased creativity and technical fluency while cathodal stimulation apparently didn't produce any significant behavioral modifications. Accordingly, authors concluded that there is at least preliminary evidence that the M1 region contributes to musical fluency and creativity. However, due to the sample size, future research should better explore these issues to identify significant statistical effects. Being a primary motor region $\mathrm{M} 1$ is, without any doubt, related to an executive component of musical creativity. Thus, the excitatory processes within this area could also, in this case, lead to enactment. Considering the underlying neurophysiological mechanisms of such processes, previous research (Nitsche et al., 2005) underlined that the corticospinal excitability modulation provided by tDCS over M1 can be attributable to membrane polarization, and less on synaptic modifications. On the other side, the engagement of intracortical synaptic mechanisms has been found for tDCS after-effects.

\section{CONCLUSION: SWITCHING ON THE CREATIVITY STATE}

Starting from the evidence discussed above, we conclude this review with a model aimed at explaining how tDCS, as well as other neuromodulation techniques, may favor creativity. We begin with the consideration that creativity is influenced by both stable characteristics and contextual conditions. Regarding stable traits, (Aron and Aron, 1997) suggested that people could differ in the way they neurologically transmit and process sensory information (Gerstenberg, 2012). It is the case of sensory processing sensitivity (SPS), described as a personality trait modulated by genetic factors, which allows people to feel and process more information at one time, and in a deeper way. Such sensitivity is referred to both external and internal stimuli (Jagiellowicz et al., 2011). Accordingly, Highly Sensitive Persons (Aron and Aron, 1997) are more inclined to experience higher arousal during exposure to environmental stimuli such as bright lights, strong smells, noise and chaotic situations. Moreover, they startle easily and are strongly sensitive to caffeine and time pressures (Jagiellowicz et al., 2011). Finally, those with SPS are more reactive to interpersonal and emotional cues. For example, they are more susceptible to the presence of external observers when performing tasks, and more aware of others' moods. These peculiar reactions are already present at the very first stages of life (Aron et al., 2012; Davidson et al., 2002) and are associated with specific neurobiological markers (Herberner et al., 1989; Aron et al., 2010). Such sensitivity could sometimes result in a sensorial overload with subsequent negative effects, like exertion and fatigue. Thus, it is possible that they could more often feel the urge to rest alone and sleep longer throughout the day (Aron and Aron, 1997). The existence of these differences is noteworthy since a tDCS program aimed to improve creativity may have different effects on different individuals. Not checking for these differences might also lead to different study results.

However, our idea is that creativity, though influenced by stable individual characteristics, may be seen as a transient property of the cognitive system, a mental configuration we may call a "creativity-on" state, which may be spontaneously activated and/or evoked by external conditions (e.g., by a task that asks individuals to find creative semantic connections between words). So far, people may be trained to have easier access to this state when they need it. We may define a state of creative-on, as opposed to a state of creative-off. The latter is essentially based on ordinary functioning, e.g., the search for information in long-term memory or the use of prompt cognitive algorithms. From a neurofunctional point of view, the creative-on/off dynamic is substantially based on the interaction between the DMN and the cognitive control system (Beaty et al., 2014, 2016). The core structures of DMN are the mPFC, the PCC precuneus (prec), and the posterior inferior parietal lobule (pIBL). The role of the network is multifaceted since it is involved in a number of specific cognitive processes. However, different studies showed that an increase of DMN activity, in particular, the connection strength between $\mathrm{mPFC}$ and PCC/pre, is involved in creative processes (Beaty et al., 2015). Conversely, the cognitive control system involves the left IFG and its role is to inhibit cognitive processes not linked to the ongoing cognitive plan. In particular, the modulatory role of the IFG is active in the ATL, in particular, the temporal pole (TP), which is considered a sort of semantic hub. When the left IFG system activity is high, the semantic search and integration will involve only short-range semantic connections (Green et al., 2010). The left IFG and ATL are involved in the process of conceptual expansion (Abraham et al., 2012). Consequently, we define the creative-on/off as the balance between the DMN and the IFG network. When the former predominates over the latter, we enter into a creative-on state; otherwise, the cognitive system works in a routine creative-off state. Distinguishing these two states is interesting since it allows understanding the cognitive and behavioral differences we observe in an individual facing the same situation in two different moments. The creative-off state is a goal-oriented, routine-based, efficient system that generally produces good cognitive and/or behavioral outcomes. This state is governed by rules, such as the path-of-least-resistance rule, which allow the brain to achieve good solutions to typical problems in a short time. On the contrary, when an easy solution to a problem is not available, or when an individual is forced to go beyond the usual semantic or analogical associations, then the creative-off state cannot serve the purpose anymore and the usual and stable neurofunctional organization breaks down. The DMN takes the lead, and the short-range smallworld organization leaves the field to long-range connections. In this way, the brain may expand its cognitive repertoire by linking together concepts, images, and experiences, thus allowing for more flexible thinking. It's in this state that we may experience the semantic expansion that strings together concepts 
apparently distinguished to produce new ideas. Because the creativity-on state obviously requires more energy and a greater cognitive load, it is, therefore, less efficient than the creativityoff, which instead has been fine-tuned by evolution, education, and specific training. Naturally, the dynamic interaction between the DMN and the IFG network also involves many other neural networks. In particular, it is interesting to note that the attention network may be modulated by the DMN activity. When within the DMN the connection strength between mPFC and the PCC/prec is high, then the attention disengages from the external environment, thus shifting toward inner processes. This may favor mind-wandering as well as imagery activity. Instead, when a focus on external stimuli must be kept high, then PIPL-PCC/prec become predominant, thus inhibiting mind-wandering and other unrelated activities. In addition, the IFG network may modulate the attention focus when a complex task requires both concentration and the inhibition of distracting stimuli at the same time. The creative-on state is roughly similar to how the infant's brain works. Indeed, Gardner (1982) defined the preschooler phase (between 3 and 6-year-old) as the Golden age of creativity, since infants show spontaneous and vibrant creativity in this period. This may be linked to the immaturity of pre-frontal cortices (Miller et al., 2012). Of course, creativity is different in childhood and adulthood. Adults' creativity involves a number of different psychological mechanisms and is generally aimed to generate a well-defined product (Charles and Runco, 2001). That's why the creativity-on state does not imply a generalized deactivation of prefrontal cortices, but a partial adjustment that permits both divergent and convergent thinking.

Considering the role of tDCS in the framework of creativeon/off, it is also interesting to appreciate how neurostimulation might be useful for promoting creativity outside the lab. In fact, we have seen that different tasks require different cortical targets, meaning that the multifaceted nature of creativity cannot be approached by a single tDCS program. For example, we could target the PFC to promote a more flexible cognitive process, and the ATL to promote verbal creativity. However, in real-life applications (e.g., to improve a manager's creative decision making at the workplace) it's difficult to define which creative mechanisms are the most important and which tDCS target will lead to better outcomes. These parameters are modulated by the individual's characteristics, by contextual factors, and

\section{REFERENCES}

Abraham, A., Pieritz, K., Thybusch, K., Rutter, B., Kröger, S., Schweckendiek, J., et al. (2012). Creativity and the brain: uncovering the neural signature of conceptual expansion. Neuropsychologia 50, 1906-1917. doi: 10.1016/j. neuropsychologia.2012.04.015

Aihara, T., Ogawa, T., Shimokawa, T., and Yamashita, O. (2017). Anodal transcranial direct current stimulation of the right anterior temporal lobe did not significantly affect verbal insight. PLoS One 12:e184749. doi: 10.1371/journal.pone.0184749

Amabile, T. M. (1983). The social psychology of creativity: a componential conceptualization. J. Pers. Soc. Psychol. 45, 357-376. doi: 10.1037/0022-3514. 45.2.357

Amabile, T. M. (1996). Creativity in Context: Update to the Social Psychology of Creativity. UK: Hachette. by the actual requirements of a given task. Furthermore, some creativity-related mechanisms are served by brain areas that are not possible to directly stimulate by non-invasive techniques. We argue that tDCS might be more useful in promoting a more general creative-on state, which will allow a spontaneous reorganization of the whole cognitive system. At this aim, it's plausible to suggest that the IFG system will play a vital role in future programs that are intended to promote a creative-on state through tDCS, while other areas may be useful for specific purposes. Indeed, as revealed in a study by Meinzer et al. (2012) using fMRI after tDCS, the stimulation of the left IFG produced effects on a large network, including the anterior insula (salience network), the anterior temporal areas (language network) and the medial prefrontal cortex (DMN). Finally, it is noteworthy that the neurostimulation effect heavily depends on the cognitive task used. Thus, it will be important to test which task is able to elicit creative thoughts during which tDCS protocol.

In conclusion, our proposal is that starting by the creativityon/off dynamic is quite easy to understand how tDCS or even other neurostimulation techniques may favor creativity. Indeed, since the creativity-on state may be considered a prodromal state, a kind of neural habitat suitable for creative ideas to survive, the role of $\mathrm{tDCS}$ is quite unspecific, modulating only the likelihood of a more flexible and imaginative thinking to arise. A modulation that might be useful in several different contexts. So, what kind of creativity is possible to stimulate? We argue that, following the classification by Kaufman and Beghetto (2009), promoting the elicitation of a creativity-on state by the inhibition of the IFG system and using proper cognitive tasks is possible to promote all form of creativity, from "mini creativity" (typical of infants) to everyday life "little creativity," from "Pro-creativity," (the one request in professional contexts) to "Big Creativity" (linked to object and important achievements). Of course, the actual results will depend also on contextual and personal characteristics not influenced by neurostimulation. Thus, it would be important for future research to address the topic of creativity within a more comprehensive framework.

\section{AUTHOR CONTRIBUTIONS}

The work was equally distributed between authors, each providing a substantial and intellectual contribution to the work, and approved it for publication.

Andrews-Hanna, J. R. (2012). The brain's default network and its adaptive role in internal mentation. Neuroscientist 18, 251-270. doi: $10.1177 / 1073858411403316$

Andrews-Hanna, J. R., Smallwood, J., and Spreng, R. N. (2014). The default network and self-generated thought: component processes, dynamic control, and clinical relevance. Ann. N Y Acad. Sci. 1316, 29-52. doi: 10.1111/nyas. 12360

Anic, A., Thompson, W. F., and Olsen, K. N. (2017). "Stimulation of the primary motor cortex enhances creativity and technical fluency of piano improvisations," in Proceedings of the 10th International Conference of Students of Systematic Musicology (SysMus17), ed. P. M. C. Harrison (London: SysMus, University of London).

Aron, E. N., and Aron, A. (1997). Sensory-processing sensitivity and its relation to introversion and emotionality. J. Pers. Soc. Psychol. 73, 345-368. doi: 10.1037/0022-3514.73.2.345 
Aron, E. N., Aron, A., and Jagiellowicz, J. (2012). Sensory processing sensitivity: a review in the light of the evolution of biological responsivity. Personal. Soc. Psychol. Rev. 16, 262-282. doi: 10.1177/1088868311434213

Aron, A. R., Fletcher, P. C., Bullmore, E. T., Sahakian, B. J., and Robbins, T. W. (2003). Stop-signal inhibition disrupted by damage to right inferior frontal gyrus in humans. Nat. Neurosci. 6, 115-116. doi: 10.1038/nn1003

Aron, A., Ketay, S., Hedden, T., Aron, E. N., Markus, H. R., and Gabrieli, J. D. E. (2010). Temperament trait of sensory processing sensitivity moderates cultural differences in neural response. Soc. Cogn. Affect. Neurosci. 5, 219-226. doi: 10.1093/scan/nsq028

Axelrod, V., Rees, G., Lavidor, M., and Bar, M. (2015). Increasing propensity to mind-wander with transcranial direct current stimulation. Proc. Natl. Acad. Sci. U S A 112, 3314-3319. doi: 10.1073/pnas.1421435112

Barron, F. (1955). The disposition toward originality. J. Abnorm. Soc. Psychol. 51, 478-485. doi: 10.1037/h0048073

Batey, M., and Furnham, A. (2006). Creativity, intelligence, and personality: a critical review of the scattered literature. Genet. Soc. Gen. Psychol. Monogr. 132, 355-429. doi: 10.3200/mono.132.4.355-430

Beaty, R. E., Benedek, M., Kaufman, S. B., and Silvia, P. J. (2015). Default and executive network coupling supports creative idea production. Sci. Rep. 5:10964. doi: 10.1038/srep10964

Beaty, R. E., Benedek, M., Silvia, P. J., and Schacter, D. L. (2016). Creative cognition and brain network dynamics. Trends Cogn. Sci. 20, 87-95. doi: 10.1016/j.tics. 2015.10.004

Beaty, R. E., Benedek, M., Wilkins, R. W., Jauk, E., Fink, A., Silvia, P. J., et al. (2014). Creativity and the default network: a functional connectivity analysis of the creative brain at rest. Neuropsychologia 64, 92-98. doi: 10.1016/j. neuropsychologia.2014.09.019

Behrmann, M., Geng, J. J., and Shomstein, S. (2004). Parietal cortex and attention. Curr. Opin. Neurobiol. 14, 212-217. doi: 10.1016/j.conb.2004.03.012

Bertossi, E., Peccenini, L., Solmi, A., Avenanti, A., and Ciaramelli, E. (2017). Transcranial direct current stimulation of the medial prefrontal cortex dampens mind-wandering in men. Sci. Rep. 7:16962. doi: 10.1038/s41598-01717267-4

Cerruti, C., and Schlaug, G. (2009). Anodal transcranial direct current stimulation of the prefrontal cortex enhances complex verbal associative thought. J. Cogn. Neurosci. 21, 1980-1987. doi: 10.1162/jocn.2008.21143

Charles, R. E., and Runco, M. A. (2001). Developmental trends in the evaluative and divergent thinking of children. Creat. Res. J. 13, 417-437. doi: 10.1207/s15326934crj1334_19

Chen, J. L., and Schlaug, G. (2016). Increased resting state connectivity between ipsilesional motor cortex and contralesional premotor cortex after transcranial direct current stimulation with physical therapy. Sci. Rep. 6:23271. doi: 10.1038/srep23271

Chi, R. P., and Snyder, A. W. (2011). Facilitate insight by non-invasive brain stimulation. PLoS One 6:e16655. doi: 10.1371/journal.pone.0016655

Chrysikou, E. G., Hamilton, R. H., Coslett, H. B., Datta, A., Bikson, M., and Thompson-Schill, S. L. (2013). Noninvasive transcranial direct current stimulation over the left prefrontal cortex facilitates cognitive flexibility in tool use. Cogn. Neurosci. 4, 81-89. doi: 10.1080/17588928.2013.768221

Colombo, B., Bartesaghi, N., Simonelli, L., and Antonietti, A. (2015). The combined effects of neurostimulation and priming on creative thinking. A preliminary tDCS study on dorsolateral prefrontal cortex. Front. Hum. Neurosci. 9:403. doi: 10.3389/fnhum.2015.00403

Csikszentmihalyi, M. (1988). "The flow experience and its significance for human psychology," in Optimal Experience: Psychological Studies of Flow in Consciousness, eds M. Csikszentmihalyi and I. S. Csikszentmihalyi (New York, NY: Cambridge University Press), 15-35.

Davidson, R. J., Lewis, D. A., Alloy, L. B., Amaral, D. G., Bush, G., Cohen, J. D., et al. (2002). Neural and behavioral substrates of mood and mood regulation. Biol. Psychiatry 52, 478-502. doi: 10.1016/s0006-3223(02)01458-0

Dietrich, A. (2004). Neurocognitive mechanisms underlying the experience of flow. Conscious. Cogn. 13, 746-761. doi: 10.1016/j.concog.2004.07.002

Finke, R. A., Ward, T. B., and Smith, S. M. (1992). Creative Cognition: Theory, Research and Applications. Cambridge, MA: MIT Press.

Friedman, R. S., Fishbach, A., Förster, J., and Werth, L. (2003). Attentional priming effects on creativity. Creat. Res. J. 15, 277-286. doi: 10.1080/10400419.2003. 9651420
Gardner, H. (1982). Art Mind and Brain: A Cognitive Approach to Creativity. New York, NY: Basic Books.

Gerstenberg, F. X. R. (2012). Sensory-processing sensitivity predicts performance on a visual search task followed by an increase in perceived stress. Pers. Individ. Dif. 53, 496-500. doi: 10.1016/j.paid.2012.04.019

Ghanavati, E., Nejati, V., and Salehinejad, M. A. (2017). Transcranial direct current stimulation over the posterior parietal cortex (PPC) enhances figural fluency: implications for creative cognition. J. Cogn. Enhanc. 2, 88-96. doi: 10.1007/s41465-017-0059-7

Goel, V., Eimontaite, I., Goel, A., and Schindler, I. (2015). Differential modulation of performance in insight and divergent thinking tasks with tDCS. J. Probl. Solving 8:2. doi: 10.7771/1932-6246.1172

Green, A. E., Cohen, M. S., Raab, H. A., Yedibalian, C. G., and Gray, J. R. (2015). Frontopolar activity and connectivity support dynamic conscious augmentation of creative state. Hum. Brain Mapp. 36, 923-934. doi: $10.1002 / \mathrm{hbm} .22676$

Green, A. E., Kraemer, D. J. M., Fugelsang, J. A., Gray, J. R., and Dunbar, K. N. (2010). Connecting long distance: semantic distance in analogical reasoning modulates frontopolar cortex activity. Cereb. Cortex 20, 70-76. doi: 10.1093/cercor/bhp081

Green, A. E., Spiegel, K. A., Giangrande, E. J., Weinberger, A. B., Gallagher, N. M., and Turkeltaub, P. E. (2017). Thinking cap plus thinking zap: tDCS of frontopolar cortex improves creative analogical reasoning and facilitates conscious augmentation of state creativity in verb generation. Cereb. Cortex 27, 2628-2639. doi: 10.1093/cercor/bhw080

Guilford, J. P. (1957). Creative abilities in the arts. Psychol. Rev. 64, 110-118. doi: $10.1037 / \mathrm{h} 0048280$

Guilford, J. P., Christensen, P. R., Merrifield, P. R., and Wison, R. C. (1978). Alternate Uses: Manual of Instructions and Interpretation. Orange, CA: Sheridan Psychological Services.

Herberner, E. S., Kagan, J., and Cohen, M. (1989). Shyness and olfactory threshold. Pers. Individ. Dif. 10, 1159-1163. doi: 10.1016/0191-8869(89)90079-2

Hermundstad, A. M., Bassett, D. S., Brown, K. S., Aminoff, E. M., Clewett, D., Freeman, S., et al. (2012). Structural foundations of resting-state and task-based functional connectivity in the human brain. Proc. Natl. Acad. Sci. U S A 110, 6169-6174. doi: 10.1073/pnas.1219562110

Ivancovsky, T., Kurman, J., Morio, H., and Shamay-Tsoory, S. (2018). Transcranial direct current stimulation (tDCS) targeting the left inferior frontal gyrus: effects on creativity across cultures. Soc. Neurosci. 4:2. doi: 10.1080/17470919.2018. 1464505

Jagiellowicz, J., Xu, X., Aron, A., Aron, E., Cao, G., Feng, T., et al. (2011). The trait of sensory processing sensitivity and neural responses to changes in visual scenes. Soc. Cogn. Affect. Neurosci. 6, 38-47. doi: 10.1093/scan/ nsq001

Jung, R. E., Mead, B. S., Carrasco, J., and Flores, R. A. (2013). The structure of creative cognition in the human brain. Front. Hum. Neurosci. 7:330. doi: 10.3389/fnhum.2013.00330

Kajimura, S., Kochiyama, T., Nakai, R., Abe, N., and Nomura, M. (2016). Causal relationship between effective connectivity within the default mode network and mind-wandering regulation and facilitation. Neuroimage 133, 21-30. doi: 10.1016/j.neuroimage.2016.03.009

Kaufman, J. C., and Beghetto, R. A. (2009). Beyond big and little: the four C model of creativity. Rev. Gen. Psychol. 13, 1-12. doi: 10.1037/a0013688

Kim, S., Stephenson, M. C., Morris, P. G., and Jackson, S. R. (2014). tDCS-induced alterations in GABA concentration within primary motor cortex predict motor learning and motor memory: a $7 \mathrm{~T}$ magnetic resonance spectroscopy study. Neuroimage 99, 237-243. doi: 10.1016/j.neuroimage.2014.05.070

Leite, J., Carvalho, S., Fregni, F., and Gonçalves, Ó. F. (2011). Task-specific effects of tDCS-induced cortical excitability changes on cognitive and motor sequence set shifting performance. PLoS One 6:e24140. doi: 10.1371/journal. pone.0024140

Marangolo, P., Fiori, V., Sabatini, U., De Pasquale, G., Razzano, C., Caltagirone, C., et al. (2016). Bilateral transcranial direct current stimulation language treatment enhances functional connectivity in the left hemisphere: preliminary data from aphasia. J. Cogn. Neurosci. 28, 724-738. doi: 10.1162/jocn_a_ 00927

Mayseless, N., and Shamay-Tsoory, S. G. (2015). Enhancing verbal creativity: modulating creativity by altering the balance between right and left 
inferior frontal gyrus with tDCS. Neuroscience 291, 167-176. doi: 10.1016/j. neuroscience.2015.01.061

Meinzer, M., Antonenko, D., Lindenberg, R., Hetzer, S., Ulm, L., Avirame, K., et al. (2012). Electrical brain stimulation improves cognitive performance by modulating functional connectivity and task-specific activation. J. Neurosci. 32, 1859-1866. doi: 10.1523/JNEUROSCI.4812-11.2012

Miller, D. J., Duka, T., Stimpson, C. D., Schapiro, S. J., Baze, W. B., McArthur, M. J., et al. (2012). Prolonged myelination in human neocortical evolution. Proc. Natl. Acad. Sci. U S A 109, 16480-16485. doi: 10.1073/pnas.1117943109

Mumford, M. D., and Gustafson, S. B. (1988). Creativity syndrome: integration, application, and innovation. Psychol. Bull. 103, 27-43. doi: 10.1037//0033-2909. 103.1.27

Nitsche, M. A., Seeber, A., Frommann, K., Klein, C. C., Rochford, C., Nitsche, M. S., et al. (2005). Modulating parameters of excitability during and after transcranial direct current stimulation of the human motor cortex. J. Physiol. 568, 291-303. doi: 10.1113/jphysiol.2005.092429

Rhodes, M. (1987). “An analysis of creativity," in Frontiers of Creativity Research: Beyond the Basics, ed. S. G. Isaksen (Buffalo, NY: Bearly, Original work published 1961), 216-222.

Ruggiero, F., Lavazza, A., Vergari, M., Priori, A., and Ferrucci, R. (2018). Transcranial direct current stimulation of the left temporal lobe modulates insight. Creat. Res. J. 30, 143-151. doi: 10.1080/10400419.2018.1446817

Rosen, D. S., Erickson, B., Kim, Y. E., Mirman, D., Hamilton, R. H., and Kounios, J. (2016). Anodal tDCS to right dorsolateral prefrontal cortex facilitates performance for novice jazz improvisers but hinders experts. Front. Hum. Neurosci. 10:579. doi: 10.3389/fnhum.2016.00579

Sehm, B., Schäfer, A., Kipping, J., Margulies, D., Conde, V., Taubert, M., et al. (2012). Dynamic modulation of intrinsic functional connectivity by transcranial direct current stimulation. J. Neurophysiol. 108, 3253-3263. doi: 10.1152/jn.00606.2012

Simis, M., Bravo, G. L., Boggio, P. S., Devido, M., Gagliardi, R. J., and Fregni, F. (2014). Transcranial direct current stimulation in de novo artistic ability after stroke. Neuromodulation 17, 497-501. doi: 10.1111/ner.12140

Stagg, C. J., O’Shea, J., Kincses, Z. T., Woolrich, M., Matthews, P. M., and Johansen-Berg, H. (2009). Modulation of movement-associated cortical activation by transcranial direct current stimulation. Eur. J. Neurosci. 30, 1412-1423. doi: 10.1111/j.1460-9568.2009.06937.x

Sternberg, R. J. (1999). Handbook of Creativity. Cambridge: Cambridge University Press.

Swick, D., Ashley, V., and Turken, A. U. (2008). Left inferior frontal gyrus is critical for response inhibition. BMC Neurosci. 9:102. doi: 10.1186/14712202-9-102

Takeuchi, H., Taki, Y., Hashizume, H., Sassa, Y., Nagase, T., Nouchi, R., et al. (2012). The association between resting functional connectivity and creativity. Cereb. Cortex 22, 2921-2929. doi: 10.1093/cercor/bhr371

Toynbee, A. (1964). "Is America neglecting her creative minority?" in Widening Horizons in Creativity: The Proceedings of the 5th Utah Creativity Research Conference, ed. C. W. Taylor (New York, NY: John Wylie \& Sons Inc.), 3-9.

Uddin, L. Q. (2015). Salience processing and insular cortical function and dysfunction. Nat. Rev. Neurosci. 16, 55-61. doi: 10.1038/nrn3857

Wei, D., Yang, J., Li, W., Wang, K., Zhang, Q., and Qiu, J. (2014). Increased resting functional connectivity of the medial prefrontal cortex in creativity by means of cognitive stimulation. Cortex 51, 92-102. doi: 10.1016/j.cortex.2013. 09.004

Zmigrod, S., Colzato, L. S., and Hommel, B. (2015). Stimulating creativity: modulation of convergent and divergent thinking by transcranial direct current stimulation (tDCS). Creat. Res. J. 27, 353-360. doi: 10.1080/10400419.2015. 1087280

Conflict of Interest Statement: The authors declare that the research was conducted in the absence of any commercial or financial relationships that could be construed as a potential conflict of interest.

Copyright (C) 2018 Lucchiari, Sala and Vanutelli. This is an open-access article distributed under the terms of the Creative Commons Attribution License (CC BY). The use, distribution or reproduction in other forums is permitted, provided the original author(s) and the copyright owner(s) are credited and that the original publication in this journal is cited, in accordance with accepted academic practice. No use, distribution or reproduction is permitted which does not comply with these terms. 
OPEN ACCESS

Edited by:

Claudio Lucchiari,

Università degli Studi di Milano, Italy

Reviewed by:

Mark Schram Christensen University of Copenhagen, Denmark

Nicholas J. Kelley,

Northwestern University,

United States

*Correspondence:

Davide Crivelli

davide.crivelli@unicatt.it

Received: 31 August 2017 Accepted: 06 November 2017

Published: 20 November 2017

Citation:

Crivelli D and Balconi M (2017) The Agent Brain: A Review of

Non-invasive Brain Stimulation

Studies on Sensing Agency.

Front. Behav. Neurosci. 11:229.

doi: 10.3389/fnbeh.2017.00229

\section{The Agent Brain: A Review of Non-invasive Brain Stimulation Studies on Sensing Agency}

\author{
Davide Crivelli ${ }^{1,2 *}$ and Michela Balconi ${ }^{1,2}$ \\ ${ }^{1}$ Research Unit in Affective and Social Neuroscience, Catholic University of the Sacred Heart, Milan, Italy, ${ }^{2}$ Department \\ of Psychology, Catholic University of the Sacred Heart, Milan, Italy
}

According to philosophy of mind and neuroscientific models, the sense of agency can be defined as the sense that I am the one that is generating an action and causing its effects. Such ability to sense ourselves as causal agents is critical for the definition of intentional behavior and is a primary root for human interaction skills. The present mini-review aims at discussing evidences from non-invasive brain stimulation (NIBS) studies targeting functional correlates of different aspects of agency and evidences on the way stimulation techniques affect such core feature of human subjective experience. Clinical and brain imaging studies helped in defining a neural network mediating agency-related processes, which includes the dorsolateral prefrontal cortex (dIPFC), the cingulate cortex (CC), the supplementary and pre-supplementary motor areas (SMA and pre-SMA), the posterior parietal cortex (PPC) and its inferior regions and the cerebellum. However, while the plurality of those structures mirrors the complexity of the phenomenon, their actual roles with respect to different components of the experience of agency have been primarily explored via correlational techniques, without a clear evidence about their causal significance with respect to the integration of sensorimotor information, intentionalization, and action monitoring processes. Therefore, insights into the specific causal role of different cortical structures can be specified by using NIBS techniques, in order to provide improved understanding into the bases of our ability vs. inability to properly act in complex social contexts.

Keywords: sense of agency, NIBS, TMS, tES, tDCS, social understanding, body ownership

\section{INTRODUCTION}

When we do act and observe the consequences of our action, we tend to be sure that we were the ones who were acting. That kind of awareness mirrors the common experience of being an agent. That feeling - together with unified subjective experiences and with the first-person point-of-view from where we perceive and qualify those experiences-is what defines human self-consciousness (Metzinger, 2003). The feeling of being agents able to influence our environment is one of the main roots that sustain and support that being-in-the-world. Our representations of the context, of ourselves and of their relationship play, in turn, a fundamental role for the definition of our agentive stance (Haggard, 2008, 2017; Synofzik et al., 2008b; Carruthers, 2009; Balconi, 2010a,b; Frith, 2013; Smith, 2016).

The richness of debates on the experience of being an agent and its physiological, other than phenomenological, correlates have been fuelled by a remarkable set of evidences obtained via neuroscientific techniques such as neuroimaging, electrophysiology and brain stimulation. The majority of data concerning neural structures supporting the components of the experience 
of agency have been, to date, primarily explored via correlational techniques, such as functional imaging (David, 2010). Differently, non-invasive brain stimulation (NIBS) techniques can provide insight concerning the specific causal role of different cortical structures with regard to a mental process or a specific function, and then foster our understanding of the bases of our ability vs. inability to sense and judge the authorship of our behavior and its outcomes. After an introduction on core aspects of the construct of agency and on the neural network that is thought to let us experience agency, we will critically compare the potential of correlational and different brain stimulation techniques as investigation tools. Finally, we will focus on NIBS studies targeting functional correlates of different aspects of agency and evidences on the way stimulation techniques affect such core feature of human subjective experience.

\section{AGENCY AS A CORE FEATURE OF HUMAN EXPERIENCE}

Here, we define the sense of agency as the sense that I am the one that is generating an action, would it be physical or mental, and causing its effects (Gallagher, 2000). Such theoretical construct is deeply bound to the one of sense of ownership, which can, instead, be defined as the sense that I am the one who is undergoing an experience. Nonetheless, the sense of agency and the sense of ownership can be experienced as separate entities and can be independently affected by experimental manipulation and pathology. Neurological and psychiatric disorders, such as the alien hand syndrome and schizophrenia, can be, for example, associated to ownership and agency disruption (Jahanshahi, 1998; Bottini et al., 2002; Synofzik et al., 2008b; Jardri et al., 2009; Jeannerod, 2009; Synofzik and Voss, 2010).

Human experience is shaped by the potential to be aware of the causal power we can exert on the external world and by self-attribution of a primary agentive role with regard to our actions (from its simpler manifestations like basic goal-directed motor acts to its more complex manifestations like moral reasoning and reflective judgments concerning responsibilities). Accordingly, modern phenomenology posits that experience is grounded "in neural activity in embodied action in appropriate surroundings" (Smith, 2016). Assuming that agency is core feature for human experience (Metzinger, 2003; Haggard, 2008; Smith, 2016), it has also been suggested that in the very moment we sense ourselves as intentional agents and we consciously self-attribute an agentive stance, we have to assume that other individuals might share the same capability and a similar stance. Further, besides being able to sense and judge the authorship of our behavior and its outcomes, we also need to be able to apply those detection and attribution processes to other potential inter-agents. Those skills are critical to adequately act in a social context and to intentionally exert control on a social interactions (see Crivelli and Balconi, 2010, 2015; Crivelli, 2016), would them be dyadic or collective-such as in team sports. Again they might act as the soil where higher social understanding skills such as inter-personal co-regulation and mind-reading sink their roots (Gallagher and Meltzoff, 1996; Saxe, 2006).
Agency is, then, a complex phenomenon influenced by intention, goals and desires but also grounded on somatosensory signals and afferent sensory information flow. The integration of different levels is necessary for the rising of a complete experience. Body image and intentional structure merge to generate unified agents capable of perceiving, influencing, and exerting causal power on each other and on the environment, understood as a complex system including both objects to act with and subjects to interact with.

\section{THE AGENT BRAIN}

Many neural structures have been associated to specific attributes of agency experience or to specific steps of the process that leads to sense agency. The collection of neural structures that are thought to mediate agency-related processes is rather wide and includes: the dorsolateral prefrontal cortex (dlPFC), the cingulate cortex (CC), the supplementary and pre-supplementary motor areas (SMA and pre-SMA), the posterior parietal cortex (PPC) and its inferior regions and the cerebellum (Gehring et al., 1990; Lee et al., 1999; Blakemore et al., 2001; Chaminade and Decety, 2002; Cunnington et al., 2002; Farrer and Frith, 2002; Blakemore and Sirigu, 2003; Farrer et al., 2003; Lau et al., 2004, 2006; Synofzik et al., 2008a; Balconi and Crivelli, 2009, 2010a,b; Balconi and Scioli, 2012; Balconi et al., 2017). The plurality of those structures and their distribution over the whole brain (see Figure 1) likely mirrors the complexity of the phenomenon and the different methodological and experimental approaches devised to study its facets (for a review see also David, 2010). Again, they are due to the contribution of multiple mechanisms in the coupling of behavior with mental states and sensory effects. Those mechanisms-and the structures that subserve them-can be traced back to overarching functions: monitoring of sensorimotor congruence and multimodal integration, intentionalization (i.e., elaboration and implementation of intentions), action monitoring and ownership/agency attribution.

\section{STIMULATING THE AGENT BRAIN: NON-INVASIVE BRAIN STIMULATION EVIDENCES}

Neuroimaging and electrophysiological methods can be both enlisted among the correlational techniques (Walsh and Cowey, 2000), which allow for qualifying and quantifying ongoing neural activity during implicit or explicit tasks to compare it with co-occurrent subjective experience, cognitive performances, or behavior. By superimposing and integrating those series of data, it is possible to draw conclusions on anatomical-functional correlates of investigated functions and processes by means of association. Conversely, non-invasive stimulation methods can be enlisted among interference or causal techniques, which grant the advantage of drawing conclusion on neural causation and on the effective role of neural structures in supporting or modulating a specific function or process (Woods et al., 2016). Indeed, NIBS can be used to perturb the ongoing activity of a target structure during implicit or explicit tasks and then 


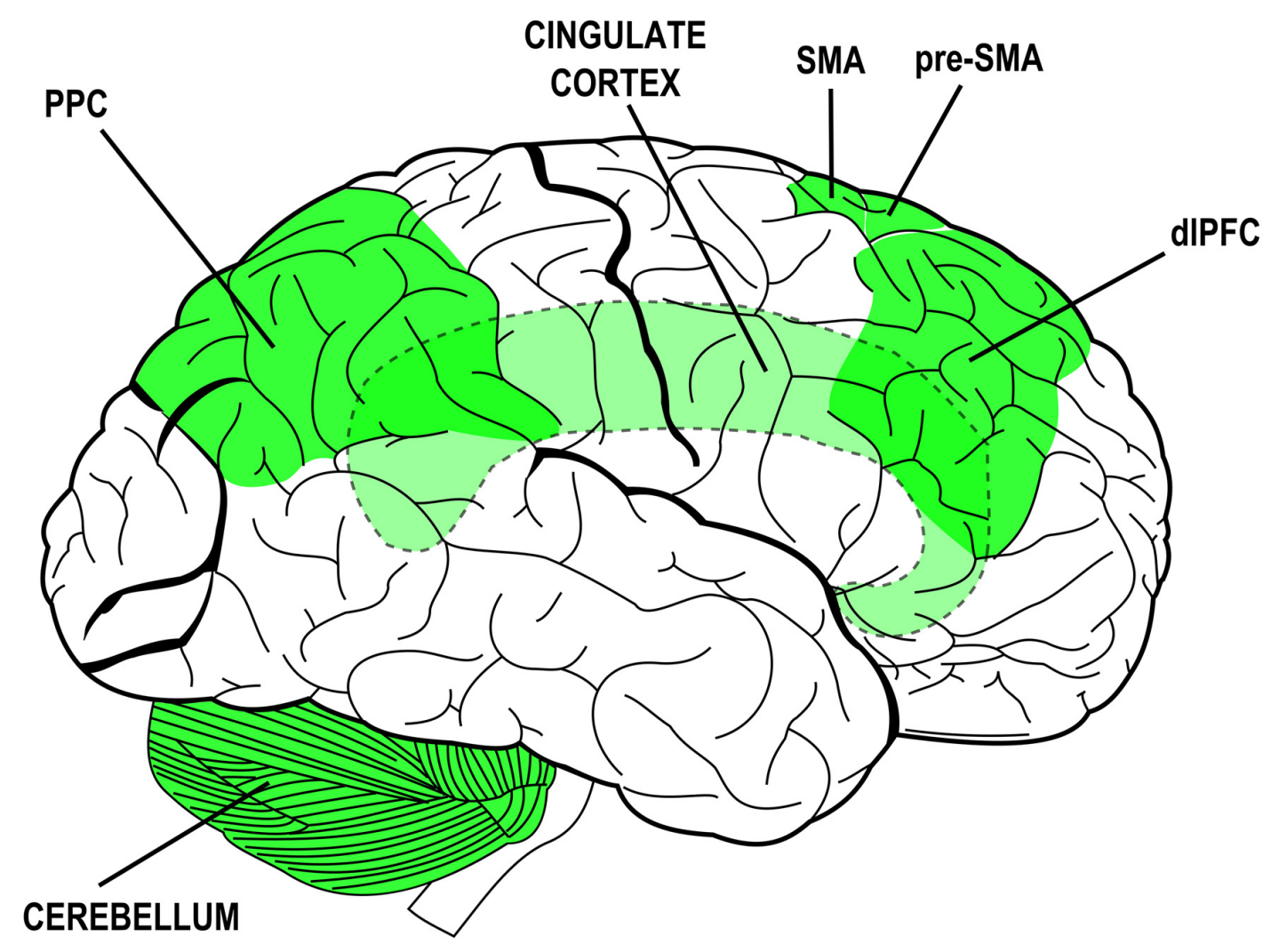

FIGURE 1 | Brain structures associated to functions and processes subserving the experience of agency and their distribution over the brain (lateral view). Simplified schematic representation. Dashed outlines and lighter colors indicate subcortical or hidden structures. PPC, posterior parietal cortex; SMA, supplementary motor area; pre-SMA, pre-supplementary motor area; dIPFC, dorsolateral prefrontal cortex.

observe the consequences of such perturbation on behavior and/or neural activity (e.g., by means of EEG). It is worth noting that conclusions that can be drawn thanks to NIBS studies also show fewer potential biases than those deriving from clinical lesion studies (Walsh and Cowey, 2000). Relevant for the present discussion, NIBS techniques then present notably greater cognitive resolution-defined as the ability to tell something new about brain processes and to answer a wide range of questions on cognitive functioning and its physiological correlates (Walsh and Pascual-Leone, 2003) - with respect to other investigation tools.

Within the panorama of NIBS, two main families of techniques have been used in research on agency: transcranial magnetic stimulations (TMS) and transcranial electrical stimulations (tES). Both TMS and tES inform us on brain functioning by interfering with neural activity, but they are thought to do so in different ways. In particular, TMS is deemed as a neurostimulation tool in that it is able to actually initiate action potentials in a nerve cell or axon by inducing non-invasively transient electrical currents at the level of cortical tissues. The mechanism of action grounds on the Faraday's law of electromagnetic induction and stimulation is induced by brief magnetic pulses delivered through a coil. Instead, tES (with lowercase $t$ so to refer to techniques using low-intensity electrical stimulation, instead of high-voltage TES) is deemed as a neuromodulation tool in that it is able to modulate spontaneous neuronal firing rates by causing small changes in the membrane potential of the nerve cells or axons (Woods et al., 2016). The mechanism of action has been linked to long-term potentiation/depression mechanisms, even though the integration of the stimulation effects with a second input (e.g., neural oscillatory activity and thalamo-cortical stimulation) is thought to be needed to induce such phenomena (Fritsch et al., 2010). TMS and tES also differ in terms of spatial and time resolution and in terms of usability and side effects. TMS, when used in single-pulse or paired-stimulation protocols, show greater time resolution than tES applications and can then be more aptly used to investigate mental chronometry. Again, while TMS applications allow for stimulating quite focal portions of the cortical tissues, tES is able to modulate only broader populations of neurons or to influence transmission of information in neural networks. At the same time, tES applications lead to fewer unwanted side effects and are less noisy than magnetic stimulations, thus exerting minimal disturbance during task execution. The one technique or the other has then been chosen in different studies depending on the research question and technical-methodological needs.

The few NIBS investigations on the neural processes and mechanisms that make us sense agency essentially focused on the role of parietal and prefrontal areas with regard to sensorimotor integration, to the link between intentions and 
behavior, and to ownership definition processes underlying agency attribution.

\section{NIBS, Sensorimotor Congruence and Agency}

There are evidences that offline low-frequency repetitive TMS - a stimulation protocol thought to have inhibition effects on the activity of stimulated portions of cortical tissues-applied to the left PPC, lead to an impairment in assessing asynchrony between a movement and its visual feedback, specifically when the movement is active and voluntary (MacDonald and Paus, 2003). At the same time, online high-frequency repetitive TMS $(10 \mathrm{~Hz})$ applied to the right inferior parietal cortex proved to induce healthy participants to misperceive their agentive role during self-controlled movements and to experience them as being externally perturbed even if it was not the case (RitterbandRosenbaum et al., 2014).

The critical role of parietal structures for the integration and comparison of sensorimotor information flows to correctly attribute movements agency has been underlined even by using non-repetitive magnetic stimulation protocols. Preston and Newport (2008), indeed, observed that when the activity of right inferior parietal lobule (IPL) is temporarily and focally tampered, healthy participants tend to attribute agency to external sources, often erroneously, regardless of the fact that perceivable visual feedbacks are consistent with executed actions or not. Again, single-pulse TMS has been recently used to explore the role of left inferior parietal areas in prospective components of the sense of agency, linked to action selection and programming processes independently from action effects (Chambon et al., 2015). By combining TMS with subliminal priming of action selection, the authors showed that the perturbation of the activity of left inferior parietal regions at the time of action selection and execution reduces participants' perceived control over subsequent action effects. The importance of parietal/pre-motor connections to solve sensorimotor conflicts and finalize agencyattribution processes has been underlined even by Karabanov et al. (2017) by using a paired-pulse stimulation protocol. The authors, by implementing a motor version of the rubber hand illusion, observed similar inhibitory parietal-to-motor connectivity at rest and during illusory conditions inducing both sense of agency and ownership.

\section{NIBS, Action Control and Agency}

To our best knowledge, the few NIBS investigations of the causal link between prefrontal action control and intentionalization processes and agency, used electrical stimulation techniques to modulate cortical activity and intentional binding to implicitly measure changes of the sense of agency. The intentional binding effect has to do with the reduction of perceived time interval between a voluntary action and its external sensory consequence when we do sense to be the primary agent of such action (Tsakiris and Haggard, 2003).

In particular, a recent meta-analysis of previously performed studies, highlighted that anodal stimulation-a continuous current electrical stimulation protocol thought to enhance cortical excitability and responsivity-applied to left dlPFC increases the intentional binding effect and, thus, implicit sense of agency, but only when people are free to act as they prefer (Khalighinejad et al., 2016). Again, even pre-SMA seems to contribute to implicit feeling of being an agent, thought results still need to be examined in depth. Indeed, Cavazzana et al. (2015) showed that both anodal and cathodal stimulation-a stimulation protocol thought to lower cortical excitability and responsivity-of pre-SMA areas leads to a relevant reduction of the intentional binding effect, hinting at the contribution of a medial frontal-prefrontal network for awareness and control of voluntary action. Finally, the perception of the temporal relationship between voluntary action and its perceivable consequences seems to be negatively affected by anodal stimulation of the left angular gyrus, critical for explicit agency judgments (Khalighinejad and Haggard, 2015).

A few final works did report studies where NIBS has not been properly used as an investigation technique but as a tool to create ad hoc experimental conditions. Namely, in the next two examples, TMS has been used to make participants move involuntarily and to deliver a complex action feedback. In the first case, the phenomenon of sensory attenuation linked to self-initiated actions and its contribution to differentiation of one's own from others' actions have been investigated by comparing neural responses to perceivable consequences (sounds) of voluntary and TMS-induced actions (Timm et al., 2014). The second case has to do with one of the first empirical investigation of the phenomenon of intentional binding. TMS has been used to deliver a complex somatosensory feedback (a muscular twitch) as a consequence of voluntary (self-initiated finger movements) and involuntary (passive finger movements caused by an external device) actions (Tsakiris and Haggard, 2003).

\section{NIBS, Multimodal Integration, Agency and Body Ownership}

Empirical investigation of visual-tactile-proprioceptive integration via NIBS globally highlighted the role of inferior parietal areas and higher visual areas for a correct self-attribution of body ownership. The mostly used experimental paradigm to investigate neural and phenomenological correlates of body ownership is the rubber hand illusion-RHI (Botvinick and Cohen, 1998). Each of the few NIBS studies specifically targeting neural basis of body ownership attribution used magnetic stimulation techniques. Namely, in a recent repetitive TMS study, it has been reported that it is possible to strengthen the illusory experience by applying an inhibitory interference to the left extrastriate body area -EBA (Wold et al., 2014). The authors concluded that EBA is involved in multimodal integration for the definition of the experience of body ownership. The body ownership illusion seems, instead, to be weakened by the inhibitory stimulation of the IPL. Low-frequency repetitive stimulation applied to left IPL was indeed found to reduce the relocation of the real hand toward the fake one, even if only immediately after the RHI induction (Kammers et al., 2009). The role of inferior parietal areas-namely, the right temporo-parietal junction, TPJ-was corroborated even by a single-pulse TMS investigation (Tsakiris et al., 2008). By perturbing the activity 
of right TPJ after brief repeated RHI-inducing stimulations, the authors observed a reduction of the embodiment of the fake hand, as indirectly measured by a reduction of the proprioceptive drift towards the real hand. Processes mediated by inferior parietal areas seem then critical for the definition of the boundaries of our bodies and for the distinction of such objects from external objects and other agents.

\section{CONCLUSION}

To sum up, NIBS studies mainly focused on the investigation of selected parietal and prefrontal structures, following previous neuroimaging evidences. Nonetheless, a few critical points are worth further discussion.

First, published reports of NIBS investigations are still limited and evidences in literature are fragmented and sometimes contrasting. One of the consequent main limitations has to do with the fact that different NIBS techniques have been used to investigate different function or structures. Physical unwanted effects of TMS are, indeed, usually reported to become more intense and bothering when moving from posterior to anterior areas. tES, instead, can be more comfortably used to stimulate anterior structures since it shows less unwanted effects. Still, the two families of techniques also differ, as above-discussed, in terms of cognitive, spatial, and time resolution. Future research should, then, take advantage of those differences to single out and better understand different facets and features of the agency experience.

Again, NIBS research on agency might benefit from a systematization of methods and measures. Even though the experience of agency pervades our everyday activities, its complex and multi-level nature lead to the development of different elegant experimental design. Nonetheless, a critical discussion on potential indices and measures to qualify and

\section{REFERENCES}

Balconi, M. (2010a). Neuropsychology of the Sense of Agency. New York, NY: Nova Science Publishers.

Balconi, M. (Ed.). (2010b). Neuropsychology of the Sense of Agency. From Consciousness to Action. Milano: Springer-Verlag.

Balconi, M., and Crivelli, D. (2009). Spatial and temporal cognition for the sense of agency: neuropsychological evidences. Cogn. Process. 10, S182-S184. doi: 10.1007/s10339-009-0281-z

Balconi, M., and Crivelli, D. (2010a). FRN and P300 ERP effect modulation in response to feedback sensitivity: the contribution of punishment-reward system (BIS/BAS) and behaviour identification of action. Neurosci. Res. 66, 162-172. doi: 10.1016/j.neures.2009.10.011

Balconi, M., and Crivelli, D. (2010b). Veridical and false feedback sensitivity and punishment-reward system (BIS/BAS): ERP amplitude and theta frequency band analysis. Clin. Neurophysiol. 121, 1502-1510. doi: 10.1016/j.clinph.2010. 03.015

Balconi, M., Crivelli, D., and Bove, M. (2017). 'Eppur si move': the association between electrophysiological and psychophysical signatures of perceived movement illusions. J. Mot. Behav. doi: 10.1080/00222895.2016.1271305 [Epub ahead of print].

Balconi, M., and Scioli, S. (2012). Error monitoring functions in response to an external feedback when an explicit judgement is required: ERP modulation and cortical source localisation. Int. J. Psychophysiol. 83, 295-301. doi: 10.1016/j. ijpsycho.2011.11.005 quantify different aspects of sensing agency may help to reduce the fragmentation of empirical findings. Here we began to suggest a potential systematization of empirical findings according to three main facets of research on agency: coding and monitoring of sensorimotor congruence during action, implementation of motor intentions and action control, multimodal integration for self-attribution of body ad action ownership. Such systematization remains valid even if the analysis of present NIBS literature is guided by a criterion concerning experimental methods. Indeed, studies related to the first topic are globally based on visuomotor matching and action attribution paradigms. Studies related to the second topic are essentially based on the intentional binding protocol. Finally, studies related to the third topic are basically based on the RHI protocol. Furthermore, it is worth noting that the vast majority of NIBS investigation of how we sense agency was grounded in indirect and implicit measures of the phenomenon, with no systematic and clear evidence for explicit measures concerning the aware reflexive level of such experience. A systematization might then contribute to find new ways to overcome such methodological limitation and to better understand all the facets of the agency experience, as well as the actual causal role of different cortical structures in relation to both prereflexive/implicit and reflexive/explicit agency-related processes.

\section{AUTHOR CONTRIBUTIONS}

Both DC and MB substantially contributed to the conception of the work and to drafting the work or revising it critically for important intellectual content. Both DC and MB gave their final approval of the version to be published and agreed to be accountable for all aspects of the work in ensuring that questions related to the accuracy or integrity of any part of the work are appropriately investigated and resolved.

Blakemore, S.-J., Frith, C. D., and Wolpert, D. M. (2001). The cerebellum is involved in predicting the sensory consequences of action. Neuroreport 12 , 1879-1884. doi: 10.1097/00001756-200107030-00023

Blakemore, S.-J., and Sirigu, A. (2003). Action prediction in the cerebellum and in the parietal lobe. Exp. Brain Res. 153, 239-245. doi: 10.1007/s00221-0031597-z

Bottini, G., Bisiach, E., Sterzi, R., and Vallar, G. (2002). Feeling touches in someone else's hand. Neuroreport 13, 249-252. doi: 10.1097/00001756-20020211000015

Botvinick, M., and Cohen, J. (1998). Rubber hands 'feel' touch that eyes see. Nature 391:756. doi: 10.1038/35784

Carruthers, G. (2009). Commentary on synofzik, vosgerau and newen 2008. Conscious. Cogn. 18, 515-520. doi: 10.1016/j.concog.2008.05.006

Cavazzana, A., Penolazzi, B., Begliomini, C., and Bisiacchi, P. S. (2015). Neural underpinnings of the 'agent brain': new evidence from transcranial direct current stimulation. Eur. J. Neurosci. 42, 1889-1894. doi: 10.1111/ejn.12937

Chambon, V., Moore, J. W., and Haggard, P. (2015). TMS stimulation over the inferior parietal cortex disrupts prospective sense of agency. Brain Struct. Funct. 220, 3627-3639. doi: 10.1007/s00429-014-0878-6

Chaminade, T., and Decety, J. (2002). Leader or follower? Involvement of the inferior parietal lobule in agency. Neuroreport 13, 1975-1978. doi: 10.1097/00001756-200210280-00029

Crivelli, D. (2016). Electrophysiological correlates of social information processing for detecting agents in social interaction scenes: P200 and N250 components. Neuropsychol. Trends 19, 45-69. doi: 10.7358/neur-2016-019-criv 
Crivelli, D., and Balconi, M. (2010). "Agency and inter-agency, action and joint action: theoretical and neuropsychological evidences," in Neuropsychology of the Sense of Agency. From Consciousness to Action, ed. M. Balconi (New York, NY: Springer), 107-122.

Crivelli, D., and Balconi, M. (2015). The "social" and "interpersonal" body in spatial cognition. The role of agency and interagency. Cogn. Process. 16, 193-196. doi: 10.1007/s10339-015-0695-8

Cunnington, R., Windischberger, C., Deecke, L., and Moser, E. (2002). The preparation and execution of self-initiated and externally-triggered movement: a study of event-related fMRI. Neuroimage 15, 373-385. doi: 10.1006/nimg. 2001.0976

David, N. (2010). "Functional anatomy of the sense of agency: past evidence and future directions," in Neuropsychology of the Sense of Agency. From Consciousness to Action, ed. M. Balconi (New York, NY: Springer), 69-80.

Farrer, C., Franck, N., Georgieff, N., Frith, C. D., Decety, J., and Jeannerod, M. (2003). Modulating the experience of agency: a positron emission tomography study. Neuroimage 18, 324-333. doi: 10.1016/S1053-8119(02)00041-1

Farrer, C., and Frith, C. D. (2002). Experiencing oneself vs. another person as being the cause of an action: the neural correlates of the experience of agency. Neuroimage 15, 596-603. doi: 10.1006/nimg.2001.1009

Frith, C. D. (2013). The psychology of volition. Exp. Brain Res. 229, 289-299. doi: 10.1007/s00221-013-3407-6

Fritsch, B., Reis, J., Martinowich, K., Schambra, H. M., Ji, Y., Cohen, L. G., et al. (2010). Direct current stimulation promotes BDNF-dependent synaptic plasticity: potential implications for motor learning. Neuron 66, 198-204. doi: 10.1016/j.neuron.2010.03.035

Gallagher, S. (2000). Philosophical conceptions of the self: implications for cognitive science. Trends Cogn. Sci. 4, 14-21. doi: 10.1016/s13646613(99)01417-5

Gallagher, S., and Meltzoff, A. N. (1996). The earliest sense of self and others: Merleau-Ponty and recent developmental studies. Philos. Psychol. 9, 211-233. doi: 10.1080/09515089608573181

Gehring, W. J., Coles, M. G. H., Meyer, D. E., and Donchin, E. (1990). The error-related negativity: an event-related brain potential accompanying errors. Psychophysiology 27:s34.

Haggard, P. (2008). Human volition: towards a neuroscience of will. Nat. Rev. Neurosci. 9, 934-946. doi: 10.1038/nrn2497

Haggard, P. (2017). Sense of agency in the human brain. Nat. Rev. Neurosci. 18, 196-207. doi: 10.1038/nrn.2017.14

Jahanshahi, M. (1998). Willed action and its impairments. Cogn. Neuropsychol. 15, 483-533. doi: 10.1080/026432998381005

Jardri, R., Delevoye-Turrell, Y., Lucas, B., Pins, D., Bulot, V., Delmaire, C., et al. (2009). Clinical practice of rTMS reveals a functional dissociation between agency and hallucinations in schizophrenia. Neuropsychologia 47, 132-138. doi: 10.1016/j.neuropsychologia.2008.08.006

Jeannerod, M. (2009). The sense of agency and its disturbances in schizophrenia: a reappraisal. Exp. Brain Res. 192, 527-532. doi: 10.1007/s00221-008-1533-3

Kammers, M. P. M., Verhagen, L., Dijkerman, H. C., Hogendoorn, H., De Vignemont, F., and Schutter, D. J. (2009). Is this hand for real? attenuation of the rubber hand illusion by transcranial magnetic stimulation over the inferior parietal lobule. J. Cogn. Neurosci. 21, 1311-1320. doi: 10.1162/jocn.2009.21095

Karabanov, A. N., Ritterband-Rosenbaum, A., Christensen, M. S., Siebner, H. R., and Nielsen, J. B. (2017). Modulation of fronto-parietal connections during the rubber hand illusion. Eur. J. Neurosci. 45, 964-974. doi: 10.1111/ejn.13538

Khalighinejad, N., Di Costa, S., and Haggard, P. (2016). Endogenous action selection processes in dorsolateral prefrontal cortex contribute to sense of agency: a meta-analysis of tDCS studies of 'Intentional Binding'. Brain Stimul. 9, 372-379. doi: 10.1016/j.brs.2016.01.005

Khalighinejad, N., and Haggard, P. (2015). Modulating human sense of agency with non-invasive brain stimulation. Cortex 69, 93-103. doi: 10.1016/j.cortex. 2015.04.015

Lau, H. C., Rogers, R. D., Haggard, P., and Passingham, R. E. (2004). Attention to intention. Science 303, 1208-1210. doi: 10.1126/science.1090973

Lau, H. C., Rogers, R. D., and Passingham, R. E. (2006). On measuring the perceived onsets of spontaneous actions. J. Neurosci. 26, 7265-7271. doi: 10.1523/JNEUROSCI.1138-06.2006
Lee, K.-M., Chang, K.-H., and Roh, J.-K. (1999). Subregions within the supplementary motor area activated at different stages of movement preparation and execution. Neuroimage 9, 117-123. doi: 10.1006/nimg. 1998.0393

MacDonald, P. A., and Paus, T. (2003). The role of parietal cortex in awareness of self-generated movements: a transcranial magnetic stimulation study. Cereb. Cortex 13, 962-967. doi: 10.1093/cercor/13.9.962

Metzinger, T. (2003). Being No One: The Self-Model Theory of Subjectivity. Cambridge, MA: MIT Press.

Preston, C., and Newport, R. (2008). Misattribution of movement agency following right parietal TMS. Soc. Cogn. Affect. Neurosci. 3, 26-32. doi: $10.1093 /$ scan/nsm036

Ritterband-Rosenbaum, A., Karabanov, A. N., Christensen, M. S., and Nielsen, J. B. (2014). $10 \mathrm{~Hz}$ rTMS over right parietal cortex alters sense of agency during self-controlled movements. Front. Hum. Neurosci. 8:471. doi: 10.3389/fnhum. 2014.00471

Saxe, R. (2006). Uniquely human social cognition. Curr. Opin. Neurobiol. 16, 235-239. doi: 10.1016/j.conb.2006.03.001

Smith, D. W. (2016). "Phenomenology," in The Stanford Encyclopedia of Philosophy, ed. E. N. Zalta. Available online at: https://plato.stanford.edu/ archives/win2016/entries/phenomenology/

Synofzik, M., Lindner, A., and Thier, P. (2008a). The cerebellum updates predictions about the visual consequences of one's behavior. Curr. Biol. 18, 814-818. doi: 10.1016/j.cub.2008.04.071

Synofzik, M., Vosgerau, G., and Newen, A. (2008b). I move, therefore I am: a new theoretical framework to investigate agency and ownership. Conscious. Cogn. 17, 411-424. doi: 10.1016/j.concog.2008.03.008

Synofzik, M., and Voss, M. (2010). "Disturbances of the sense of agency in schizophrenia," in Neuropsychology of the Sense of Agency. From Consciousness to Action, ed. M. Balconi (New York, NY: Springer), 145-155.

Timm, J., SanMiguel, I., Keil, J., Schröger, E., and Schönwiesner, M. (2014). Motor intention determines sensory attenuation of brain responses to self-initiated sounds. J. Cogn. Neurosci. 26, 1481-1489. doi: 10.1162/jocn_a_ 00552

Tsakiris, M., Costantini, M., and Haggard, P. (2008). The role of the right temporo-parietal junction in maintaining a coherent sense of one's body. Neuropsychologia 46, 3014-3018. doi: 10.1016/j.neuropsychologia.2008. 06.004

Tsakiris, M., and Haggard, P. (2003). Awareness of somatic events associated with a voluntary action. Exp. Brain Res. 149, 439-446. doi: 10.1007/s00221-0031386-8

Walsh, V., and Cowey, A. (2000). Transcranial magnetic stimulation and cognitive neuroscience. Nat. Rev. Neurosci. 1, 73-79. doi: 10.1038/350 36239

Walsh, V., and Pascual-Leone, A. (2003). Transcranial Magnetic Stimulation: A Neurochronometrics of Mind. Cambridge, MA: The MIT Press.

Wold, A., Limanowski, J., Walter, H., and Blankenburg, F. (2014). Proprioceptive drift in the rubber hand illusion is intensified following $1 \mathrm{~Hz}$ TMS of the left EBA. Front. Hum. Neurosci. 8:390. doi: 10.3389/fnhum.2014. 00390

Woods, A. J., Antal, A., Bikson, M., Boggio, P. S., Brunoni, A. R., Celnik, P., et al. (2016). A technical guide to tDCS and related non-invasive brain stimulation tools. Clin. Neurophysiol. 127, 1031-1048. doi: 10.1016/j.clinph. 2015.11.012

Conflict of Interest Statement: The authors declare that the research was conducted in the absence of any commercial or financial relationships that could be construed as a potential conflict of interest.

Copyright (C) 2017 Crivelli and Balconi. This is an open-access article distributed under the terms of the Creative Commons Attribution License (CC BY). The use, distribution or reproduction in other forums is permitted, provided the original author(s) or licensor are credited and that the original publication in this journal is cited, in accordance with accepted academic practice. No use, distribution or reproduction is permitted which does not comply with these terms. 


\section{Stimulating Self-Regulation: A Review of Non-invasive Brain Stimulation Studies of Goal-Directed Behavior}

\author{
Nicholas J. Kelley ${ }^{1 *}$, Alessia Gallucci ${ }^{2}$, Paolo Riva ${ }^{2}$, Leonor Josefina Romero Lauro ${ }^{2}$ and \\ Brandon J. Schmeichel ${ }^{3}$ \\ ' Department of Psychology, Northwestern University, Evanston, IL, United States, ${ }^{2}$ Department of Psychology, University \\ of Milano-Bicocca, Milan, Italy, ${ }^{3}$ Department of Psychological and Brain Sciences, Texas A\&M University, College Station, \\ TX, United States
}

Self-regulation enables individuals to guide their thoughts, feelings, and behaviors in a purposeful manner. Self-regulation is thus crucial for goal-directed behavior and contributes to many consequential outcomes in life including physical health, psychological well-being, ethical decision making, and strong interpersonal relationships. Neuroscientific research has revealed that the prefrontal cortex plays a central role in self-regulation, specifically by exerting top-down control over subcortical regions involved in reward (e.g., striatum) and emotion (e.g., amygdala). To orient readers, we first offer a methodological overview of tDCS and then review experiments using non-invasive brain stimulation techniques (especially transcranial direct current stimulation) to target prefrontal brain regions implicated in self-regulation. We focus on brain stimulation studies of self-regulatory behavior across three broad domains of response: persistence, delay behavior, and impulse control. We suggest that stimulating the prefrontal cortex promotes successful self-regulation by altering the balance in activity between the prefrontal cortex and subcortical regions involved in emotion and reward processing.

Keywords: transcranial direct current stimulation, self-regulation, emotion-regulation, goal-directed behavior, dorsolateral prefrontal cortex

\section{INTRODUCTION}

At least since Walter Mischel's seminal work on delay of gratification (Mischel, 1958; Mischel et al., 1972), the practical and the theoretical implications of self-regulation have been important topics of study in psychological science (Carver and Scheier, 1982; Metcalfe and Mischel, 1999; Muraven and Baumeister, 2000). Self-regulation refers to the conscious and non-conscious processes that enable individuals to guide their thoughts, feelings, and behaviors in a purposeful manner. Self-regulation is crucial for goal-directed behavior and has been related to many consequential outcomes in life including physical and mental health, psychological well-being, ethical decision making, and strong interpersonal relationships. Likewise, failures at self-regulation are thought to contribute to alcohol and drug addiction, personal debt, obesity, and other outcomes that carry both personal and societal costs (for a review, see Vohs and Baumeister, 2016). Because of its consequences for so many crucial outcomes, the scientific study of self-regulation spans many of subfields in psychological science and frequently centers on a relatively simple question: How can we improve self-regulatory abilities? 
One possible answer is by stimulating the brain with electrical current. Self-regulation is typically assessed with laboratory analogs of common challenges encountered in daily life, such as persisting at difficult tasks, choosing between immediate versus more delayed rewards, and managing emotional impulses. These examples represent paradigmatic forms of self-regulation, namely persistence, or the sustained performance of aversive behavior (e.g., tolerating pain, coping with failure); delay behavior, which refers to choices that favor more longterm investments at the expense of short-term gains; and impulse control, which involves the purposeful inhibition of emotive response tendencies. Below we focus on these three paradigmatic forms of self-regulation and review experiments that have used brain stimulation techniques to try to improve them. We then consider inconsistencies in findings, address unresolved questions, and point to new directions for future research.

\section{Neural Correlates of Self-Regulation}

Convergent evidence from social, cognitive, and affective neuroscience research reveals that the interplay between the prefrontal cortex and subcortical threat and reward processing is crucial for self-regulation (for a review see: Heatherton and Wagner, 2011; Kelley et al., 2015b; Berkman, 2017; Wagner and Heatherton, 2017). Numerous studies have associated successful self-regulation with top-down control from the prefrontal cortex over subcortical regions involved in reward and threat processing (e.g., Dambacher et al., 2014; Giuliani et al., 2014; Vijayakumar et al., 2014; Lopez et al., 2017). By contrast, self-regulatory failure occurs when top-down control is diminished or when the balance in activity favors threat and reward systems (e.g., Demos et al., 2012; Wagner and Heatherton, 2012; Wagner et al., 2013; Chester and DeWall, 2014; Lopez et al., 2014; Meyer and Bucci, 2016).

As an illustrative example, in a 40-year longitudinal followup with children who participated in Mischel's seminal delay of gratification work, children who successfully delayed gratification exhibited preferential recruitment of the prefrontal cortex (PFC) during a task requiring inhibitory control as adults. In contrast, children who were unsuccessful at delaying gratification showed preferential recruitment of the ventral striatum in a delay task as adults (Casey et al., 2011). These findings highlight the importance of the balance or relative activity levels in the prefrontal cortex and subcortical regions, respectively, and how shifts in this balance can tip the scales between short-term and long-term goal regulation.

Several models of emotion regulation similarly emphasize the balance in activity between prefrontal and subcortical brain regions. For example, Ochsner and Gross (2007) proposed a seminal model of emotion that distinguishes between bottomup affective processing, mediated by limbic system structures such as amygdala, and top-down appraisal processes that involve prefrontal regions. Success at cognitive reappraisal, a form of emotion regulation that involves generating cool cognitive representations of affective states or changing the meaning of emotional events, is associated with increased activity in dorsomedial and dorsolateral prefrontal cortices, alongside corresponding decreases limbic system (i.e., amygdala) activity.
Another relevant model of emotion regulation highlighted the key role of a cortico-subcortical network consisting in a dorsal system and a ventral system (Phillips, 2003; Phillips et al., 2008). The dorsal system encompasses the hippocampus, the dorsal regions of anterior cingulate gyrus, and the DLPFC and is involved in executive functions, particularly planning, attention control, and effortful regulation of affective states. The ventral system includes the amygdala, the insula, the ventral striatum, the anterior cingulated cortex, the orbitofrontal cortex, and the ventrolateral prefrontal cortex (VLPFC) and is predominantly recruited for the automatic regulation of affective reactions and for the recognition of emotional valence of stimuli.

Consistent with and prior to these models of emotion regulation, Botvinick and colleagues (Cohen et al., 2000; Botvinick et al., 2001, 2004; Shenhav et al., 2013) discussed cortical-subcortical balance as it relates to cognitive control. Cognitive control refers to a constellation of functions that orient cognitive subsystems to perform difficult and novel tasks (Botvinick et al., 2004). Botvinick and colleagues' conflict monitoring hypothesis proposed that response conflict activates cortical brain regions (i.e., the dorsal ACC), thereby signaling the need for conflict resolution to facilitate effective behavior (see MacDonald et al., 2000; Botvinick et al., 2001; Ochsner et al., 2009). The theoretical models reviewed above suggest that regulation of both cognition and emotion is contingent upon the balance in activity between cortical and subcortical regions. Brain stimulation techniques targeting cortical regions may thus influence self-regulation by influencing cortical-subcortical balance.

\section{Overview and Goals}

We propose that non-invasive brain stimulation targeting the prefrontal cortex holds promise for improving human selfregulation. To orient the reader to neuromodulation, we first provide a methodological overview of transcranial direct current stimulation (tDCS) - one of the most popular brain stimulation techniques in psychological science. Then we review behavioral evidence that non-invasive brain stimulation targeting the PFC may enhance three paradigmatic forms of self-regulation: persistence, delay behavior, and impulse control (see Table 1). We conclude our review by highlighting inconsistencies in findings, unresolved questions, and new directions for future research.

\section{A METHODOLOGICAL OVERVIEW OF tDCS}

Effects of weak electrical currents on brain and neuronal function were first described more than two centuries ago (Priori, 2003; Nitsche et al., 2008; Zago et al., 2008). Systematic studies with animals showed the efficacy of inducing modifications, enhancements, or diminutions of cortical activity by delivering weak direct currents to the brains of laboratory rats. Specifically, passing currents through the scalp polarized the brain region beneath the electrodes and altered the firing rate of neurons. These effects were detected immediately after the stimulation and seemed to last beyond the stimulation period (Bindman et al., 
TABLE 1 | Study features, stimulation parameters, and key outcomes of all studies reviewed.

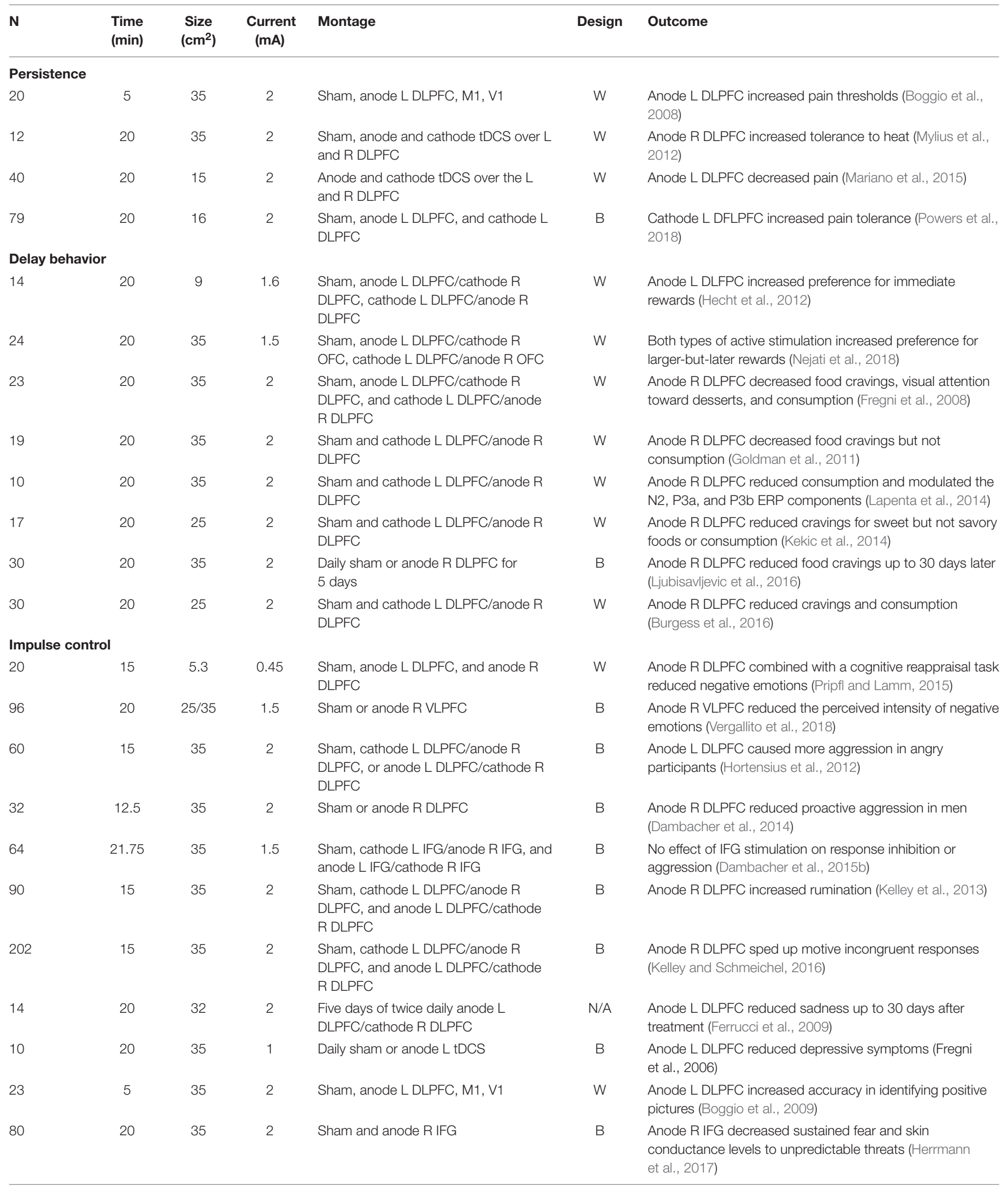




\begin{tabular}{|c|c|c|c|c|c|c|}
\hline $\mathbf{N}$ & $\begin{array}{l}\text { Time } \\
\text { (min) }\end{array}$ & $\begin{array}{c}\text { Size } \\
\left(\mathrm{cm}^{2}\right)\end{array}$ & $\begin{array}{l}\text { Current } \\
(\mathrm{mA})\end{array}$ & Montage & Design & Outcome \\
\hline 45 & 30 & 25 & 1 & $\begin{array}{l}\text { Sham and either cathode L DLPFC/anode R } \\
\text { DLPFC, or anode L DLPFC/cathode R DLPFC }\end{array}$ & W & $\begin{array}{l}\text { Anode L DLPFC improved performance and decreased } \\
\text { cortisol among participants high in ma anxiety (Sarkar et al., } \\
\text { 2014) }\end{array}$ \\
\hline 47 & 20 & 25 & 1 & Sham and anode L DLPFC & $\mathrm{B}$ & $\begin{array}{l}\text { Anode L DLPFC enhanced fear memories (Mungee et al., } \\
\text { 2014) }\end{array}$ \\
\hline 17 & 20 & 25 & 1 & Sham and cathode R DLPFC & $\mathrm{B}$ & $\begin{array}{l}\text { No effect of cathode R DLPFC on fear memories (Mungee } \\
\text { et al., 2016) }\end{array}$ \\
\hline 80 & 20 & $25 / 35$ & 1.5 & Sham and anode R VLPFC & $\mathrm{B}$ & $\begin{array}{l}\text { Anode R VLPFC reduced aggression after social exclusion } \\
\text { (Riva et al., 2012) }\end{array}$ \\
\hline \multirow[t]{2}{*}{79} & 15 & $25 / 35$ & 1.5 & Sham and anode R VLPFC & B & $\begin{array}{l}\text { Anode R VLPFC reduced negative feelings after social } \\
\text { exclusion (Riva et al., 2014a) }\end{array}$ \\
\hline & 20 & $25 / 50$ & 1.5 & Sham and anode R VLPFC & $\mathrm{B}$ & $\begin{array}{l}\text { Anode R VLPFC reduced unproved aggression in } \\
\text { violent-game players (Riva et al., 2017) }\end{array}$ \\
\hline 92 & 15 & 35 & 2 & $\begin{array}{l}\text { Sham, cathode L DLPFC/anode DLPFC, and } \\
\text { anode L DLPFC/cathode R DLPFC }\end{array}$ & $\mathrm{B}$ & $\begin{array}{l}\text { Anode L DLPFC increased jealousy after social exclusion } \\
\text { (Kelley et al., 2015a) }\end{array}$ \\
\hline 16 & 20 & 35 & 1 & Sham and anode L DLPFC & W & $\begin{array}{l}\text { Negative pictures rated less negative after anode tDCS over } \\
\text { the L DLPFC (Peña-Gómez et al., 2011) }\end{array}$ \\
\hline 48 & 20 & $35 / 100$ & 1.5 & Sham and anode R DLPFC & $\mathrm{B}$ & $\begin{array}{l}\text { Anode } R \text { DLPFC enhanced cognitive control during } \\
\text { emotion regulation (Feeser et al., 2014) }\end{array}$ \\
\hline $35 / 12$ & 20 & 35 & 2 & S1: Bilateral DLPFC S2: Unilateral DLPFC & $\mathrm{B}$ & $\begin{array}{l}\text { Bilateral DLPFC tDCS modulated decision making (Fecteau } \\
\text { et al., 2007b) }\end{array}$ \\
\hline 36 & & 35 & 2 & $\begin{array}{l}\text { Sham, cathode L DLPFC/anode DLPFC, and } \\
\text { anode L DLPFC/cathode R DLPFC }\end{array}$ & $\mathrm{B}$ & Anode R DLPFC reduced risk taking (Fecteau et al., 2007a) \\
\hline 16 & 19 & 35 & 2 & $\begin{array}{l}\text { Sham, cathode DLPFC/anode R DLPFC, and } \\
\text { anode L DLPFC/cathode R DLPFC }\end{array}$ & W & $\begin{array}{l}\text { Anode stimulation over the R DLPFC reduced risky } \\
\text { decision-king (Cheng and Lee, 2016) }\end{array}$ \\
\hline 30 & 25 & 25 & 2 & $\begin{array}{l}\text { Twice daily sham or anode R DLPFC/cathode } L \\
\text { DLPFC for } 5 \text { days }\end{array}$ & $\mathrm{B}$ & $\begin{array}{l}\text { Active tDCS paired with the cognitive task reduced } \\
\text { risk-taking This effects persisted } 2 \text { months (Gilmore et al., } \\
\text { 2017) }\end{array}$ \\
\hline 20 & 15 & 25 & 1.5 & Sham or anode R DLPFC/cathode L DLPFC & $\mathrm{B}$ & $\begin{array}{l}\text { Anode R DLPFC caused greater R DLPFC-whole brain } \\
\text { connectivity which was associated with reduced risk-taking } \\
\text { (Wacker et al., 2008) }\end{array}$ \\
\hline 24 & 15 & 35 & 1 & $\begin{array}{l}\text { Anode and cathode R DLPFC or anode and } \\
\text { cathode L DLPFC }\end{array}$ & W & $\begin{array}{l}\text { Anode DLPFC (either } L \text { or } R \text { ) led to reduced risk taking on a } \\
\text { driving simulation (Beeli et al., 2008) }\end{array}$ \\
\hline
\end{tabular}

DLPFC, dorsolateral prefrontal cortex; VLPFC, ventrolateral prefrontal cortex; L, left; $R$, right; $B$, between-subjects; W, within-subjects.

1964; Gartside, 1968; Hattori et al., 1990; Islam et al., 1995). Early research in human samples investigated the application of electrical currents in mood disorders treatment, with some evidence suggesting a reduction in symptoms of depression and mania (Costain et al., 1964; Carney, 1969). But subsequent studies and null findings contributed to skepticism in the efficacy of running weak electrical currents into the brain as an effective tool for symptom reduction (Lolas, 1977; Nitsche et al., 2003b). More recently, researchers have shed light on bidirectional, time, and polarity-dependent excitability changes following tDCS (Priori et al., 1998; Nitsche and Paulus, 2000).

\section{How Does tDCS Work?}

The tDCS device consists of an electric stimulator, which delivers a constant current and an isolation current, linked to a pair of electrodes positioned on the scalp over cortical regions of interest. The electrodes, namely an anode and a cathode, are typically covered by sponges soaked in $\mathrm{NaCI}$ solution (or electrode cream) to increase conductivity, reduce resistance, and improve the homogeneity of the electric field under the electrodes.
Transcranial direct current stimulation differs from other brain stimulation techniques, such as transcranial electrical stimulation (TES) and transcranial magnetic stimulation (TMS), because tDCS does not induce action potentials in neuronal membrane. Instead, tDCS transiently modifies spontaneous neuronal excitability by depolarizing or hyperpolarizing neurons' resting membrane potentials, producing ionic concentration shifts within the extracellular fluid (Creutzfeldt et al., 1962; Purpura and McMurtry, 1965). Anodal stimulation typically depolarizes local neurons, which in turn will require less dendritic input to fire, whilst cathodal stimulation hyperpolarizes neurons' typical resting membrane potentials so that increasing dendritic input is required (Nitsche and Paulus, 2000). This mechanism of action generally occurs both during stimulation and for a short period of time $(<5 \mathrm{~min})$ thereafter.

Transcranial direct current stimulation has been found to involve more complex mechanisms including long-term potentiation (LTP) and long-term depression (LTD) mechanisms at the synaptic level, affecting hyper-communicative activity through the anode and hypo-communicative activity through the cathode. These tDCS-driven changes in LTP and LTD may 
be mediated by a number of synaptic mechanisms including: NMDA ( $N$-methyl-D-aspartate) receptors, GABAergic activity, glutamatergic activity, intracellular CA2 + concentration, brainderived neurotrophic factor (BDNF) secretion, and tropomyosinrelated kinase B (TrkB) activation (Liebetanz et al., 2002; Nitsche et al., 2003a, 2004; Stagg et al., 2009; Fritsch et al., 2010; Stagg and Nitsche, 2011). Also, non-synaptic mechanisms, such as changes in $\mathrm{pH}$ and transmembrane proteins, seem to be involved in long-term effects of tDCS (Ardolino et al., 2005).

\section{Parameters Influencing Stimulation Efficacy}

The efficacy of eliciting changes in brain activity using tDCS depends on several physical parameters including current density, stimulation duration, and the orientation and focality of the electrical field. These parameters constitute the tDCS dosage.

\section{Current Density}

Current density represents the ratio between current strength (normally up to $2 \mathrm{~mA}$ ) and electrode size (normally reference electrode varies from 25 to $35 \mathrm{~cm}^{2}$ ). Current density determines the delivered electrical field strength (Purpura and McMurtry, 1965).

\section{Stimulation Duration}

Stimulation duration refers to the amount of time participants undergo stimulation. It is based on LTP and LTD mechanisms and is related to the occurrence and length of aftereffects. Generally, keeping current density constant, brief exposure to tDCS stimulation (few seconds) does not induce long-lasting effects, whereas tDCS stimulations of about $10 \mathrm{~min}$ (up to $30 \mathrm{~min}$ ) typically do elicit aftereffects (Nitsche et al., 2003c; Ardolino et al., 2005).

\section{Orientation of the Electric Field}

The orientation of the electric field normally depends on electrodes' polarity and position. As already described, tDCS produces polarity-dependent effects whereby anodal stimulation increases the activity of the stimulated area, whereas cathodal stimulation decreases it. Several studies of both the primary motor and visual cortices have found that different electrode positions modulate different neuronal groups and elicit different evoked potentials (Priori et al., 1998; Antal et al., 2004; Accornero et al., 2007) suggesting that electrode position is a crucial tDCS parameter.

Indeed, not only may electrode position affect the amount of current delivered to the brain and the direction of current flow, but also it may determine effects on the targeted brain region, due to electrical field interactions associated with neuronal geometry (Nitsche and Paulus, 2000; Nitsche et al., 2008). Whereas the coupling of anodal-excitatory and cathodal-inhibitory effects is well established in the sensorimotor domain, evidence gets more controversial when addressing higher cognitive functions (Jacobson et al., 2012). This lack of consistency between sensorimotor and cognitive functions is at this output level. It is not that the inconsistencies arise from differences in the effects of stimulation on sensorimotor cortices versus prefrontal cortices themselves. In other words, the inconsistency is not the result in differences in the effects of the stimulation protocol on activity or function but rather the consequence of those changes. Indeed, when dealing with more complex functions, likely represented by large and interconnected neural networks comprising both excitatory and inhibitory connections, it is more difficult to obtain a predictable outcome, hence it is not always the case that anodal tDCS leads to an enhancement (e.g., better performance in a task) and cathodal tDCS leads to a diminution of the assessed cognitive function (Fertonani and Miniussi, 2017).

\section{Induced Electric Field Focality}

Another important tDCS parameter is the induced electric field focality. Generally, large electrodes and bipolar scalp electrode arrangements limit tDCS focality (Gandiga et al., 2006), in part because large electrodes may alter activity in areas adjacent to the stimulated region. Moreover, with an intracephalic montage, the so-called reference electrode (the secondary electrode with regard to a specific experimental setting), being located on the scalp, is not entirely inert. The lack of spatial focality of tDCS effects suggests the need for arrangements to increase focality. For instance, to increase tDCS focality it is possible to use smaller target electrodes. Another option is to increase the reference electrode size so that, due to decreased current density, it becomes practically inert. Alternatively, using an extracephalic montage may increase stimulation focality (Nitsche et al., 2007, 2008; Ferrucci et al., 2008), although in this case the spread of current flow could be hardly traceable. In any case, widespread (nonfocal) effects of tDCS should be taken into account, considering that functionally active cortical targets may be more susceptible to excitability changes induced by tDCS. Lack of spatial focality may undermine tDCS effectiveness in performing cortical mapping, but the idea that widespread cortical networks are affected by tDCS could explain the strength of the observed behavioral effects, in some cases lasting for months after several stimulation sessions are performed, thus supporting the possible benefits of this technique for clinical applications.

\section{Underlying Neural Activity}

Studies using fMRI to assess online and offline tDCS effects have found that anodal and cathodal stimulation elicit, respectively, an increment and a decrement of perfusion in a wide set of brain areas, including cortical and subcortical structures, even at some distance from the target area (e.g., Stagg et al., 2013). Similarly, computational models of current flow have indicated that strong electric fields occur not only underneath and near the stimulating electrodes but also in the regions between them (Miranda et al., 2013). Consistent with the computational modeling studies noted above, recent findings have observed tDCS effects on both structural and functional connectivity (Romero Lauro et al., 2014, 2016; Pisoni et al., 2017).

\section{Practical Considerations}

\section{Sham Stimulation}

Regarding its practical applications, tDCS, allows for more effective placebo stimulation-controlled studies compared to TMS where notable issues with placebo effects and limited 
blinding success (Duecker and Sack, 2015). Placebo (sham) stimulation can be delivered for few seconds and subjects experience the same physical sensations as real stimulation (e.g., itching sensation), without substantial neural or behavioral effects (Gandiga et al., 2006; Nitsche et al., 2008).

\section{Individual Differences}

Another practical consideration concerns individual differences. Following seminal work in the tDCS literature (e.g., Nitsche et al., 2008) we recommend that participants be matched on individual differences like sex or age that can influence tDCS efficacy (Pitcher et al., 2003; Kuo et al., 2006; Quartarone et al., 2007; Chaieb et al., 2008). In addition to demographic factors, researchers should also consider whether participants respond to tDCS protocols. In two motor cortex studies, anodal stimulation increased cortical excitability in $50-64 \%$ of participants (Wiethoff et al., 2014; López-Alonso et al., 2015). These individual differences highlight the need to develop more robust stimulation protocols and well-powered studies which can systematically test for the moderating role of individual differences.

\section{Safety}

Transcranial direct current stimulation is a safe and non-invasive neuromodulatory technique. Several neuroimaging and EEG studies have demonstrated that tDCS does not cause adverse effects on the brain (Nitsche et al., 2004; Iyer et al., 2005). The most common side effects include mild tingling sensations, moderate fatigue and light itching sensations, especially at stimulation onset (e.g., Poreisz et al., 2007) and stimulation sessions up to $50 \mathrm{~min}$ do not cause serious consequences (Nitsche et al., 2008). Moreover, ramping up and ramping down current at the beginning and at the end of a stimulation session is useful to avoid brief retinal phosphenes or startle-like phenomenon caused by sudden neuronal firings.

To summarize, tDCS is a safe, inexpensive, well-tolerated, and easy to use. Its physiological consequences have led to an increasing interest in using tDCS to alter psychological processes. With an appreciation for the history and mechanics of tDCS, the next section will explore the consequences of prefrontal tDCS psychological processes in the domain of self-regulation.

\section{TRANSCRANIAL DIRECTION CURRENT STIMULATION AND SELF-REGULATION}

\section{Persistence}

Self-regulation enables individuals to guide their thoughts, feelings, and behaviors in a goal-directed fashion. Self-regulation can be aversive both physically and psychologically. Goal directed behavior often entails persistence, including the sustained performance of aversive behavior. For example, physical exercise sometimes elicits short-term pain or physical discomfort but also brings more long-term appearance- and health-related benefits. Individuals who are better able to endure the discomfort presumably exercise longer and more frequently. As a result, these same individuals may be more successful at achieving their fitness goals. Other situations may require individuals to endure psychologically aversive states in the interest of accomplishing one's goals. For example, academic and occupational successes may entail many failed attempts to solve complex problems. The ability to endure these aversive states seems likely to facilitate goal-directed behavior and thus may constitute an important facet of self-regulation.

\section{Pain Tolerance}

Research suggests that the DLPFC is a key brain region for various aspect of pain, including pain tolerance (see Seminowicz and Moayedi, 2017). Consistent with this viewpoint, several studies have used prefrontal tDCS to modulate the experience of pain in laboratory tasks. For example, Mylius et al. (2012) found that anodal tDCS over the right DLPFC increases tolerance to heat pain as measured by the temperature of a thermode applied to the forearm. Similar results were obtained by Boggio et al. (2008), who found that excitatory stimulation of the left DLPFC increases pain thresholds. Taken together, these findings suggest that stimulation to the prefrontal cortex in either hemisphere may increase pain tolerance - a classic form of self-regulation.

More recently, a study by Mariano et al. (2016) applied anodal versus sham tDCS during both a cold pressor task and a breath holding task (see also Mariano et al., 2015, for similar work probing the dorsal anterior cingulate cortex). These two tasks represent commonly used laboratory pain paradigms. Participants' pain ratings were assessed before and after stimulation using the Defense and Veterans Pain Rating Scale (DVPRS), which asks participants to rate their pain on an 11-point visual analog scale from $0=$ no pain to $10=$ severe pain. Ratings were obtained after the first $7 \mathrm{~min}$ of tDCS in each testing block and immediately after the cold pressor and breath holding tasks. Stimulation did not influence performance on the cold pressor task as measured by threshold, tolerance, or endurance, nor did it influence breath holding time. However, anodal stimulation over the left DLPFC decreased the experience of pain as measured by the DVPRS. This study suggests that excitatory stimulation of the DLPFC may influence pain perception.

Another relevant pilot study by Powers et al. (2018) paired anodal, cathodal, or sham stimulation over the left DLPFC with either pain education or a 3-min audio recording designed to mimic key components of Cognitive-Behavioral Therapy for pain. Afterward, participants completed five trials of a heat tolerance pain test. Regardless of which intervention was paired with tDCS, cathodal stimulation over the left DFLPFC increased pain tolerance. But several limitations in this study mar the interpretability of the results. First, the study included 6 experimental conditions with 79 total participants, resulting in small sample sizes in each condition and thus relatively low statistical power to detect anything but very large effects of tDCS. Second, the electrode montage involved placing the anode or cathode over the F3 region and the other electrode on the right shoulder. The study by Mariano et al. (2016) reviewed above, which found reduced pain experience but not increased pain tolerance, used the mastoid as a reference, whereas the study by Mylius and colleagues, which found increased pain tolerance, involved placing the reference electrode over the contralateral supraorbital area. Thus, inconsistencies in the 
electrode montages used across studies of prefrontal tDCS and pain, along with differences in methods used to induce and measure pain, hamper our ability to draw clear conclusions about the effects of $\mathrm{tDCS}$ on pain experience and pain tolerance.

\section{Delay Behavior}

Often one's end goals are situated far in the future, and pursuing such goals comes at the cost of satisfying more immediate desires. Saving for the future comes at the cost of spending today. Maintaining a healthy physique comes at the expense of delicious desserts. Academic achievement often comes at the expense of fraternization. Delay behavior refers to choices that favor more long-term investments at the expense of short-term gains. To the extent that individuals stifle or subdue their immediate urges, the better they are in striving toward their long-term goals.

\section{Delay Discounting}

Delay discounting refers to the reduction in the present value of a reward with delayed receipt. The basic idea is that the valuation of rewards degrades over time. So, for example, gaining $\$ 10$ today would typically be valued more than gaining $\$ 10$ in a month from today. For each unit of time increase in the delay to receipt, the value of a reward decreases (or is discounted) by a non-fixed proportion. In other words, the effect of delay on value is not the same across the range of delays. At short delays value decreases less steeply, whereas at longer delays value degrades more steeply. A hyperbolic discounting function captures the pattern that at shorter delays reward valuation degrades less than it does at longer delay periods. The steepness of the slope within this hyperbolic model reflects the extent to which people prefer smaller-but-immediate (compared to largerbut-delayed) rewards. A steep slope (i.e., a larger hyperbolic $k$ ) reflects a stronger preference for smaller-but-immediate rewards. A less steep slope (i.e., a smaller hyperbolic $k$ ) reflects a stronger preference for larger-but-delayed rewards. Typically, a preference for smaller-but-immediate rewards is thought to reflect impulsivity (poor self-regulation) whereas a preference for larger-but-delayed rewards is thought to reflect self-control (good self-regulation).

Hecht et al. (2012) paired prefrontal tDCS with a delay discounting task. Specifically, participants received anodal right DLPFC/cathodal left DLPFC, cathodal right DLPFC/anodal left DLFPC, or sham stimulation. They observed a greater preference for smaller-but-sooner rewards when participants had received anodal stimulation over the left DLFPC, suggesting that this pattern of stimulation increased impulsivity or reduced selfregulation. Similar results have been obtained in TMS studies disrupting right DLPFC activity (Figner et al., 2010; Smittenaar et al., 2013). More recently, a study by Nejati et al. (2018) paired DLPFC stimulation with OFC stimulation prior to a delay discounting task. Specifically, participants received sham stimulation, anodal left DLPFC/cathodal right OFC, and cathodal left DLPFC/anodal right OFC. They observed that relative to sham stimulation, both active stimulation conditions caused a greater preference for delayed rewards as reflected in a smaller $k$ value. The results of this study hinge upon the interaction between the DLPFC and OFC and as a result we cannot determine to what extent they were driven by the DLPFC (or OFC). Because of this, the study by Nejati and colleagues differs in a critical way from the discounting studies reviewed above as those studies speak moreso to the role of hemispheric asymmetry in discounting behavior. Thus, the majority of evidence here suggests that stimulation to shift the balance in neural activity toward the left prefrontal cortex increases delay discounting in a manner that suggests poorer self-regulation.

\section{Food Choice}

Food choice and eating entail delay behavior insofar as choosing nutritious foods (e.g., vegetables) over tempting ones (e.g., chocolate cake) represents a choice favoring long-term investments in health at the expense of a short-term hedonic gain. Fregni et al. (2008) compared excitatory right DLPFC stimulation (cathode over F3/anode over F4), excitatory left DLPFC stimulation (anode over F3/cathode over F4), and sham stimulation in the context of food. Self-report measures of food craving and craving in response to food in the laboratory were assessed before and after tDCS. Additionally, after tDCS, participants had their gaze patterns recorded while they viewed an array of nature scenes and images of tempting foods (e.g., desserts). Last, participants had the opportunity to ingest foods and the number of calories ingested was recorded. Results indicated that excitatory right DLPFC stimulation decreased food cravings, decreased visual attention toward tempting desserts, and decreased caloric consumption relative sham stimulation.

Goldman et al. (2011) conducted a similar experiment in a group of healthy individuals with frequent food cravings. Participants viewed food images from the International Affective Picture System (IAPS; Lang et al., 2008) before and after tDCS. Additionally, after stimulation, participants were free to eat a variety of tempting foods including chips, cookies, chocolate, and donuts. Consistent with the results of Fregni et al. (2008), Goldman and colleagues found that excitatory right DLPFC stimulation (cathode over F3/anode over F4) decreases food cravings, especially for sweets. Unlike the study by Fregni et al., however, the study by Goldman et al. did not find that tDCS influences food consumption. Thus, stimulation over the right prefrontal cortex appears to influence self-regulation in the context of desire for tempting foods but has seemingly inconsistent effects on consumption.

More recently, a study by Lapenta et al. (2014) found that excitatory stimulation over the right DLPFC reduces caloric ingestion and food intake (e.g., cakes and sweets). Additionally, after stimulation participants completed a GO/NO-GO task while EEG was recorded. Excitatory stimulation over the right DLPFC modulated the N2, P3a, and P3b ERP components. All three of these components have been implicated in successful inhibitory control during GO/NO-GO tasks (e.g., Albert et al., 2013). This study thus provides more direct evidence that tDCS over prefrontal cortex influences regulatory mechanisms, which in turn influence food choice behavior.

Kekic et al. (2014) compared excitatory right DLPFC stimulation (cathode over F3/anode over F4) to sham stimulation as participants completed both a food craving questionnaire and a food challenge task before and after stimulation. The food 
challenge task consisted of two parts. First, participants watched two short videos of tempting foods (e.g., chocolate). Next, each of the foods from the videos was made available to participants in the laboratory. At this point participants rated their desire to eat and emotional reactions to each of the presented foods. After stimulation, participants were left alone with the foods and instructed to eat what they would like while the experimenter was out of the room. Consistent with past research (e.g., Fregni et al., 2008; Goldman et al., 2011), excitatory right DLPFC stimulation reduced cravings for sweet but not savory foods on the food challenge task. Much like the findings from Goldman et al. these effects did not extend to influence actual consumption during the free eating portion of the task. Kekic et al. further reported that the tDCS-driven reductions in cravings were more pronounced among participants with low (versus high) delay discounting tendencies. This pattern suggests that it was easier to modulate food-related cravings in individual relatively low in impulsivity (high in self-control).

Ljubisavljevic et al. (2016) explored the consequences of repeated stimulation over the DLPFC on food craving. In their study, participants received excitatory right DLPFC stimulation for five consecutive days. Replicating past research, a single session of excitatory right DLPFC stimulation reduced the intensity of food craving. The effects of five consecutive days of stimulation reduced cravings both immediately and 30 days later. The craving reductions were most pronounced for fast foods and desserts. These effects were not moderated by participants' weight at the beginning of the study, nor did the stimulation protocol influence weight assessed 30 days later. Another study using a repeated stimulation design found that stimulation for 8 straight days reduced both self-reported appetite and caloric consumption during a free eating buffet on the last day of stimulation (Jauch-Chara et al., 2014).

Inspired by the evidence linking excitatory right DLPFC stimulation to reductions in food cravings, Burgess et al. (2016) extended this body of research by testing a sample of participants with clinical or subclinical binge eating disorder. They observed that excitatory right DLPFC stimulation not only reduced cravings across food categories but also decreased desire to binge eat and decreased food consumption. In summary, research using tDCS to influence delay behavior has occasionally tested delay discounting for monetary rewards, and the evidence suggests that a left lateralized pattern of prefrontal stimulation induces a more impulsive preference for immediate rewards. Even more studies have concentrated on the domain of food craving and food consumption. Much of this work has found that excitatory stimulation over the right DLPFC decreases cravings for unhealthy foods, and in some cases this stimulation pattern also decreased actual consumption.

Why does tDCS seem more likely to reduce craving but not consumption as the studies above suggest? It may be that cravings or subjective responses are easier to modulate with tDCS (and other interventions) than consumption or behavior. The fact that more of the studies reviewed above find craving effects than consumption effects is consistent with this view.

In the case of studies where cravings are reduced but not reduced consumption, in these studies it may be the case that craving was not reduced enough to reduce eating. Additional consumption without craving (or low levels of craving) may reflect a form of dysregulation suggesting more automatic/habitual factors may influence consumption independent of craving (e.g., mindless eating). These reasons suggest that many contextual factors may make detecting links between impulse and consumption more difficult.

\section{Impulse Control}

Impulse control involves the inhibition of emotive response tendencies. It plays a key role in diverse behavioral domains including emotion regulation, prosocial behavior, and risk taking.

\section{Emotion Regulation}

Emotions are not always functional. For example, emotions may work against one's goals when they are expressed at the wrong time or at an inappropriate level of intensity (Taylor and Liberzon, 2007). Emotion regulation refers to the conscious and non-conscious processes individuals use to influence the intensity, variety, and duration of their emotions (Gross, 1998, 2001). Antecedent-focused emotion regulation strategies aim to modulate emotional responses before they solidify, whereas response-focused strategies aim to modulate emotional responses that have already been initiated (Gross, 1998, 2001). Neurobiological models of emotion regulation distinguish between bottom-up emotion regulation (e.g., expressive suppression) mediated by limbic system structures, such as amygdala, and top-down emotion regulation (e.g., cognitive reappraisal), which involves prefrontal regions (e.g., Ochsner and Gross, 2007).

Current neural models of emotion regulation (e.g., Phillips, 2003; Phillips et al., 2008) further distinguish between deliberate, conscious emotion regulation processes, which involve specifically the DLPFC, and more automatic, implicit regulation, which predominantly recruits the VLPFC (Chaiken and Trope, 1999; Strack and Deutsch, 2004; Mauss et al., 2007). Although emotion regulation typically recruits a wide range of cortical and subcortical structures, the DLPFC and the VLPFC constitute two central hubs in emotion regulation processes. These two regions contribute also to other forms of self-control, including motor control, risk-taking behavior regulation, task switching, response inhibition, and conflict monitoring (Braver et al., 2003; Aron et al., 2004; Berkman et al., 2009).

Evidence from fMRI research regarding the contributions of VLPFC and DLPFC to emotion regulation paved the way for the application of non-invasive brain stimulation to these regions to investigate stimulation effects on both explicit and implicit control strategies. For instance, one study tested the hypothesis that a top-down form of antecedent-focused emotion regulation - cognitive reappraisal - involves DLPFC. Indeed, anodal tDCS over the right DLPFC combined with a cognitive reappraisal task reduced negative emotions (but not positive emotions or craving) compared to anodal tDCS over the left hemisphere and sham stimulation (Pripfl and Lamm, 2015). This pattern of finding corroborates the widely established role of the right hemisphere in painful or aversive feelings (Canli et al., 1998; Kalisch et al., 2006; Baek et al., 2012; Herrmann et al., 
2016, 2017; Salas et al., 2016; Mattavelli et al., 2017), but in this case stimulation to increase activity over right DLPFC reduced negative emotions. This perspective is further supported by the evidence that anodal tDCS over the right VLPFC, compared with sham stimulation, reduces the perceived intensity of negative (i.e., fear, anxiety, and sadness) but not positive or neutral emotions induced by film clips (Vergallito et al., 2018). In this study, participants were not explicitly instructed to apply any regulation strategy, suggesting a role for the VLPFC in incidental or implicit emotion regulation. In the next paragraphs we review tDCS effects on negative emotions, empathy, and social pain. Most of the reported studies have tested tDCS application on prefrontal cortex, mainly on DLPFC and VLPFC.

\section{Anger and Aggression}

Anger and aggression are associated with the activation of the behavioral approach system (Harmon-Jones and Sigelman, 2001; Harmon-Jones, 2003). The behavioral approach system is associated with greater left than right frontal cortical activity (Wacker et al., 2008; Zinner et al., 2008; Harmon-Jones et al., 2010). Using tDCS in laboratory aggression paradigms, several researchers have found additional support for a causal relationship between greater relative left frontal cortical activity and approach motivation.

Hortensius et al. (2012) asked participants to write a short essay on a controversial topic (e.g., abortion) before receiving insulting feedback on their essay from another ostensibly real participant. After writing the essay but before receiving the insulting feedback, participants received $15 \mathrm{~min}$ of tDCS. By random assignment some participants received stimulation to increase in relative left frontal cortical excitability (anodal over F3/cathode over the F4), increase in relative right frontal cortical excitability (cathode over F3/anode over F4), or sham stimulation. After tDCS, participants played a competitive reaction time game against the purported insulter. The game was based on the Taylor aggression paradigm (Taylor, 1967). Aggression was operationalized as the duration and intensity of a noxious noise blast given to the other participant. Participants also reported how much anger they felt both pre- and post-insult. Results indicated that after receiving tDCS to increase relative left frontal cortical activity, individuals behaved more aggressively toward the other participant, but only when they also reported high insult-related anger. In other words, stimulation to increase relative left frontal activity strengthened the link between anger and aggression.

Dambacher et al. (2015b) also combined tDCS over the DLFPC with the Taylor aggression paradigm. They found that stimulation to increase relative right frontal activity reduced aggression. Taken together with the results of Hortensius et al. (2012), these results suggest that manipulating frontal asymmetry with tDCS can modulate aggressive behaviors in a manner consistent with previous correlational work linking aggression to relative left frontal asymmetry: increasing relative left frontal activity increases aggression (especially among angry individuals; Hortensius et al., 2012) whereas increasing relative right frontal activity reduces aggression using the same Taylor aggression paradigm (Dambacher et al., 2015b). Unfortunately, Dambacher and colleagues did not include a condition to increase relative left frontal activity, so, they were unable to test whether increased left frontal activity increases aggressive behavior, as was the case for angry individuals in the study by Hortensius et al. (2012).

One difference between the results of Hortensius et al. (2012) and Dambacher et al. (2015b) is that the former found a main effect of tDCS on aggressive behavior whereas the latter did not. Rather, Hortensius et al. found a moderated pattern of results whereby anodal stimulation over the left DLPFC/cathodal stimulation over the right DLPFC increased aggression only for those high in insult-related anger. The studies differed insofar as Dambacher et al.'s study included two experimental conditions, whereas Hortensius study included three conditions. Moreover, another study (Dambacher et al., 2015a) did not find an effect of stimulation condition on aggressive behavior. Although this latter study did include all three stimulation conditions as in Hortensius et al. (2012), stimulation occurred over the F7/F8 prefrontal regions, whereas Hortensius et al. and Dambacher stimulated over the F3/F4 prefrontal regions. Thus, methodological differences preclude a direct comparison of these studies. Despite modest support for the effect of asymmetrical frontal cortical activity on aggressive behavior, further research is needed.

One common response to negative emotions, like anger, is rumination, which is an automatic cognitive process characterized by repetitive and distressful thoughts (Denson et al., 2006; Wade et al., 2008). Parallel literatures on depression (e.g., Heller et al., 1995; Nolen-Hoeksema, 2000) and anger (e.g., Bushman, 2002) have developed competing hypotheses about how rumination relates to lateralized patterns of prefrontal cortical activity. The depression literature links rumination to an increase in right frontal cortical activity, whereas the anger literature links rumination to an increase in left frontal cortical activity.

A study by Kelley et al. (2013) tested these two competing hypotheses by delivering anodal or cathodal tDCS over the right or left frontal cortex while participants received negative (insulting) ratings on their essay writing. Results supported the rumination-depression literature in showing that anodal stimulation over the right frontal cortex, compared with the other stimulation conditions, caused enhanced rumination. This study left open the question of what this increase in rumination means. One option flowing from the rumination-depression literature is that an increase in right lateralized frontal brain activity reflects an increase in avoidance motivation; the stronger the avoidance motivation, the greater the rumination. Another possibility, which may align with the rumination-aggression literature, is that the increase in right frontal activity reflects an increase in inhibition rather than avoidance motivation. Therefore, when considering the increasing of right frontal cortex activity, anger is still experienced, but inhibited; conversely left frontal cortex activation is linked to an approach-oriented motivation. Consistent with the latter interpretation, Kelley et al. observed an association between rumination and behavioral inhibition sensitivity (BIS; Carver and White, 1994), which is thought to reflect inhibition more so than avoidance (e.g., Neal and Gable, 2017). 
A follow-up study further explored the consequences of excitatory tDCS over the right DLPFC for inhibitory processing. A meta-analysis of neuroimaging studies using a Go/No-Go task found a broad pattern of right frontal cortical activation during response inhibition (Swick et al., 2011). A separate EEG literature has often found that right lateralized frontal EEG alpha asymmetry is associated with exaggerated avoidance-motivated reactions to aversive events (Tomarken et al., 1990; Dawson et al., 1992; Wheeler et al., 1993; Kalin et al., 1998; Coan et al., 2001). These literatures suggest competing hypotheses regarding the psychological correlates of activity in the right frontal lobe.

Kelley and Schmeichel (2016) used tDCS to test the competing hypotheses. One hypothesis, flowing from the frontal EEG literature, is that excitatory stimulation over the right DLPFC should increase avoidance motivation. A second hypothesis, flowing from the neuroimaging findings, is that excitatory stimulation over the right DLPFC should increase response inhibition. The key to differentiating these two hypotheses is to test the effects of stimulation over right DLPFC on the inhibition of avoidance-oriented impulses. Kelley and Schmeichel paired tDCS with an approach-avoidance task whereby participants enacted motive incongruent motor responses to appetitive and aversive IAPS images. Specifically, participants in the positive emotion condition pushed away positive images whereas those in the negative emotion condition pulled negative images toward them. These responses require inhibitory control insofar as avoiding rewards and approaching threats requires one to override a predominant response tendency.

The results revealed that anodal stimulation over the right frontal cortex facilitates the inhibition of both approachincongruent and avoidance-incongruent responding. These results are not readily explained by an increase in avoidance motivation. Indeed, an increase in avoidance motivation would have slowed the enactment of an avoidance-incongruent response. But Kelley and Schmeichel (2016) found that stimulation over the right DLPFC sped up both avoidance- and approach-incongruent responding. This study thus supported the hypothesis that increasing right frontal cortical activity increases response inhibition.

\section{Sadness}

Several studies have concentrated on testing tDCS efficacy in sadness regulation, which has important implications for considering tDCS as a treatment device for major depression. Indeed, it has been widely observed that major depression is associated with an asymmetry in prefrontal cortical activity, specifically hypoactivity in the left DLPFC and hyperactivity in the right DLPFC. For instance, one study found that five sessions of bilateral, twice-a-day tDCS over the DLFC (anode left/cathode right) decreased self-reported sadness in a group of patients with severe, drug-resistant major depression. These improvements lasted up to a month after the end of the treatment (Ferrucci et al., 2009). These findings are in line with a growing body of evidence that tDCS can be effective for treating the symptoms of major depression (for a review, see Nitsche et al., 2009). Indeed, a randomized, double-blinded, sham-controlled study found mood symptoms ameliorations after five session of active prefrontal
tDCS in a group of newly diagnosed patients (Fregni et al., 2006b). Moreover, a study by Boggio et al. (2009) found that a single tDCS session over prefrontal cortex, but not occipital or sham tDCS, improved accuracy in identifying emotionally positive visual material in a sample of participants with major depression.

\section{Fear}

Another major, consequential negative emotion is fear. Fear plays a crucial role in the onset and maintenance of chronic pain (Riva et al., 2014b) and several mental disorders, including anxiety and post-traumatic stress disorder (Mungee et al., 2014). Regarding fear regulation, one study using an unpredictable threat paradigm found that anodal tDCS over right prefrontal cortex decreased sustained fear and skin conductance levels in the context of unpredictable threats (Herrmann et al., 2017). This finding lends additional support to the idea that right PFC activation increases response inhibition rather than avoidance motivation. Another study, this one using a bipolar montage over the DLPFC with the anode over the left DLPFC and cathode over the right DLPFC, found improved reaction times on simple arithmetic decisions and decreased cortisol concentrations among participants high in math anxiety (Sarkar et al., 2014).

On the other hand, a recent tDCS study on the consolidation of fear memories observed that greater right frontal cortical activity may enhance fear-related responding. Mungee et al. (2014) paired a fear-conditioning paradigm with either cathodal stimulation (i.e., stimulation to decrease activity) over the right dorsolateral prefrontal cortex, anodal stimulation (i.e., stimulation to increase activity) over the right dorsolateral prefrontal cortex, or sham stimulation. Fear was measured via skin conductance responses to the conditioned stimulus. Results revealed that anodal stimulation over the right dorsolateral prefrontal cortex increased memory for the conditioned feared stimulus as measured via skin conductance responses. These results suggested that increasing activation of the right dorsolateral prefrontal cortex increases fear memory consolidation (see also Mungee et al., 2016). However, this study did not simultaneously pair anodal stimulation to the right dorsolateral prefrontal cortex with cathodal stimulation to the left dorsolateral prefrontal cortex to create an asymmetric pattern of activity, as was done in the studies described in the previous paragraphs in this section. Given that the effects of anodal stimulation over the right dorsolateral prefrontal cortex in conjunction with cathodal stimulation over the left dorsolateral prefrontal cortex have yet to be tested in the study of fear memory consolidation, the causal relationship between greater relative right frontal cortical asymmetry and avoidance motivation remains unclear. Collectively, the evidence on prefrontal tDCS effects on the regulation of fear-related responses remains mixed. Future studies should clarify the extent to which excitatory stimulation over the right DLPFC helps or hinders fear regulation.

\section{Social Pain}

Social pain refers to the hurt feelings caused by rejection or ostracism from the group. These aversive social experiences 
generally cause negative emotions and result in a loss of sense of belonging, control, and self-esteem (Williams, 2009). Some studies have found a role for the dorsal anterior cingulated cortex (dACC) and the right VLPFC in experienced and observed social exclusion conditions, suggesting the presence of a partially overlapping neural network involved both in physical and social pain (Eisenberger et al., 2003; Kross et al., 2007; Wager et al., 2008; Masten et al., 2009). The right VLPFC, in addition to being broadly involved in emotion regulation, has also been found to have a crucial role also in regulating the pain associated with social exclusion (Onoda et al., 2010).

These findings have lead researchers to test the effects of tDCS over brain regions involved in social pain regulation, mainly the right VLPFC. For example, participants in a study by Riva et al. (2012) received $15 \mathrm{~min}$ of anodal tDCS over the right VLPFC. At the end of the stimulation, participants were randomly assigned to be included or excluded in a virtual ball-tossing game called Cyberball (Williams et al., 2000). In the inclusion condition, subjects received a ball the same number of times as the other two players, whereas in the exclusion condition participants received the ball only on the first throws. Actually, participants did not play with real players; a computer program controlled the ball. Results revealed that anodal tDCS over right VLPFC, compared to sham stimulation, reduced levels of pain and hurt feelings among excluded subjects.

In a similar study, the same research group (Riva et al., 2014a) tasted the effects of tDCS on behavioral aggression following social inclusion or exclusion condition in the Cyberball paradigm. Participants received anodal or sham stimulation during the Cyberball game. At the end of the stimulation, subjects had to choose the amount of hot sauce for their ostensible partners to taste. This is the well-validated hot-sauce paradigm for studying aggression (e.g., Lieberman et al., 1999). Findings indicated that increasing right VLPFC cortical activity with tDCS reduced behavioral aggression among excluded participants, who were no more aggressive than included ones. These findings were replicated in a subsequent study (Riva et al., 2017), which found that brain polarization through anodal tDCS over the right VLPFC reduced unprovoked aggression as measured by the Taylor aggression paradigm (Taylor, 1967).

Similar results were obtained by Kelley et al. (2015a), who also paired a Cyberball game with prefrontal tDCS. In this modified version of the game, participants first chose a partner from a group of images of eight opposite-sex individuals. A third Cyberball player was assigned by the experimenter and was always the same sex as the participant. Harmon-Jones et al. (2009) had found that this Cyberball game evokes jealousy and that self-reported jealousy after being excluded by a desired partner correlates with relative left frontal cortical activity. Kelley et al. had participants choose a partner in the modified Cyberball paradigm and play a practice version of the game before receiving 15 min of tDCS in one of three conditions: excitatory left DLPFC stimulation (anode over F3/cathode over the F4), excitatory right DLPFC stimulation (cathode over F3/anode over F4), or sham stimulation. Stimulation to increase relative left frontal cortical activity increased self-reported jealousy. Because the direct manipulation of cortical excitability with tDCS produced the same outcome as the correlational finding reported HarmonJones et al. (2009), this study suggests that tDCS over the dorsolateral prefrontal cortex does indeed modulate emotive responses associated with social exclusion and asymmetric frontal cortical activity.

Collectively, these studies highlight the effects of tDCS over the prefrontal cortex on regulating responses to social exclusion. This work may have clinical implications for disorders characterized by maladaptive responses to social exclusion (e.g., borderline personality disorder). Future research should test the extent to which the increased negative affect and autonomic arousal (Kopala-Sibley et al., 2012) and inappropriate coping strategies and impulsive behaviors (Dixon-Gordon et al., 2011; Coifman et al., 2012) associated with these disorders can be modified by tDCS.

\section{Empathy}

Recently, some research has observed that personal physical suffering and empathy for the pain of others share the same neural network, involving prefrontal cortex as well as somatosensory cortex, anterior cingulate cortex, amygdala, and anterior insula (Eisenberger, 2012). To investigate the role of prefrontal cortex in empathic pain regulation, Boggio et al. (2009) conducted a study in which participants judged the unpleasantness of pictures showing human beings under painful conditions. Anodal tDCS was applied over the left DLPFC, the primary motor cortex, and the primary visual cortex (control site) in three experimental sessions. Findings revealed decreased unpleasantness and discomfort during anodal stimulation over left DLPFC compared to sham stimulation. Moreover, compared to a previous study in which tDCS was delivered together with an electrical peripheral stimulation (Boggio et al., 2008), no significant effects were found in the primary motor cortex stimulation condition, suggesting that DLPFC specifically contributes to the emotional processing of empathic pain.

These findings were expanded by a similar study comparing emotional reactions to negative, positive, and neutral human pictures (Peña-Gómez et al., 2011). This study found that anodal tDCS over the left DLPFC reduces only painful and not positive or neutral affects. Based on previously mentioned neural models (Phillips, 2003; Phillips et al., 2008; Kohn et al., 2014) and previous neuroimaging evidences regarding DLPFC involvement in more cognitive forms of emotion regulation (Blair et al., 2007; Boggio et al., 2007a; Ochsner et al., 2009; Peña-Gómez et al., 2012), the authors suggested that increasing activity in the DLPFC may enhance cognitive control of emotional reactions. Moreover, the tDCS effect was more noticeable for participants with higher subclinical scores on the introversion personality dimension, perhaps due to their enhanced ability to control emotion expression, which correlates with the increased cortical activity, compared with extraverts, especially in the frontal lobes (Suslow et al., 2010).

A similar finding was reported also by Feeser et al. (2014), who applied anodal tDCS over the right DLPFC while participants applied cognitive reappraisal strategies to downor up-regulate the emotions elicited by negative or neutral pictures. Skin conductance responses were also assessed. Anodal 
tDCS, compared to sham stimulation, increased emotional and autonomic reactions when the reappraisal instruction was to upregulate emotions and decreased emotional and autonomic responses when participants attempted down-regulation. Taken together, the studies reviewed here implicate DLPFC and VLPFC in emotion regulation when observing or experiencing painful situations, thereby highlighting the feasibility of tDCS application for the study of pain and empathy for pain.

\section{Risk Taking}

In addition to emotion and mood manipulations, another way researchers have explored the consequences of prefrontal brain stimulation on emotion regulation is by studying risk taking. Risk taking involves the possibility of punishment or potential harm in the pursuit of rewards or goal-relevant actions. From an emotion-regulation perspective, risk-taking involves managing the emotions associated with the anticipation of winning and losing. In a seminal tDCS study, Fecteau et al. (2007a) used tDCS over the DLPFC during a risk task (Rogers et al., 1999). Across 100 trials of a gambling task, participants viewed 6 horizontal boxes. Some boxes were blue, and some were pink, and the ratio of blue to pink boxes varied from trial to trial. The ratio could be 5:1, 4:2, or 3:3. Of the two options, the high likelihood option was always associated with a small reward whereas the low likelihood option was always associated with a large reward. Participants were to indicate which color box contained a token. Each trial selecting the winning color box earned a reward but selecting the incorrect color box incurred a penalty. Larger rewards were always paired with riskier decisions such that correctly choosing a pink box with a low win probability (1/6) would result in a large reward whereas making that same choice and losing was associated with same magnitude of a loss. Thus, participant's tendency to choose high-risk/unlikely rewards over low risk/likely rewards was the measure of risk-taking. Results indicated that excitatory right DLPFC stimulation increased the number of participants earned by decreasing risk taking on the task. These results suggest that excitatory stimulation over the right DLPFC tilted participants toward safer, less risky choices, as though they were less tempted by the larger, riskier rewards.

These findings have been partially replicated by Cheng and Lee (2016), who found that the excitatory stimulation over the right DLPFC influences performance on the Risky Gains Task (RGT; Paulus et al., 2003), but not on the Balloon Analogue Risk Task (BART; Lejuez et al., 2002). Regarding the RGT task, participant's goal was to win as many points as possible. To accomplish this goal, they made quick (1 s) decisions between taking a reward (i.e., points) now or waiting for a larger point value later. However, this later-but-larger reward also came with the risk of being punished (i.e., losing points equivalent to the later-butlarger reward). Risk-taking was quantified as the rate at which participants selected trials for which punishment was possible whereas safe decision-making was the rate at which participants chose point values with no possibility of punishment. The effect of excitatory right DLPFC stimulation on risky (but not safe) trials was moderated by individual differences in impulsivity, such that greater risk-taking was associated with greater (versus lower) impulsivity. More recently these results have recently been conceptually replicated in a clinically impulsive sample. Gilmore et al. (2017) paired excitatory stimulation over the right DLPFC (twice a day for 5 days) with a balloon analog risk taking (BART) task. They found that excitatory stimulation over the right DLPC reduced risk-taking by $46 \%$. This diminished risk-taking persisted at a two-month follow-up.

Another study probed the underlying neurocircuitry that may be driving the changes in risk taking. Weber et al. (2014) used excitatory stimulation over the right DLPFC, prior to functional MRI during which participants completed the BART. They found that excitatory stimulation over the right DLPFC increased activity in the right DLPFC and the ACC as well. Additionally, this pattern of stimulation also influenced how these two regions connected with the rest of the brain. Specifically, greater right DLPFC-whole brain connectivity was associated with diminished risk-taking on the BART. These results suggest that the diminished risk-taking linked to excitatory right DLPF stimulation influences the neurocircuitry implicated in successful self-regulation.

In addition to laboratory risk-taking paradigms, an innovative study paired prefrontal tDCS with a more ecologically valid form of risk taking: driving behavior. In a driving simulator study, Beeli et al. (2008) observed that excitatory stimulation of the DLPFC (either left or right) led participants to keep a safer distance behind a lead driver and reduced the number of speeding errors. This pattern of stimulation did not influence average speed or revolutions per minute. These results are broadly consistent with reduced risk taking, but they differ from the risk-taking findings reviewed above in a crucial way: the electrode montage used. Whereas the risk-taking studies described above paired excitatory stimulation over the right DLPFC with inhibitory stimulation over the left DLPFC, the study be Beeli and colleagues placed the cathode over the ipsilateral mastoid. Because of this difference in electrode montages it is unclear to what extent these results are comparable to the risk-taking studies above. Future studies should explore the extent to which anodal right DLPFC/cathodal left DLPFC stimulation influences risk-taking in this domain.

In summary, numerous studies have observed enhanced self-regulation after anodal tDCS over right DLPFC (cathodal over left). Improved self-regulation manifested in a variety of ways including greater pain tolerance, healthier food choices, less impulsive decision-making, improved emotion regulation, reduced anger and aggression, less sadness and fear, more empathy, and less risk taking. Collectively, these results anodal tDCS over right DLPFC may be one tool that shows promise in helping individuals live happier, healthier lives.

\section{Limitations and Future Directions}

We have reviewed evidence suggesting that non-invasive brain stimulation over the prefrontal cortex may improve human selfregulation. First, we detailed a methodological review of tDCS highlighting that tDCS is a safe, inexpensive, and easy to use technique that can be used to study higher-order cognition, emotion, and clinical phenomena. We also reviewed stimulation parameters that may help or hinder tDCS efficacy including current density, stimulation duration, and the orientation and focality of the electrical field. Second, we reviewed findings 
from experiments observing that non-invasive brain stimulation over the PFC can enhance three paradigmatic forms of selfregulation: persistence, delay behavior, and impulse control. Although the current review focused predominantly on tDCS, other techniques such as low energy neurofeedback (e.g., Ochs, 2006) and transcranial ultrasound (e.g., Tufail et al., 2011) also represent low-cost, non-invasive techniques for investigating the causal role of neural activation in various forms of self-regulation.

Although much of this review highlighted the ability of brain stimulation to promote self-regulation, under the right circumstances brain stimulation may also undermine selfregulation. For example, we highlighted studies wherein excitatory stimulation over the right DLPFC enhances inhibition (e.g., Kelley and Schmeichel, 2016) whereas excitatory stimulation over the left DLPFC increases approach motivated negative emotions like anger (e.g., Hortensius et al., 2012) and jealousy (e.g., Kelley et al., 2015a,b). These contrasting findings imply that excitatory tDCS is not an unmitigated good that improves self-regulation in all cases. In fact, these findings emphasize the need to identify precise stimulation parameters that help versus hinder self-regulation. Below, we discuss inconsistencies, unresolved questions, and new directions emanating from the above review.

\section{tDCS Dosage}

The efficacy of eliciting changes in brain activity using tDCS depends on several physical parameters including current density, stimulation duration, and the orientation and focality of the electrical field. These parameters constitute the tDCS dosage. As we noted above, different electrode positions modulate different neuronal groups and elicit different evoked potentials in the case of primary motor cortex and primary visual cortex stimulation, respectively (Priori et al., 1998; Antal et al., 2004; Accornero et al., 2007), and these different electrode positions may also determine effects on the targeted brain region due to electrical field interactions associated with neuronal geometry (Nitsche and Paulus, 2000; Nitsche et al., 2008).

Whereas the coupling of anodal-excitatory and cathodalinhibitory effects is well established in the sensorimotor domain, the evidence pertaining to higher cognitive functions is more controversial (Jacobson et al., 2012). Indeed, when dealing with more complex functions represented by large and interconnected neural networks comprising both excitatory and inhibitory connections, it is more difficult to obtain predictable outcomes especially when different electrode montages have been used across studies. Hence it is not always the case that anodal tDCS leads to an enhancement (e.g., better performance in a task) and cathodal tDCS leads to a diminution of the assessed cognitive function (Fertonani and Miniussi, 2017).

To illustrate this point, consider the studies above on physical pain tolerance. These studies found increased pain tolerance as the result of excitatory stimulation of the right DLPFC (Mylius et al., 2012), excitatory stimulation over the left DLPFC (Boggio et al., 2008; Mariano et al., 2016), and inhibitory stimulation over the left DLPFC (Powers et al., 2018). Of the four studies we reviewed, three difference electrode montages were used. Notably, the study by Powers and colleagues, unlike the other pain studies, paired tDCS with clinical interventions. These methodological differences likely contributed to the differences in results across studies and thus makes interpreting the effects of tDCS difficult. Future studies should more precisely optimize the stimulation protocols that accentuate versus undermine selfregulation for easier comparison across studies.

More broadly, however, the findings from the pain studies are congruent with the findings from studies of other selfregulatory domains in suggesting that stimulation over right DLPFC facilitates self-regulation. Whether such stimulation is most profitably paired with inhibitory (cathodal) stimulation over left DLPFC remains to be seen. And the effects of excitatory (anodal) stimulation over left DLPFC on self-regulation are even more uncertain, with some studies finding better and some finding worse self-regulation after excitatory stimulation over left DLPFC.

\section{Individual Differences}

Another inconsistency in past research concerns individual differences. Despite seminal work suggesting that participants should be matched on individual differences like sex or age that can influence tDCS efficacy (Pitcher et al., 2003; Kuo et al., 2006; Quartarone et al., 2007; Chaieb et al., 2008; Nitsche et al., 2008), many of the studies reviewed above did not use this strategy, and we recommend future studies implement matching procedures to reduce error variance and increase power to detect tDCS effects.

In addition to demographic factors, which may subtly influence tDCS efficacy, many of the studies we reviewed also did not consider the extent to which participants are responsive to tDCS. Previous research has found that between 50 and 64 percent of participants are responsive to tDCS protocols, which leaves a substantial proportion of individuals who are not particularly responsive to tDCS (Wiethoff et al., 2014; LópezAlonso et al., 2015). Studies that include non-responders and studies that do not systematically test for differences between responders and non-responders many obscure effects of tDCS on regulatory behavior. Further, only few studies have considered individual differences traits, though these are also likely to moderate tDCS effects. For example, the study by Kekic et al. (2014) found that tDCS-driven reductions in food craving were more pronounced among participants with low (versus high) delay discounting tendencies. Evidence of this sort highlights the need to develop more robust stimulation protocols in wellpowered studies that can systematically test for the moderating role of individual differences.

\section{Impulse-Behavior Relationship}

Another inconsistency in the research reviewed above concerns whether changes in emotive responding extend to changes in behavior. We summarized evidence that tDCS may alter food craving without altering food consumption (although sometime consumption changes, too; e.g., Fregni et al., 2008). Similarly, we summarized evidence that changes in emotional responses (e.g., anger) may occur in both the absence of and presence of corresponding changes in behavior.

The path from impulse to behavior entails multiple determinants, and thus impulses and behaviors may diverge for 
several reasons. Practical considerations including variability in stimulation dosage may explain why tDCS sometimes does and sometimes does not influence behavior. For example, one important aspect of the tDCS dosage is electrode size. The study by Kekic et al. (2014) who did not find effects on food consumption used $25 \mathrm{~cm}^{2}$ electrodes whereas Fregni et al. (2008) used $35 \mathrm{~cm}^{2}$ electrodes. Smaller electrodes are more spatially precise compared to larger electrodes. The larger electrodes may affect distal brain regions that are less affected in studies using smaller electrodes possibly accounting for why the study by Fregni et al. founds effects on consumption, but Kekic did not. Beyond these practical considerations idiosyncratic food preferences may have played a role in the inconsistent results. The studies assessing compulsion offered actual food to participants, but those foods were not specifically tailored to the participant. Instead participants were offered foods that are generally (but not universally) well liked, such as chips, cookies, and chocolate. This may be problematic insofar as some participants may not prefer any of the food options offered by the experimenter. Future studies using more ecologically valid measures of food consumption and others types of regulatory behavior (e.g., naturalistic observation) may prove useful in clarifying the extent to which tDCS-induced changes in emotions/impulses influence subsequent behavior. However, we must also consider the possibility that cravings/subjective states are more readily influenced by tDCS, whereas behaviors may be harder to change as they have a multiple of determinates above and beyond the preceding subjective state.

\section{How Does tDCS Effect Underlying Brain Activity?}

The effects of tDCS on underlying brain activity are not well understood and subject to ongoing debate. It remains to be seen how tDCS affects brain activity and how these changes relate to changes in self-regulation. One way to advance research on this topic is to pair concurrent measures of brain function with electrical stimulation. Candidate neural processes mediating links between tDCS and self-regulation include changes in prefrontal EEG alpha and functional connectivity between the prefrontal cortex and subcortical regions.

\section{Prefrontal Alpha}

One potential mediator driving many of the effects reported in the current review is EEG alpha activity. Specifically, lateralized patterns of alpha activity may reflect a person's motivational orientation with left lateralized EEG alpha activity reflecting approach-motivation (Tomarken et al., 1992; Harmon-Jones and Allen, 1997, 1998; Sutton and Davidson, 1997; Harmon-Jones and Sigelman, 2001; Harmon-Jones et al., 2002, 2006; Coan and Allen, 2003; Harmon-Jones, 2007) and right lateralized pattern of EEG alpha activity has been linked to withdrawal or avoidance motivation (Davidson et al., 1990; Tomarken et al., 1990; Dawson et al., 1992; Kalin et al., 1998; Coan et al., 2001). Self-regulatory failure tends to occur when individuals have strong impulses such as strong impulses to engage in approach or avoidance motivated behavior. Likewise successful self-regulation tends to occur when impulses are weaker. To the extent that asymmetric patterns of alpha activity in the frontal cortex reflect the strength of appetitive and aversive impulses they may be a good candidate linking tDCS to changes in self-regulation.

Indirect support for the role of EEG alpha activity in tDCS effects comes from parallel findings using EEG and tDCS, respectively, to study the emotion of jealousy. Harmon-Jones et al. (2009) found that feelings of jealousy during a Cyberball game correlate with greater left frontal alpha activity, and Kelley et al. $(2015 \mathrm{a}, \mathrm{b})$ found the same pattern by manipulating (rather than measuring) brain activity with tDCS. More specifically, Kelley et al. observed that excitatory stimulation of the right DLPFC paired with inhibitory stimulation of the left DLPFC increases feelings of jealousy during a Cyberball game. By finding the congruent effects with both measured and manipulated brain activity, the pair of results together suggest that EEG alpha activity may mediate the link between prefrontal tDCS and behavioral self-regulation.

In an innovative new study, Vöröslakos et al. (2018) developed an intersectional short-pulse (ISP) stimulation paradigm in cadaver and rodent studies. ISP delivers shorts bursts (less than $10 \mu \mathrm{s}$ ) of high intensity stimulation from multiple electrode pairs centered on a stimulation site of interest. ISP thus offers superior spatial focality plus higher current densities (7-9 MA) more easily tolerated than traditional tDCS paradigms ( $>2 \mathrm{MA}$ ), with relatively low charge densities and scalp sensations. Pairing Vöröslakos and colleagues then paired ISP with concurrent EEG measurement and observed that tDCS affects the amplitude of simultaneously recorded EEG alpha waves. This work provides an important example of how technical studies in mouse and cadaver models can be used to improve existing tDCS protocols. Future research should consider implementing innovate stimulation paradigms like ISP to increase the efficacy of existing stimulation paradigms and to trace the neural mediators of tDCS effects.

\section{Functional Connectivity}

The self-regulation findings we reviewed may also be mediated by frontal cortical-subcortical interactions. Due to its high spatial resolution, functional MRI is perhaps best suited to probe such possibilities. Research along these lines has already begun. Weber et al. (2014) administered excitatory stimulation over the right DLPFC prior to functional MRI of a risk-taking task. They found that excitatory stimulation over the right DLPFC influences how the right DLPFC connects to the rest of the brain. Specifically, stronger right DLPFC-whole brain connectivity and diminished risk-taking emerged in participants who had received excitatory stimulation over the right DLPFC. Although this study did not measure brain function during tDCS, the finding that tDCS administered immediately prior to a risktaking task influences connectivity patterns revealed by fMRI does highlight that stimulation may influence communication among neural networks. Future studies should continue to probe the extent to which prefrontal stimulation influences connectivity to reward-relevant (e.g., ventral striatum) and threat-relevant (e.g., amygdala) subcortical regions.

Potential neural aftereffects of tDCS, such as changes in prefrontal alpha asymmetry or functional connectivity, may mediate the relationship between tDCS and regulatory behavior. 
What remains to be established is the extent to which alpha asymmetry or functional connectivity carry the causal effects of tDCS on self-regulation, or whether they merely correlate with observed changes in regulatory behavior.

\section{How Do Impulse and Control Strength Contribute to Regulatory Behavior?}

Imagine a person who, when offered a chocolate treat, decides not to eat it. Does foregoing chocolate reflect the application of control to resist chocolate consumption, a low desire for chocolate, or some combination of both things? Social psychologists have traditionally viewed self-regulatory success as the consequence of applying self-regulatory strength (see Baumeister et al., 1994, 1998, 2007). In this view, a person forgoes chocolate by resisting the temptation to eat it. But impulse strength also contributes to self-regulatory outcomes. Presumably, weaker impulses are easier to control or hardly require control at all (e.g., Inzlicht and Schmeichel, 2012; Kelley et al., 2018). Successful self-regulation has been associated both with increased activity in areas associated with top-down control, including the prefrontal cortex, and reduced activity in subcortical regions involved in reward and threat processing. These patterns also implicate and potentially confound strong control and weak impulses. Thus, an unresolved issue concerns the relative contribution of control strength and impulse strength to self-regulatory outcomes. By paring tDCS with measures of concurrent brain function, it may be possible to compare the contributions of neural activity caused by (1) stimulation to areas associated with impulse strength, and (2) stimulation to increase activity in control regions.

\section{What Are the Long-Term Consequent of Brain Stimulation on Regulatory Behavior?}

In addition to limited understanding of the effects of tDCS on underlying neural processes, we also have a limited understanding of the long-term effects of tDCS. Most of the studies we reviewed involved just a single session of tDCS. Although some studies have involved the administration of tDCS once a day for multiple days (Fregni et al., 2006a; Boggio et al., 2007b), those studies have primarily been conducted with patients recovering from spiral cord injury or stroke. Pairing repeated stimulation with measures of self-regulation in nonpatient samples may yield stronger, more consistent effects.

Self-regulation research touches on alcohol and drug addiction, personal debt, obesity, and a variety of other consequential outcomes. It is important to acknowledge that these outcomes are not the result of one decision or behavior but instead results from multiple patterns of behavior unfolding over long periods of time. Few studies have examined long-term

\section{REFERENCES}

Accornero, N., Li Voti, P., La Riccia, M., and Gregori, B. (2007). Visual evoked potentials modulation during direct current cortical polarization. Exp. Brain Res. 178, 261-266. doi: 10.1007/s00221-006-0733-y downstream effects of stimulation. One notable exception was a study by Gilmore et al. (2017), who found that excitatory stimulation over the right DLPFC (twice a day for 5 days) reduced risk-taking at the end of the stimulation period and at a two-month follow-up. We encourage future studies to follow the example of Gilmore and colleagues and use both repeated stimulation paradigms and longitudinal designs to examine changes in behavior. Extend this work to include also concurrent changes in brain function would help to more precisely map the long-term consequences of tDCS on regulatory behavior.

\section{CONCLUSION}

Self-regulation is crucial for goal-directed behavior and contributes to many consequential outcomes in life including physical and mental health, decision making, and interpersonal functioning. Likewise, failure at self-regulation is thought to contribute to alcohol and drug addiction, personal debt, obesity, and other outcomes that carry both personal and societal costs. Based on the evidence reviewed here, we conclude that brain stimulation techniques hold promise for improving selfregulation across three broad domains of response: persistence, delay behavior, and impulse control. To maximize the utility of tDCS in future research, we encourage researchers be especially mindful of issues such as dosage and individual differences issues that have been commonly been neglected in past research and have likely contributed to inconsistencies in past findings. Future research that takes these concerns into account and incorporates concurrent measures of brain activity will bring the promise of tDCS into sharper focus and help to reveal underlying neural mechanisms.

\section{AUTHOR CONTRIBUTIONS}

All authors contributed to the conception, writing, and revising of this manuscript.

\section{FUNDING}

Preparation of this manuscript was supported by National Institute of Health (NIH) grant T32 NS047987 to NK.

\section{ACKNOWLEDGMENTS}

We wish to thank Andrew R. Kittleson and Emily E. Smith for their feedback on previous versions of this manuscript.

Albert, J., López-Martín, S., Hinojosa, J. A., and Carretié, L. (2013). Spatiotemporal characterization of response inhibition. Neuroimage 76, 272-281. doi: 10.1016/ j.neuroimage.2013.03.011

Antal, A., Kincses, T. Z., Nitsche, M. A., Bartfai, O., and Paulus, W. (2004). Excitability changes induced in the human primary visual cortex by 
transcranial direct current stimulation: direct electrophysiological evidence. Invest. Ophthalmol. Vis. Sci. 45, 702-707. doi: 10.1167/iovs.03-0688

Ardolino, G., Bossi, B., Barbieri, S., and Priori, A. (2005). Non-synaptic mechanisms underlie the after effects of cathodal transcutaneous direct current stimulation of the human brain. J. Physiol. 568, 653-663. doi: 10.1113/jphysiol. 2005.088310

Aron, A. R., Robbins, T. W., and Poldrack, R. A. (2004). Inhibition and the right inferior frontal cortex. Trends Cogn. Sci. 8, 170-177. doi: 10.1016/j.tics.2004. 02.010

Baek, K., Chae, J. H., and Jeong, J. (2012). The effect of repetitive transcranial magnetic stimulation on fear extinction in rats. Neuroscience 200, 159-165. doi: 10.1016/j.neuroscience.2011.09.050

Baumeister, R. F., Bratslavsky, E., Muraven, M., and Tice, D. M. (1998). Ego depletion: Is the active self a limited resource? J. Pers. Soc. Psychol. 74, 12521265. doi: 10.1037/0022-3514.74.5.1252

Baumeister, R. F., Heatherton, T. F., and Tice, D. M. (1994). Losing Control: How and Why People Fail at Self-Regulation. Cambridge, MA: Academic press.

Baumeister, R. F., Vohs, K. D., and Tice, D. M. (2007). The strength model of self-control. Curr. Dir. Psychol. Sci. 16, 351-355. doi: 10.1111/j.1467-8721.2007. 00534.x

Beeli, G., Koeneke, S., Gasser, K., and Jancke, L. (2008). Brain stimulation modulates driving behavior. Behav. Brain Funct. 4:34. doi: 10.1186/1744-90814-34

Berkman, E. T. (2017). "Routledge international handbook of self-control in health and well-being," in The Neuroscience of Self-Control, eds D. de Ridder, M. Adriaanse, and K. Fujita (Abingdon: Routledge).

Berkman, E. T., Burklund, L., and Lieberman, M. D. (2009). Inhibitory spillover: intentional motor inhibition produces incidental limbic inhibition via right inferior frontal cortex. Neuroimage 47, 705-712. doi: 10.1016/j.neuroimage. 2009.04.084

Bindman, L. J., Lippold, O. C. J., and Redfearn, J. W. T. (1964). The action of brief polarizing currents on the cerebral cortex of the rat (1) during current flow and (2) in the production of long-lasting after-effects. J. Physiol. 172, 369-382. doi: 10.1113/jphysiol.1964.sp007425

Blair, K. S., Smith, B. W., Mitchell, D. G. V., Morton, J., Vythilingam, M., Pessoa, L., et al. (2007). Modulation of emotion by cognition and cognition by emotion. Neuroimage 35, 430-440. doi: 10.1016/j.neuroimage.2006.11.048

Boggio, P. S., Bermpohl, F., Vergara, A. O., Muniz, A. L., Nahas, F. H., Leme, P. B., et al. (2007a). Go-no-go task performance improvement after anodal transcranial DC stimulation of the left dorsolateral prefrontal cortex in major depression. J. Affect. Disord. 101, 91-98.

Boggio, P. S., Nunes, A., Rigonatti, S. P., Nitsche, M. A., Pascual-Leone, A., and Fregni, F. (2007b). Repeated sessions of noninvasive brain DC stimulation is associated with motor function improvement in stroke patients. Restor. Neurol. Neurosci. 25, 123-129.

Boggio, P. S., Zaghi, S., and Fregni, F. (2009). Modulation of emotions associated with images of human pain using anodal transcranial direct current stimulation (tDCS). Neuropsychologia 47, 212-217. doi: 10.1016/j.neuropsychologia.2008. 07.022

Boggio, P. S., Zaghi, S., Lopes, M., and Fregni, F. (2008). Modulatory effects of anodal transcranial direct current stimulation on perception and pain thresholds in healthy volunteers. Eur. J. Neurol. 15, 1124-1130. doi: 10.1111/ j.1468-1331.2008.02270.x

Botvinick, M. M., Braver, T. S., Barch, D. M., Carter, C. S., and Cohen, J. D. (2001). Conflict monitoring and cognitive control. Psychol. Rev. 108, 624-652. doi: 10.1037/0033-295X.108.3.624

Botvinick, M. M., Cohen, J. D., and Carter, C. S. (2004). Conflict monitoring and anterior cingulate cortex: an update. Trends Cognit. Sci. 8, 539-546. doi: $10.1016 /$ j.tics.2004.10.003

Braver, T. S., Reynolds, J. R., and Donaldson, D. I. (2003). Neural mechanisms of transient and sustained cognitive control during task switching. Neuron 39, 713-726. doi: 10.1016/S0896-6273(03)00466-5

Burgess, E. E., Sylvester, M. D., Morse, K. E., Amthor, F. R., Mrug, S., Lokken, K. L., et al. (2016). Effects of transcranial direct current stimulation (tDCS) on binge-eating disorder. Int. J. Eat. Disord. 49, 930-936. doi: 10.1002/eat.22554

Bushman, B. J. (2002). Does venting anger feed or extinguish the flame? Catharsis, rumination, distraction, anger, and aggressive responding. Pers. Soc. Psychol. Bull. 28, 724-731. doi: 10.1177/0146167202289002
Canli, T., Desmond, J. E., Zhao, Z., Glover, G., and Gabrieli, J. D. (1998). Hemispheric asymmetry for emotional stimuli detected with fMRI. Neuroreport 9, 3233-3239. doi: 10.1097/00001756-199810050-00019

Carney, M. W. P. (1969). Negative polarisation of the brain in the treatment of manic states. Ir. J. Med. Sci. 2, 133-135. doi: 10.1007/BF02958921

Carver, C. S., and Scheier, M. F. (1982). Control theory: a useful conceptual framework for personality-social, clinical, and health psychology. Psychol. Bull. 92, 111-135. doi: 10.1037/0033-2909.92.1.111

Carver, C. S., and White, T. L. (1994). Behavioral inhibition, behavioral activation, and affective responses to impending reward and punishment: the BIS/BAS Scales. J. Pers. Soc. Psychol. 67, 319-333. doi: 10.1037/0022-3514.67.2.319

Casey, B. J., Somerville, L. H., Gotlib, I. H., Ayduk, O., Franklin, N. T., Askren, M. K., et al. (2011). Behavioral and neural correlates of delay of gratification 40 years later. Proc. Natl. Acad. Sci. U.S.A. 108, 14998-15003. doi: 10.1073/pnas. 1108561108

Chaieb, L., Antal, A., and Paulus, W. (2008). Gender-specific modulation of shortterm neuroplasticity in the visual cortex induced by transcranial direct current stimulation. Visual Neuroscience 25, 77-81. doi: 10.1017/S0952523808080097

Chaiken, S., and Trope, Y. (eds). (1999). Dual-Process Theories in Social Psychology. New York, NY: Guilford Press.

Cheng, G. L., and Lee, T. M. (2016). Altering risky decision-making: influence of impulsivity on the neuromodulation of prefrontal cortex. Soc. Neurosci. 11, 353-364. doi: 10.1080/17470919.2015.1085895

Chester, D. S., and DeWall, C. N. (2014). Prefrontal recruitment during social rejection predicts greater subsequent self-regulatory imbalance and impairment: neural and longitudinal evidence. Neuroimage 101, 485-493. doi: 10.1016/j.neuroimage.2014.07.054

Coan, J. A., and Allen, J. J. (2003). Frontal EEG asymmetry and the behavioral activation and inhibition systems. Psychophysiology 40, 106-114. doi: 10.1111/ 1469-8986.00011

Coan, J. A., Allen, J. J., and Harmon-Jones, E. (2001). Voluntary facial expression and hemispheric asymmetry over the frontal cortex. Psychophysiology 38, 912-925. doi: 10.1111/1469-8986.3860912

Cohen, J. D., Botvinick, M., and Carter, C. S. (2000). Anterior cingulate and prefrontal cortex: who's in control? Nat. Neurosci. 3, 421-423. doi: 10.1038/ 74783

Coifman, K. G., Berenson, K. R., Rafaeli, E., and Downey, G. (2012). From negative to positive and back again: polarized affective and relational experience in borderline personality disorder. J. Abnorm. Psychol. 121, 668-679. doi: 10.1037/ a0028502

Costain, R., Redfearn, J. W. T., and Lippold, O. C. J. (1964). A controlled trial of the therapeutic effects of polarization of the brain in depressive illness. Br. J. Psychiatry 110, 786-789. doi: 10.1192/bjp.110.469.786

Creutzfeldt, O. D., Fromm, G. H., and Kapp, H. (1962). Influence of transcortical dc currents on cortical neuronal activity. Exp. Neurol. 5, 436-452. doi: 10.1016/ 0014-4886(62)90056-0

Dambacher, F., Sack, A. T., Lobbestael, J., Arntz, A., Brugman, S., and Schuhmann, T. (2014). The role of right prefrontal and medial cortex in response inhibition: interfering with action restraint and action cancellation using transcranial magnetic brain stimulation. J. Cogn. Neurosci. 26, 1775-1784. doi: 10.1162/jocn_a_00595

Dambacher, F., Schuhmann, T., Lobbestael, J., Arntz, A., Brugman, S., and Sack, A. T. (2015a). No effects of bilateral tDCS over inferior frontal gyrus on response inhibition and aggression. PLoS One 10:e132170. doi: 10.1371/ journal.pone.0132170

Dambacher, F., Schuhmann, T., Lobbestael, J., Arntz, A., Brugman, S., and Sack, A. T. (2015b). Reducing proactive aggression through non-invasive brain stimulation. Soc. Cogn. Affect. Neurosci. 10, 1303-1309. doi: 10.1093/scan/ nsv018

Davidson, R. J., Ekman, P., Saron, C. D., Senulis, J. A., and Friesen, W. V. (1990). Approach-withdrawal and cerebral asymmetry: emotional expression and brain physiology: I. J. Pers. Soc. Psychol. 58, 330-341. doi: 10.1037/0022-3514.58. 2.330

Dawson, G., Panagiotides, H., Klinger, L. G., and Hill, D. (1992). The role of frontal lobe functioning in the development of infant self-regulatory behavior. Brain Cogn. 20, 152-175. doi: 10.1016/0278-2626(92)90066-U

Demos, K. E., Heatherton, T. F., and Kelley, W. M. (2012). Individual differences in nucleus accumbens activity to food and sexual images predict weight gain and 
sexual behavior. J. Neurosci. 32, 5549-5552. doi: 10.1523/JNEUROSCI.5958-11. 2012

Denson, T. F., Pedersen, W. C., and Miller, N. (2006). The displaced aggression questionnaire. J. Pers. Soc. Psychol. 90, 1032-1051. doi: 10.1037/0022-3514.90. 6.1032

Dixon-Gordon, K. L., Chapman, A. L., Lovasz, N., and Walters, K. (2011). Too upset to think: the interplay of borderline personality features, negative emotions, and social problem solving in the laboratory. Pers. Disord. 2, 243-260. doi: $10.1037 / \mathrm{a} 0021799$

Duecker, F., and Sack, A. T. (2015). Rethinking the role of sham TMS. Front. Psychol. 6:210. doi: 10.3389/fpsyg.2015.00210

Eisenberger, N. I. (2012). The pain of social disconnection: examining the shared neural underpinnings of physical and social pain. Nat. Rev. Neurosci. 13, 421-434. doi: 10.1038/nrn3231

Eisenberger, N. I., Lieberman, M. D., and Williams, K. D. (2003). Does rejection hurt? An fMRI study of social exclusion. Science 302, 290-292. doi: 10.1126/ science. 1089134

Fecteau, S., Knoch, D., Fregni, F., Sultani, N., Boggio, P., and PascualLeone, A. (2007a). Diminishing risk-taking behavior by modulating activity in the prefrontal cortex: a direct current stimulation study. J. Neurosci. 27, 12500-12505.

Fecteau, S., Pascual-Leone, A., Zald, D. H., Liguori, P., Theoret, H., Boggio, P. S., et al. (2007b). Activation of prefrontal cortex by transcranial direct current stimulation reduces appetite for risk during ambiguous decision making. J. Neurosci. 27, 6212-6218. doi: 10.1523/JNEUROSCI.0314-07.2007

Feeser, M., Prehn, K., Kazzer, P., Mungee, A., and Bajbouj, M. (2014). Transcranial direct current stimulation enhances cognitive control during emotion regulation. Brain Stimul. 7, 105-112. doi: 10.1016/j.brs.2013.08.006

Ferrucci, R., Bortolomasi, M., Vergari, M., Tadini, L., Salvoro, B., Giacopuzzi, M., et al. (2009). Transcranial direct current stimulation in severe, drug-resistant major depression. J. Affect. Disord. 118, 215-219. doi: 10.1016/j.jad.2009.02.015

Ferrucci, R., Marceglia, S., Vergari, M., Cogiamanian, F., Mrakic-Sposta, S., Mameli, F. E. E. A., et al. (2008). Cerebellar transcranial direct current stimulation impairs the practice-dependent proficiency increase in working memory. J. Cogn. Neurosci. 20, 1687-1697. doi: 10.1162/jocn.2008.20112

Fertonani, A., and Miniussi, C. (2017). Transcranial electrical stimulation: what we know and do not know about mechanisms. Neuroscientist 23, 109-123. doi: $10.1177 / 1073858416631966$

Figner, B., Knoch, D., Johnson, E. J., Krosch, A. R., Lisanby, S. H., Fehr, E., et al. (2010). Lateral prefrontal cortex and self-control in intertemporal choice. Nat. Neurosci. 13, 538-539. doi: 10.1038/nn.2516

Fregni, F., Boggio, P. S., Lima, M. C., Ferreira, M. J., Wagner, T., Rigonatti, S. P., et al. (2006a). A sham-controlled, phase II trial of transcranial direct current stimulation for the treatment of central pain in traumatic spinal cord injury. Pain 122, 197-209.

Fregni, F., Boggio, P. S., Nitsche, M. A., Marcolin, M. A., Rigonatti, S. P., and Pascual-Leone, A. (2006b). Treatment of major depression with transcranial direct current stimulation. Bipolar Disord. 8, 203-204. doi: 10.1111/j.1399-5618.2006.00291.x

Fregni, F., Orsati, F., Pedrosa, W., Fecteau, S., Tome, F. A., Nitsche, M. A., et al. (2008). Transcranial direct current stimulation of the prefrontal cortex modulates the desire for specific foods. Appetite 51, 34-41. doi: 10.1016/j.appet. 2007.09.016

Fritsch, B., Reis, J., Martinowich, K., Schambra, H. M., Ji, Y., Cohen, L. G., et al. (2010). Direct current stimulation promotes BDNF-dependent synaptic plasticity: potential implications for motor learning. Neuron 66, 198-204. doi: 10.1016/j.neuron.2010.03.035

Gandiga, P. C., Hummel, F. C., and Cohen, L. G. (2006). Transcranial DC stimulation (tDCS): a tool for double-blind sham-controlled clinical studies in brain stimulation. Clin. Neurophysiol. 117, 845-850. doi: 10.1016/j.clinph.2005. 12.003

Gartside, I. B. (1968). Mechanisms of sustained increases of firing rate of neurons in the rat Cerebral cortex after polarization: role of protein synthesis. Nature 220, 383-384. doi: 10.1038/220383a0

Gilmore, C. S., Dickmann, P. J., Nelson, B. G., Lamberty, G. J., and Lim, K. O. (2017). Transcranial Direct Current Stimulation (tDCS) paired with a decisionmaking task reduces risk-taking in a clinically impulsive sample. Brain Stimul. 11, 302-309. doi: 10.1016/j.brs.2017.11.011
Giuliani, N. R., Mann, T., Tomiyama, A. J., and Berkman, E. T. (2014). Neural systems underlying the reappraisal of personally craved foods. J. Cogn. Neurosci. 26, 1390-1402. doi: 10.1162/jocn_a_00563

Goldman, R. L., Borckardt, J. J., Frohman, H. A., O’Neil, P. M., Madan, A., Campbell, L. K., et al. (2011). Prefrontal cortex transcranial direct current stimulation (tDCS) temporarily reduces food cravings and increases the selfreported ability to resist food in adults with frequent food craving. Appetite 56, 741-746. doi: 10.1016/j.appet.2011.02.013

Gross, J. J. (1998). Antecedent-and response-focused emotion regulation: divergent consequences for experience, expression, and physiology. J. Pers. Soc. Psychol. 74, 224-237. doi: 10.1037/0022-3514.74.1.224

Gross, J. J. (2001). Emotion regulation in adulthood: timing is everything. Curr. Dir. Psychol. Sci. 10, 214-219. doi: 10.1111/1467-8721.00152

Harmon-Jones, E. (2003). Clarifying the emotive functions of asymmetrical frontal cortical activity. Psychophysiology 40, 838-848. doi: 10.1111/1469-8986.00121

Harmon-Jones, E. (2007). Trait anger predicts relative left frontal cortical activation to anger-inducing stimuli. Int. J. Psychophysiol. 66, 154-160. doi: 10.1016/j. ijpsycho.2007.03.020

Harmon-Jones, E., Abramson, L. Y., Sigelman, J., Bohlig, A., Hogan, M. E., and Harmon-Jones, C. (2002). Proneness to hypomania/mania or depression and asymmetrical frontal cortical responses to an anger-evoking event. J. Pers. Soc. Psychol. 82, 610-618.

Harmon-Jones, E., and Allen, J. J. (1997). Behavioral activation sensitivity and resting frontal EEG asymmetry: covariation of putative indicators related to risk for mood disorders. J. Abnorm. Psychol. 106, 159-163. doi: 10.1037/0021-843X. 106.1.159

Harmon-Jones, E., and Allen, J. J. (1998). Anger and frontal brain activity: EEG asymmetry consistent with approach motivation despite negative affective valence. J. Pers. Soc. Psychol. 74, 1310-1316. doi: 10.1037/0022-3514.74.5. 1310

Harmon-Jones, E., Gable, P. A., and Peterson, C. K. (2010). The role of asymmetric frontal cortical activity in emotion-related phenomena: a review and update. Biol. Psychol. 84, 451-462. doi: 10.1016/j.biopsycho.2009.08.010

Harmon-Jones, E., Lueck, L., Fearn, M., and Harmon-Jones, C. (2006). The effect of personal relevance and approach-related action expectation on relative left frontal cortical activity. Psychol. Sci. 17, 434-440. doi: 10.1111/j.1467-9280. 2006.01724.x

Harmon-Jones, E., Peterson, C. K., and Harris, C. R. (2009). Jealousy: novel methods and neural correlates. Emotion 9, 113-117. doi: 10.1037/a001 4117

Harmon-Jones, E., and Sigelman, J. (2001). State anger and prefrontal brain activity: evidence that insult-related relative left-prefrontal activation is associated with experienced anger and aggression. J. Pers. Soc. Psychol. 80, 797-803. doi: 10.1037/0022-3514.80.5.797

Hattori, Y., Moriwaki, A., and Hori, Y. (1990). Biphasic effects of polarizing current on adenosine-sensitive generation of cyclic AMP in rat cerebral cortex. Neurosci. Lett. 116, 320-324. doi: 10.1016/0304-3940(90)90094-P

Heatherton, T. F., and Wagner, D. D. (2011). Cognitive neuroscience of selfregulation failure. Trends Cogn. Sci. 15, 132-139. doi: 10.1016/j.tics.2010. 12.005

Hecht, D., Walsh, V., and Lavidor, M. (2012). Bi-frontal direct current stimulation affects delay discounting choices. Cogn. Neurosci. 4, 7-11. doi: 10.1080/ 17588928.2011.638139

Heller, W., Etienne, M. A., and Miller, G. A. (1995). Patterns of perceptual asymmetry in depression and anxiety: implications for neuropsychological models of emotion and psychopathology. J. Abnorm. Psychol. 104, 327-327. doi: 10.1037/0021-843X.104.2.327

Herrmann, M. J., Beier, J. S., Simons, B., and Polak, T. (2016). Transcranial Direct Current Stimulation (tDCS) of the right inferior frontal gyrus attenuates skin conductance responses to unpredictable threat conditions. Front. Hum. Neurosci. 10:352. doi: 10.3389/fnhum.2016.00352

Herrmann, M. J., Simons, B., and Polak, T. (2017). Transcranial direct current stimulation (tDCS) of the right inferior frontal gyrus attenuates sustained fear. Brain Stimul. 10, 376-376. doi: 10.3389/fnhum.2016.00352

Hortensius, R., Schutter, D. J., and Harmon-Jones, E. (2012). When anger leads to aggression: induction of relative left frontal cortical activity with transcranial direct current stimulation increases the anger-aggression relationship. Soc. Cogn. Affect. Neurosci. 7, 342-347. doi: 10.1093/scan/nsr012 
Inzlicht, M., and Schmeichel, B. J. (2012). What is ego depletion? Toward a mechanistic revision of the resource model of self-control. Perspect. Psychol. Sci. 7, 450-463. doi: 10.1177/1745691612454134

Islam, N., Aftabuddin, M., Moriwaki, A., Hattori, Y., and Hori, Y. (1995). Increase in the calcium level following anodal polarization in the rat brain. Brain Res. 684, 206-208. doi: 10.1016/0006-8993(95)00434-R

Iyer, M. B., Mattu, U., Grafman, J., Lomarev, M., Sato, S., and Wassermann, E. M. (2005). Safety and cognitive effect of frontal DC brain polarization in healthy individuals. Neurology 64, 872-875. doi: 10.1212/01.WNL.0000152986. 07469.E9

Jacobson, L., Koslowsky, M., and Lavidor, M. (2012). tDCS polarity effects in motor and cognitive domains: a meta-analytical review. Exp. Brain Res. 216, 1-10. doi: 10.1007/s00221-011-2891-9

Jauch-Chara, K., Kistenmacher, A., Herzog, N., Schwarz, M., Schweiger, U., and Oltmanns, K. M. (2014). Repetitive electric brain stimulation reduces food intake in humans. Am. J. Clin. Nutr. 100, 1003-1009. doi: 10.3945/ajcn.113. 075481

Kalin, N. H., Larson, C., Shelton, S. E., and Davidson, R. J. (1998). Asymmetric frontal brain activity, cortisol, and behavior associated with fearful temperament in rhesus monkeys. Behav. Neurosci. 112, 286-292. doi: 10.1037/ 0735-7044.112.2.286

Kalisch, R., Korenfeld, E., Stephan, K. E., Weiskopf, N., Seymour, B., and Dolan, R. J. (2006). Context-dependent human extinction memory is mediated by a ventromedial prefrontal and hippocampal network. J. Neurosci. 26, 9503-9511. doi: 10.1523/JNEUROSCI.2021-06.2006

Kekic, M., McClelland, J., Campbell, I., Nestler, S., Rubia, K., David, A. S., et al. (2014). The effects of prefrontal cortex transcranial direct current stimulation (tDCS) on food craving and temporal discounting in women with frequent food cravings. Appetite 78, 55-62. doi: 10.1016/j.appet.2014.03.010

Kelley, N. J., Eastwick, P. W., Harmon-jones, E., and Schmeichel, B. J. (2015a). Jealousy increased by induced relative left frontal cortical activity. Emotion 15, 550-555. doi: 10.1037/emo0000068

Kelley, N. J., Finley, A. J., and Schmeichel, B. J. (2018). Aftereffects of SelfControl: The Reward Responsivity Hypothesis. College Station, TX: Texas A\&M University.

Kelley, N. J., Hortensius, R., and Harmon-Jones, E. (2013). When anger leads to rumination: induction of relative right frontal cortical activity with transcranial direct current stimulation increases anger-related rumination. Psychol. Sci. 24, 475-481. doi: 10.1177/0956797612457384

Kelley, N. J., and Schmeichel, B. J. (2016). Noninvasive stimulation over the dorsolateral prefrontal cortex facilitates the inhibition of motivated responding. J. Exp. Psychol. 145, 1702-1712. doi: 10.1037/xge0000238

Kelley, W. M., Wagner, D. D., and Heatherton, T. F. (2015b). In search of a human self-regulation system. Ann. Rev. Neurosci. 38, 389-411. doi: 10.1146/annurevneuro-071013-014243

Kohn, N., Eickhoff, S. B., Scheller, M., Laird, A. R., Fox, P. T., and Habel, U. (2014). Neural network of cognitive emotion regulation - an ALE meta-analysis and MACM analysis. Neuroimage 87, 345-355. doi: 10.1016/j.neuroimage.2013. 11.001

Kopala-Sibley, D. C., Zuroff, D. C., Russell, J. J., Moskowitz, D. S., and Paris, J. (2012). Understanding heterogeneity in borderline personality disorder: differences in affective reactivity explained by the traits of dependency and self-criticism. J. Abnorm. Psychol. 121, 680-691. doi: 10.1037/a002 8513

Kross, E., Egner, T., Ochsner, K., Hirsch, J., and Downey, G. (2007). Neural dynamics of rejection sensitivity. J. Cogn. Neurosci. 19, 945-956. doi: 10.1162/ jocn.2007.19.6.945

Kuo, M. F., Paulus, W., and Nitsche, M. A. (2006). Sex differences of cortical neuroplasticity in humans. Neuroreport 17, 1703-1707. doi: 10.1097/01.wnr. 0000239955.68319.c2

Lang, P. J., Bradley, M. M., and Cuthbert, B. N. (2008). International Affective Picture System (IAPS): Affective Ratings of Pictures and Instruction Manual. Technical Report, No. A-8. Gainesville, FL: University of Florida.

Lapenta, O. M., Di Sierve, K., de Macedo, E. C., Fregni, F., and Boggio, P. S. (2014). Transcranial direct current stimulation modulates ERP-indexed inhibitory control and reduces food consumption. Appetite 83, 42-48. doi: 10.1016/j.appet. 2014.08.005
Lejuez, C. W., Read, J. P., Kahler, C. W., Richards, J. B., Ramsey, S. E., Stuart, G. L., et al. (2002). Evaluation of a behavioral measure of risk taking: the Balloon Analogue Risk Task (BART). J. Exp. Psychol. 8, 75-84. doi: 10.1037/1076-898X. 8.2.75

Lieberman, J. D., Solomon, S., Greenberg, J., and McGregor, H. A. (1999). A hot new way to measure aggression: hot sauce allocation. Aggress. Behav. 25, 331-348. doi: 10.1002/(SICI) 1098-2337(1999)25:5<331::AID-AB2>3.0.CO;2- 1

Liebetanz, D., Nitsche, M. A., Tergau, F., and Paulus, W. (2002). Pharmacological approach to the mechanisms of transcranial DC-stimulation-induced after effects of human motor cortex excitability. Brain 125, 2238-2247. doi: 10.1093/ brain/awf 238

Ljubisavljevic, M., Maxood, K., Bjekic, J., Oommen, J., and Nagelkerke, N. (2016). Long-term effects of repeated prefrontal cortex transcranial direct current stimulation (tDCS) on food craving in normal and overweight young adults. Brain Stimul. 9, 826-833. doi: 10.1016/j.brs.2016.07.002

Lolas, F. (1977). Brain polarization: behavioral and therapeutic effects. Biol. Psychiatry 12, 37-47.

Lopez, R. B., Chen, P. H. A., Huckins, J. F., Hofmann, W., Kelley, W. M., and Heatherton, T. F. (2017). A balance of activity in brain control and reward systems predicts self-regulatory outcomes. Soc. Cogn. Affect. Neurosci. 12, 832-838. doi: 10.1093/scan/nsx004

Lopez, R. B., Hofmann, W., Wagner, D. D., Kelley, W. M., and Heatherton, T. F. (2014). Neural predictors of giving in to temptation in daily life. Psychol. Sci. 25, 1337-1344. doi: 10.1177/0956797614531492

López-Alonso, V., Fernández-del-Olmo, M., Costantini, A., Gonzalez-Henriquez, J. J., and Cheeran, B. (2015). Intra-individual variability in the response to anodal transcranial direct current stimulation. Clin. Neurophysiol. 126, 2342-2347. doi: 10.1016/j.clinph.2015.03.022

MacDonald, A. W., Cohen, J. D., Stenger, V. A., and Carter, C. S. (2000). Dissociating the role of the dorsolateral prefrontal and anterior cingulate cortex in cognitive control. Science 288, 1835-1838. doi: 10.1126/science.288.5472. 1835

Mariano, T. Y., van't Wout, M., Garnaat, S. L., Rasmussen, S. A., and Greenberg, B. D. (2016). Transcranial direct current stimulation (tDCS) targeting left dorsolateral prefrontal cortex modulates task-induced acute pain in healthy volunteers. Pain Med. 17, 737-745. doi: 10.1093/pm/pnv042

Mariano, T. Y., van't Wout, M., Jacobson, B. L., Garnaat, S. L., Kirschner, J. L., Rasmussen, S. A., et al. (2015). Effects of transcranial direct current stimulation (tDCS) on pain distress tolerance: a preliminary study. Pain Med. 16, 15801588. doi: 10.1111/pme.12798

Masten, C. L., Eisenberger, N. I., Borofsky, L. A., Pfeifer, J. H., McNealy, K., Mazziotta, J. C., et al. (2009). Neural correlates of social exclusion during adolescence: understanding the distress of peer rejection. Soc. Cogn. Affect. Neurosci. 4, 143-157. doi: 10.1093/scan/nsp007

Mattavelli, G., Pisoni, A., Casarotti, A., Comi, A., Sera, G., Riva, M., et al. (2017). Consequences of brain tumour resection on emotion recognition. J. Neuropsychol. doi: 10.1111/jnp.12130 [Epub ahead of print].

Mauss, I. B., Bunge, S. A., and Gross, J. J. (2007). Automatic emotion regulation. Soc. Pers. Psychol. Compass 1, 146-167. doi: 10.1111/j.1751-9004.2007.00005.x

Metcalfe, J., and Mischel, W. (1999). A hot/cool-system analysis of delay of gratification: dynamics of willpower. Psychol. Rev. 106, 3-19. doi: 10.1037/0033295X.106.1.3

Meyer, H. C., and Bucci, D. J. (2016). Imbalanced activity in the orbitofrontal cortex and nucleus accumbens impairs behavioral inhibition. Curr. Biol. 26, 2834-2839. doi: 10.1016/j.cub.2016.08.034

Miranda, P. C., Mekonnen, A., Salvador, R., and Ruffini, G. (2013). The electric field in the cortex during transcranial current stimulation. Neuroimage 70, 48-58. doi: 10.1016/j.neuroimage.2012.12.034

Mischel, W. (1958). Preference for delayed reinforcement: an experimental study of a cultural observation. Journal of Abnormal and Social Psychology 56, 57-61. doi: $10.1037 / \mathrm{h} 0041895$

Mischel, W., Ebbesen, E. B., and Zeiss, A. R. (1972). Cognitive and attentional mechanisms in delay of gratification. J. Pers. Soc. Psychol. 21, 204-218. doi: 10.1037/h0032198

Mungee, A., Burger, M., and Bajbouj, M. (2016). No effect of cathodal transcranial direct current stimulation on fear memory in healthy human subjects. Brain Sci. 6, 55. doi: 10.3390/brainsci6040055 
Mungee, A., Kazzer, P., Feeser, M., Nitsche, M. A., Schiller, D., and Bajbouj, M. (2014). Transcranial direct current stimulation of the prefrontal cortex: a means to modulate fear memories. Neuroreport 25, 480-484. doi: 10.1097/WNR. 0000000000000119

Muraven, M., and Baumeister, R. F. (2000). Self-regulation and depletion of limited resources: Does self-control resemble a muscle? Psychol. Bull. 126, 247-259. doi: 10.1037/0033-2909.126.2.247

Mylius, V., Jung, M., Menzler, K., Haag, A., Khader, P. H., Oertel, W. H., et al. (2012). Effects of transcranial direct current stimulation on pain perception and working memory. Eur. J. Pain 16, 974-982. doi: 10.1002/j.1532-2149.2011. 00105.x

Neal, L. B., and Gable, P. A. (2017). Regulatory control and impulsivity relate to resting frontal activity. Soc. Cogn. Affect. Neurosci. 12, 1377-1383. doi: 10.1093/ scan/nsx080

Nejati, V., Salehinejad, M. A., and Nitsche, M. A. (2018). Interaction of the left dorsolateral prefrontal cortex (l-DLPFC) and right orbitofrontal cortex (OFC) in hot and cold executive functions: evidence from transcranial direct current stimulation (tDCS). Neuroscience 369, 109-123. doi: 10.1016/j.neuroscience. 2017.10.042

Nitsche, M. A., Boggio, P. S., Fregni, F., and Pascual-Leone, A. (2009). Treatment of depression with transcranial direct current stimulation (tDCS): a review. Experimental neurology 219, 14-19. doi: 10.1016/j.expneurol.2009.03.038

Nitsche, M. A., Cohen, L. G., Wassermann, E. M., Priori, A., Lang, N., Antal, A., et al. (2008). Transcranial direct current stimulation: state of the art 2008. Brain Stimul. 1, 206-223. doi: 10.1016/j.brs.2008.06.004

Nitsche, M. A., Doemkes, S., Karakoese, T., Antal, A., Liebetanz, D., Lang, N., et al. (2007). Shaping the effects of transcranial direct current stimulation of the human motor cortex. J. Neurophysiol. 97, 3109-3117. doi: 10.1152/jn.01312. 2006

Nitsche, M. A., Fricke, K., Henschke, U., Schlitterlau, A., Liebetanz, D., Lang, N., et al. (2003a). Pharmacological modulation of cortical excitability shifts induced by transcranial direct current stimulation in humans. J. Physiol. 553, 293-301. doi: 10.1113/jphysiol.2003.049916

Nitsche, M. A., Liebetanz, D., Antal, A., Lang, N., Tergau, F., and Paulus, W. (2003b). Modulation of cortical excitability by weak direct current stimulationtechnical, safety and functional aspects. Suppl. Clin. Neurophysiol. 56, 255-276. doi: 10.1016/S1567-424X(09)70230-2

Nitsche, M. A., Niehaus, L., Hoffmann, K. T., Hengst, S., Liebetanz, D., Paulus, W., et al. (2004). MRI study of human brain exposed to weak direct current stimulation of the frontal cortex. Clin. Neurophysiol. 115, 2419-2423. doi: 10. 1016/j.clinph.2004.05.001

Nitsche, M. A., Nitsche, M. S., Klein, C. C., Tergau, F., Rothwell, J. C., and Paulus, W. (2003c). Level of action of cathodal DC polarisation induced inhibition of the human motor cortex. Clin. Neurophysiol. 114, 600-604.

Nitsche, M. A., and Paulus, W. (2000). Excitability changes induced in the human motor cortex by weak transcranial direct current stimulation. J. Physiol. 527, 633-639. doi: 10.1111/j.1469-7793.2000.t01-1-00633.x

Nolen-Hoeksema, S. (2000). The role of rumination in depressive disorders and mixed anxiety/depressive symptoms. J. Abnorm. Psychol. 109, 504-511. doi: 10.1037/0021-843X.109.3.504

Ochs, L. (2006). The low energy neurofeedback system (LENS): theory, background, and introduction. J. Neurother. 10, 5-39. doi: 10.1300/ J184v10n02_02

Ochsner, K. N., and Gross, J. J. (2007). "The neural architecture of emotion regulation," in Handbook of Emotion Regulation, ed. J. J. Gross (New York, NY: Guilford Press), 87-109.

Ochsner, K. N., Hughes, B., Robertson, E. R., Cooper, J. C., and Gabrieli, J. D. (2009). Neural systems supporting the control of affective and cognitive conflicts. J. Cogn. Neurosci. 21, 1841-1854. doi: 10.1162/jocn.2009.21129

Onoda, K., Okamoto, Y., Nakashima, K. I., Nittono, H., Yoshimura, S., Yamawaki, S., et al. (2010). Does low self-esteem enhance social pain? The relationship between trait self-esteem and anterior cingulate cortex activation induced by ostracism. Soc. Cogn. Affect. Neurosci. 5, 385-391. doi: 10.1093/scan/ nsq002

Paulus, M. P., Rogalsky, C., Simmons, A., Feinstein, J. S., and Stein, M. B. (2003). Increased activation in the right insula during risk-taking decision making is related to harm avoidance and neuroticism. Neuroimage 19, 1439-1448. doi: 10.1016/S1053-8119(03)00251-9
Peña-Gómez, C., Sala-Lonch, R., Junqué, C., Clemente, I. C., Vidal, D., Bargalló, N., et al. (2012). Modulation of large-scale brain networks by transcranial direct current stimulation evidenced by resting-state functional MRI. Brain Stimul. 5, 252-263. doi: 10.1016/j.brs.2011.08.006

Peña-Gómez, C., Vidal-Piñeiro, D., Clemente, I. C., Pascual-Leone, Á, and Bartrés-Faz, D. (2011). Down-regulation of negative emotional processing by transcranial direct current stimulation: effects of personality characteristics. PLoS One 6:e22812. doi: 10.1371/journal.pone.0022812

Phillips, M. L. (2003). Understanding the neurobiology of emotion perception: implications for psychiatry. Br. J. Psychiatry 182, 190-192. doi: 10.1192/bjp.182. 3.190

Phillips, M. L., Ladouceur, C. D., and Drevets, W. C. (2008). A neural model of voluntary and automatic emotion regulation: implications for understanding the pathophysiology and neurodevelopment of bipolar disorder. Mol. Psychiatry 13, 829-857. doi: 10.1038/mp.2008.65

Pisoni, A., Mattavelli, G., Papagno, C., Rosanova, M., Casali, A. G., and Romero Lauro, L. J. (2017). Cognitive enhancement induced by anodal tDCS drives circuit-specific cortical plasticity. Cereb. Cortex 28, 1132-1140. doi: 10.1093/ cercor/bhx021

Pitcher, J. B., Ogston, K. M., and Miles, T. S. (2003). Age and sex differences in human motor cortex input-output characteristics. J. Physiol. 546, 605-613. doi: 10.1113/jphysiol.2002.029454

Poreisz, C., Boros, K., Antal, A., and Paulus, W. (2007). Safety aspects of transcranial direct current stimulation concerning healthy subjects and patients. Brain Res. Bull. 72, 208-214. doi: 10.1016/j.brainresbull.2007. 01.004

Powers, A., Madan, A., Hilbert, M., Reeves, S. T., George, M., Nash, M. R., et al. (2018). Effects of combining a brief cognitive intervention with transcranial direct current stimulation on pain tolerance: a randomized controlled pilot study. Pain Med. 19, 677-685. doi: 10.1093/pm/pnx098

Priori, A. (2003). Brain polarization in humans: a reappraisal of an old tool for prolonged non-invasive modulation of brain excitability. Clin. Neurophysiol. 114, 589-595. doi: 10.1016/S1388-2457(02)00437-6

Priori, A., Berardelli, A., Rona, S., Accornero, N., and Manfredi, M. (1998). Polarization of the human motor cortex through the scalp. Neuroreport 9, 2257-2260. doi: 10.1097/00001756-199807130-00020

Pripfl, J., and Lamm, C. (2015). Focused transcranial direct current stimulation (tDCS) over thedorsolateral prefrontal cortex modulates specific domains of self-regulation. Neurosci. Res. 91, 41-47. doi: 10.1016/j.neures.2014.09.007

Purpura, D. P., and McMurtry, J. G. (1965). Intracellular activities and evoked potential changesduring polarization of motor cortex. J. Neurophysiol. 28, 166-185. doi: 10.1152/jn.1965.28.1.166

Quartarone, A., Lang, N., Rizzo, V., Bagnato, S., Morgante, F., Sant'Angelo, A., et al. (2007). Motor cortex abnormalities in amyotrophic lateral sclerosis with transcranial direct current stimulation. Muscle Nerve 35, 620-624. doi: 10.1002/ mus. 20737

Riva, P., Gabbiadini, A., Lauro, L. J. R., Andrighetto, L., Volpato, C., and Bushman, B. J. (2017). Neuromodulation can reduce aggressive behavior elicited by violent video games. Cogn. Affect. Behav. Neurosci. 17, 452-459. doi: 10.3758/s13415016-0490-8

Riva, P., Romero Lauro, L. J., DeWall, C. N., and Bushman, B. J. (2012). Buffer the pain away: stimulating the right ventrolateral prefrontal cortex reduces pain following social exclusion. Psychol. Sci. 23, 1473-1475. doi: 10.1177/ 0956797612450894

Riva, P., Romero Lauro, L. J., DeWall, C. N., Chester, D. S., and Bushman, B. J. (2014a). Reducing aggressive responses to social exclusion using transcranial direct current stimulation. Soc. Cogn. Affect. Neurosci. 10, 352-356. doi: 10. 1093/scan/nsu053

Riva, P., Wesselmann, E. D., Wirth, J. H., Carter-Sowell, A. R., and Williams, K. D. (2014b). When pain does not heal: the common antecedents and consequences of chronic social and physical pain. Basic Appl. Soc. Psychol. 36, 329-346. doi: 10.1080/01973533.2014.917975

Rogers, R. D., Owen, A. M., Middleton, H. C., Williams, E. J., Pickard, J. D., Sahakian, B. J., et al. (1999). Choosing between small, likely rewards and large, unlikely rewards activates inferior and orbital prefrontal cortex. J. Neurosci. 19, 9029-9038. doi: 10.1523/JNEUROSCI.19-20-09029.1999

Romero Lauro, L., Pisoni, A., Rosanova, M., Casarotto, S., Mattavelli, G., Bolognini, N., et al. (2016). Localizing the effects of anodal tDCS at the 
level of cortical sources: a Reply to Bailey et al., 2015. Cortex 74, 323-328. doi: 10.1016/j.cortex.2015.04.023

Romero Lauro, L. J., Rosanova, M., Mattavelli, G., Convento, S., Pisoni, A., Opitz, A., et al. (2014). TDCS increases cortical excitability: direct evidence from TMS-EEG. Cortex 58, 99-111. doi: 10.1016/j.cortex.2014.05.003

Salas, C. E., Castro, O., Yuen, K. S., Radovic, D., d'Avossa, G., and Turnbull, O. H. (2016). 'Just can't hide it': a behavioral and lesion study on emotional response modulation after right prefrontal damage. Soc. Cogn. Affect. Neurosci. 11, 1528-1540. doi: 10.1093/scan/nsw075

Sarkar, A., Dowker, A., and Kadosh, R. C. (2014). Cognitive enhancement or cognitive cost: trait-specific outcomes of brain stimulation in the case of mathematics anxiety. J. Neurosci. 34, 16605-16610. doi: 10.1523/JNEUROSCI. 3129-14.2014

Seminowicz, D. A., and Moayedi, M. (2017). The dorsolateral prefrontal cortex in acute and chronic pain. J. Pain 18, 1027-1035. doi: 10.1016/j.jpain.2017.03.008

Shenhav, A., Botvinick, M. M., and Cohen, J. D. (2013). The expected value of control: an integrative theory of anterior cingulate cortex function. Neuron 79, 217-240. doi: 10.1016/j.neuron.2013.07.007

Smittenaar, P., FitzGerald, T. H., Romei, V., Wright, N. D., and Dolan, R. J. (2013). Disruption of dorsolateral prefrontal cortex decreases model-based in favor of model-free control in humans. Neuron 80, 914-919. doi: 10.1016/j.neuron.2013. 08.009

Stagg, C. J., Best, J. G., Stephenson, M. C., O'Shea, J., Wylezinska, M., Kincses, Z. T., et al. (2009). Polarity-sensitive modulation of cortical neurotransmitters by transcranial stimulation. J. Neurosci. 29, 5202-5206. doi: 10.1523/JNEUROSCI. 4432-08.2009

Stagg, C. J., Lin, R. L., Mezue, M., Segerdahl, A., Kong, Y., Xie, J., et al. (2013). Widespread modulation of cerebral perfusion induced during and after transcranial direct current stimulation applied to the left dorsolateral prefrontal cortex. J. Neurosci. 33, 11425-11431. doi: 10.1523/JNEUROSCI.3887-12.2013

Stagg, C. J., and Nitsche, M. A. (2011). Physiological basis of transcranial direct current stimulation. Neuroscientist 17, 37-53. doi: 10.1177/1073858410386614

Strack, F., and Deutsch, R. (2004). Reflective and impulsive determinants of social behavior. Pers. Soc. Psychol. Rev. 8, 220-247. doi: 10.1207/s15327957pspr0803_1

Suslow, T., Kugel, H., Reber, H., Bauer, J., Dannlowski, U., Kersting, A., et al. (2010). Automatic brain response to facial emotion as a function of implicitly and explicitly measured extraversion. Neuroscience 167, 111-123. doi: 10.1016/ j.neuroscience.2010.01.038

Sutton, S. K., and Davidson, R. J. (1997). Prefrontal brain asymmetry: a biological substrate of the behavioral approach and inhibition systems. Psychol. Sci. 8, 204-210. doi: 10.1111/j.1467-9280.1997.tb00413.x

Swick, D., Ashley, V., and Turken, U. (2011). Are the neural correlates of stopping and not going identical? Quantitative meta-analysis of two response inhibition tasks. Neuroimage 56, 1655-1665. doi: 10.1016/j.neuroimage.2011.02.070

Taylor, S. F., and Liberzon, I. (2007). Neural correlates of emotion regulation in psychopathology. Trends Cogn. Sci. 11, 413-418. doi: 10.1016/j.tics.2007.08.006

Taylor, S. P. (1967). Aggressive behavior and physiological arousal as a function of provocation and the tendency to inhibit aggression. J. Pers. 35, 297-310. doi: 10.1111/j.1467-6494.1967.tb01430.x

Tomarken, A. J., Davidson, R. J., and Henriques, J. B. (1990). Resting frontal brain asymmetry predicts affective responses to films. J. Pers. Soc. Psychol. 59, 791-801. doi: 10.1037/0022-3514.59.4.791

Tomarken, A. J., Davidson, R. J., Wheeler, R. E., and Doss, R. C. (1992). Individual differences in anterior brain asymmetry and fundamental dimensions of emotion. J. Pers. Soc. Psychol. 62, 676-687. doi: 10.1037/0022-3514.62.4.676

Tufail, Y., Yoshihiro, A., Pati, S., Li, M. M., and Tyler, W. J. (2011). Ultrasonic neuromodulation by brain stimulation with transcranial ultrasound. Nat. Protocols 6, 1453-1470. doi: 10.1038/nprot.2011.371

Vergallito, A., Riva, P., Pisoni, A., and Lauro, L. J. R. (2018). Modulation of negative emotions through anodal tDCS over the right ventrolateral prefrontal cortex. Neuropsychologia 119, 128-135. doi: 10.1016/j.neuropsychologia.2018. 07.037

Vijayakumar, N., Whittle, S., Yücel, M., Dennison, M., Simmons, J., and Allen, N. B. (2014). Prefrontal structural correlates of cognitive control during adolescent development: a 4-year longitudinal study. J. Cogn. Neurosci. 26, 1118-1130. doi: 10.1162/jocn_a_00549

Vohs, K. D., and Baumeister, R. F. (eds). (2016). Handbook of Self-Regulation Research, Theory, and Applications. New York, NY: Guilford Publications.

Vöröslakos, M., Takeuchi, Y., Brinyiczki, K., Zombori, T., Oliva, A., FernándezRuiz, A., et al. (2018). Direct effects of transcranial electric stimulation on brain circuits in rats and humans. Nat. Commun. 9:483. doi: 10.1038/s41467-01802928-3

Wacker, J., Chavanon, M. L., Leue, A., and Stemmler, G. (2008). Is running away right? The behavioral activation-behavioral inhibition model of anterior asymmetry. Emotion 8, 232-249. doi: 10.1037/1528-3542.8.2.232

Wade, N. G., Vogel, D. L., Liao, K. Y. H., and Goldman, D. B. (2008). Measuring state-specific rumination: development of the rumination about an interpersonal offense scale. J. Couns. Psychol. 55, 419-426. doi: 10.1037/00220167.55.3.419

Wager, T. D., Davidson, M. L., Hughes, B. L., Lindquist, M. A., and Ochsner, K. N. (2008). Prefrontal subcortical pathways mediating successful emotion regulation. Neuron 59, 1037-1050. doi: 10.1016/j.neuron.2008.09.006

Wagner, D. D., Altman, M., Boswell, R. G., Kelley, W. M., and Heatherton, T. F. (2013). Self-regulatory depletion enhances neural responses to rewards and impairs top-down control. Psychol. Sci. 24, 2262-2271. doi: 10.1177/ 0956797613492985

Wagner, D. D., and Heatherton, T. F. (2012). Self-regulatory depletion increases emotional reactivity in the amygdala. Soc. Cogn. Affect. Neurosci. 8, 410-417. doi: $10.1093 /$ scan/nss082

Wagner, D. D., and Heatherton, T. F. (2017). "The cognitive neuroscience of self-regulatory failure," in Handbook of Self-Regulation: Research, Theory, and Applications, eds K. D. Vohs and R. F. Baumeister (New York, NY: Guilford Press), 111.

Weber, M. J., Messing, S. B., Rao, H., Detre, J. A., and Thompson-Schill, S. L. (2014). Prefrontal transcranial direct current stimulation alters activation and connectivity in cortical and subcortical reward systems: a tDCS fMRI study. Hum. Brain Mapp. 35, 3673-3686. doi: 10.1002/hbm.22429

Wheeler, R. E., Davidson, R. J., and Tomarken, A. J. (1993). Frontal brain asymmetry and emotional reactivity: a biological substrate of affective style. Psychophysiology 30, 82-89. doi: 10.1111/j.1469-8986.1993.tb03207.x

Wiethoff, S., Hamada, M., and Rothwell, J. C. (2014). Variability in response to transcranial direct current stimulation of the motor cortex. Brain Stimul. 7, 468-475. doi: 10.1016/j.brs.2014.02.003

Williams, K. D. (2009). Ostracism: a temporal need-threat model. Adv. Exp. Soc. Psychol. 41, 275-314.

Williams, K. D., Cheung, C. K., and Choi, W. (2000). Cyberostracism: effects of being ignored over the Internet. J. Pers. Soc. Psychol. 79, 748-762. doi: 10.1037/ 0022-3514.79.5.748

Zago, S., Ferrucci, R., Fregni, F., and Priori, A. (2008). Bartholow, Sciamanna, Alberti: pioneers in the electrical stimulation of the exposed human cerebral cortex. Neuroscientist 14, 521-528. doi: 10.1177/10738584073 11101

Zinner, L. R., Brodish, A. B., Devine, P. G., and Harmon-Jones, E. (2008). Anger and asymmetrical frontal cortical activity: evidence for an anger-withdrawal relationship. Cogn. Emot. 22, 1081-1093. doi: 10.1080/0269993070162 2961

Conflict of Interest Statement: The authors declare that the research was conducted in the absence of any commercial or financial relationships that could be construed as a potential conflict of interest.

Copyright (๑) 2019 Kelley, Gallucci, Riva, Romero Lauro and Schmeichel. This is an open-access article distributed under the terms of the Creative Commons Attribution License (CC BY). The use, distribution or reproduction in other forums is permitted, provided the original author(s) and the copyright owner(s) are credited and that the original publication in this journal is cited, in accordance with accepted academic practice. No use, distribution or reproduction is permitted which does not comply with these terms. 


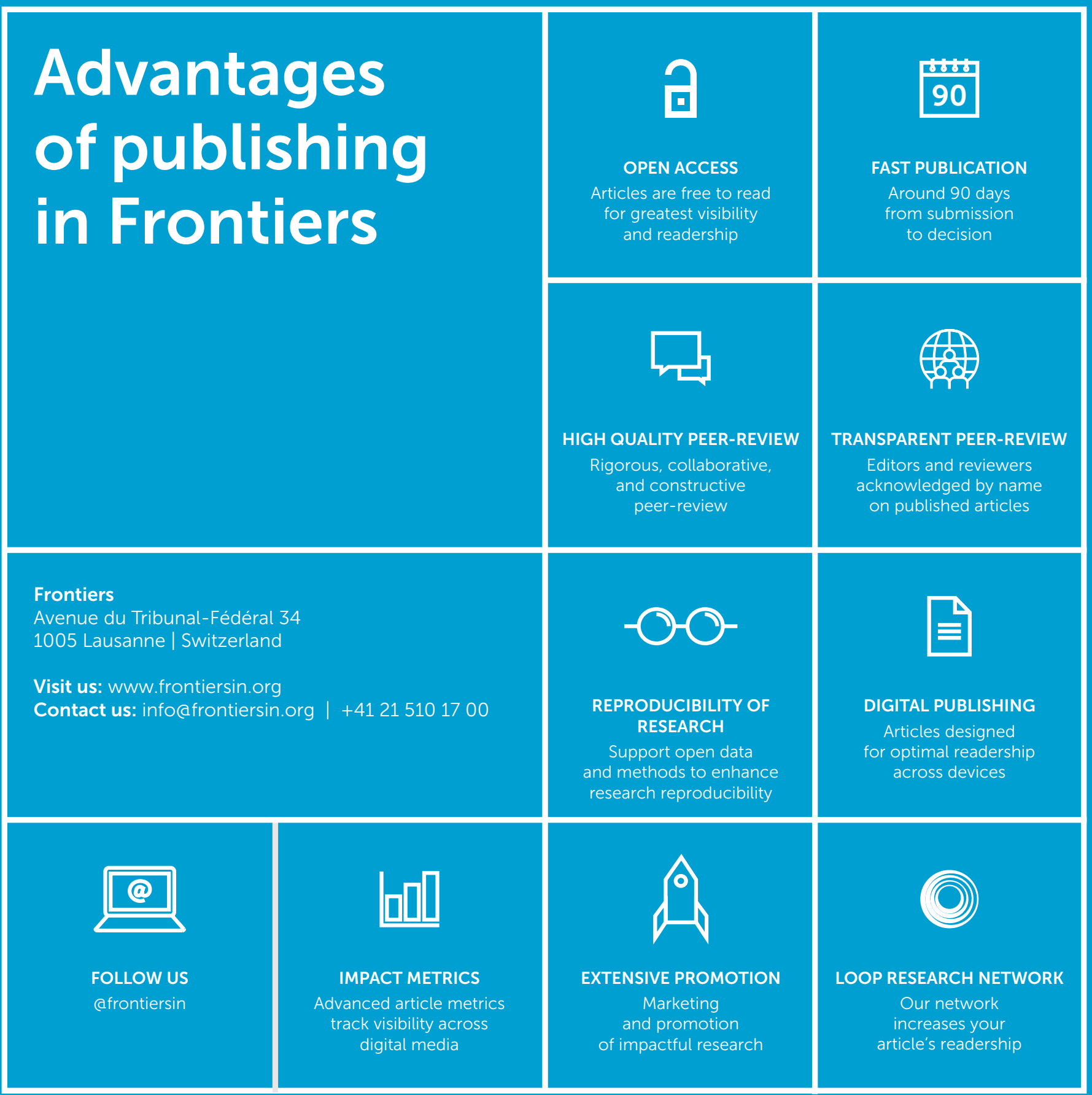

